ARCHIVES

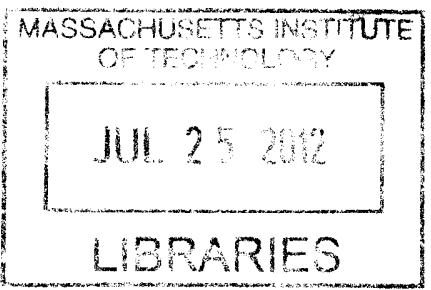

\title{
A Fission-Fusion Hybrid Reactor in Steady-State L-mode Tokamak Configuration with Natural Uranium
}

\author{
Mark Wilbert Reed \\ S.B. Physics, Massachusetts Institute of Technology, 2010 \\ Submitted in Partial Fulfillment of the Requirements \\ for the Degrees of \\ MASTER OF SCIENCE IN NUCLEAR SCIENCE AND ENGINEERING \\ and \\ BACHELOR OF SCIENCE IN NUCLEAR SCIENCE AND ENGINEERING \\ at the \\ MASSACHUSETTS INSTITUTE OF TECHNOLOGY \\ February 2011 \\ (C)2011 Massachusetts Institute of Technology \\ All rights reserved
}

Signature of Author: ...

Department of Nuclear Science and Engineering

January 10, 2011

Certified by:

Ronald R. Parker, Thesis Supervisor

Professor of Nuclear Science and Engineering

Professor of Electrical Engineering and Computer Science

Certified by: ......

Benoit Forget, Thesis Reader $\longrightarrow$

Assistant Professor of Nuclear Science and Engineering

Accepted by:

Mujid S. Kazimi, Chairman of Committee fon fraduate Students
Professor of Nuclear Science and Engineermg 


\title{
A Fission-Fusion Hybrid Reactor in Steady-State L-mode Tokamak Configuration with Natural Uranium
}

\author{
Mark Wilbert Reed \\ Massachusetts Institute of Technology
}

Submitted to the Department of Nuclear Science and Engineering on January 10, 2011 in Partial Fulfillment of the Requirements for the Degrees of Master of Science and Bachelor of Science in Nuclear Science and Engineering

\begin{abstract}
The most prevalent criticism of fission-fusion hybrids is simply that they are too exotic - that they would exacerbate the challenges of both fission and fusion. This is not really true. Intriguingly, hybrids could actually be more viable than stand-alone fusion reactors while mitigating many challenges of fission. This work develops a conceptual design for a fission-fusion hybrid reactor in steady-state L-mode tokamak configuration with a subcritical natural or depleted uranium pebble bed blanket. A liquid lithiumlead alloy breeds enough tritium to replenish that consumed by the D-T fusion reaction. Subcritical operation could obviate the most challenging fuel cycle aspects of fission. The fission blanket augments the fusion power such that the fusion core itself need not have a high power gain, thus allowing for fully non-inductive (steady-state) low confinement mode (L-mode) operation at relatively small physical dimensions.

A neutron transport Monte Carlo code models the natural uranium fission blanket. Maximizing the fission power while breeding sufficient tritium allows for the selection of an optimal set of blanket parameters, which yields a maximum prudent fission power gain of 7.7 .

A 0-D tokamak model suffices to analyze approximate tokamak operating conditions. If the definition of a "reactor" is a device with a total power gain of 40 , then this fission blanket would allow the fusion component of a hybrid reactor with the same dimensions as ITER to operate in steady-state L-mode very comfortably with a fusion power gain of 6.7 and a thermal fusion power of $2.1 \mathrm{GW}$. Taking this further can determine the approximate minimum scale for a steady-state L-mode tokamak hybrid reactor, which is a major radius of $5.2 \mathrm{~m}$ and an aspect ratio of 2.8 . This minimum scale device operates barely within the steady-state L-mode realm with a thermal fusion power of $1.7 \mathrm{GW}$.

This hybrid, with its very fast neutron spectrum, could be superior to pure fission reactors in terms of breeding fissile fuel and transmuting deleterious fission products. It could operate either as a breeder, producing fuel for pure fission reactors from natural or depleted uranium, or as a deep burner, fissioning heavy metal and transmuting waste with a cycle time of decades. Despite a plethora of potential functions, its primary
\end{abstract}


mission is deemed to be that of a deep burner producing baseload commercial power with a once-through fuel cycle. Although hybrids are often purported a priori to pose an elevated proliferation risk, this reactor breeds plutonium that could actually be more proliferation-resistant than that bred by fast reactors. Furthermore, a novel method (the "variable fixed source method") can maintain constant total hybrid power output as burnup proceeds by varying the neutron source strength.

As for engineering feasibility, basic thermal hydraulic analysis demonstrates that pressurized helium could cool the pebble bed fission blanket with a flow rate below 10 $\mathrm{m} / \mathrm{s}$. The Brayton cycle thermal efficiency is $41 \%$.

This device is dubbed the Steady-State L-Mode Non-Enriched Uranium Tokamak Hybrid (SLEUTH). The purpose of this work is not any sort of elaborate design, but rather the exploration of an idea coupled with corroborating numerical analysis. At this point in the hybrid debate, viable conceptual designs are persuasive while intricate build-ready designs are superfluous. This work conceives such a conceptual design, demonstrates its viability, and will perhaps, incidentally, spur a profusion of pro-fusion sentiment!

Thesis Supervisor: Ronald R. Parker

Title: $\quad$ Professor of Nuclear Science and Engineering

Professor of Electrical Engineering and Computer Science

Thesis Reader: Benoit Forget

Title:

Assistant Professor of Nuclear Science and Engineering 


\section{Acknowledgements}

Foremost, I must thank my thesis advisor Professor Ron Parker, who graciously agreed to advise me in this endeavor and chose to judge me based on my abilities and the quality of my work, not frivolities. Without his facilitation and fairness, I would have completed neither this thesis nor my master's degree. I would also like to thank Professor Ben Forget, who taught me neutron transport and who agreed to act as my thesis reader even though he was already exorbitantly busy. My interactions with Doctor Leslie Bromberg, Professor Michael Driscoll, and Professor Eugene Shwageraus were helpful in evaluating the feasibility of my ideas.

Professor Alan Guth, my undergraduate advisor in the Department of Physics, has been very encouraging throughout my time at MIT. Despite his brilliance and illustrious accomplishments, he remains humble and helpful in a way that is salubriously refreshing.

My office comrades Jake DeWitte, Bryan Herman, and Joe Yurko have made me feel welcome here and tolerated my eccentricities as I worked assiduously to complete nearly all of this work in less than three months. The couch in our office is devastatingly comfortable.

My family in Yakima has always been extremely supportive regardless of what choices or mistakes I made. Although there have been many talented engineers in my family, I am the first to earn an engineering degree. I have achieved what my grandfather, who became a successful mechanical engineer with only a $10^{\text {th }}$ grade education, always wished he'd had the opportunity to achieve. I have achieved what my father, who could not afford college, worked hard to provide me with the opportunity to achieve.

I would not be where I am today without good friends in Seattle, Boston, and around the world. In particular, Brian Myhre, Emily Richmond, Karlen Ruleman, and Stephanie Tharp are the kindest, highest-quality people I have ever known. Next to them, I am an abject ogre. I must also thank Tyler Smith for eleven years of friendship, for co-founding a company, and for saga-worthy expeditions in Canada, Iceland, and Japan. It's remarkable that we're still alive. Finally, I must thank Stephen James for seventeen years of friendship, for partnership in various shenanigans, and especially for instilling in me a curiosity for and drive to discover what's beyond my current sphere of knowledge, whether it be the next hill in our neighborhood or the next perplexity in my research. 
To the Cascade Mountains, whose peaks, valleys, and pristine alpine lakes have always been sources of inspiration, health, and happiness.

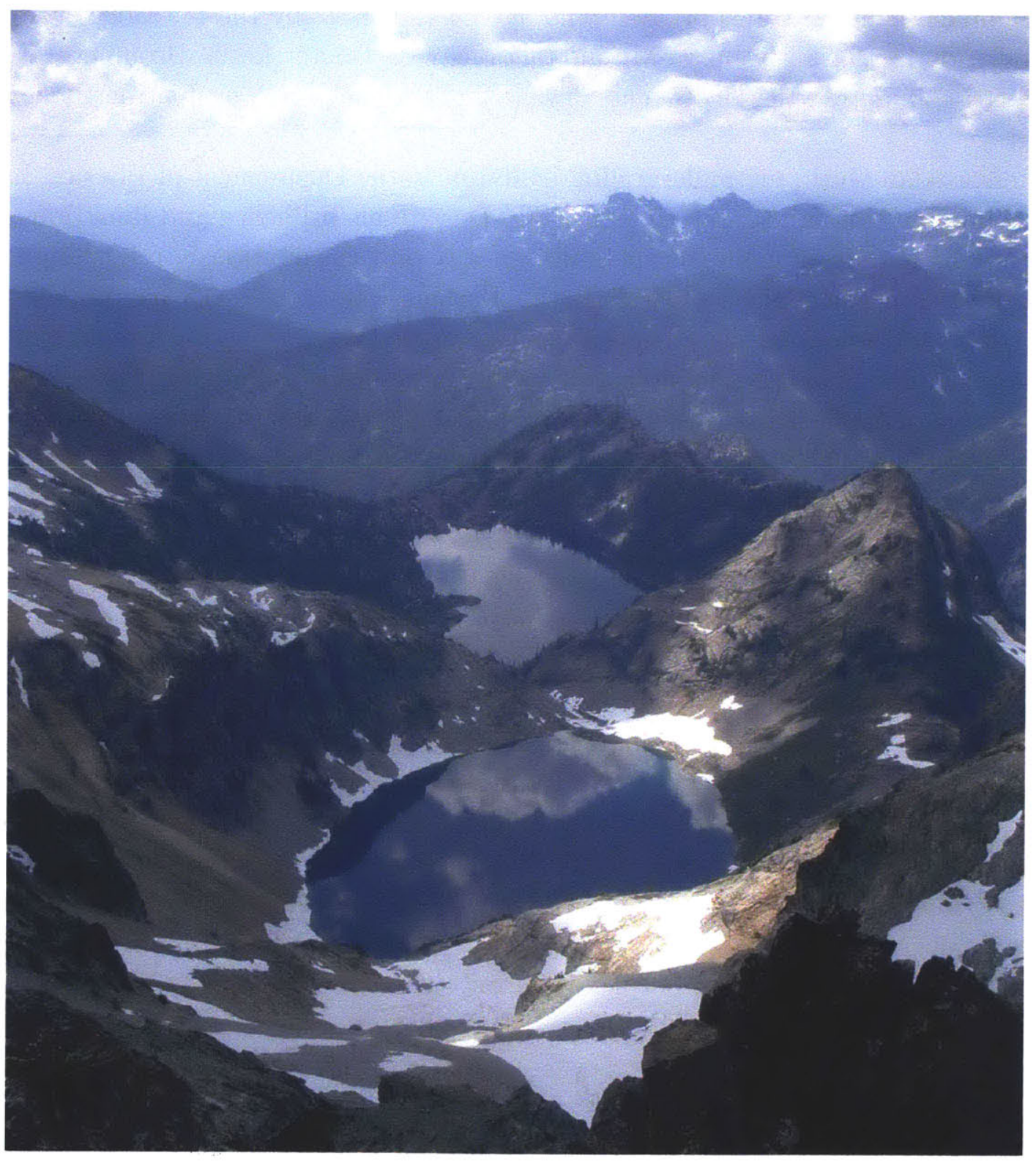




\section{Contents}

1 The Fission-Fusion Concept $\quad 17$

1.1 Hybrid History . . . . . . . . . . . . . . . . . . . . . . . . 17

1.1 .1 Nascence . . . . . . . . . . . . . . . . . . . 17

1.1 .2 Renaissance . . . . . . . . . . . . . . . . . 18

1.2 Present Limitations . . . . . . . . . . . . . . . . . . . . . . . . . . . 19

1.2.1 The Limitation of Fusion: Plasma Stability . . . . . . . . . . . . . . . 19

1.2 .2 The Limitation of Fission: Criticality . . . . . . . . . . . . . . . . . . 20

1.3 A New Conceptual Design . . . . . . . . . . . . . . . . . . . . 20

1.3.1 Geometry and Fission Power Gain . . . . . . . . . . . . . . . . . . . 21

1.3.2 Pebble Bed Blanket . . . . . . . . . . . . . . . . . . 23

1.3.3 Subcritical Operation . . . . . . . . . . . . . . . . . 23

1.3.4 Fast Spectrum . . . . . . . . . . . . . . . . . . 24

1.3.5 Natural or Depleted Uranium . . . . . . . . . . . . . . . . . . . . 24

1.3.6 Tritium Breeding . . . . . . . . . . . . . . . . . . . . 28

1.3 .7 Shielding. . . . . . . . . . . . . . . . . . 28

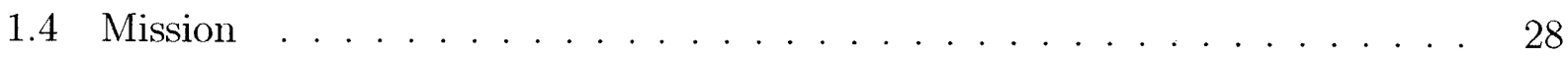

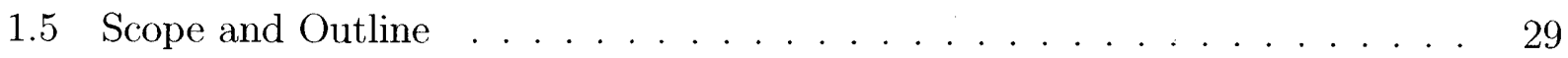

2 Fission Blanket Monte Carlo Code $\quad 30$

2.1 Path Length Sampling in Toroidal Geometry . . . . . . . . . . . . . . . . . 30

2.1.1 Flight Distance in Toroidal Geometry . . . . . . . . . . . . . . . . 30

2.1 .2 Sampling Algorithm . . . . . . . . . . . . . . . . . . . . . . . . . . . . . . . . . . 33

2.1.3 Tokamak Wall Neutron Flux . . . . . . . . . . . . . . . . . 35

2.1.4 Quartic Solution Comparison . . . . . . . . . . . . . 38

2.1.5 Cylindrical Comparison . . . . . . . . . . . . . . . 39

2.2 Monte Carlo Methodology . . . . . . . . . . . . . . . . . . . . . . 40

2.2.1 ENDF Cross-Sections . . . . . . . . . . . . . . . . . . . . . . . . . . . . . . . . . . .

2.2 .2 Elastic Scattering . . . . . . . . . . . . . . . . . . . . . . . . . . . . . . . . . 40

$2.2 .3(\mathrm{n}, \mathrm{xn})$ and $(\mathrm{n}, \alpha)$ Reactions . . . . . . . . . . . . . . . . . . . . . . . . . . . . . . 43

2.2 .4 Inelastic Scattering . . . . . . . . . . . . . . . . . 44

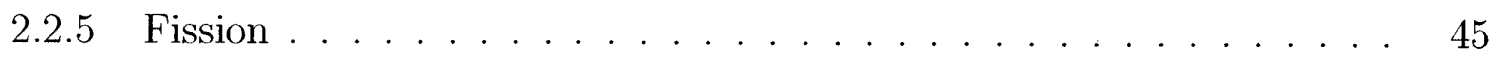

2.3 MCNP Benchmark . . . . . . . . . . . . . . . . . . . . . . 46

2.4 Pebble Homogenization . . . . . . . . . . . . . . . . . . . 48

3 Fission Blanket Analysis $\quad 66$

3.1 Blanket Parameter Analysis . . . . . . . . . . . . . . . . . . . 66

3.1.1 Uranium Layer Thickness . . . . . . . . . . . . . . . 68 
3.1 .2 Lithium Layer Thickness . . . . . . . . . . . . . . . . . 71

3.1 .3 Lithium Enrichment . . . . . . . . . . . . . . . . . 72

3.1 .4 Layer Positioning . . . . . . . . . . . . . . . . . . . . 77

$3.1 .5 \mathrm{Li}-\mathrm{Pb}$ Content . . . . . . . . . . . . . . . . . . . . . 81

3.2 The Optimal Fission Blanket . . . . . . . . . . . . . . 83

4 A Tokamak Fusion Core Model $\quad 96$

4.1 Concept and Geometry . . . . . . . . . . . . . . . . . . . 96

$4.2 \quad$ 0-D Core Model Overview . . . . . . . . . . . . . . . . . . . . . 98

4.3 0-D Core Model System Parameters . . . . . . . . . . . . . . . . . . 98

4.3.1 D-T Fusion Rate Coefficient . . . . . . . . . . . . . . . . . . 102

4.3 .2 Elongation vs. Aspect Ratio . . . . . . . . . . . . . . . 102

4.3.3 Plasma Current and Sustainment . . . . . . . . . . . . . . . . 102

4.4 L-mode and H-mode . . . . . . . . . . . . . . . . . . 105

5 Pure Fusion Core Analysis $\quad 107$

5.1 0-D Allowable Parameter Space . . . . . . . . . . . . . . . . . . 107

5.1 .1 Allowable $\left[R, q^{*}, F_{G}\right]$ Space $\ldots \ldots \ldots \ldots \ldots \ldots \ldots$

$5.1 .2 \quad$ Allowable $\left[R, q^{*}, R / a\right]$ Space $\ldots \ldots \ldots \ldots \ldots \ldots$

5.1 .3 Allowable $\left[R, q^{*}, R / a, Q\right]$ Space . . . . . . . . . . . . . . 121

5.2 Minimum Tokamak Scale Defined . . . . . . . . . . . . . . . . . 121

5.3 Technology Limits . . . . . . . . . . . . . . . . . . . . . . . . 124

5.3.1 Maximum On-Coil Magnetic Field $B_{\max } \ldots \ldots \ldots \ldots . \ldots . . \ldots 124$

5.3 .2 Blanket Fusion Power Density $P_{F} / A_{S} \ldots \ldots \ldots \ldots 127$

5.4 Aspect Ratio $R / a$ Considerations . . . . . . . . . . . . . . . . . . . . . . . . 129

5.5 A "Reactor" Defined . . . . . . . . . . . . . . . . . . . . . . 132

6 Coupled Fission-Fusion Analysis $\quad 135$

6.1 Effect of Tokamak Geometry on Fission Blanket . . . . . . . . . . . . 135

6.2 Solenoid Size vs. Blanket Thickness . . . . . . . . . . . . . . . . . . 135

6.3 Steady-State L-mode ITER-PBR . . . . . . . . . . . . . . . . . . 137

6.4 Minimum Scale Steady-State L-mode Fission-Fusion Hybrid . . . . . . . . 143

6.4 .1 1-D Profiles . . . . . . . . . . . . . . . . . . . . 147

6.4 .2 Radiative Power . . . . . . . . . . . . . . . . . . . 155

6.4 .3 Operating Point Access . . . . . . . . . . . . . . . . 156

6.5 Implications . . . . . . . . . . . . . . . . . 158 
7 Burnup and Fuel Cycle $\quad 159$

7.1 Subcritical Burnup Implementation . . . . . . . . . . . . . . . . . . . 159

7.2 Maintaining Constant Hybrid Power . . . . . . . . . . . . . . . . 160

7.3 Breeding . . . . . . . . . . . . . . . . . . . . . . . . . . . . . . . . . . . . . . . . . . . . .

7.4 Viability of a Thorium Fuel Cycle . . . . . . . . . . . . . . . . 173

7.5 Non-Proliferation . . . . . . . . . . . . . . . . . . . 176

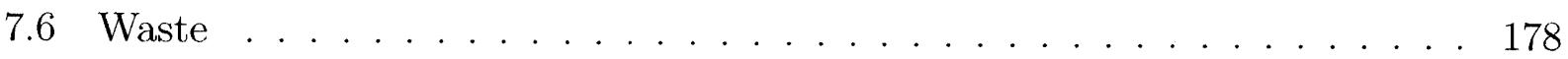

7.7 Cycle Time . . . . . . . . . . . . . . . . . . . . . 180

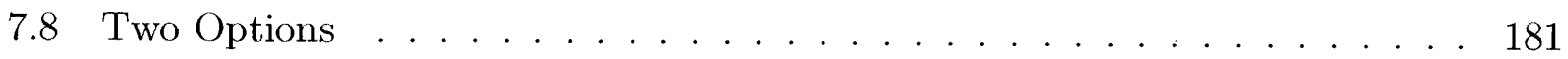

8 Pebble Structure, Thermal Hydraulics, and Safety 182

8.1 Pebble Structure . . . . . . . . . . . . . . . . . . . . . . . . 182

8.2 Thermal Hydraulic Analysis . . . . . . . . . . . . . . . . . . . . 185

8.2.1 Coolant Flow Rate and Pressure Drop . . . . . . . . . . . . . . 187

8.2.2 Heat Transfer and Temperature . . . . . . . . . . . . . . . . . . 194

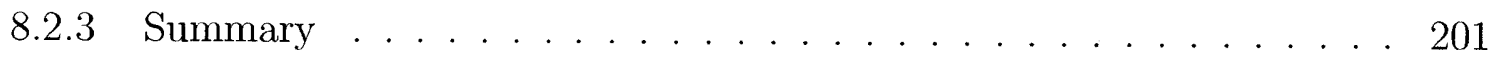

8.3 Brayton Power Cycle and Electric Power . . . . . . . . . . . . . . . . . . 202

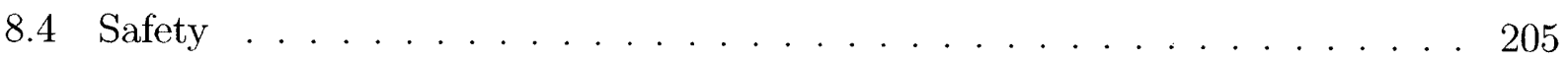

9 Ramifications $\quad 206$

9.1 Comparison to Other Studies . . . . . . . . . . . . . . . . . . . . . . 206

9.1.1 Tokamak Pebble Bed Hybrids (ITER-PBR) . . . . . . . . . . . . . . 206

9.1 .2 Other Tokamak Hybrids (SABR) . . . . . . . . . . . . . . . . 209

9.1.3 Inertial Confinement Hybrids (LIFE) . . . . . . . . . . . . . . . 211

9.2 The Hybrid Debate . . . . . . . . . . . . . . . . . . . . . . . . 213

9.3 Overarching Conclusions . . . . . . . . . . . . . . . . . . 213

$\begin{array}{ll}\text { A Fusion Model } & 219\end{array}$

A.1 0 -D Core Model . . . . . . . . . . . . . . . . . . . . . . . . . 219

A.2 1-D Density, Temperature, and Power Profiles . . . . . . . . . . . . . . . . 222

A.3 1-D Current Profiles . . . . . . . . . . . . . . . . . . . . . 224

A.4 1-D q Profile . . . . . . . . . . . . . . . . . . . . . 226

A.5 Auxiliary Power for Operating Point Access . . . . . . . . . . . . . . . . . 228

B Toroidal Monte Carlo Code $\quad 232$

C Cylindrical Monte Carlo Code $\quad 265$ 
D Plasma Surface Neutron Flux Code $\quad 269$

D.1 Toroidal Flux Monte Carlo . . . . . . . . . . . . . . . . . . . . . 269

D.2 Toroidal Volume - Spiric Sections . . . . . . . . . . . . . . . . 274

$\begin{array}{lll}\text { E } & k_{\infty} \text { Monte Carlo Code } & 275\end{array}$

$\begin{array}{lrl}\text { F } & \text { Elastic Scattering } & 285\end{array}$

G Thermal Hydraulics Model $\quad 286$

$\begin{array}{ll}\text { H Conversion Ratio Model . } & 289\end{array}$

I MCNP Input Files 293

I.1 Full Toroidal Hybrid Blanket . . . . . . . . . . . . . . . . . . . . . . 293

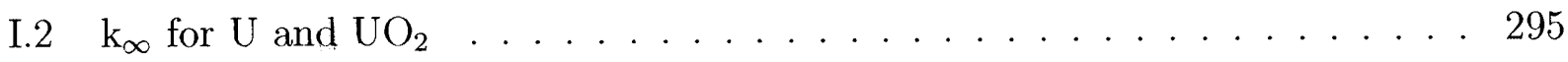

I.3 Infinite Array of $\mathrm{UO}_{2}$ Pebbles in $\mathrm{H}_{2} \mathrm{O}$ Pool (Simple Cubic) $\ldots \ldots \ldots$. . . . 296

I.4 Typical Fast Reactor Spectrum _. . . . . . . . . . . . . . . . . . . 297 


\section{List of Tables}

3.1 Optimal Blanket Parameters . . . . . . . . . . . . . . . . . . . . . . . 83

6.1 Steady-State L-mode ITER-PBR Operating Parameters . . . . . . . . . . . . 142

6.2 Minimum Scale Steady-State L-mode Operating Parameters . . . . . . . . 146

7.1 Fissile Nuclide Conversion Ratios . . . . . . . . . . . . . . . . . . . . . 172

7.2 Comparison of Thorium and Uranium Breeding Ratios . . . . . . . . . 175

$7.3{ }^{239} \mathrm{Pu}$ One-Group Cross-Sections (barns) . . . . . . . . . . . . . . . 177

7.4 Fission Product Equilibrium Concentrations . . . . . . . . . . . . . . . . 179

8.1 Pebble Bed Blanket Thermal Hydraulic Parameters . . . . . . . . . . . . . . 201

8.2 Ideal Brayton Cycle Parameters . . . . . . . . . . . . . . . . . . . 203

9.1 Comparison of Graphite Matrix Pebbles to $\mathrm{UO}_{2}$ Pebbles . . . . . . . . . 208 


\section{List of Figures}

1.1 A fission blanket coats a tokamak. . . . . . . . . . . . . . . 21

1.2 The total ${ }^{238} \mathrm{U}$ cross-section showing constituent parts as a function of energy. 25

1.3 Normalized constituent parts of the total ${ }^{238} \mathrm{U}$ cross-section as a function of energy. . . . . . . . . . . . . . . . . . 26

1.4 Normalized constituent parts of the total ${ }^{235} \mathrm{U}$ cross-section as a function of energy. . . . . . . . . . . . . . . . . . . . 27

2.1 Simple elongated toroidal geometry. . . . . . . . . . . . . 31

2.2 A toroidal cross-section of a torus showing a neutron's initial position $\left(x_{0}, y_{0}, z_{0}\right)$ and direction $\left(\mu_{x}, \mu_{y}, \mu_{z}\right) \ldots \ldots \ldots \ldots \ldots \ldots \ldots \ldots \ldots \ldots \ldots$

2.3 A poloidal cross-section of concentric tori showing neutron initial positions, directions, and intersection points with the tori. . . . . . . . 36

2.4 Scalar neutron flux at the plasma surface as a function of poloidal angle $\Theta$. . 49

2.5 The ratio of scalar neutron flux at a tokamak's outermost and innermost surface points as a function of aspect ratio $R / a \ldots \ldots \ldots \ldots$

2.6 The ratio of scalar neutron flux at a tokamak's outermost and innermost surface points as a function of elongation $\kappa \ldots \ldots \ldots \ldots$

2.7 The poloidal angle $\Theta$ of maximum scalar neutron flux as a function of tokamak elongation $\kappa, \ldots \ldots \ldots \ldots \ldots \ldots \ldots \ldots \ldots \ldots \ldots$

2.8 The angular neutron flux distribution at the outermost point on the plasma

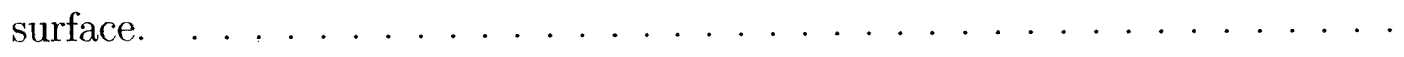

2.9 "Visible" regions of the plasma volume from which D-T fusion neutrons can impinge on a given point on the tokamak surface. . . . . . . . .

2.10 The angular neutron flux distribution at the innermost point on the plasma

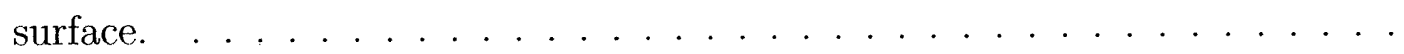

2.11 The angular neutron flux distribution at the topmost point on the plasma

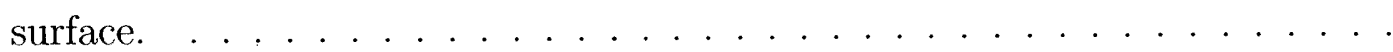

2.12 Five unique spiric sections of a torus. . . . . . . . . . . .

2.13 A CPU runtime analysis of our tokamak surface neutron flux Monte Carlo code comparing the MATLAB function roots to a direct calculation of Ferrari's method. . . . . . . . . . . . . . . . .

2.14 A CPU runtime analysis of our entire hybrid Monte Carlo simulation comparing the MATLAB function roots to a direct calculation of Ferrari's method.

2.15 Scalar neutron flux at the plasma edge (an elliptic cylindrical surface) as a

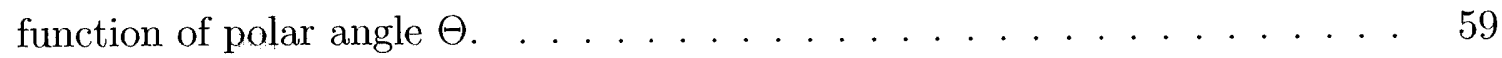

2.16 Elastic scattering $P\left(\mu_{L}\right)$ for ${ }^{1} \mathrm{H},{ }^{4} \mathrm{He},{ }^{16} \mathrm{O}$, and ${ }^{56} \mathrm{Fe} \ldots \ldots \ldots \ldots .60$

2.17 The analytical solution for $P\left(\mu_{L}\right)$ as a smooth surface from $A=1$ and 10. . 61

$2.18 P\left(\mu_{L}=1\right) / P\left(\mu_{L}=-1\right)=\alpha$ as a function of $A$ from $A=4$ to $200 . \ldots 62$ 
2.19 A comparison of our Monte Carlo model to MCNP with $k_{\infty}$ as a function of uranium enrichment for $\mathrm{UO}_{2}$ and pure $\mathrm{U}$ metal. . . . . . . . . . . . . .

2.20 A comparison of our full hybrid Monte Carlo model to MCNP with the initial neutron multiplication $k_{0}$ and the asymptotic neutron multiplication $k$ as a function of uranium pebble layer thickness. . . . . . . . . . . . . . .

$2.21 k_{\infty}$ for an infinite standard cubic array of $\mathrm{UO}_{2}$ pebbles in an infinite $\mathrm{H}_{2} \mathrm{O}$ pool as a function of pebble size.

3.1 A basic schematic of a poloidal cross-section of our neutron transport hybrid model.

3.2 Fissions and bred tritons per fusion-born neutron as a function of uranium pebble layer thickness. . . . . . . . . . . . . . . . . . .

3.3 The fission multiplication factors $k_{0}$ (first generation) and $k$ (successive generations) as a function of uranium pebble layer thickness. . . . . . . . . . .

3.4 Fissions and bred tritons per fusion-born neutron as a function of lithium layer thickness. . . . . . . . . . . . . . . . . . . . 71

3.5 The total ${ }^{6} \mathrm{Li}$ cross-section showing constituent parts as a function of energy. $\quad 73$

3.6 Normalized constituent parts of the total ${ }^{6} \mathrm{Li}$ cross-section as a function of energy. . . . . . . . . . . . . . . . . . . . 74

3.7 Normalized constituent parts of the total ${ }^{7} \mathrm{Li}$ cross-section as a function of energy. . . . . . . . . . . . . . . . . . . 75

3.8 Fissions and bred tritons per fusion-born neutron as a function of ${ }^{6} \mathrm{Li}$ enrichment. 76

3.9 Fissions and bred tritons per fusion-born neutron as a function of ${ }^{6} \mathrm{Li}$ enrichment. 78

3.10 Fissions and bred tritons per fusion-born neutron as a function of uranium pebble layer thickness. . . . . . . . . . . . . . . . . . . . . . . . 79

3.11 Fissions and bred tritons per fusion-born neutron as a function of lithium layer thickness. . . . . . . . . . . . . . . . . . 80

3.12 Fissions and bred tritons per fusion-born neutron as a function of lithium atomic fraction in Li-Pb. . . . . . . . . . . . . . . . . . . . . 82

3.13 The number of neutrons in each generation throughout the hybrid system. $\quad 85$

3.14 The composition of the total hybrid power from various sources. . . . . . . . 87

3.15 The fate of fusion-born (1st generation) neutrons. . . . . . . . . . . . . 88

3.16 The total neutron energy spectrum in the uranium pebble layer. . . . . . . . 89

3.17 The total neutron energy spectrum and the fission-inducing neutron energy spectrum in the uranium pebble layer. . . . . . . . . . . . . . . . 90

3.18 The total neutron energy spectrum in the Li-Pb layer. . . . . . . . . . . . . . 91

3.19 The total neutron energy spectrum and the tritium-breeding neutron energy spectrum in the $\mathrm{Li}-\mathrm{Pb}$ layer. . . . . . . . . . . . . . . . . . . . . 92 
3.20 A distribution of the number of collisions a fusion-born neutron undergoes before breeding tritium. . . . . . . . . . . .

3.21 The total neutron flux in the uranium pebble layer as a function of poloidal angle. . . . . . . . . . . . . . . . . . . . . 94

3.22 The total neutron flux in the uranium pebble layer as a function of radius. . 95

4.1 A simple elliptical torus model for tokamak geometry. . . . . . . . . . . . 97

4.2 The D-T reactivity coefficient $\langle\sigma v\rangle$ as a function of temperature $T$. . . . 103

4.3 The 1989 ITER scaling for $H$ vs. radiative power fraction. . . . . . . . . 105

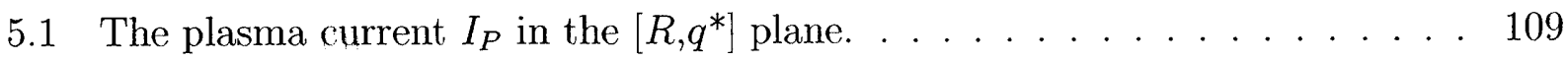

5.2 The total ion density $n$ in the $\left[R, q^{*}\right]$ plane. . . . . . . . . . . 110

5.3 The temperature $T$ in the $\left[R, q^{*}\right]$ plane revealing the unphysical reactivity region. 111

$5.4 \quad \beta_{N}$ in the $\left[R, q^{*}\right]$ plane. . . . . . . . . . . . . . . . . . . . 112

$5.5 \quad H$ in the $\left[R, q^{*}\right]$ plane. . . . . . . . . . . . . . . . . 113

5.6 Areas in the $\left[R, q^{*}\right]$ plane that satisfy and do not satisfy the parameter constraints. . . . . . . . . . . . . . . . . . 115

5.7 Areas in the $\left[R, F_{G}\right]$ plane that satisfy and do not satisfy the parameter constraints. . . . . . . . . . . . . . . . . 116

5.8 The maximum value of $q^{*}$ (at whichever $R$ that maximum occurs) as a function

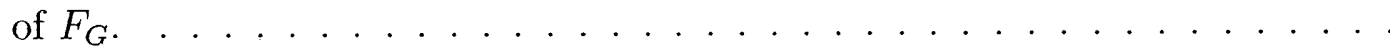

5.9 Areas in the $\left[R, q^{*}\right]$ plane that satisfy the parameter constraints for $R / a$ values of $2.5,3$, and $3.5 \ldots \ldots \ldots \ldots \ldots \ldots \ldots$

5.10 The maximum value of $q^{*}$ (at whichever $R$ that maximum occurs) as a function

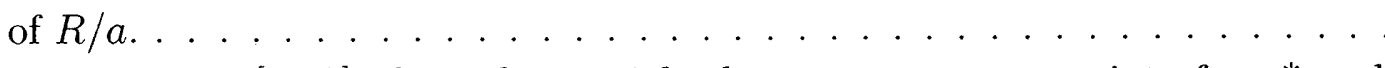

5.11 Areas in the $[R, Q]$ plane that satisfy the parameter constraints for $q^{*}$ and

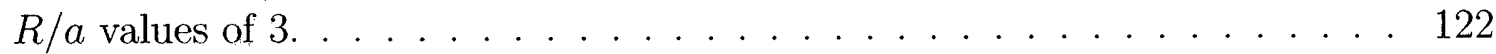

$5.12 f_{N I}$ for areas in the $\left[R, q^{*}\right]$ plane that satisfy the parameter constraints. . . 123

5.13 Areas in the $\left[R, q^{*}\right]$ plane that satisfy the parameter constraints for maximum on-coil magnetic field $B_{\max }$ values of $13,14.5$, and $16 \mathrm{~T} \ldots \ldots \ldots$

5.14 The minimum $R$ in the $\left[R, q^{*}\right]$ plane for fully non-inductive L-mode operation

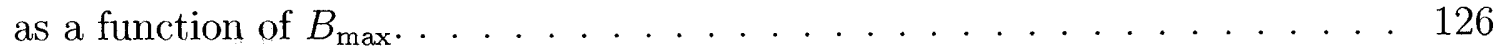

5.15 Areas in the $\left[R, q^{*}\right]$ plane that satisfy the parameter constraints for fusion power per surface area $P_{F} / A_{S}$ values of 5 and $7 \mathrm{MW} / \mathrm{m}^{2} \ldots \ldots \ldots 127$

5.16 The minimum $R$ in the $\left[R, q^{*}\right]$ plane for fully non-inductive L-mode operation

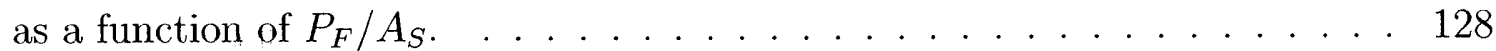

5.17 Areas in the $\left[R, q^{*}\right]$ plane that satisfy the parameter constraints for aspect ratio $R / a$ values of $2.3,2.6$, and 2.9 .

5.18 The minimum $R$ in the $\left[R, q^{*}\right]$ plane for fully non-inductive L-mode operation as a function of $R / a$. 
5.19 The maximum $Q$ in the $\left[Q, q^{*}\right]$ plane for fully non-inductive L-mode operation as a function of $R$

5.20 The maximum $Q$ in the $\left[Q, q^{*}\right]$ plane for fully non-inductive L-mode operation as a function of $R$ for the ITER aspect ratio of 3.1.

6.1 The fission and tritium breeding ratios as a function of elongation $\kappa$ for ITER tokamak dimensions and the optimal blanket thicknesses. Only tritium breeding is sensitive to $\kappa \ldots \ldots \ldots \ldots \ldots \ldots \ldots$

6.2 A "phase diagram" over $\left[R, q^{*}\right]$ showing L-mode, H-mode, and areas forbidden by reactivity and the Troyon limit.

6.3 A "phase diagram" over $\left[R, q^{*}\right]$ showing L-mode, H-mode, and areas forbidden by reactivity and the Troyon limit.

6.4 A "phase diagram" over $\left[R, q^{*}\right]$ showing L-mode, H-mode, and areas forbidden by reactivity and the Troyon limit.

6.5 A "phase diagram" over $\left[R, q^{*}\right]$ showing L-mode, H-mode, and areas forbidden by reactivity and the Troyon limit.

6.6 Minimum major radius $R$ (for steady-state L-mode) and flux ratio $\Phi_{\text {sol }} / \Phi_{P}$ as a function of aspect ratio $R / a$ at $q *=3.0 \ldots \ldots \ldots \ldots \ldots$

6.7 A "phase diagram" over $\left[R, q^{*}\right]$ showing L-mode, H-mode, and areas forbidden by reactivity and the Troyon limit. . . . . . . . . . . . . . . . 145

6.8 Ion density $n$ as a function of minor radius $r$ with an offset fraction of 0.25 . 147

6.9 Plasma temperature $T$ as a function of minor radius $r$ with an edge temperature of $0.15 \mathrm{keV} \ldots \ldots \ldots \ldots$. . . . . . . . . . . . . . . 148

6.10 The D-T fusion reactivity rate coefficient $\langle\sigma v\rangle$ as a function of minor radius $r .149$

6.11 Fusion power $P_{F}$ density as a function of minor radius $r \ldots \ldots \ldots 150$

6.12 Bootstrap current density $I_{\text {boot }}$ as a function of minor radius $r \ldots \ldots 152$

6.13 The total current density $I_{P}$ (with constituent parts $I_{C D}$ and $I_{\text {boot }}$ ) as a function of minor radius $r \ldots \ldots \ldots \ldots \ldots \ldots \ldots \ldots$

6.14 The safety factor $q$ profile as a function of minor radius $r$. . . . . . 154

6.15 Auxiliary power $P_{\text {aux }}$ contours as a function of density $n$ and temperature $T$ for the minimum scale reactor. . . . . . . . . . . . . . . 157

7.1 Fractional share of neutrons from each generation in the entire hybrid system. 163

7.2 Causation relationships between basic core quantities in critical and subcritical systems. . . . . . . . . . . . . . . . . . . . . . . 164

7.3 The ${ }^{238} \mathrm{U}$ transmutation-decay chain. . . . . . . . . . . . . . . . 165

7.4 Monoenergetic ${ }^{239} \mathrm{Pu}$ conversion ratio as a function of time for $5 \%$ uranium enrichment. . . . . . . . . . . . . . . . . . . . . 167

7.5 Monoenergetic ${ }^{239} \mathrm{Pu}$ conversion ratio as a function of energy at $5 \%$ enrichment. 168

7.6 Monoenergetic conversion ratios for ${ }^{239} \mathrm{Pu},{ }^{237} \mathrm{~Np}$, and ${ }^{233} \mathrm{U} \ldots \ldots . . . . .170$ 
7.7 Our fission-fusion hybrid spectrum overlaid on typical spectra for fast and thermal reactors. . . . . . . . . . . . . . . . . . . 171

7.8 Effective ${ }^{239} \mathrm{Pu}$ and ${ }^{233} \mathrm{U}$ breeding cross-sections for uranium and thorium fuel, respectively. . . . . . . . . . . . . . . . . . . . . . 174

8.1 Pink wiffle balls. . . . . . . . . . . . . . . . . . . . . . . . . 184

8.2 A basic schematic of the hybrid concept showing helium coolant flow paths. . 186

8.3 The ratio of the helium pressure drop $\Delta P$ to the ambient pressure $P_{0}$ as a function of $P_{0} \ldots \ldots \ldots \ldots \ldots \ldots \ldots \ldots \ldots \ldots$

8.4 The ratio of the helium pressure drop $\Delta P$ to the ambient pressure $P_{0}$ as a function of fuel pebble diameter. . . . . . . . . . . . . . . . 190

8.5 The Reynolds number as a function of pebble size. . . . . . . . . . . . . . 191

8.6 The helium coolant velocity as a function of its temperature increase through the core. . . . . . . . . . . . . . . . . . . . . . 192

8.7 The helium coolant velocity as a function of ambient pressure. . . . . . . . 193

8.8 A phase diagram for $\mathrm{Pb}$ content in Li. . . . . . . . . . . . . . 195

8.9 $T_{\mathrm{Li}}(r)$ near the coolant outlet for zero heat conduction at $r=a_{4}$ and for equal heat conduction at $r=a_{3}$ and $a_{4} \ldots \ldots \ldots \ldots \ldots \ldots \ldots$

8.10 Temperature extrema as functions of fuel pebble diameter. . . . . . . . . . 199

8.11 Temperature extrema as functions of helium temperature gain. . . . . . . . . 200

8.12 A generic Brayton power cycle with real components and duct pressure losses. 204

9.1 A schematic of the Subcritical Advanced Burner Reactor (SABR) conceived at the Georgia Institute of Technology. . . . . . . . . . . . . . . 210

9.2 A schematic of the Laser Inertial Confinement Fusion-Fission Energy (LIFE) Reactor conceived at Lawrence Livermore National Laboratory (LLNL). . . . 212 


\section{The Fission-Fusion Concept}

Fusion boasts a neutron plethora; fission copes with a neutron dearth. Fission requires neutrons; fusion produces neutrons. This is why people talk about fission-fusion hybrids: they couple a deficit with a surplus. D-T fusion reactions produce $14 \mathrm{MeV}$ neutrons that serve no purpose other than to breed tritium from lithium (through a primarily thermal reaction!). These uncommonly-high-energy neutrons are essentially wasted. On the other hand, fission reactions require neutrons and are limited completely and utterly by a delicate neutron balance. So there is clearly some mutual benefit to exploit from these two branches of nuclear engineering - the challenge is how to optimally configure the fission and fusion components.

The geometry of a fission-fusion hybrid is usually constrained by the fusion component, as the magnetic topology. necessary to confine the plasma overrides any geometric constraints in fission. Thus, the fission component conforms to the geometry of the fusion component in such a way as to maximize the neutron fluence it intercepts. In the case of tokamaks, the fission component is usually some sort of "blanket" coating the outer surface of the toroid.

It is essential to understand that the interaction between fusion and fission components is not two-way but one-way. Fusion drives, fission. Fusion produces neutrons, which drive fission, while fission produces nothing that influences fusion. The procession of burnup within fission component will not alter the fusion reaction, but any perturbation of the fusion reaction will have a direct and immediate affect on the fission reaction. Thus, the flow of information is exclusively from fusion to fission. Fusion is the master, fission the slave.

\subsection{Hybrid History}

This is not a new idea. Scientists began pondering hybrids little more than ten years after they first achieved fission beneath those fabled bleachers in Chicago. To date, the only fission-fusion hybrid actually constructed is the hydrogen bomb, first tested in 1952. Less excitingly, the history of peaceable fission-fusion hybrids has consisted entirely of design proposals. Here we will briefly outline some of the history.

\subsubsection{Nascence}

Lawrence M. Lidsky best chronicles the early evolution of the hybrid concept from its inception in the early 1950's until 1975 [34]. The concept of a power-multiplying blanket surrounding a fusion reactor first arose from attempts to breed sufficient tritium to replenish that consumed by D-T fusion. From this, the natural train of thought was to consider what else such a marvelous $14 \mathrm{MeV}$ neutron source could accomplish. People soon realized that 
fissionable material in the blanket would have the effect of both multiplying and slowing the neutrons in order to facilitate tritium breeding from ${ }^{6} \mathrm{Li}$ (which occurs primarily at thermal energies). Most of these very early studies proposed depleted uranium, which is ideally suited for (very) fast fission. In 1955, J. D. Lawson published a report claiming that fast fission of a ${ }^{238} \mathrm{U}$ blanket could augment a tokamak's power approximately fivefold.

Subsequently, for the remainder of the 1950's and throughout the 1960's, a host of elaborate designs burst onto the scene. There were proposals for aqueous liquid fuel $\left(\mathrm{UO}_{2}-\mathrm{SO}_{4}\right)$ circulating annularly about a stellarator. There were proposals for $\mathrm{UF}_{4}$ salt doubling as a coolant and a power-multiplier. There were proposals for thorium blankets doubling as a breeders and power-multipliers. There were proposals for breeding blankets cooled with lithium metal. There were proposals for graphite-moderated thermal blankets intended primarily for breeding. Some designs in this litany were clearly more practical than others. Many ignored such crucial considerations as corrosion and fission product buildup. See Lidsky's 1975 review paper for details and references for all of these [34].

Lidsky classifies fission-fusion systems into three categories: hybrid, symbiotic, and augean. All three of these contain a fusion reactor and at least some surrounding fissionable material. Hybrid systems contain fissile or fissionable material and produce power. Symbiotic systems contain fissionable material and utilize the fusion neutron source to breed fissile material for pure fission reactors. Augean systems contain spent nuclear fuel and dispose of waste through the transmutation of fission products or the fission of actinides. These three categories overlap, as any fission-fusion system will accomplish each to at least some degree. However, each fission-fusion system will likely tout only one of these as its primary mission.

\subsubsection{Renaissance}

The eminent physicist Hans Bethe became interested in fission-fusion hybrids and continued to advocate them until the end of the 1970's [40]. Subsequently, enthusiasm for hybrids waned. Surprisingly, it was their least-touted augean mission of that revived them. Beginning in the late 1990's, woes of the Yucca Mountain political stagnation spurred renewed interest in fast neutron sources to transmute high-level waste. However, this renaissance has blossomed into studies with breeding or power-producing missions as well. At the Georgia Institute of Technology, Professor Weston Stacey has led a series of hybrid design projects over the past ten years culminating in the Subcritical Advanced Burner Reactor (SABR) [32]. Here at the MIT Plasma Science and Fusion Center, Vincent Tang wrote a 2002 master's thesis under the auspices of Professor Ronald Parker analyzing a pebble bed blanket for ITER [22] and went as far as to quantify temperature transients in loss of coolant accidents (LOCAs) [44]. He also investigated the viability of thorium tokamak blankets [39]. The University of Illinois has also looked into the thorium blanket idea [41]. Beyond magnetic confinement, Lawrence Livermore National Laboratory (LLNL) and the University of Cal- 
ifornia at Berkeley have conceived the Laser Inertial Confinement Fusion-Fission Energy (LIFE) Reactor [31]. At the conclusion of this work, we will compare our conceptual design to those proposed by Stacey, Tang, and LLNL.

Most recently, MIT Professors Jeffrey Freidberg and Andrew Kadak broached the hybrid topic with a 2009 article in Nature Physics [46]. They concluded that the most effective short-term (25-30 years) mission of hybrids is to transmute waste. The most effective longterm (50-100 years) mission is to breed fissile fuel for pure fission reactors as natural uranium reserves begin to run low.

A few months later, Freidberg chaired a conference entitled "Research Needs for FusionFission Hybrid Systems" in Gaithersburg, Maryland [21]. This produced a comprehensive report on the current status of hybrid research and debate. This report identifies three potential hybrids missions: energy production, fuel supply, and waste management. Note that these correspond precisely to Lidsky's prescient classification of hybrid, symbiotic, and augean systems.

\subsection{Present Limitations}

The fission-fusion hybrid is a plausible solution for an existing set of problems, not (as some would claim) a rogue solution that begs for a problem. Here we will outline the limitations of both fusion and fission and articulate how hybrids could overcome or at least mitigate them.

\subsubsection{The Limitation of Fusion: Plasma Stability}

Putting things together is harder than tearing things apart. To fission heavy atoms, the atoms need only be stationary neutron targets. To fuse light atoms, the atoms themselves must be in high-energy motion. This requires plasma, which we must confine. The great challenge of fusion is simply to confine the plasma such that it is stable.

We will focus on magnetic confinement in the tokamak configuration. Tokamaks have two distinct "modes" of operation: low confinement mode (L-mode) and high confinement mode (H-mode). We will expound on these in Section 4.4, but for now we will state that H-mode yields higher power densities while L-mode yields lower power densities. Unfortunately, $\mathrm{H}$ mode is also vulnerable to cantankerous instabilities called edge-localized modes (ELMs) near the plasma "edge". These ELMs are the bane of current fusion research. Operating in L-mode would circumvent ELMs, but L-mode does not allow for large enough power gain to constitute a reactor [7].

However, the addition of a fission blanket would multiply the fusion power such that the hybrid system could operate in L-mode while still achieving a sufficiently high power gain. We will show this with rudimentary physics in Section 1.3.1 and far more rigorously with 
our 0-D tokamak model in Sections 6.3 and 6.4. We certainly do not claim that L-mode is any sort of panacea for fusion, but we do contend that it would ease certain constraints and thus enhance the viability of fusion.

\subsubsection{The Limitation of Fission: Criticality}

This subtitle might seem strange to some readers. Criticality is not a limitation of fission - it is precisely what makes fission possible! This is true if we take the narrow-minded approach of considering only pure fission systems with no external neutron sources. In those systems, criticality is clearly a constraint but not really a limitation. However, if we think outside the box and take on a broader perspective of all possible fission systems (critical and subcritical), we see that criticality is indeed a severe limitation on what fission can accomplish. In a pure fission reactor, criticality dictates nearly everything - enrichment level, flux shape, moderator choice, reactivity control systems, burnup level, fuel cycle, and waste composition. Subcritical fission obviates all of these constraints.

Critics often claim that subcritical operation has no real advantage over critical operation in terms of power production, because criticality accidents are not a major concern for pure fission reactors. Light water reactors (LWRs) have negative fuel and coolant temperature coefficients of reactivity. Most fast reactors have very small positive coolant temperature coefficients but preponderantly negative fuel temperature coefficients. Loss of coolant accidents (LOCAs) are much more ominous. This is all very true, and we accept that subcritical operation offers only marginal safety improvements (although it does completely eliminate the complexity and cost associated with reactivity control). However, subcritical operation is not preferable due to safety - rather, it is preferable due to the numerous constraints it lifts.

With subcritical operation, neither enrichment nor moderation is necessary. Only materials limit burnup and cycle time. As we will see in Section 7.3, it opens up the fuel cycle in such a way as to allow for breeding tremendous quantities of fissile fuel.

Subcritical operation also facilitates transmutation of nuclear waste. The fusion neutron source can bombard mixtures of actinides and fission products that are very far below criticality. Additionally, a fission-fusion hybrid spectrum, which contains $14 \mathrm{MeV}$ neutrons, would transmute fission products and fission actinides more effectively than a typical fast reactor spectrum. We will show this quantitatively in Section 7.6.

\subsection{A New Conceptual Design}

We have argued that fission-fusion hybrids could be mutually beneficial for both fission and fusion, that they could obviate significant constraints and challenges for both. We now propose a new conceptual fission-fusion hybrid design that takes advantage of these mutual 
benefits. The primary mission of this design is to produce power, although we will also explore its potential to breed fissile fuel and transmute waste. Here we will outline its distinguishing features.

\subsubsection{Geometry and Fission Power Gain}

Tokamaks are the most advanced, well-understood fusion devices. Although we will not assume any specific tokamak design in this thesis, we will at least need to assume that the fusion component is in fact some sort of tokamak. All the more specific properties of the tokamak (size, geometry, magnetic field strength, etc.) will be free parameters. The fission blanket will coat the tokamak surface. Figure 1.1 shows this very basic geometry.

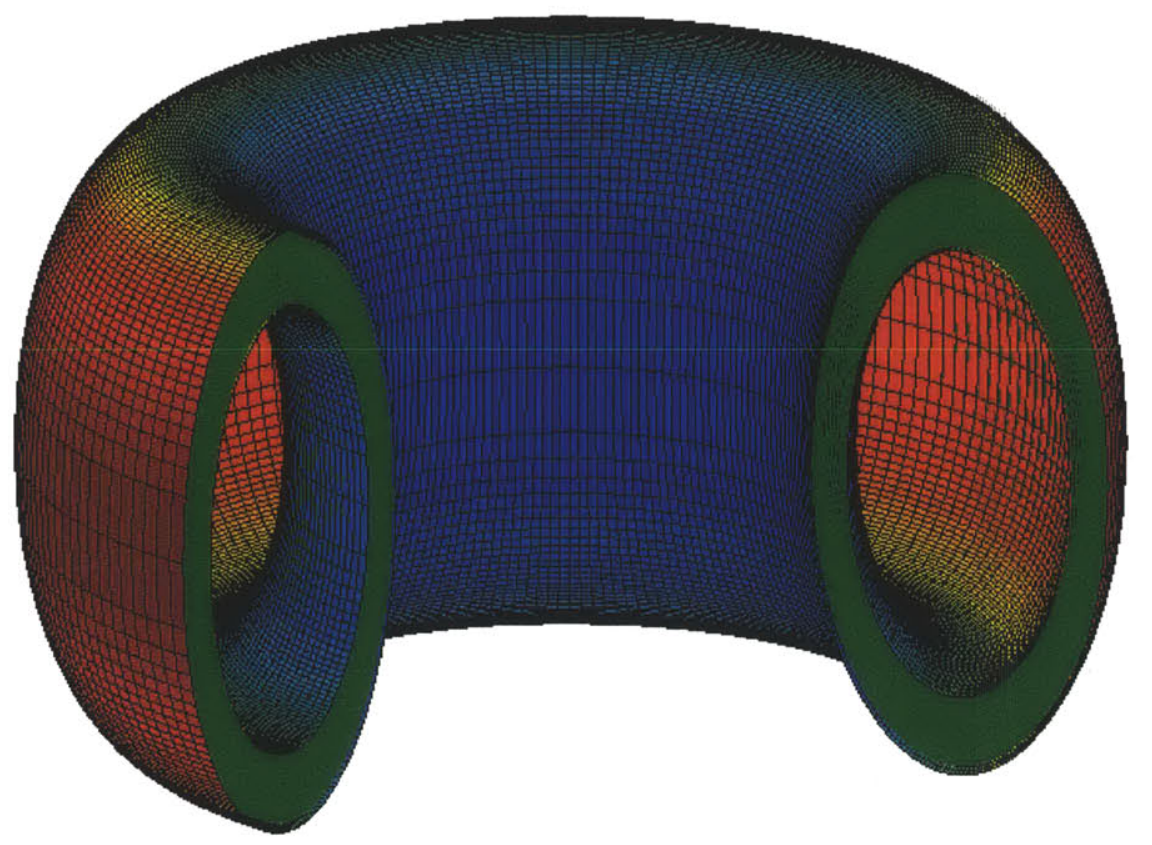

Figure 1.1: A fission blanket (green) coats a tokamak. This is a very simple conceptual diagram. Practically, the fission blanket would not coat the entire tokamak surface.

To show how a fission blanket could dramatically increase the power gain of a tokamak, we need only to walk through some simple physics. In the D-T fusion reaction, the neutron and $\alpha$-particle carry $4 / 5$ and $1 / 5$ of the total energy, respectively. The $\alpha$-particle is confined by the magnetic fields and deposits its energy within the plasma. The neutron, however, exits the plasma and deposits its energy in the blanket materials. As tokamak plasmas are many orders of magnitude less dense than typical solids, it is reasonable to assume that the neutrons never collide in the plasma. 


$$
{ }_{1}^{2} \mathrm{H}+{ }_{1}^{3} \mathrm{H} \rightarrow{ }_{2}^{4} \mathrm{He}(3.5 \mathrm{MeV})+{ }_{0}^{1} \mathrm{n}(14.1 \mathrm{MeV})
$$

It is common to express the fusion power gain $Q_{\text {fus }}$ as a ratio of the total produced fusion power $P_{\text {fus }}$ to the total externally applied auxiliary power $P_{\text {aux }}$. Here all powers have units of Watts.

$$
Q_{\text {fus }}=\frac{\text { total fusion power }}{\text { total supplied auxiliary power }}=\frac{P_{\text {fus }}}{P_{\text {aux }}}
$$

Let us express fission power gain $Q_{\text {fis }}$ as the ratio of the total fission power $P_{\text {fis }}$ produced in the blanket to the total power of all the $\mathrm{D}$ - $\mathrm{T}$ neutrons that enter the fission blanket. Of course, not all the neutrons will enter the fission blanket. The fission blanket cannot practically coat the entire tokamak, and many neutrons are needed for tritium breeding. We will call the fraction of all D-T neutrons that enter the fission blanket $\eta$, which will depend on the geometry and materials of the particular device. Also, not all neutrons that enter the blanket will spur fission, but $Q_{\text {fis }}$ accounts for that. Now we can say that the total D-T neutron power entering the fission blanket is $(4 / 5) \eta P_{\text {fus }}$ and write a simple expression for $Q_{\text {fis. }}$

$$
Q_{\text {fis }}=\frac{\text { total fission power }}{\text { total power of all D-T neutrons entering fission blanket }}=\frac{P_{\text {fis }}}{\frac{4}{5} \eta P_{\text {fus }}}
$$

Now let us define $Q_{\text {hyb }}$ as the total power gain for the entire fission-fusion device. The total power produced has three components: the fusion power from $\alpha$-particles, the fusion power from neutrons that do not enter the fission blanket, and the fission power $P_{\text {fis }}$. The total power consumed is still just the tokamak auxiliary power $P_{\text {aux }}$.

$$
Q_{\text {hyb }}=\frac{\frac{1}{5} P_{\text {fus }}+\frac{4}{5}(1-\eta) P_{\text {fus }}+P_{\text {fis }}}{P_{\text {aux }}}
$$

Some simple algebra yields various expressions for $Q_{\text {hyb }}$ in terms of $Q_{\text {fus }}, Q_{\text {fis }}$, and $\eta$. Below are two of them, the first more intuitive and the second more beautiful. Note that when $Q_{\text {fis }}=1, Q_{\text {hyb }}=Q_{\text {fus }}$ as if the tokamak were bare and had no fission blanket.

$$
\begin{aligned}
& Q_{\mathrm{hyb}}=Q_{\text {fus }}\left[\frac{1}{5}+\frac{4}{5}(1-\eta)+\frac{4}{5} \eta Q_{\mathrm{fis}}\right] \\
& Q_{\mathrm{hyb}}=Q_{\text {fus }}\left[\frac{4}{5}\left(\eta\left(Q_{\mathrm{fis}}-1\right)+1\right)+\frac{1}{5}\right]
\end{aligned}
$$

This is the crux of this thesis. We can clearly see that the fission blanket augments the power gain of a pure fusion tokamak. The fission blanket multiplies the pure fusion power gain by a factor that is linearly dependent upon the fission power gain. This means 
that for a given set of tokamak parameters, $Q_{\text {hyb }}$ can be much greater than $Q_{\text {fus }}$ such that a relatively small L-mode tokamak could operate at high $Q_{\text {hyb }}$. The main goal of this thesis is to determine approximately how small such an L-mode tokamak could be while still producing a high $Q_{\text {hyb. }}$

A fission blanket, which essentially augments the fusion power gain, could allow a fairly small L-mode tokamak to achieve a power gain sufficient to operate as a reactor. So instead of complicating the already difficult challenges of fusion with a fission blanket, hybrids could actually simplify a major challenge of fusion by allowing for L-mode operation.

\subsubsection{Pebble Bed Blanket}

Due to the unusual nature of toroidal geometry, which is unheard of in fission design, conventional cylindrical fuel elements would be an awkward choice. Instead, we opt for small fuel pebbles (spheres), which can be "poured" into any odd-shaped volume. Given our geometric constraints, pebbles are the natural choice and perhaps the only feasible choice.

However, while the choice of fuel shape might be straightforward, the choice of fuel composition is more open-ended. Pure fission pebble bed reactors use numerous tristructuralisotropic (TRISO) particles embedded within graphite matrix pebbles. Each TRISO particle is coated with silicon carbide to retain fission products, and the graphite matrix is impervious to melting.

As we discussed above, a larger fission power multiplication $Q_{\text {fis }}$ would allow for a smaller fusion power multiplication $Q_{\text {fus }}$, which is our goal. Thus, we will choose $\mathrm{UO}_{2}$ pebbles over graphite matrix pebbles due to their significantly higher power density (which naturally corresponds to higher power multiplication). There are a number of engineering concerns with this choice, which we will discuss in Section 8.1. We will compare and contrast $\mathrm{UO}_{2}$ pebbles with graphite matrix pebbles in Section 9.1.1.

\subsubsection{Subcritical Operation}

We have already lauded the advantages of subcritical operation. In short, criticality accidents are impossible (as long as $k_{\text {eff }}$ is not near 1 ), and no traditional criticality control systems (such as control rods or neutron poison injection) are necessary. The whole science of pointkinetics and delayed neutrons is irrelevant.

In our introductory statement, we emphasized that fusion drives fission. Indeed, the fission power level is proportional to the tokamak power level. We can control the fission reaction indirectly through control of the fusion reaction. 


\subsubsection{Fast Spectrum}

Since we have no desire to achieve criticality, there is no reason to moderate neutrons in order to exploit the $1 / v$ region of the ${ }^{235} \mathrm{U}$ fission cross-section. We are free to shape our spectrum however we please. Although our primary mission is power production, we would still like to leave our options open in terms of fissile fuel breeding and waste transmutation. Ss we will see in Section 7.6, fast spectra are likely preferable for transmuting hazardous fission products. They are also superior for fissioning actinides that are fissionable (as opposed to fissile). The very fast $14 \mathrm{MeV}$ neutrons are precisely why fission-fusion hybrids are touted as prolific waste transmuters, and it would be a shame to weaken that argument by softening the spectrum. We prefer the spectrum to be hard.

Since we wish to avoid moderation, we will select helium gas as the blanket coolant. This is also the coolant of choice (along with molten salt) for pure fission pebble beds, so its thermal hydraulic aspects have already been analyzed in this geometry. Although supercritical $\mathrm{CO}_{2}$ has the potential to increase Brayon cycle efficiency, we will stick with helium because it is inert and virtually transparent to neutrons.

\subsubsection{Natural or Depleted Uranium}

A lack of concern for criticality also leaves us no compelling reason to enrich uranium. Fission-fusion hybrids could run on natural or depleted uranium. At $14.1 \mathrm{MeV}$, the fission cross-sections for ${ }^{235} \mathrm{U}$ and ${ }^{238} \mathrm{U}$ are not significantly different. Let us examine them.

Figure 1.2 shows the total ${ }^{238} \mathrm{U}$ cross-section with constituent parts as a function of energy. Fission virtually never occurs below approximately $1.3 \mathrm{MeV}$, and the fission cross-section increases monotonically with energy.

Figure 1.3 shows the same data as Figure 1.2, but this time the total neutron cross-section is normalized to 1.0. We can interpret this plot as showing the probability of each type of neutron interaction (given that a collision occurs) as a function of energy. We have also overlaid the fission $\chi(E)$ spectrum and the $14 \mathrm{MeV}$ neutrons in red. Although fast reactors (and all reactors, really) will fission ${ }^{238} \mathrm{U}$ to some extent, it is the $14 \mathrm{MeV}$ neutrons that make fissioning ${ }^{238} \mathrm{U}$ worthwhile. We will see in Section 3.2 that the initial generation of fusion-born $14 \mathrm{MeV}$ neutrons actually fissions enough ${ }^{238} \mathrm{U}$ to produce a larger number of second generation fission-born neutrons. Also, the $14 \mathrm{MeV}$ neutrons also induce $(\mathrm{n}, 2 \mathrm{n})$ and $(\mathrm{n}, 3 \mathrm{n})$ reactions on ${ }^{238} \mathrm{U}$ that complicate the uranium transmutation-decay chains (and thus the whole fuel cycle) in ways that pure fission neutron spectra do not. We will analyze this in Section 7.3.

Figure 1.4 shows for the same information for ${ }^{235} \mathrm{U}$. Although it is fissile, it will still fission more readily in the presence of $14 \mathrm{MeV}$ neutrons than in a typical fast spectrum.

As we will show conclusively in Section 7.3 , natural uranium $\left(0.7 \%{ }^{235} \mathrm{U}\right)$ is sufficient to 
yield significant power multiplication due primarily to the fissioning of ${ }^{238} \mathrm{U}$ with $14 \mathrm{MeV}$ neutrons. Thus, we have no need for ${ }^{235} \mathrm{U}$. Even pure ${ }^{238} \mathrm{U}$ would be sufficient. Although we will perform our quantitative analysis with natural uranium, our design would not change in any significant way were we to select depleted uranium $\left(0.2 \%-0.4 \%{ }^{235} \mathrm{U}\right)$ instead.

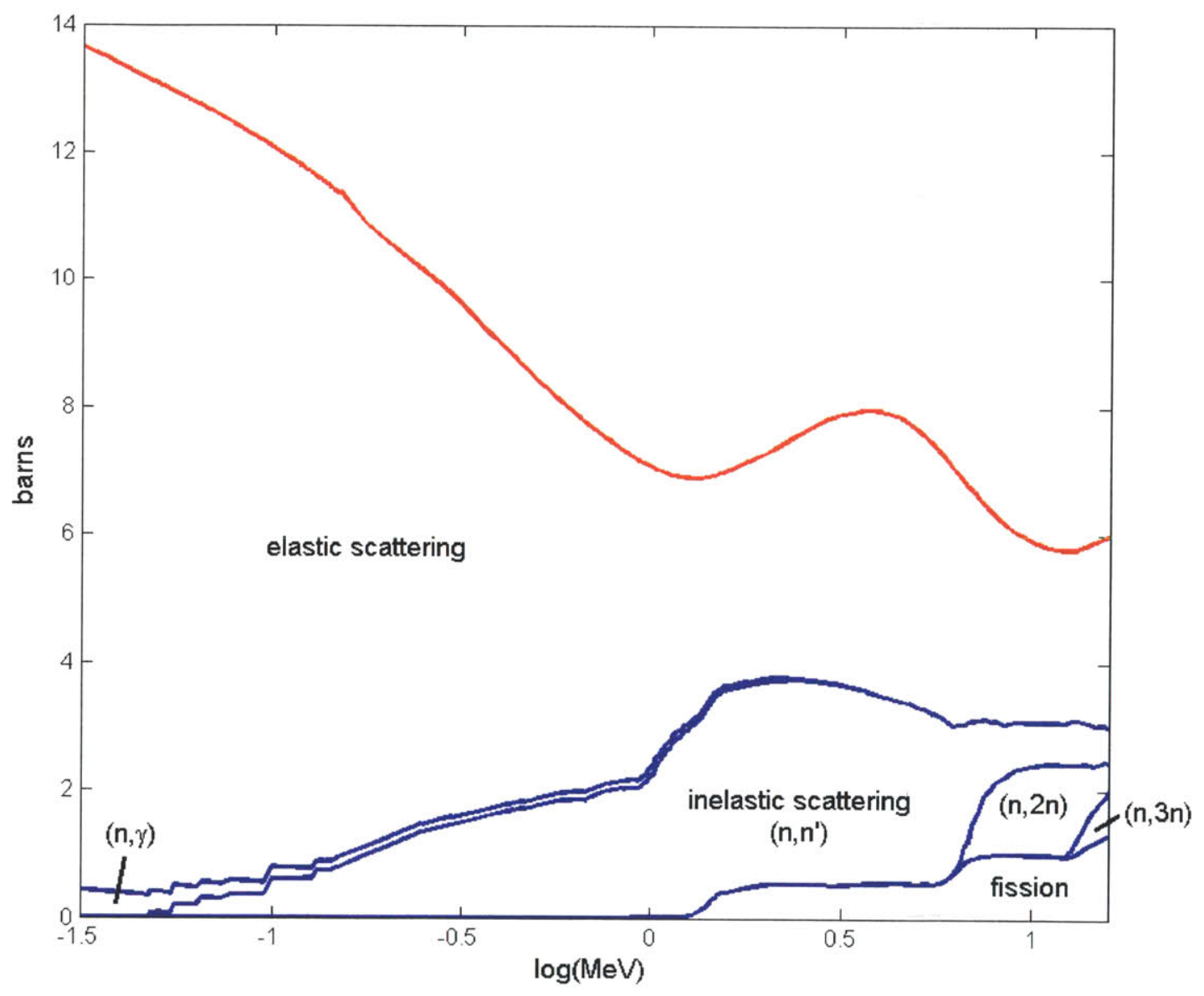

Figure 1.2: The total ${ }^{238} \mathrm{U}$ cross-section showing constituent parts as a function of energy. The total cross-section is in red. 


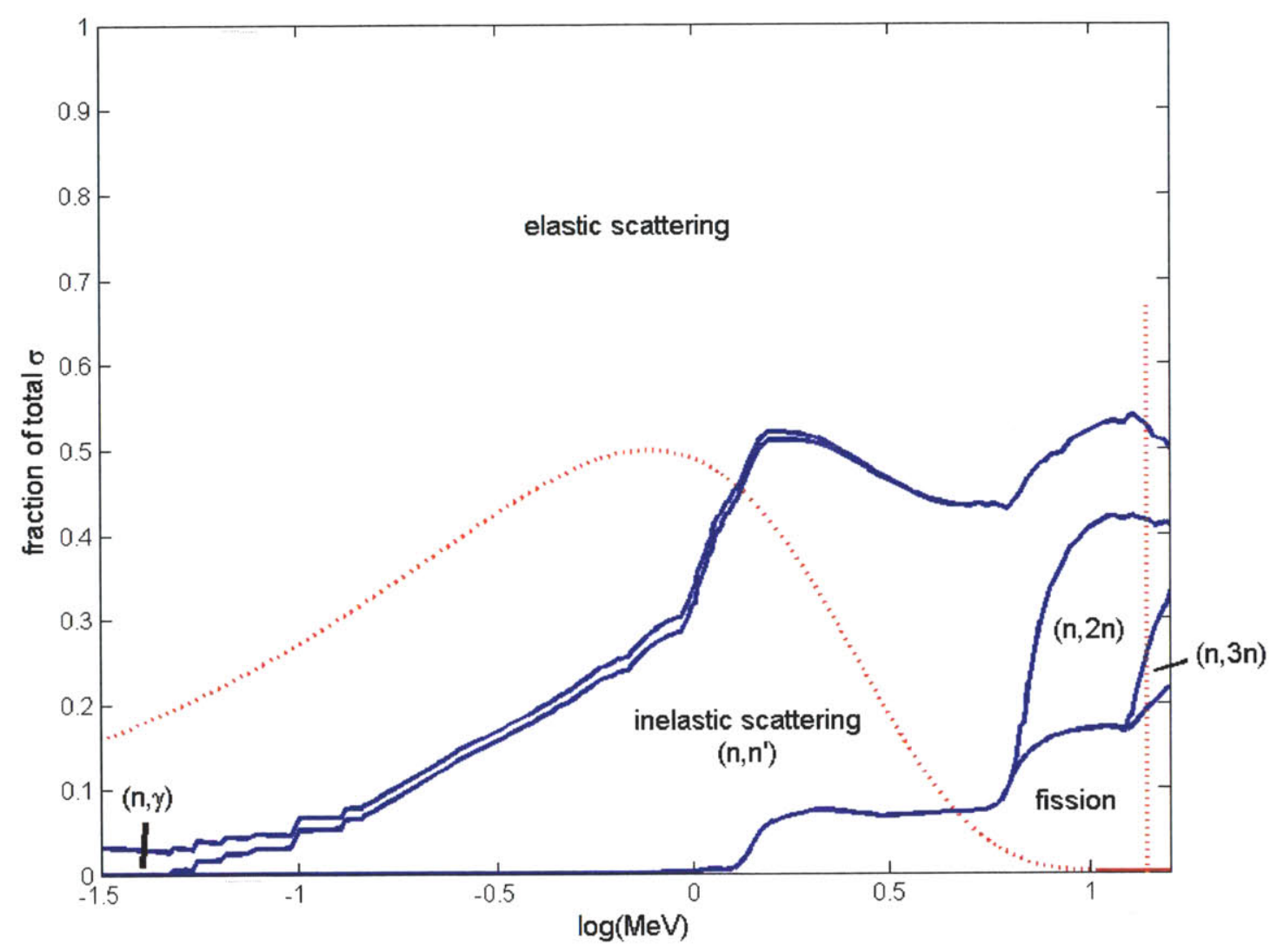

Figure 1.3: Normalized constituent parts of the total ${ }^{238} \mathrm{U}$ cross-section as a function of energy. Here the total cross-section is always 1.0. We have outlined the fission $\chi(E)$ spectrum and the 14 $\mathrm{MeV}$ fusion-born neutrons in red. ${ }^{238} \mathrm{U}$ is fissionable. 


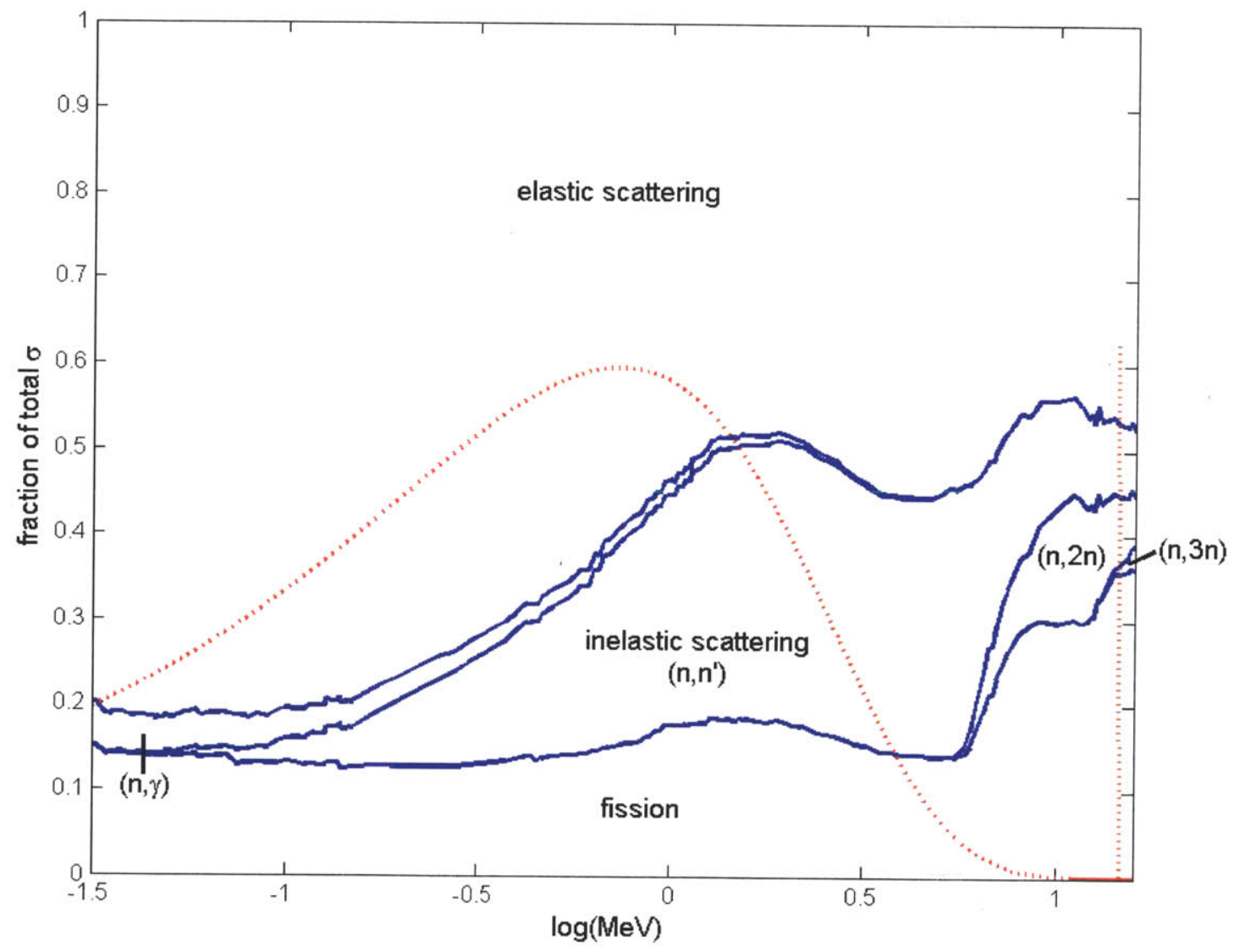

Figure 1.4: Normalized constituent parts of the total ${ }^{235} \mathrm{U}$ cross-section as a function of energy. Here the total cross-section is always 1.0. We have outlined the fission $\chi(E)$ spectrum and the 14 $\mathrm{MeV}$ fusion-born neutrons in red. ${ }^{235} \mathrm{U}$ is fissile. 


\subsubsection{Tritium Breeding}

Tritium is a precious and hazardous commodity. Since it is difficult to obtain and decays with a half-life of 12.3 years, we must produce just as much of it as we consume. Since it is hazardous, we must not produce much more of it than we consume. So for every triton consumed in a D-T fusion reaction, we must breed one triton in the blanket. Thus, our fission blanket must contain not only uranium fuel but also a tritium-breeding material. The only viable choice is lithium, which is naturally composed of $92.5 \%{ }^{7} \mathrm{Li}$ and $7.5 \%{ }^{6} \mathrm{Li}$. Both isotopes breed tritium, but ${ }^{6} \mathrm{Li}$ breeds much more than ${ }^{7} \mathrm{Li}$. We will discuss these reactions and show how to meet the tritium breeding requirement in Section 3.

\subsubsection{Shielding}

We must note that the superconducting magnets (which must be exterior to the fission blanket) can tolerate very little neutron fluence. The limit for $\mathrm{Nb}_{3} \mathrm{Sn}$ magnets is $3 \times 10^{22}$ $\mathrm{n} / \mathrm{m}^{2}$ [5]. Thus, we will need to incorporate an effective shield between the fission blanket and the magnets. This will increase the required total blanket thickness

\subsection{Mission}

The primary mission of this fission-fusion hybrid is to produce power, which could be used for electricity, hydrogen production, or any other suitable purpose. Our goal is to maximize the power multiplication of a natural uranium blanket such that we can minimize the physical size of a steady-state L-mode tokamak while still achieving a net hybrid power gain worthy of a commercial reactor. Steady-state L-mode operation is advantageous for plasma stability, and smaller size is economically advantageous. Subcritical operation is advantageous for fission, primarily in the context of fuel cycle. It obviates enrichment and allows for extremely high burnup through ${ }^{238} \mathrm{U}$ fission. Fission-fusion hybrids can extract more than an order of magnitude more energy from the world's uranium resources than pure fission reactors. We will dub this conceptual design the Steady-State L-Mode Non-Enriched Uranium Tokamak Hybrid (SLEUTH).

Plausible alternative missions include (1) transmuting long-lived waste products in order to reduce the necessary storage capacity of geologic waste repositories and (2) breeding fissile fuel for pure fission reactors to solve the future fuel supply problem. Although these two alternative missions do not constitute our main focus, we will analyze them as well and demonstrate that they are indeed worthy pursuits. 


\subsection{Scope and Outline}

We will accomplish our mission by creating two models: a Monte Carlo fission blanket model coupled with a 0-D tokamak fusion model. We will develop an original Monte Carlo simulation in MATLAB that determines fission power and tritium breeding given a neutron

source in the plasma. Section 2 describes this methodology in detail. We will perform some basic tests to ensure that it matches our intuition, and we will benchmark it with MCNP.

In Section 3, we will optimize various parameters (such as blanket layer thickness and lithium enrichment) to maximize the fission power multiplication. We will also perform detailed neutronics analysis to show that ${ }^{238} \mathrm{U}$ alone can substantially multiply the fission power.

In Section 4, we will turn to fusion and describe our 0-D tokamak model.

We will demonstrate how this model applies to pure fusion reactors in Section 5. This will cultivate intuition and understanding of how our 0-D model works. We will repeat some results of earlier work for instructive purposes.

In Section 6, we will couple our fission and fusion models and show quantitatively how a power-multiplying fission blanket allows for steady-state L-mode operation with relatively small physical dimensions. This will be the crux of our work. We will specify two designs: a steady-state L-mode hybrid the same size as ITER and a minimum-scale steady-state L-mode hybrid dubbed SLEUTH.

Although we do not perform a blanket burnup calculation, we propose a novel method for maintaining constant hybrid power as burnup proceeds. We will perform fuel cycle analysis to show that this natural uranium hybrid could be a prolific breeder of fissile fuel as well as an excellent transmuter of fission product waste. We will also touch on the possibility of a thorium cycle as well as some non-proliferation implications.

Finally, we will justify the engineering feasibility of our fission blanket through basic thermal hydraulic analysis. Although this is certainly not our focus, it is essential that we at least demonstrate that the general tokamak hybrid configuration is thermally practical. 


\section{Fission Blanket Monte Carlo Code}

To perform reactor physics analysis, we develop a neutron transport Monte Carlo code from scratch. This produces criticality and tally results that are very consistent with the Monte Carlo N-Particle (MCNP) Transport Code. We will explain significant aspects of the methodology, delving into more or less detail as we feel so inclined.

It is true that MCNP is capable of performing all the analysis that our new code performs. We chose this arduous route for a number of reasons, some practical and some preferential. First, we tailored this code specifically for this configuration, and so the structure is efficient. Second, this code expedites data analysis, because we have written it entirely in MATLAB. The data is simple to extract, and the code is simple to modify. It is flexible. Third, the overarching reason was to acquire a deep understanding of reactor physics and Monte Carlo methods as well as particular appreciation for this problem. Anyone can run MCNP with minimal grasp of the underlying physics, but developing a code from scratch cultivates insight.

Our Monte Carlo code is completely analog except for fission and $(\mathrm{n}, \mathrm{xn})$ reactions. We can, however, introduce limited variance reduction through fission, which we will discuss below.

\subsection{Path Length Sampling in Toroidal Geometry}

We must solve the problem of neutron path length sampling in toroidal geometry, which is not trivial. Here we will develop from scratch and test our sampling algorithm.

\subsubsection{Flight Distance in Toroidal Geometry}

Figure 2.1 shows the basic toroidal geometry with which we model the tokamak configuration. This is identical to the geometry in our 0-D fusion tokamak model. $R$ is the major radius, and $a$ is the minor radius. The torus has an elongation $\kappa$ so that its poloidal cross-section is an ellipse. $\Phi$ is the toroidal angle, and $\Theta$ the poloidal angle. We can write a sample expression for a toroidal surface in cartesian $(x, y, z)$ coordinates.

$$
\left(\sqrt{x^{2}+y^{2}}-R\right)^{2}+\left(\frac{z}{\kappa}\right)^{2}=a^{2}
$$

The problem of neutron path length sampling boils down to solving the distance from a given point to a toroidal surface in a given direction. When a neutron is born or scatters, it has a known position $\left(x_{0}, y_{0}, z_{0}\right)$ as well as a known direction that we can easily sample. If we define the unknown quantity $s$ as the distance such a neutron must travel to intersect the toroidal surface, we can specify the $(x, y, z)$ location of that intersection point. 


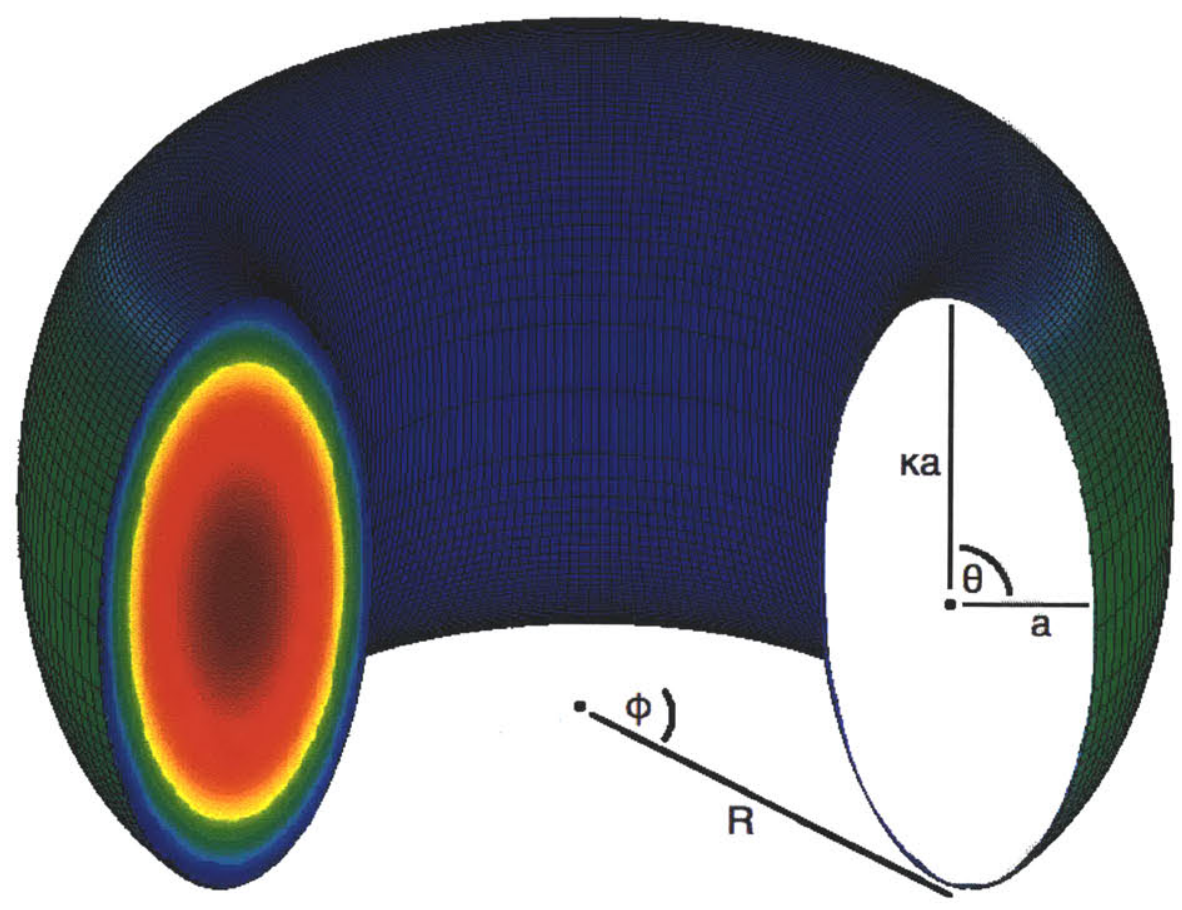

Figure 2.1: Simple toroidal geometry. $R$ is the major radius and $a$ the minor radius. The torus has an elongation $\kappa$ so that its poloidal cross-section is an ellipse. $\Phi$ is the toroidal angle and $\Theta$ the poloidal angle.

$$
\begin{aligned}
& x=x_{0}+s \mu_{x} \\
& y=y_{0}+s \mu_{y} \\
& z=z_{0}+s \mu_{z}
\end{aligned}
$$

Here $\left(\mu_{x}, \mu_{y}, \mu_{z}\right)$ are the unit vector components of the neutron's direction, and they are simple to express in terms of $\theta$ and $\phi$.

$$
\begin{aligned}
& \mu_{x}=\sin \theta \cos \phi \\
& \mu_{y}=\sin \theta \sin \phi \\
& \mu_{z}=\cos \theta
\end{aligned}
$$

We use this notation for elegance and algebraic convenience. $\mu_{z}$ is ubiquitous in neutron transport theory, usually written as just $\mu$. To avoid confusion, we will always use $(\theta, \phi)$ for a neutron's direction in spherical coordinates and $(\Theta, \Phi)$ for the fixed toroidal coordinates shown above in Figure 2.1. 
We can combine Equations 2-4 with Equation 1 to yield a quartic equation in $s$ with coefficients $A, B, C, D$, and $E$.

$$
A s^{4}+B s^{3}+C s^{2}+D s+E=0
$$

Determining the five coefficients requires a bit of convoluted algebra, but the result is not so ugly. The three parameters $L, M$, and $N$ naturally arise and distinguish themselves. $L$ is a function of only the neutron's initial position squared $\left(x_{0}^{2}, y_{0}^{2}, z_{0}^{2}\right)$ with units of length squared. $N$ is a function of only the neutron's initial direction squared $\left(\mu_{x}^{2}, \mu_{y}^{2}, \mu_{z}^{2}\right)$ and is unitless. $M$ is a function of only the intermediate quantity $\left(x_{0} \mu_{x}, y_{0} \mu_{y}, z_{0} \mu_{z}\right)$ with units of length.

$$
\begin{aligned}
& L=R^{2}-a^{2}+x_{0}^{2}+y_{0}^{2}+\left(\frac{z_{0}}{\kappa}\right)^{2} \\
& M=2 x_{0} \mu_{x}+2 y_{0} \mu_{y}+2\left(\frac{z_{0} \mu_{z}}{\kappa^{2}}\right) \\
& N=\mu_{x}^{2}+\mu_{y}^{2}+\left(\frac{\mu_{z}}{\kappa}\right)^{2}
\end{aligned}
$$

Now we can compile $L, M$, and $N$ to express the five quartic coefficients. We will not attempt to impart much intuition here, although it is interesting to note that $x_{0}^{2}+y_{0}^{2}=r_{0}^{2}$ and $\mu_{x}^{2}+\mu_{y}^{2}=\mu_{r}^{2}$ if we define $r^{2}=x^{2}+y^{2}$ and $\mu_{r}=\sin \theta$.

$$
\begin{aligned}
& A=N^{2} \\
& B=2 N M \\
& C=2 N L+M^{2}-4 R^{2}\left(\mu_{x}^{2}+\mu_{y}^{2}\right) \\
& D=2 M L-8 R^{2}\left(x_{0} \mu_{x}+y_{0} \mu_{y}\right) \\
& E=L^{2}-4 R^{2}\left(x_{0}^{2}+y_{0}^{2}\right)
\end{aligned}
$$

Now that our equation is in standard form, there exists a plethora of techniques for solving it. MATLAB has a function roots that solves any polynomial in standard form with matrix eigenvalues. However, as we will show in Section 3.3.4, roots is not optimal for our purposes. Instead, we will employ Ferrari's method, conceived by the Italian mathematician Lodovico Ferrari in the $16^{\text {th }}$ century. This standard widely-known method can solve any quartic equation with simple algebraic relationships, which constitute a mere 18 lines of code (see Appendix B source code).

That the expression is quartic in $s$ is intuitive, because a line can intersect a torus at a maximum of four points. If we assume that a randomly sampled line will never be exactly tangent to the torus, then we can say that an infinite line will always intersect the torus at zero, two, or four points. This translates into zero, two, or four real values of $s$. In the 
context of neutrons, $\left(x_{0}, y_{0}, z_{0}\right)$ and $\left(\mu_{x}, \mu_{y}, \mu_{z}\right)$ define an infinite neutron path. Positive real values of $s$ represent distances the neutron must travel forward along $\left(\mu_{x}, \mu_{y}, \mu_{z}\right)$ to intersect the torus. Negative real values of $s$ represent distances the neutron would travel backward along $\left(-\mu_{x},-\mu_{y},-\mu_{z}\right)$ to intersect the torus (if that were its direction). Naturally, we only care about the positive real values of $s$. If the neutron begins inside the torus, there will be either one or three intersection points. If the neutron begins outside the torus, there will be zero, two, or four intersection points. Figure 2.2 illustrates this nicely.

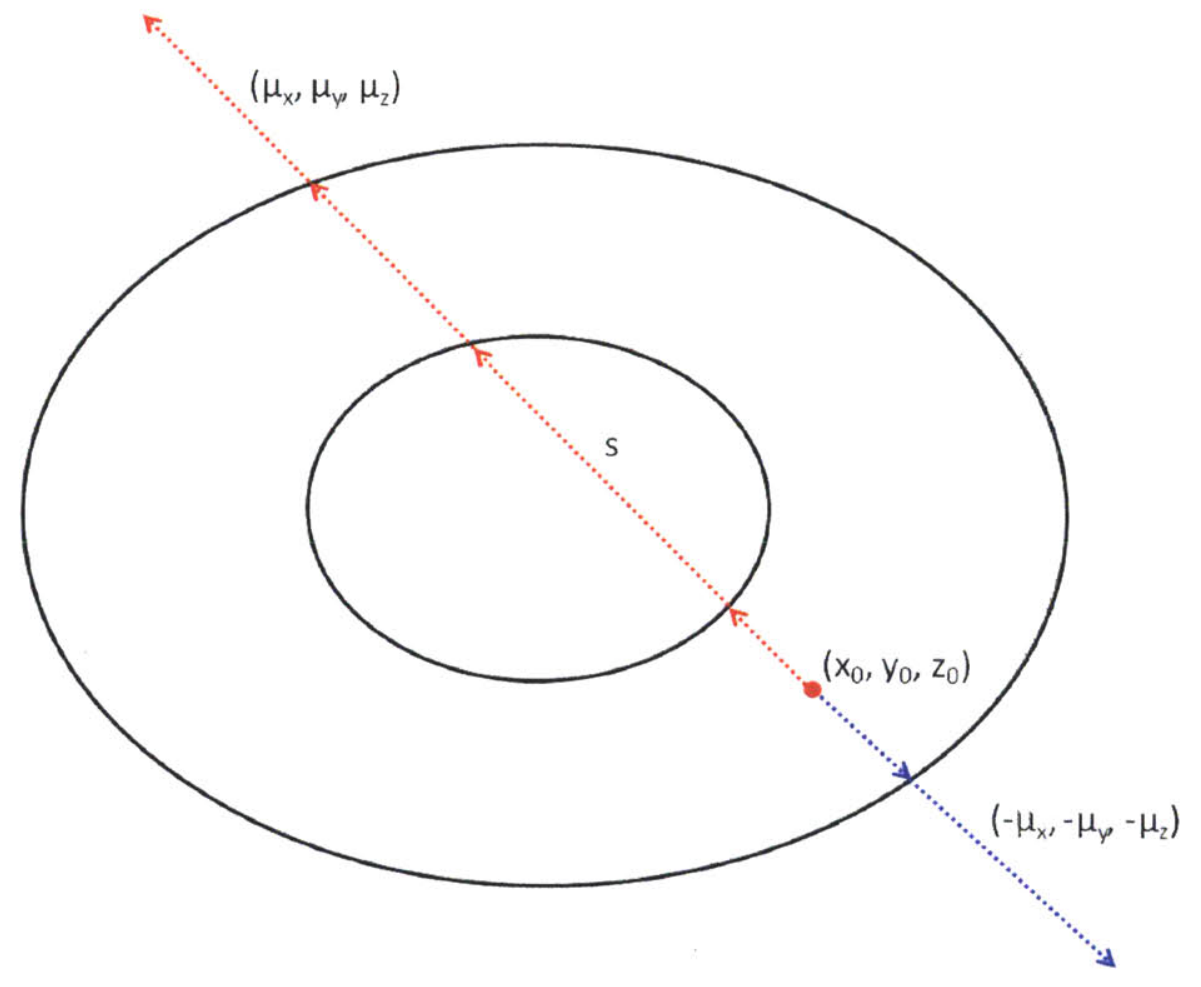

Figure 2.2: A toroidal cross-section of a torus showing a neutron's initial position $\left(x_{0}, y_{0}, z_{0}\right)$ and direction $\left(\mu_{x}, \mu_{y}, \mu_{z}\right)$. Here all four solutions for $s$ are real, three positive and one negative. Since the negative solution corresponds to backward motion $\left(-\mu_{x},-\mu_{y},-\mu_{z}\right)$, we are only interested in the positive solutions.

\subsubsection{Sampling Algorithm}

Now that we have shown how to determine $s$, the distance a neutron must travel to intersect a torus, let us specify the full path length sampling algorithm. Our fission-fusion hybrid model consists of concentric tori. Referring back to Figure 2.1, the parameters $R$, $a$, and $\kappa$ fully define an elongated torus. Our tori all have the same values $R$ and $\kappa$ but different 
values of $a$. The poloidal cross-sections of concentric tori are concentric ellipses.

Suppose there are $n$ concentric tori. These $n$ tori enclose $n$ finite regions: one solid toroid and $n-1$ annular toroids. Each of these regions has a different total neutron cross-section $\Sigma_{t}$. If each of these regions were infinite, we could sample the neutron path length like this, where $\xi$ is a random number on $[0,1]$.

$$
s_{\infty}=\frac{-\ln \xi}{\Sigma_{t}}
$$

Now the basic algorithm proceeds like this:

1. Given an initial $\left(x_{0}, y_{0}, z_{0}\right)$ and $\left(\mu_{x}, \mu_{y}, \mu_{z}\right)$ for a neutron, solve the quartic equation for all $n$ tori. This will yield $4 n$ values of $s$.

2. Discard imaginary and negative $s$ values.

3. Sort all positive real values of $s$ from smallest to largest, keeping track of which tori each $s$ value corresponds to.

4. Sample $s_{\infty 1}$ with $\Sigma_{t 1}$ for the initial region that $\left(x_{0}, y_{0}, z_{0}\right)$ falls within. Here subscripts denote successive regions defined by successive tori intersections.

5. If $s_{\infty 1}$ is less than the smallest value $s_{1}$, the neutron travels a total distance $s_{\infty, 1}$ and stops in region 1. The sampling is complete. If $s_{\infty 1}$ is greater than $s_{1}$, sample $s_{\infty 2}$ in region 2. If $s_{\infty 2}$ is less than $s_{2}-s_{1}$, the neutron travels a total distance $s_{2}-s_{1}+s_{\infty 2}$ and stops in region 2. If $s_{\infty 2}$ is greater than $s_{2}-s_{1}$, sample $s_{\infty 3}$ in region 3 . Keep repeating this for all $s$ values in ascending order.

6. If the neutron reaches the outermost torus, we kill it. In reality, a neutron could exit the outermost torus and subsequently reenter it. However, given that the outermost region is a shield, we will assume that the reentrant neutrons are negligible. Of course, neutrons often exit and reenter the inner tori.

Here is a short code segment that shows the heart of this algorithm. Here points contains the $s$ values, and path contains the segment length of $s$ within each region so that path(1) $=s_{1}$ and $\operatorname{path}(2)=s_{2}-s_{1}$. reg contains the region index associated with each segment, and shell contains the index associated with each shell. sigma_t(n) is $\Sigma_{t}$ in region $n$. points, path, reg, and shell have the same length. The rest of this code segment should be self-explanatory. Figure 2.3 illustrates this algorithm.

pathlength_tot $=0$;

for $\mathrm{m}=1:$ length (points) 


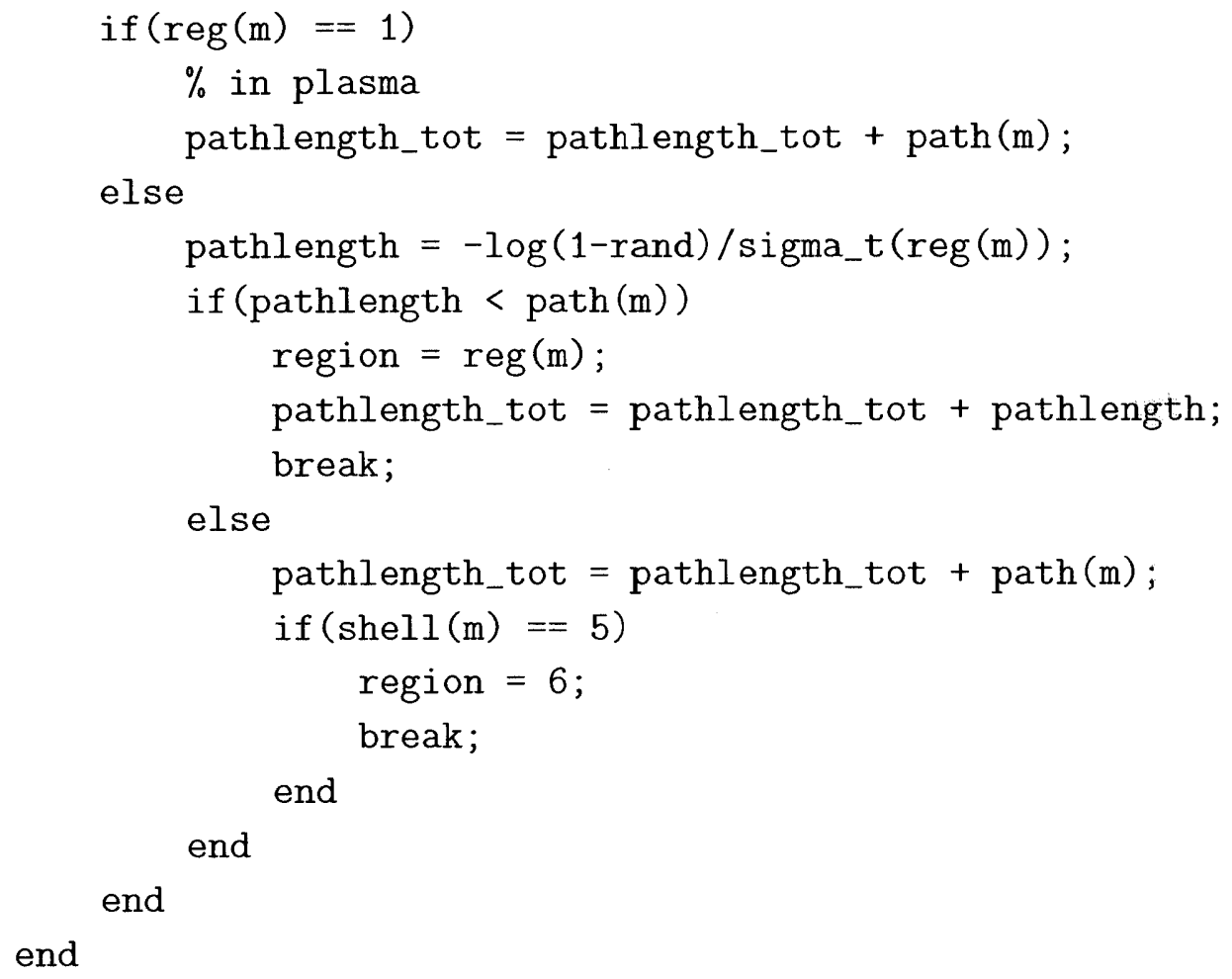

Note that we do not sample $s_{\infty}$ in region 1 . In our hybrid model, the innermost torus is filled with plasma, which we approximate as a vacuum. A typical D-T plasma ion density for ITER is $7.5 \times 10^{19} \mathrm{~m}^{-2}$. At $1 \mathrm{MeV}$, this corresponds to a neutron mean free path of about about $59,000 \mathrm{~km}$, which is over 4.6 times the diameter of the earth. At $14 \mathrm{MeV}$, the mean free path is about $152,000 \mathrm{~km}$, approximately $40 \%$ of the distance from the earth to the moon. Since we currently have no plans to build a device quite that large, we can safely assume that no neutrons collide in the plasma.

\subsubsection{Tokamak Wall Neutron Flux}

Now that we have specified our geometry algorithm, it makes sense to test it independently of other modules in our code (such cross-sections, scattering, and fission). Conveniently, our model of neutron transport through the plasma is purely geometric - no collisions occur. We can determine the $14-\mathrm{MeV}$ neutron flux at any point on the toroidal plasma surface. This constitutes the first step in each neutron history for our full Monte Carlo simulation.

1. Sample fusion sites uniformly in the plasma volume using rejection sampling. In reality, the fusion power density is not quite spatially uniform, but this will be a decent approximation for our purposes. Each $14 \mathrm{MeV}$ neutron is emitted isotropically from the fusion reaction. 


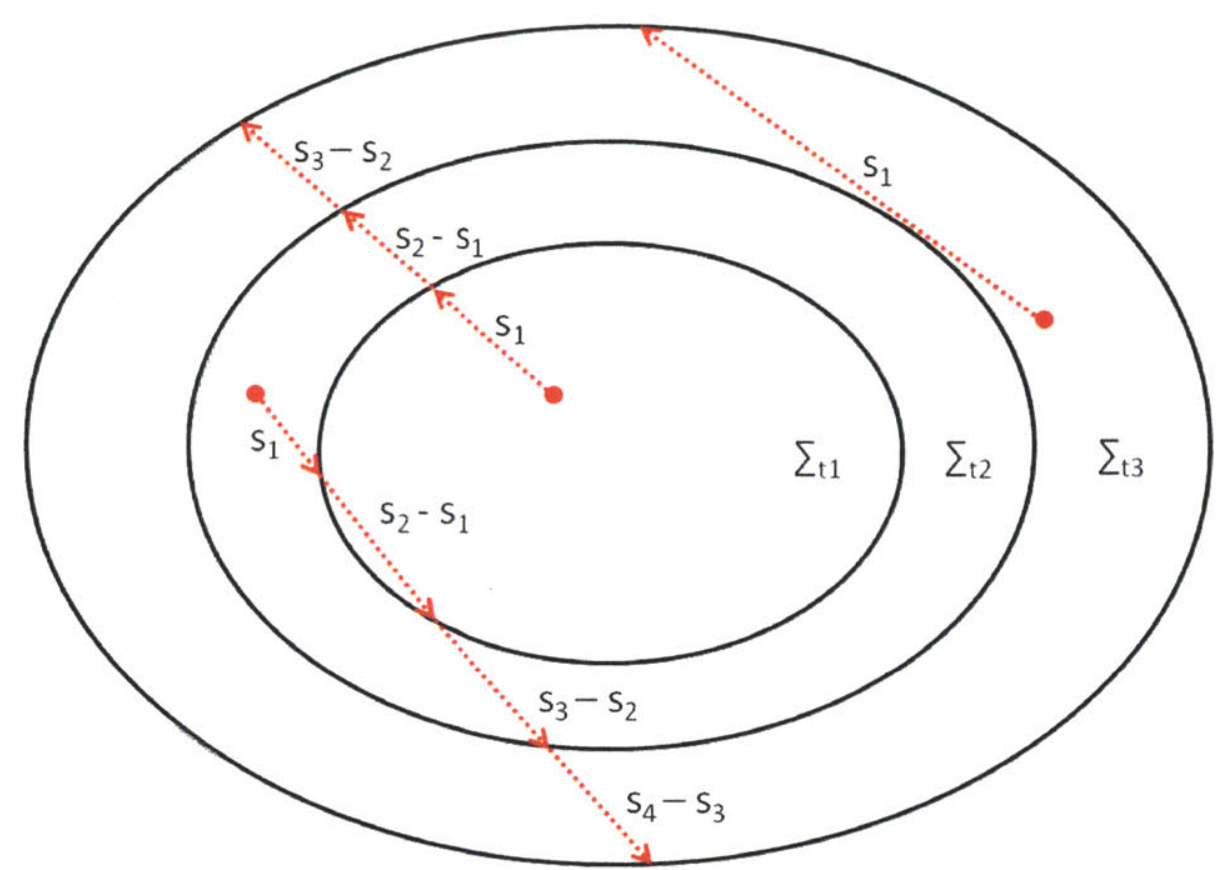

Figure 2.3: A poloidal cross-section of concentric tori showing neutron initial positions, directions, and intersection points with the tori. Each region between tori has a different total cross-section $\Sigma_{t}$. If the quartic solution $s_{n}$ is the distance to a neutron's $n^{\text {th }}$ intersection point, then the total distance that neutron would travel from its $(n-1)^{\text {th }}$ intersection point to its $n^{\text {th }}$ intersection point (if no collisions occur) is $s_{n}-s_{n-1}$. For convention, $s_{0}=0$. We kill neutrons at the outermost torus.

2. Given the initial position and initial direction, solve the quartic equation for the point of when the neutron first intersects the plasma surface.

3. Tally the neutron's direction $(\theta, \phi)$ in association with that intersection point $(\Theta, \Phi)$.

4. Transform and condense the intersection points according to toroidal symmetry. For example, the torus is axisymmetric in $\Phi$, so the neutron's directions at different $\Phi$ values must be transformed to be consistent relative to the surface the neutron's path intersects.

Of course, the actual flux at the plasma surface will include more than just the first intersections of the fusion-born neutrons. Many fusion-born neutrons will travel back into the plasma after leaving it, and many fission-born neutrons will also traverse the plasma. However, this $14 \mathrm{MeV}$ neutron flux is still useful for testing purposes, as it is easy to judge whether the distributions accurately reflect toroidal geometry. 
Figure 2.4 shows the scalar neutron flux at the toroidal plasma surface as a function of poloidal angle $\Theta$. The ratio of flux at the outermost point to flux at the innermost point is about 2, which is consistent with toroidal geometry in that much more of the plasma is "visible" to points on the outboard edge than to points on the inboard edge. See Figure 2.9 for an illustration of this. There is also a maximum flux that occurs at approximately $\Theta=1.2$. These two quantities of interest, the flux ratio and the angular location of maximum flux, vary with geometric parameters. Figure 2.5 shows the flux ratio as a function of aspect ratio $R / a$. As $R / a$ grows very large, the toroid resembles a cylinder, and the flux ratio approaches 1 . At low $R / a$, the ratio grows rapidly, because a greater portion of the toroid volume is "visible" to a point on the outboard edge. Figure 2.6 shows the flux ratio as a function of elongation $\kappa$. The flux ratio has only a weak dependence on this. Figure 2.7 shows the $\Theta$ of maximum flux as a function of elongation. When $\kappa=1$, the tokamak's poloidal cross-section is a circle, and the maximum flux occurs at the outermost point $\Theta=$ 0 . Thus, Figure 2.4 would be a continuously decreasing function for $\kappa=0$. As $\kappa$ increases, the angle of maximum flux approaches 1.2 radians. All these results match our geometric intuition and verify that we have correctly derived and computed our quartic solutions.

We can proceed further by computing the angular neutron flux at the toroidal surface at certain unique points of interest: the outermost point $\left(x^{2}+y^{2}=(R+a)^{2}\right)$, the innermost point $\left(x^{2}+y^{2}=(R-a)^{2}\right)$, and the topmost point $\left(x^{2}+y^{2}=R^{2}, z= \pm \kappa a\right)$. We compute all these distributions assuming the standard ITER parameters of $R / a=3.1$ and $\kappa=1.75$. Figures 2.8, 2.10, and 2.11 show the neutron flux distribution as a function of standard fixed spherical coordinates $\theta$ and $\phi$ at these three points of interest. The azimuthal $\phi$ distributions at the outermost and topmost points exhibit Bactrian camelback shapes. These shapes arise not from the barren steppes of central Asia but from simple geometry. These angular distributions are really nothing more than muddled reflections of how much toroidal volume is "visible" in each direction from the surface point under consideration. See Figure 2.9 for an illustration of this. Since the practical purpose of these plots is to ensure that our numerical solutions match our intuition, it is sufficient to grasp how the general shapes arise from geometry.

A naive way to interpret the scalar distribution in Figures 2.8, 2.10, and 2.11 would be to suppose that the relative flux magnitudes at each poloidal angle are precisely proportional to the total plasma volume that is "visible" from that point. While the "visible" concept provides general intuition, it is not actually precise. Fusion neutrons that are born far from a surface point are less likely to hit the close vicinity of that surface point than neutrons born closer. Since fusion reactions produce neutrons isotropically, the probability that a fusion-born neutron will hit a small area $d A$ on the plasma surface is $d A / r^{2}$, where $r$ is the distance between that small area and the fusion reaction location. Thus, if we desire to calculate neutron scalar fluxes based on "visible" volumes, we must weight the differential 
volume element $d V$ with some factor that depends on distance.

Nevertheless, computing volumes "visible" to a point on a torus is still useful in that it shows us the volume of plasma that contributes to the neutron flux at that point. Let us begin with the innermost torus point defined by $\Theta=0$ or $x^{2}+y^{2}=(R-a)^{2}$. We can define total toroidal volume "visible" to this point as the toroidal volume on one side of the plane $x=R-a$.

Slicing tori with planes is something people have thought about for a while, even predating contemporary bagel shops. In fact, there exists an entire taxonomy for the various quartic curves that comprise intersections of tori and planes. The intersection of a torus with any plane is a toric section. The intersection of a torus with a plane parallel to the $s$ axis is a spiric section. Figure 2.12 illustrates various spiric sections. The unique spiric section formed by the $z=R-a$ plane is shaped like a figure-eight. We could also rightly call it a lemniscate. This is the spiric section that bounds the toroidal volume that is "visible" to the innermost point, which is $z>R-a$. For the dimensions of $R / a=3.1$ and $\kappa=1$, this volume is is about $1 / 4$ the total plasma volume. We determine this value by first determining spiric sections as a function of $z$ and then integrating the areas enclosed by those spiric sections from $z=R-a$ to $z=R+a$. We could also determine the plasma volume "visible" to the outermost point by evaluating other spiric sections. The ratio of the volumes "visible" to the outermost and innermost points is approximately the ratio we determined with Monte Carlo in Figures 2.5 and 2.6. To analytically evaluate this ratio precisely, we would need to introduce the convoluted distance weighting factors discussed above, but we will gladly relegate that effort to a future study.

\subsubsection{Quartic Solution Comparison}

We have already described how to convert neutron path length sampling in toroidal coordinates into a standard quartic equation. We have also stated that we employ Ferrari's method to solve it. However, it is worth comparing the performance of our direct implementation of Ferrari's method to reputable polynomial solvers.

MATLAB contains the built-in function roots, which computes the roots of any polynomial in standard form. roots does this by solving eigenvalues of a companion matrix. While this method might be superior for polynomials of higher degree, it is inferior to a direct implementation of Ferrari's method for quartic polynomials. Figures 2.13 and 2.14 show CPU runtime analyses for our tokamak neutron flux Monte Carlo code and our entire hybrid Monte Carlo code, respectively. Ferrari's method reduces the runtime of our entire hybrid Monte Carlo simulation by about $27 \%$, which is delightful.

The only drawback to an algebraic implementation of Ferrari's method is that it introduces rounding error. This is a problem for neutrons that just barely graze any of the toroidal surfaces. Their paths are very nearly tangential to the tori. This causes some quartic 
solutions to be very slightly real or very slightly imaginary to the point where it is difficult to resolve whether the solution is truly real or only real due to rounding error. We can introduce tolerance thresholds, but with millions of flight paths, the error magnitudes will always overlap with the actual values in a few cases. Thus, we must introduce error trapping. If a neutron path's intersections with successive tori becomes out-of-order or irrational in some other specific way, we immediately kill that neutron. Fortunately, this only occurs a few times for every ten thousand source neutrons. roots does not exhibit this problem at all, but we judge that the runtime advantage overrides this issue.

\subsubsection{Cylindrical Comparison}

It is common practice to model a tokamak as a cylinder, which is most accurate for large aspect ratios. This is the approach Vincent Tang took in his 2002 master's thesis [22]. We have chosen to implement the full toroidal geometry. While there is no question that a toroidal model is be more accurate than a cylindrical model, it is worth some time to examine how much more accurate it is. Solving quartic equations in toroidal geometry is much more computational expensive than solving quadratic equations in cylindrical geometry, and it is important to ask whether the gain in precision is worth the computational expense.

Since we used elongated tori in our toroidal model, we will use elliptic cylindrical shells in our cylindrical model. In this way, a radial cross-section of our cylinder will be identical to a poloidal cross-section of our toroid. We can define the elliptic cylinder as

$$
x^{2}+\left(\frac{y}{\kappa}\right)^{2}=a^{2}
$$

We can follow the same general procedure as in toroidal geometry to derive expressions for the three coefficients in a standard quadratic equation $A s^{2}+B s+C=0$.

$$
\begin{aligned}
& A=\mu_{x}^{2}+\left(\frac{\mu_{y}}{\kappa}\right)^{2} \\
& B=2\left[x_{0} \mu_{x}+\frac{y_{0} \mu_{y}}{\kappa^{2}}\right] \\
& C=x_{0}^{2}+\left(\frac{y_{0}}{\kappa}\right)^{2}-a^{2}
\end{aligned}
$$

Now we can use the same basic toroidal geometry algorithm to determine neutron flight paths, except that now there are only two solutions instead of four. Figure 2.15 shows the scalar flux as a function of polar angle. The maximum flux occurs at the elongated end of the ellipse. That this flux distribution is not flat utterly debunks any assertion that "visible" plasma volume is an accurate predictor of neutron flux. In an elliptic cylinder, the entire plasma is visible to every surface point. Yet the distribution is very far from flat due 
to distance weights of the form $1 / r^{2}$ as discussed in the previous section. Of course, the distribution is flat for a circular cylinder $(\kappa=1)$ due to symmetry.

In order to test cylindrical geometry in our full hybrid model, we replaced our quartic solver method with this quadratic solver method while keeping shell radii all the same. The total fission and tritium breeding tallies were not substantially different (within 10\%) than in the case of ITER aspect ratio $(R / a=3.1)$. However, while a cylindrical model might yield very approximate values averaged throughout the entire device, it does not yield accurate values in localized regions of the hybrid blanket. Comparing Figure 2.15 with Figure 2.4 shows that toroidal geometry yields many more fission and tritium breeding events in the outboard blanket than in the inboard blanket, while cylindrical geometry yields equal values on both sides. This consequence of the toroidal model is important in terms of burnup, because the inboard blanket will need to be replaced less frequently than the outboard blanket. Since the inboard blanket would be much more difficult to replace, this is advantageous. We conclude that although cylindrical geometry is a decent approximation in terms of cumulative values, it does not sufficiently capture spatial dependence.

\subsection{Monte Carlo Methodology}

Now that we have exhausted geometry concerns, we will turn to the more generic aspects of neutron transport Monte Carlo, including cross-section evaluation, scattering, and fission.

\subsubsection{ENDF Cross-Sections}

We employ cross-section data from the Evaluated Nuclear Data File (ENDF). To evaluate a cross-section, we perform a binary search of energy values and select the corresponding cross-section value. In order to optimize our code performance, we align all cross-sections on the same set of energy values, approximately 20,000 in number. In this way, we only need to perform one binary search per collision. With a thermal neutron spectrum, the temperature dependence of resonance broadening would be a major concern. However, our spectrum is fast, and so the effects of this will be negligible.

\subsubsection{Elastic Scattering}

In any Monte Carlo transport simulation, when to approximate scattering (in the lab frame) as isotropic is always an important question. Here will take an in-depth look at the angular distribution of elastic scattering. It is common knowledge that elastic scattering is virtually isotropic for heavy nuclei but quite anisotropic for light nuclei. For hydrogen, elastic backscattering is not even theoretically possible. However, it is instructive to perform some analysis here to quantify how heavy a nucleus must be for scattering off it to be considered isotropic. 
Let us begin with energy. The standard probability distribution for energy shift $P(E \rightarrow$ $\left.E^{\prime}\right)$ is

$$
P\left(E \rightarrow E^{\prime}\right)=\left\{\begin{array}{lc}
\frac{1}{(1-\alpha) E} & \alpha E<E^{\prime}<E \\
0 & 0<E^{\prime}<\alpha E
\end{array}\right.
$$

Here $\alpha=(A-1)^{2} /(A+1)^{2}$ as usual, where $A$ is the nuclide mass number. Given a certain lab frame energy shift $E_{L}^{\prime} / E_{L}$, we can conveniently derive the scattering angle in center of mass coordinates, where everything is isotropic. The standard relationship between lab frame energy shift and center of mass scattering angle is

$$
\frac{E_{L}^{\prime}}{E_{L}}=\frac{(1+\alpha)+(1-\alpha) \mu_{C}}{2}
$$

Here $\mu=\cos \theta$ as usual, and subscripts $\mathrm{L}$ and $\mathrm{C}$ denote lab and center of mass coordinates. So once we sample the energy shift, we can quickly determine $\mu_{C}$, which will always be evenly distributed on $[-1,1]$. However, since we are really only interested in $\mu_{L}$, we must employ the law of cosines to relate scattering angles in the two frames.

$$
\tan \theta_{L}=\frac{\sin \theta_{C}}{1 / A+\cos \theta_{C}}
$$

Now we have fully specified the sampling process for $\mu_{L}$, which is nothing new. Of course, we always sample the azimuthal angle $\phi$ uniformly on $[0,2 \pi]$. To develop intuition for how $P\left(\mu_{L}\right)$ varies with $A$, we can run a simple Monte Carlo simulation to determine $P\left(\mu_{L}\right)$ for various values of $A$. Figure 2.16 shows this for $A=1,4,16$, and 56. Obviously, elastic scattering for ${ }^{1} \mathrm{H}$ is very anisotropic. Backscattering is not even possible. As $A$ increases, the scattering becomes more isotropic. For ${ }^{56} \mathrm{Fe}$, the scattering is nearly isotropic. These distributions are well-known, and they are effective in providing insight into isotropy.

However, eyeing distribution shapes is quite arbitrary, and a much better measure of how isotropic scattering is would be the quantity $P\left(\mu_{L}=1\right) / P\left(\mu_{L}=-1\right)$, the ratio of forward scattering to backward scattering. If this ratio is close to 1 , the scattering is isotropic. If it is much greater than 1 , the scattering is anisotropic. There are a number of ways to calculate this ratio as a function of $A$, but we will proceed with our own derivation. Since $P\left(\mu_{L}\right)$ and $P\left(\mu_{C}\right)$ are the same distribution of two related variables, it is a mathematical fact that

$$
P\left(\mu_{L}\right) d \mu_{L}=P\left(\mu_{C}\right) d \mu_{C}
$$

Now we know that $P\left(\mu_{C}\right)$ is a constant, because scattering is always isotropic in center of mass coordinates. Its value is $1 / 2$ on $[-1,1]$. So we say with confidence that $P\left(\mu_{L}\right)$ is proportional to $d \mu_{C} / d \mu_{L}$. Now we can reexamine our relationship between $\mu_{L}$ and $\mu_{C}$ and express it free of trigonometric functions to avoid phase and range ambiguity. 


$$
\frac{\sqrt{1-\mu_{L}^{2}}}{\mu_{L}}=\frac{\sqrt{1-\mu_{C}^{2}}}{1 / A+\mu_{C}}
$$

Now let the left side of this equation be $f\left(\mu_{L}\right)$ and the right side be $g\left(\mu_{C}\right)$. Since $f$ and $g$ are equal, we can express $d \mu_{C} / d \mu_{L}$ as

$$
\frac{d \mu_{C}}{d \mu_{L}}=\frac{d f / d \mu_{L}}{d g / d \mu_{C}}
$$

For the sake of showing the convoluted explicit expression for $P\left(\mu_{L}\right)$, here it is.

$$
P\left(\mu_{L}\right)=\frac{1}{2} \frac{d \mu_{C}}{d \mu_{L}}=\frac{1}{2} \frac{\frac{1}{\sqrt{1-\mu_{L}^{2}}}+\frac{\sqrt{1-\mu_{L}^{2}}}{\mu_{L}^{2}}}{\frac{\mu_{C}}{\left(1 / A+\mu_{C}\right) \sqrt{1-\mu_{C}^{2}}}+\frac{\sqrt{1-\mu_{C}^{2}}}{\left(1 / A+\mu_{C}\right)^{2}}}
$$

Of course, to practically evaluate this, we need to replace $\mu_{C}$ with $\mu_{C}\left(\mu_{L}\right)$, which we can solve numerically. We can also express this more elegantly in terms of $\theta_{L}$ and $\theta_{C}$.

$$
P\left(\mu_{L}\right)=\frac{1}{2}\left[\frac{\sin \theta_{C}}{\sin \theta_{L}}\right]\left[\frac{\cot \theta_{L}+\tan \theta_{L}}{\cot \theta_{C}+\tan \theta_{L}}\right]
$$

Although $A$ no longer appears here directly, it is here implicitly in that $A$ is necessary to convert between $\theta_{L}$ and $\theta_{C}$. A bit more messy algebra and trigonometry can yield $P\left(\mu_{L}\right)$ as a function of only $\mu_{L}$ and $A$.

$$
P\left(\mu_{L}\right)=\frac{1}{2 A} \frac{\left(\mu_{L}+\sqrt{\mu_{L}^{2}+A^{2}-1}\right)^{2}}{\sqrt{\mu_{L}^{2}+A^{2}-1}}
$$

This is the standard form that appears in some new reactor physics textbook (such as Applied Reactor Physics by Alain Hebert [32]) but is surprisingly absent from many older ones.[32]. This satisfies Equation 2.25.

For the raw pleasure of it, we can now generate a surface plot of $P\left(\mu_{L}\right)$ as a function of $A$. Figure 2.17 shows this surface, which is consistent with its four slices in Figure 2.16. We can discern how the shape of $P\left(\mu_{L}\right)$ smoothly evolves as $A$ increases.

We must now painfully avert our eyes from the this mesmerizing surface to continue with the task at hand - quantifying $P\left(\mu_{L}=1\right) / P\left(\mu_{L}=-1\right)$. The difficult way to do this is with Equation 2.28. The limits are necessary, because $d f / d \mu_{L}$ and $d f / d \mu_{C}$ both approach zero at -1 and 1.

$$
\frac{P\left(\mu_{L}=1\right)}{P\left(\mu_{L}=-1\right)}=\frac{\lim _{\mu_{L} \rightarrow 1} \frac{d \mu_{C}}{d \mu_{L}}}{\lim _{\mu_{L} \rightarrow-1} \frac{d \mu_{C}}{d \mu_{L}}}
$$


However, it is much more efficient to simply evaluate Equation 2.30 , which yields a very elegant result.

$$
\frac{P\left(\mu_{L}=-1\right)}{P\left(\mu_{L}=1\right)}=\left(\frac{A-1}{A+1}\right)^{2}=\alpha
$$

That the ratio of the probability of backward scattering $\left(d \mu_{L}\right.$ about $\left.\mu_{L}=-1\right)$ to the probability of forward scattering ( $d \mu_{L}$ about $\mu_{L}=1$ ) is $\alpha$ should not be terribly surprising. $\alpha$ arises from simple collision kinematics, and $\alpha$ is also the ratio of the final and initial neutron energies when $\mu_{L}=\mu_{C}=-1$. This is an interesting and instructive way to define $\alpha$, although few (if any) reactor physics texts touch on it.

Figure 2.18 shows $P\left(\mu_{L}=1\right) / P\left(\mu_{L}=-1\right)=\alpha$ as a function of $A$ from $A=4$ to 200 . The ratio becomes less than 1.1 at $A \approx 50$. However, we will require that the ratio be less than 1.05 , which occurs at $A \approx 100$. Thus, we will treat all elastic scattering collisions with $A>100$ as isotropic and collisions with $A<100$ as anisotropic.

Now that we have quantified isotropy and chosen a reasonable boundary between isotropic and anisotropic scattering, we should explain how we transform angular coordinates in the case of anisotropic scattering. In isotropic scattering, we can easily sample the post-collision angles independently of the pre-collision angles. In anisotropic scattering, we can only sample the angle shifts, and then we must transform those shifts into our fixed angular coordinate system. We can derive the mathematical relationships that define such a coordinate transformations from rotation matrices, but we will not delve into that here. The manual for PENELOPE, an electron and photon transport code, explains this very well [27]. We will simply write it down. Let $\mu$ and $\phi$ represent the sampled angular shifts.

$$
\begin{aligned}
& \mu_{x 2}=\mu_{x 1} \mu+\sqrt{\frac{1-\mu^{2}}{1-\mu_{z 1}^{2}}}\left(\mu_{x 1} \mu_{z 1} \cos \phi-\mu_{y 1} \sin \phi\right) \\
& \mu_{y 2}=\mu_{y 1} \mu+\sqrt{\frac{1-\mu^{2}}{1-\mu_{z 1}^{2}}}\left(\mu_{y 1} \mu_{z 1} \cos \phi+\mu_{x 1} \sin \phi\right) \\
& \mu_{z 2}=\mu_{z 1} \mu-\sqrt{\left(1-\mu^{2}\right)\left(1-\mu_{z 1}^{2}\right)} \cos \phi
\end{aligned}
$$

\subsection{3 (n,xn) and $(\mathbf{n}, \alpha)$ Reactions}

There are a number of interactions that we must model very approximately, in far less intricacy than elastic scattering. First let us consider $(n, x n)$ reactions, in which a nucleus absorbs a neutron and subsequently emits at least two. In our set of hybrid blanket materials, we only encounter $(n, 2 n)$ and $(n, 3 n)$ reactions of significant magnitude. Since this is not a scattering interaction, the neutrons are emitted isotropically. We do not kill or split the particle 
but simply increase its weight by $x$. Other than fission, this is the only circumstance under which our Monte Carlo simulation is not strictly analog. For a crude approximation of the emitted neutron energies, we sample them from a simple truncated Maxwellian distribution with an average energy 10 times less than the incident neutron energy. This is very roughly consistent with ENDF energy distributions.

$$
{ }_{Z}^{A} \mathrm{Q}+\mathrm{n} \rightarrow{ }_{Z}^{A-X} \mathrm{Q}+\mathrm{Xn}
$$

Now let us turn to the tritium breeding reactions. In the case of ${ }^{6} \mathrm{Li}$, the incident neutron is absorbed, and no neutrons are emitted. It is straightforward to tally tritium production. This reaction produces $4.8 \mathrm{MeV}$, which contributes to the total hybrid power.

$$
{ }_{3}^{6} \mathrm{Li}+\mathrm{n} \rightarrow{ }_{2}^{4} \mathrm{He}+{ }_{1}^{3} \mathrm{H}
$$

In the case of the ${ }^{7} \mathrm{Li}$ reaction, a neutron is emitted along with the tritium and $\alpha$-particle. Interestingly, this reaction consumes $2.5 \mathrm{MeV}$ and only occurs at incident energies above that threshold. We compute the emitted neutron energy as inversely proportional to its mass share, just as in a $\mathrm{D}-\mathrm{T}$ fusion reaction. So the emitted neutron energy $E$ in terms of the incident neutron energy $E_{0}$ is $E=\left(E_{0}-2.5 \mathrm{MeV}\right)(12 / 19)$.

$$
{ }_{3}^{7} \mathrm{Li}+\mathrm{n} \rightarrow{ }_{2}^{4} \mathrm{He}+{ }_{1}^{3} \mathrm{H}+\mathrm{n}
$$

\subsubsection{Inelastic Scattering}

From a quantum perspective, inelastic scattering is a misnomer. Physically, it is $(\mathrm{n}, 1 \mathrm{n})$. The nucleus absorbs a neutron, forms a compound nucleus, and ejects a neutron. The ejected neutron is not necessarily the same neutron that was absorbed - that is unknowable, because identical nucleons are indistinguishable. In the case of elastic potential scattering, we know that the incident and scattered neutron are one and the same.

Unlike $(n, 2 n)$ and $(n, 3 n)$ reactions, inelastic scattering comprises a substantial portion of the uranium cross-sections at high energy. While elastic scattering by uranium has only a very small effect on the flux energy distribution, inelastic scattering causes neutrons to lose large fractions of their energies and thus has an enormous effect on the flux energy distri-

bution. In the case of ${ }^{238} \mathrm{U}$, inelastic scattering effectively "pushes" the neutron spectrum away from the fissile threshold. If the ${ }^{238} \mathrm{U}$ cross-section were renormalized without inelastic scattering, the natural uranium $k_{\infty}$ would be well above 1.0 (the actual value is less than $0.3)$.

We will assume that inelastic scattering is isotropic, just like the emission of secondary neutrons in $(\mathrm{n}, 2 \mathrm{n})$ and $(\mathrm{n}, 3 \mathrm{n})$ reactions. The sampling of energy loss in inelastic scattering is convoluted. The ENDF repository contains a plethora of applicable distributions, but 
in the interest of keeping our code clean and simple, we will introduce an average energy loss parameter. This parameter is the ratio of the ejected neutron energy to the absorbed neutron energy. We calibrate it with MCNP, and it is typically near 0.4 .

\subsubsection{Fission}

Although our Monte Carlo simulation is largely analog, we do employ weights for fission and $(\mathrm{n}, \mathrm{xn})$ reactions. In $(\mathrm{n}, \mathrm{xn})$ reactions, we simply multiply the neutron weight by $x$. This is acceptable, because $(\mathrm{n}, \mathrm{xn})$ reactions constitute only a small portion of neutron multiplication. However, in the case of fission, simply multiplying neutron weights by $\nu$ would be extremely problematic for $k$ convergence. In this code, we define $k$ as the ratio of the number of neutrons produced by fission in each successive generation (except the first generation, which is produced by fusion). We tally them at the instant they are produced. Imagine a situation with $k=1.2, \nu=3.0$, and 0.4 fissions per neutron in each generation. Then the number of neutron histories in the $n$th generation would be $0.4^{n}$, and the total weights of those neutrons would be $1.2^{n}$. Eventually, only a few particles would hold tremendous weight. The simulation would end prematurely when the last particle, holding the entire weight of the system, is absorbed. This would be ridiculous. It would be "variance expansion", the antithesis of variance reduction.

So we must split particles at fission. We could do this the purely analog way with a discrete distribution function for the number of particles that are released in fission (this would average to $\nu$ ). However, a more interesting and flexible way to manage fission is to stipulate that each fission-inducing neutron splits into a fixed number of next-generation neutrons. We will call this fixed integer $\Psi$, because we like pictograms. We can vary $\Psi$ depending on the criticality of our system and convergence preferences. Large $\Psi$ values will increase variance reduction, while small $\Psi$ values will decrease variance reduction ("variance expansion"). If the weight of each fission-inducing particle is $w_{0}$, then the weight of each resulting fission-born neutron is

$$
w=w_{0}\left(\frac{\nu}{\Psi}\right)
$$

For any given value of $k$, we can choose $\Psi$ such that the number of particle histories (not particle weights) increases or decreases with each subsequent generation. If $\Psi$ is below a certain threshold, the number of particle histories will continually decrease to zero, ending the simulation. If $\Psi$ is above that same threshold, the number of particle histories will increase, the simulation will be an infinite loop, and we will need to truncate it after a certain number of generations. The threshold is

$$
\Psi=\frac{k}{\bar{\nu}}
$$


Here $\bar{\nu}$ is $\nu$ averaged over the energy spectrum. When this equality holds, the number of histories will remain roughly constant in each generation. It now makes sense to define a new kind of $k$, one that applies not to total particle weight but to the number of particle histories. Let this be $k_{H}$.

$$
k_{H}=\frac{k \Psi}{\bar{\nu}}
$$

$k_{H}$ describes the number of particle histories in precisely the same way that $k$ describes the total particle weight. $k_{H}$ is the ratio of particle histories in one generation to the previous generation. When $k_{H}<1$, all particles will eventually be absorbed. When $k_{H} \approx 1$, the number of particle histories remains constant in each generation, and the system is "simulation critical". When $\Phi=\bar{\nu}, k_{H}=k$, and the system is nearly analog in the sense that all particle weights remain near 1.0. In the case of our subcritical hybrid blanket, we know that $k$ is much less than 1 , actually near 0.3 . It makes sense for us to set $k_{H}$ much higher at around 0.9 . We would like to keep $k_{H}<1$ so that our simulation terminates itself when all neutrons are absorbed, but we want $k_{H}$ to be higher than $k$ in order to take advantage of variance reduction. Otherwise, a much larger number of initial neutrons would be required to converge a system with such a low $k$.

To summarize, there are two important properties of $k_{H}$. First, variance reduction occurs when $k_{H}>k$. Second, a Monte Carlo simulation will terminate itself when $k_{H}<1$ regardless of $k$, because all neutrons will eventually be absorbed.

Our Monte Carlo code tracks one generation at a time. When a fission occurs, we kill the incident neutron, sample the emitted neutron properties, and store the fission location until the subsequent generation.

\subsection{MCNP Benchmark}

Now that we have explained our Monte Carlo model, we must ensure that it is accurate. We must compare it to a reputable code. We choose MCNP.

First, in order to test our cross-sections, tracking, and fission methodology, we compare $k_{\infty}$ as a function of uranium enrichment for $\mathrm{UO}_{2}$ and pure $\mathrm{U}$ metal. Since $k_{\infty}$ is purely a function of material properties (in a homogenous medium), this eliminates concerns relating to our geometry model and algorithms. Figure 2.19 shows the results. Evidently, our code yields results quite consistent with $\mathrm{MCNP}$. $\mathrm{UO}_{2}$ has a slightly lower $k_{\infty}$, because the oxygen moderates neutrons enough to push the spectrum away from the high ${ }^{238} \mathrm{U}$ cross-section but not enough to push it anywhere near the thermal region. Infinite $\mathrm{UO}_{2}$ becomes critical at about $8 \%$ enrichment, while $\mathrm{U}$ metal becomes critical at about $6 \%$ enrichment. Both materials have $k_{\infty} \approx 2.25$ at $100 \%$ enrichment. 
Now that we are confident that our cross-sections, tracking, and fission methodology are correct, we can ensure that our geometry is correct by comparing our complete hybrid model to MCNP. Figure 2.20 shows the results. Here we compare the initial neutron multiplication $k_{0}$ and the asymptotic neutron multiplication $k$ for varying uranium pebble layer thickness. For an in-depth discussion of these quantities, see the Section 5.2. Clearly, these quantities are in fair agreement with MCNP. There is certainly some deviation, but this is much smaller than the deviation that would occur between cylindrical and toroidal models. We deem this close enough for a scoping study. 


\subsection{Pebble Homogenization}

Until now, we have assumed that homogenizing our uranium pebbles and helium coolant does not significantly affect the reactor physics. This is a reasonable assumption, because the neutron mean free path in helium is orders of magnitude larger than that in $\mathrm{UO}_{2}$. Also, since the neutron spectrum is fast, mean free paths will generally be larger than the pebble diameter.

It is still interesting to quantify the validity of pebble bed homogenization. We perform kcode calculations in MCNP for $\mathrm{UO}_{2}$ pebbles of varying diameter in helium. These pebbles are in an infinite standard cubic array. We find that pebble size and homogenization make virtually no difference in this case of helium-cooled $\mathrm{UO}_{2}$ pebbles, so there is no problem with homogenization.

However, we were interested in studying pebble bed homogenization further, so we performed the same MCNP runs with water in place of helium. Figure 2.21 shows $k_{\infty}$ as a function of pebble size. Again, the pebbles are in infinite standard cubic formation. The zero pebble radius limit corresponds to homogenization. $k_{\infty}$ initially increases as the pebbles grow larger, and the $k_{\text {eff }}$ for each individual pebble increases. When the pebble radius is roughly equal to the $1 \mathrm{MeV}$ neutron mean free path in $\mathrm{UO}_{2}$ and $\mathrm{H}_{2} \mathrm{O}$ (which is approximately $1.8 \mathrm{~cm}$ for both), $k_{\infty}$ attains a maximum and subsequently decreases far below the homogenized $k_{\infty}$. This is because neutrons can no longer easily traverse the gaps between pebbles. In this case of $\mathrm{UO}_{2}$ pebbles in $\mathrm{H}_{2} \mathrm{O}$, a pebble radius of less than $0.1 \mathrm{~cm}$ is necessary for homogenization to be an accurate approximation. Although this particular example is not applicable to our hybrid, it provides physical insight into why homogenization is generally valid. 


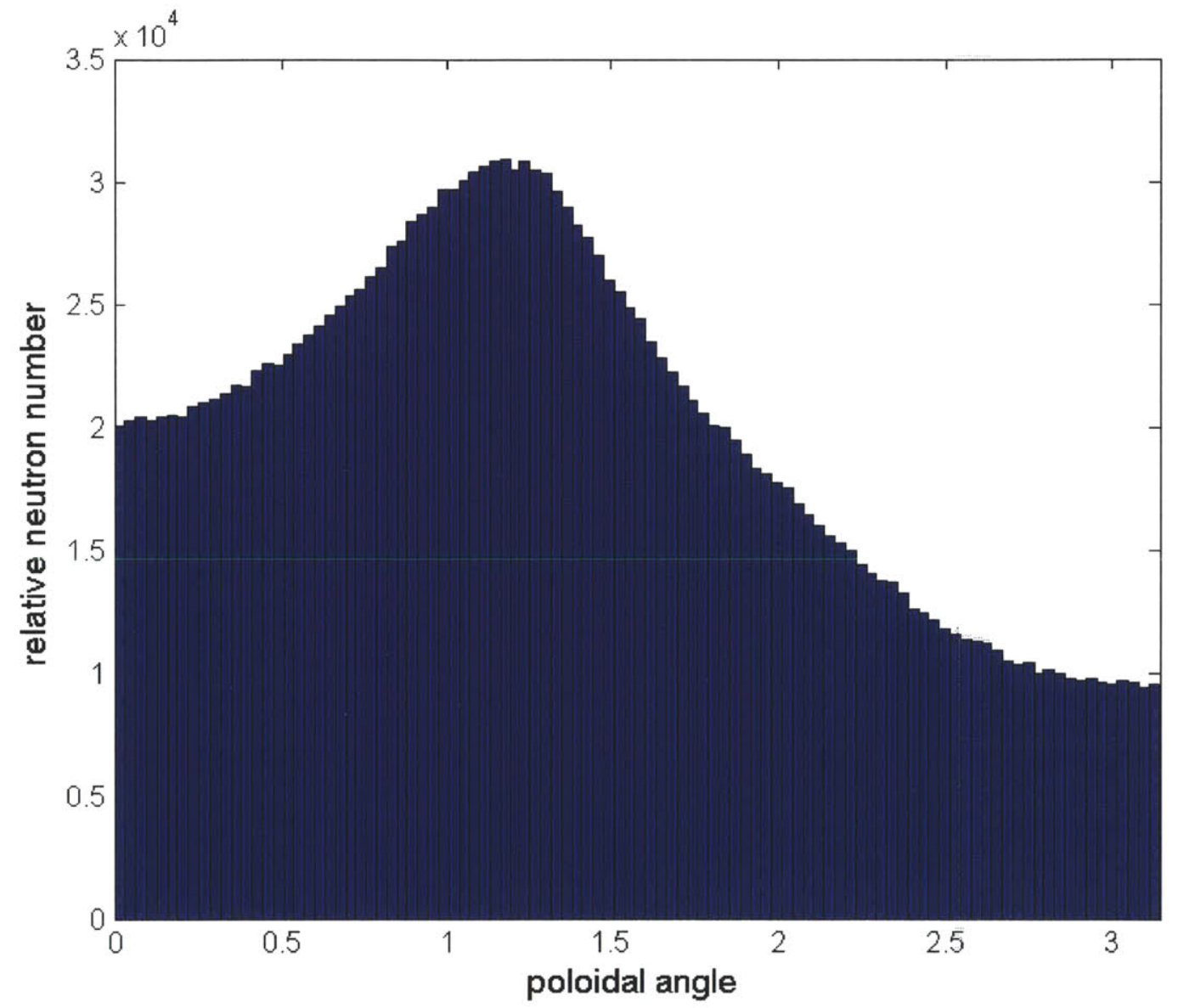

Figure 2.4: Scalar neutron flux at the plasma surface as a function of poloidal angle $\Theta$. $\Theta=0$ corresponds to the outermost point on the tori $\left(x^{2}+y^{2}=(R+a)^{2}\right)$, while $\Theta=\pi$ corresponds to the innermost point $\left(x^{2}+y^{2}=(R-a)^{2}\right)$. The flux is about twice as large at the outermost point than at the innermost point, and there is a maximum at approximately 1.2 radians. 


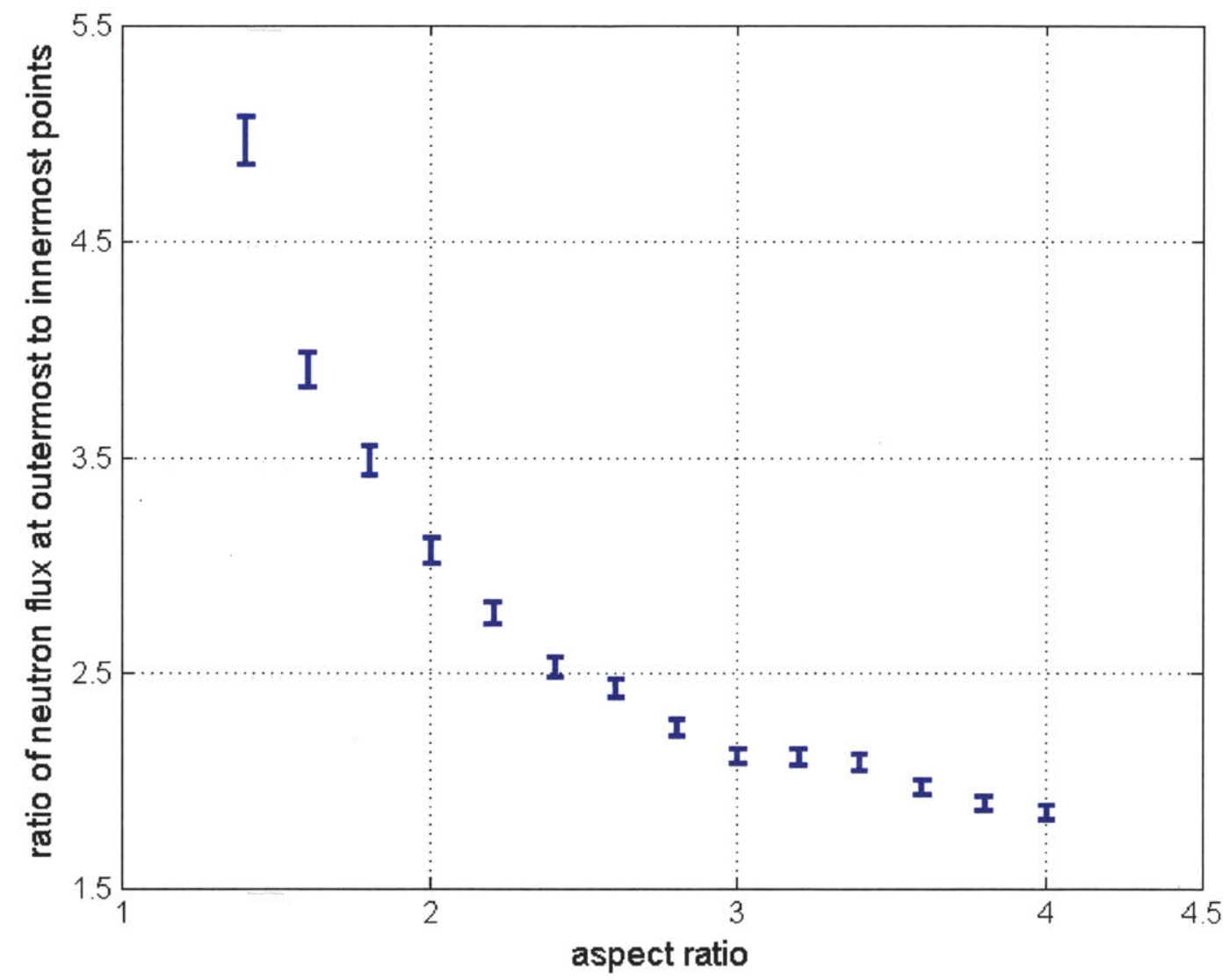

Figure 2.5: The ratio of scalar neutron flux at the outermost point $\left(x^{2}+y^{2}=(R+a)^{2}\right)$ to the innermost point $\left(x^{2}+y^{2}=(R-a)^{2}\right)$ as a function of tokamak aspect ratio $R / a$. As $R / a$ grows very large, the toroid resembles a cylinder, and the flux ratio approaches 1 . Here the elongation $\kappa$ is 1.75 . 


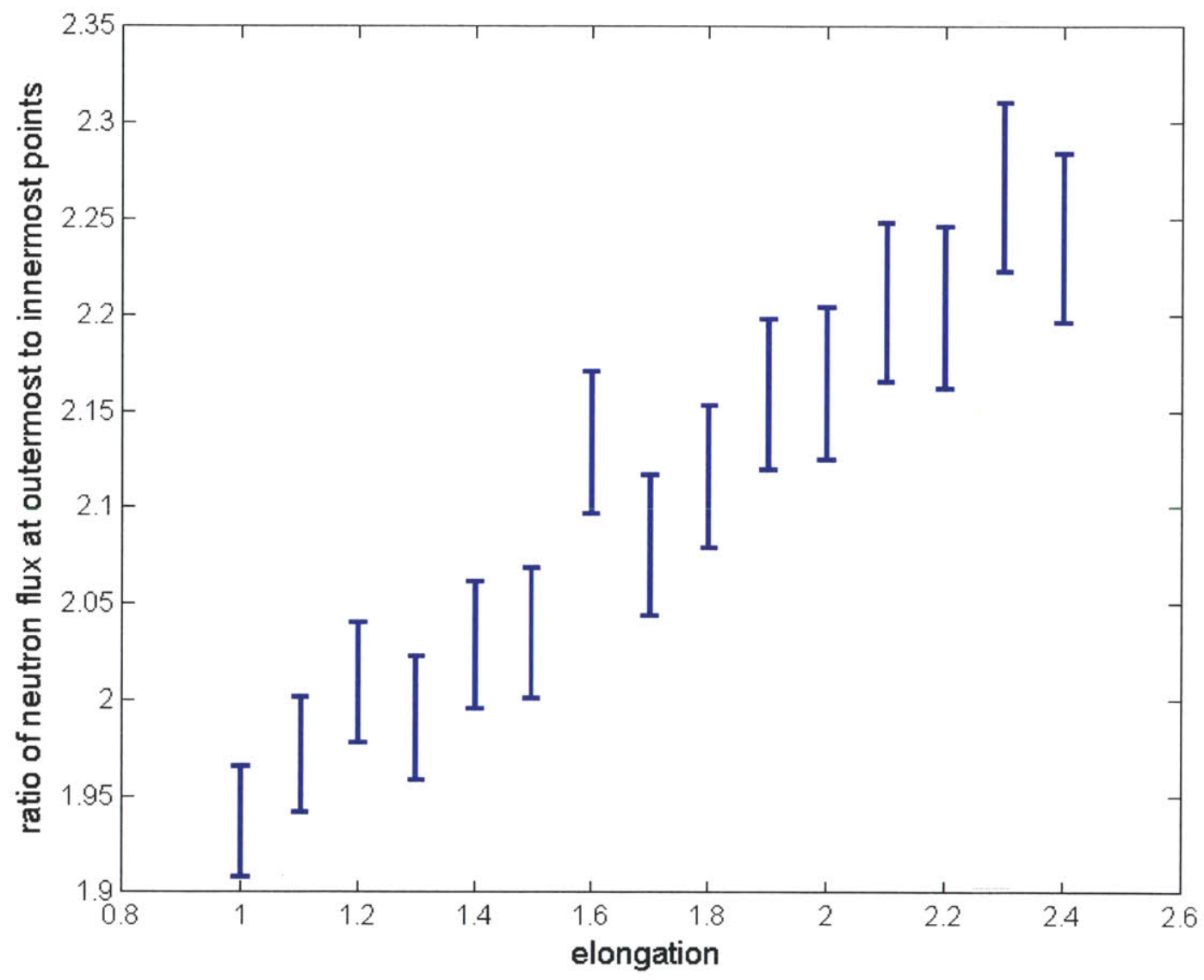

Figure 2.6: The ratio of scalar neutron flux at the outermost point $\left(x^{2}+y^{2}=(R+a)^{2}\right)$ to the innermost point $\left(x^{2}+y^{2}=(R-a)^{2}\right)$ as a function of tokamak elongation $\kappa$. As $\kappa$ increases, the flux ratio increases moderately. Here the aspect ratio $R / a$ is 3.1 . 


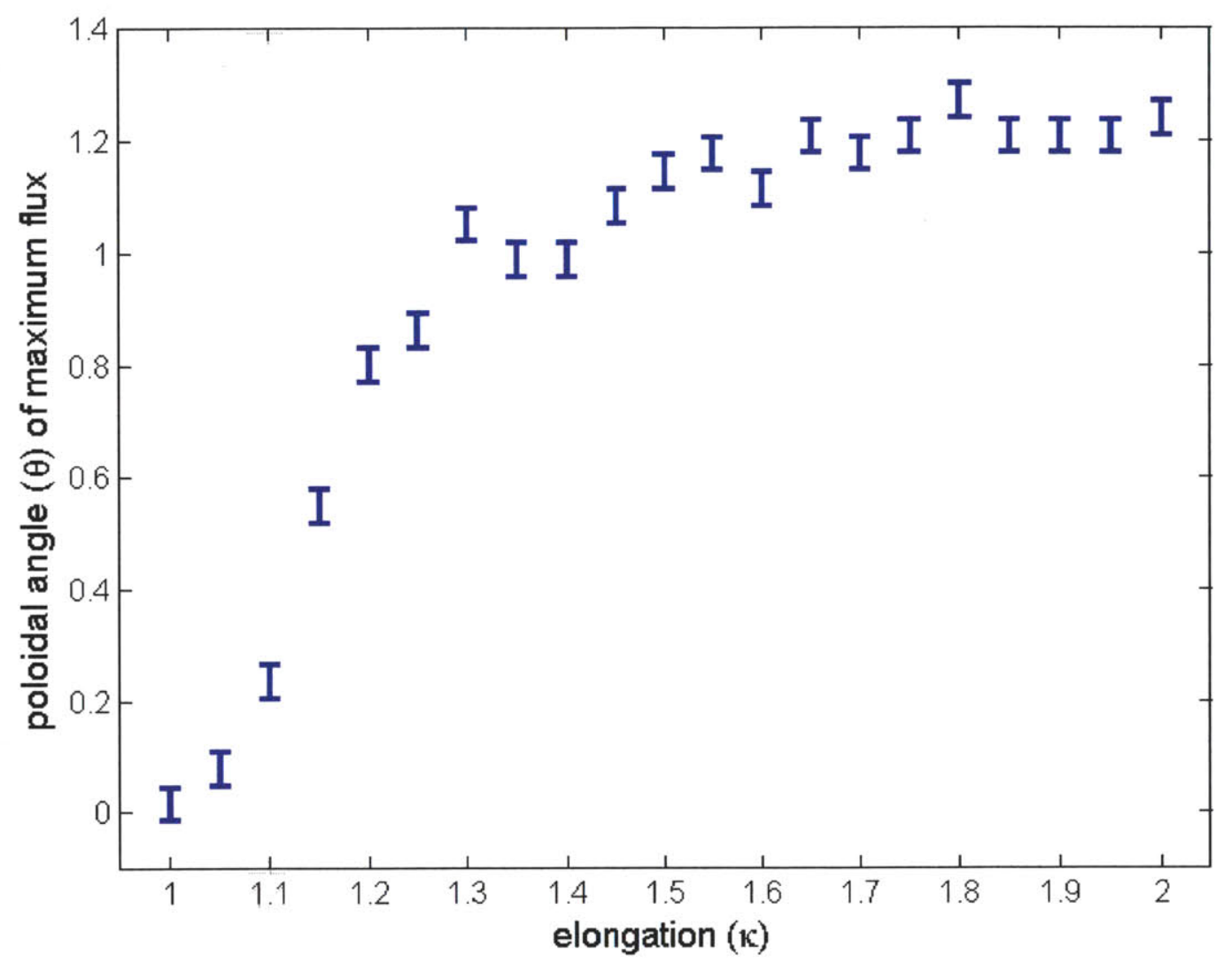

Figure 2.7: The poloidal angle $\Theta$ of maximum scalar neutron flux as a function of tokamak elongation $\kappa$. Here the aspect ratio $R / a$ is 3.1 . When $\kappa=1$, the tokamak's poloidal cross-section is a circle, and the maximum flux occurs at the outermost point $\Theta=0$. Thus, Figure 2.4 would be a continuously decreasing function for $\kappa=0$. As $\kappa$ increases, the angle of maximum flux approaches 1.2 radians. 

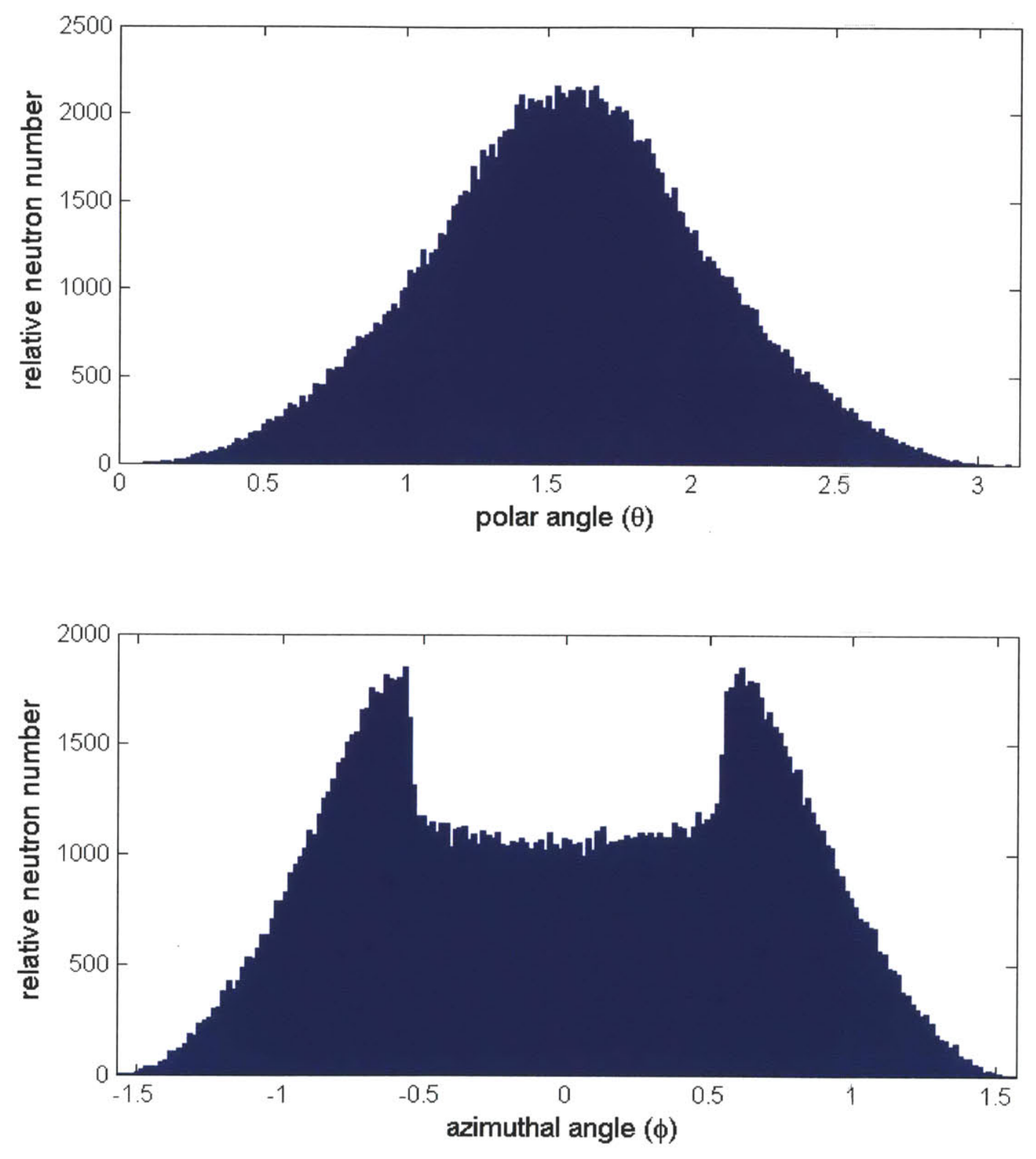

Figure 2.8: The angular neutron flux distribution in standard spherical coordinates $(\theta, \phi)$ at the outermost point on the plasma surface $(\Theta=0)$. The camelback shape of the azimuthal distribution reflects the two "arms" of the toroid visible from this point. See Figure 2.9 for further illumination on this. 

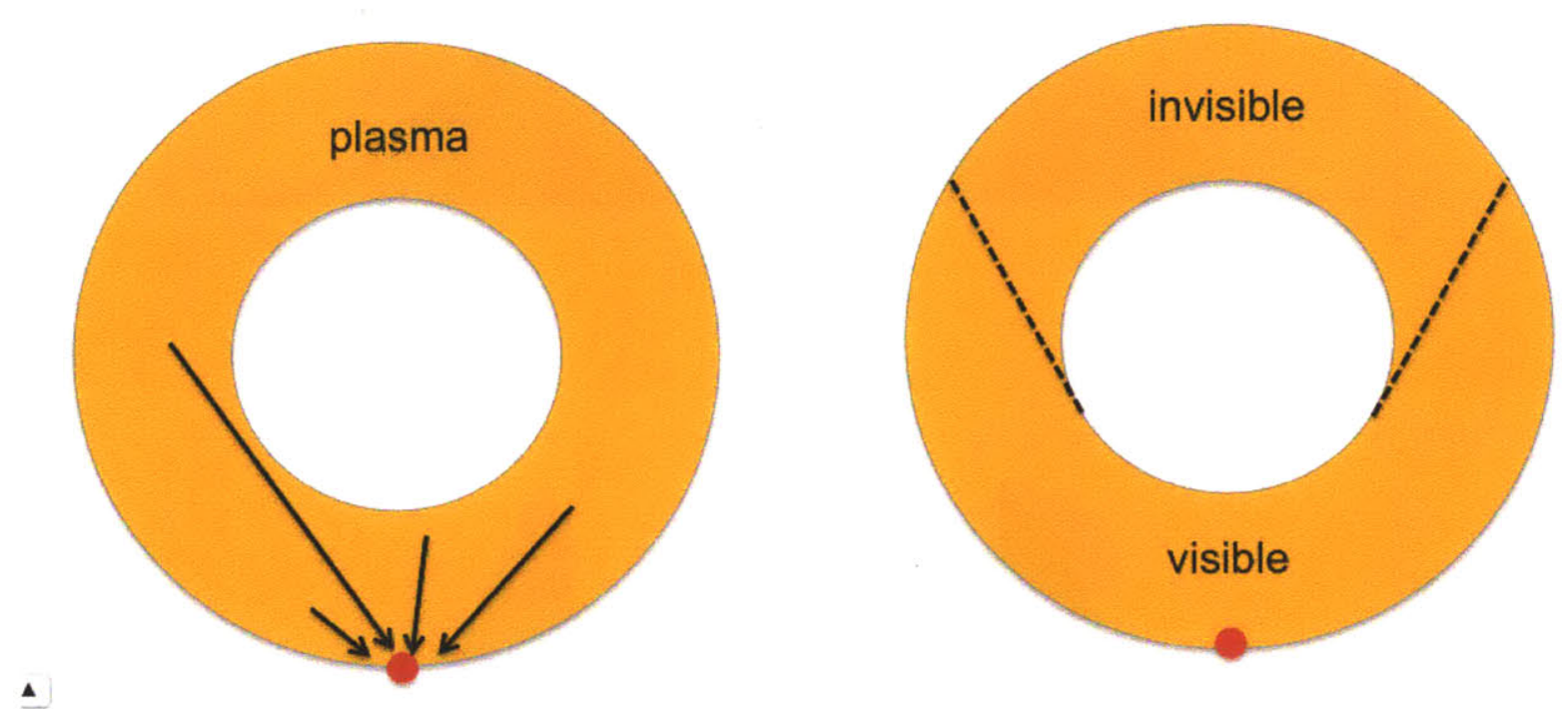

Figure 2.9: Neutrons that impinge on a given point on a tokamak surface originate from D-T fusion reactions at all points in the plasma that are "visible" from that surface point. This clarifies the camelback shape of the azimuthal distribution in Figure 2.8. Of course, this "visible" concept only applies to neutrons that intersect the tokamak surface for the first time. 

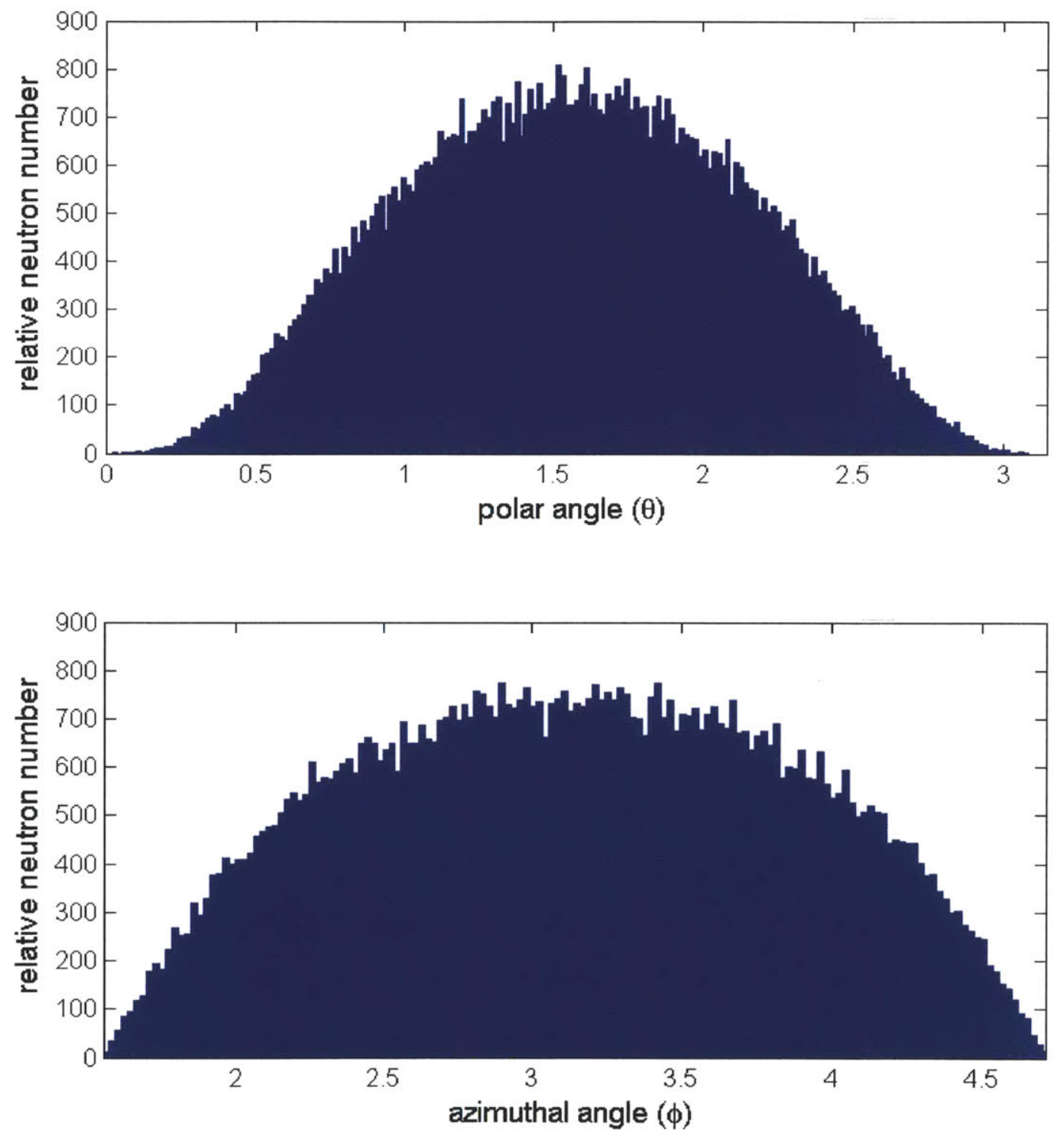

Figure 2.10: The angular neutron flux distribution in standard spherical coordinates $(\theta, \phi)$ at the innermost point on the plasma surface $(\Theta=\pi)$. Now there is no camelback shape, because most of the tokamak curvature is not visible from this point. 

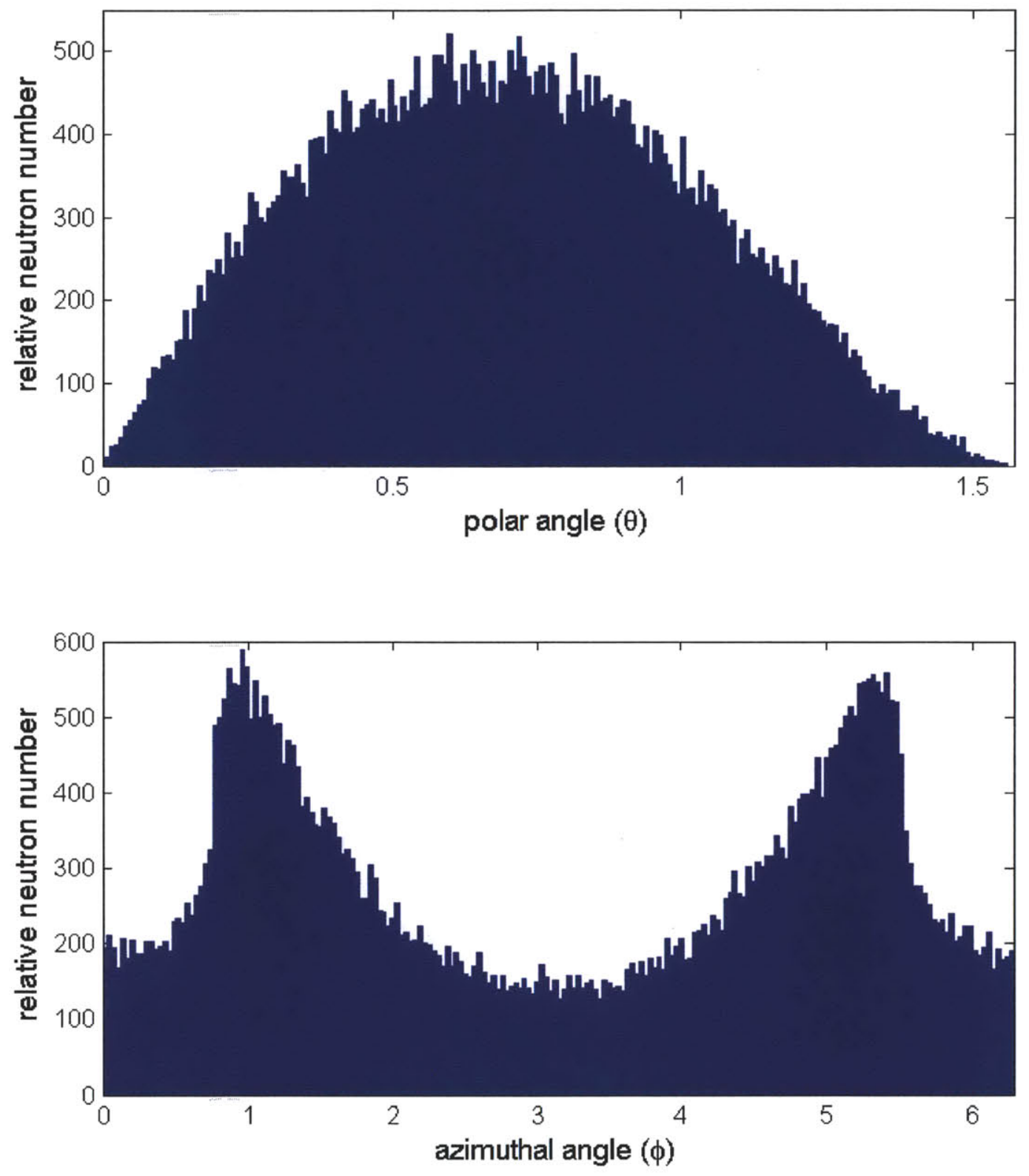

Figure 2.11: The angular neutron flux distribution in standard spherical coordinates $(\theta, \phi)$ at the topmost point on the plasma surface $(\Theta=\pi / 2)$. The camelback shape of the azimuthal distribution reflects the two "arms" of the toroid visible from this point. See Figure 2.9 for further illumination on this. The azimuthal distribution exhibits a camelback shape similar to Figure 8 and reveals the inner curvature of the tokamak quite nicely. The polar angle distribution approaches at $\theta=0$ only because the spherical integrand $\sin \theta d \theta$ approaches zero. 


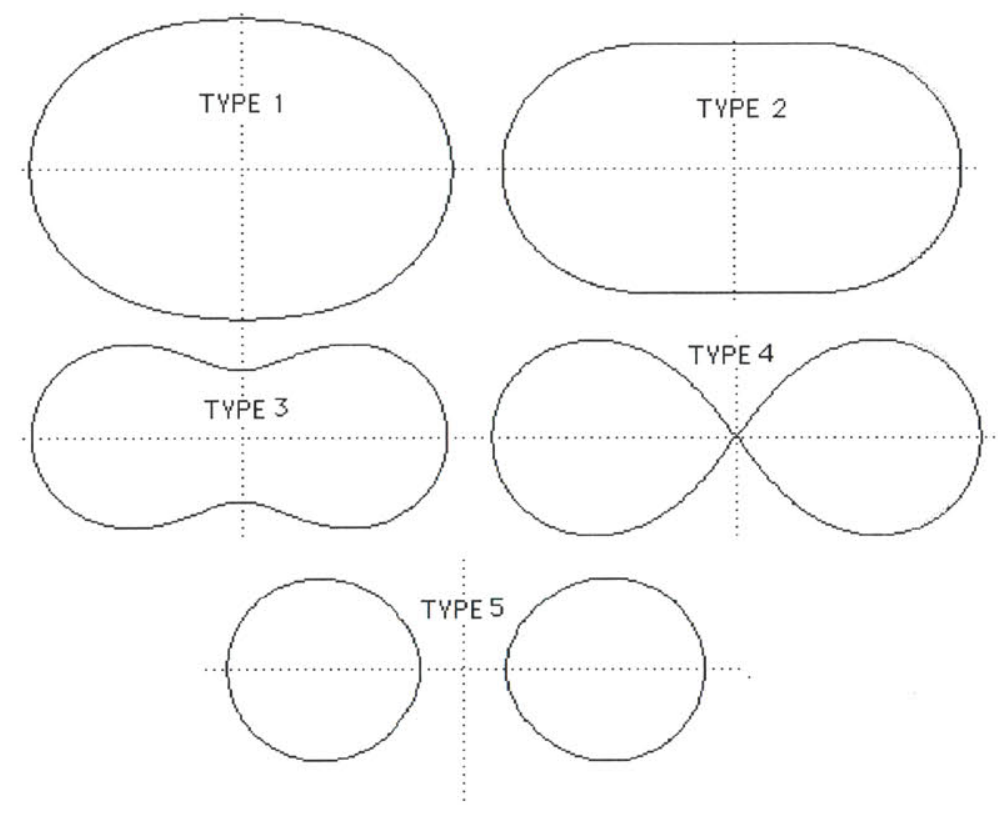

Figure 2.12: Five spiric sections of a torus. These are the five general shapes that arise from intersections of tori with planes parallel to the toroidal axis (the $z$ direction).

Profile Summary
Generated 21-Jul-2010 18: 13:45 using cpu time.
$\underline{\text { Function Name }}$
$\underline{\text { toroidalMatrix }}$
$\underline{\underline{\text { toroidalAlgebraic }}}$
$\underline{\text { roots }}$
$\underline{\text { quarticAlgebraic }}$
$\underline{\text { toroidalMatrix }>\text { inplasma }}$
toroidalAlgebraic>inplasma

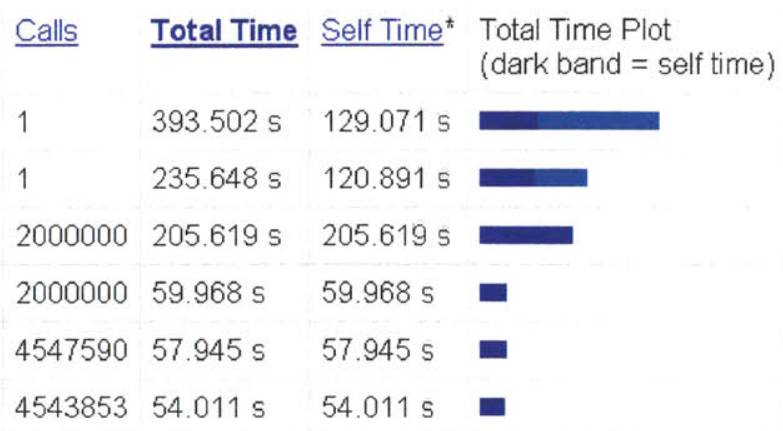

Figure 2.13: A CPU runtime analysis of our tokamak surface neutron flux Monte Carlo code comparing the MATLAB function roots to a direct calculation of Ferrari's method. toroidalMatrix is the entire code using roots, and toroidalAlgebraic is the entire code using quarticAlgebraic, which contains Ferrari's method. Clearly, a direct implementation of Ferrari's method is superior to roots and reduces runtime by $27 \%$. 


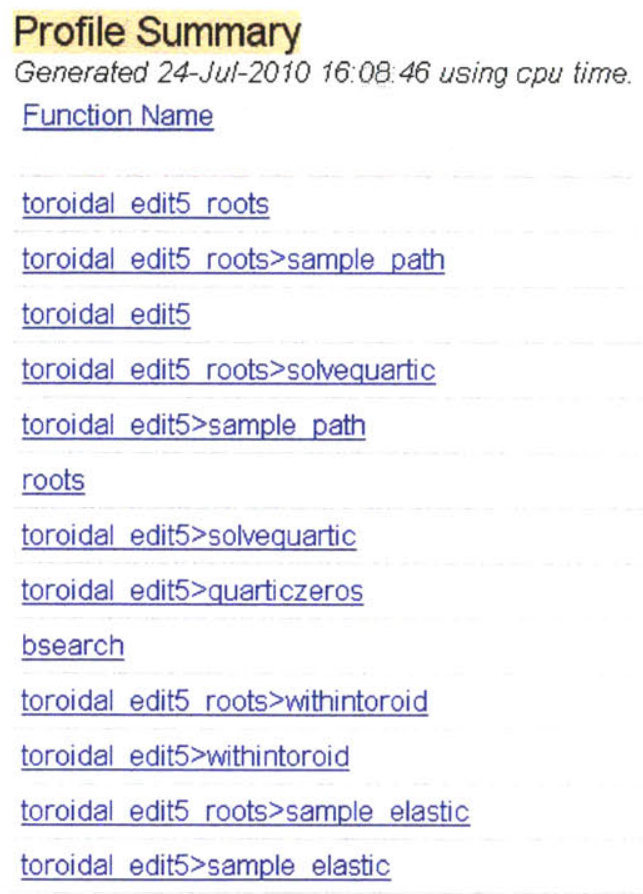

\begin{tabular}{ll|lll}
\hline Calls & Total Time & Self Time $^{*}$ & $\begin{array}{l}\text { Total Time Plot } \\
\text { (dark band = self time) }\end{array}$ \\
\hline 1 & $29492.277 \mathrm{~s}$ & $2570.775 \mathrm{~s}$ & $\mathbf{}$ \\
\hline 15357168 & $24184.381 \mathrm{~s}$ & $3543.529 \mathrm{~s}$ & \\
\hline 1 & $21617.886 \mathrm{~s}$ & $2525.610 \mathrm{~s}$ & \\
\hline 76785840 & $20640.852 \mathrm{~s}$ & $7262.997 \mathrm{~s}$ & \\
\hline 15337679 & $16409.436 \mathrm{~s}$ & $3451.095 \mathrm{~s}$ & \\
\hline 76785840 & $13377.855 \mathrm{~s}$ & $13377.855 \mathrm{~s}$ & \\
\hline 76688395 & $12958.341 \mathrm{~s}$ & $6873.371 \mathrm{~s}$ & \\
\hline 76688395 & $6084.969 \mathrm{~s}$ & $6084.969 \mathrm{~s}$ & \\
\hline 30694847 & $3448.438 \mathrm{~s}$ & $3448.438 \mathrm{~s}$ & $\mathbf{0}$ \\
\hline 15584351 & $691.097 \mathrm{~s}$ & $691.097 \mathrm{~s}$ & $\mathbf{1}$ \\
\hline 15565119 & $660.618 \mathrm{~s}$ & $660.618 \mathrm{~s}$ & $\mathrm{I}$ \\
\hline 6379514 & $219.259 \mathrm{~s}$ & $219.259 \mathrm{~s}$ & $\mathrm{I}$ \\
\hline 6378266 & $218.263 \mathrm{~s}$ & $218.263 \mathrm{~s}$ & $\mathrm{I}$
\end{tabular}

Figure 2.14: A CPU runtime analysis of our entire hybrid Monte Carlo simulation comparing the MATLAB function roots to a direct calculation of Ferrari's method. toroidal_edit5_roots is the entire code using roots, and toroidal_edit5 is the entire code using quarticAlgebraic, which contains Ferrari's method. Clearly, a direct implementation of Ferrari's method is superior to roots and reduces the total runtime time by over $25 \%$. 


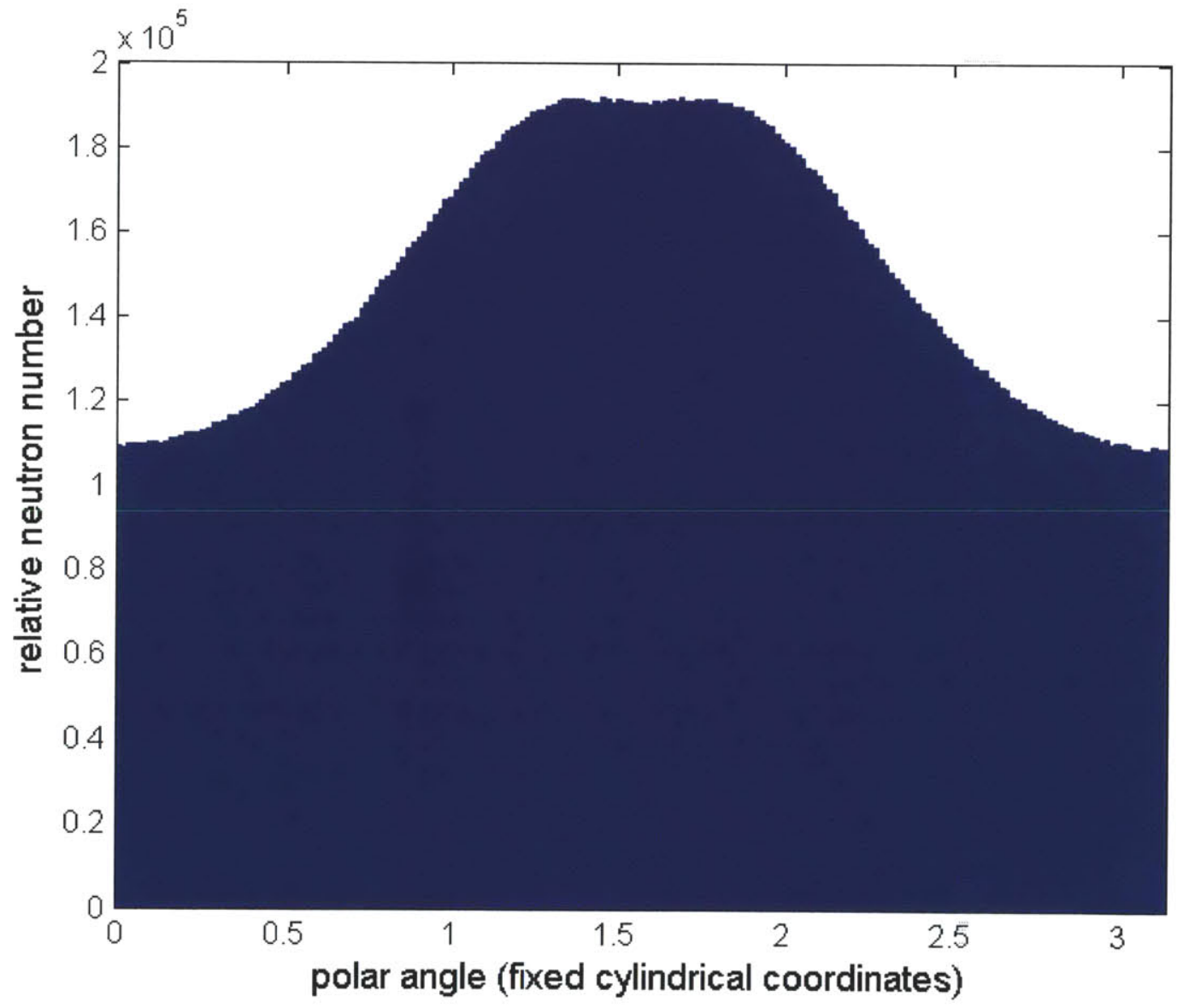

Figure 2.15: Scalar neutron flux at the plasma edge (an elliptic cylindrical surface) as a function of polar angle $\Theta$. The flux achieves its maximum at the elongated ends of the elliptic cylinder. 

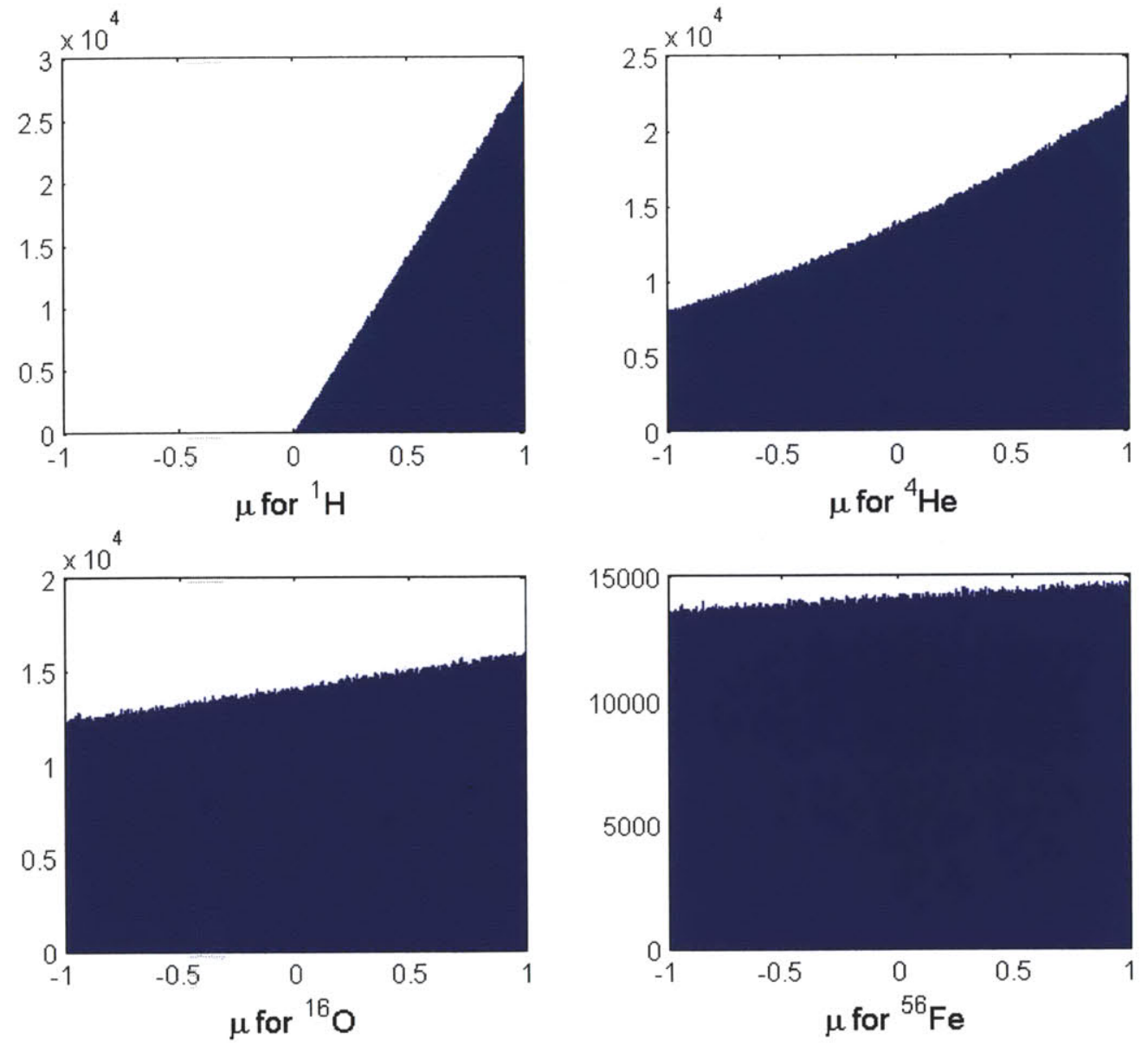

Figure 2.16: Elastic scattering $P\left(\mu_{L}\right)$ for ${ }^{1} \mathrm{H},{ }^{4} \mathrm{He},{ }^{16} \mathrm{O}$, and ${ }^{56} \mathrm{Fe}$. As $A$ increases, the scattering becomes more isotropic. In the case of ${ }^{1} \mathrm{H}$, scattering is anisotropic to the point that backscattering is impossible. 


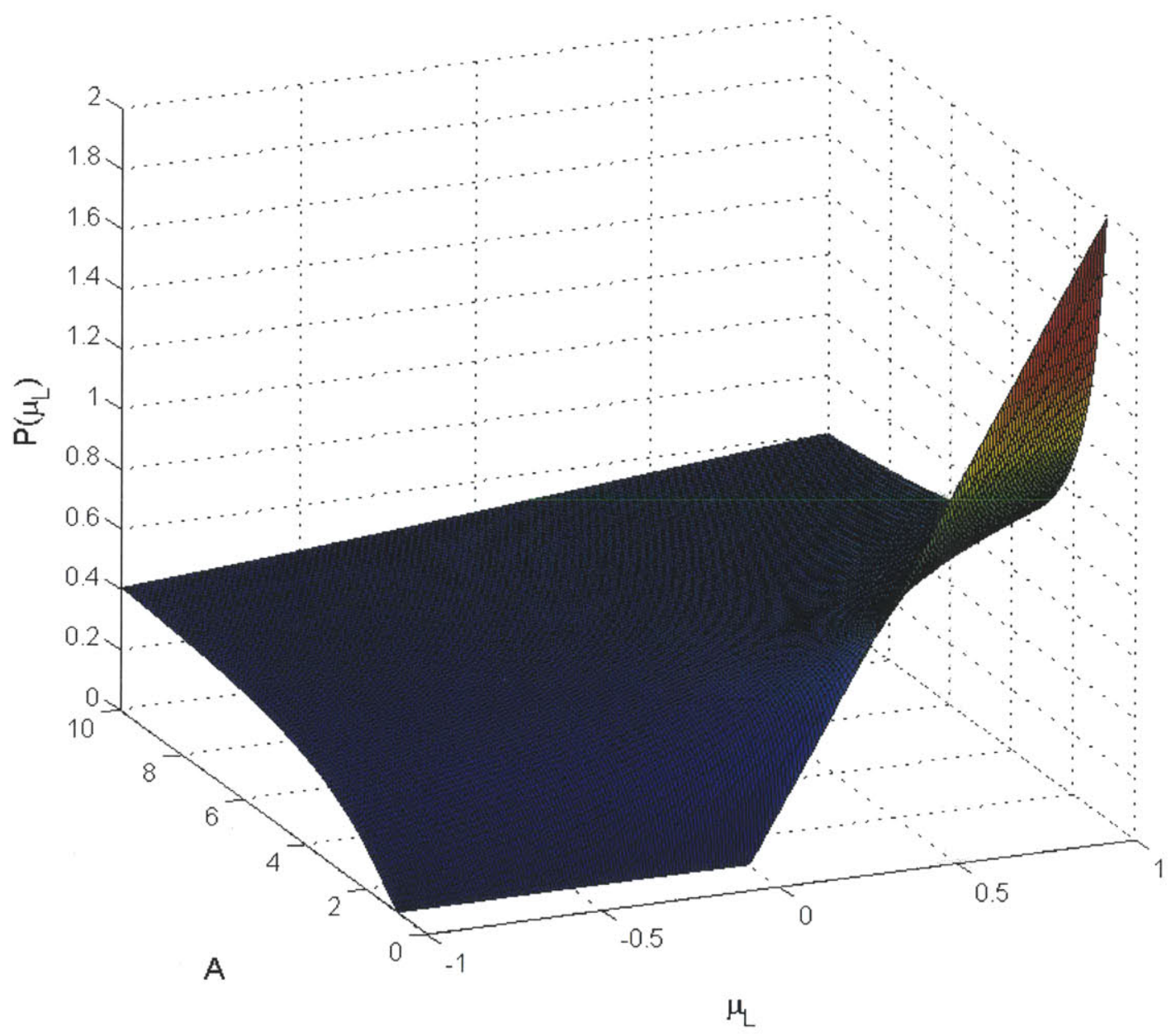

Figure 2.17: The analytical solution for $P\left(\mu_{L}\right)$ for $A=1$ to 10 . We display this as a surface function of both $\mu_{L}$ and $A$. This is consistent with Figure 2.16, which we created with Monte Carlo. 


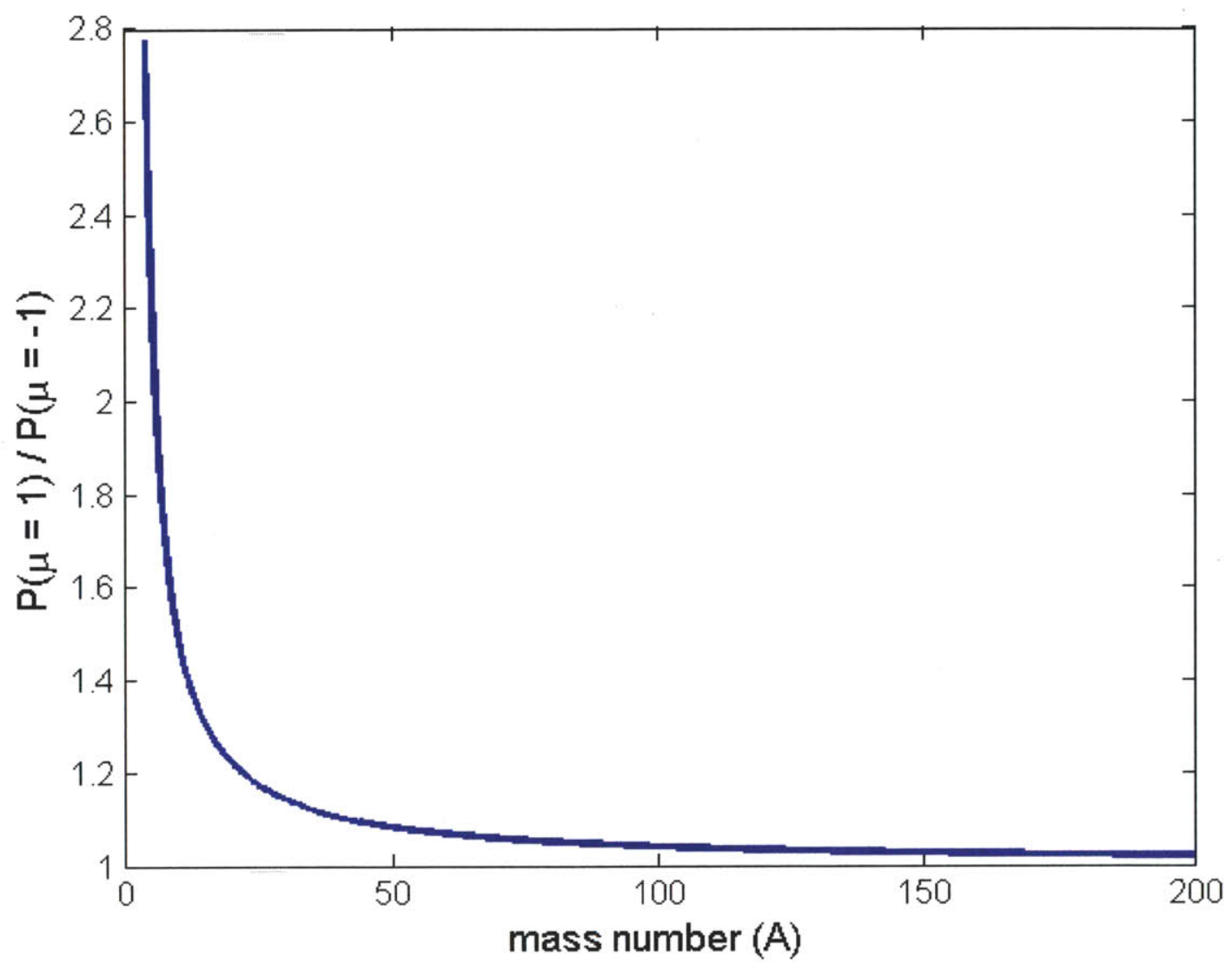

Figure 2.18: $P\left(\mu_{L}=1\right) / P\left(\mu_{L}=-1\right)=\alpha$ as a function of $A$ from $A=4$ to 200. This ratio $\alpha$ is an excellent way to quantify the degree of isotropy of a nuclide. $\alpha$ becomes less than 1.1 at $A \approx 50$. 


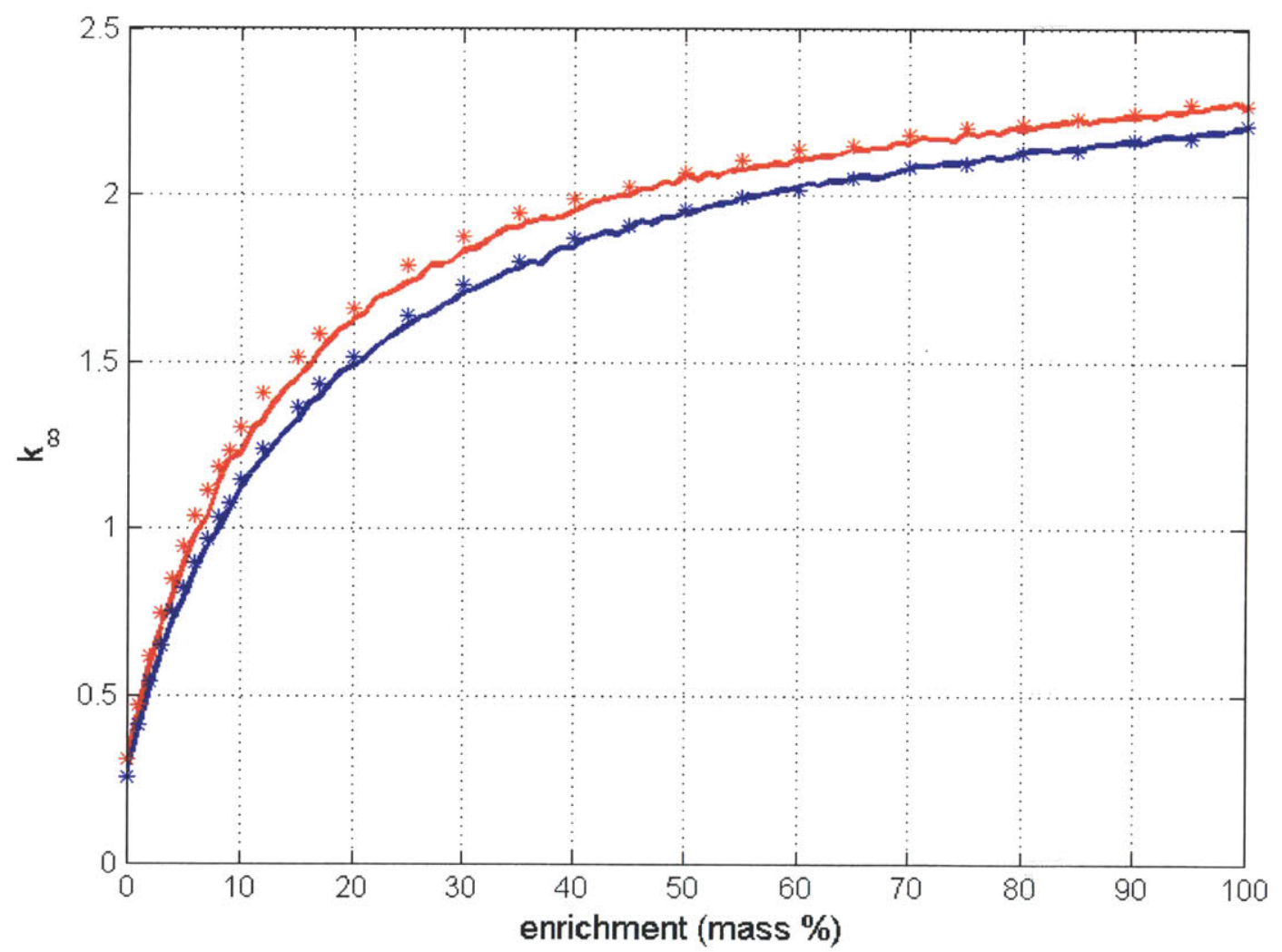

Figure 2.19: $k_{\infty}$ as a function of uranium enrichment for $\mathrm{UO}_{2}$ (blue) and pure $\mathrm{U}$ metal (red). The solid lines represent our Monte Carlo model, and the asterisks represent MCNP. 


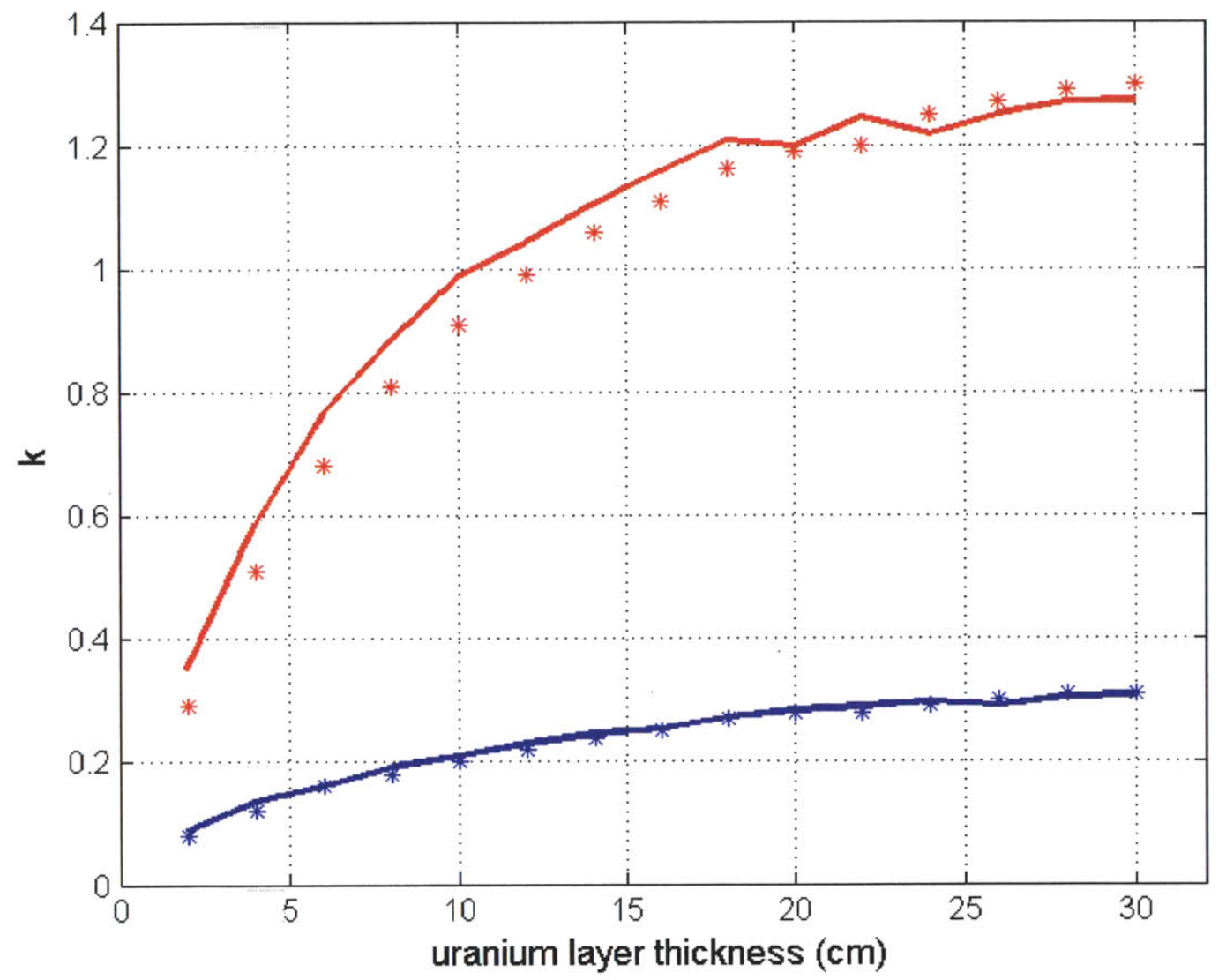

Figure 2.20: The initial neutron multiplication $k_{0}$ (red) and the asymptotic neutron multiplication $k$ (blue) in our hybrid model as a function of uranium pebble layer thickness. The solid lines represent our Monte Carlo model, and the asterisks represent MCNP. 


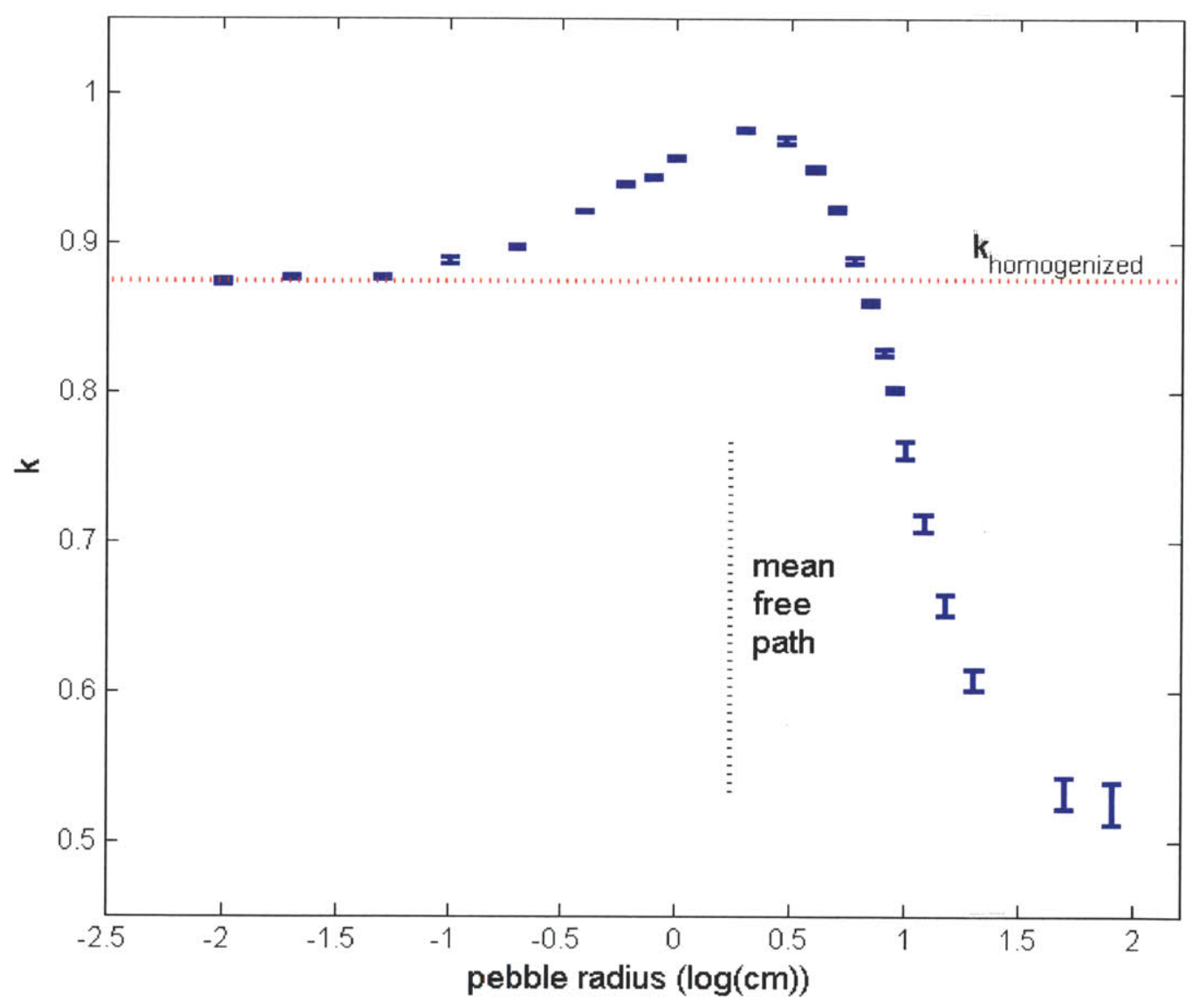

Figure 2.21: $k_{\infty}$ for an infinite standard cubic array of $\mathrm{UO}_{2}$ pebbles in an infinite $\mathrm{H}_{2} \mathrm{O}$ pool. For very small pebble sizes, $k_{\infty}$ corresponds to the homogenized $k_{\infty}$. As the pebbles grow larger, $k_{\infty}$ initially increases but subsequently decreases after the pebble size exceeds the neutron mean free path in $\mathrm{H}_{2} \mathrm{O}$. 


\section{Fission Blanket Analysis}

Now that we have expounded on our Monte Carlo fission blanket model, all that remains is to run it repeatedly to ascertain how fissioning and tritium breeding change with various system parameters. In this section, we will focus on toroidal layer thicknesses, relative positioning of toroidal layers, lithium enrichment, and lithium content in the Li- $\mathrm{Pb}$ alloy.

\subsection{Blanket Parameter Analysis}

Our neutronics model consists of five concentric tori that bound layers of $\mathrm{SiC}$ (the first wall), $\mathrm{UO}_{2}$ pebbles (natural uranium) immersed in helium coolant, liquid Li- $\mathrm{Pb}$ tritium breeder, and a steel and $\mathrm{H}_{2} \mathrm{O}$ shield. Figure 3.1 shows a poloidal cross-section of this model. Of course, an actual device would include additional structural components, but this will suffice as a simple model. It is sufficient for our purposes. See Section 1 for a more detailed description as well as our motivation for choosing this conceptual design. 


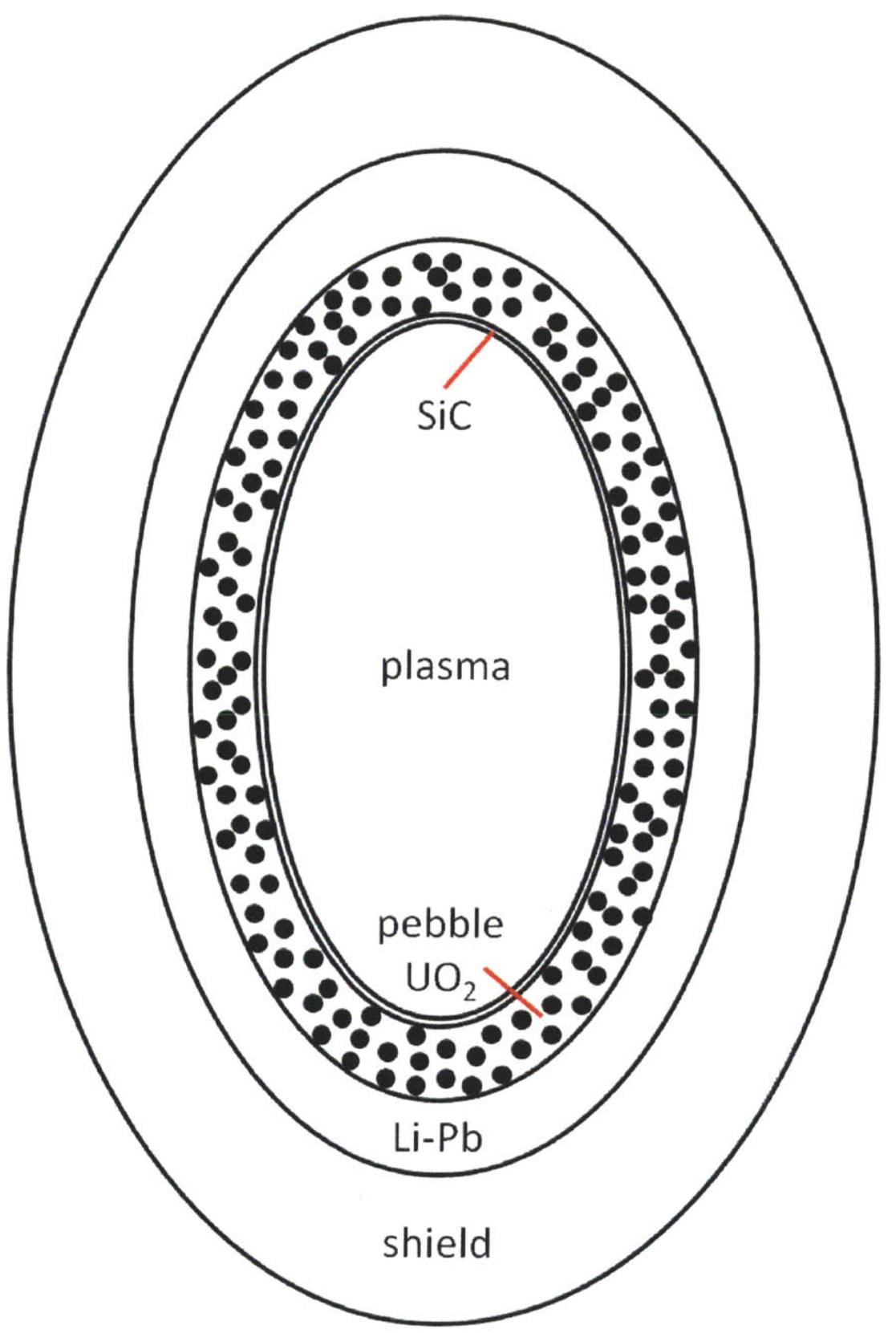

Figure 3.1: A basic schematic of a poloidal cross-section of our neutron transport hybrid model. The toroidal plasma is incased within four toroidal layers: a silicon carbide first wall, a $\mathrm{UO}_{2}$ pebble fission layer with helium coolant, a liquid lithium-lead alloy, and a steel shield. Five concentric tori bound these layers. Layer thicknesses and pebble sizes are not to scale. 


\subsubsection{Uranium Layer Thickness}

First we will show what happens when we vary the uranium pebble layer thickness while holding all other parameters constant. Figure 3.2 shows the two quantities of most interest - fissions per fusion neutron and bred tritons per fusion neutron - as a function of the uranium pebble layer thickness. We will call these two quantities the fission ratio and tritium breeding ratio. When there is no uranium layer, each fusion neutron breeds about 0.65 tritons. As the uranium layer thickens, the each fusion neutron can spur up to about 0.75 fissions. However, after a thickness of about $25 \mathrm{~cm}$, there is little to gain from further thickening. The effect on tritium breeding is much more interesting. When the uranium layer thickens, two competing effects are at play. First, the uranium multiplies the fusion neutrons. Second, the uranium pushes the lithium away from the fusion neutron source. For thin uranium layers (less than $8 \mathrm{~cm}$ ), the first effect is preponderant such that the tritium breeding per fusion neutron more than doubles. However, as the uranium layer thickness surpasses a certain threshold, the second effect dominates such that the tritium breeding per fusion neutron gradually decreases. A uranium layer thickness of $27 \mathrm{~cm}$ would yield the same tritium breeding ratio as in the case of no uranium layer at all. This neat fact is convenient for us. We can insert a sizable fissionable layer without any cost in terms of tritium breeding.

Figure 3.3 shows the initial fission multiplication factors $k_{0}$ (first generation) and $k$ (successive generations) as a function of uranium pebble layer thickness. $k_{0}$ is the ratio of the number of neutrons in the first fission-born generation to the number of neutrons in the initial fusion-born generation. Due to the high fission cross-section of ${ }^{238} \mathrm{U}$ at high energies, $k_{0}$ is well above $1 . k$ is the standard multiplication factor for fission-born neutrons in all successive generations when the flux distribution has converged. $k$ is precisely equivalent to

$k_{\text {eff }}$, which is the common denotation in reactor physics texts. Since we use natural uranium and rely mostly on the fusion-born neutrons to achieve a larger power gain, $k$ is much lower than $k_{0}$. The fission ratio in Figure 3.2 is an increasing function of both $k_{0}$ and $k$, and it is directly proportional to $k_{0}$.

Our goal in this analysis is to maximize the fission ratio while still achieving a tritium breeding ratio slightly greater than 1 . It is paramount that we breed at least one recoverable triton per every triton consumed in a fusion reaction (or per fusion-born neutron). Looking at Figure 3.2, we could easily breed a plethora of tritium, but our fission ratio would be fairly low. We could also achieve a high fission ratio with a very thick uranium layer, but that would thwart tritium breeding altogether. It is simple to see that a uranium layer thickness of slightly less than $20 \mathrm{~cm}$ is optimal. This would produce a fission ratio of about 0.5 (which corresponds to a power gain of about 7) while satisfying the tritium breeding requirements. Of course, here we have held other parameters constant, and so these curves will shift around a bit when we vary those. However, the basic shapes of the curves will not 


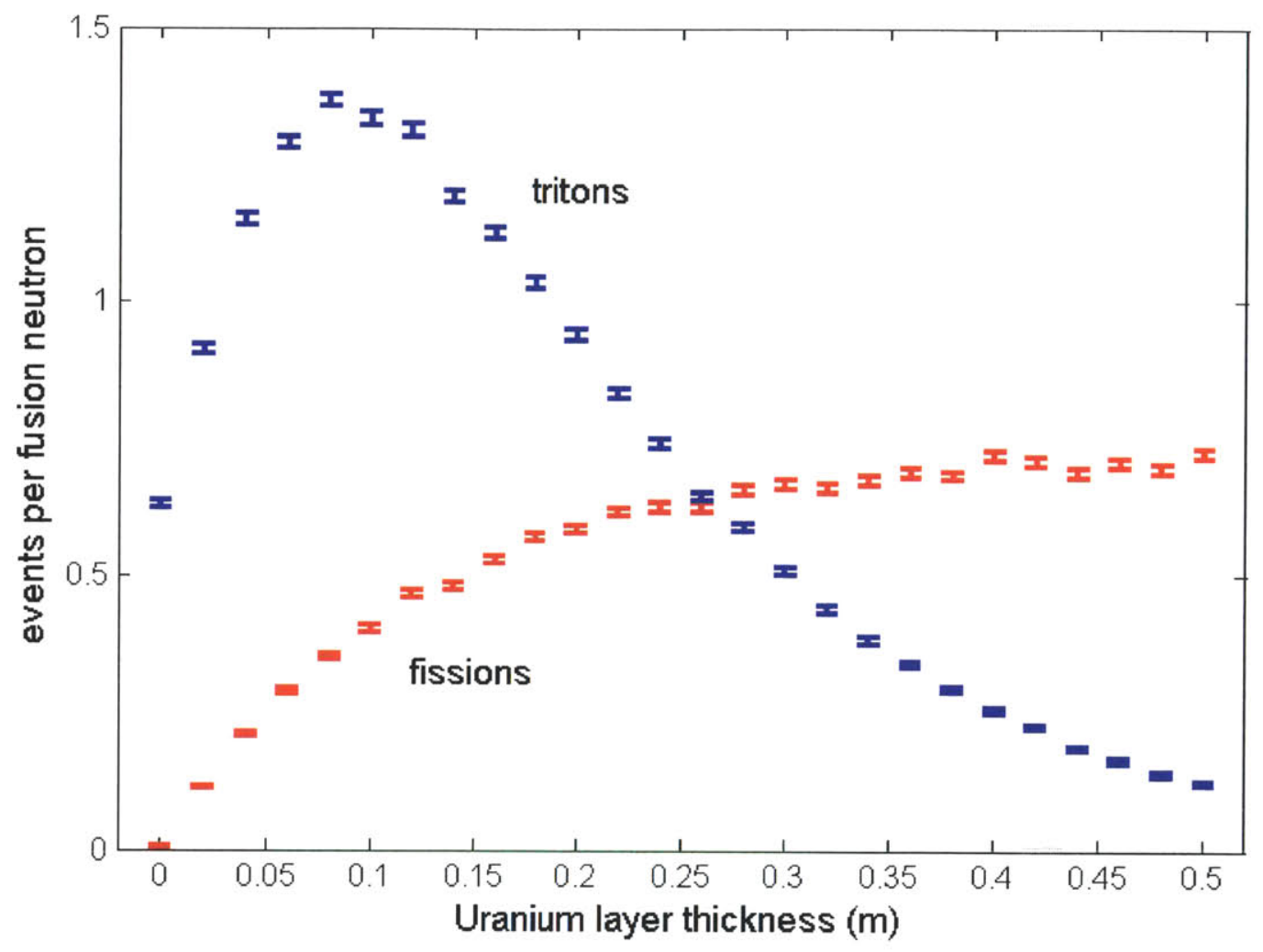

Figure 3.2: Fissions and bred tritons per fusion-born neutron as a function of uranium pebble layer thickness. Here the lithium layer is $30 \mathrm{~cm}$ thick with $90 \%{ }^{6} \mathrm{Li}$ enrichment. The error bars represent Monte Carlo uncertainty.

significantly change. 


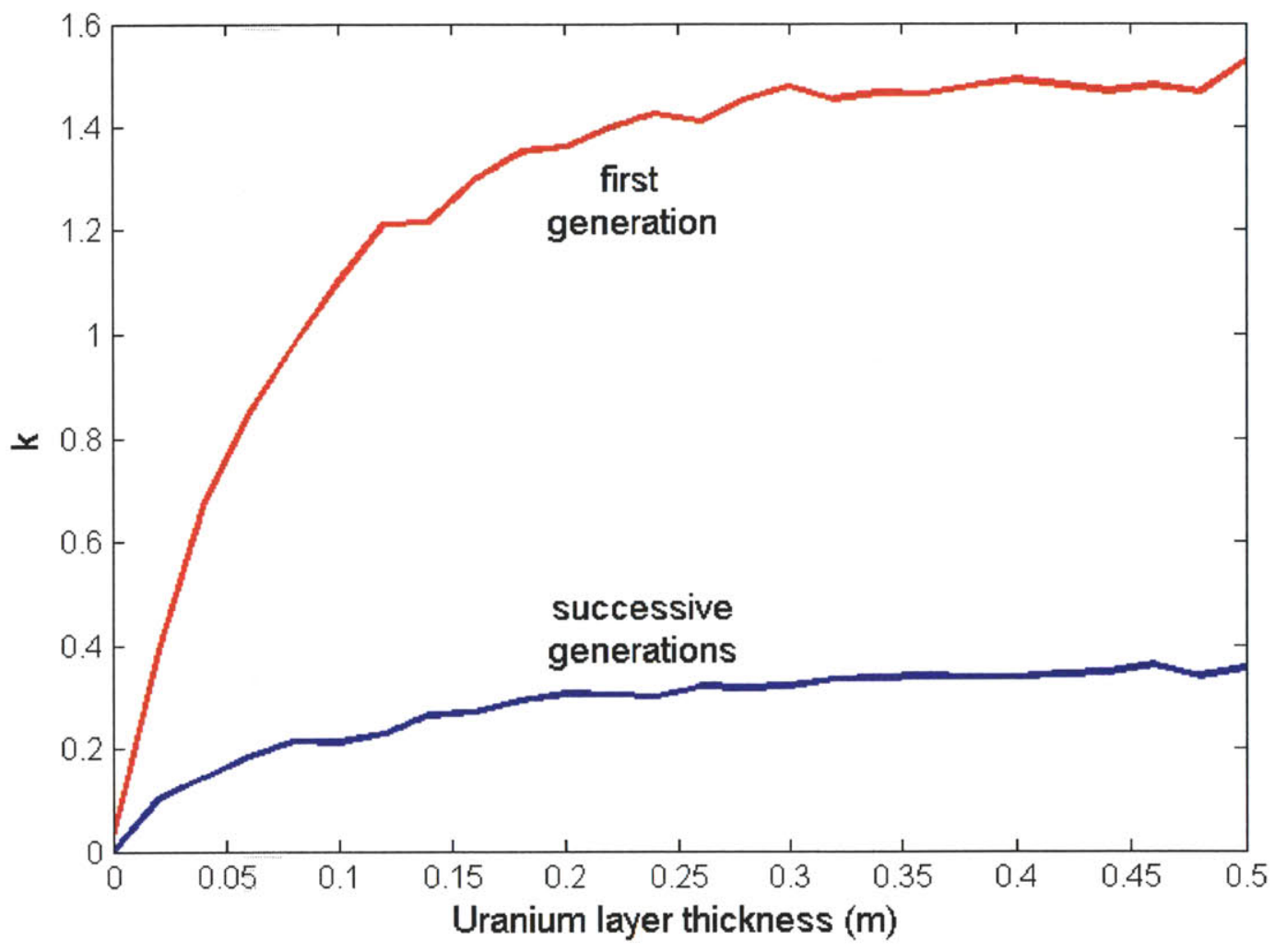

Figure 3.3: The fission multiplication factors $k_{0}$ (first generation) and $k$ (successive generations) as a function of uranium pebble layer thickness. Here the lithium layer is $30 \mathrm{~cm}$ thick with $90 \%$ ${ }^{6} \mathrm{Li}$ enrichment. 


\subsubsection{Lithium Layer Thickness}

Now we will vary the lithium layer thickness while holding the fission layer thickness constant at our favored $15 \mathrm{~cm}$. See Figure 3.4. Unsurprisingly, a thicker lithium layer produces a higher tritium breeding ratio. Somewhat less unsurprising, the lithium layer thickness has virtually no effect on the fission ratio. Of course, a small number of neutrons do backscatter into the uranium layer from the fission layer, but these neutrons, having scattered from lithium, are nearly all below the fissionable energy of ${ }^{238} \mathrm{U}$. Of course, there is also lead in the lithium layer, but lead has a sizable inelastic scattering cross-section that is comparable to its elastic scattering cross-section. Under the conditions in Figure 3.4, we would need the lithium layer to be at least $15 \mathrm{~cm}$ thick to achieve a tritium breeding ratio of 1.

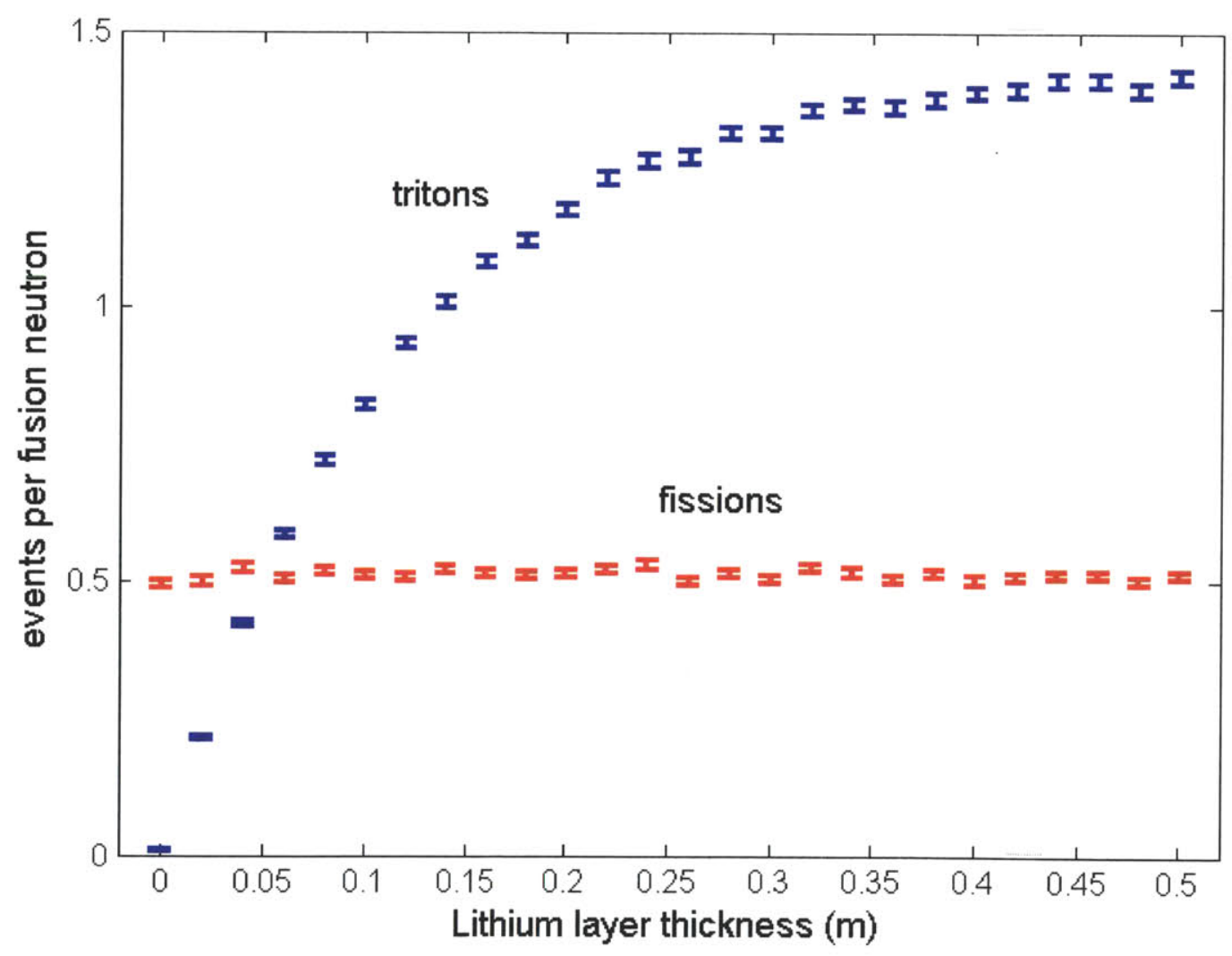

Figure 3.4: Fissions and bred tritons per fusion-born neutron as a function of lithium layer thickness. Here the uranium layer is $15 \mathrm{~cm}$ thick, and the ${ }^{6} \mathrm{Li}$ enrichment is $90 \%$. 


\subsubsection{Lithium Enrichment}

Beyond physical dimensions of the layers, we must also consider the material compositions of the layers. We have decided that the uranium layer should be $\mathrm{UO}_{2}$ pebbles with natural uranium, so we must only examine the $\mathrm{Li}-\mathrm{Pb}$ composition in detail. First we will analyze the effect of lithium enrichment. Natural lithium is $92.5 \%{ }^{7} \mathrm{Li}$ and $7.5 \%{ }^{6} \mathrm{Li}$ (atomic fraction). Both isotopes breed tritium. In pure fusion reactors, the lithium is enriched to $90 \%{ }^{6} \mathrm{Li}$. The reason for this is evident from the cross-sections.

Figure 3.5 shows the ${ }^{6} \mathrm{Li}$ total cross-section broken down into constituent parts as a function of energy. The tritium breeding component, denoted ${ }^{6} \mathrm{Li}(\mathrm{n}, \mathrm{t}) \alpha$, is small at high energies but extremely large at low energies. There is a large elastic scattering component at high energies that serves to scatter neutrons to lower energies where they can more readily breed tritium. Figure 3.6 shows the same ${ }^{6} \mathrm{Li}$ cross-section, but now we have normalized it so that the total cross-section is always 1. Here the scale is not linear (not logarithmic), so we can see the relative probabilities of various reactions as a function of energy. We have outlined the fission energy spectrum $\chi(E)$ and the $14 \mathrm{MeV}$ neutrons with dotted red lines. The unscattered $(14 \mathrm{MeV})$ fusion-born neutrons will breed only a negligible quantity of tritium. However, since the total scattering cross-section comprises over $90 \%$ of the total cross-section above $3 \mathrm{MeV}$, the fusion-born neutrons will scatter to lower energies where the tritium breeding cross-section comprises over half of the total scattering-cross-section.

Figure 3.7 shows the normalized constituent parts of the ${ }^{6} \mathrm{Li}$ total cross-section as a function of energy. The tritium breeding reaction ${ }^{7} \operatorname{Li}(\mathrm{n}, \mathrm{t}+\mathrm{n}) \alpha$ only occurs at very high energies, above $4 \mathrm{MeV}$. In this high energy range, tritium breeding is actually a bit more probable per $\mathrm{n}^{7} \mathrm{Li}$ collision than per $\mathrm{n}^{6} \mathrm{Li}$ collision. However, virtually no fission-born neutrons will breed tritium with ${ }^{7} \mathrm{Li}$. Even in a pure fusion reactor, the neutrons scatter and slow down far below $4 \mathrm{MeV}$ such that it is difficult to breed tritium with natural lithium. Thus, pure fusion reactors usually enrich lithium to $90 \%{ }^{6} \mathrm{Li}$, a near reversal of the natural abundances. We strongly prefer ${ }^{6} \mathrm{Li}$ to ${ }^{7} \mathrm{Li}$.

However, there is one caveat in the case of fission-fusion hybrids. Although ${ }^{6} \mathrm{Li}$ tritium breeding consumes a neutron, ${ }^{7} \mathrm{Li}$ tritium breeding does not. Here are the reactions for both isotopes.

$$
\begin{aligned}
& { }_{3}^{6} \mathrm{Li}+\mathrm{n} \rightarrow{ }_{2}^{4} \mathrm{He}+{ }_{1}^{3} \mathrm{H}+4.8 \mathrm{MeV} \\
& { }_{3}^{7} \mathrm{Li}+\mathrm{n} \rightarrow{ }_{2}^{4} \mathrm{He}+{ }_{1}^{3} \mathrm{H}+\mathrm{n}-2.5 \mathrm{MeV}
\end{aligned}
$$

In pure fusion reactors, no one cares about the extra neutron. In fact, eliminating neutrons is preferable. Furthermore, ${ }^{6} \operatorname{Li}(\mathrm{n}, \mathrm{t}) \alpha$ produces energy, while ${ }^{7} \operatorname{Li}(\mathrm{n}, \mathrm{t}+\mathrm{n}) \alpha$ consumes energy. In contrast, we prefer more neutrons in a fission-fusion hybrid. Although ${ }^{7} \operatorname{Li}(n, t+n) \alpha$ only 


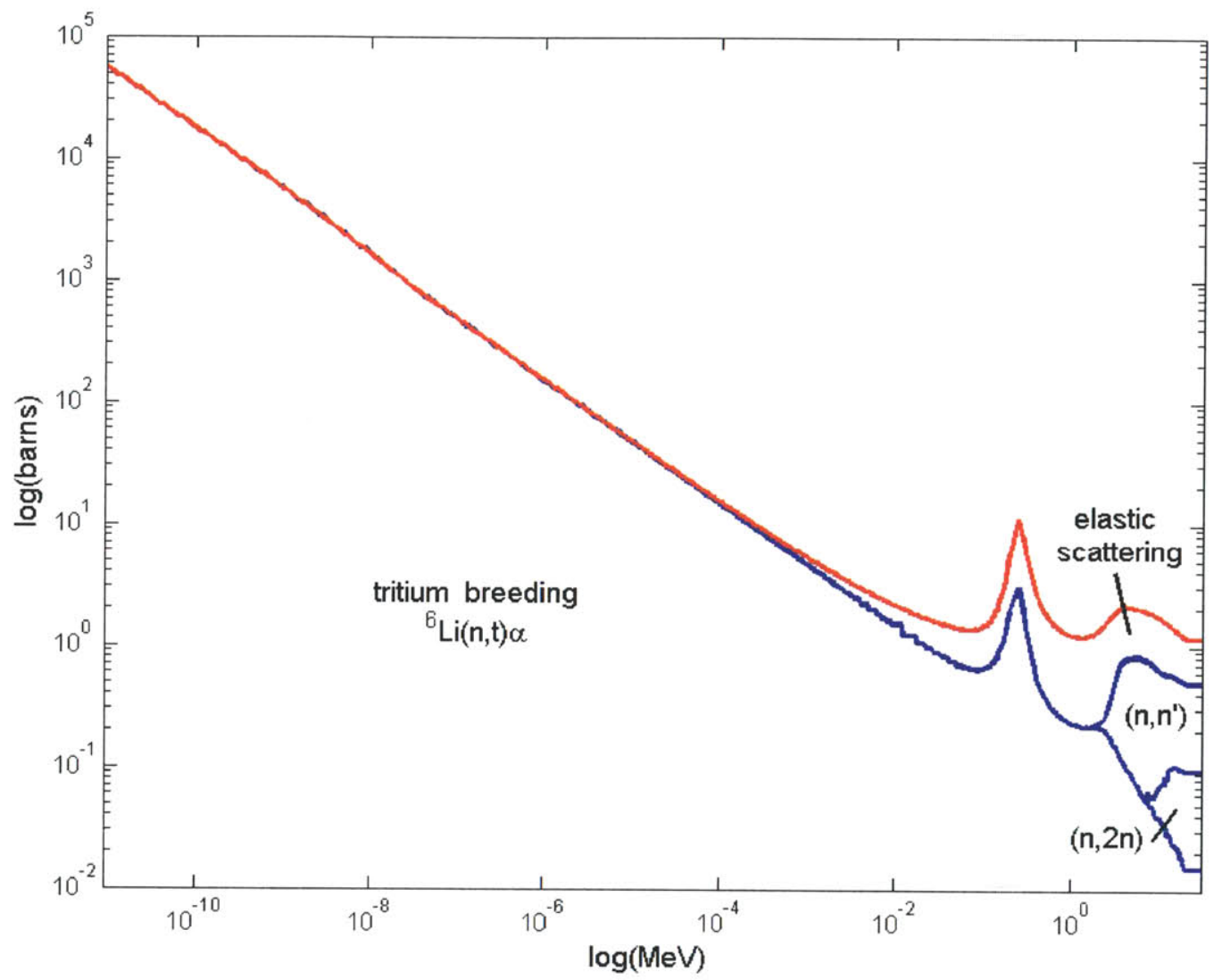

Figure 3.5: The total ${ }^{6} \mathrm{Li}$ cross-section showing constituent parts as a function of energy. The total cross-section is in red.

occurs at high energies, the extra neutron per bred triton will spur more fission. The question is whether this extra neutron is worth the drawbacks of ${ }^{7} \mathrm{Li}$.

Figure 3.8 shows the fission and tritium breeding ratios as a function of lithium enrichment (atomic fraction ${ }^{6} \mathrm{Li}$ ). Clearly, ${ }^{6} \mathrm{Li}$ is vastly superior to ${ }^{7} \mathrm{Li}$ in terms of tritium breeding. In terms of fission, the effect seems to be negligible, because backscattering into the uranium layer from the lithium layer is rare to begin with. Given all these facts, we will prudently stick with the lithium enrichment of $90 \%{ }^{6} \mathrm{Li}$ in pure fusion reactors. More ${ }^{6} \mathrm{Li}$ is always better, but there comes a point where the cost of enrichment outweighs the benefits. As enrichment becomes very high (above $90 \%$ ), it becomes exponentially more difficult to filter out the rarefied ${ }^{7} \mathrm{Li}$ atoms. 


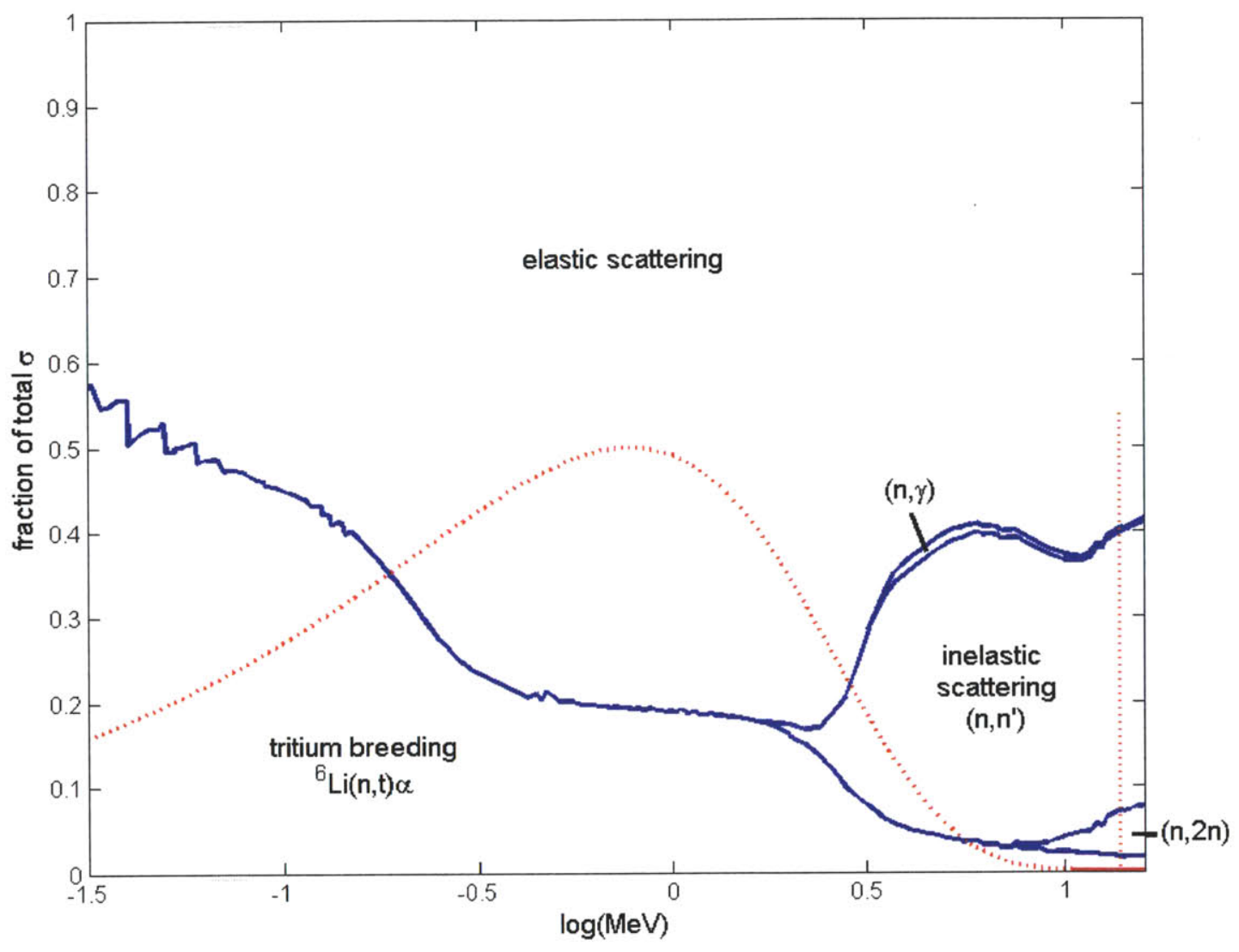

Figure 3.6: Normalized constituent parts of the total ${ }^{6} \mathrm{Li}$ cross-section as a function of energy. Here the total cross-section is always 1.0. We have outlined the fission $\chi(E)$ spectrum and the 14 $\mathrm{MeV}$ fusion-born neutrons in red. 


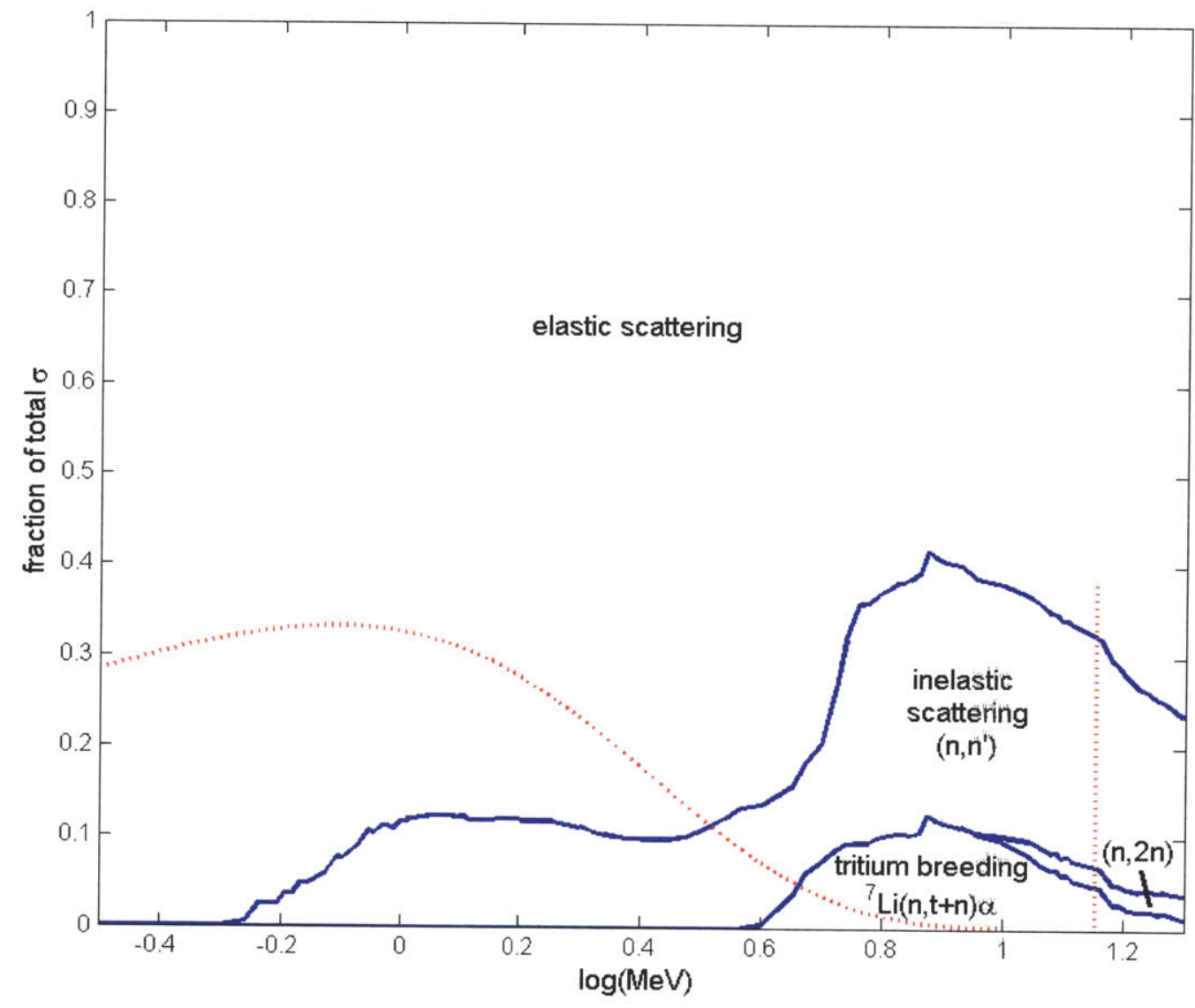

Figure 3.7: Normalized constituent parts of the total ${ }^{7} \mathrm{Li}$ cross-section as a function of energy. Here the total cross-section is always 1.0. We have outlined the fission $\chi(E)$ spectrum and the 14 $\mathrm{MeV}$ fusion-born neutrons in red. 


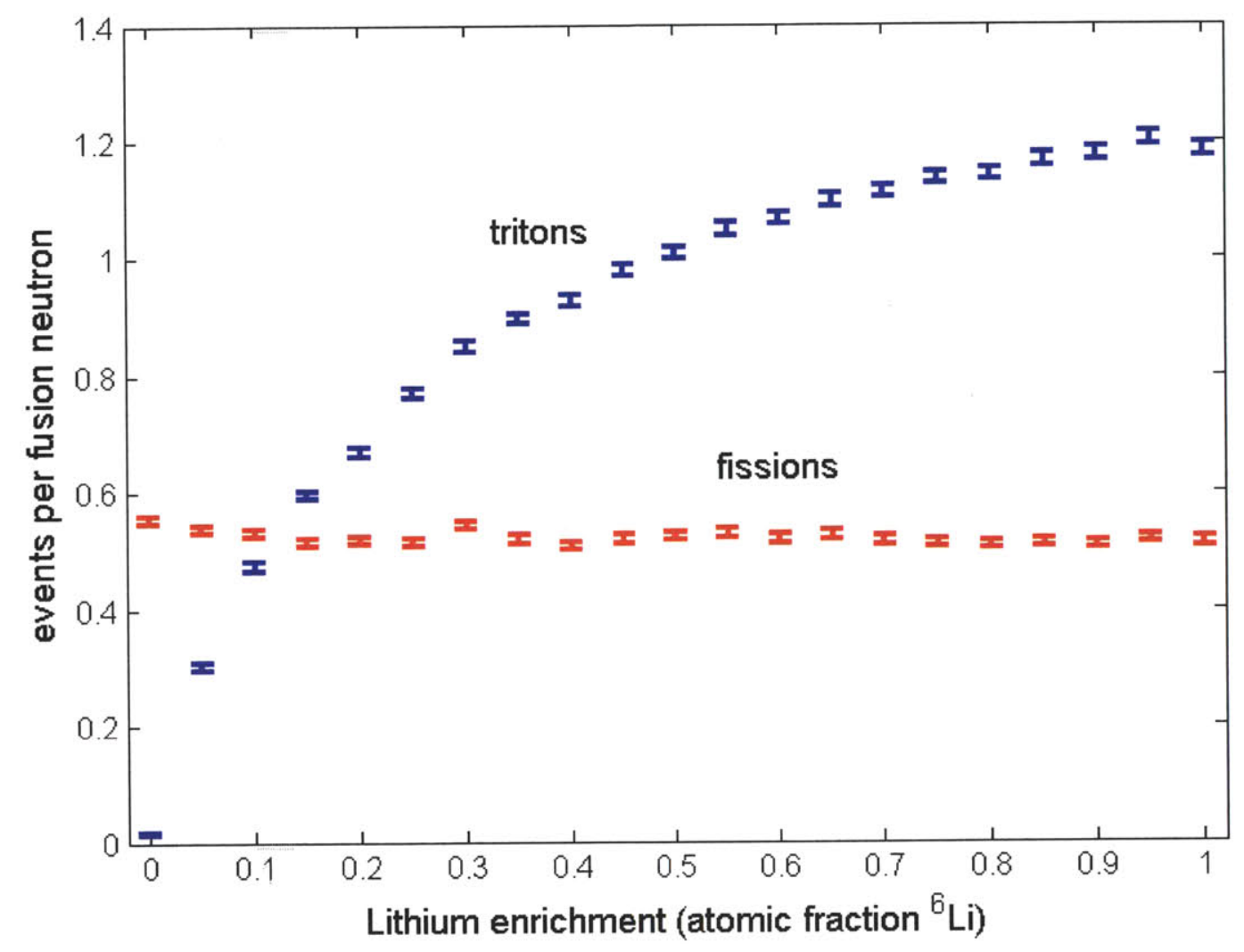

Figure 3.8: Fissions and bred tritons per fusion-born neutron as a function of ${ }^{6} \mathrm{Li}$ enrichment. Here the uranium and lithium layers are $20 \mathrm{~cm}$ and $30 \mathrm{~cm}$ thick, respectively. 


\subsubsection{Layer Positioning}

Until now, we have assumed that the lithium layer is exterior to the uranium layer. This works well, because the uranium layer acts like both a neutron multiplier and a neutron "moderator' in the sense that fission-born neutrons are much slower than fusion-born neutrons (the ${ }^{6} \mathrm{Li}(\mathrm{n}, \mathrm{t}) \alpha$ cross-section is exponentially higher at lower energies). It is a nice little system. However, to be thorough, we should at least quantify how much more favorable this arrangement is than the reverse - uranium exterior to lithium.

Figure 3.9 shows the fission and tritium breeding ratios as a function of lithium enrichment for a $7 \mathrm{~cm}$ lithium layer interior to $40 \mathrm{~cm}$ uranium layer so that the lithium abuts the tokamak first wall. While we saw that lithium enrichment has only a negligible effect on fission when the lithium is exterior to the uranium, in this case there is a noticeable difference. In this case, all fusion-born neutrons that spur fission must traverse the lithium layer. The extra neutron from ${ }^{7} \mathrm{Li}(\mathrm{n}, \mathrm{t}+\mathrm{n}) \alpha$ contributes to the number of neutrons that fully traverse the lithium layer. Of course, this neutron is emitted isotropically, but even if its direction is directly away from the uranium, it will simply traverse the plasma and enter the uranium layer on the opposite side (if it does not collide in the lithium a second time). Furthermore, ${ }^{7} \mathrm{Li}(\mathrm{n}, \mathrm{t}+\mathrm{n}) \alpha$ is more likely to occur in the very fast neutron spectrum that forms when the lithium abuts the plasma. However, even though the ${ }^{7} \operatorname{Li}(n, t+n) \alpha$ neutron contributes to fission, ${ }^{7} \mathrm{Li}$ still has no advantage in terms of tritium breeding. As Figure 3.9 shows, even though pure ${ }^{7} \mathrm{Li}$ yields a fission ratio about $30 \%$ higher than pure ${ }^{6} \mathrm{Li}$, ${ }^{7} \mathrm{Li}$ is devastating for tritium breeding. Even in this reversed layer positioning, we prefer the lithium enrichment to be as high as possible.

Figure 3.10 shows the fission and tritium breeding ratios as a function of uranium layer thickness for a $30 \mathrm{~cm}$ lithium layer. Here we hold lithium enrichment constant at the favored $90 \%$. As the uranium layer (which is exterior to the lithium layer) thickens, more fissions occur and more tritium is bred. This is similar to Figure 3.2, except that there is no subsequent drop in tritium production, because the uranium does not separate the lithium from the fusion-born neutron source. However, it is very difficult to achieve both a tritium breeding ratio of 1 and a reasonably high fission ratio. Note that the tritium breeding ratio just barely reaches 1 and that the fission ratio is only about 0.1 , which would correspond to a measly power gain of 1.4. If we make the tritium layer much thinner and the uranium layer much thicker, as in Figure 3.9, then it is possible to achieve a fission ratio of 0.3 with a tritium breeding ratio of 1 . However, this requires more than twice as much $\mathrm{UO}_{2}$ fuel (40 $\mathrm{cm}$ at a larger poloidal radius versus $20 \mathrm{~cm}$ at a smaller poloidal radius). It also poses a technical challenge in that the liquid $\mathrm{Li}-\mathrm{Pb}$ would be asinine to periodically remove if it is interior to the uranium layer. Lastly, 0.3 is still significantly less than the 0.5 we achieve with the uranium layer abutting the first wall.

Figure 3.11 shows the fission and tritium breeding ratios as a function of lithium layer 


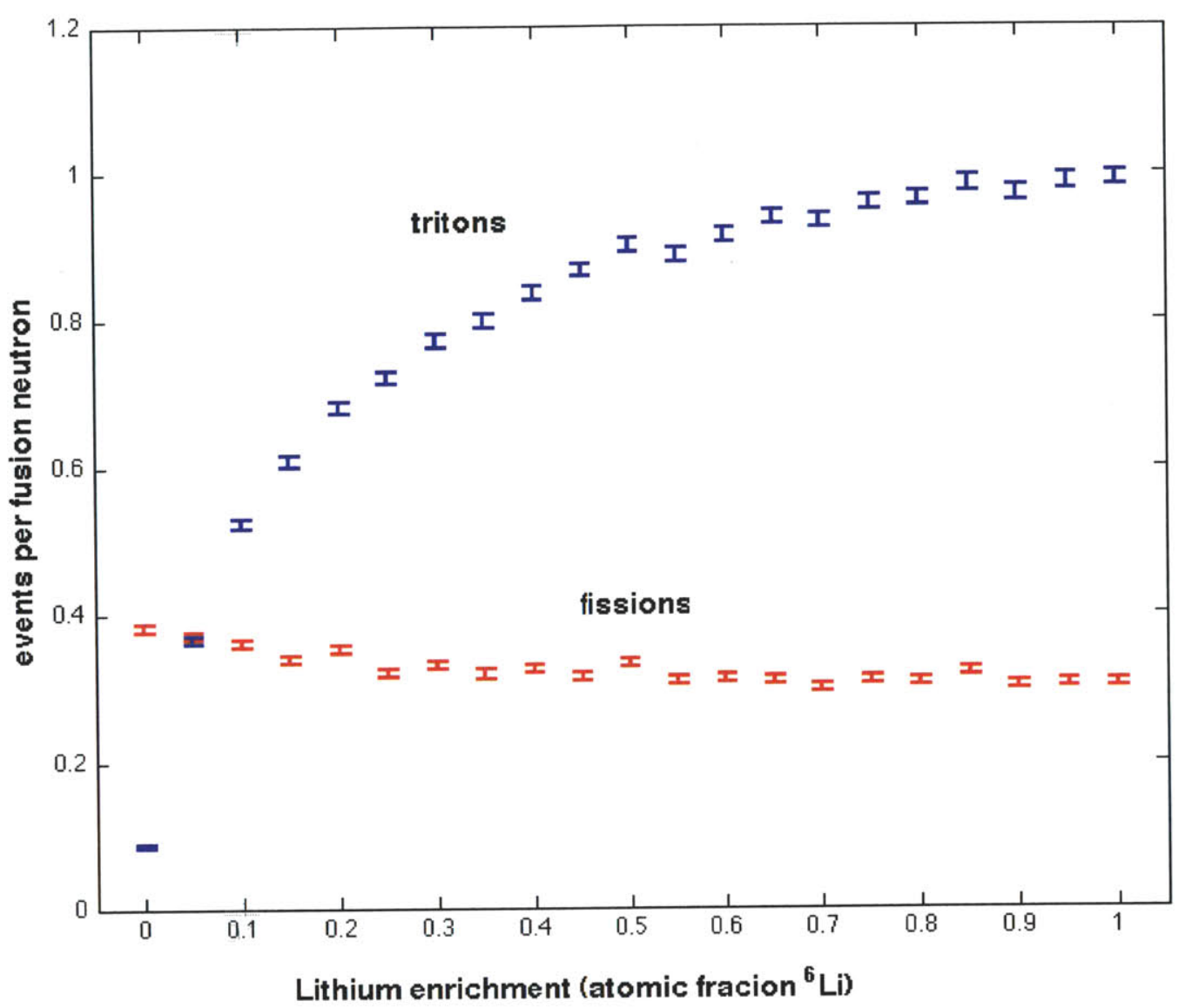

Figure 3.9: Fissions and bred tritons per fusion-born neutron as a function of ${ }^{6} \mathrm{Li}$ enrichment. Here the uranium layer is exterior to the lithium layer. The uranium and lithium layers and $40 \mathrm{~cm}$ and $7 \mathrm{~cm}$ thick, respectively.

thickness for a $20 \mathrm{~cm}$ lithium layer. Interestingly, the tritium breeding ratio rises precipitously to 0.75 with a thickness of only $5 \mathrm{~cm}$, while a thickness of $25 \mathrm{~cm}$ is necessary for a breeding ratio of 1 . In that same thickness range, the fission ratio falls exponentially to approach zero. This plot capture exactly why this positioning of layers is unworkable - it pits fission and tritium breeding at odds with each other. Compare this to Figure 3.2, in which fission enhances the tritium breeding (for thin uranium layers). We conclude that a uranium layer abutting the first wall and an exterior lithium layer is the superior option. 


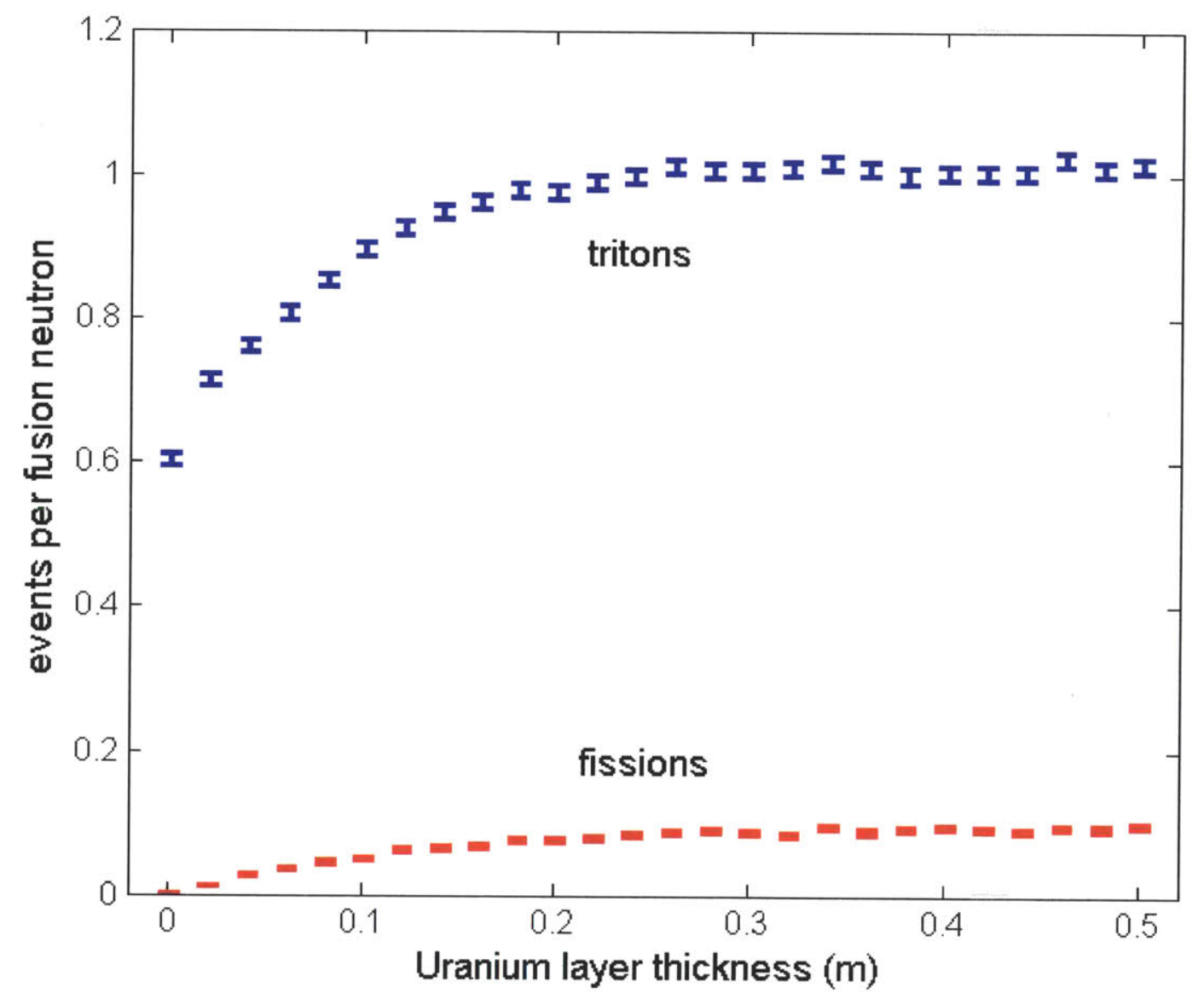

Figure 3.10: Fissions and bred tritons per fusion-born neutron as a function of uranium pebble layer thickness. Here the uranium layer is exterior to the lithium layer, which is $30 \mathrm{~cm}$ thick with $90 \%{ }^{6} \mathrm{Li}$ enrichment. 


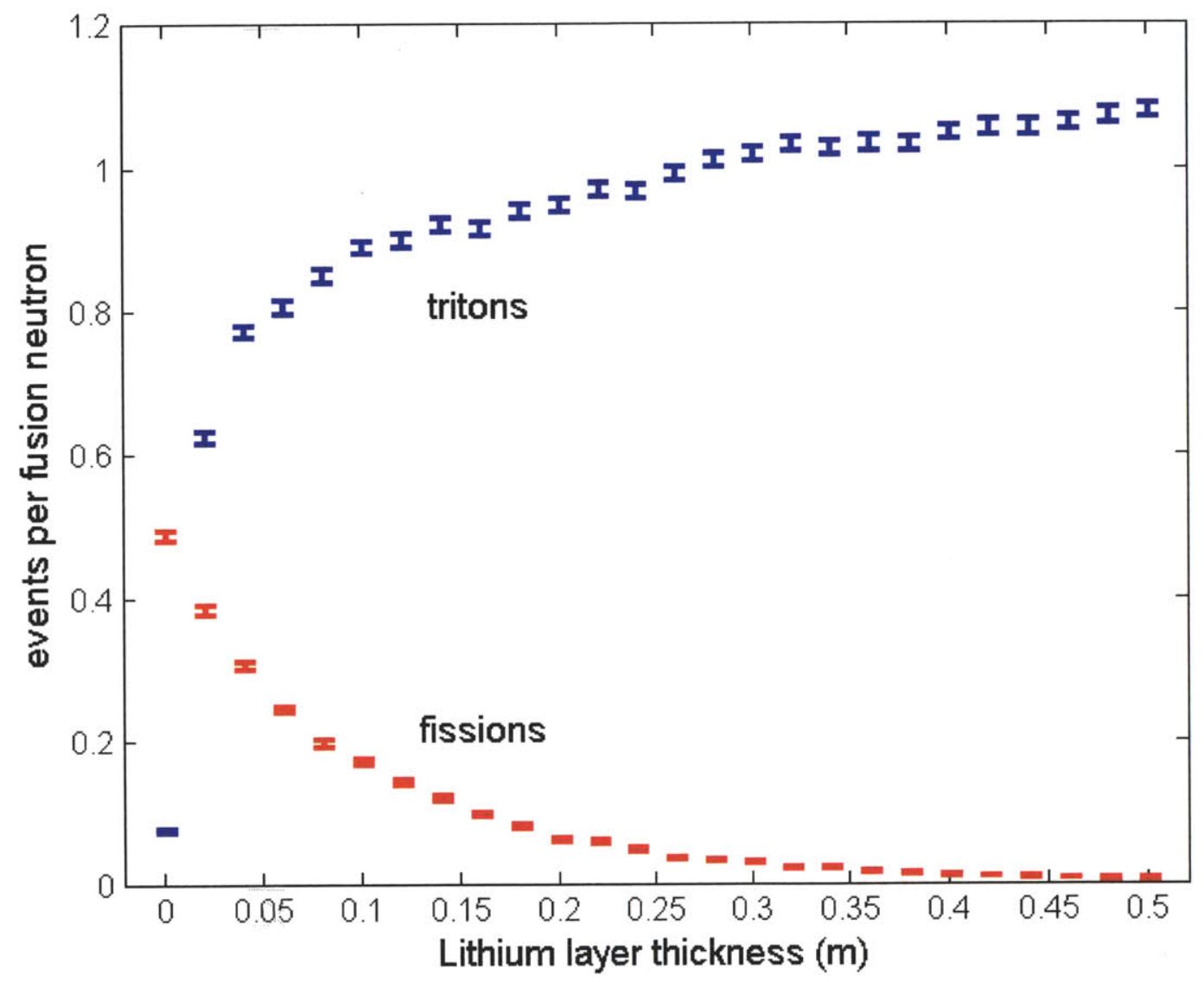

Figure 3.11: Fissions and bred tritons per fusion-born neutron as a function of lithium layer thickness. Here the uranium layer is exterior to the lithium layer. The uranium layer is $20 \mathrm{~cm}$ thick, and the lithium is enriched to $90 \%{ }^{6} \mathrm{Li}$. 


\subsubsection{Li-Pb Content}

Now one last parameter remains - the lithium content in the liquid Li- $\mathrm{Pb}$ alloy. Li- $\mathrm{Pb}$ is standard in pure fusion tokamak designs, because it is chemically benign and relatively easy to extract the bred tritium from. Pure lithium would pose safety issues due to its ravenous corrosive behavior. Pure fusion designs use the eutectic $\mathrm{Pb}_{84} \mathrm{Li}_{16}$ (84\% lead by atomic fraction) for its low melting point. $\mathrm{Li}-\mathrm{Pb}$ with lead content below $5 \%$ also has a low melting point, but then there might be corrosive danger.

Figure 3.12 shows the fission and tritium breeding ratios as a function of lithium content in $\mathrm{Li}-\mathrm{Pb}$ (atomic fraction). Here we fix the layer thickness near our optimal values of $20 \mathrm{~cm}$ for uranium and $30 \mathrm{~cm}$ for lithium. The fission ratio increases very slightly for higher lead content, because the lead causes more backscattering (inelastic as well as isotropic elastic) from the lithium layer to the uranium layer. However, high lead content is devastating to tritium breeding. $\mathrm{Pb}_{84} \mathrm{Li}_{16}$ yields a tritium breeding ratio that is less than half that of pure lithium. If we were designing a pure fusion reactor, this could be overcome with a much thicker Li-Pb layer. However, with a $20 \mathrm{~cm}$ uranium layer between the neutron source and the Li-Pb, there is no thickness of $\mathrm{Pb}_{84} \mathrm{Li}_{16}$ that will yield a breeding ratio of 1 . The lithium atomic density is too low, and the isotropic lead scattering prevents deep neutron diffusion. Thus, we cannot use $\mathrm{Pb}_{84} \mathrm{Li}_{16}$ for this hybrid. An addition of a neutron multiplier (such as beryllium) might help the situation somewhat, but we leave that analysis to future work. Tang's 2002 thesis uses solid lithium titanate as a tritium breeder with a beryllium neutron multiplier [22]. These were also in pebble form, so his design is a "double pebble bed". We chose to stick with the more conventional liquid Li-Pb material for this thesis, because it is more expedient to remove the bred tritium from a liquid than from solid pebbles.

Since $\mathrm{Pb}_{84} \mathrm{Li}_{16}$ is not feasible, we will need to use a lower lead content. As we will discuss in more depth in our thermal hydraulic analysis (see Section 12), the Li-Pb melting point becomes far too large at lead contents between $5 \%$ and $60 \%$. Consequently, we are forced to work with $5 \%$ lead. Corrosion could be a problem, although we do not know the lead content at which $\mathrm{Li}-\mathrm{Pb}$ becomes benign. 


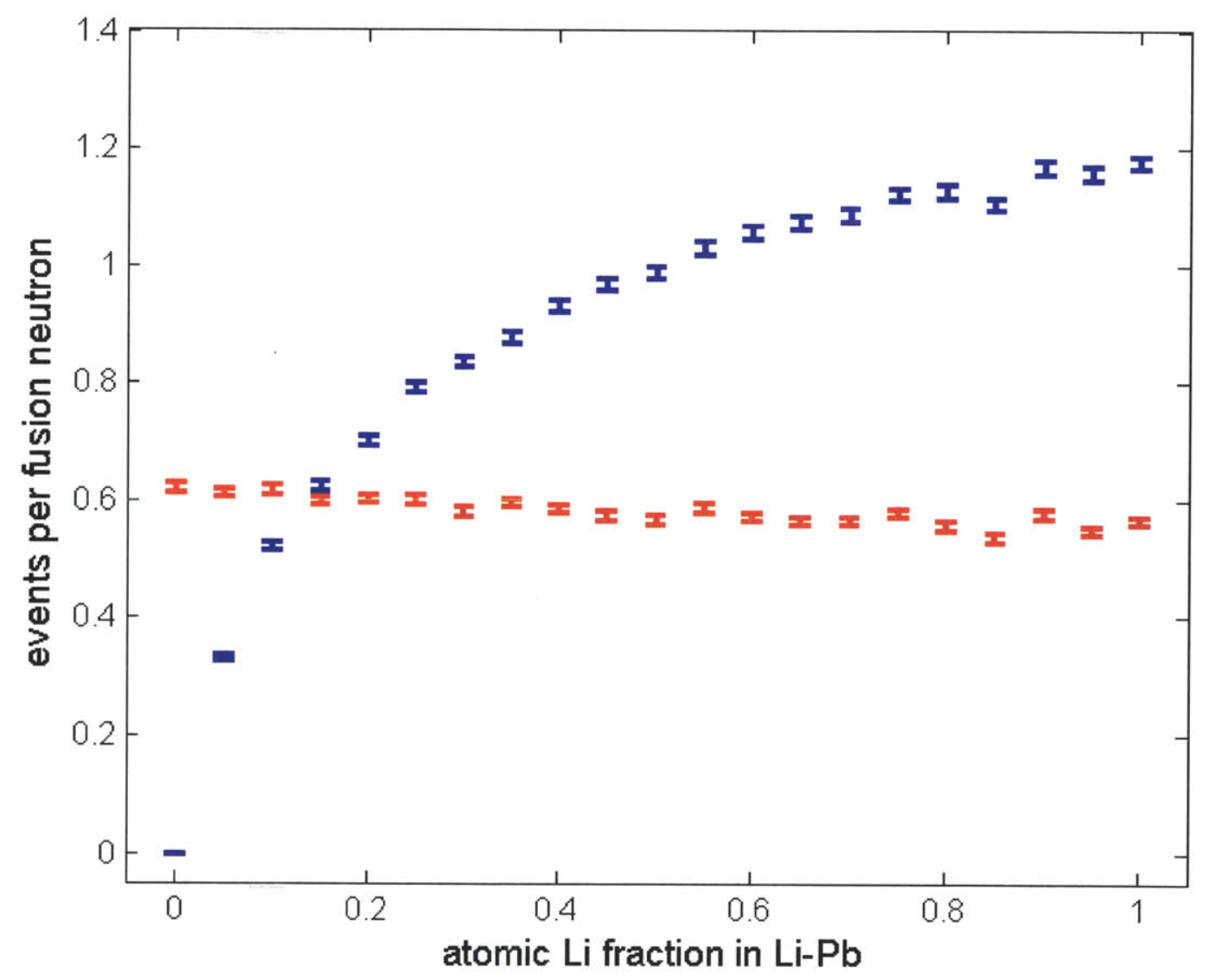

Figure 3.12: Fissions and bred tritons per fusion-born neutron as a function of lithium atomic fraction in $\mathrm{Li}-\mathrm{Pb}$. Here the uranium and lithium layers are $20 \mathrm{~cm}$ and $30 \mathrm{~cm}$ thick, respectively, and the ${ }^{6} \mathrm{Li}$ enrichment is $90 \%$. The lithium layer is exterior to the uranium layer. 


\subsection{The Optimal Fission Blanket}

Now that we have performed the bulk of our blanket analysis, we can settle on an approximate set of optimal parameters as shown in Table 3.1. A uranium thickness of $18 \mathrm{~cm}$ and a lithium thickness of $25 \mathrm{~cm}$ produces the highest fission ratio while maintaining a tritium breeding ratio above 1. The fission ratio is 0.47 , and the total fission gain $Q_{\text {fis }}$ is 7.7 . $Q_{\text {fis }}$ is a bit higher than the fission ratio indicates, because tritium breeding also produces substantial energy. We tally the energy generation very precisely in our Monte Carlo code. The fusion-born neutron multiplication $k_{0}$ is 1.16 , while the converged $k$ is a mere 0.27 .

Table 3.1: Optimal Blanket Parameters

$\begin{array}{cc}\mathrm{UO}_{2} \text { pebble layer thickness } & 18 \mathrm{~cm} \\ \mathrm{Li}-\mathrm{Pb} \text { layer thickness } & 25 \mathrm{~cm} \\ \mathrm{Li} \text { enrichment } & 90 \% \\ \mathrm{Li} \text { atomic fraction in } \mathrm{Li}-\mathrm{Pb} & 10 \% \\ \text { fission ratio } & 0.47 \\ Q_{\text {fis }} & 7.7 \\ \text { tritium breeding ratio } & 1.05 \\ \mathrm{k}_{0} & 1.19 \\ \mathrm{k} & 0.27\end{array}$

Even though we have selected an approximate set of optimal parameters, our work is not yet done. Since this is an unusual type of fission reactor, and since we have developed our own code from scratch to model it, we should perform additional analysis to convince ourselves that what we have done is correct. We must perform a "sanity check". From here forward, we will assume the optimal blanket parameters shown in Table 3.1.

Figure 3.13 shows the relative number of neutrons in each neutron generation, beginning with the fusion-born neutrons (generation 1). As we expect, the first generation of fissionborn neutrons (generation 2) are a factor of $k_{0}=1.2$ greater in number than the fusion-born neutrons. After generation 2, each successive generation of neutrons is smaller by a factor of $k=0.27$. Of course, the fission-born neutron spectrum does not converge precisely at generation 2. Instead, $k$ will not converge to precisely 0.27 until after many generations. However, $k$ does come quite close to its asymptotic value beginning with generation 2 . The most interesting thing about this plot is that it reveals just how tremendously important the fusion-born neutrons are. It is clear that they produce a huge portion of the total fission power. These fusion-born neutrons are not merely a source that spurs something more interesting - they are the real engine of this system. The fission-born neutrons are a 
convenient side-effect.

Figure 3.14 shows this from a different perspective. This shows the entire hybrid power broken down into sources. We tally these quantities with our Monte Carlo code. Fission spurred by fusion-born neutrons comprise $55 \%$ of the total system power, while all subsequent generations of fission-born neutrons comprise less than half that $(27 \%)$. This is consistent with Figure 3.13. The large ${ }^{238} \mathrm{U}$ cross-section at high energies empowers the fusion-born neutrons. Continuing on, the next-largest energy source is the slowing-down energy of fusion-born neutrons prior to fission. Many of these neutrons do not spur fission, and many of those that do slow significantly beforehand. The total energy deposition of fusion-born neutrons prior to fission comprises $10 \%$. Amazingly, this means that the fusionborn neutrons directly produce $65 \%$ of the total hybrid power. The net energy production of the exothermic tritium breeding reaction ${ }^{6} \mathrm{Li}(\mathrm{n}, \mathrm{t}) \alpha$ and the endothermic tritium breeding reaction ${ }^{7} \mathrm{Li}(\mathrm{n}, \mathrm{t}+\mathrm{n}) \alpha$ comprises $4 \%$. The fusion $\alpha$-particles comprise $3 \%$, which is deposited directly in the plasma.

Some might still be skeptical of these data, especially the huge amount of energy produced by the fusion-born neutrons. To dispel this, we have created Figure 3.15, which shows the fate of the fusion-born neutrons. $8 \%$ spur fission without any scattering. $23 \%$ spur fission after at least one scattering event. Note that these neutrons can backscatter, traverse the plasma, and still spur fission on the opposite side of the blanket. So a total of $31 \%$ of fusion-born neutrons eventually spur fission. Since $\nu$ is near 4 at these high energies, this is consistent with $k_{0}$ in Figure 3.13. A surprisingly large fraction (41\%) breed tritium but nearly all do so after multiple scattering events. The fraction of fusion-born neutrons that breed tritium without prior scattering is negligible. Finally, 28\% leak to freedom or (more likely) eventually succumb to absorption.

To further corroborate our data, we should note that the neutron mean free paths in our homogenized $\mathrm{UO}_{2}$ pebble layer are $7.1 \mathrm{~cm}(14 \mathrm{MeV})$ and $2.7 \mathrm{~cm}(1 \mathrm{MeV})$. These numbers are consistent with our analysis here.

Figure 3.16 shows the full neutron energy spectrum in the uranium pebble layer. We derive this from a collision tally in our Monte Carlo code and then construct this weighted histogram. The large spike at $14 \mathrm{MeV}$ represents the fusion-born neutrons before collision. The smaller spike to its left is an artificial result of our inelastic scattering approximation. Oxygen resonances cause conspicuous flux dips at lower energies. The average energy is 0.94 $\mathrm{MeV}$, and so we can rightfully call this spectrum fast. This average energy is significantly higher than typical fast spectrum energies due to the $14 \mathrm{MeV}$ neutrons and the very low $k$. The spectrum peak occurs at $0.1 \mathrm{MeV}$, which is more typical.

It serves our intuition to take this further by superimposing the fission-inducing energy spectrum on top of the total energy spectrum. We compute this fission-inducing energy spectrum by tallying each incident neutron energy in all fission events. Figure 3.17 shows 


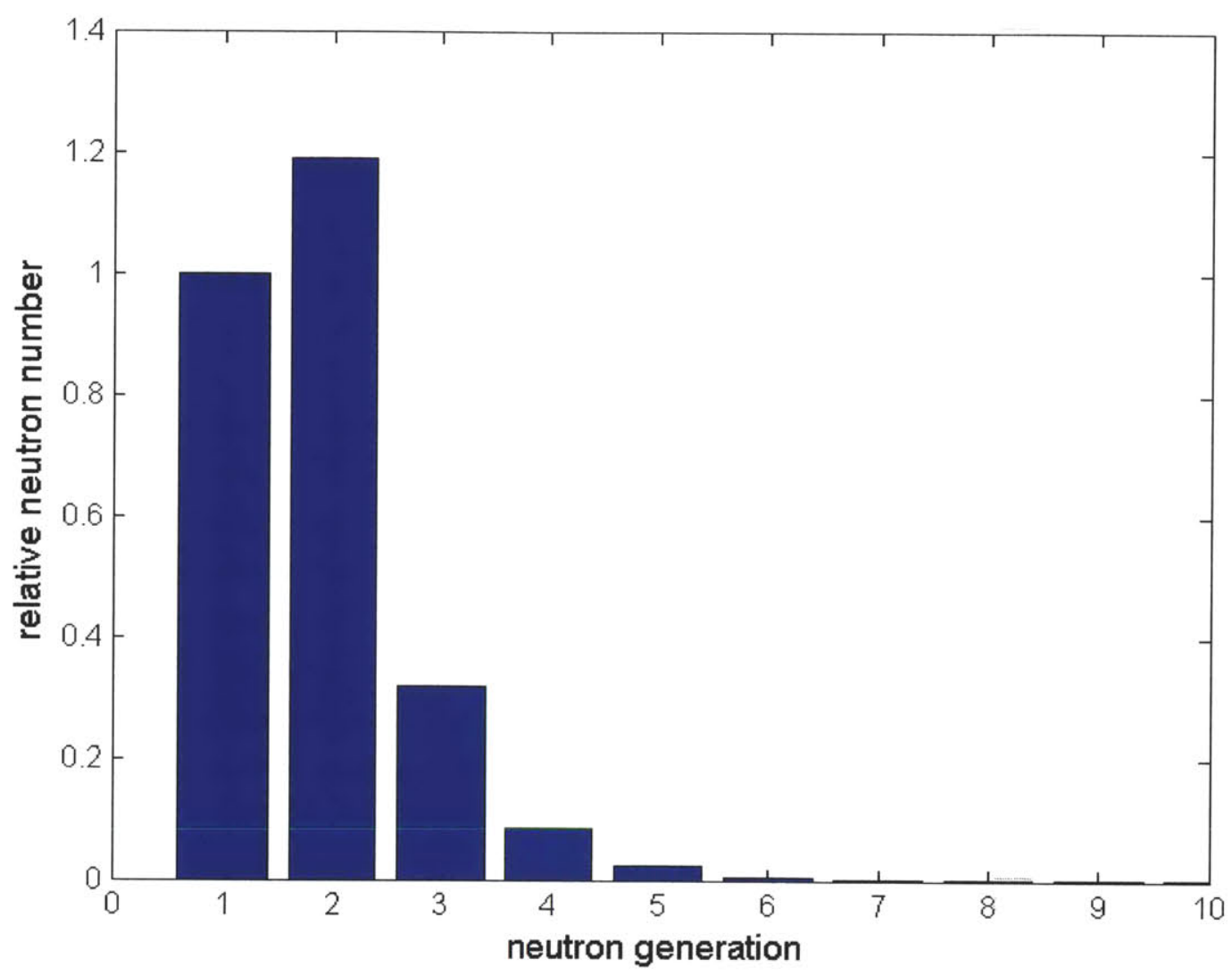

Figure 3.13: The number of neutrons in each generation throughout the hybrid system. The first generations contains only fusion-born neutrons, and all subsequent generations contain only fission-born neutrons. The multiplication factor $k_{0}$ is the ratio of generation 2 to generation 1 , while the multiplication factor $k$ is the ratio of generation $n$ to generation $n-1$ for $n>2$.

this superposition. The total spectrum (identical to Figure 3.15) is in red, and the fissioninducing spectrum is in blue. The magnitudes of the two spectra are not to scale - we only wish to analyze their distributions. The average fission-inducing energy is $9.9 \mathrm{MeV}$ ! This is consistent with Figures 3.14 and 3.15, from which we can infer that fusion-born neutrons spur $67 \%$ of all fissions. Furthermore, $17 \%$ of all fissions are spurred by virgin fusion-born neutrons at $14 \mathrm{MeV}$ (no scattering).

Figure 3.18 shows the total neutron energy spectrum in the Li-Pb layer. The fusionborn neutrons spike is evident but not nearly as prominent as in the uranium layer. The massive chasm centered around $0.3 \mathrm{MeV}$ is the result of a large resonance in ${ }^{6} \mathrm{Li}$. The average neutron energy here is $0.77 \mathrm{MeV}$, so the spectrum is a bit slower than in the uranium layer. Figure 3.19 shows a superposition of the tritium-breeding spectrum (blue) on top of the total 
spectrum (red). The tritium-breeding spectrum is notably slower with an average energy of $0.29 \mathrm{MeV}$. This is due to the high ${ }^{6} \mathrm{Li}(\mathrm{n}, \mathrm{t}) \alpha$ cross-section at lower energies.

Although we can infer form Figures 3.15 and 3.17 that the fission-inducing neutrons do not undergo many scattering events, we can demonstrate that the tritium-breeding neutrons undergo numerous scattering events. Figure 3.20 shows a distribution of the number of collisions a tritium-breeding fusion-born neutron undergoes prior to breeding tritium. The average value is about 33 . This shows that the fusion-born neutrons scatter off the lead and lithium numerous times until they reach lower energies where the ${ }^{6} \mathrm{Li}(\mathrm{n}, \mathrm{t}) \alpha$ cross-section is high.

The main thing to take away from this is that our neutron spectrum straddles the high ${ }^{6} \mathrm{Li}$ tritium breeding cross-section at low energies and the high ${ }^{238} \mathrm{U}$ fission cross-section at high energies. The uranium layer capitalizes on the high-energy fusion-born neutrons to induce fission, while the lithium layer slows the neutrons to breed tritium. The spectrum slows as it moves further away from the fusion source. This is the fundamental reason why the lithium layer should be external to the uranium layer. It just makes sense.

Finally, we can perform a bit of analysis with the spatial distribution of the neutron flux. Figure 3.21 shows the poloidal angular distribution of neutron flux in the uranium layer. We produce this with a collision tally. This figure is very important, because it shows how our toroidal model captures what a cylindrical model does not. This distribution is similar to the tokamak wall neutron flux distribution we computed in Section 2. This distribution is similar to the fission power distribution, which we would need to take into account were we to perform detailed thermal hydraulic analysis. Since the flux magnitude varies by a factor of 2, this distribution would also be very important for spatially-depenent burnup analysis.

Figure 3.22 shows the radial distribution of neutron flux. Here we have corrected for elongation by normalizing the radii as if $\kappa=1$.

$$
r^{\prime}=r \sqrt{\frac{\sin ^{2} \Theta}{\kappa^{2}}+\cos ^{2} \Theta}
$$

We have plotted the distribution as a function of $r^{\prime}$, and $\Theta$ is the poloidal angle of each tally at poloidal radius $r$. In diffusion theory, the shape of this curve would be a superposition of modified Bessel functions $I_{0}(r)$ and $K_{0}(r)$. It is noteworthy that the flux magnitude attenuates by a factor of 6 through the $20 \mathrm{~cm}$ layer, which is consistent with what we expect for a subcritical reactor with a source coming from the left. This also shows that there would be paltry gain from thickening the layer any further. These spatially-dependent flux plots are the only place in the subsection where we use a uranium layer thickness of $20 \mathrm{~cm}$ instead of the optimal $18 \mathrm{~cm}$, but there is little substantial difference. 


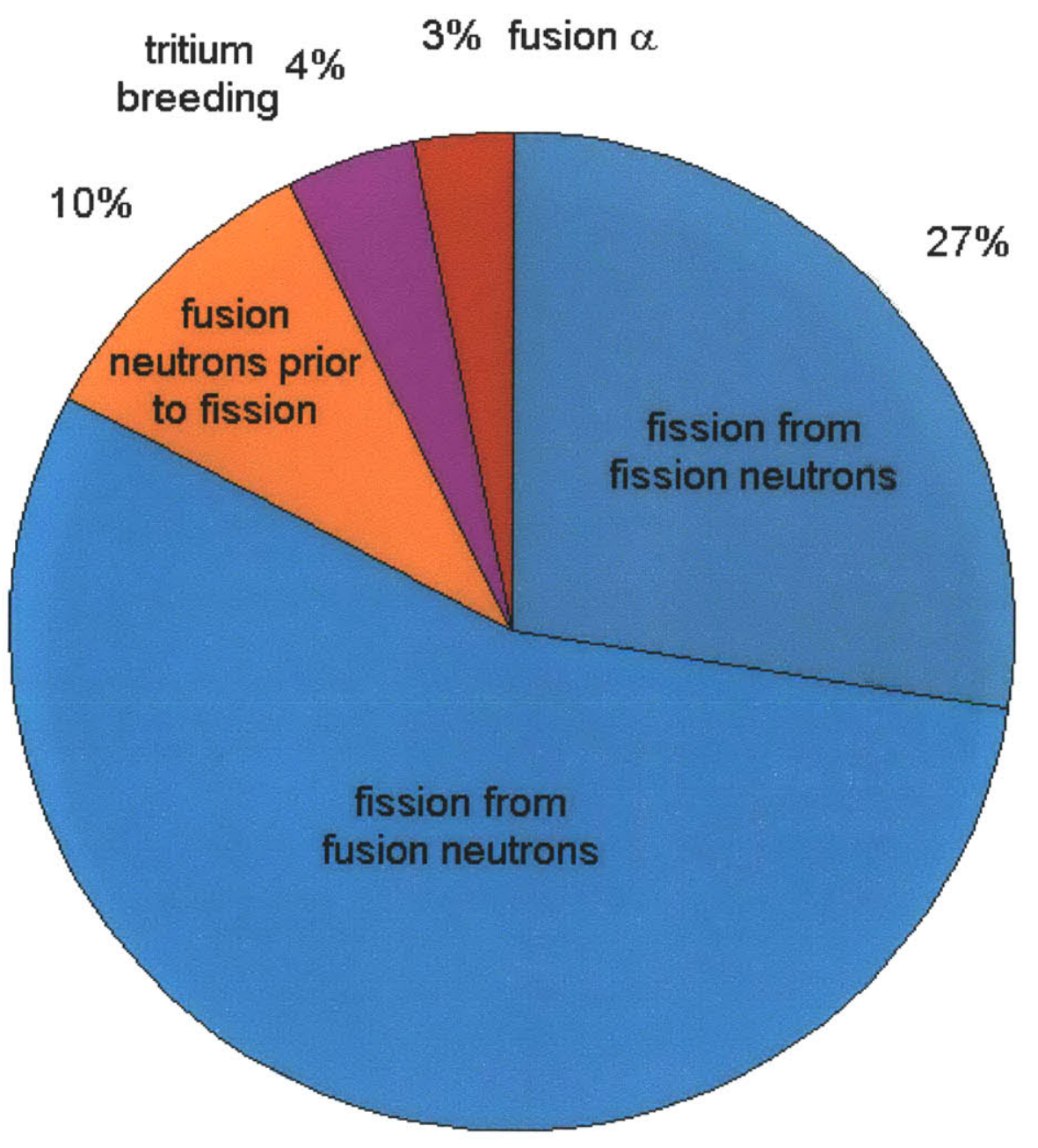

$55 \%$

Figure 3.14: The composition of the total hybrid power from various sources. Astonishingly, fission spurred by the 1st generation fusion-born neutrons generates over half the total hybrid power. Fission spurred by all other (fission-born) neutrons accounts for only about one quarter. A substantial fraction $(1 / 10)$ is due to the slowing down of fusion-born neutrons prior to fission. The remainder is from exothermic tritium breeding reactions and $\alpha$-particles, which deposit their energy in the plasma. 


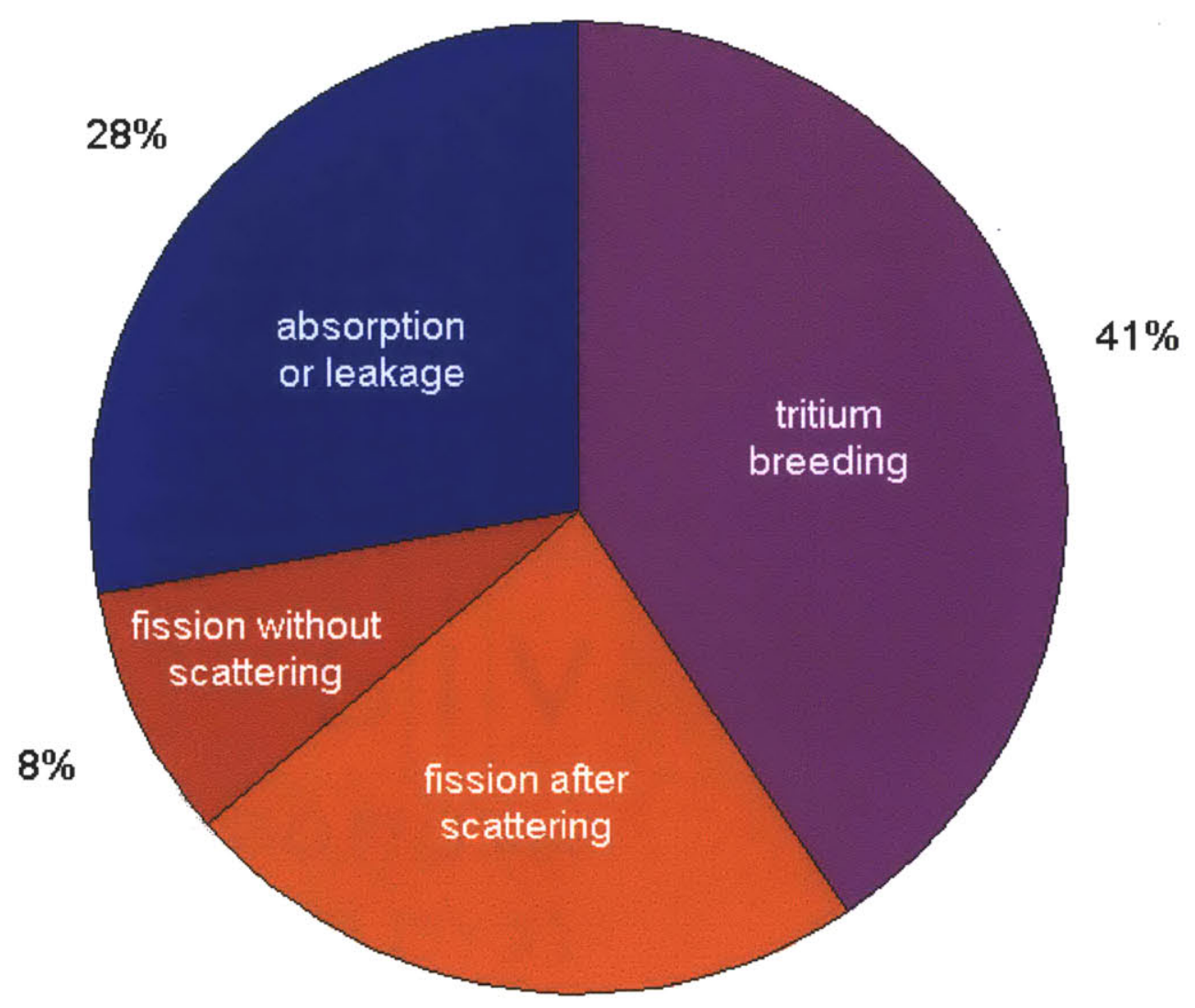

$23 \%$

Figure 3.15: The fate of fusion-born (1st generation) neutrons. $8 \%$ spur fission on their first collision, while $31 \%$ eventually spur fission. $41 \%$ pass through the uranium layer to breed tritium, and the remaining $28 \%$ are absorbed or leaked. 


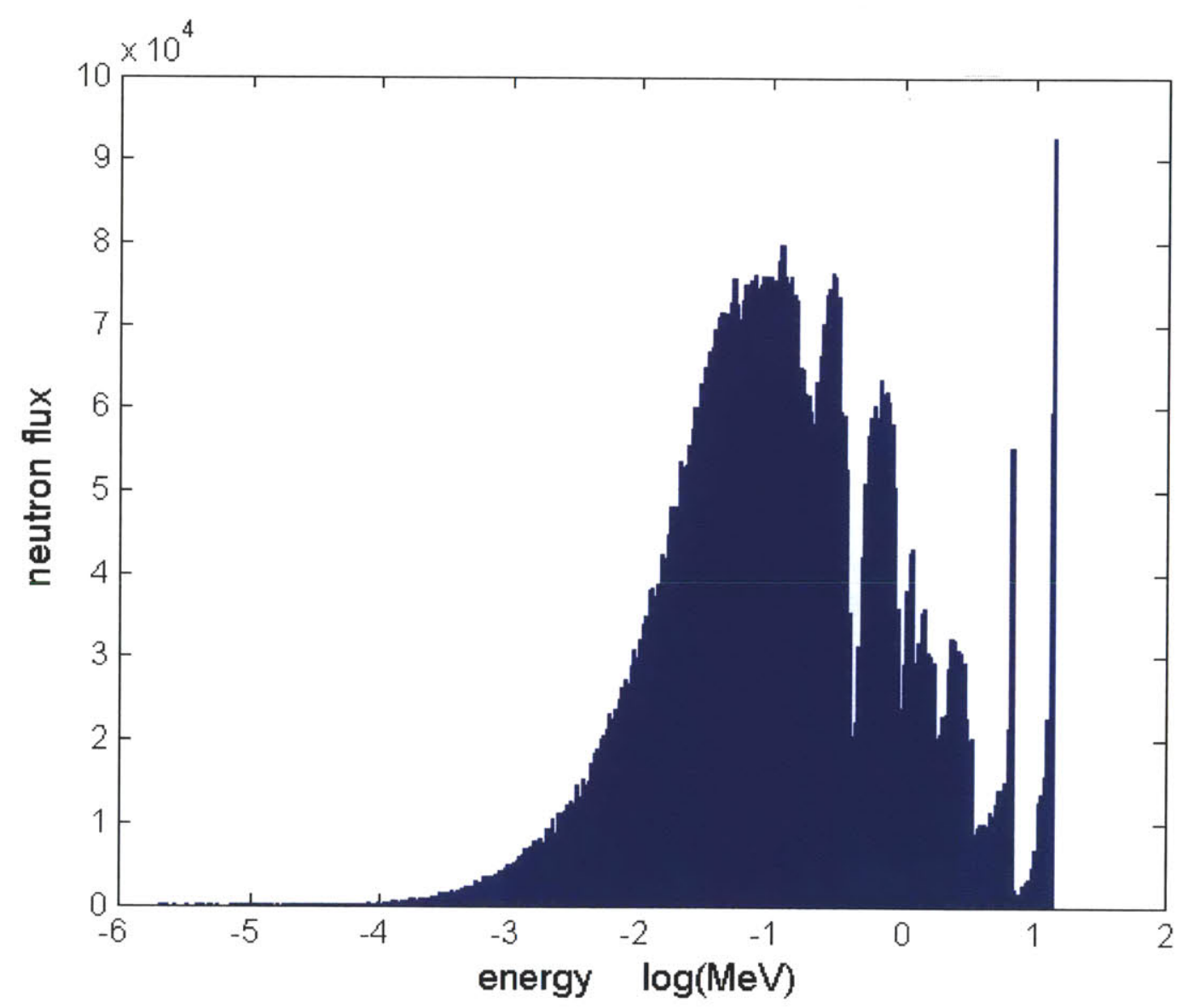

Figure 3.16: The total neutron energy spectrum in the uranium pebble layer. The large spike at $14 \mathrm{MeV}$ represents the fusion-born neutrons before collision. The smaller spike to its left is an artificial result of our inelastic scattering approximation. Oxygen resonances cause conspicuous flux dips at lower energies. The average energy is $0.94 \mathrm{MeV}$, and so we can rightfully call this spectrum fast. 


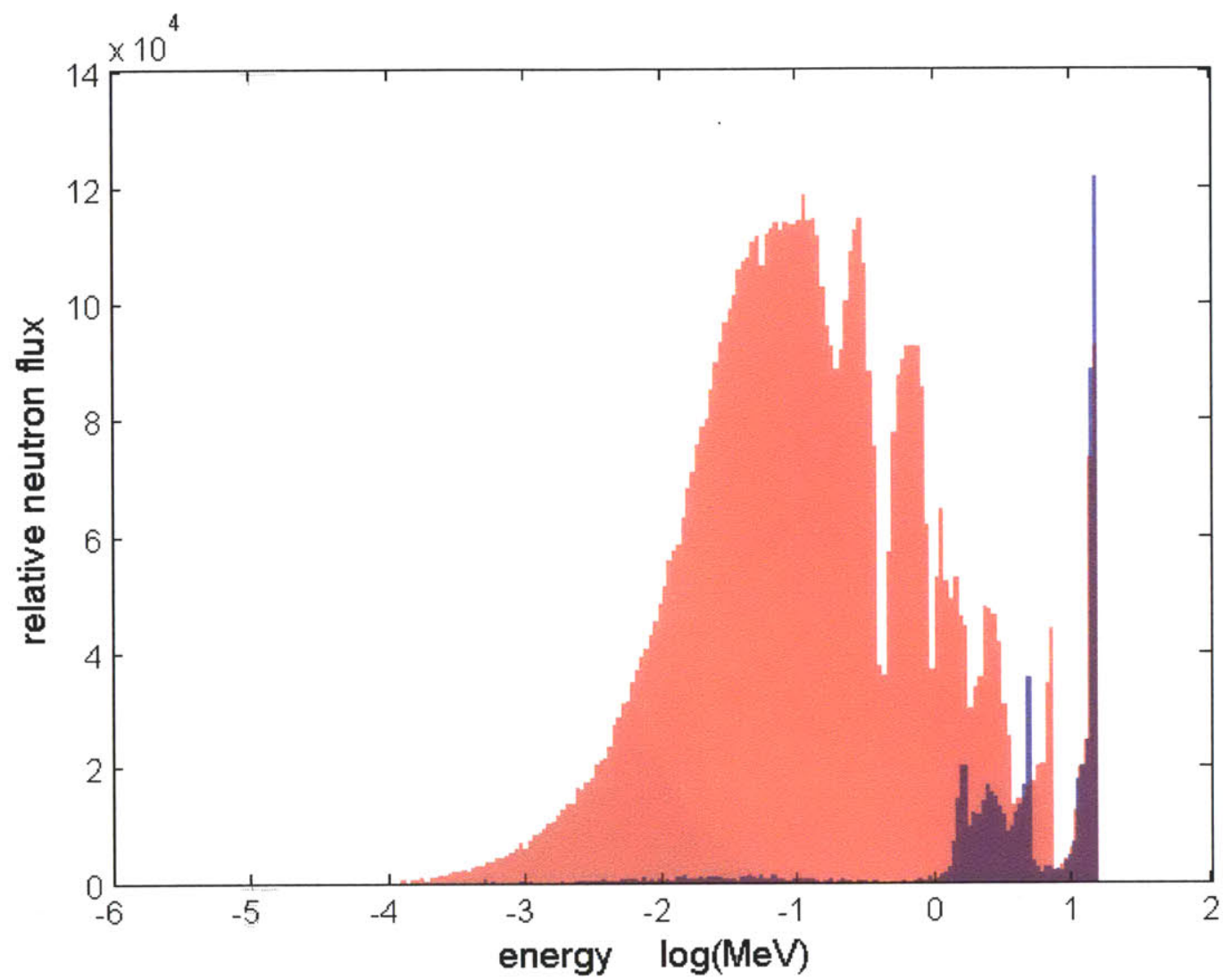

Figure 3.17: The total neutron energy spectrum (red) and the fission-inducing neutron energy spectrum (blue) in the uranium pebble layer. To obtain the latter, we tally the energies of all incident neutrons in fission events. While the average neutron energy is $0.94 \mathrm{MeV}$, the average fission-inducing neutron energy is $9.90 \mathrm{MeV}$ ! The spectra magnitudes are not to scale with each other. 


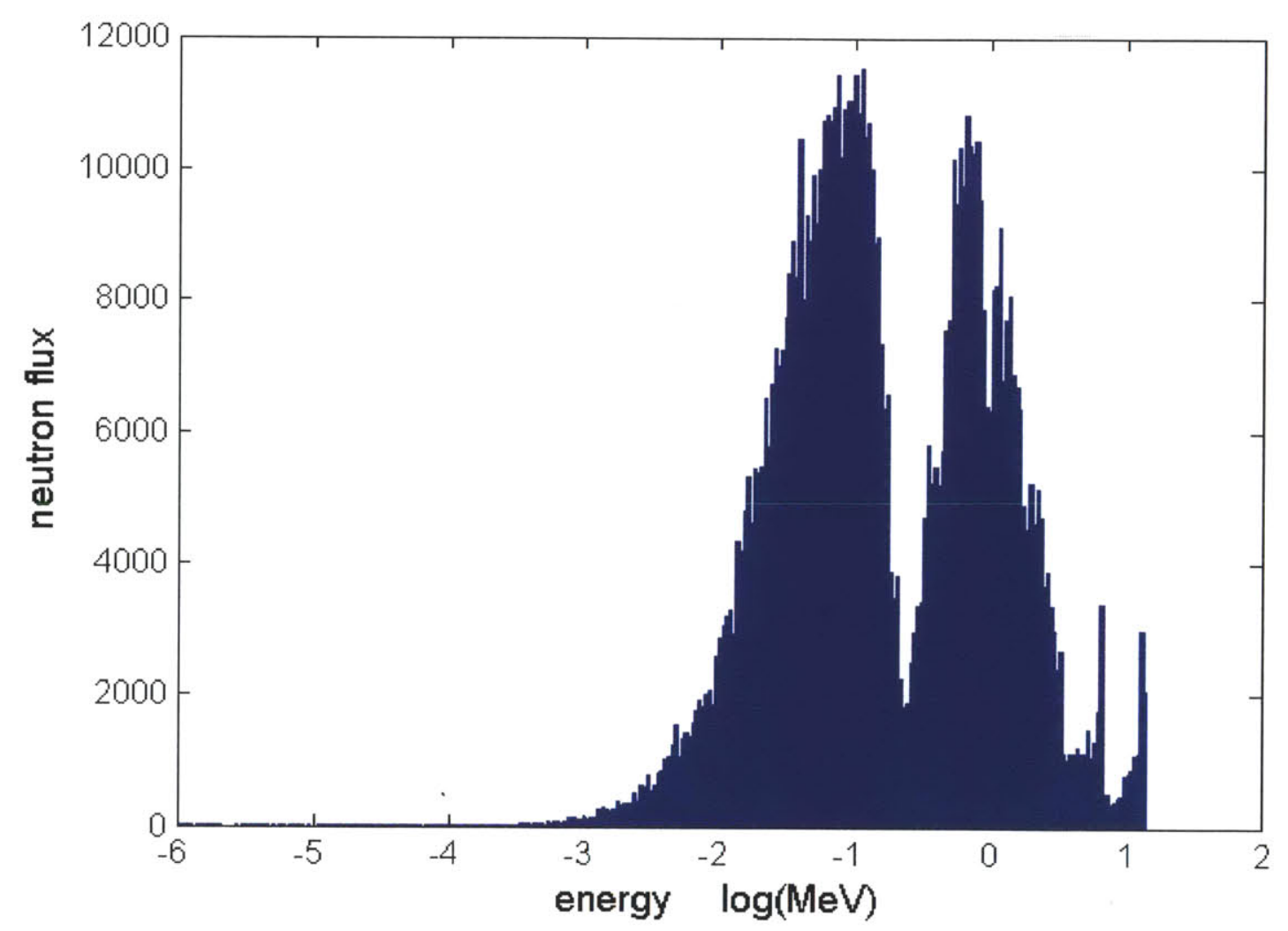

Figure 3.18: The total neutron energy spectrum in the Li-Pb layer. The fusion-born neutrons spike is evident but not nearly as prominent as in the uranium layer. The massive chasm centered around $0.3 \mathrm{MeV}$ is the result of a large resonance in ${ }^{6} \mathrm{Li}$. The average neutron energy here is 0.77 $\mathrm{MeV}$, so the spectrum is a bit slower than in the uranium layer. 


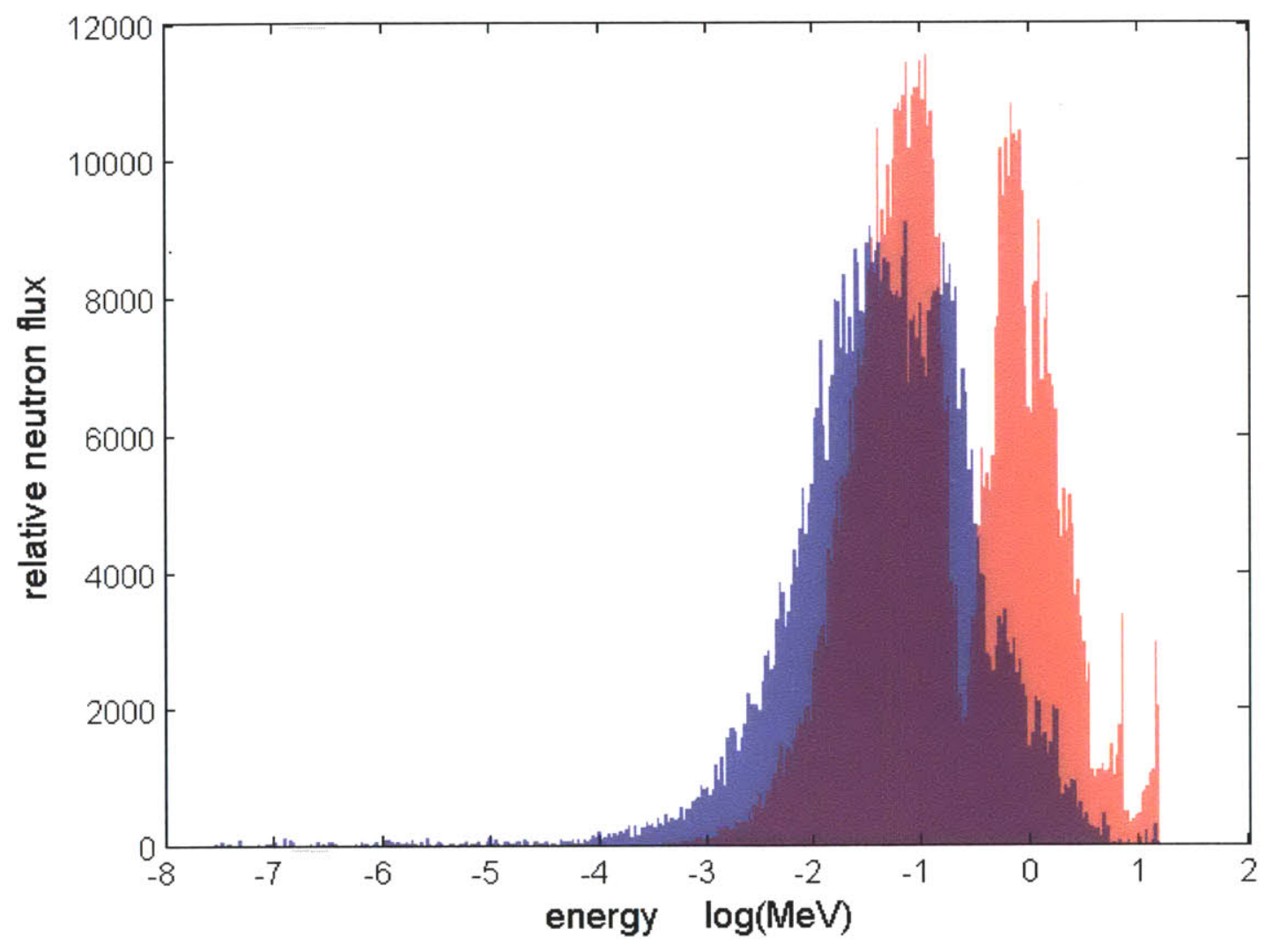

Figure 3.19: The total neutron energy spectrum (red) and the tritium-breeding neutron energy spectrum (blue) in the $\mathrm{Li}-\mathrm{Pb}$ layer. To obtain the latter, we tally the energies of all incident neutrons in tritium breeding events. While the average neutron energy is $0.77 \mathrm{MeV}$, the average fission-inducing neutron energy is $0.29 \mathrm{MeV}$. The spectra magnitudes are not to scale with each other. 


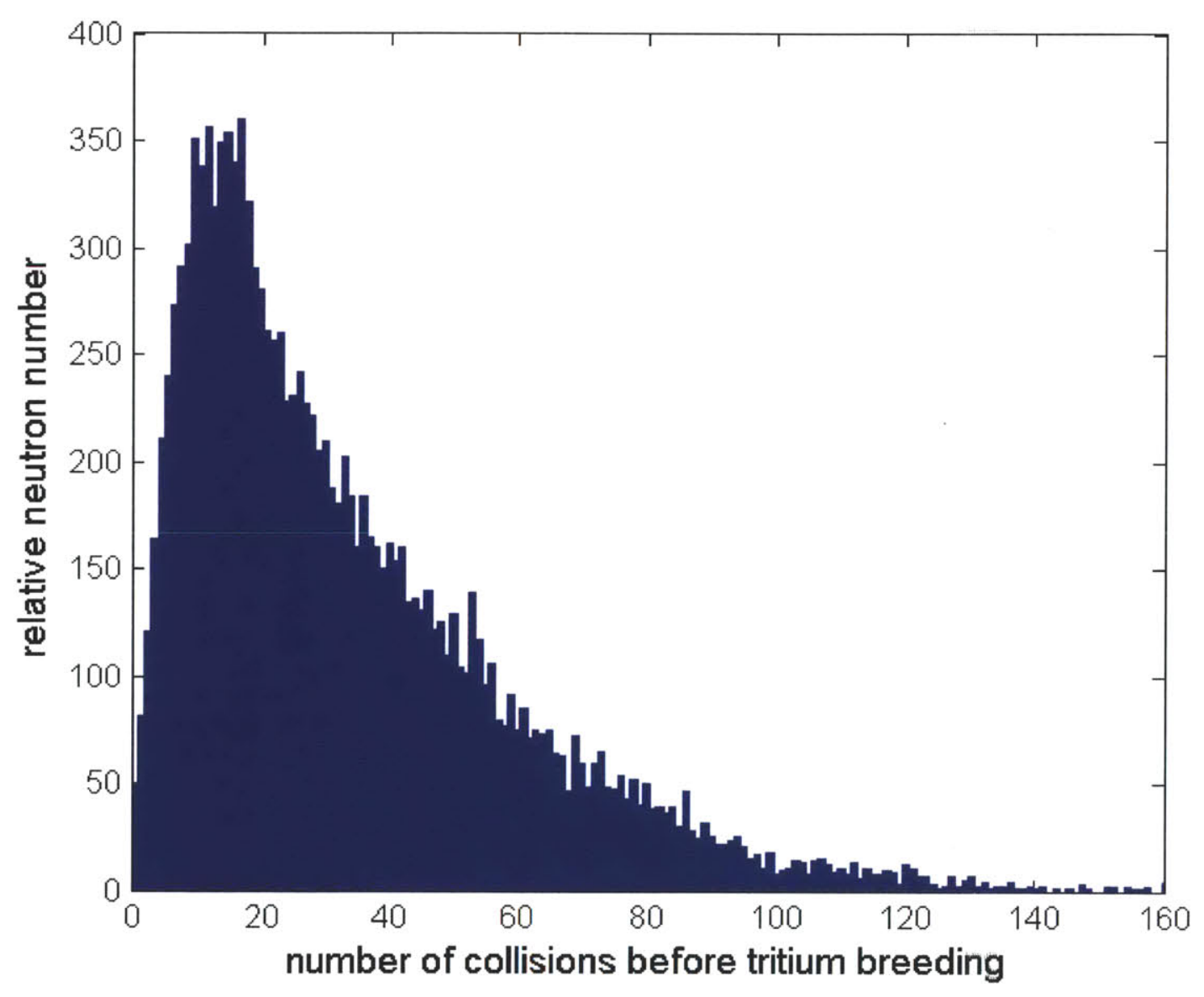

Figure 3.20: A distribution of the number of collisions a fusion-born neutron undergoes before breeding tritium (considering only the neutron that do eventually breed tritium, of course). The neutrons typically scatter off the lead and lithium numerous times until they reach lower energies where the ${ }^{6} \mathrm{Li}$ tritium breeding cross-section is high. 


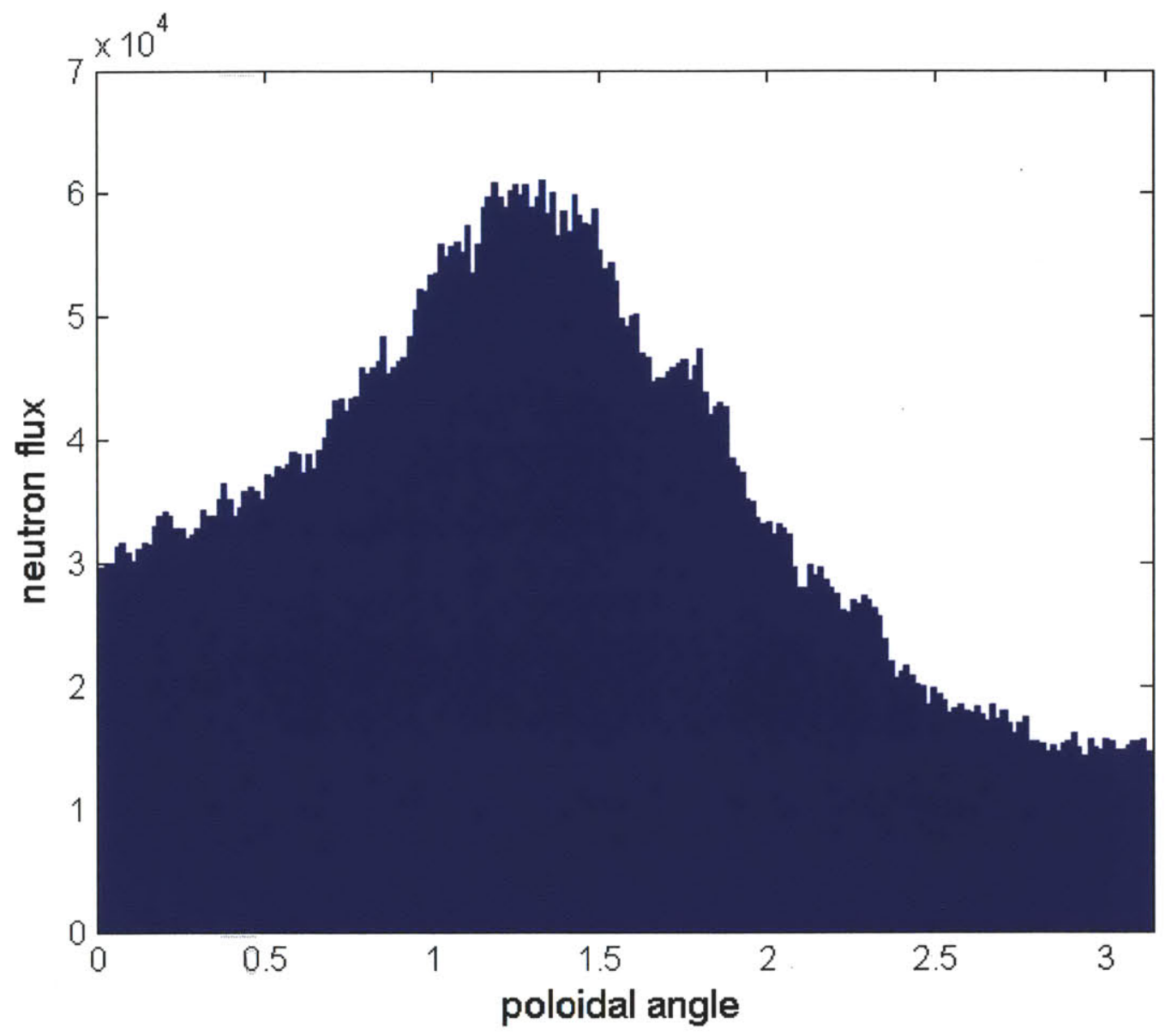

Figure 3.21: The total neutron flux in the uranium pebble layer as a function of poloidal angle. This generally reflects the neutron flux distribution on toroidal surfaces that we computed in Section 2 . 


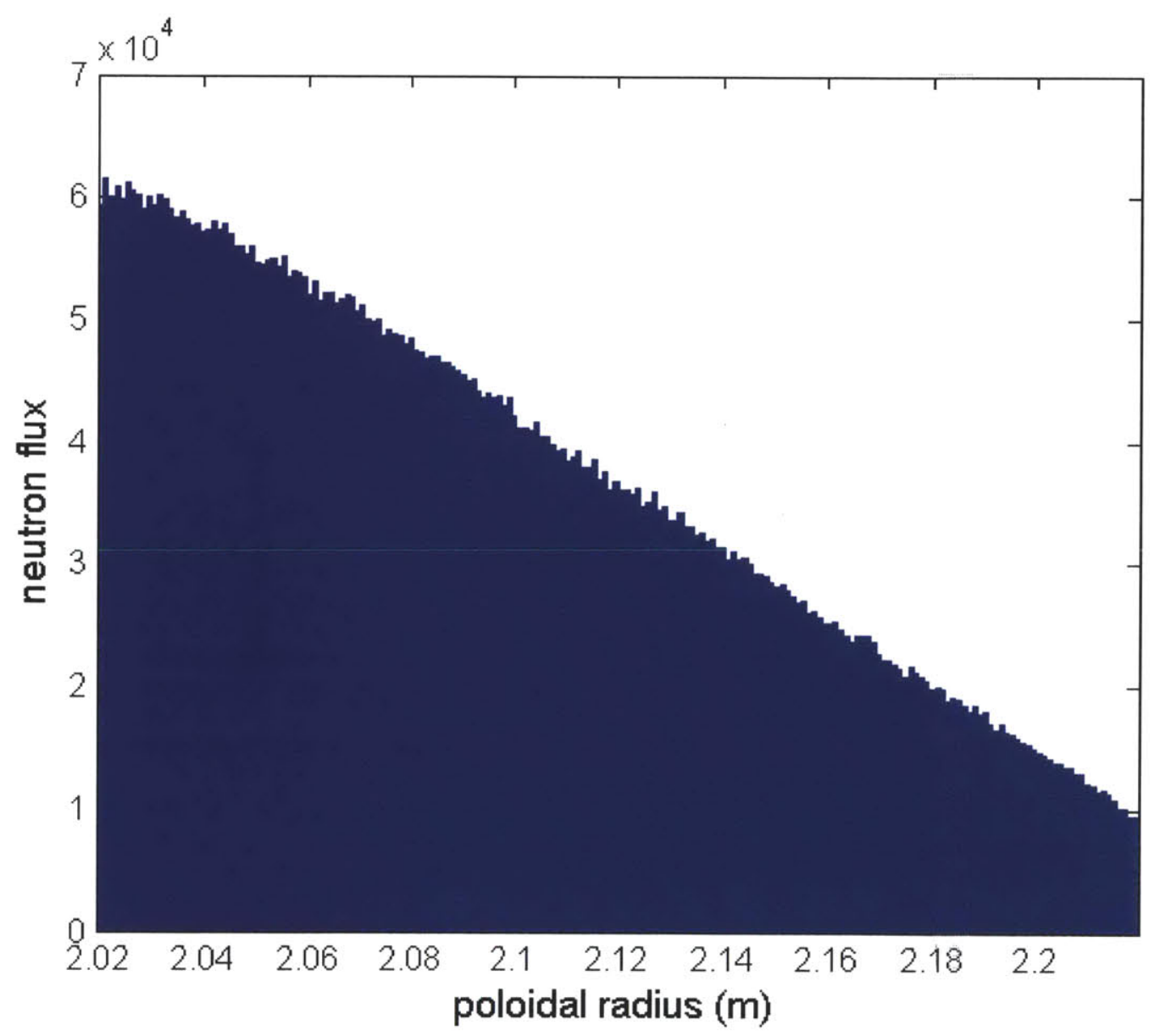

Figure 3.22: The total neutron flux in the uranium pebble layer as a function of radius (normalized as if $\kappa=1$ ). This is consistent with what we expect for a subcritical reactor with a source coming from the left. 


\section{A Tokamak Fusion Core Model}

We have completed our fission blanket analysis in toroidal geometry, but we have not yet said anything substantial about the fusion core. We have optimized the blanket layer thicknesses, but we have not optimized the basic physical dimensions of the tokamak $(R, a$, and $\kappa)$. In order to proceed, we have developed a simple model to relate various tokamak parameters such as total fusion power, magnetic field strength, physical dimensions, and input auxiliary power. We explain that model here in this section.

We originally developed this model as part of the Fall 2008 MIT Nuclear Science and Engineering Design Project, which produced the HYPERION conceptual design [4]. We expanded it for our May 2009 Bachelor of Science thesis in the Department of Physics [28]. In those studies, we analyzed various tokamak parameters as well as economic concerns to determine the optimal tokamak size and minimum tokamak size that allow for steady-state L-mode operation. In subsequent sections of this thesis, we will couple this tokamak model to our new fission blanket model to explore how the fission power gain eases constraints on tokamak operation.

\subsection{Concept and Geometry}

Tokamaks are toroidal chambers which magnetically confine plasma. Figure 4.1 shows the basic geometry of a tokamak. We also used this in Section 2, but it is pertinent again here. $R$ is the major radius, and $a$ is the minor radius. $\Phi$ and $\Theta$ represent the toroidal and poloidal angular directions, respectively. In practice, tokamaks usually have D-shaped poloidal crosssections to achieve favorable magnetic topologies. For simplicity, we will model the poloidal cross-section as an ellipse with elongation $\kappa$, which is equal to the ratio of the major axis $\kappa a$ to the minor axis $a$.

Superconducting coils are wound around the tokamak poloidally (not helically, as in stellarators) to produce a purely toroidal ( $\Phi$ direction) magnetic field. A large solenoid filling the center of the tokamak produces flux swing and thus induces a toroidal current in the plasma. This toroidal current in turn produces a poloidal ( $\Theta$ direction) magnetic field. The toroidal and poloidal magnetic fields confine the plasma such that it can, with sufficient temperature and density, produce fusion reactions to generate enormous thermal power.

We will focus on deuterium-tritium (D-T) fusion reactions and assume that the plasma consists of half deuterium and half tritium. The fusion reaction is

$$
{ }_{1}^{2} \mathrm{H}+{ }_{1}^{3} \mathrm{H} \rightarrow{ }_{2}^{4} \mathrm{He}(3.5 \mathrm{MeV})+{ }_{0}^{1} \mathrm{n}(14.1 \mathrm{MeV})
$$

It is important to note that while the charged $\alpha$-particle is confined by the magnetic fields, the uncharged neutron is not. The plasma absorbs the $\alpha$-particle energy, which is $1 / 5$ the total energy produced by the fusion reaction. 


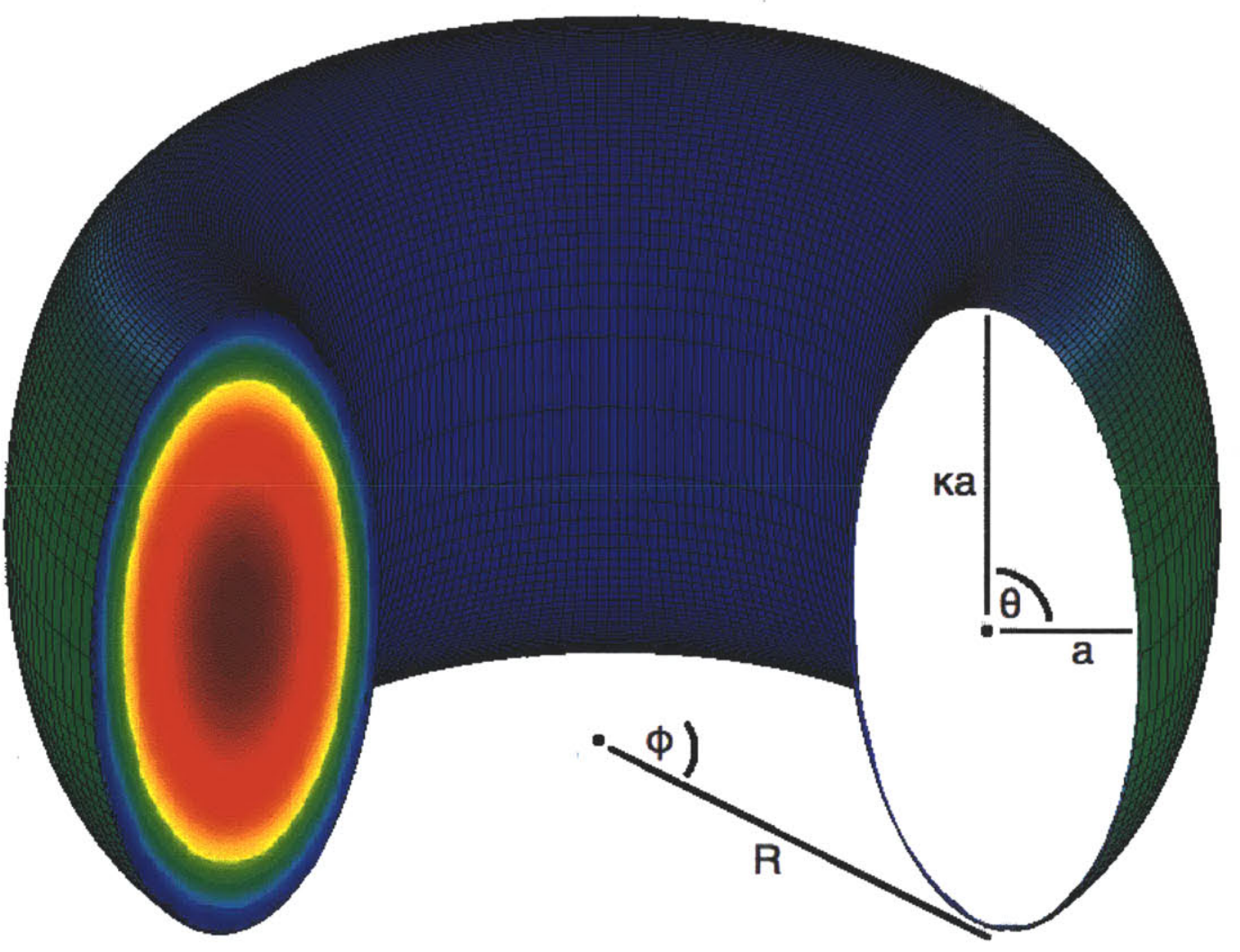

Figure 4.1: An elliptical torus model for a tokamak. $R$ is the major radius, $a$ the minor radius, and $\kappa$ the elongation. $\Phi$ and $\Theta$ represent the toroidal and poloidal angular directions, respectively. 


\section{$4.2 \quad$ 0-D Core Model Overview}

A "perfect" model of a tokamak would include 1-D or 2-D distributions. However, for the purpose of obtaining a model that is computationally simple enough to perform extensive analysis with, a 0-D model is the most efficient approach. Since we plan to formulate only an approximate conceptual design, 0-D will both meet our design goals and allow us to provide general insight into tokamak design.

0-D analysis assumes the plasma has only volume-averaged parameters, meaning that all its properties (temperature, pressure, density, magnetic fields, etc.) are represented by a single "average" $0-\mathrm{D}$ value rather than by a spatial distribution. The plasma is fully ionized and consists of half deuterium and half tritium with negligible densities of $\alpha$-particles and impurities.

Though not precise, the 0-D model greatly simplifies computational analysis so that we can take more considerations into account and explore wider parameter spaces at higher resolution. Later on, we will verify the chosen 0 -D operating point with 1-D analysis.

\subsection{0-D Core Model System Parameters}

Here we will define specify all the interrelationships of our entire $0-\mathrm{D}$ model. Let $R / a$ be the aspect ratio of the tokamak. The aspect ratio is the most distinguishing geometric parameter, as it determines if the tokamak looks like sphere, a doughnut, or a hula hoop. Any tokamak model will be very sensitive to $R / a$.

The poloidal cross-sectional area is simply

$$
A_{C S}=\pi \kappa a^{2}
$$

The toroidal volume is approximately that area multiplied by the toroidal circumference:

$$
V=(2 \pi R) A_{C S}
$$

Using a good approximation for the perimeter of an ellipse as $2 \pi a\left[\left(1+\kappa^{2}\right) / 2\right]^{1 / 2}$, the toroidal surface area is

$$
A_{S}=(2 \pi R)(2 \pi a)\left(\frac{1+\kappa^{2}}{2}\right)^{1 / 2}
$$

It is also important to note that a shielding blanket of width $w_{B}$ covers this entire toroidal surface. In the case of a hybrid, $w_{B}$ is the fission blanket thickness.

Now that the geometry is defined, consider the toroidal magnetic field $B_{\Phi}$. It is generated by current-carrying coils wrapped around the torus in the poloidal plane. We can calculate its magnitude in the toroidal direction using a simple application of Ampere's law (with a 
toroidal loop) to find that $B_{\Phi}$ has a $1 / R$ (inverse major radius) dependence. Assuming that $B_{\Phi}$ has some value $B_{\max }$ on the coil surface, $B_{\Phi}(R)$ is

$$
B_{\Phi}=\left(\frac{R-a-w_{B}}{R}\right) B_{\max }
$$

In 0-D analysis, this is the "average" $B_{\Phi}$. For simplicity, we will often call it $B$.

A very important quantity is the safety factor $q$, which is a measure of how tightly wound the magnetic field lines are about the torus. It is quantified as the inverse of the number of poloidal revolutions per toroidal revolution $(\Delta \Theta / 2 \pi)^{-1}$ along a magnetic field line [7]. This is related to $d \Phi / d \Theta$ along a field line. If $q$ is low, the magnetic field is primarily poloidal and confines the plasma quite well but is potentially vulnerable to current-driven instabilities. If $q$ is high, the magnetic field is primarily toroidal and confines the plasma less well but is not vulnerable to such instabilities. Essentially, the toroidal current (which the poloidal field is proportional to) must not be too large relative to the toroidal field. Thus, $q$ is a measure of how stable the plasma is against current-driven instabilities, and we prefer it to be high, even at the expense of confinement.

$$
q(\psi)=\frac{R_{0} B_{\Phi}}{2 \pi} \oint \frac{d \ell_{\Theta}}{R^{2} B_{\Theta}} \approx\left[\frac{B_{\Phi}}{\wp_{\Phi}}\right]\left[\frac{\wp_{\Theta}}{B_{\Theta}}\right]=\left[\frac{2 \pi}{\langle\Delta \Theta\rangle}\right]_{\text {field line }}
$$

Here $R_{0}$ is the fixed major radius, and $d \ell_{\Theta}$ the differential poloidal length. $\wp_{\Theta}$ and $\wp_{\Phi}$ are the toroidal and poloidal cross-section perimeters, respectively. In order to make this approximation, we have assumed that all magnetic fields have "average" 0 -D values. We treat this as a cylindrical "screw pinch".

Although $q$ has only one value for a whole tokamak and is a function of the plasma flux $q(\psi)$, we can define it locally as a function of poloidal radius $q(r)$ if we assume that each plasma flux $\psi$ contour maps directly to a unique radial position $r$. This assumes that the flux contours are concentric with the plasma poloidal cross-section. Of course, this cross-section is elliptic rather than circular, so we must also assume that the flux $\psi(r)$ in the horizontal direction maps to the flux $\psi(\kappa r)$ in the vertical direction and to the flux $\psi\left(r \sqrt{\cos ^{2} \Theta+\kappa^{2} \sin ^{2} \Theta}\right)$ in any arbitrary direction within the poloidal plane. These are significant simplifications, but they are adequate for a 0-D or 1-D model. In 0-D analysis, $q$ is constant and equal to $q^{*}$, which in turn is equal to $q(r=a)$ as long as $R / a$ is not too large [7]. We can calculate the "average" $B_{\ominus}$ with another simple application of Ampere's law (this time with a poloidal loop) given that the total plasma current is $I_{P}$. Let the toroidal and poloidal cross-section perimeters be $\wp_{\Phi}=2 \pi R$ and $\wp_{\Theta}=2 \pi a\left[\left(1+\kappa^{2}\right) / 2\right]^{1 / 2}$. Equation 4.7 expresses $q^{*}$ in terms of $B_{\Phi}, I_{P}$, and geometry. 


$$
q * \approx q(a) \approx\left[\frac{B_{\Phi}}{\wp_{\Phi}}\right]\left[\frac{\wp_{\Theta}}{B_{\Theta}}\right]=\left[\frac{B_{\Phi}}{2 \pi R}\right]\left[\frac{2 \pi a\left(\frac{1+\kappa^{2}}{2}\right)^{1 / 2}}{\mu_{0} I_{P} / 2 \pi a\left(\frac{1+\kappa^{2}}{2}\right)^{1 / 2}}\right]=\frac{B_{\Phi}}{\mu_{0} I_{P}} \frac{\pi a^{2}}{R}\left(1+\kappa^{2}\right)
$$

Given the total plasma current and the poloidal cross-section, the total ion density $n$ is

$$
n=n_{e}=F_{G}\left(\frac{I_{P}}{\pi a^{2}}\right)
$$

$F_{G}$ is the Greenwald fraction, the ratio of plasma density to the Greenwald empirical density limit of $I_{P} / \pi a^{2}$, with $I_{P}$ in MA and $n$ in $10^{20} / \mathrm{m}^{3} . F_{G}$ can range from 0 to 1 and sets a limit on the plasma density available without disruption [7].

Power balance requires that the total power lost $P_{\text {loss }}$ balance the total power consumed, which is the auxiliary heating power $P_{\text {aux }}$ and the $\alpha$-particle power $P_{\alpha}[15]$. We neglect radiative power in $0-\mathrm{D}$.

$$
P_{\text {aux }}+P_{\alpha}=P_{\text {loss }}
$$

Since the $\alpha$-particles are confined and eventually transfer all their energy to the plasma, we can express $P_{\alpha}$ as the product of the $\alpha$-particle energy $E_{\alpha}(\mathrm{J})$ and the reaction frequency $f_{\text {reac }}\left(\mathrm{s}^{-1}\right)[15]$. The density of each colliding particle is $n / 2$, and the D-T reactivity rate coefficient $\left(\mathrm{m}^{3} / \mathrm{s}\right)$ is $\langle\sigma v\rangle$. Equation 4.10 expresses $n$ in single particles per cubic meter and $P_{\alpha}$ in watts.

$$
P_{\alpha}=E_{\alpha} f_{\text {reac }}=\frac{n^{2}}{4}\langle\sigma v\rangle E_{\alpha} V
$$

$P_{\text {loss }}$ represents the natural rate of internal energy loss in the plasma. The internal energy of a plasma is $3 n k T$, where $T$ is the temperature in Kelvin and $k$ is the Boltzmann constant. $\tau_{e}$ is the energy confinement time in seconds, the $e$-folding time of internal energy $(\mathrm{W})$ decay due to heat conduction [15].

$$
P_{\text {loss }}=\frac{W}{\tau_{e}}=\frac{3 n k T}{\tau_{e}} V
$$

Then the explicit power balance is [15]

$$
P_{\text {aux }}+\frac{n^{2}}{4}\langle\sigma v\rangle E_{\alpha} V=\frac{3 n k T}{\tau_{e}} V
$$

$P_{\text {aux }}$ is the externally-applied power, and so the plasma must "ignite" and sustain itself when $P_{\text {aux }}=0$. The ignition condition (often called the Lawson criterion) is thus [15] 


$$
n \tau_{e} \geq \frac{12 k T}{\langle\sigma v\rangle E_{\alpha}}
$$

$Q$ is the fusion power gain, the ratio of the total fusion power $P_{F}$ to the total externallyapplied power $P_{\text {aux }}$. It is important to note that $P_{F}$ is always five times greater than $P_{\alpha}$, because the energy released in each fusion reaction is five times the $\alpha$-particle energy (see Equation 4.1). Most of the fusion power does not contribute to heating, because the uncharged product particles (neutrons) are not confined [15].

$$
Q=\frac{P_{F}}{P_{\text {aux }}}=\frac{5 P_{\alpha}}{P_{\text {aux }}}=\frac{\left(n^{2} / 4\right)\langle\sigma v\rangle\left(5 E_{\alpha}\right) V}{P_{\text {aux }}}
$$

The fusion power per surface area $P_{F} / A_{S}$ is another important quantity. The blanket material will, after exhausting power for a long enough time, inevitably require replacement. Also, the blanket must be feasible to cool from a thermal hydraulics perspective. This puts a practical limit on $P_{F} / A_{S}$ that the HYPERION team determined with economic and sustainability analysis. Thus, fusion power is a function of the reactor size only.

A useful scaling parameter is the enhancement factor $H$, which defines the tokamak operating mode (L-mode or $\mathrm{H}$-mode) and has been empirically determined as a function of operating parameters. Equation 4.15 shows the 1989 scaling for $H$, which we will use throughout this analysis [7].

$$
\tau_{e}=(0.048) H R^{1.2} I_{P}^{0.85} \kappa^{0.5} M^{0.5} a^{0.3} B^{0.2} n^{0.1}\left(P_{\text {aux }}+P_{\alpha}\right)^{-0.5}
$$

$M$ is the average atomic mass of the plasma nuclei, which is $2.5 \mathrm{amu}$ for D-T fusion. Equation 4.15 expresses $n$ in $10^{20} / \mathrm{m}^{3}, M$ in amu, $I_{P}$ in MA, $R$ and $a$ in meters, $B$ in Tesla, $P_{\text {aux }}$ and $P_{\alpha}$ in MW, and $\tau_{e}$ in seconds.

In 0-D analysis, the volume-averaged pressure $\left(\mathrm{MJ} / \mathrm{m}^{3}\right)$ is due to heating power. It is on the order of a few bar.

$$
\langle p\rangle=\frac{2}{3} \frac{\left(P_{\mathrm{aux}}+P_{\alpha}\right) \tau_{e}}{V}
$$

The normalized plasma pressure $\beta$ is the ratio of the kinetic plasma pressure to the magnetic pressure. It is a measure of how well the magnetic field confines the plasma and thus how stable the plasma is. We can now calculate the toroidal and poloidal $\beta$ values $\beta_{T}$ and $\beta_{P}$. We evaluate $B_{\Phi}(a)$ and $B_{\Theta}(a)$ as shown previously [7].

$$
\begin{aligned}
& \beta_{T}=\frac{\langle p\rangle}{B^{2} / 2 \mu_{0}} \\
& \beta_{P}=\frac{\langle p\rangle}{B_{\Theta}^{2}(a) / 2 \mu_{0}}=\frac{4 \pi^{2} a^{2}\left(1+\kappa^{2}\right)\langle p\rangle}{\mu_{0} I_{P}^{2}}
\end{aligned}
$$


Another pressure parameter is $\beta_{N}$, which has been found to be the most useful measure of stability in tokamaks. $\beta_{N}$ is $\beta_{T}$ normalized with a ratio of the poloidal field to the toroidal field at the outermost edge of the toroid, where the toroidal field is weakest. Even though the toroidal field is uniform in 0-D analysis, $\beta_{N}$ is still quite important.

$$
\beta_{N}=\frac{\beta_{T}(\%)}{\mu_{0} I_{P} / a B}=\frac{a\langle p\rangle}{50 I_{P} B}
$$

\subsubsection{D-T Fusion Rate Coefficient}

The D-T fusion reaction rate coefficient $\langle\sigma v\rangle$ is a critical part of the $0-\mathrm{D}$ model. It depends only on $T$ but has no simple analytic form. The Naval Research Laboratory (NRL) Plasma Formulary lists empirical values of $\langle\sigma v\rangle$ for values of $T$ between 1 and $1000 \mathrm{keV}[10]$. We fit a logarithmic polynomial to this data:

$$
\log _{10}\langle\sigma v\rangle=-\frac{\left(\log _{10} T\right)^{5}}{16.61}+\frac{\left(\log _{10} T\right)^{4}}{1.78}-\frac{\left(\log _{10} T\right)^{3}}{0.64}+\frac{\log _{10} T}{0.19}-26.11
$$

$\langle\sigma v\rangle$ is expressed in $\mathrm{m}^{3} / \mathrm{s}$ and $T$ in $\mathrm{keV}$. Figure 4.2 shows that this fit function and the NRL data points are very consistent for $1 \mathrm{keV}<T<1000 \mathrm{keV}$.

\subsubsection{Elongation vs. Aspect Ratio}

To reduce the number of free parameters, it is useful to express the elongation $\kappa$ in terms of $R / a$ by realizing that there is a maximum $\kappa$ that depends on $R / a$. When $R / a$ is very large, the tokamak can be treated like a cylindrical "screw pinch" with $\kappa=1$. As $R / a$ decreases, the maximum allowed $\kappa$ increases. Examining $R / a$ and $\kappa$ for the C-Mod, the DIII-D, and two NHTX tokamaks shows that an excellent model for the relationship is

$$
\kappa \leq 5.276\left(\frac{R}{a}\right)^{-0.985}
$$

We desire high $\kappa$, because it yields high surface area and thus high fusion power without affecting the density-current relationship (Equation 4.8). We will set $\kappa$ at this limit.

\subsubsection{Plasma Current and Sustainment}

The total plasma current $I_{P}$ is a sum of three currents from three different sources.

$$
I_{P}=I_{C D}+I_{\text {boot }}+I_{\text {induced }}
$$

$I_{\text {induced }}$ is the current induced by the solenoid. $I_{C D}$ is the externally-driven current from the complex processes of electron-cyclotron heating and optical steering, which we will not 


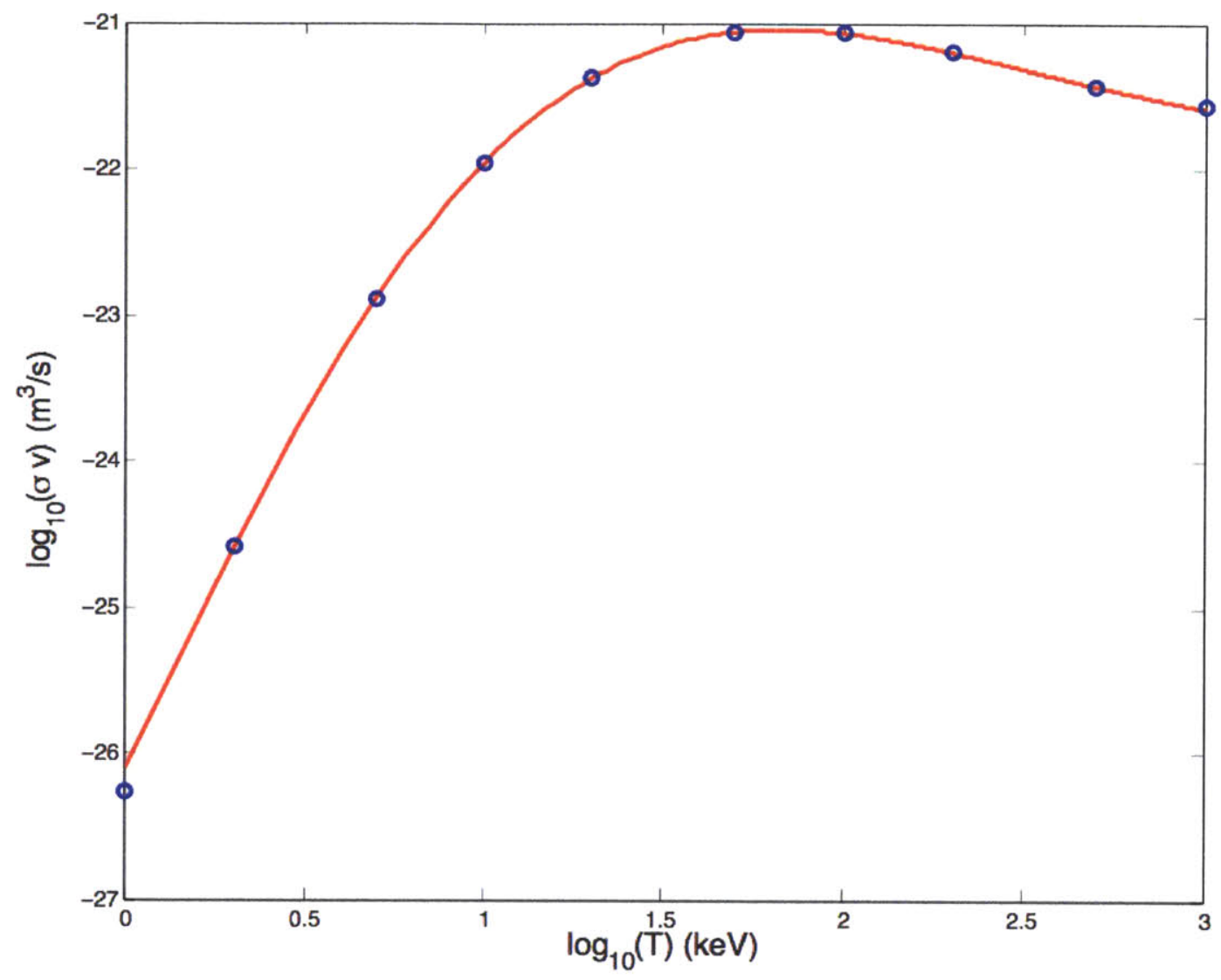

Figure 4.2: A log-log plot of the D-T reactivity coefficient $\langle\sigma v\rangle$ as a function of temperature $T$. The polynomial fit function is shown in red, and the NRL data points are shown in blue. This fit is valid for $1 \mathrm{keV}<T<1000 \mathrm{keV}$. Note that $\langle\sigma v\rangle$ has an absolute maximum at approximately 65 $\mathrm{keV}$.

describe in detail here [6]. We can express $I_{C D}$ quantitatively as $P_{F} / n R$ with a current-drive efficiency $\eta_{C D}$, which is approximately constant at $0.3 \times 10^{20} \mathrm{~A} / \mathrm{Wm}^{2}$ for temperatures above $20 \mathrm{keV} \mathrm{[9]} \mathrm{[12].}$

$$
I_{C D}=\frac{\eta_{C D} P_{F}}{n R}
$$

$I_{\text {boot }}$ is the "bootstrap current", which naturally arises from neoclassical transport through density and temperature gradients [7]. A common expression for the total $I_{\text {boot }}$ is

$$
I_{\text {boot }}=\left(\frac{\beta_{N}}{100}\right) \frac{(12.5) c_{B S} a^{2} B\left(1+\kappa^{2}\right)}{(a R)^{1 / 2}}
$$

$c_{B S}$ is a fitting constant, and we can assume it is approximately 0.8 in 0-D analysis [12] [7]. 
$f_{C D}=I_{C D} / I_{P}$ and $f_{\text {boot }}=I_{\text {boot }} / I_{P}$ are the current-drive and bootstrap fractions, respectively. The non-inductive current fraction $f_{N I}$ (the fraction of current that is not induced by the solenoid) is

$$
f_{N I}=\frac{I_{C D}+I_{\mathrm{boot}}}{I_{P}}=f_{C D}+f_{\mathrm{boot}}
$$

When $f_{N I}$ is 1 or greater, the tokamak is "fully non-inductive" and "steady-state", because it can recharge the solenoid without ceasing operation. $f_{N I}>1$ is also called "overdrive", and it is a primary design goal.

In order to externally drive current with electron-cyclotron heating, the electron-cyclotron frequency $\omega_{c e}$ absolutely must be greater than the electron plasma frequency $\omega_{p e}$ so that the electron-cyclotron waves can propogate without interference [7]. These two frequencies are standard in basic plasma physics [3].

$$
\begin{aligned}
& \omega_{p e}=\left(\frac{n e^{2}}{\epsilon_{0} m_{e}}\right)^{1 / 2} \\
& \omega_{c e}=\frac{e B}{m_{e}}
\end{aligned}
$$

The tokamak begins operating by "ramping up" induced current with the solenoid. For this process to work, the solenoid flux swing $\Delta \Phi_{\text {sol }}$ must be sufficiently larger than the plasma flux $\Phi_{P}$. ITER studies suggest that $\Delta \Phi_{\text {sol }} / \Phi_{P}$ must be at least $\sim 2$ [4]. We can simply express $\Delta \Phi_{\text {sol }}$ as

$$
\begin{aligned}
& \Delta \Phi_{\mathrm{sol}}=\left(2 B_{\max }\right)\left(\pi r_{\mathrm{sol}}^{2}\right) \\
& r_{\mathrm{sol}}=R-a-w_{B}-\frac{R}{6.2 \mathrm{~m}}(0.5 \mathrm{~m})
\end{aligned}
$$

The maximum field within the solenoid is simply $B_{\max }$. The factor of 2 represents the fact that the flux "swings" from $-B_{\max }$ to $+B_{\max }$. The maximum solenoid radius $r_{\text {sol }}$ is the difference between $R$ and the sum of $a$, the blanket width $w_{B}$, and the coil thickness. Given that the coil thickness for ITER $(R=6.2 \mathrm{~m})$ is $0.5 \mathrm{~m}$, we scale it up in proportion to R. Determining $\Phi_{P}$ requires deriving the toroidal inductance of the tokamak. The result is approximately [4]

$$
\Phi_{P}=\mu_{0} R I_{P}\left[\ln \left(\frac{R}{(\kappa a)^{1 / 2}}\right)+\frac{1}{2}\right]
$$

Ensuring that $\omega_{c e} / \omega_{p e}>1$ and $\Delta \Phi_{\text {sol }} / \Phi_{P} \sim 2$ is essential. 


\subsection{L-mode and H-mode}

Low confinement mode (L-mode) and high-confinement mode (H-mode) are two distinct operating modes of a tokamak plasma. L-mode is characterized by smooth temperature, density, and power profiles. It is predictable and well-understood physically. At one time, it was the only known operating mode. As one increases the auxiliary power $P_{\text {aux }}$ in an L-mode tokamak plasma, a sudden transition occurs in which the density profile becomes nearly flat throughout the plasma. The temperature profile also flattens to a degree. The high edge density and edge temperature cause a high edge pressure, called an "edge pedestal". This in turn slows energy loss through the plasma surface, increasing the energy confinement time $\tau_{e}$. These changes characterize H-mode, a high confinement mode [7]. The physical basis for $\mathrm{H}$-mode and the sudden L-H transition are not fully understood. The enhancement factor $H$ (see Equation 4.15) was developed in part to quantify this transition. There are different scalings for $H$, but we will always use the 1989 scaling. Figure 4.3 shows that H-mode is only possible for $H$ greater than approximately 1.5 .

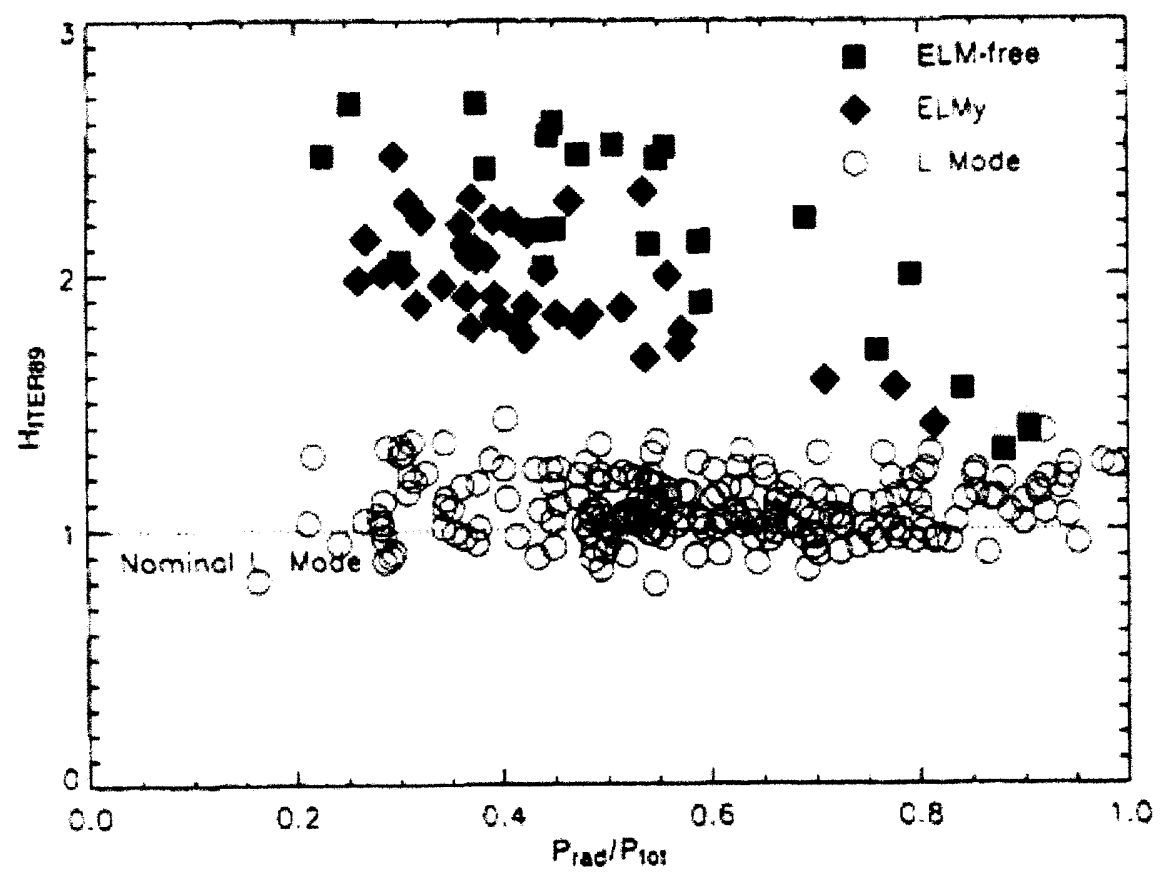

Figure 4.3: The 1989 ITER scaling for $H$ vs. radiative power fraction. There is a distinct division between $\mathrm{H}$-mode (ELM-free and $\mathrm{ELM}_{y}$ ) and L-mode at approximately $H=1.5$. Higher radiative power fractions tend to drive $\mathrm{H}$-mode plasmas into L-mode [8].

H-mode has natural advantages and disadvantages, and the choice of operating mode depends on one's design priorities. $\tau_{e}$ is about twice as long in H-mode as in L-mode. 
However, the H-mode "edge pedestal" can cause instabilities known as edge-localized modes (ELMs). Though ELMs are the focus of a large portion of current plasma physics research, it is still not possible to predict ELM behavior. Thus, ELMs pose significant challenges for H-mode tokamaks [7].

Since we intend to analyze large scale tokamaks, we can assume that $\tau_{e}$, which generally increases with size, will be sufficiently large regardless of operational mode. With that consideration off the table, we prefer L-mode in order to avoid the challenges of ELMs. Also, the addition of a fission blanket (which augments the fusion power) allows for lower $Q$. Thus, we will require $H$ to be less than 1.5 throughout this analysis. 


\section{Pure Fusion Core Analysis}

Now that we have formulated a feasible 0-D model, we can apply it to any standard tokamak. We could plunge headlong into our fission-fusion hybrid analysis, but that would obscure the advantages of hybrids relative to pure fusion reactors. First we will show how to use this model in the context of pure fusion tokamaks, and we will show how to determine the minimum scale of a tokamak. Once we have done this in Section 5, our coupled fission-fusion analysis in Section 6 will be much more lucid. This analysis follows our 2009 Bachelor of Science thesis in the Department of Physics [28].

\subsection{0-D Allowable Parameter Space}

The first step in the analysis is to determine which combinations of system parameters yield "allowable" plasma conditions given our design specifications. It is most efficient to narrow down the parameter space in this way before we do any optimization work. In order to avoid eliminating any potentially favorable parameter space, we impose only the most essential constraints. Of course, all physical parameters must be real and positive, and the other constraints are:

- $T>10 \mathrm{keV}$ is necessary to achieve a sufficient D-T fusion rate coefficient (see Figure $4.2)$.

- $q^{*}>2$ is necessary to ensure confinement and prevent current-driven instabilities. The toroidal field must be sufficiently large relative to the poloidal field [7].

- $\beta_{N}<3$ is the $\beta$ "no-wall" Troyon stability limit that applies when no conducting wall is present near the plasma [7].

- $H<1.5$ is necessary to operate in L-mode and thus avoid instabilities and other complications associated with H-mode (see Section 4.4) [8].

To determine a preliminary allowable parameter space, we first stipulate that many parameters have values consistent with existing tokamaks. We will hold these values constant throughout the 0-D allowable parameter space analysis.

- $B_{\max }=13 \mathrm{~T}$ is the peak on-coil magnetic field possible with niobium-tin superconductors (see Section 5.3.1) [11] [7].

- $w_{B}=1 \mathrm{~m}$ is necessary to stop a high fraction of $14.1 \mathrm{MeV}$ neutrons from D-T fusion reactions [7]. Later on, we will match this to our optimal fission blanket thickness.

- $P_{F} / A_{S}=5 \mathrm{MW} / \mathrm{m}^{2}$ is a reasonable limit for the blanket material (see Section 5.3.2) [13] [12]. 
Given these constants, we can manipulate the equations in Section 4.3 to write all system parameters as some function $f$ of five parameters: $R, q^{*}, F_{G}, R / a$, and $Q$.

$$
\left[R, I_{P}, n, B, P_{F}, \beta_{N}, H\right]=f\left(R, q *, F_{G}, R / a, Q\right)
$$

If $R$ and $R / a$ are known, then all the tokamak geometry is known (Equations 4.2-4 and 4.21). If $q^{*}$ is known, then the plasma current is known (Equation 4.7). Then if $F_{G}$ is known, the density is known (Equation 4.8). If $P_{F} / A_{S}$ and $Q$ are known, then all terms of the power balance are known (Equation 4.9). Given the scaling for $H$ and the relationship between $\langle\sigma v\rangle$ and $T$, all other parameters can be known. This analysis will determine which parameter "spaces" in the 5 -D parameters space $\left[R, q^{*}, F_{G}, R / a, Q\right]$ meet our constraints.

\subsubsection{Allowable $\left[R, q^{*}, F_{G}\right]$ Space}

To begin, we examine just the 3 -D parameter space $\left[R, q^{*}, F_{G}\right]$ for $R / a$ and $Q$ fixed:

- $R / a=3$ is close to ITER (3.1) and Alcator C-Mod (3.05) [11].

- $Q=40$ is typical for fusion reactor designs that are intended to be economically viable. See Section 5.5 for a more in-depth discussion of this.

Now we can write

$$
\left[R, I_{P}, n, B, P_{F}, \beta_{N}, H\right]=f\left(R, q *, F_{G}\right)
$$

So we can define every property of the plasma throughout the parameter space $\left[R, q^{*}, F_{G}\right]$ by some function $f$. The following contour plots show $I_{P}, n, T, \beta_{N}$, and $H$ in the $\left[R, q^{*}\right]$ plane at a fixed $F_{G}$ value of 0.9 .

Due to a fixed $B_{\max }, B$ depends only on $R$ and asymptotically approaches $(1-a / R) B_{\max }$ (see Equation 4.5). Due to fixed $P_{F} / A_{S}$ and $R / a, P_{F}$ also depends only on $R$ and increases as $\sim R^{2}$.

Figure 5.1 shows $I_{P}\left(R, q^{*}\right)$ for fixed $F_{G}$. It is roughly proportional to $R$ (note the $R$ dependence of $B$ ) and exactly inversely proportional to $q^{*}$ (see Equation 4.7).

Figure 5.2 shows $n\left(R, q^{*}\right)$ for fixed $F_{G}$. Given that $n \sim I_{P} / R^{2}$ (see Equation 4.8), $n$ has the same inverse $q^{*}$ dependence as $I_{P}$. However, its $R$ dependence is roughly $\sim 1 / R$.

Figure 5.3 shows volume-averaged $T\left(R, q^{*}\right)$ for fixed $F_{G}$. Many areas in $\left[R, q^{*}\right]$ space require a reactivity rate coefficient $\langle\sigma v\rangle$ that is unphysically large, meaning that it is greater than the maximum shown in Figure 4.2. Thus, there is no solution for $T$, and the reactor is not viable. These unphysical areas occupy the high $R$ and high $q^{*}$ space. At the boundary of this area, $R\left(q^{*} / B\right)^{2}$ is constant. Furthermore, since $B$ is constant at large $R$, we can say 
that $R q^{* 2}$ is approximately constant at the boundary for large $R$. This shows that when we consider only reactivity, the maximum possible $q^{*}$ decreases as $\sim R^{-1 / 2}$.

Figures 5.4 and 5.5 show $\beta_{N}\left(R, q^{*}\right)$ and $H\left(R, q^{*}\right)$, respectively. They exhibit complex behavior but have approximately the same form as each other. At a fixed $q^{*}$, both $\beta_{N}(R)$ and $H(R)$ decrease rapidly for $R<10$ meters. This means that confinement improves significantly with reactor size when $R$ is less than about 10 meters, allowing large reactors to operate in L-mode.

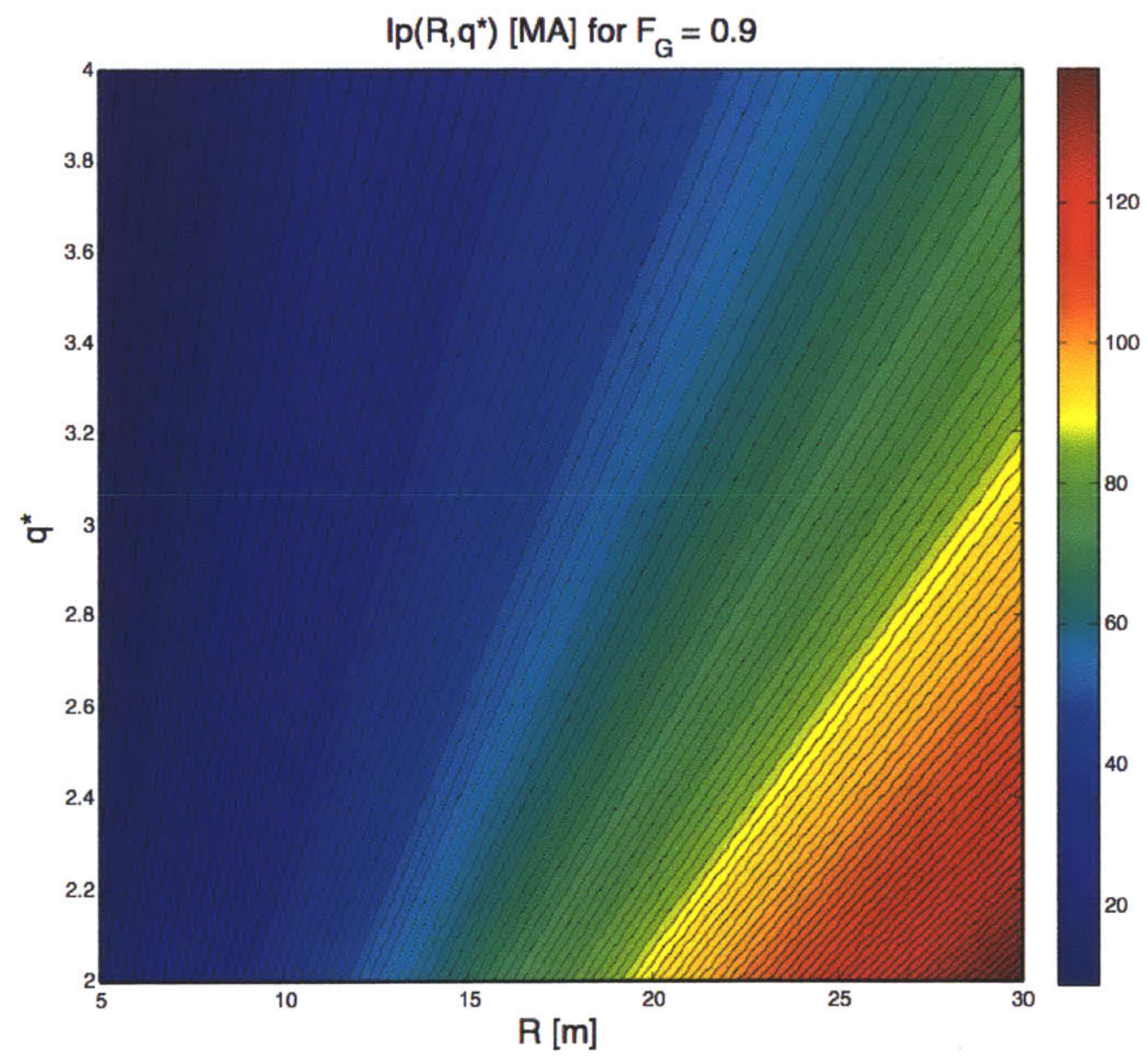

Figure 5.1: The plasma current $I_{P}$ in the $\left[R, q^{*}\right]$ plane. It is inversely proportional to $q^{*}$ and roughly proportional to $R$. 


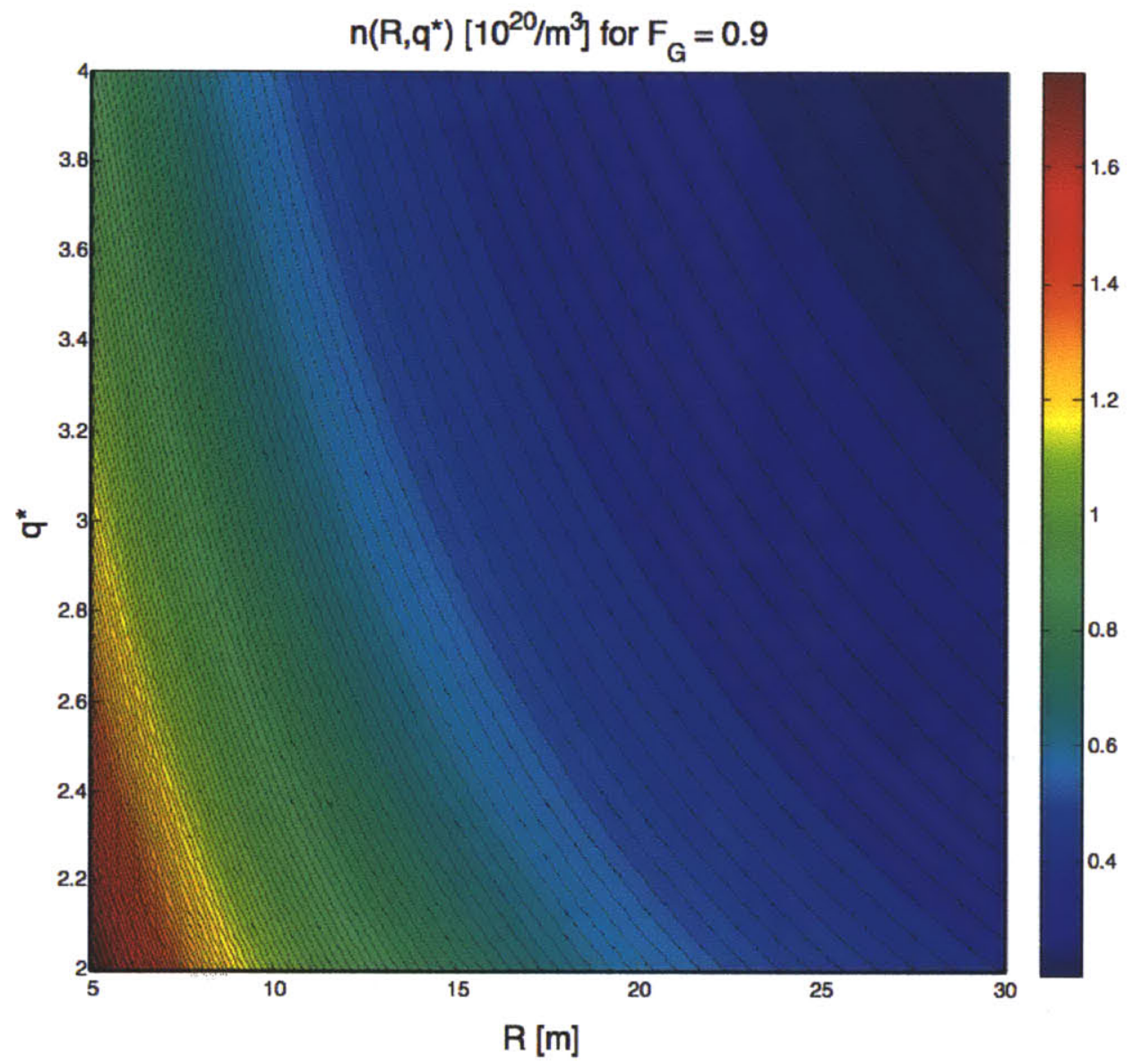

Figure 5.2: The total ion density $n$ in the $\left[R, q^{*}\right]$ plane. It is inversely proportional to $q^{*}$ and roughly inversely proportional to $R$. 


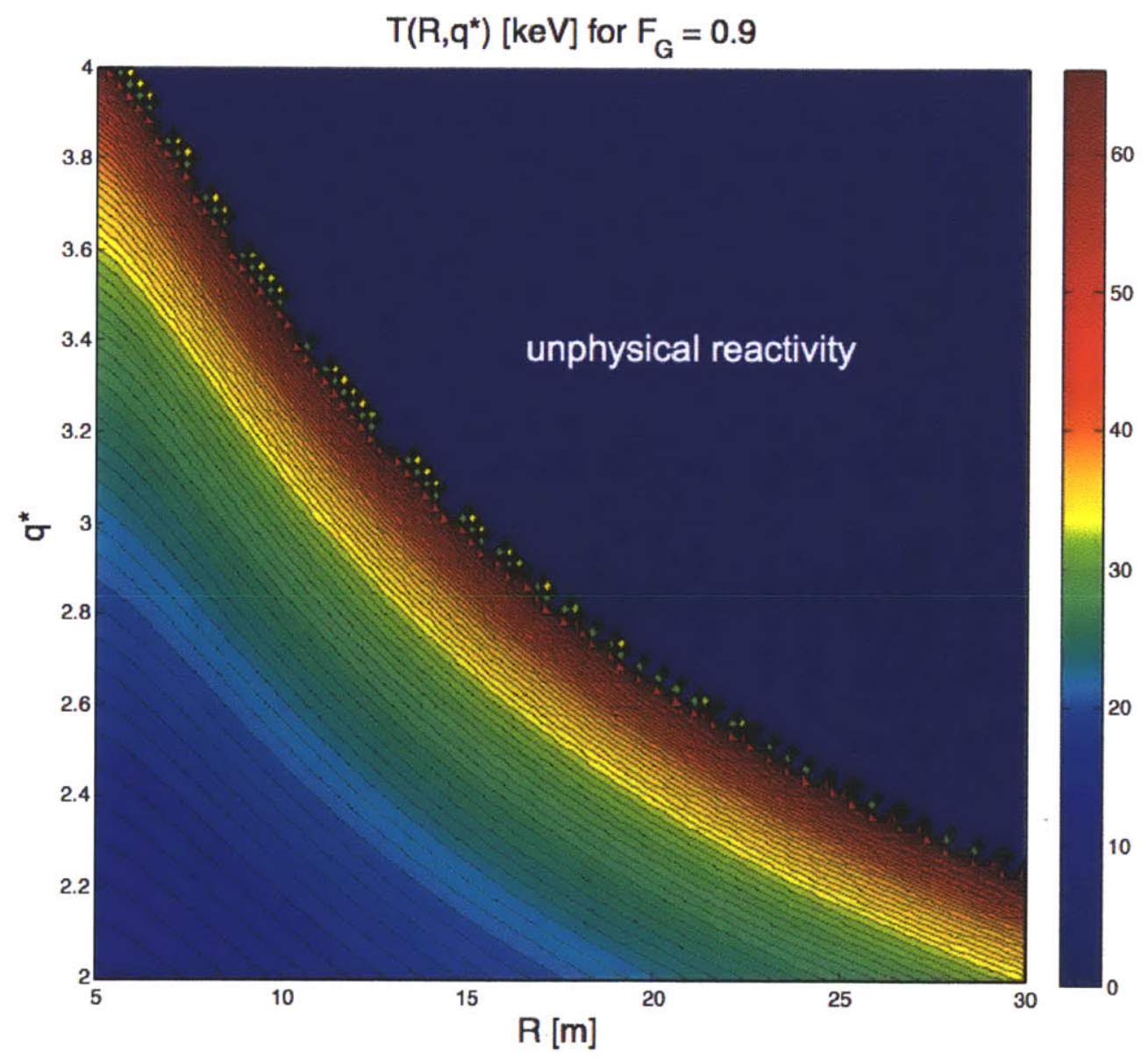

Figure 5.3: The temperature $T$ in the $\left[R, q^{*}\right]$ plane. Many combinations of $R$ and $q^{*}$ in this space require a $\mathrm{D}-\mathrm{T}$ cross-section $\langle\sigma v\rangle$ that is larger than the maximum possible $\langle\sigma v\rangle$ (see Figure 4.2), meaning that those values of $R$ and $q^{*}$ are not feasible. In such cases, $T$ has no solution and is set to zero. The large dark blue area represents the unphysical areas, and the thick dark line represents the boundary between the physical and unphysical areas. 


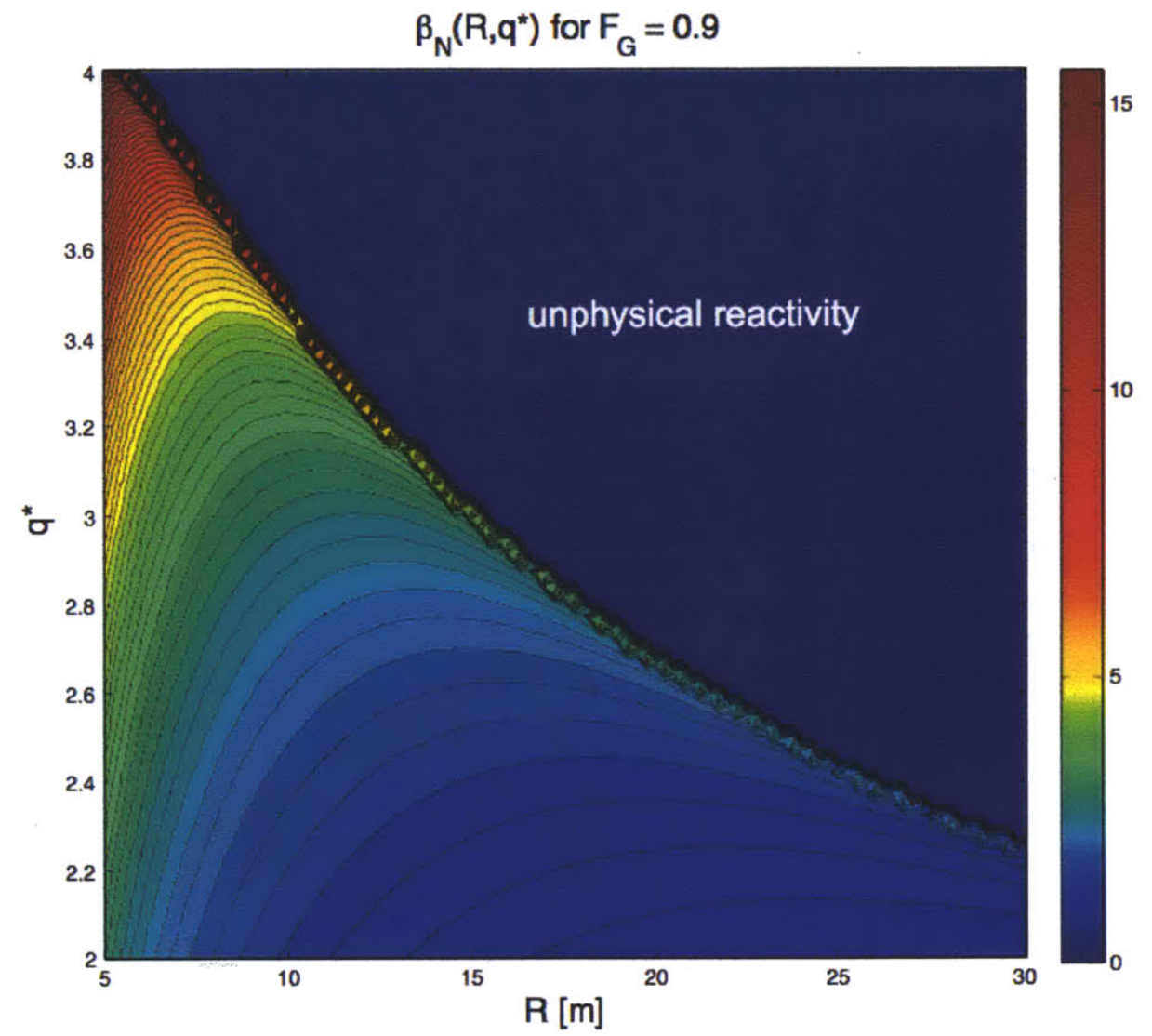

Figure 5.4: $\beta_{N}$ in the $\left[R, q^{*}\right]$ plane. The unphysical areas in which $T$ has no solution are also represented here by dark blue, as $\beta_{N}$ requires a solution for $T$. Again, the thick dark line represents the boundary between the physical and unphysical areas. 


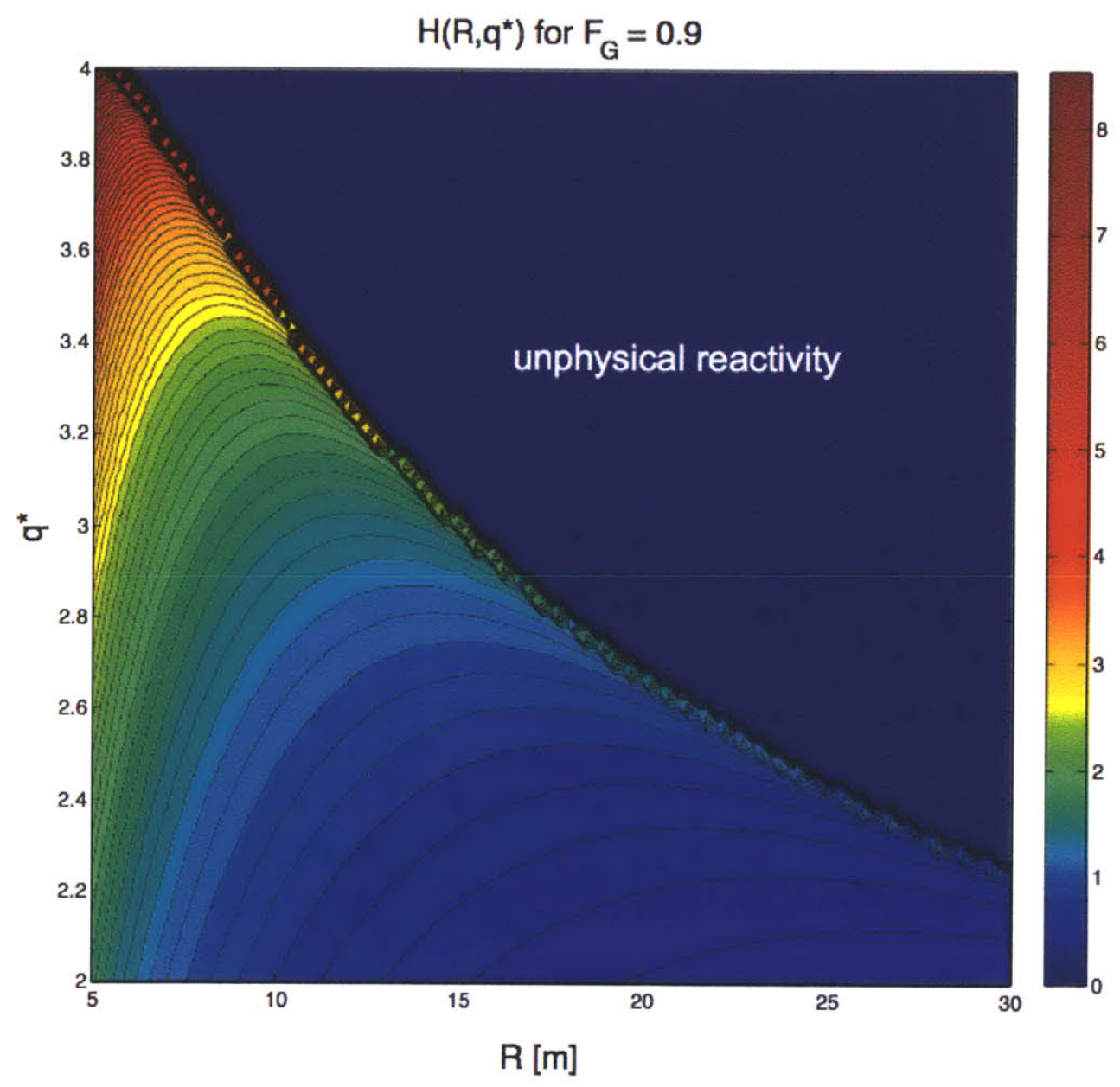

Figure 5.5: $H$ in the $\left[R, q^{*}\right]$ plane. The unphysical areas in which $T$ has no solution are also represented here by dark blue, as $H$ requires a solution for $T$. Again, the thick dark line represents the boundary between the physical and unphysical areas. 
Now it is simple, given the constraints in Section $5.1\left(H<1.5\right.$ for L-mode and $\beta_{N}<3$ for the "no-wall" limit), to determine what subspace of $\left[R, q^{*}, F_{G}\right]$ is allowable. The following plots show cross-sections of this allowable subspace in the $\left[R, q^{*}\right],\left[R, F_{G}\right]$, and $\left[q^{*}, F_{G}\right]$ planes.

Figure 5.6 shows the allowable subspace (red) in the $\left[R, q^{*}\right]$ plane for $F_{G}$ fixed at 0.9 . The maximum allowable $q^{*}$ as a function of $R$ has a broad maximum between 10 and 15 meters. The allowable area is limited by unphysical reactivity at high $R$ and by confinement (high $\beta_{N}$ and high $H$ ) at low $R$. This is a critical result, because it means that to achieve a certain level of stability (a certain $q^{*}$ value), we must design the tokamak within a finite range of major radii. It also shows that only large tokamaks $(R \sim 10$ meters) can operate in L-mode with high $q^{*}$. Tokamaks the size of ITER (6.2 meters) or smaller are forced to operate in H-mode in order to achieve high $q^{*}$.

There is a crucial advantage associated with this result. To achieve maximum $q^{*}$, the operating point should be located at the peak shown in Figure 5.6 (approximately $R=13$ $\mathrm{m}$ and $q^{*}=3$ ). Referring back to Figure 5.3, such an operating point is located near the boundary of the unphysical region, near where $T$ quickly becomes large. This means that it is also located not too far below the maximum reactivity coefficient shown in Figure 4.2. Were $T$ to suddenly increase, the reactivity would only increase slightly before decreasing. The tokamak would then have a negative reactivity coefficient and naturally return to a lower $T$. Thus, the tokamak is intrinsically stable with respect to temperature instabilities.

Figure 5.7 shows a similar allowable parameter space in the $\left[R, F_{G}\right]$ plane for $q^{*}$ fixed at 2.5. It shows that as $F_{G}$ increases, the finite range of allowable $R$ increases in width. Figure 5.8 shows the height of the $q^{*}$ maximum as a function of $F_{G}$. One can see that $q^{*}$ is 3 when $F_{G}$ is 0.9 , which is consistent with Figure 5.6. This shows that the height of this $q^{*}$ maximum increases with $F_{G}$. Thus, as $F_{G}$ increases, both the width and height of the $q^{*}$ maximum also increase. Indeed, the curve in Figure 5.6 shifts upward as $F_{G}$ increases. This is in part because $n^{2}\langle\sigma v\rangle$ is constant for fixed size, and so reactivity must decrease as the Greenwald fraction and density increase. As Figure 5.8 reveals, t he relationship is linear, meaning that the highest allowable $q^{*}$ is proportional to $F_{G}$. Thus, we prefer to operate near the Greenwald limit $\left(F_{G}<1\right)$ and will choose $F_{G}=0.9$. 


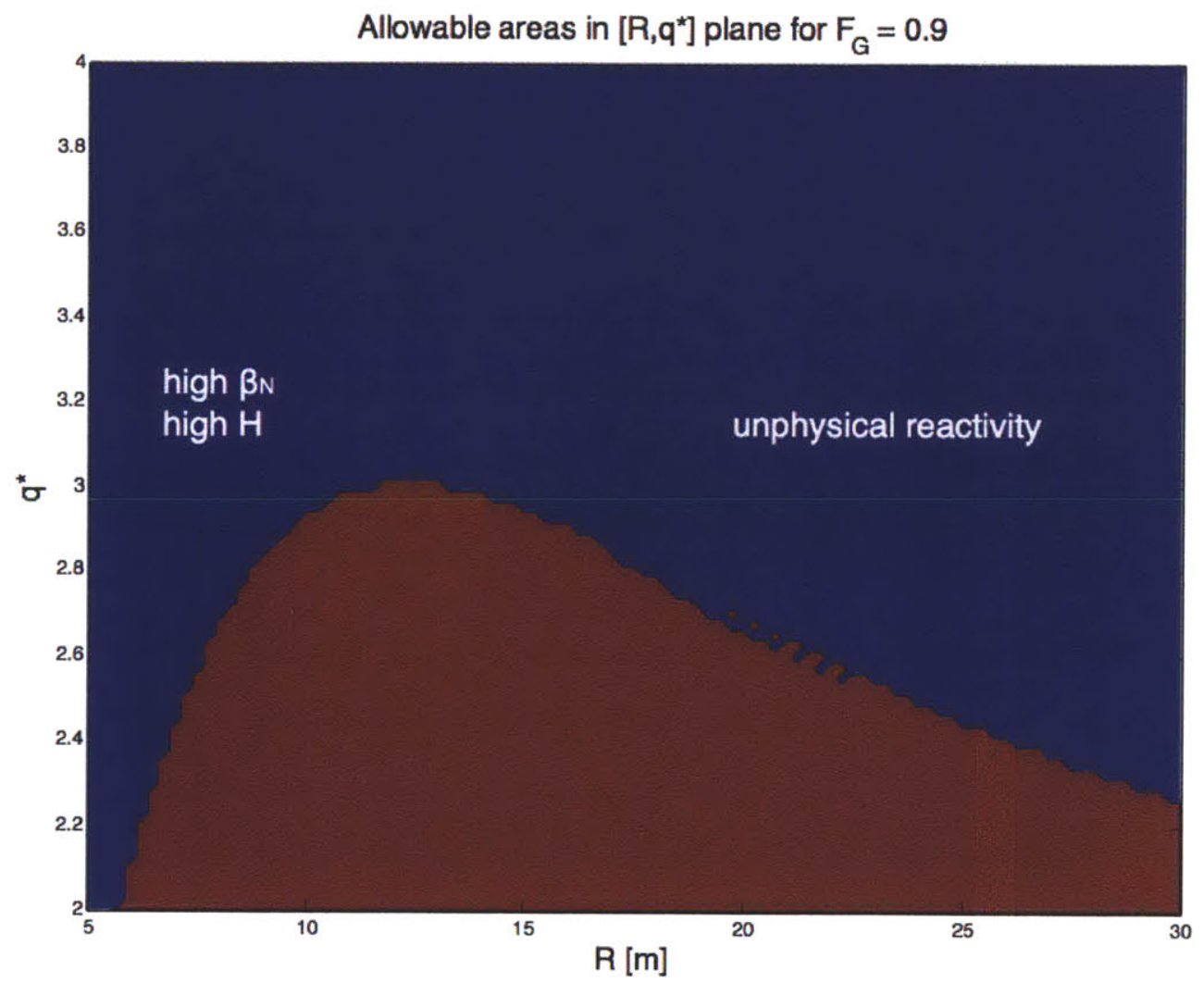

Figure 5.6: Areas in the $\left[R, q^{*}\right]$ plane that satisfy (red) and do not satisfy (blue) the parameter constraints. This shows that $R$ between 10 and 15 meters will yield a maximal $q^{*}$. 


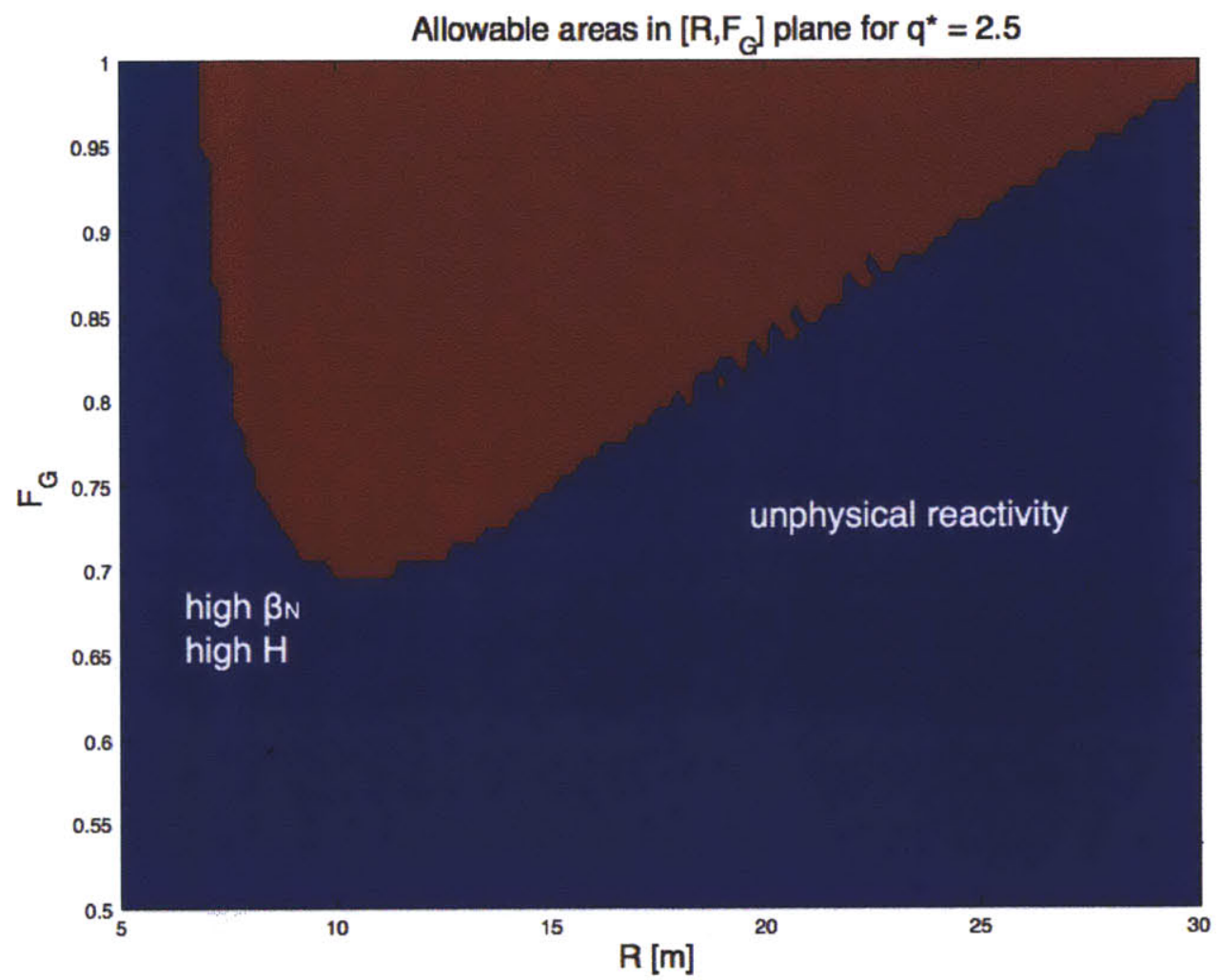

Figure 5.7: Areas in the $\left[R, F_{G}\right]$ plane that satisfy (red) and do not satisfy (blue) the parameter constraints. This shows that a high $F_{G}$ requires an $R$ between 10 and 15 meters. 


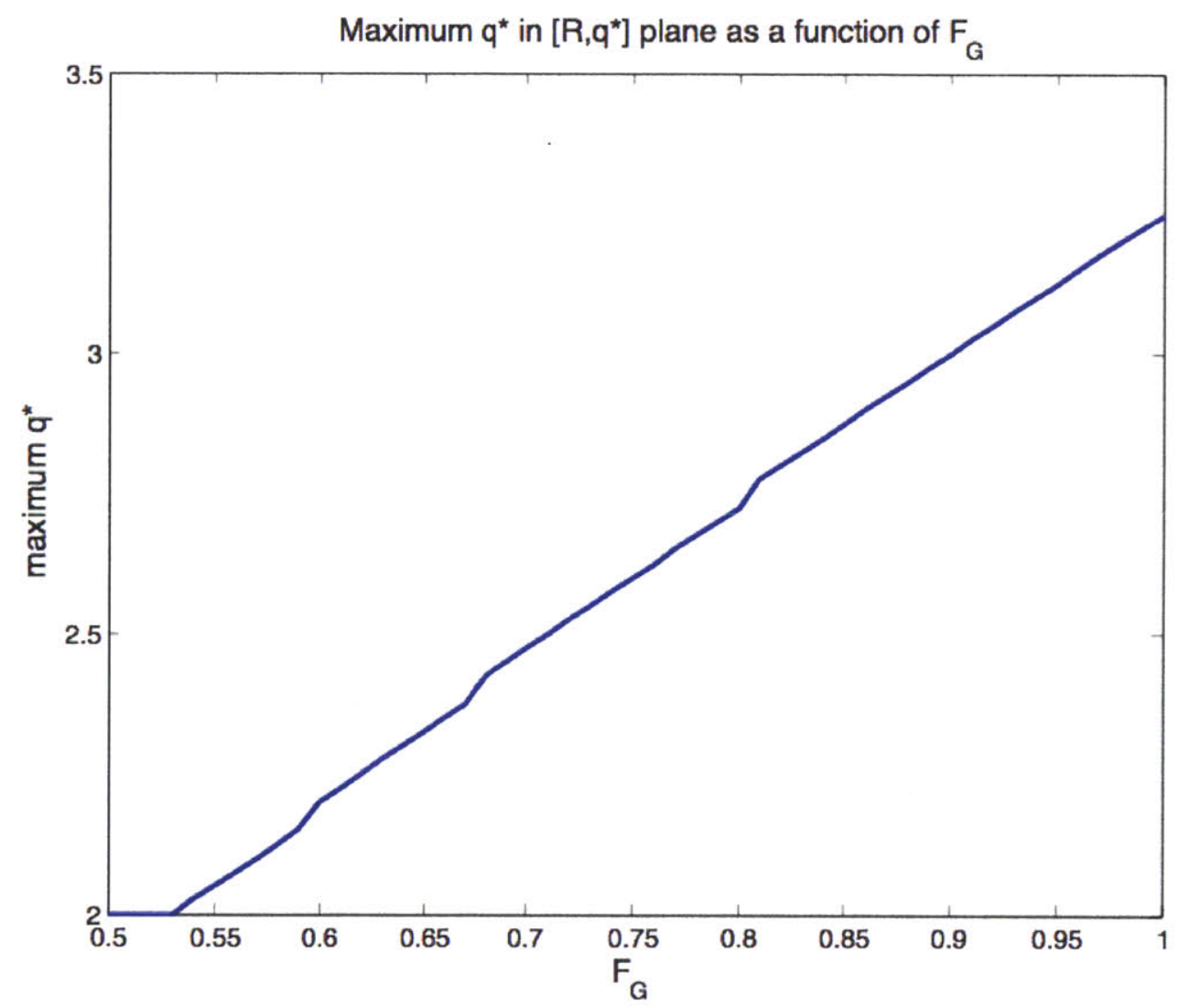

Figure 5.8: The maximum value of $q^{*}$ (at whichever $R$ that maximum occurs) as a function of $F_{G}$. The relationship is roughly linear. 
From this $\left[R, q^{*}, F_{G}\right]$ space analysis, it is clear that we prefer high $F_{G}$ in order to achieve high $q^{*}$. From this point forward, we will assume $F_{G}=0.9$. It is also clear that $R$ should lie in or near the range of 10 to 15 meters to maximize $q^{*}$, though other performance factors will determine a more precise $R$.

\subsubsection{Allowable $\left[R, q^{*}, R / a\right]$ Space}

Throughout Section 5.1.1, we held $R / a$ constant at 3 . Now that as $F_{G}$ is fixed at 0.9 , we will vary $R / a$ and repeat the same analysis. We can write

$$
\left[R, I_{P}, n, B, P_{F}, \beta_{N}, H\right]=f(R, q *, R / a)
$$

So every property of the plasma is defined throughout the parameter space $\left[R, q^{*}, R / a\right]$ by some new function $f$.

Figure 5.9 shows the allowable parameter space in the $\left[R, q^{*}\right]$ plane for three different values of $R / a$. Clearly, the same broad $q^{*}$ maximum appears between 10 and 15 meters. $R / a$ changes the height and width of this maximum in the same way that $F_{G}$ does. This time, however, the maximum $q^{*}$ decreases as $R / a$ increases.

To see the exact form of this relationship, Figure 5.10 shows the maximum $q^{*}$ as a function of $R / a$ in the same way that Figure 5.8 shows the maximum $q^{*}$ as a function of $F_{G}$. This time, the relationship is not linear but closer to $\sim(R / a)^{-1}$. In large part, this is due to the $\sim(R / a)^{-1}$ dependence of the elongation $\kappa$. Smaller $R / a$ and larger $\kappa$ imply larger surface area, larger fusion power, and a larger volume to surface area ratio, which obviate the need for a high $\langle\sigma v\rangle$. So clearly, in the interest of stability, we desire a low $R / a$. However, since $R / a$ affects much more than just stability, choosing a precise $R / a$ requires sustainability analysis.

From this $\left[R, q^{*}, R / a\right]$ space analysis, it is clear that varying $R / a$ does not significantly affect the key findings of the previous $\left[R, q^{*}, F_{G}\right]$ space analysis. It is also clear that an $R / a$ lower than 3 (our starting point) is favorable. 


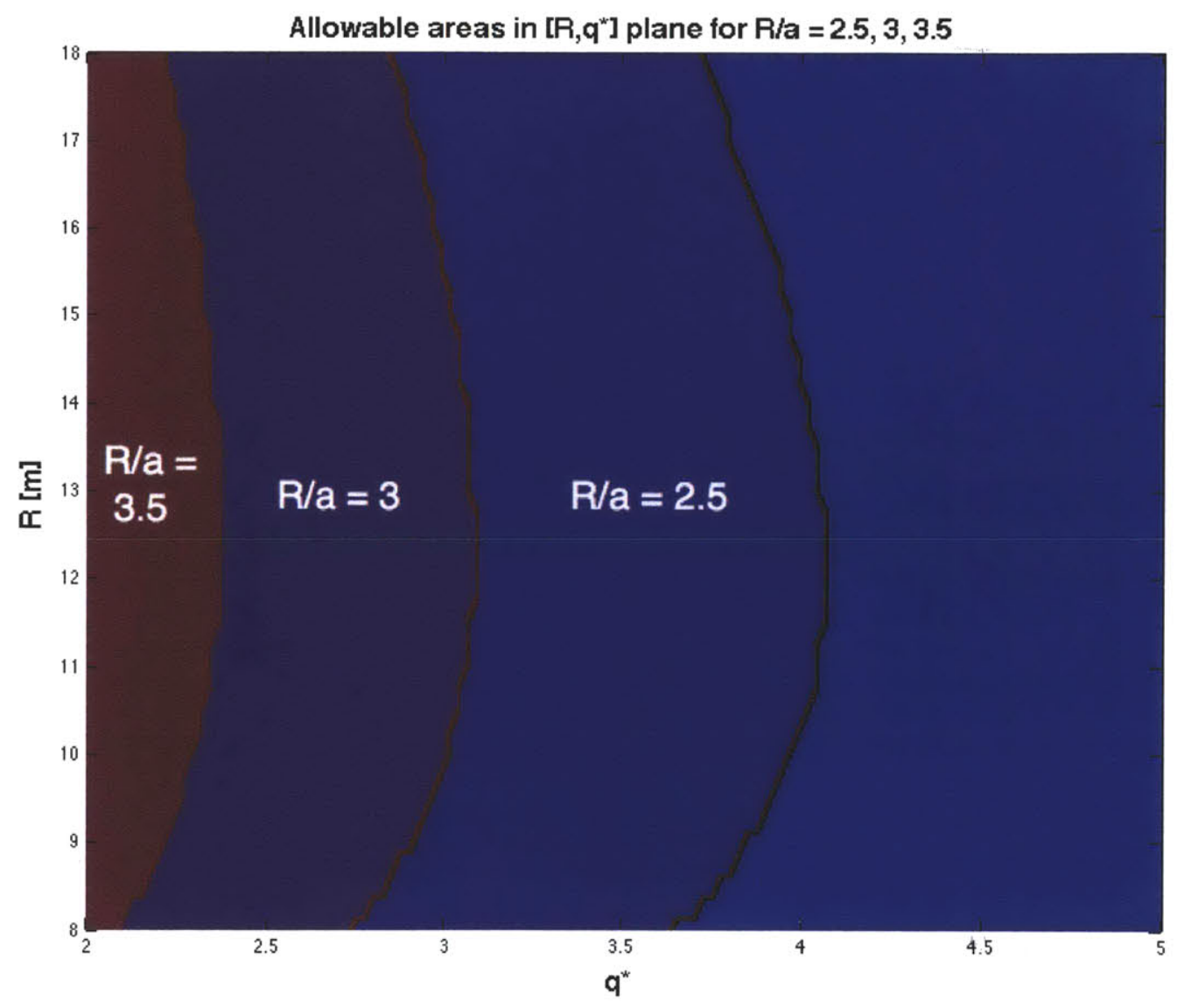

Figure 5.9: Areas in the $\left[R, q^{*}\right]$ plane that satisfy the parameter constraints for $R / a$ values of $2.5,3$, and 3.5. The $R$-location of the $q^{*}$ maximum is independent of $R / a$, and the maximum $q^{*}$ increases dramatically as $R / a$ decreases. 


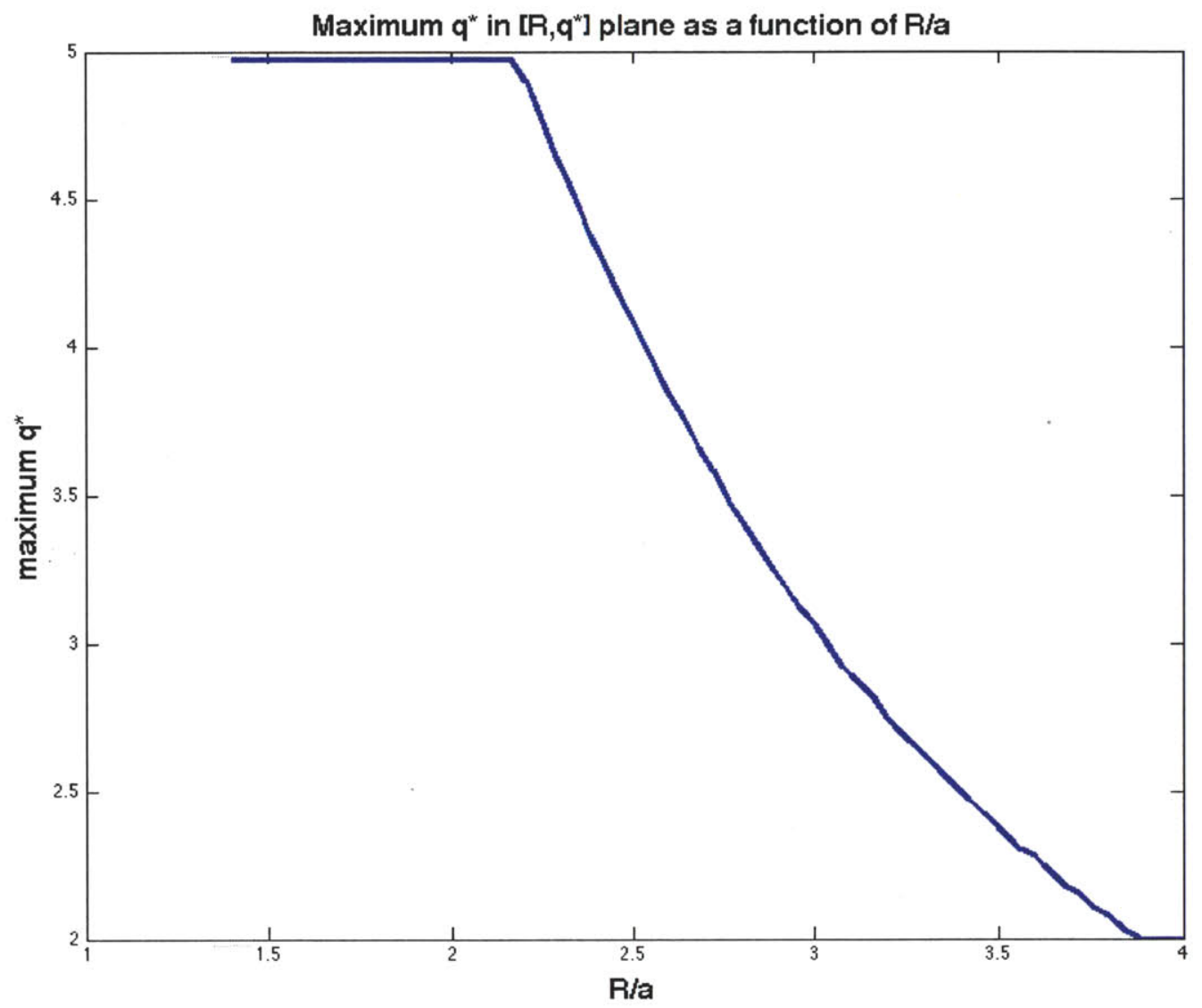

Figure 5.10: The maximum value of $q^{*}$ (at whichever $R$ that maximum occurs) as a function of $R / a$. The relationship is roughly of the form $\sim(R / a)^{-1}$. 


\subsubsection{Allowable $\left[R, q^{*}, R / a, Q\right]$ Space}

As a final check of the parameter space, it is prudent to test the sensitivity of the allowable areas to changes in the gain $Q$. We perform a 4 -D analysis in $\left[R, q^{*}, R / a, Q\right]$ space in order to determine how changes in $Q$ affect the $\left[R, q^{*}\right]$ plane shown in Figure 5.6.

$$
\left[R, I_{P}, n, B, P_{F}, \beta_{N}, H\right]=f(R, q *, R / a, Q)
$$

Examination of the $\left[q^{*}, Q\right]$ and $[R / a, Q]$ planes show that $Q$ has no effect on the allowable ranges of $q^{*}$ or $R / a$ when other parameters are held constant. However, examination of the $[R, Q]$ plane shows that $Q$ does have a marginal effect on the allowable range of $R$. Figure 5.11 shows that for $q^{*}$ and $R / a$ both fixed at 3 , the range of allowable $R$ (the width of the maximum shown in Figure 5.6) decreases as $Q$ increases. Nevertheless, this increase is slight as long as $Q$ is much greater than 5 so that the ratio of total heating power to fusion power $(1 / 5+1 / Q)$ changes little with $Q$. We can conclude that $Q$ is not an important contributor to the allowable parameter space. However, $Q$ will become much lower (and much closer to 5) when we perform the coupled fission-fusion analysis in Section 6.

\subsection{Minimum Tokamak Scale Defined}

For each point in any of these parameter spaces, we can also compute plasma current quantities. The most important quantity for us is the non-inductive current fraction $f_{N I}$. We defined this in Equation 4.25. We desire $f_{N I}>1$ in order to achieve "steady-state" operation. Figure 5.12 shows $f_{N I}$ in the $\left[R, q^{*}\right]$ plane for the HYPERION operating parameters [4]. The fully non-inductive $\left(f_{N I}>1\right)$ region is outlined in white. There is clearly a sharply-defined minimum fully non-inductive $R$ at approximately $9 \mathrm{~m}$. This is precisely what we refer to when we write "minimum scale" or "minimum $R$ ". This is important for design purposes, as capital cost usually scales with $R$. As we will see in the following sections, varying system parameters (especially $Q$ ) can dramatically alter this minimum $R$. 


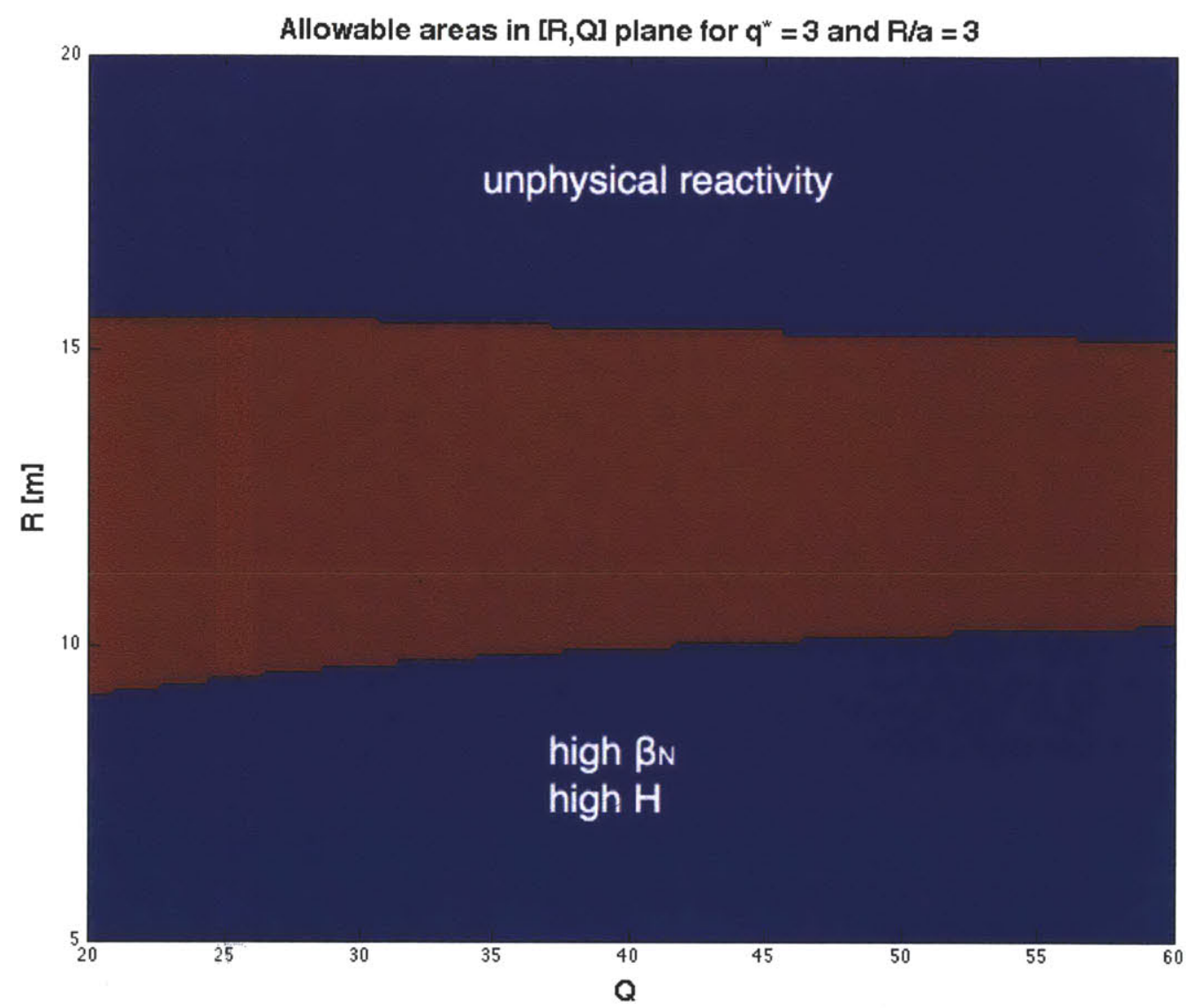

Figure 5.11: Areas in the $[R, Q]$ plane that satisfy the parameter constraints for $q^{*}$ and $R / a$ values of 3 . This shows that the width of the broad $q^{*}$ maximum (see Figure 5.6) decreases slightly as $Q$ increases. This is also a more intuitive way to show that $R$ is limited by confinement on the low end and by reactivity on the high end. 


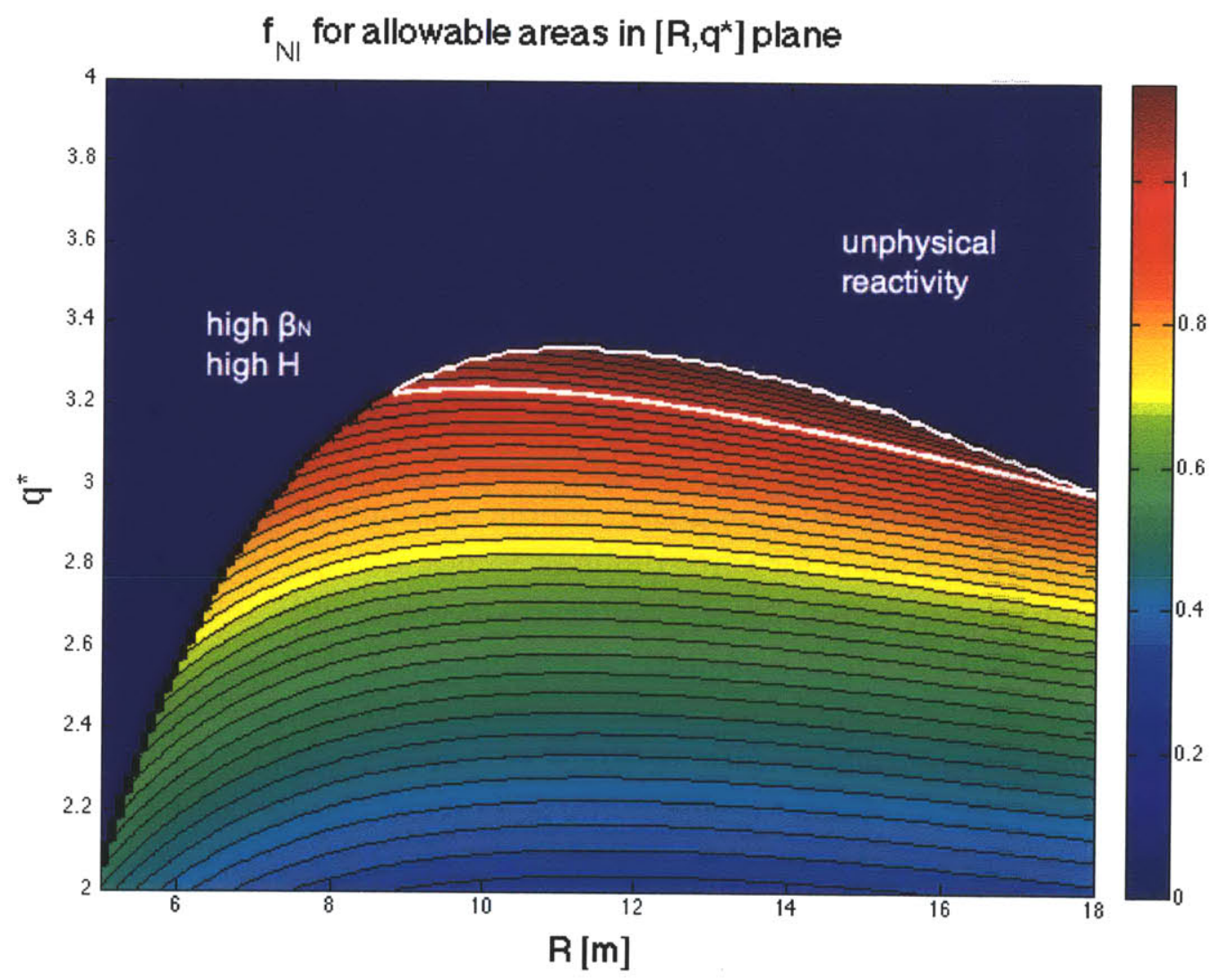

Figure 5.12: $f_{N I}$ for areas in the $\left[R, q^{*}\right]$ plane that satisfy the parameter constraints. The parameters are those of the large scale HYPERION operating point: $R / a=2.6, Q=40$ and $P_{F} / A_{S}=7 \mathrm{MW} / \mathrm{m}^{2}$. The fully non-inductive $\left(f_{N I}>1\right)$ region is outlined in white. In this case, the minimum $R$ for fully non-inductive L-mode operation is slightly less than $9 \mathrm{~m}$. The shape of this plot is similar to that in Figure 5.6. 


\subsection{Technology Limits}

\subsubsection{Maximum On-Coil Magnetic Field $B_{\max }$}

In the HYPERION design, $B_{\max }$ is $13 \mathrm{~T}$, the approximate maximum on-coil field for niobiumtin $\left(\mathrm{Nb}_{3} \mathrm{Sn}\right)$ superconductors in ITER [11] [7]. It is difficult to achieve fields higher than this with conventional superconductors (critical temperatures $T_{C} \leq 20 \mathrm{~K}$ ). This is because a certain magnetic field strength can "quench" superconductivity at a certain temperature below $T_{C}$. This certain magnetic field strength is proportional to $\left[1-T / T_{C}\right]^{2}$ so that the further a superconductor is cooled below $T_{C}$, the higher the magnetic field it can withstand while remaining superconducting [16]. Of course, material stress also poses significant limitations. High-temperature superconductors (HTS), usually cuprate (containing copper oxide), often have $T_{C}$ values much higher than $20 \mathrm{~K}$. Yttrium barium copper oxide $\left(\mathrm{YBa}_{2} \mathrm{Cu}_{3} \mathrm{O}_{7}\right)$, often abbreviated $\mathrm{YBCO}$, has $T_{C} \approx 90 \mathrm{~K}[16]$. By cooling YBCO to $T<10 \mathrm{~K}$, we can produce significantly higher fields [1] [5]. Considering material stress limitations and economic factors, HTS tokamak studies have shown that the highest sensible field strength is approximately $16 \mathrm{~T}$ [1].

Figure 5.13 shows the effect of varying $B_{\max }$ on the minimum $R$. This shows the familiar allowable area in the $\left[R, q^{*}\right]$ plane for three different values of $B_{\max }$. The fully non-inductive area $\left(f_{N I}>1\right)$ is outlined in white. As $B_{\max }$ increases, the allowable area in the $\left[R, q^{*}\right]$ plane moves to higher $q^{*}$, and the minimum fully non-inductive $R$ decreases. This is intuitive, because higher magnetic fields increase plasma current and thus (indirectly) confinement, allowing the tokamak to be smaller without losing energy too quickly. Given a fixed point in the $\left[R, q^{*}\right]$ plane, a higher magnetic field requires a higher plasma current, which in turn requires a higher density and a lower temperature.

Figure 5.14 shows this minimum $R$ as a function of $B_{\max }$. It decreases rapidly for $B_{\max }$ $\leq 14 \mathrm{~T}$ but more gradually at higher $B_{\max }$. Consequently, it is important to ensure $B_{\max } \geq$ $14 \mathrm{~T}$ by using HTS technology. Beyond that point, higher $B_{\max }$ is certainly favorable but not essential. However, since we are minimizing $R$, we will choose $B_{\max }=16 \mathrm{~T}$ consistent with the HTS tokamak studies. We will assume this choice for the remainder of this pure fusion analysis. 


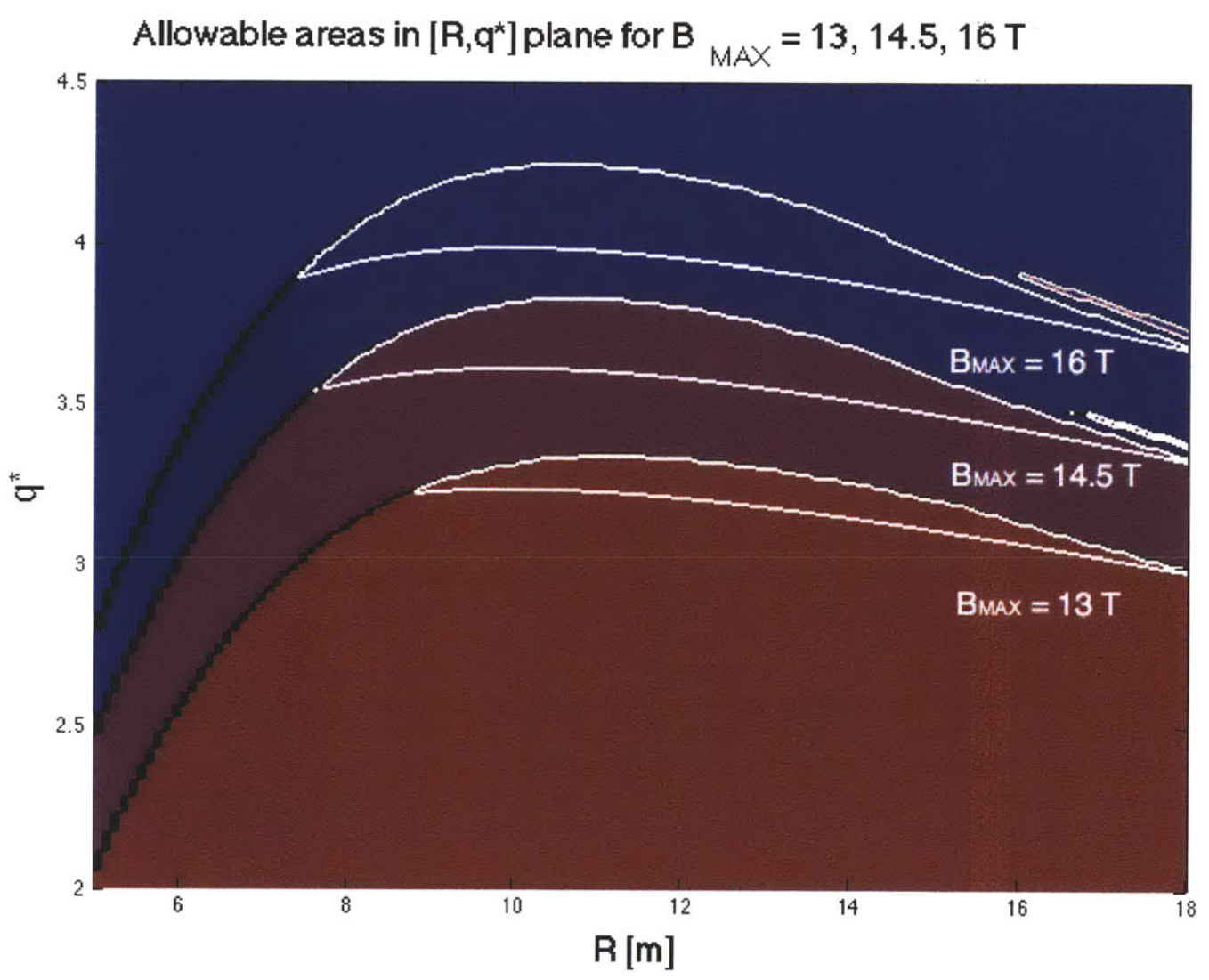

Figure 5.13: Areas in the $\left[R, q^{*}\right]$ plane that satisfy the parameter constraints for maximum on-coil magnetic field $B_{\max }$ values of $13,14.5$, and $16 \mathrm{~T}$. Here $R / a=2.6, P_{F} / A_{S}=7 \mathrm{MW} / \mathrm{m}^{2}$, and $Q=$ 40. As $B_{\max }$ increases, the highest available $q^{*}$ increases while the minimum (fully non-inductive) $R$ decreases. Consequently, we prefer higher $B_{\max }$. 


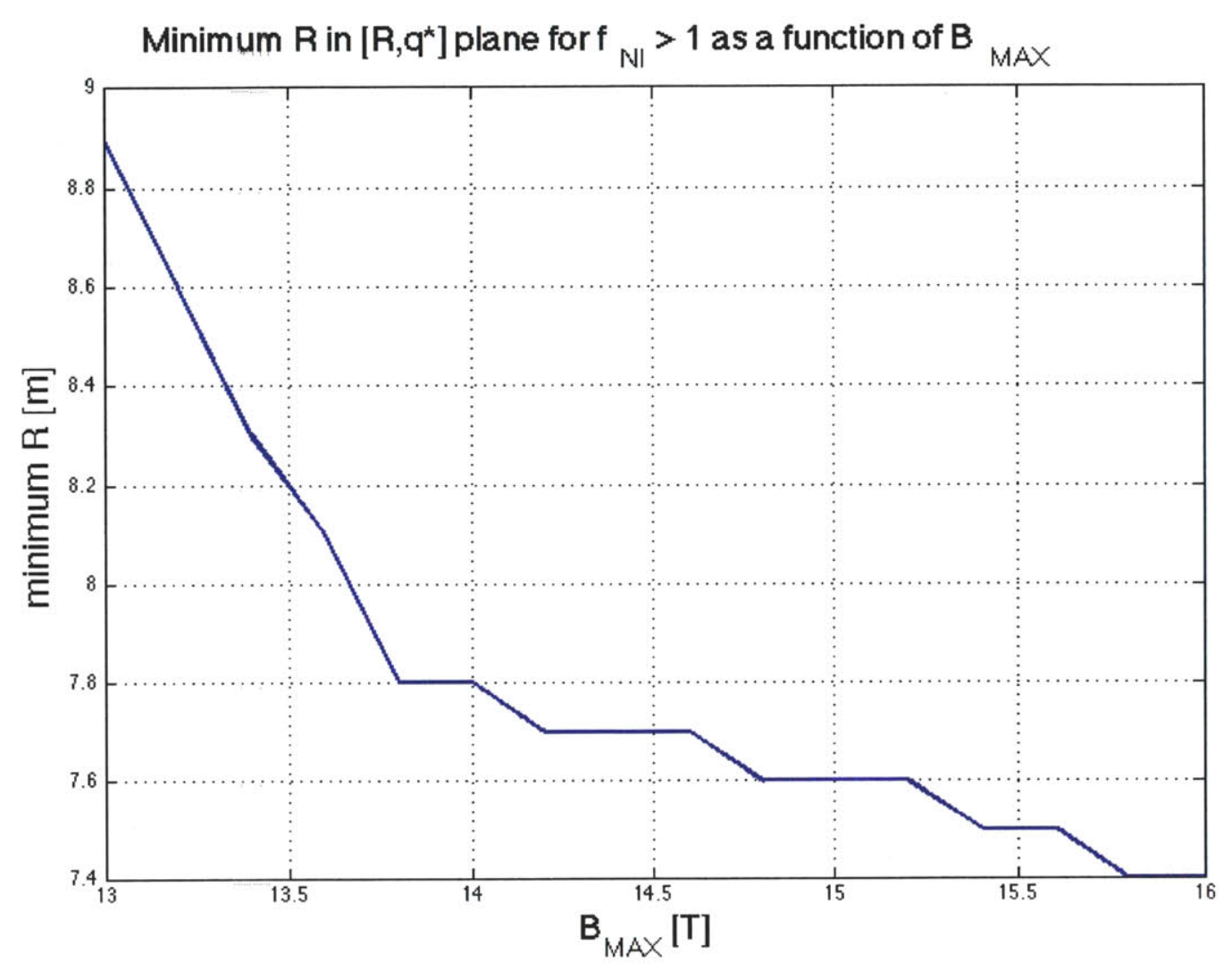

Figure 5.14: The minimum $R$ in the $\left[R, q^{*}\right]$ plane for fully non-inductive L-mode operation as a function of $B_{\max }$. The minimum $R$ decreases dramatically for $B_{\max } \leq 14 \mathrm{~T}$ but only marginally for $B_{\max } \geq 14 \mathrm{~T}$. The parameters here are the same as those in Figure 5.13. 


\subsubsection{Blanket Fusion Power Density $P_{F} / A_{S}$}

In the HYPERION design, $P_{F} / A_{S}$ is $7 \mathrm{MW} / \mathrm{m}^{2}$, which resulted primarily from economic analysis [4]. While $7 \mathrm{MW} / \mathrm{m}^{2}$ may certainly be possible, ARIES-AT blanket performance studies have shown maximum power loading of only $4.8 \mathrm{MW} / \mathrm{m}^{2}$ on a silicon carbide $(\mathrm{SiC}$ ) blanket [13]. Given this, it would not be prudent to let $P_{F} / A_{S}$ exceed $\approx 5 \mathrm{MW} / \mathrm{m}^{2}[12]$.

Figure 5.15 shows the effect of varying $P_{F} / A_{S}$ on the minimum $R$. The allowable area in the $\left[R, q^{*}\right]$ plane shifts to lower $q^{*}$ when $P_{F} / A_{S}$ increases from $5 \mathrm{MW} / \mathrm{m}^{2}$ to $7 \mathrm{MW} / \mathrm{m}^{2}$. This is intuitive, because given constant geometry and constant $\langle\sigma v\rangle$, increasing power requires increasing density, which in turn requires increasing plasma current and decreasing $q^{*}$. The interesting thing here is that while the allowable area shifts to lower $q^{*}$ for increasing $P_{F} / A_{S}$; the minimum $R$ decreases.

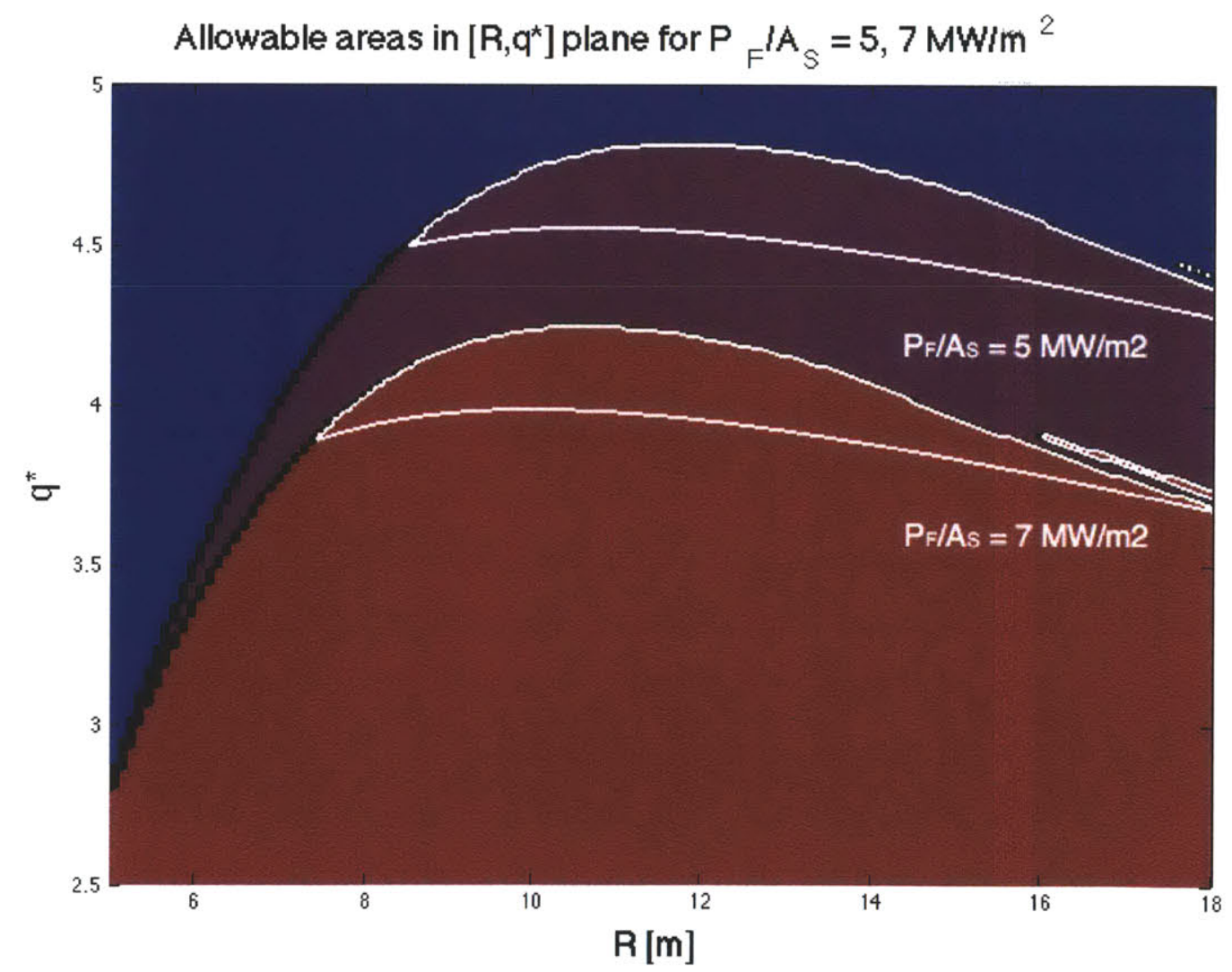

Figure 5.15: Areas in the $\left[R, q^{*}\right]$ plane that satisfy the parameter constraints for fusion power per surface area $P_{F} / A_{S}$ values of 5 and $7 \mathrm{MW} / \mathrm{m}^{2}$. Here $R / a=2.6, B_{\max }=16 \mathrm{~T}$, and $Q=40$. As $P_{F} / A_{S}$ increases, the highest available $q^{*}$ decreases while the minimum (fully non-inductive) $R$ also decreases. Consequently, we prefer higher $P_{F} / A_{S}$. 
Figure 5.16 shows this decrease in minimum $R$ as a function of $P_{F} / A_{S}$. The relationship is somewhat linear, and thus higher $P_{F} / A_{S}$ is obviously favorable. However, given the uncertainty of whether $P_{F} / A_{S} \geq 5 \mathrm{MW} / \mathrm{m}^{2}$ is practically workable, we will choose $P_{F} / A_{S}$ $=5 \mathrm{MW} / \mathrm{m}^{2}$. We will assume this choice for the remainder of this thesis.

It is interesting to examine the relationship between $P_{F} / A_{S}$ and the total fusion power $P_{F}$ at minimum scale. Equations 4.4 and 4.21 show that $P_{F} \propto R^{2}\left(P_{F} / A_{S}\right)$ at fixed $R / a$, and Figure 5.16 shows that $R$ decreases linearly with $P_{F} / A_{S}$. Therefore, $P_{F}$ does not necessarily increase with $P_{F} / A_{S}$. It may in fact decrease, a counterintuitive result.

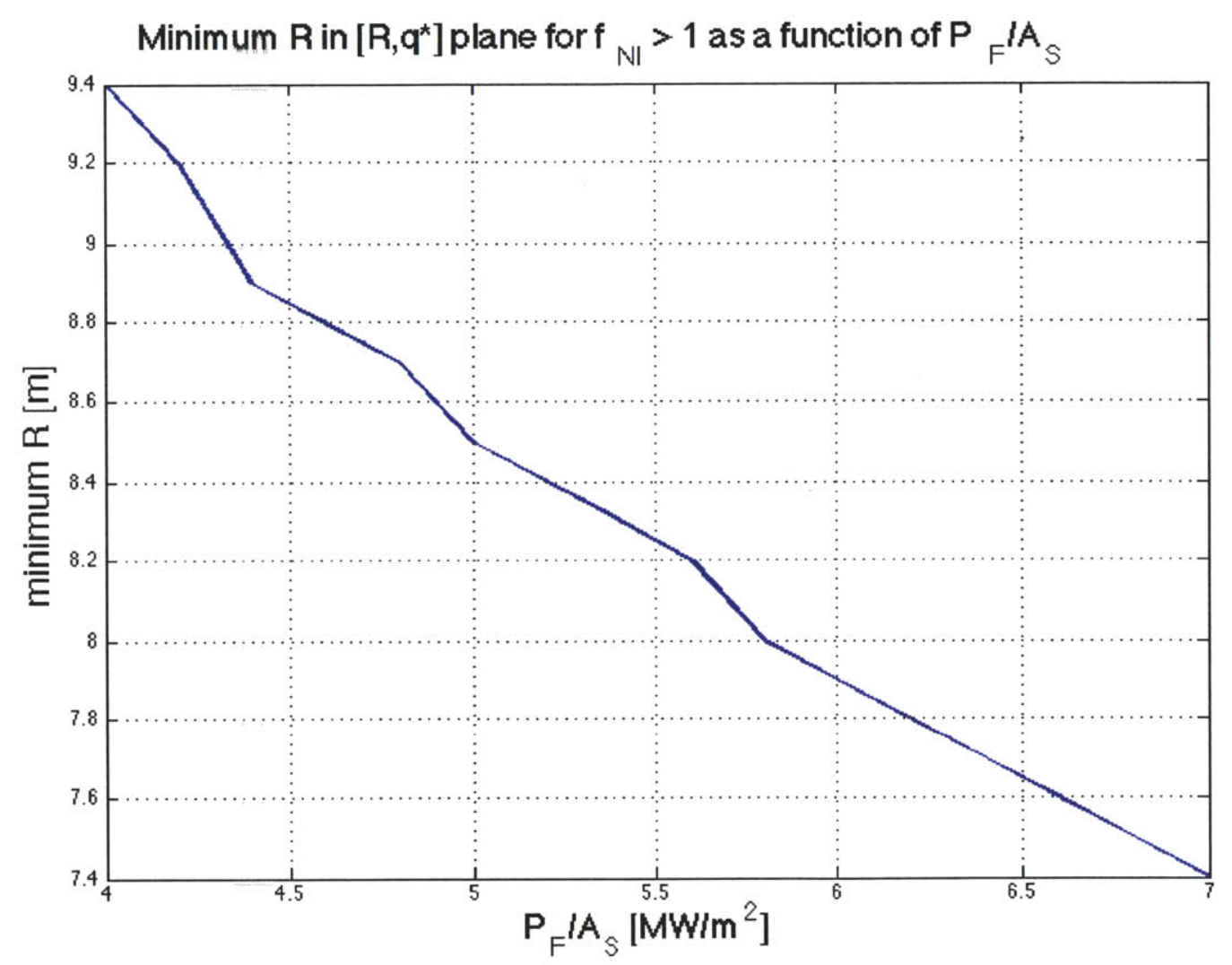

Figure 5.16: The minimum $R$ in the $\left[R, q^{*}\right]$ plane for fully non-inductive L-mode operation as a function of $P_{F} / A_{S}$. The minimum $R$ decreases fairly linearly with $P_{F} / A_{S}$. The parameters here are the same as those in Figure 5.15. 


\subsection{Aspect Ratio $R / a$ Considerations}

In the HYPERION design, $R / a$ is 2.6. We made this choice based on the requirement that $\Delta \Phi_{\text {sol }} / \Phi_{P}$ be at least in the vicinity of $2[4]$. As $R / a$ decreases given a fixed $R$, the solenoid area becomes too small to generate a sufficient flux swing $\Delta \Phi_{\text {sol }}$. The only hard limit on $R / a$ is of course that it must exceed 1 , though the solenoid may require a more complex model for $R / a \sim 2$.

Figure 5.17 shows the effect of varying $R / a$ on the minimum $R$. The allowable area in the $\left[R, q^{*}\right]$ plane shifts to higher $q^{*}$ when $R / a$ decreases, which we have already shown in Figure 5.9. Now we also show that the minimum $R$ decreases as $R / a$ decreases. This is intuitive, because tokamaks with smaller $R / a$ have higher densities (and lower temperatures) due to larger $\kappa$.

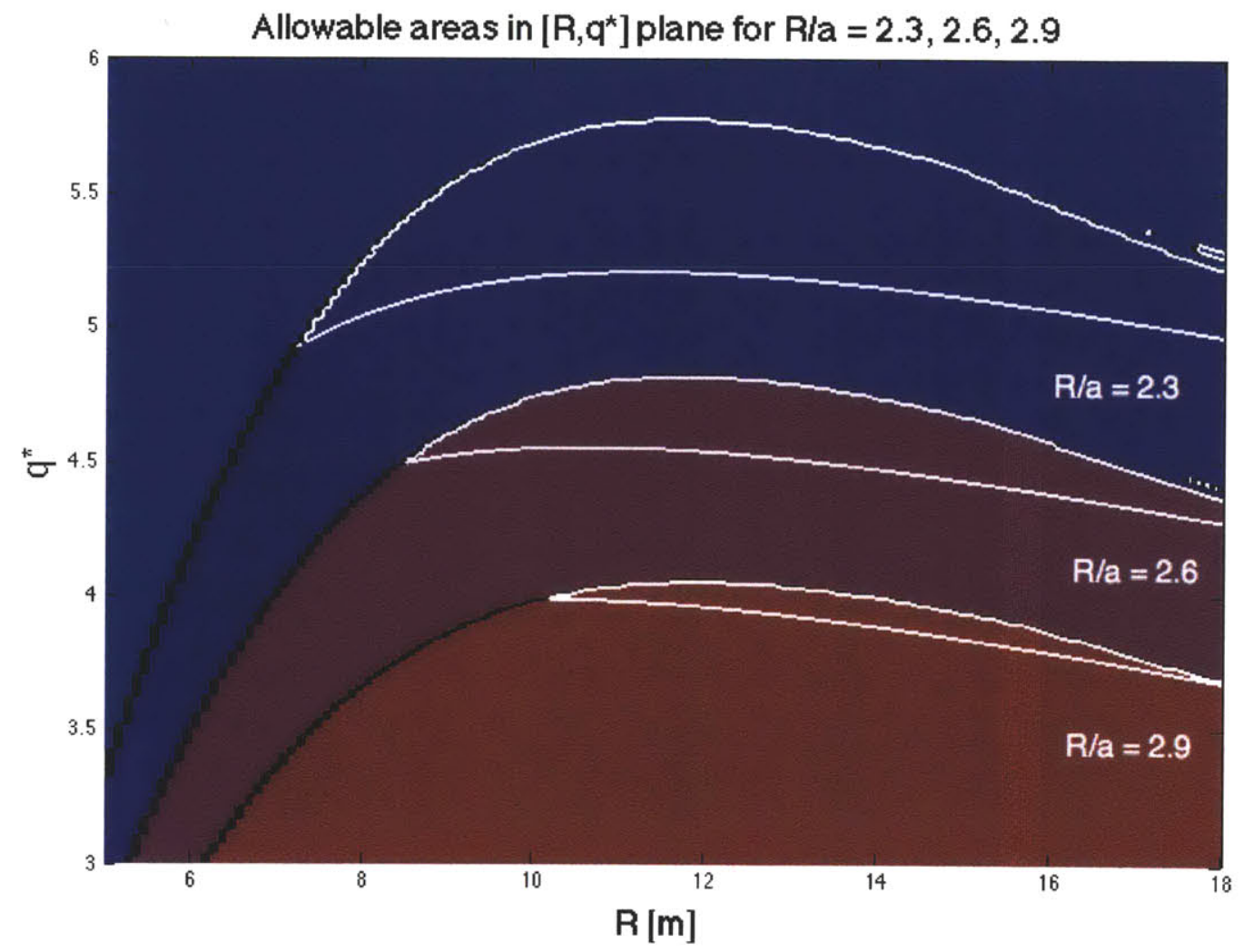

Figure 5.17: Areas in the $\left[R, q^{*}\right]$ plane that satisfy the parameter constraints for aspect ratio $R / a$ values of $2.3,2.6$, and 2.9 . Here $B_{\max }=16 \mathrm{~T}, P_{F} / A_{S}=5 \mathrm{MW} / \mathrm{m}^{2}$, and $Q=40$. The allowable area is especially sensitive to $R / a$. As $R / a$ increases, the highest available $q^{*}$ decreases while the minimum (fully non-inductive) $R$ increases. Consequently, we prefer lower $R / a$.

Figure 5.18 shows minimum $R$ as a function of $R / a$. Minimum $R$ increases more rapidly 
with $R / a$ at large $R / a \geq 2.8$. Note that for $\operatorname{ITER}(R / a=3.1)$, the minimum $R$ is over 12 $\mathrm{m}$. The HYPERION choice of $R / a=2.6$ avoids the high-slope region, though lowering $R / a$ below 2.6 could still certainly provide a modest decrease in minimum $R$.

To make any choice for $R / a$, we must consider flux swing. For the purposes of a pure fusion reactor with $Q=40$, flux swing will not constrict $R / a$ any more than the basic assumptions of our 0-D model already do. However, this is not the case for much low $Q$, as we will address in our coupled fission-fusion analysis in Section 6.2.

All this suggests that we should dramatically lower $R / a$. However, our $0-\mathrm{D}$ core model is based on the geometry and physics of a standard tokamak, not a spherical tokamak. In particular, our model of the blanket $\left(w_{B}=1 \mathrm{~m}\right)$ and solenoid may not be accurate for $R / a \approx 2$. Also, as $R / a$ becomes low, the poloidal cross-section must be less elliptical and more $\mathrm{D}$-shaped. These uncertainties, coupled with the fact that minimum $R$ has a much less significant dependence on $R / a$ below 2.6 than above 2.6 (see Figure 5.18), make it reasonable to keep $R / a$ fixed at 2.6. Also, since this minimum scale reactor is meant to demonstrate the viability of HYPERION, it makes sense to at least keep the geometry consistent. We will choose $R / a=2.6$ but emphasize the subjectivity of this choice. 


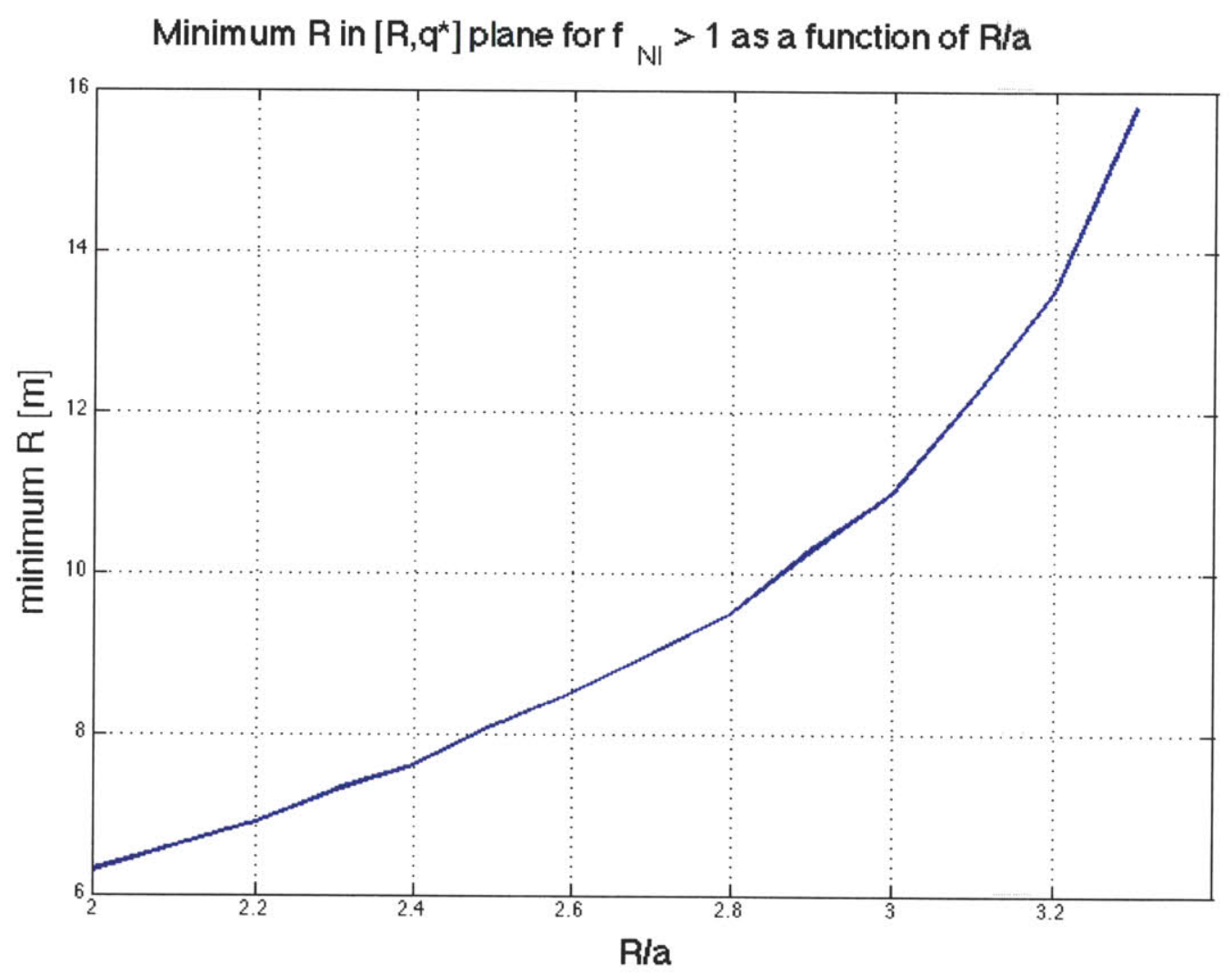

Figure 5.18: The minimum $R$ in the $\left[R, q^{*}\right]$ plane for fully non-inductive L-mode operation as a function of $R / a$. The minimum $R$ increases with $R / a$ very rapidly for $R / a \geq 3$ but much less rapidly for lower $R / a$. The parameters here are the same as those in Figure 5.17. 


\subsection{A "Reactor" Defined}

So far, we have assumed $Q=40$ to ensure that the tokamak is a "reactor". However, there is really no precise definition of a reactor in terms of tokamaks. While $Q=40$ is definitely a reactor and $Q=5$ is definitely not a reactor, there is certainly no threshold of $Q$ at which a tokamak suddenly transitions into "reactor mode" as it does into L-mode or H-mode. Thus, we investigate possible benefits of changing $Q$.

As $Q$ increases, the L-mode allowable area in the $\left[R, q^{*}\right]$ plane shrinks, eventually excluding all fully non-inductive areas. Thus, there is an absolute maximum $Q$ for fully noninductive L-mode operation. Figure 5.19 shows this maximum $Q$ (at any $q^{*}$ ) as a function of $R$. $Q$ increases fairly linearly for $R \leq 10 \mathrm{~m}$ but then levels off at $\approx 70$ for $R \geq 13 \mathrm{~m}$. This plot can be read two ways: as a maximum $Q$ corresponding to a certain $R$ or as a minimum $R$ corresponding to a certain $Q$. We are interested in the latter interpretation, though the two are equivalent. At $Q=40$, the minimum $R$ is approximately $8.5 \mathrm{~m}$. Lowering $Q$ improves the minimum $R$ by approximately $0.1 \mathrm{~m}$ per unit $Q$.

The question is by how much to lower $Q$. The answer is somewhat arbitary, but for the purpose of defining a specific operating point, we choose to decrease $Q$ to 30 so that the minimum $R$ is approximately $7.5 \mathrm{~m}$. This improves the minimum $R$ by $\approx 1 \mathrm{~m}$ while maintaining "reactor" status.

The only way for a pure fusion tokamak "reactor" at an anything-less-than-huge size is to operate in H-mode. That's ITER. Many people interested in fission-fusion hybrids have suggested modeling them after ITER, simply because ITER is what's being built right now. Unfortunately, that would miss the greatest potential advantage of hybrids: operating in L-mode at small size.

Figure 5.20 shows the same relationship as in Figure 5.19 for the ITER $R / a$ of 3.1. The maximum $Q$ at the ITER $R$ of $6.2 \mathrm{~m}$ is 12.5 . Since ITER is designed for $Q \approx 10$, this shows that ITER could potentially operate in fully non-inductive L-mode given $B_{\max } \approx 16 \mathrm{~T}$ and $P_{F} / A_{S} \approx 5 \mathrm{MW} / \mathrm{m}^{2}$.

It is important to emphasize the reality that choices of $R / a$ and $Q$ are very subjective. The choices of $R / a=2.6$ and $Q=30$ are actually quite conservative and may overestimate the true miniumum $R$. 


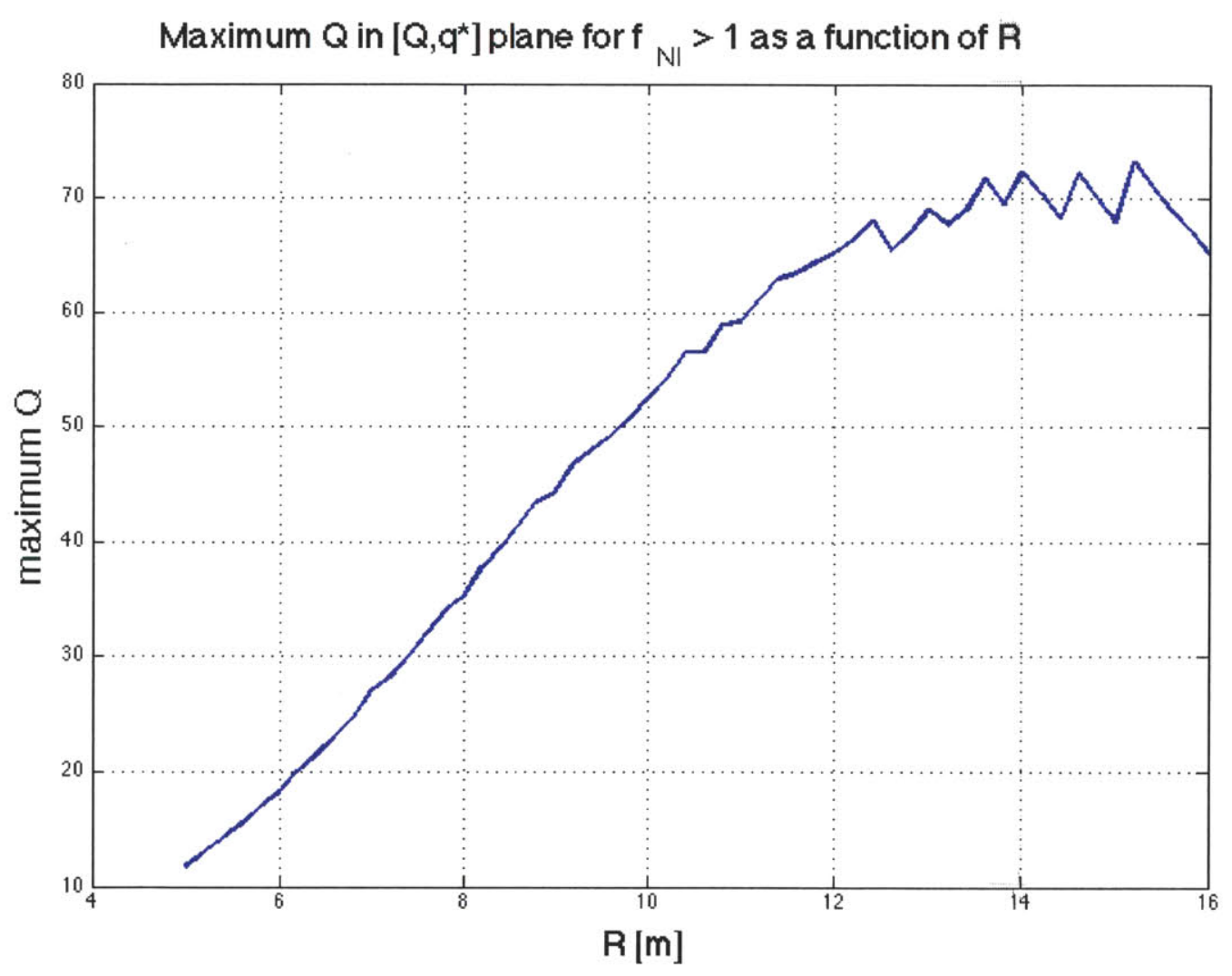

Figure 5.19: The maximum $Q$ in the $\left[Q, q^{*}\right]$ plane for fully non-inductive L-mode operation as a function of $R$. The relationshp is fairly linear for $5 \mathrm{~m} \leq R \leq 10 \mathrm{~m}$. At $Q=40$, the minimum $R$ is approximately $8.5 \mathrm{~m}$. At $Q=30$, the minimum $R$ is approximately $7.5 \mathrm{~m}$. Here $R / a=2.6$. The jaggedness at $R>12 \mathrm{~m}$ is an artificial result due to insufficient $q^{*}$ resolution. 


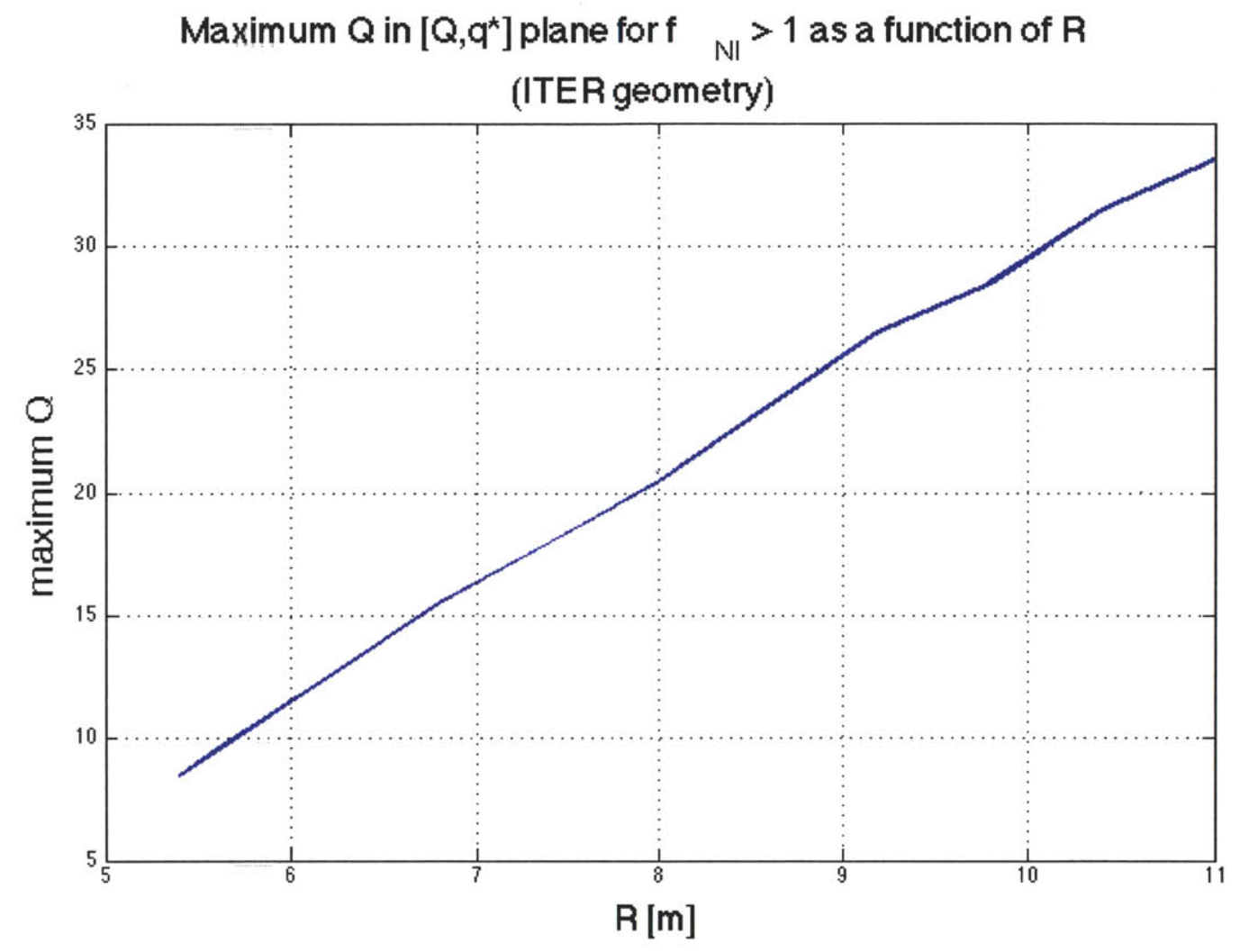

Figure 5.20: The maximum $Q$ in the $\left[Q, q^{*}\right]$ plane for fully non-inductive L-mode operation as a function of $R$ for the ITER aspect ratio of 3.1. At the ITER major radius of $6.2 \mathrm{~m}$, the maximum $Q$ is 12.5 . ITER is designed for $Q \approx 10$. 


\section{Coupled Fission-Fusion Analysis}

Thus far, we have developed a Monte Carlo neutron transport code to analyze the fission blanket as well as a 0-D tokamak model to analyze the fusion core. Now we will marry them to perform coupled fission-fusion analysis. We have already defined limiting magnetic field strength and surface power density, but now we can vary the physical dimensions ( $R$ and $a$ ) along with $q^{*}$ and $Q$ to optimize for steady-state L-mode operation in conjunction with our optimal fission blanket parameters.

\subsection{Effect of Tokamak Geometry on Fission Blanket}

In Section 3, we determined an optimal set of fission blanket layer thicknesses assuming ITER geometry $(R=6.2 \mathrm{~m}, a=2.0 \mathrm{~m}, \kappa=1.75)$. Now that we plan to vary the physical dimensions of the tokamak, we must determine to what extent these optimal blanket parameters would change for different tokamak geometry. We have run our neutronics model for varying $R, a$, and $\kappa$. Varying $R$ or $a$ while holding everything else fixed has very little effect on the fission and tritium breeding ratios as long as the aspect ratio $R / a$ remains above 2.0 and $a$ remains larger than $1.0 \mathrm{~m}$. However, varying $\kappa$ does have a notable effect on the tritium breeding ratio. Figure 6.1 shows this. The fission ratio is largely unaffected, but the tritium breeding ratio decreases markedly as $\kappa$ increases. The reason for this is that the effective thickness of the uranium layer increases near the top and bottom of the torus as $\kappa$ increases. If the thickness at the midplane is $w$, then the thickness at the top and bottom is $\kappa w$. When the subcritical uranium layer is thicker, fewer neutrons reach the lithium. However, since we have defined $\kappa$ as a function of $R / a$, we will assume that $\kappa$ varies only minimally throughout this analysis and does not have any substantial effect on the tritium breeding ratio. Also, in practice, this hybrid device would be constructed so that the layer thicknesses are constant throughout the poloidal plane (not precise ellipses).

\subsection{Solenoid Size vs. Blanket Thickness}

We have stated that the tokamak fusion core drives the fission blanket, while the fission blanket has no effect on the fusion reaction. This is absolutely true for a fixed geometry, but it is not entirely true when we vary the geometry. The size of the tokamak solenoid, which must fit within the inner "hole" of the torus, is limited by how thick the fission blanket is. When the blanket is thicker, the solenoid must have a smaller radius. Quantitatively, the maximum solenoid radius is $R-a-w_{B}-w_{M}$, where $w_{B}$ is the blanket thickness and $w_{M}$ is the magnetic coil thickness (around $0.5 \mathrm{~m}$ for ITER). In our pure fusion analysis (Sections 4 and 5 ), we set $w_{B}=1$, which is the usual thickness for pure fusion tokamaks. This is the thickness of shielding material necessary to protect the magnetic coils from neutron and 


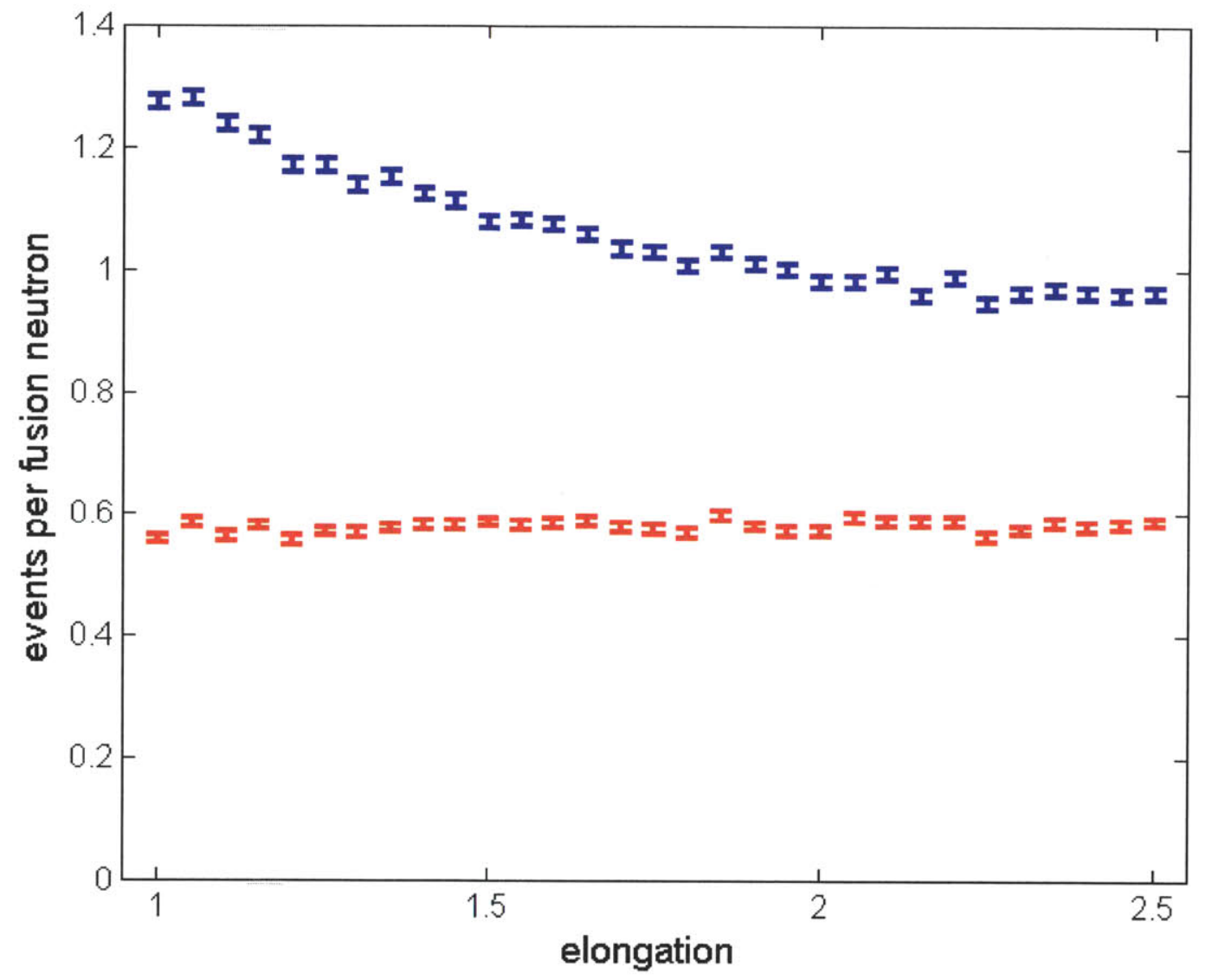

Figure 6.1: The fission and tritium breeding ratios as a function of elongation $\kappa$ for ITER tokamak dimensions and the optimal blanket thicknesses. Only tritium breeding is sensitive to $\kappa$.

gamma fluence. In this thesis, we do not perform detailed shielding calculations to determine how much thicker the blanket must be when it is fissionable. Our optimal uranium and lithium layer thicknesses sum to $45 \mathrm{~cm}$. There must also be at least $3 \mathrm{~cm}$ of material for the first wall and dividing the uranium pebbles from the lithium (in our neutronic analysis, we assumed this was silicon carbide). That brings the total blanket thickness to $48 \mathrm{~cm}$. However, since even a pure fusion tokamak must have a first-wall and a tritium breeding layer, we have only really added the $18 \mathrm{~cm}$ uranium layer to the total thickness. We believe it is reasonable to assume that a steel and water shield about $40 \mathrm{~cm}$ thick beyond the lithium will adequately protect the magnetic coils. Our neutronics model (as well as MCNP) shows that the neutron fluence will be well below the limit of $3 \times 10^{22} \mathrm{n} / \mathrm{m}^{2}$ for $\mathrm{Nb}_{3} \mathrm{Sn}$ [5]. 


\subsection{Steady-State L-mode ITER-PBR}

With our arguments in the previous two subsections, we have effectively decoupled the fission and fusion components. We have essentially argued, based on neutronics data and general reasoning, that the fission gain $Q_{\text {fis }}$ is independent of tokamak geometry within the range of geometric parameters we wish to consider. This is not wishful thinking - it is a valid approximation that, given the data, is not any less accurate than the approximations already inherent in our 0-D model. What we can now state is that for a fission-fusion hybrid "reactor" to operate with $Q_{\text {hyb }}=40$ given a fixed $Q_{\text {fis }}$, we can obtain the necessary $Q_{\text {fus }}$ from

$$
Q_{\text {hyb }}=Q_{\text {fus }}\left(\frac{1}{5}+\frac{4}{5} Q_{\text {fis }}\right)
$$

If we require $Q_{\mathrm{hyb}}=40$ and use $Q_{\mathrm{fis}}=7.7$ from Table 3.1, then $Q_{\text {fus }}$ must be approximately 6.3. This dramatically changes the analysis we performed in Section 5. Although much of that analysis is redundant from our 2009 MTT bachelor's thesis, we will expand on it here.

First, let us introduce a new variety of $R-q^{*}$ "phase diagram" to further elucidate the analysis. Figure 6.2 shows L-mode and $\mathrm{H}$-mode regions in the $R-q^{*}$ plane for a pure fusion reactor with $Q=40$. It also shows the forbidden regions where $\beta_{N}$ exceeds the Troyon limit and where the required reactivity is unphysically high. The $\mathrm{H}$-mode region is bounded by the $H=1.5$ curve and the $\beta_{N}=3$ curve. For a fixed $q^{*}$ and fixed $R / a$, L-mode is only possible in a larger tokamak than H-mode. This figure is similar to Figure 5.6, except that it shows multiple "phases" instead of only L-mode. We will call this new type of figure an $R-q^{*}$ tokamak phase diagram. These diagrams are useful for the initial planning of tokamaks.

We can expand on this with Figure 6.3, which is identical to Figure 6.2 except with an additional subdivision between pulsed and steady-state modes. The additional curve that does not appear in Figure 6.2 represents $f_{N I}=1$. This also qualifies as an ' $R$ - $q^{*}$ tokamak phase diagram". Here we can clearly see the steady-state L-mode region that was the focus of our 2009 bachelor's thesis and has a sharply-defined minimum $R$ associated with it [28].

While Figures 6.2 and 6.3 has $R / a$ fixed at 2.6, Figure 6.4 has $R / a$ fixed at 3.1 to match ITER. There is no steady-state L-mode here - that highly favorable region disappears for $R / a>3$. This is the reason why a steady-state L-mode tokamak must have an aspect ratio lower than that of ITER.

Now we will keep everything the same except $Q$ - we will lower it to 6.3 as recommended by our fission blanket analysis. This changes everything. Figure 6.5 shows the result. By lowering $Q_{\text {fus }}$ from 40 to 6.3 , we have narrowed the H-mode swath and dramatically lowered the $f_{N I}$ curve to open up a vast expanse of the steady-state L-mode phase. Comparing Figure 6.5 to Figure 5.4 shows just how powerful the addition of a fission blanket to a tokamak can be. 


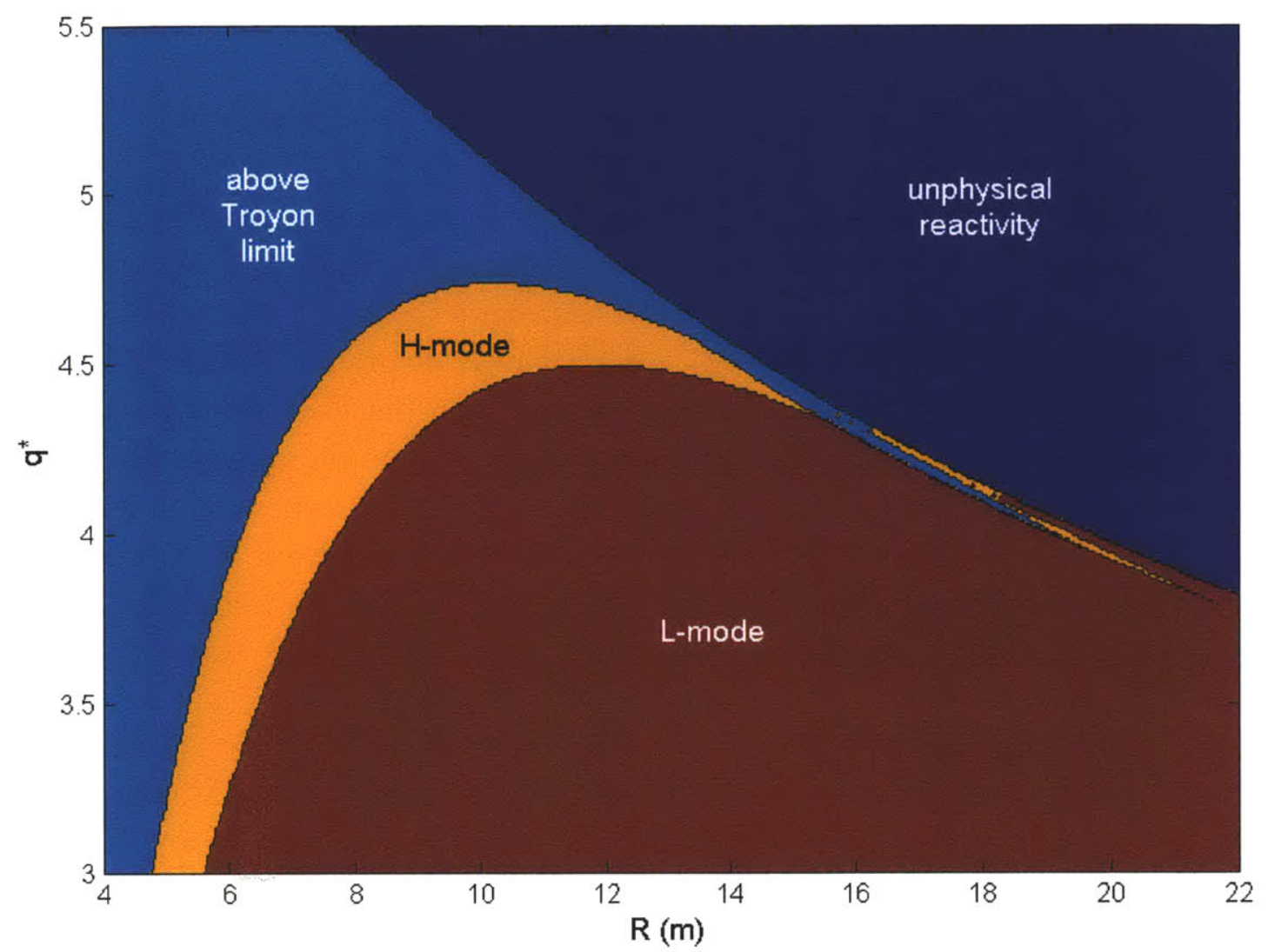

Figure 6.2: A "phase diagram" over $[R, q *]$ showing L-mode, H-mode, and areas forbidden by reactivity and the Troyon limit. This is for a pure-fusion "reactor" with $Q_{\text {fus }}=40$. Here we use $R / a=2.6, B_{\max }=15 \mathrm{~T}$, and $P_{F} / A_{S}=5 \mathrm{MW} / \mathrm{m}^{2}$. 


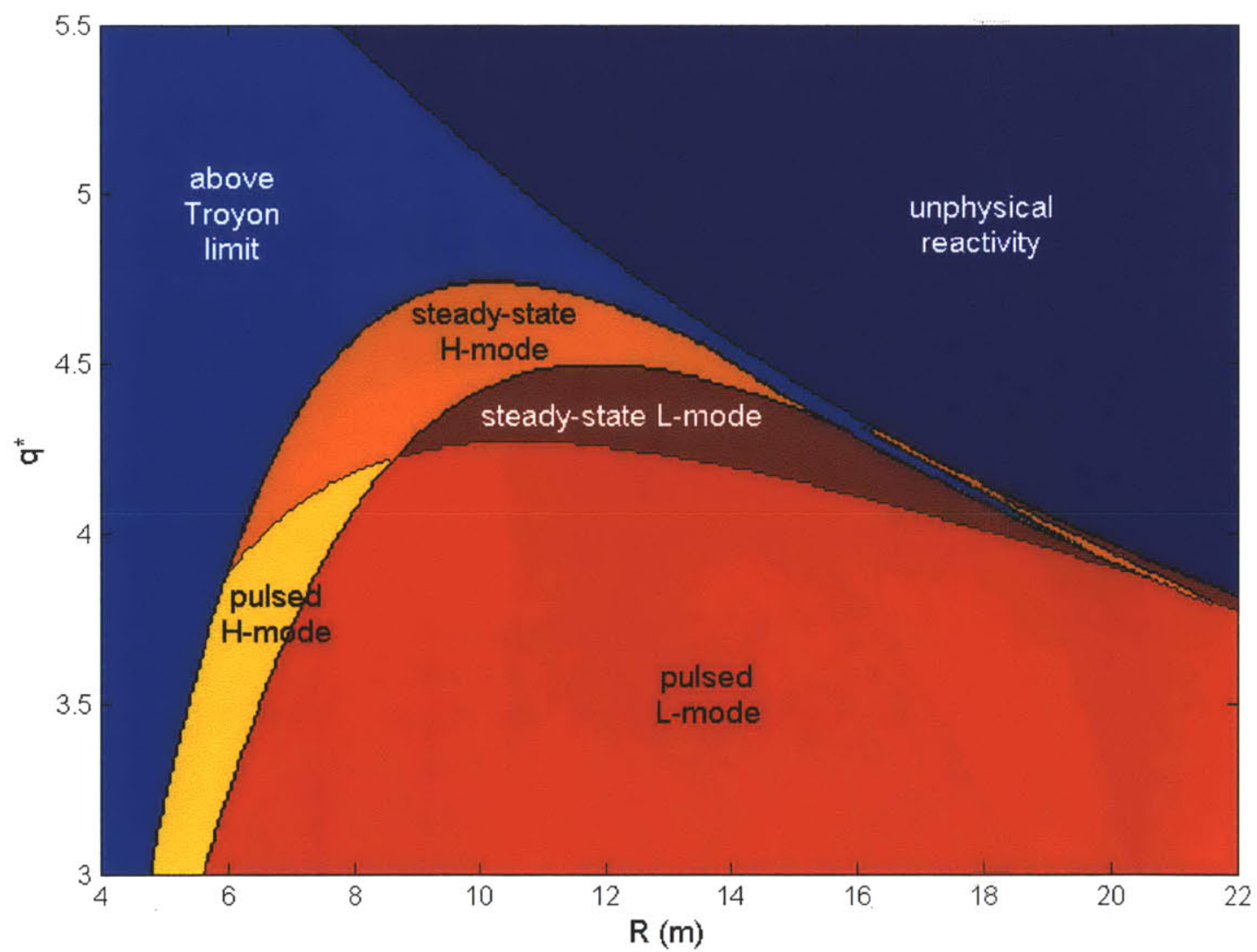

Figure 6.3: A "phase diagram" over $[R, q *]$ showing L-mode, H-mode, and areas forbidden by reactivity and the Troyon limit. This is for a pure-fusion "reactor" with $Q_{\text {fus }}=40$. We subdivide L-mode and H-mode into "pulsed" and "steady-state" zones with the curve defined by $f_{N I}=1$. Here we use $R / a=2.6, B_{\max }=15 \mathrm{~T}$, and $P_{F} / A_{S}=5 \mathrm{MW} / \mathrm{m}^{2}$. 


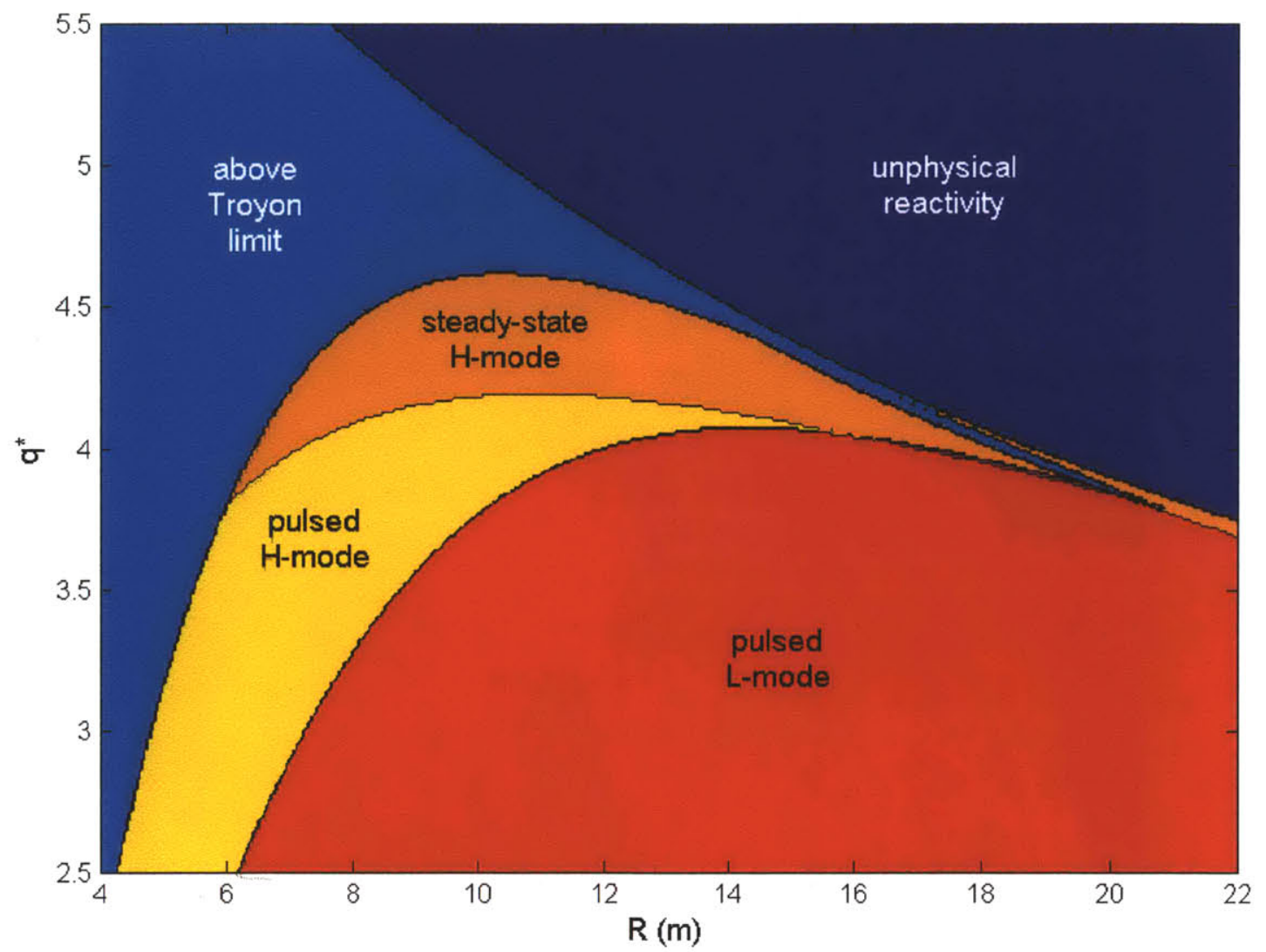

Figure 6.4: A "phase diagram" over $[R, q *]$ showing L-mode, H-mode, and areas forbidden by reactivity and the Troyon limit. This is for a pure-fusion "reactor" on the scale of ITER with $Q_{\text {fus }}$ $=40, R / a=3.1, B_{\max }=15 \mathrm{~T}$, and $P_{F} / A_{S}=3 \mathrm{MW} / \mathrm{m}^{2}$. We subdivide L-mode and H-mode into "pulsed" and "steady-state" zones with the curve defined by $f_{N I}=1$. 


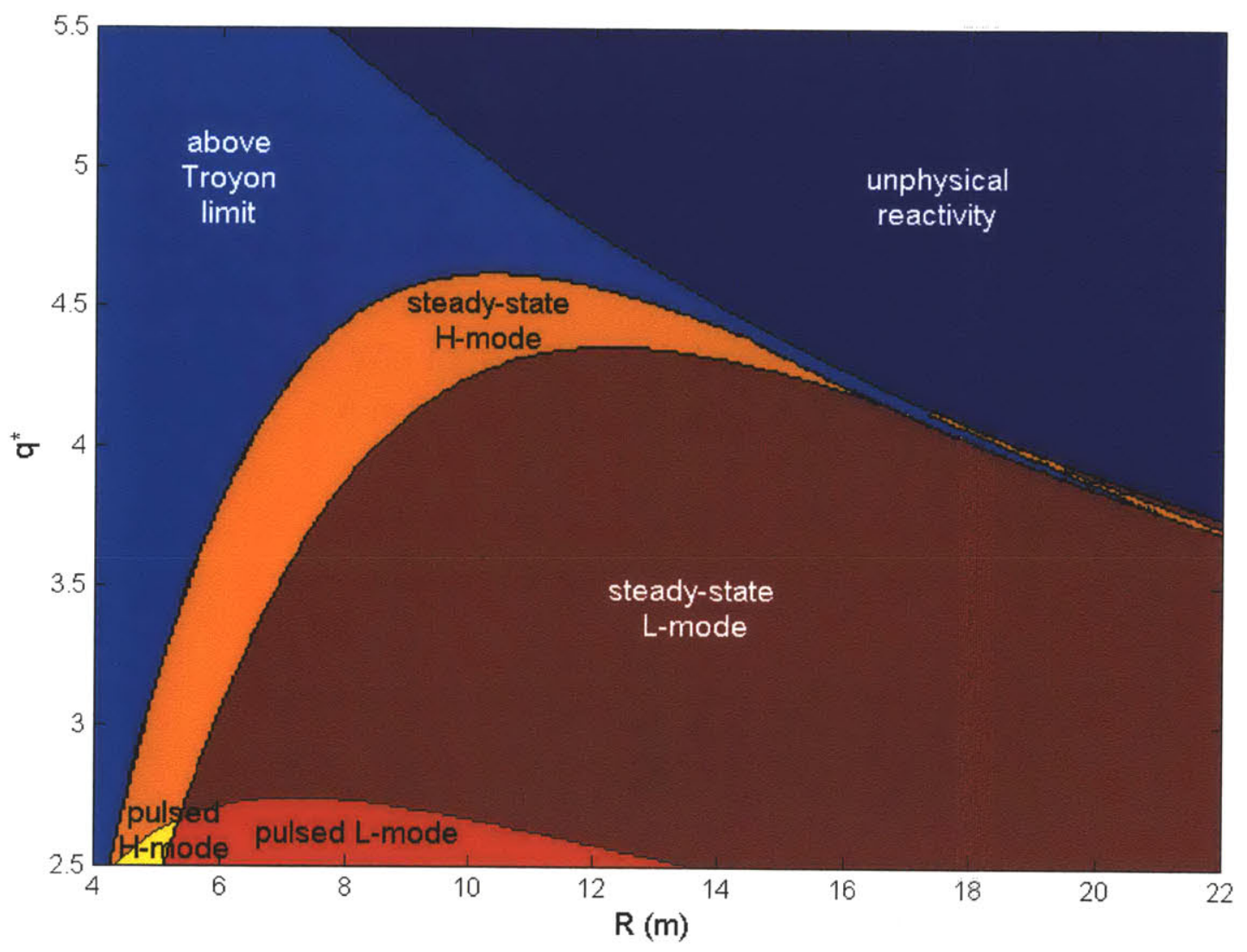

Figure 6.5: A "phase diagram" over $[R, q *]$ showing L-mode, H-mode, and areas forbidden by reactivity and the Troyon limit. We subdivide L-mode and H-mode into "pulsed" and "steadystate" zones with the curve defined by $f_{N I}=1$. This is for a fission-fusion hybrid "reactor" with a fission gain of 7.7 and $Q_{\text {fus }}=6.3$. This is on the scale of ITER with $R / a=3.1, B_{\max }=15 \mathrm{~T}$, and $P_{F} / A_{S}=3 \mathrm{MW} / \mathrm{m}^{2}$. This shows that ITER could operate in steady-state L-mode with our fission blanket. 
With the results shown in Figure 6.5, we can choose a set of approximate operating parameters for a fission-fusion hybrid based on ITER geometry $(R=6.2 \mathrm{~m}, R / a=3.1$, $\kappa=1.75)$. Figure 6.5 shows that a tokamak fission-fusion hybrid with this fixed geometry (ITER) could operate in steady-state L-mode. We show the complete set of operating points in Table 6.1.

This proves that the addition of this optimal fission blanket to ITER would allow ITER to operate in steady-state L-mode (with adjustments to its magnets and a few other parameters). Tang's ITER-PBR concept focused solely on the fission aspects of adding a fission blanket to ITER [22], but here we have shown that there could also be substantial advantages in terms of the fusion operation.

Table 6.1: Steady-State L-mode ITER-PBR Operating Parameters

$$
\begin{array}{cc}
R=6.2 \mathrm{~m} & P_{F}=2.1 \mathrm{GW} \\
R / a=3.1 & P_{\text {aux }}=310 \mathrm{MW} \\
\kappa=1.75 & H=1.4 \\
B_{\max }=15 \mathrm{~T} & \tau_{e}=1.0 \mathrm{~s} \\
B=7.7 \mathrm{~T} & T=14.5 \mathrm{keV} \\
Q=6.7 & \beta_{N}=2.2 \\
P_{F} / A_{S}=3 \mathrm{MW} / \mathrm{m}^{2} & \omega_{c e} / \omega_{p e}=2.2 \\
q^{*}=3.0 & \Delta \Phi_{\text {sol }} / \Phi_{P}=3.1 \\
I_{P}=16.6 \mathrm{MA} & f_{\text {boot }}=0.46 \\
n=1.210^{20} / \mathrm{m}^{3} & f_{C D}=0.76 \\
F_{G}=0.9 & f_{N I}=1.22
\end{array}
$$




\subsection{Minimum Scale Steady-State L-mode Fission-Fusion Hybrid}

We have analyzed the case of a steady-state L-mode hybrid with the geometry of ITER, but now we will turn to a more interesting scenario - that of minimum size. This is a point of significant interest, because capital costs of tokamaks tend to scale with major radius $R$.

In the ITER-PBR analysis, we were forced to deviate from the ITER aspect ratio in order to achieve steady-state L-mode. Now that we are more free in our geometry, we should strive to determine the optimal $R / a$ if one exists. We know from our analysis in Section 5.4 that a lower aspect ratio allows for a smaller minimum $R$, and so we should always favor low $R / a$. However, there must be a lower limit on $R / a$. Otherwise, our tokamak would evolve (or emphdevolve) into a spherical tokamak.

One property that depends largely on $R / a$ is the flux ratio $\Phi_{\text {sol }} / \Phi_{P}$, the ratio of solenoid flux to plasma flux. As we explained in Section 4.3.3, this ratio must exceed 2. If we fix $Q_{\text {fus }}$ at 6.7 as in the ITER-PBR analysis and assume other appropriate values such as $q *=$ 3.0 , we can calculate $\Phi_{\text {sol }} / \Phi_{P}$ and the minimum $R$ (for steady-state L-mode operation) as a function of $R / a$. Figure 6.6 shows this. $\Phi_{\text {sol }} / \Phi_{P}$ exceeds 2 for $R / a>2.8$, which we must now set as our lower limit on $R / a$. Since the minimum $R$ is also an increasing function of $R / a$, it is easy to see that we should set $R / a$ at its limit of 2.8 and choose our minimum scale $R$ as the corresponding value of slightly more than $5 \mathrm{~m}$.

To confirm this, we can generate yet another $R-q^{*}$ "phase diagram" for $R / a=2.8$. Figure 6.7 shows this. Clearly, at $q^{*}=3.0$, the minimum steady-state L-mode $R$ is slightly more than $5 \mathrm{~m}$. A closer look reveals that it is approximately $5.2 \mathrm{~m}$.

Table 6.2 shows the corresponding complete set of parameters for the fusion component of this minimum scale steady-state L-mode fission-fusion hybrid. Clearly, it is steady state with $f_{N I} \approx 1.0$ and L-mode with $H<1.5$.. The total fusion power is $1.7 \mathrm{GW}$. Thus, the total hybrid thermal power, given a fission blanket multiplication of 7.7 , is $10.8 \mathrm{GW}$. Since the auxiliary power is $260 \mathrm{MW}$, the net $Q_{\text {hub }}$ is an admirable 41 . This is unequivocally a true reactor. 


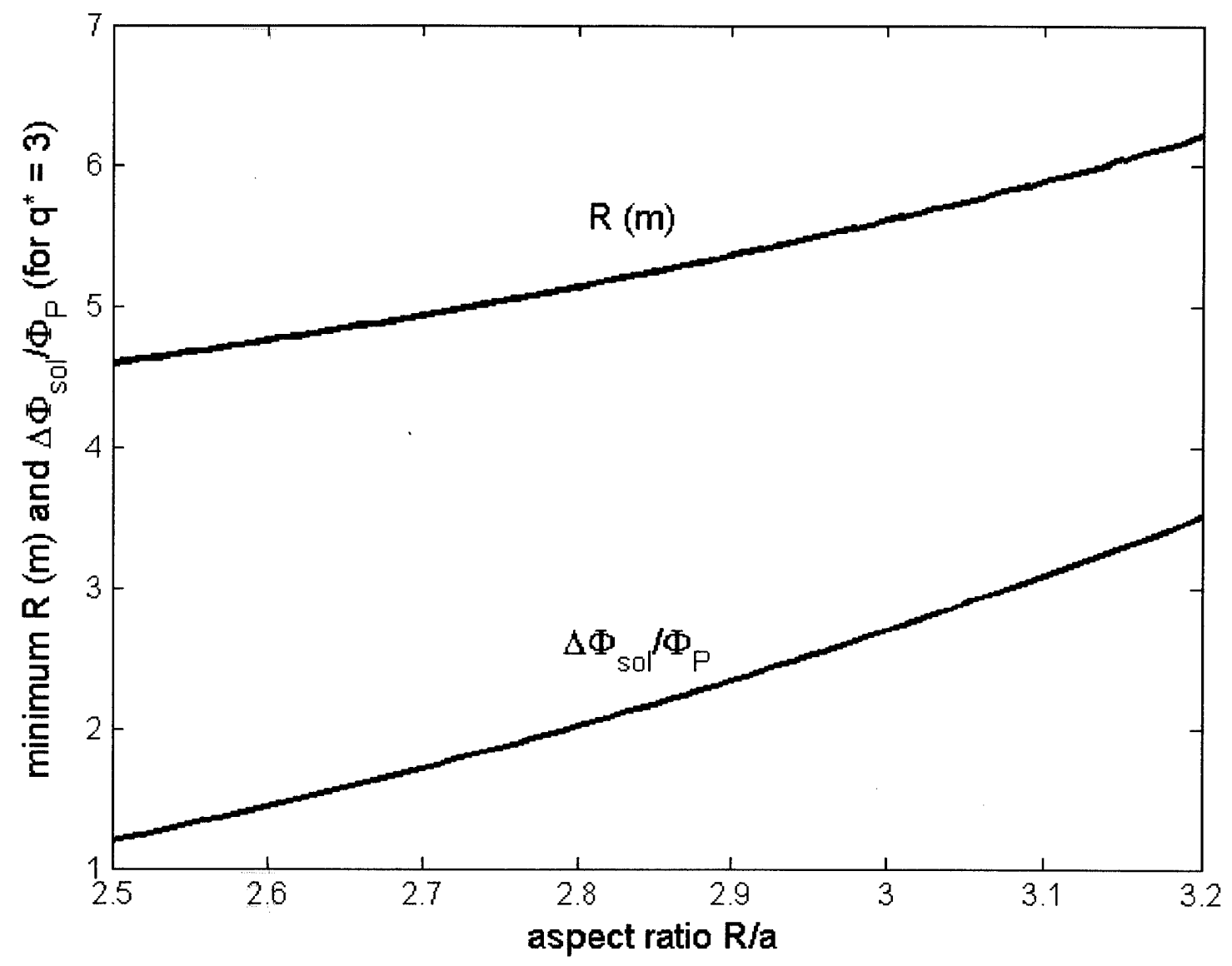

Figure 6.6: Minimum major radius $R$ (for steady-state L-mode) and flux ratio $\Phi_{\text {sol }} / \Phi_{P}$ as a function of aspect ratio $R / a$ at $q *=3.0$. Here $B_{\max }=15 \mathrm{~T}, P_{F} / A_{S}=3 \mathrm{MW} / \mathrm{m}^{2}$, and $Q_{\text {fus }}=6.7$. The flux ratio is above 2 for $R / a>2.8$, which corresponds to $R=5.2 \mathrm{~m}$. 


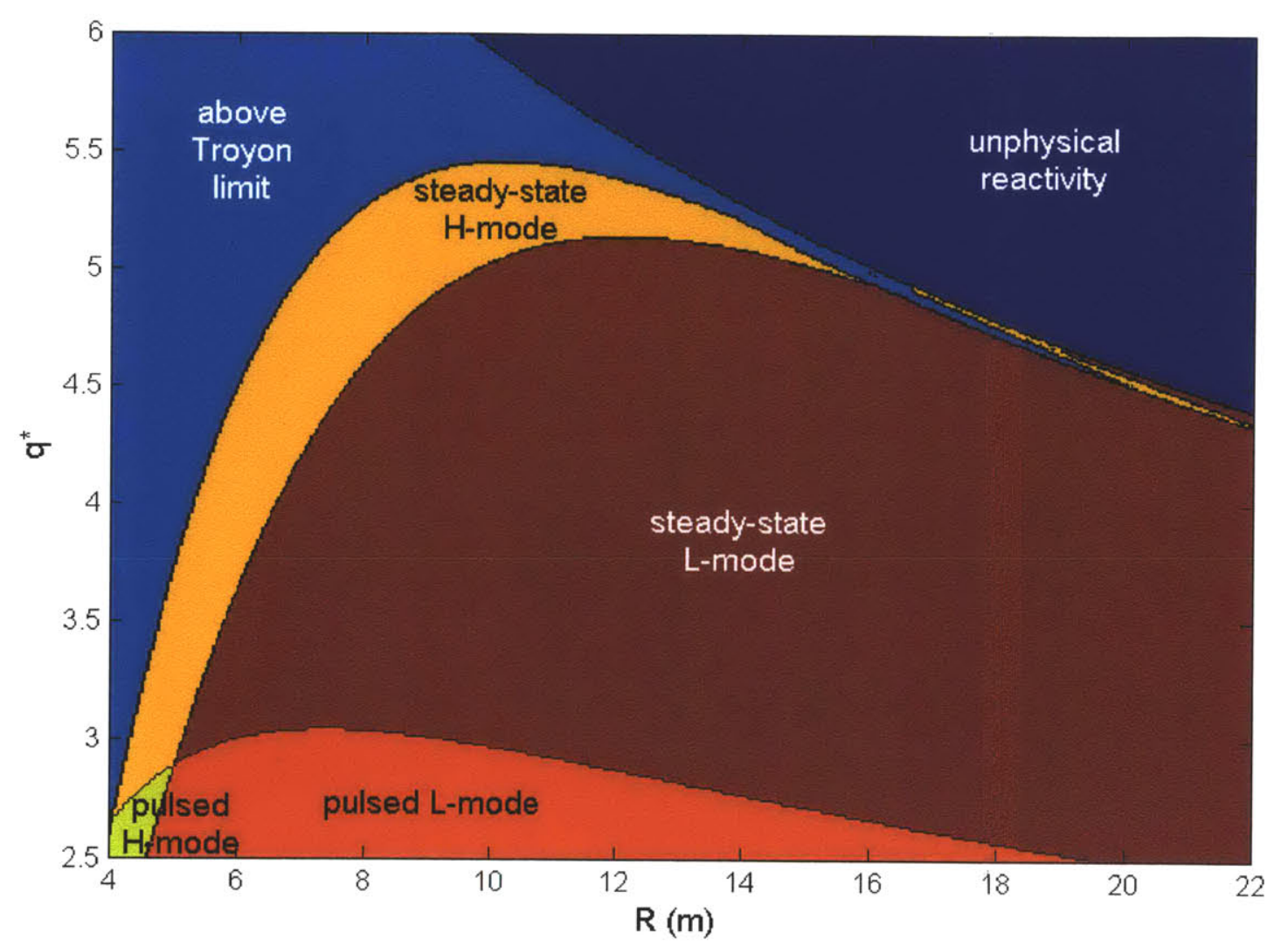

Figure 6.7: A "phase diagram" over $[R, q *]$ showing L-mode, H-mode, and areas forbidden by reactivity and the Troyon limit. We subdivide L-mode and H-mode into "pulsed" and "steadystate" zones with the curve defined by $f_{N I}=1$. This is for a fission-fusion hybrid "reactor" with a fission gain of 6.0 and $Q_{\text {fus }}=6.7$. We use the minimum feasible aspect ratio $R / a=2.8$ along with $B_{\max }=15 \mathrm{~T}$ and $P_{F} / A_{S}=3 \mathrm{MW} / \mathrm{m}^{2}$. This set of parameters would allow for the minimum scale steady-state L-mode reactor with our fission blanket. 
Table 6.2: Minimum Scale Steady-State L-mode Operating Parameters

$$
\begin{array}{cc}
R=5.2 \mathrm{~m} & P_{F}=1.7 \mathrm{GW} \\
R / a=2.8 & P_{\text {aux }}=260 \mathrm{MW} \\
\kappa=1.91 & H=1.48 \\
B_{\max }=15 \mathrm{~T} & \tau_{e}=0.94 \mathrm{~s} \\
B=6.8 \mathrm{~T} & T=12.2 \mathrm{keV} \\
Q=6.7 & \beta_{N}=2.3 \\
P_{F} / A_{S}=3 \mathrm{MW} / \mathrm{m}^{2} & \omega_{c e} / \omega_{p e}=1.8 \\
q^{*}=3.0 & \Delta \Phi_{\text {sol }} / \Phi_{P}=2.02 \\
I_{P}=17.4 \mathrm{MA} & f_{\text {boot }}=0.46 \\
n=1.410^{20} / \mathrm{m}^{3} & f_{C D}=0.60 \\
F_{G}=0.9 & f_{N I}=1.06
\end{array}
$$




\subsubsection{1-D Profiles}

To ensure the viability of this minimum scale 0-D operating point, we perform 1-D profile analysis similar to that performed on the HYPERION operating point. We can express density and temperature profiles as parabolas raised to some power $\alpha$ plus a constant edge value [4]. Equation 6.2 expresses this in terms of a generic profile $X(r)$, which could be either density or temperature. Note that $X(a)=X_{\text {edge }}$ and $X(0)=X_{0}+X_{\text {edge }}$.

$$
X(r)=X_{0}\left[1-\left(\frac{r}{a}\right)^{2}\right]^{\alpha}+X_{\text {edge }}
$$

In the case of density, we assume $\alpha=0.5$ and an edge value of $n_{\text {edge }}=0.25\langle n(r)\rangle$, where 0.25 is called the density offset fraction and $\langle n(r)\rangle$ is the volume-averaged 0-D density [4]. Figure 6.8 shows the $n(r)$ profile, normalized so that $\langle n(r)\rangle=n_{0-D}$.

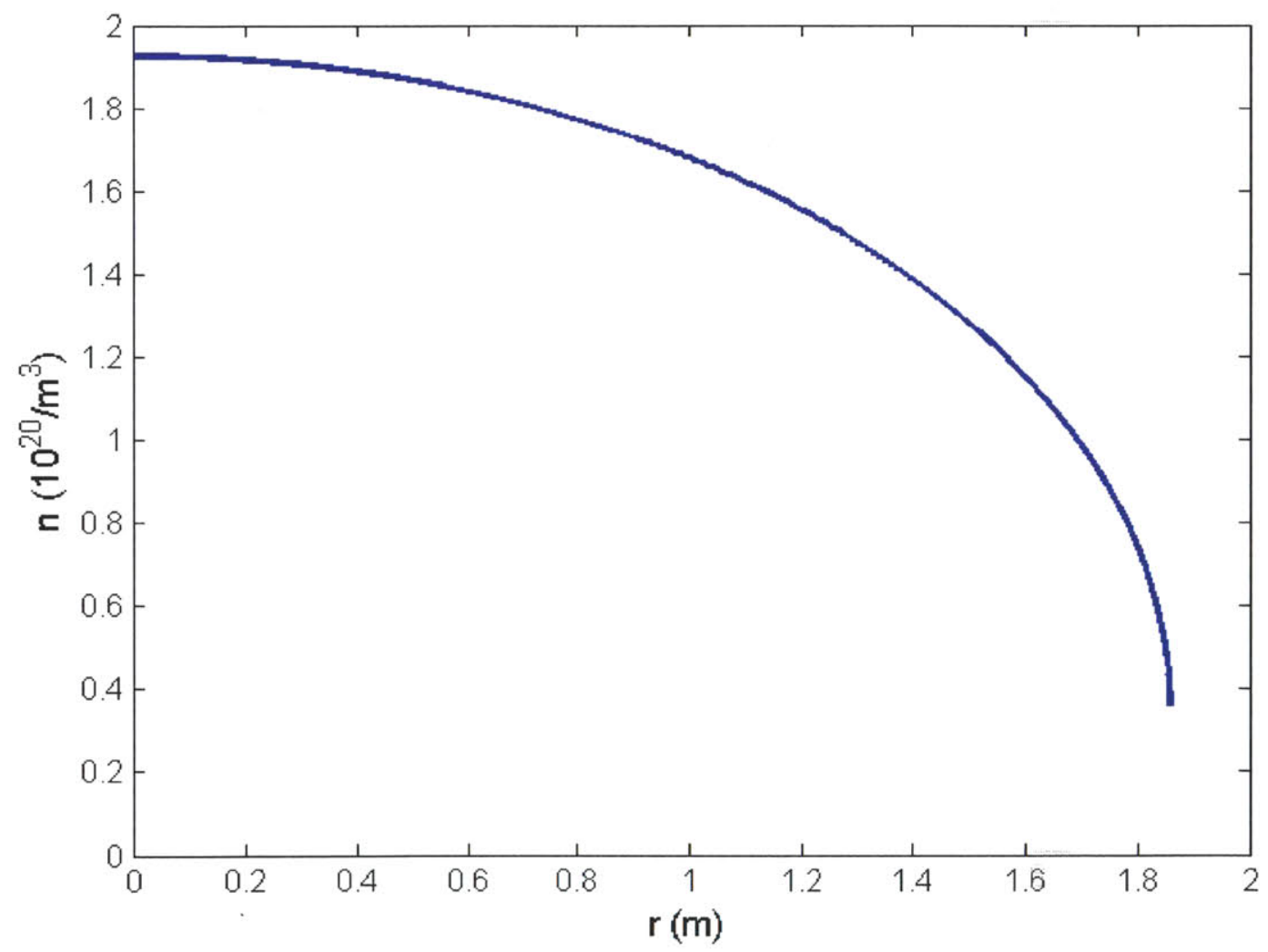

Figure 6.8: Ion density $n$ as a function of minor radius $r$ with an offset fraction of 0.25 .

In the case of temperature, we assume $\alpha=1.25$ and a small edge value of $T_{\text {edge }}=0.15$ $\mathrm{keV}[4]$. Figure 6.9 shows the $T(r)$ profile, normalized so that $\langle T(r)\rangle=T_{0-D}$. Note that 
$T(0)$ is less than $65 \mathrm{keV}$, and so no portion of the plasma reaches the maximum $\langle\sigma v\rangle$.

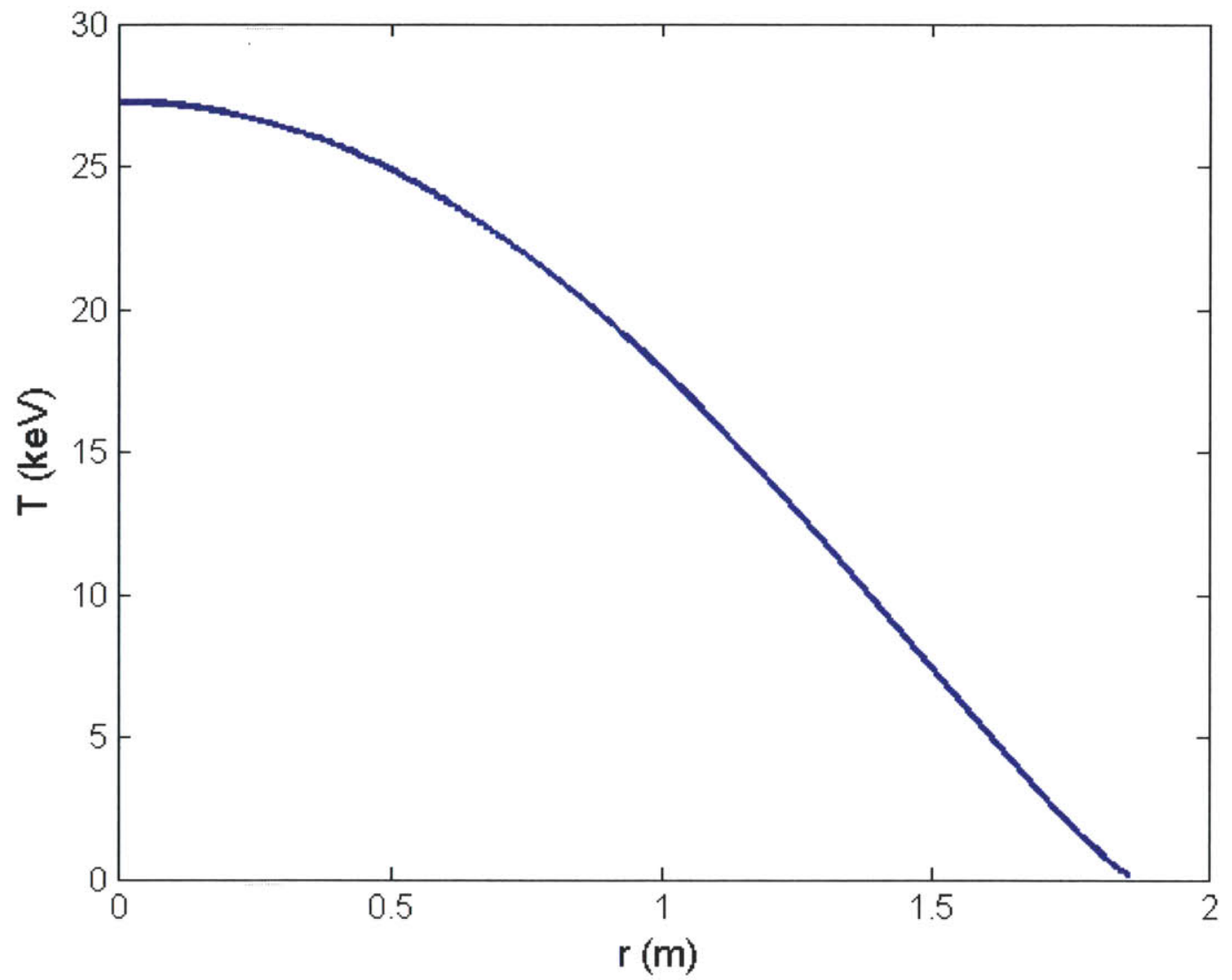

Figure 6.9: Plasma temperature $T$ as a function of minor radius $r$ with an edge temperature of $0.15 \mathrm{keV}$. In this model, we assume that the electron and ion temperatures are equal. Note that $T$ never nears $65 \mathrm{keV}$, the temperature which yields the maximum $\langle\sigma v\rangle$.

It is simple to calculate $\langle\sigma v\rangle(r)$ from $T(r)$, which we show in Figure 6.10. In HYPERION, $\langle\sigma v\rangle(r)$ is very flat for $r<a / 2$ and even a bit hollow, which provides inherent stability. This minimum scale reactor does not have such an advantage, but $\langle\sigma v\rangle(r)$ does flatten a bit as $T$ approaches its maximum.

Figure 6.11 shows the $P_{F}$ density profile, computed from $\langle\sigma v\rangle(r)$ and $n(r)$. The $P_{F}$ density is very small in the outer $0.3 \mathrm{~m}$ of the plasma, which we call the "mantle" region. In the mantle, the temperature is low enough so that radiative power losses dominate. In fact, nearly all radiative power loss occurs in the mantle, which justifies our neglect of radiation in the 0 -D model. 


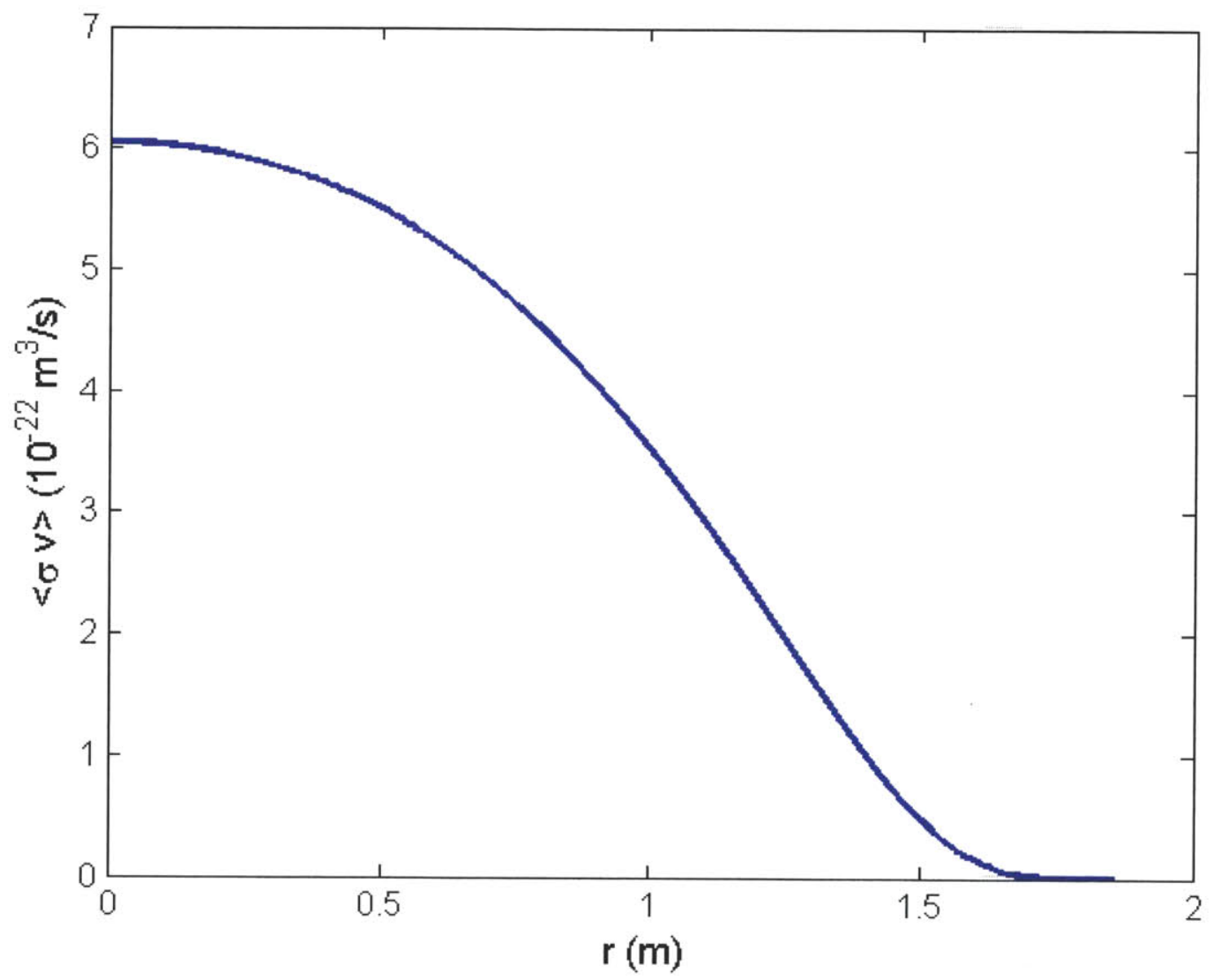

Figure 6.10: The D-T fusion reactivity rate coefficient $\langle\sigma v\rangle$ as a function of minor radius $r$. $\langle\sigma v\rangle$ never nears its physical maximum. 


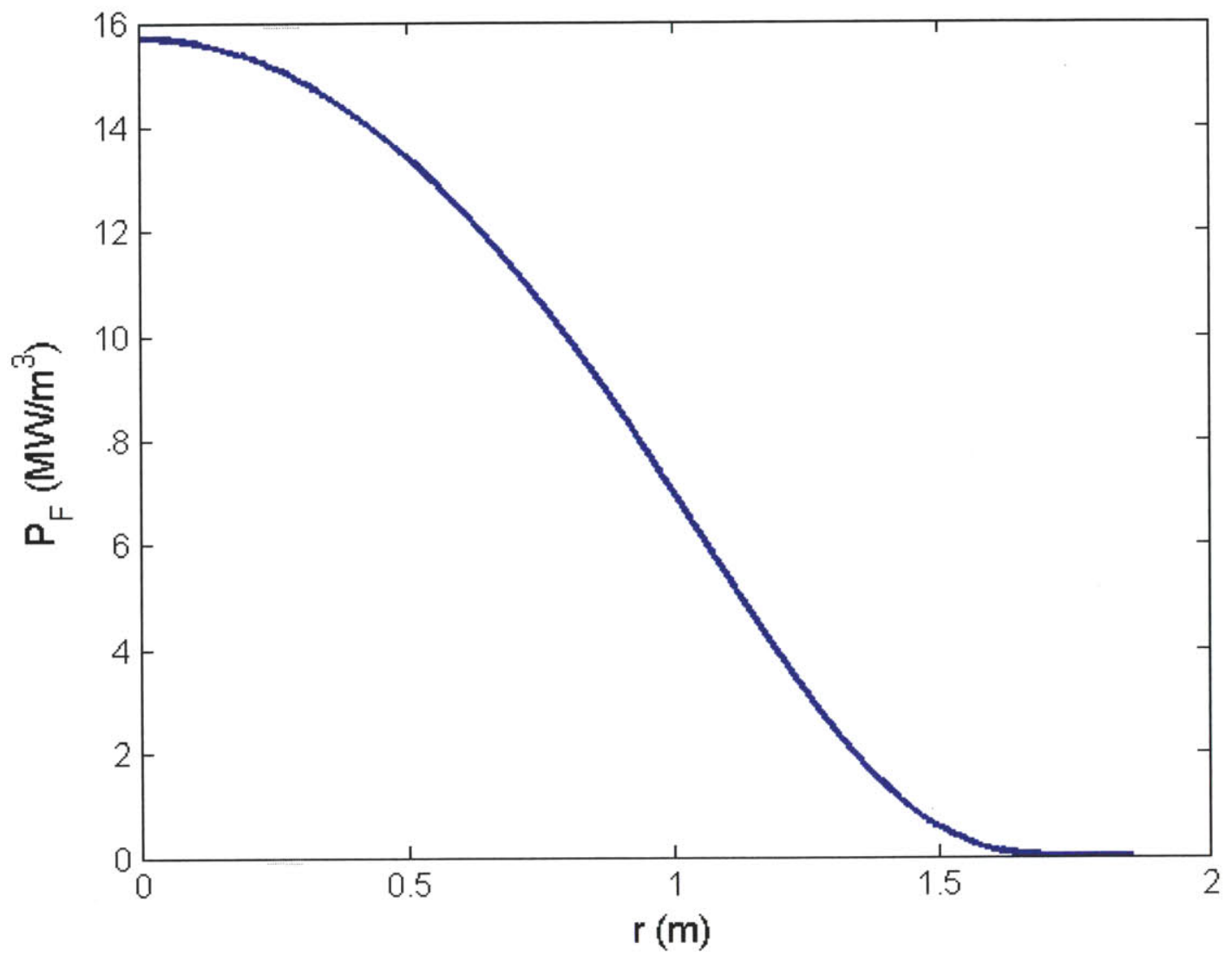

Figure 6.11: Fusion power $P_{F}$ density as a function of minor radius $r$. Very little power is generated in the outer $0.3 \mathrm{~m}$ of the plasma (the mantle). 
Current profiles are also instructive, and here we will complete an analysis similar to that in the HYPERION report [4]. Equation 6.3 shows that the bootstrap current density profile $I_{\text {boot }}(r)$ is a function of $n(r), T(r)$, the electron pressure profile $p_{e}(r)$, and the poloidal field profile $B_{\Theta}(r)[7] . p_{e}(r)$ is simply $n(r) k T(r) / 2$, where $k$ is the Boltzmann constant [3]. We can compute $B_{\Theta}(r)$ from $I_{P}(r)$, the total plasma current density. Of course, since $I_{P}(r)$ depends on $I_{\text {boot }}(r)$, we must either employ an iterative solution or assume a plausible initial $I_{P}(r)$ profile. We choose the latter approach and assume $I_{P}(r)=J_{0}\left[1-(r / a)^{2}\right]$, where $J_{0}$ is a normalization constant we choose so that the integral of $I_{P}(r)$ over the poloidal plane is equal to $I_{P}$. Equation 6.3 is derived from neoclassical transport theory [7]. Figure 6.12 shows $I_{\text {boot }}(r)$, which we renormalized to be consistent with $f_{\text {boot }}=0.46$ in Table 6.2 .

$$
\begin{gathered}
I_{\text {boot }}(r)=\left(\frac{r}{R}\right)^{1 / 2}\left(\frac{p_{e}(r)}{B_{\Theta}(r)}\right)\left[-4.88 \frac{1}{n(r)} \frac{d n(r)}{d r}-0.27 \frac{1}{T(r)} \frac{d T(r)}{d r}\right] \\
B_{\Theta}(r)=\frac{\mu_{0}}{\wp_{\Theta}} \int_{0}^{r} 2 \pi \kappa r^{\prime} I_{P}\left(r^{\prime}\right) d r^{\prime}
\end{gathered}
$$

In order to compute a total plasma current density profile $I_{P}(r)$, we must know the current drive density profile $I_{C D}(r)$. Due to electron-cyclotron current drive (ECCD) and optical steering, we can assume $I_{C D}(r)$ is concentrated in the region $r<a / 2$ [6] [9]. Given a healthy amount of current diffusion, we can assume $I_{C D}(r)$ is of the form

$$
I_{C D}(r)=C_{1} \operatorname{erfc}\left[C_{2}\left(r-\frac{a}{2}\right)\right]
$$

where $\operatorname{erfc}(x)$ is the complementary error function. $I_{C D}(r)$ is mostly flat for $r<a / 2$ and smoothly drops to zero for $r>a / 2$. $C_{2}$ represents the width of the drop. We choose $C_{2}=$ a/2. $C_{1}$ is a normalization constant that we use to ensure $I_{C D}(r)$ is consistent with $f_{C D}=$ 0.60 in Table 6.2. Figure 6.13 shows $I_{P}(r)$. For purely illustrative purposes, $I_{C D}(r)$ sits on top of $I_{\text {boot }}(r)$.

Now that we know $I_{P}(r)$, we can calculate $q(r)$ directly from Equation 6.6, which is no more than a generalized version of Equation 4.7. Figure 6.14 shows the result. $q(r)$ is a smooth profile with a minimum at approximately $r \approx a / 4$, which is called a "reversed $q$ profile". The $0-\mathrm{D} q^{*}$ value is $q^{*}=q(a)=2.9$, which confirms that our model is satisfactorily self-consistent (see Table 6.2 ). Note that $q_{0}=q(0) \approx 2.2 . q(r)$ is always greater than 2 , ensuring excellent current stability. No sawtooth or surface tearing instabilities will occur [7].

$$
q(r)=\frac{\pi r^{2}\left(1+\kappa^{2}\right)}{R} \frac{B_{\Phi}}{\mu_{0} \int_{0}^{r} 2 \pi \kappa r^{\prime} I_{P}\left(r^{\prime}\right) d r^{\prime}}
$$




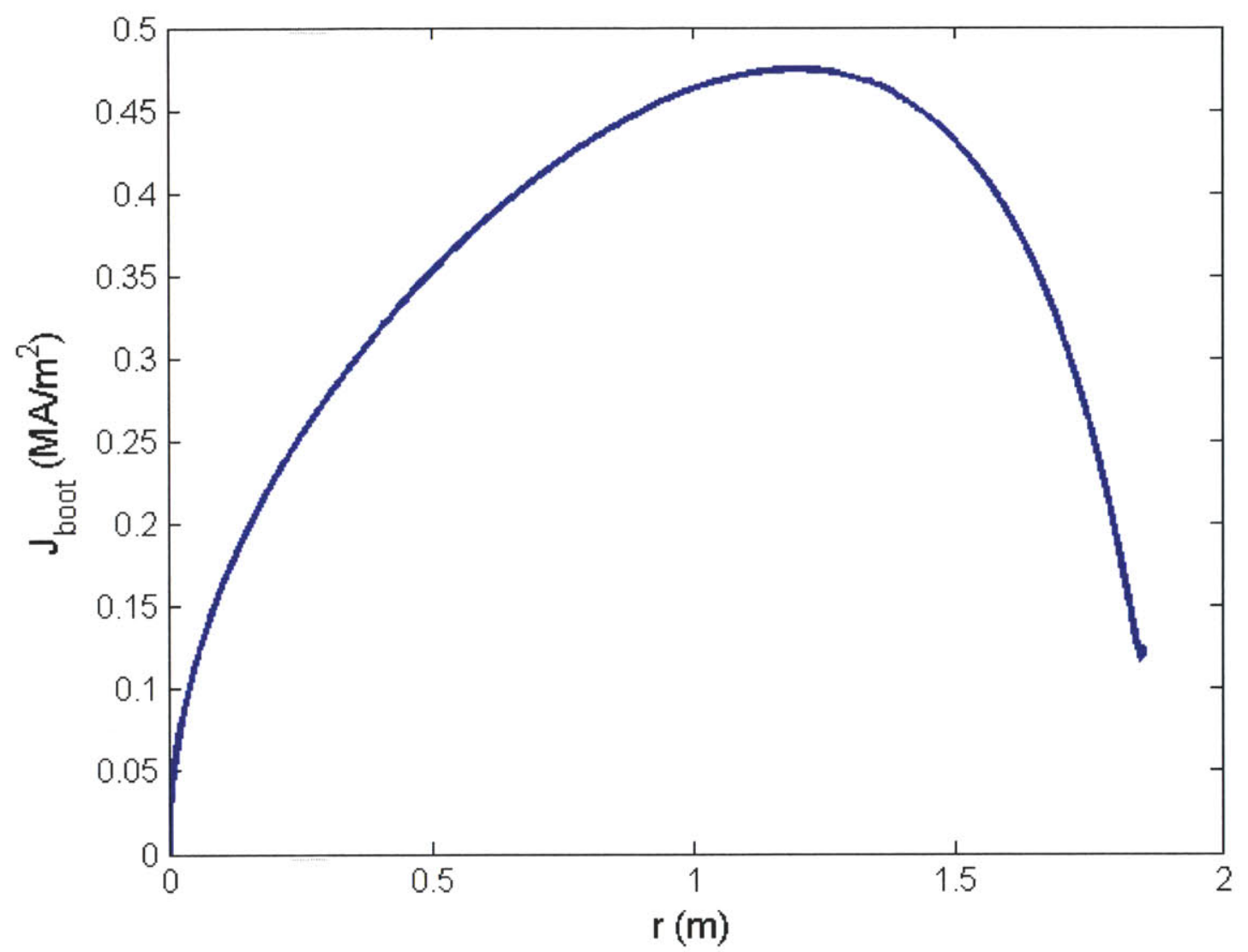

Figure 6.12: Bootstrap current density $I_{\text {boot }}$ as a function of minor radius $r$. The bootstrap current $I_{\text {boot }}$ is a fraction $f_{\text {boot }}=0.46$ of the total plasma current $I_{P}$. It is concentrated near the plasma edge. 


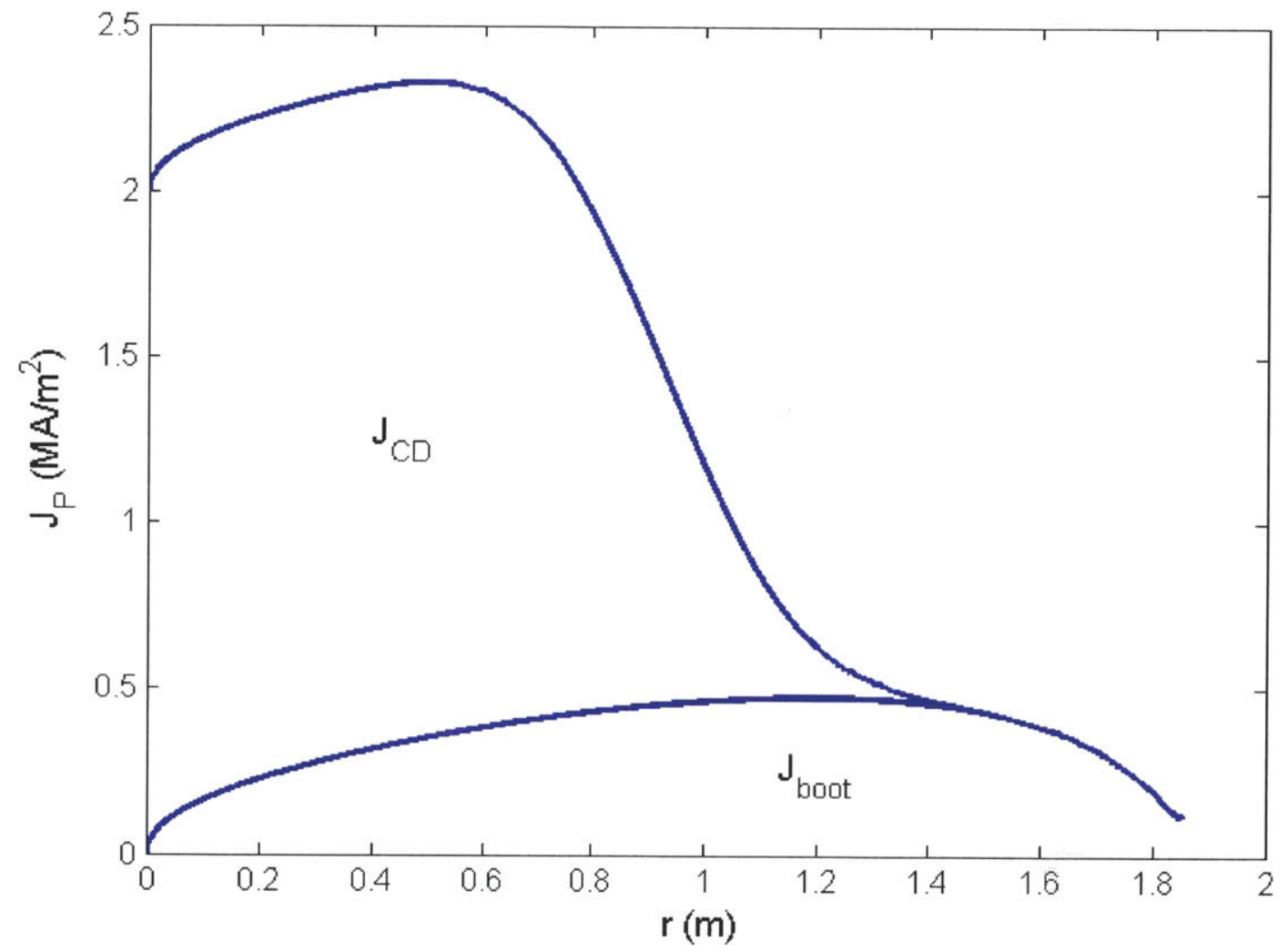

Figure 6.13: The total current density $I_{P}$ (with constituent parts $I_{C D}$ and $I_{\text {boot }}$ ) as a function of minor radius $r$. The bootstrap current density $I_{\text {boot }}$ is as shown in Figure 6.12, and Equation 6.5 is our approximation for $I_{C D}$. Here the topmost curve represents $I_{P}$, and the area between the two curves represents $I_{C D}$. The current drive density $I_{C D}$ is concentrated almost entirely in the plasma interior $r<a / 2$ with a profile of the form in Equation 6.5. This is plausible given electron-cyclotron current drive (ECCD) control. 


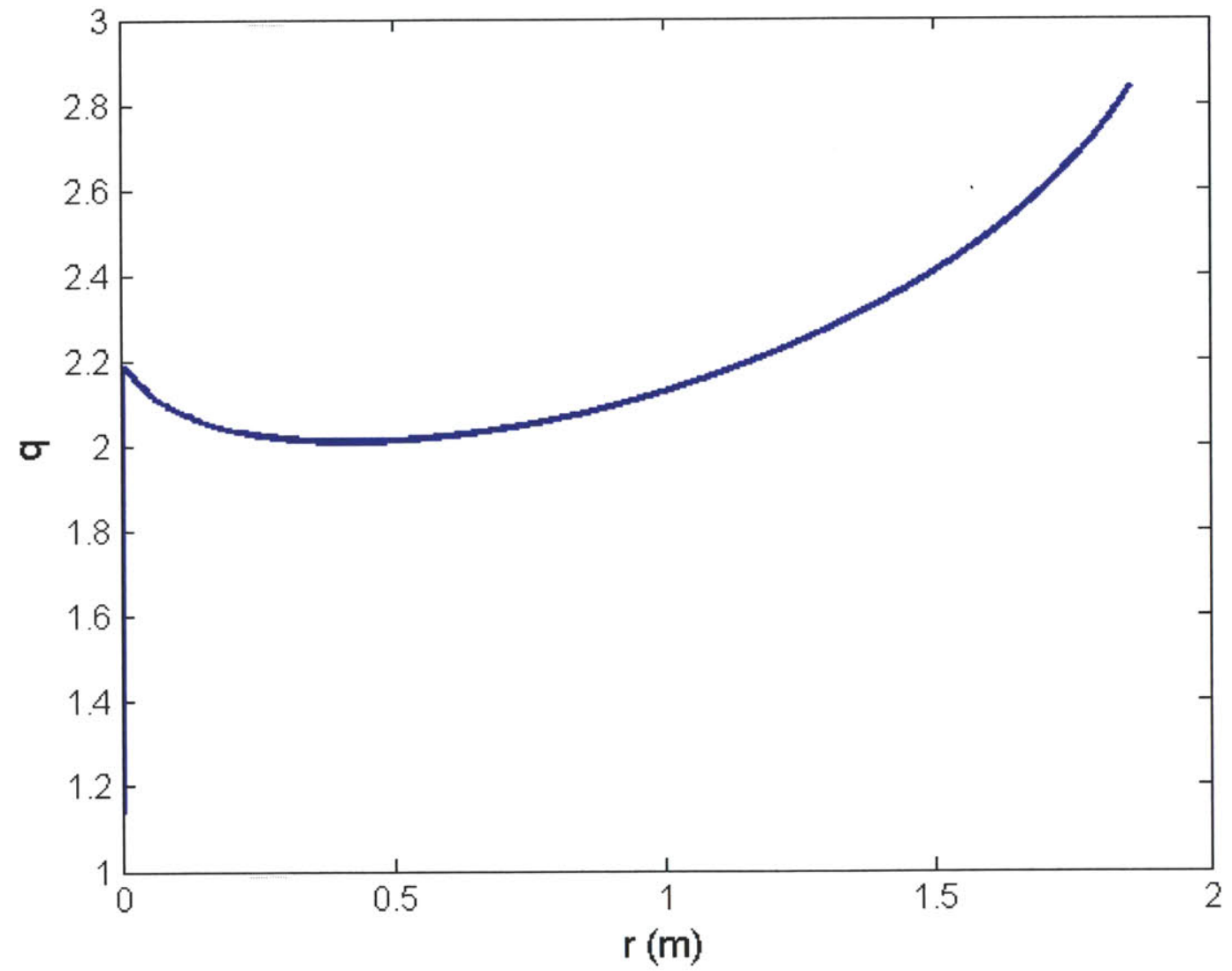

Figure 6.14: The safety factor $q$ profile as a function of minor radius $r$ given the total plasma current profile given in Figure 6.13. This is consistent with the 0 -D safety factor $q *=q(a) \approx 3$. Note that $q$ is always greater than 2 , ensuring a very stable current distribution. 


\subsubsection{Radiative Power}

Since $H=1.48$ is close to the L-H transition at $H=1.5$, it is important to confirm L-mode operation in terms of radiative power. As we described in Section 4.4, the L-H transition occurs when a certain amount of auxiliary power is applied. More specifically, it is the power conducted through the "scrape-off layer" $P_{\text {sol }}$ that causes the L-H transition [7]. The scrape-off layer is a thin zone between the mantle and the blanket where significant radiation occurs. We can express $P_{\text {sol }}$ as

$$
P_{\text {sol }}=\left(P_{\alpha}+P_{\text {aux }}\right)-P_{\text {rad }}=\left(P_{\alpha}+P_{\text {aux }}\right)\left(1-f_{\text {rad }}\right)
$$

where $f_{\text {rad }}$ is the ratio of radiative power $P_{\text {rad }}$ to input power $\left(P_{\alpha}+P_{\text {aux }}\right)$ [12]. For the L-H transition to occur, $P_{\text {sol }}$ must be greater than the $\mathrm{L}-\mathrm{H}$ transition power $P_{L-H}$. As long as $P_{\text {sol }}$ is less than $P_{L-H}$, the reactor is in L-mode. $P_{\text {sol }}$ is defined with the scaling shown in Equation 6.8.

$$
P_{L-H}=\frac{(2.84) C B^{0.82} n^{0.58} R a^{0.81}}{M}
$$

The constant $C$ is 3.5 in this case. $P_{L-H}$ is in MW, $B$ is in T, $n$ is in $10^{20} / \mathrm{m}^{3}, R$ and $a$ are in $\mathrm{m}$, and $M=2.5 \mathrm{amu}$ [4] [7]. This yields $P_{L-H}=355 \mathrm{MW}$. Then, given that $P_{\alpha}+P_{\text {aux }}$ $=1.612 \mathrm{GW}, f_{\text {rad }}$ must exceed 0.78 for the reactor to operate in L-mode. Referring back to Figure $4.3, f_{\mathrm{rad}} \geq 0.78$ is quite reasonable.

Due to the very small $P_{F}$ density (see Figure 6.11) and low temperature (see Figure 6.9) in the mantle, we can assume that nearly all radiative power losses occur in the mantle and therefore do not significantly affect the 0-D $P_{F}$ [4]. The HYPERION report shows this, and the 1-D profiles we show here (see Figures 6.8-11) indicate that the same is likely true for this minimum scale reactor. Thus, our neglect of radiative power losses in the 0-D core model is a reasonable approach. 


\subsubsection{Operating Point Access}

Just as with HYPERION, it is essential to ensure that the operating point is accessible. Figure 6.15 shows the auxiliary power $P_{\text {aux }}$ in the $[n, T]$ plane for the 0 -D operating point specified in Table 6.2. In contrast with HYPERION, there is no $P_{\text {aux }}<0$ zone within the $n$ or $T$ ranges of the operating point. There is still a saddle point, but it lies just off the plot at high $n$. Accessing the operating point is simple. We can increase $n$ and $P_{\text {aux }}$ such that the plasma simply moves directly to the operating point, which is marked with the red circle at $T=12 \mathrm{keV}, n=1.4 \times 10^{20} / \mathrm{m}^{3}$, and $P_{\text {aux }}=260 \mathrm{MW}$.

This operating point is stable for the same reasons as the HYPERION operating point. If the temperature were to decrease, the required auxiliary power would also decrease, causing the plasma to heat up and regain its original temperature. If the temperature were to increase, the required auxiliary power would also increase, causing the plasma to cool down and regain its original temperature. Given that we can directly control density, the operating point is quite secure. 


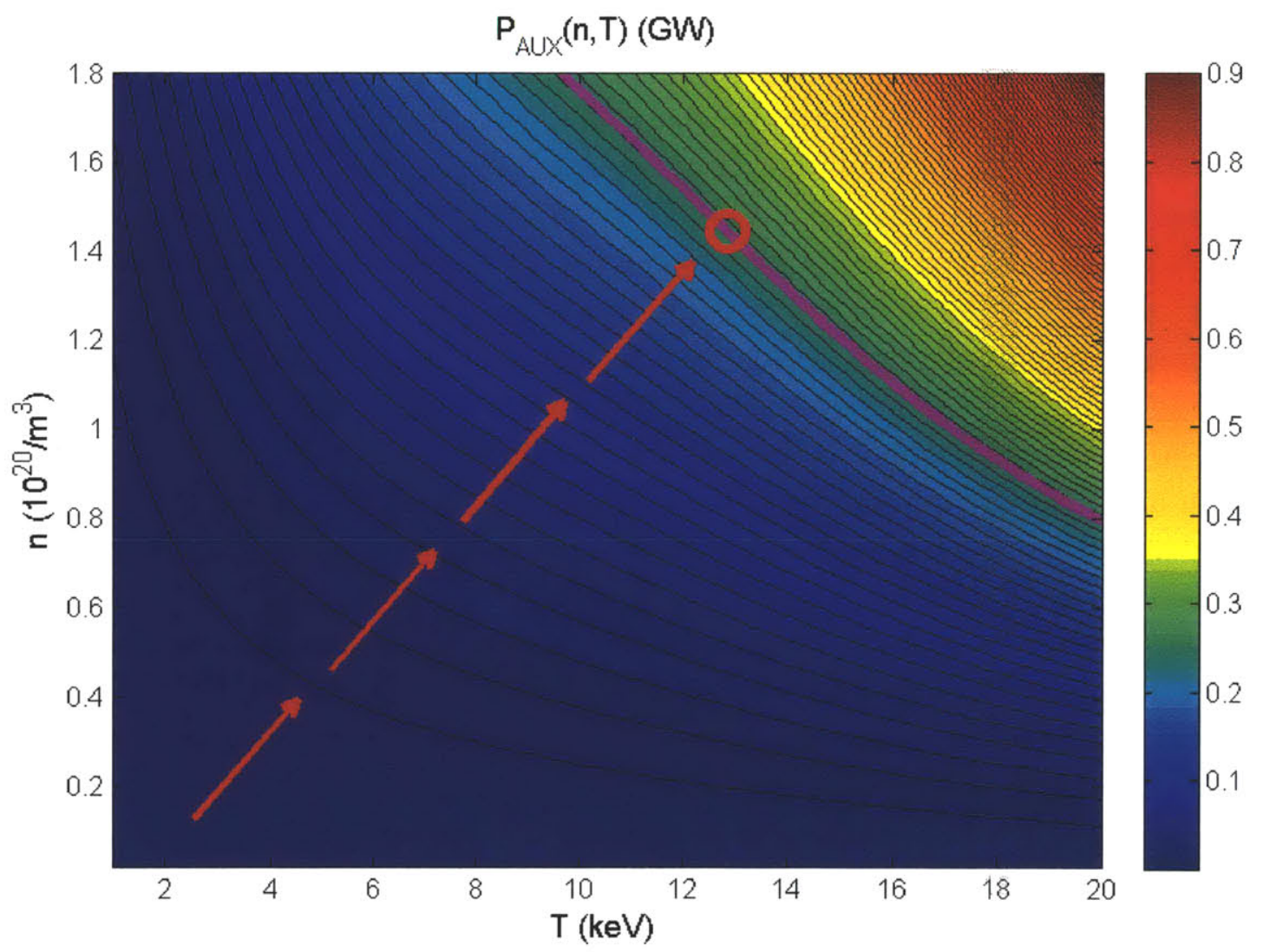

Figure 6.15: Auxiliary power $P_{\text {aux }}$ contours as a function of density $n$ and temperature $T$ for the minimum scale reactor. The operating point $\left(n=1.4 \times 10^{20} \mathrm{~m}^{-3}, T=12.2 \mathrm{keV}, P_{\text {aux }}=260\right.$ MW), is marked with the red circle. The thick, conspicuous purple contour represents when $P_{\text {aux }}$ is consistent with the operating point of $260 \mathrm{MW}$. The red arrows show the ignition path. 


\subsection{Implications}

Our minimum scale operating point has a fusion power of $1.6 \mathrm{GW}$. With a fission blanket multiplication of 7.7 , this yields a total hybrid power of $10.8 \mathrm{GW}$. If we employ a standard Brayton cycle with a thermal efficiency of $41 \%$ (see Section 8.3), then the total electric power would be $4.4 \mathrm{GW}$.

Unfortunately, this is too large for the U.S. electrical grid as it now stands. If the grid is not substantially improved in the future, we could easily employ our model to constrain the total electrical power output to $1 \mathrm{GW}$ and generate a new set of parameters similar to those in Tables 6.1 or 6.2 . The only drawback would be that the minimum scale - and thus the minimum capital cost - would not be substantially less than our results in this thesis. A $1 \mathrm{GW}$ steady-state L-mode tokamak would certainly not be anywhere near as economically viable as a similar $5 \mathrm{GW}$ device, and it might not be economically viable at all. The main conclusion of our 2009 bachelor's thesis was that tokamaks are best suited for steady-state L-mode operation only at large scales with large power outputs [28]. Thus, the grid is a constraining factor, or perhaps even a crippling factor, in sustainable tokamak design.

An alternative option would be to split the power output between commercial electricity generation and hydrogen production. We could send the maximum allowable electric power to the grid and utilize the remainder for hydrogen production (or some other chemical process that happens to be in demand).

However, this thesis is centered around how a fissionable blanket can augment the fusion power and eliminate the high $Q$ requirement such that steady-state L-mode operation becomes feasible at smaller scales. We have now shown that this is true - the blanket allows for steady-state L-mode operation at $R \approx 5.2 \mathrm{~m}$, which is about $5 / 6$ the size of ITER. Our novel coupled fission-fusion analysis demonstrates unequivocally that a fissionable blanket eases the constraints on fusion. 


\section{Burnup and Fuel Cycle}

Now that we have reamed out the initial steady-state condition, we must analyze the evolution of fuel composition - burnup. Although we do not include a quantitative burnup calculation here, we propose a novel subcritical implementation of BGCore as well as a novel technique to sustain a constant hybrid power level throughout burnup. Additionally, we perform some simple fuel cycle analysis to show that our proposed hybrid could breed fissile material or transmute fission product waste much more effectively than a pure fission fast reactor. We also touch on non-proliferation as well as the potential for a thorium fuel cycle.

Since this hybrid will breed large quantities of plutonium (replacing ${ }^{238} \mathrm{U}$ with ${ }^{239} \mathrm{Pu}$ ), the blanket $k_{\text {eff }}$ and power gain will both initially increase. Lawrence Livermore National Laboratory (LLNL) has studied inertial confinement hybrids with depleted uranium blankets (see Section 9.1.3) and concluded that $k_{\text {eff }}$ will rise to a maximum of not more than 0.7 before gradually decreasing indefinitely [31]. Although our device is substantially different, it is not terribly unreasonable to assume that the similarities between natural and depleted uranium will yield the same general trends. We will assume that there is no criticality safety problem and proceed with our discussion.

\subsection{Subcritical Burnup Implementation}

In future work, we plan to implement the burnup package BGCore, recently developed at Ben-Gurion University of the Negev [47]. We prefer BGCore for its accessible MATLAB implementation. It accepts an MCNP input file with several additional burnup parameters. At each time-step, BGCore runs MCNP to obtain the flux distribution and computes the change in fuel composition during the time-step using that flux distribution. Then BGCore runs MCNP using the new fuel composition, and the cycle repeats for as long as the user specifies.

Unfortunately, BGCore, as it currently stands, will not work for subcritical systems. BGCore requires the MCNP kcode command, which iterates a normalized flux distribution through subsequent neutron generations (so that the total number of source neutrons in each generation is constant, as in a critical system). When the $k$ eigenvalue converges after a set number of iterations, the final flux distribution that yields that converged $k$ eigenvalue is deemed the true flux distribution of the system. Essentially, the goal is to determine the fission source distribution that yields a subsequent fission source distribution (in the subsequent generation) identical to itself. Although this scheme works marvelously for critical systems, it is folly for subcritical systems. In a subcritical system, a known (nonfission) neutron source causes everything to happen. Since the source is already known, no iteration is required to determine it. Of course, the kcode command will still function for a subcritical fixed source system with $k<1$, but it will determine the flux distribution 
after many successive neutron generations, where the flux is very small in magnitude. While such a flux distribution might be physically interesting, it is useless for burnup calculations because it comprises only a very small portion of the total flux. In our system, the fusion source neutrons themselves $\left(\approx 3 \mathrm{MW} / \mathrm{m}^{2} \approx 1.3 \times 10^{14} \mathrm{n} / \mathrm{cm}^{2} / \mathrm{s}\right)$ comprise a sizable portion of the total flux. Refer back to Figure 3.13, which shows the relative number of neutrons in each generation. We can reproduce that same data here in a pie chart with Figure 7.1. Over one third of all neutrons in the system at any given time are generation 1 (fusion-born). Any flux distribution produced by a kcode iteration would not include generation $1(35 \%)$ and would probably not even be very accurate for generation 2 (46\%). Without the high-energy fusion-born neutrons, no burnup calculation can be accurate.

Fortunately, BGCore could be applicable to subcritical systems with a few slight modifications. In a critical pure-fission system, both the (fission-born) neutron source and the fuel composition evolve with each time step. In our subcritical system, the (fusion-born) neutron source remains fixed while only the fuel composition evolves. Of course, the flux distribution will evolve, but it will evolve as a consequence of fuel composition only. See Figure 7.2 to compare causation flow charts for critical and subcritical systems. Interestingly, the interrelationships between quantities in subcritical systems are less complex than those in critical systems. The only real barrier to subcritical burnup analysis is that there is little prior work on which to base it - like BGCore, most burnup codes assume criticality. However, the task of applying BGCore to a subcritical system would consist of simply modifying the parameter relationships as in Figure 7.2. The source distribution file must be constant throughout the burnup process, and the flux distribution must be obtained from MCNP without kcode. Circumventing kcode would dramatically reduce the runtime of BGCore.

To our dismay, we must postpone a subcritical implementation of BGCore until future work, as it would take us beyond the timeframe for this study. For now, we can look to other studies, such as Vincent Tang's 2002 thesis, to see other hybrid burnup calculations. Although Tang's design is quite different than ours (in ways we have already discussed), he found that his device could operate for $\approx 30$ years. Since we employ a higher fuel density with natural uranium, it is plausible that our conceptual design could yield similar results. We may perform a follow-up study with our proposed subcritical BGCore implementation.

\subsection{Maintaining Constant Hybrid Power}

One interesting property of this hybrid system is that the fusion power can be varied at will. Operators can adjust the plasma density in the tokamak to alter the $\mathrm{D}$ - $\mathrm{T}$ reaction rate. This fact happens to be extremely favorable for control of the fission component. In a pure fission reactor, various complex mechanisms are implemented to achieve and maintain criticality at a desired power level. These include control rods, chemical shim, fission product poisons, and burnable poisons. Of course, none of these mechanisms is necessary in a subcritical 
system, the advantages of which we have already discussed at length. However, even though criticality $\left(k_{\text {eff }}=1\right)$ is of no concern, we must still consider how to maintain a constant power level during long periods of time in which significant burnup occurs and $k_{\text {eff }}$ does in fact vary.

Referring to Figure 3.13, we will express the ratio of neutrons in generation 2 to generation 1 as $k_{0}$ and the ratio of neutrons in generation $n$ to generation $n-1$ for $n>2$ as $k$. Thus the ratio of neutrons in generation $n$ to generation 1 is $k_{0} k^{n-2}$ for $n>1$. Here $k_{0} \approx 1.3$ and $k \approx 0.3$. The number of fissions per neutron in generation 1 is $k_{0} / \bar{\nu}_{0}$, where $\bar{\nu}_{0}$ is simply $\nu$ averaged over the fissions spurred by generation 1 neutrons. Similarly, the number of fissions spurred by generation $n$ neutrons per generation 1 neutron is $k_{0} k^{n-2} / \bar{\nu}$, where $\bar{\nu}$ is $\nu$ averaged over the fissions spurred by generation $n>1$ neutrons. Here we are assuming that $k$ and $\nu$ have essentially converged and change very little after generation 2 .

With definitions out of the way, we can easily express the fission power multiplication $Q_{\text {fis }}$ and the total hybrid power $P_{\text {hyb }}$ as a sum of the fissions spurred by each generation of neutrons. Here we assume that the energy released in a fission event is always $193.9 \mathrm{MeV}$ and that all fusion neutrons, which constitute $4 / 5$ of the fusion power $P_{\text {fus }}$, are born at 14.1 $\mathrm{MeV}$.

$$
P_{\text {hyb }}=\frac{4}{5} P_{\text {fus }} Q_{\text {fis }}=\frac{4}{5} P_{\text {fus }}\left[\frac{193.9}{14.1}\right]\left[\frac{k_{0}}{\bar{\nu}_{0}}+\frac{k_{0} k}{\bar{\nu}}+\frac{k_{0} k^{2}}{\bar{\nu}}+\frac{k_{0} k^{3}}{\bar{\nu}}+\ldots\right]
$$

We can convert this infinite sum to an algebraic function of $k$.

$$
P_{\text {hyb }}=\frac{4}{5} P_{\text {fus }}\left[\frac{193.9}{14.1}\right] k_{0}\left[\frac{1}{\bar{\nu}_{0}}+\frac{1}{\bar{\nu}}\left(\frac{1}{1-k}-1\right)\right]
$$

Now, as burnup occurs in the fission blanket, $k$ and $\bar{\nu}$ will evolve in time. This will cause $P_{\text {hyb }}$ to evolve in time, which is not preferable.

$$
P_{\mathrm{hyb}}(t)=\frac{4}{5} P_{\text {fus }}\left[\frac{193.9}{14.1}\right] k_{0}(t)\left[\frac{1}{\bar{\nu}_{0}(t)}+\frac{1}{\bar{\nu}(t)}\left(\frac{1}{1-k(t)}-1\right)\right]
$$

However, since we have control over $P_{\text {fus }}$, we can stipulate that $P_{\text {fus }}$ evolve in time as $P_{\text {fus }}(t)$ to compensate for burnup such that $P_{\text {hyb }}$ remains constant. It is simple to see how this evolution would work:

$$
P_{\text {fus }}(t)=P_{\text {fus }}(0)\left[\frac{k_{0}(0)}{k_{0}(t)}\right]\left[\frac{\frac{1}{\bar{\nu}_{0}(0)}+\frac{1}{\bar{\nu}(0)}\left(\frac{1}{1-k(0)}-1\right)}{\frac{1}{\bar{\nu}_{0}(t)}+\frac{1}{\bar{\nu}(t)}\left(\frac{1}{1-k(t)}-1\right)}\right]
$$

So while this hybrid configuration obviates criticality, it also greatly simplifies power stability. There is no need for specialized control mechanisms, as we can manipulate the fission power indirectly through the fusion power. We recognize that this function would be extremely 
difficult to know beforehand, but burnup occurs gradually enough that $P_{\text {fus }}(t)$ could be adjusted in real-time.

Of course, it is important to note that $k(t)$ in Equation 7.4 is not the same $k(t)$ in the case of constant $P_{\text {fus }}$. Although we can keep the total blanket power $P_{\text {hyb }}$ constant throughout burnup by varying $P_{\text {fus }}(t)$, we cannot keep the spatial and energetic distribution of the flux constant as the fuel composition evolves. Let us suppose that $k(t)$ initially increases. We lower $P_{\text {fus }}(t)$ so that $P_{\text {hyb }}$ remains constant. Now the rate of increase of $k(t)$ slows, because the flux has decreased. However, if $k(t)$ initially decreases, raising $P_{\text {fus }}(t)$ will accelerate the decrease of $k(t)$. Thus, we can say with confidence that this technique moderates $k(t)$ increases and exacerbates $k(t)$ decreases.

Since this hybrid will breed large quantities of plutonium, $k(t)$ will initially increase as a function of time. $k_{0}(t)$ will also increase, although not as substantially, as ${ }^{238} \mathrm{U}$ and ${ }^{239} \mathrm{Pu}$ have similar fission cross-section magnitudes at $14 \mathrm{MeV}$ (within a factor of 2). Inertial confinement hybrid studies by Lawrence Livermore National Laboratory (LLNL) have shown that $k_{\text {eff }}$ will rise to a maximum of not more than 0.7 before gradually decreasing indefinitely [31]. Thus, there would be ample opportunity to apply this method to both $k(t)$ increases and decreases.

Finally, we should clarify a potential ambiguity. In the previous subsection and in Figure 7.2 , we defined the fusion neutron source as a fixed source. When we consider varying $P_{\text {fus }}(t)$, the fusion source is still "fixed" in the sense that it is independent of all other fission properties - Figure 7.2 is still valid. However, it is obviously not "fixed" in the sense of being constant. A more precise way to characterize $P_{\text {fus }}(t)$ would be "variable but independent". This is one of many potentially-confusing subtleties in fission-fusion hybrid dynamics. We will call this kind of source a variable fixed source, a fitting oxymoron.

We could test this variable fixed source idea with our proposed subcritical implementation of BGCore. Instead of using the same fixed source for each iteration $i$, BGCore could modify the source $S_{i}$ based on the initial system power $P_{0}$ and the system power for the prior generation $P_{i-1}$.

$$
S_{i}=S_{0} \frac{P_{0}}{P_{i-1}}
$$

Our convention here is that the source $S_{i}$ generates the power $P_{i}$ in the $i$ th iteration. Here we must stipulate that $S_{1}=S_{0}$ to initiate the recursion. 


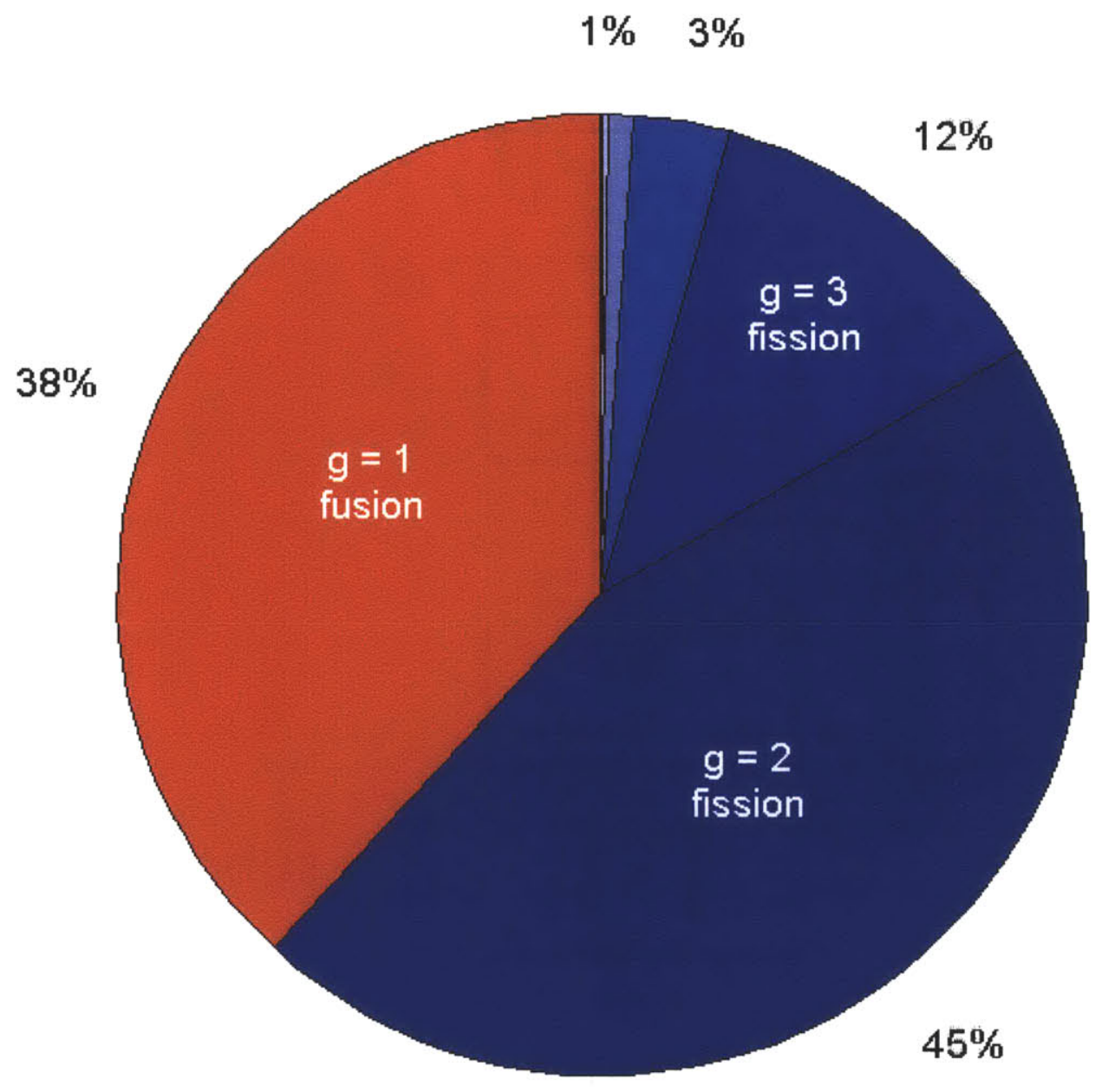

Figure 7.1: Fractional share of neutrons from each generation in the entire hybrid system. The initial fusion-born neutrons comprise $35 \%$ of all neutrons, while the first generation of fission-born neutrons comprise $46 \%$. Beginning with generation 3, each successive generation contains only $k \approx 0.3$ as many neutrons as the previous generation. 


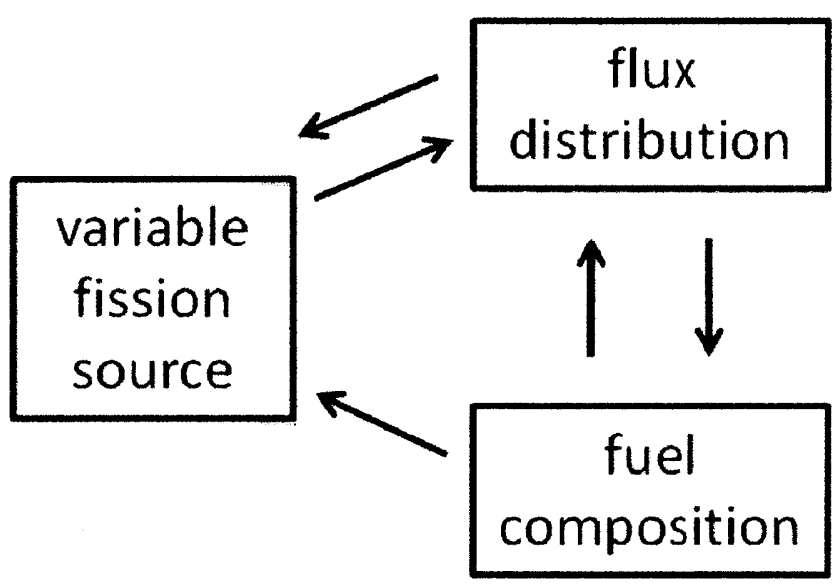

\section{critical}

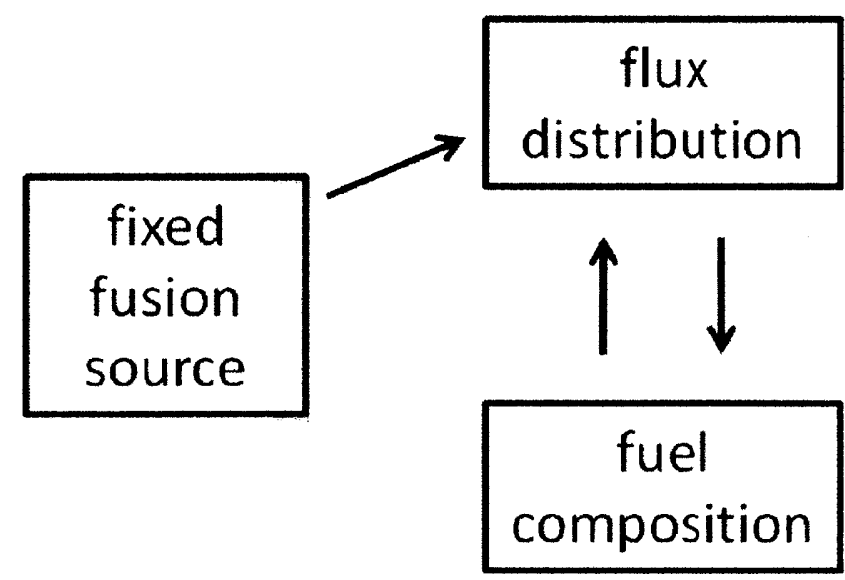

\section{subcritical}

Figure 7.2: Causation relationships between basic core quantities in critical and subcritical systems. In a critical system, the fission-born source depends on both fuel composition and the flux distribution generated by previous sources. In a subcritical system, the non-fission source is independent of all other quantities. In the hybrid context, this shows that while fusion drives fission, fission has no effect on fusion. 


\subsection{Breeding}

This natural uranium fission-fusion hybrid will breed copious fissile material. Figure 7.3 shows the portion of the ${ }^{238} \mathrm{U}$ transmutation-decay chain that is of interest to us. Any uranium-fueled reactor will breed both ${ }^{239} \mathrm{Pu}$ and ${ }^{237} \mathrm{~Np}$ from the ${ }^{238} \mathrm{U}(\mathrm{n}, \gamma)$ and ${ }^{238} \mathrm{U}(\mathrm{n}, 2 \mathrm{n})$ reactions, respectively. It will also breed many other fissile nuclides (such as ${ }^{241} \mathrm{Pu}$ and ${ }^{241} \mathrm{Am}$ ), although those will have orders of magnitude smaller concentrations due to the additional $(\mathrm{n}, \gamma)$ reactions required.

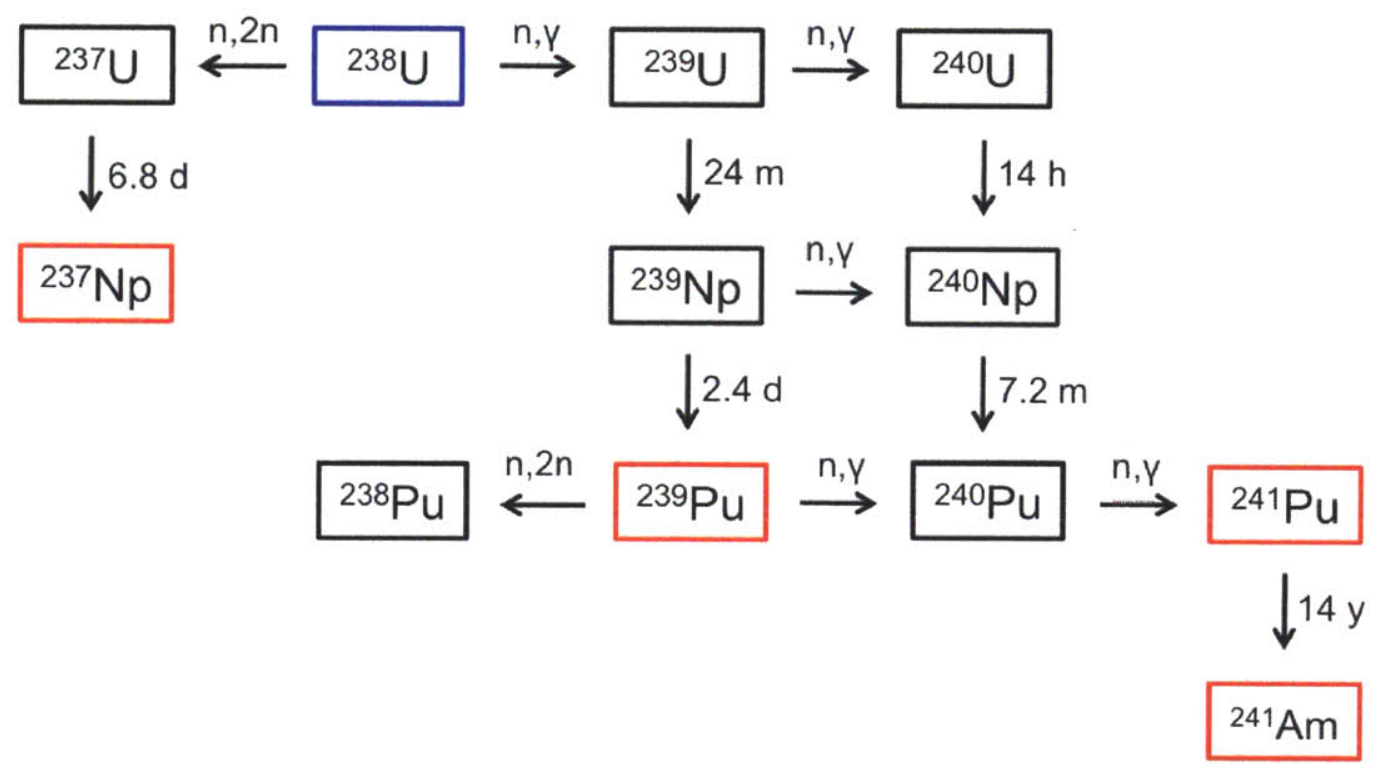

Figure 7.3: The ${ }^{238} \mathrm{U}$ transmutation-decay chain with relatively stable fissile isotopes in red. Here we show only the dominant interaction between each isotope.

When most people talk about breeding and conversion ratios in the uranium cycle, they primarily mean converting ${ }^{238} \mathrm{U}$ into ${ }^{239} \mathrm{Pu}$ and other plutonium isotopes through the ${ }^{238} \mathrm{U}(\mathrm{n}, \gamma)$ reaction. We can define a conversion ratio $C$ for each fissile isotope produced in the reactor as the ratio of the production rate of that isotope to the removal rate of the original fissile isotope(s) present in the fuel. In the case of converting ${ }^{238} \mathrm{U}$ into ${ }^{239} \mathrm{Pu}, C$ is roughly proportional to the ratio of the ${ }^{238} \mathrm{U}$ neutron one-group capture cross-section $\sigma_{c}$ to the ${ }^{235} \mathrm{U}$ one-group absorption cross-section $\sigma_{a}$. Here the absorption cross-section $\sigma_{a}$ includes fission, capture, and all other reactions that absorb a neutron except inelastic scattering. Thus, the absorption cross-section is equivalent to the total removal cross-section. We define $\epsilon$ as enrichment by atomic fraction.

$$
C \approx \frac{\Sigma_{c}^{\mathrm{U} 238}}{\sum_{a}^{\mathrm{U} 235}}=\left(\frac{1-\epsilon}{\epsilon}\right) \frac{\sigma_{c}^{\mathrm{U} 238}}{\sigma_{a}^{\mathrm{U} 235}}
$$


For illustrative purposes, it is convenient to define an energy-dependent conversion ratio $C(E)$, which is $C$ evaluated with cross-sections at one specific energy instead of with onegroup cross-sections averaged over a spectrum. $C(E)$ would be the conversion ratio for a hypothetical reactor in which all the neutrons are monoenergetic with energy E. Although not particularly physical, $C(E)$ provides an instructive spectrum-independent overview of how the conversion ratio changes with energy.

$$
C(E) \approx\left(\frac{1-\epsilon}{\epsilon}\right) \frac{\sigma_{c}^{\mathrm{U} 238}(E)}{\sigma_{a}^{\mathrm{U} 235}(E)}
$$

Although these approximations for $C$ are simple and useful, the conversion ratio is actually a time-dependent quantity that evolves as burnup proceeds. We can calculate $C$ precisely as a function of time by numerically integrating transmutation-decay differential equations - one for each nuclide. We set these up in a standard form with Figure 7.3 as a guide. The change in the concentration $N^{i}$ of a given nuclide $i$ is the difference between the one-group cumulative loss $N^{i} \sigma_{a}^{i} \phi$ and the sum of all one-group gains $N^{j} \sigma^{j \rightarrow i} \phi$ from other nuclides of index $j$. Of course, nuclide $i$ can decay at the rate $\lambda^{i} N^{i}$ and be produced from another nuclide $j$ at the rate $\lambda^{j \rightarrow i} N^{j}$. The one-group cross-sections are joyfully simple to compute in MATLAB with unionized cross-sections and flux spectra, which we will explain near the end of this subsection.

$$
\frac{d N^{i}}{d t}=-N^{i}\left[\lambda^{i}+\int_{0}^{\infty} \sigma_{a}^{i}(E) \phi(E) d E\right]+\sum_{j \neq i} N^{j}\left[\lambda^{j \rightarrow i}+\int_{0}^{\infty} \sigma^{j \rightarrow i}(E) \phi(E) d E\right]
$$

We can numerically integrate these differential equations for the system shown in Figure 7.3 to obtain the instantaneous time-dependent ${ }^{239} \mathrm{Pu}$ conversion ratio $C(t)$.

$$
C(t)=\frac{d N^{\mathrm{Pu} 239} / d t}{-d N^{\mathrm{U} 235} / d t}
$$

We can also set up a very similar set of differential equations to determine the timedependent monoenergetic nuclide concentrations $N_{m}$ at energy $E_{m}$.

$$
\frac{d N_{m}^{i}}{d t}=-N_{m}^{i}\left[\lambda^{i}+\sigma_{a}^{i}\left(E_{m}\right) \phi\left(E_{m}\right)\right]+\sum_{j \leq i} N_{m}^{j}\left[\lambda^{j \rightarrow i}+\sigma^{j \rightarrow i}\left(E_{m}\right) \phi\left(E_{m}\right)\right]
$$

Then the time-dependent monoenergetic conversion ratio $C\left(E_{m}, t\right)$ is

$$
C\left(E_{m}, t\right)=\frac{d N_{m}^{\mathrm{Pu} 239} / d t}{-d N_{m}^{\mathrm{U} 235} / d t}
$$


Figure 7.4 shows $C\left(E_{m}, t\right)$ during the first 23 days of operation for $E_{m}=0.1 \mathrm{MeV}$ and 1.0 $\mathrm{MeV}$. Here we arbitrary assume an enrichment of $5 \%$. Obviously, $C\left(E_{m}, 0\right)=0$, because the finite half-lives of ${ }^{239} \mathrm{U}$ and ${ }^{239} \mathrm{~Np}$ prevent immediate production of ${ }^{239} \mathrm{Pu}$. However, after a time much longer than the longest half-life has elapsed, $C\left(E_{m}, t\right)$ approaches an equilibrium value very close to Equation 7.7. As burnup proceeds over much longer time scales, this equilibrium will evolve. However, we will henceforth refer to this initial equilibrium value as the "monoenergetic conversion ratio".

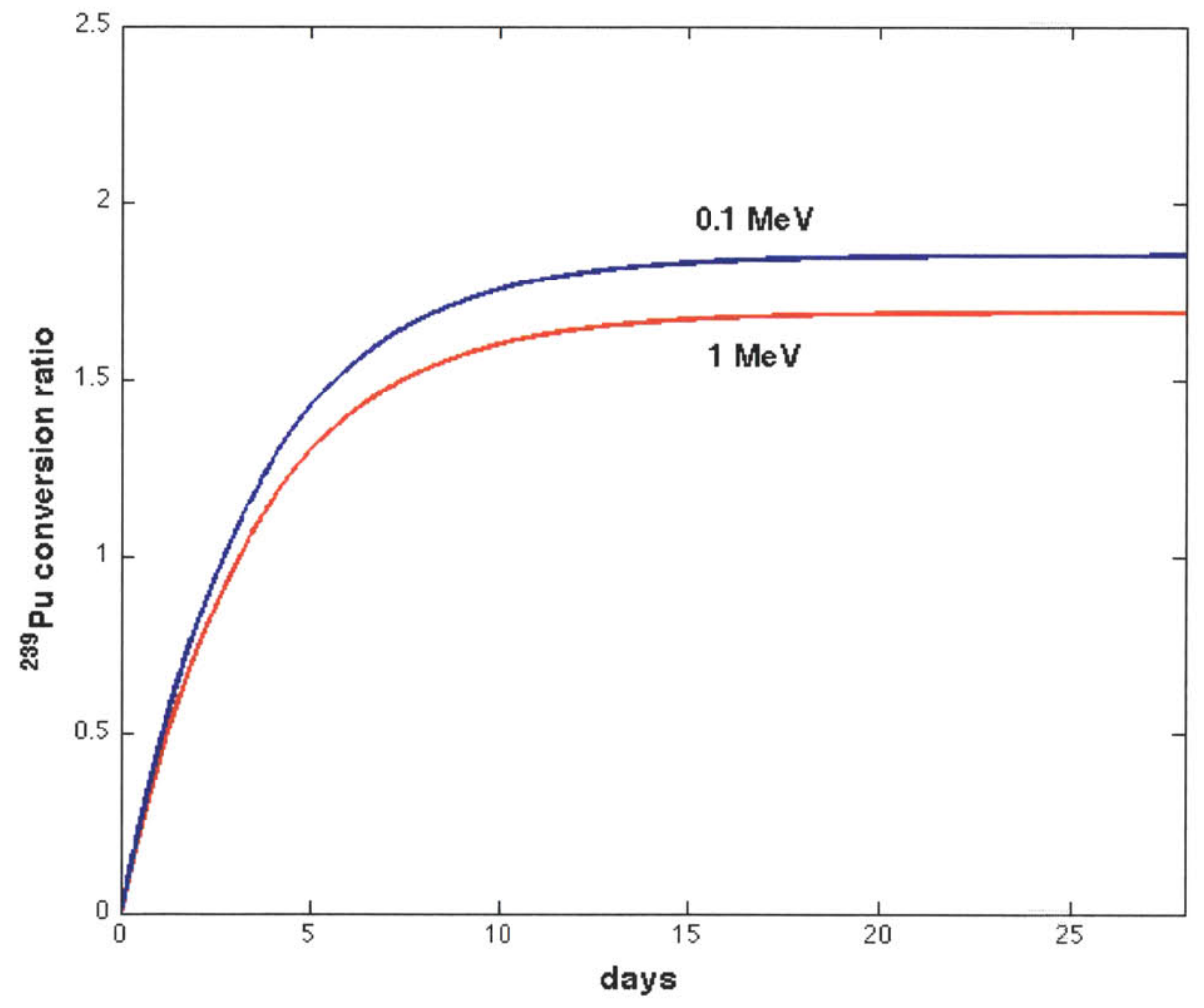

Figure 7.4: Monoenergetic ${ }^{239} \mathrm{Pu}$ conversion ratio as a function of time for $5 \%$ uranium enrichment. Here we show $1 \mathrm{MeV}$ and $100 \mathrm{keV}$. The conversion ratios asymptotically approach equilibrium after time becomes much longer than the longest half-life in the decay chain $\left(2.4\right.$ days for $\left.{ }^{239} \mathrm{~Np}\right)$.

Figure 7.5 shows the monoenergetic conversation ratio as a function of energy for $5 \%$ enrichment. This can be somewhat closely approximated by Equation 7.7, although the transmutation-decay chains contain some additional losses. Figure 7.7 encapsulates why fast reactors can breed more fissile material than they consume much more readily than thermal reactors. The monoenergetic conversation ratio $C(E)$ exceeds 1.0 in the range $0.01<E<1.0 \mathrm{MeV}$, which is approximately the range in which a typical fast reactor 
spectrum resides. Thermal reactors have a fast peak in the $C(E)>1$ region and a thermal peak in the $C(E) \approx 0.1$ region, and the spectrum-averaged $C$ tends to be greater than 0.5 but less than 1.0 .

Beyond $1 \mathrm{MeV}, C(E)$ drops by two orders of magnitude. Consequently, the $14 \mathrm{MeV}$ neutron source in our fission-fusion hybrid will breed only trace quantities of ${ }^{239} \mathrm{Pu}$. However, the portion of our hybrid spectrum below the ${ }^{238} \mathrm{U}$ fissionable threshold is nearly congruent with that of a typical fast reactor spectrum and will breed ample ${ }^{239} \mathrm{Pu}$. Furthermore, the low abundance of ${ }^{235} \mathrm{U}$ in natural uranium (0.7\%) will mean that the conversion ratio for our hybrid will be approximately 7.5 times higher than that shown in Figure 7.5. So although the $14 \mathrm{MeV}$ neutron source does not contribute directly to conversion, it contributes indirectly a great deal by obviating criticality such that the initial concentration of fissile material can be very low.

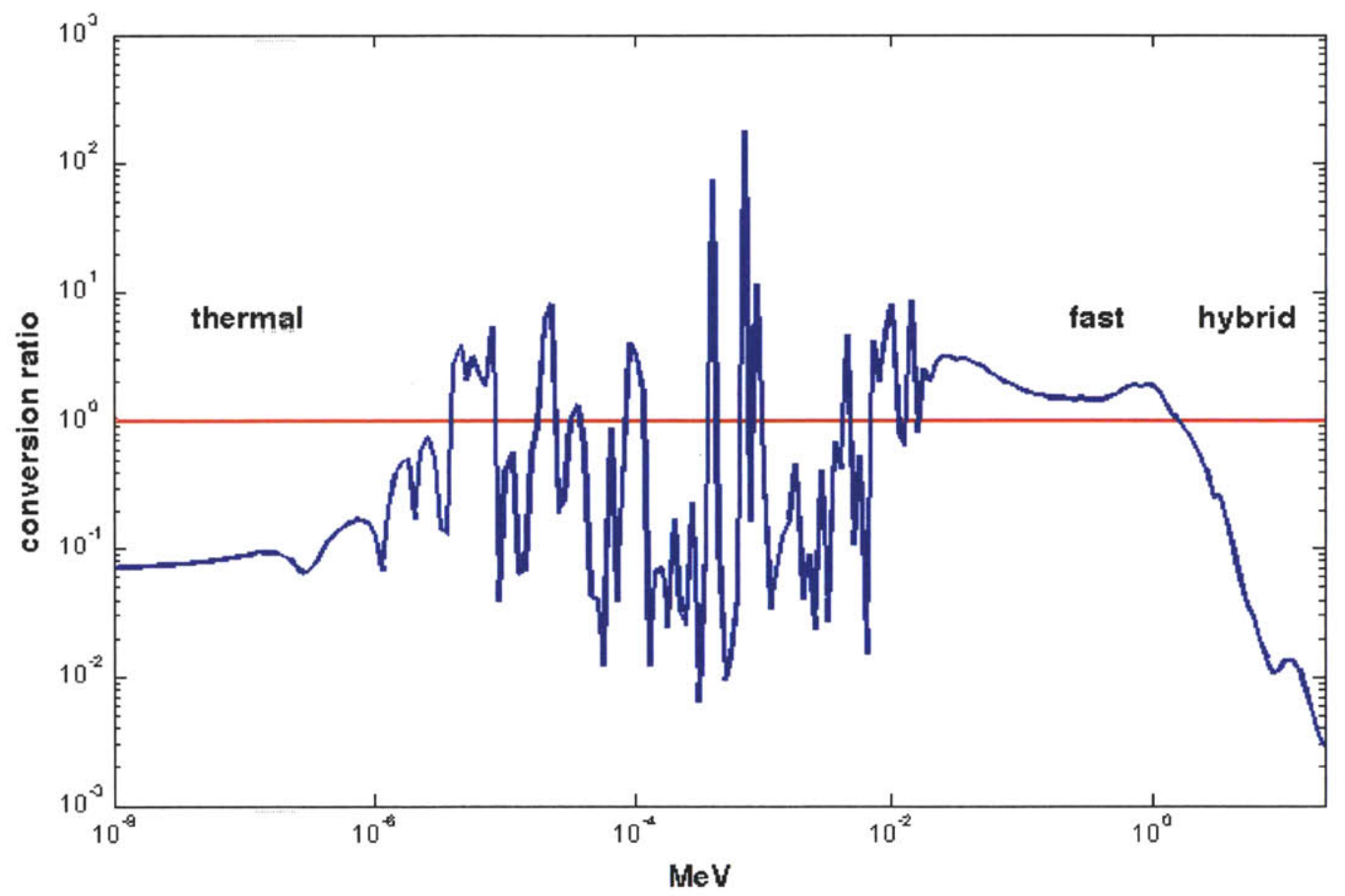

Figure 7.5: Monoenergetic ${ }^{239} \mathrm{Pu}$ conversion ratio as a function of energy at $5 \%$ enrichment. The red line denotes $C=1$. This illustrates why fast reactors can breed ${ }^{239} \mathrm{Pu}$ while thermal reactors cannot. Although conversion ratio is very low at $14 \mathrm{MeV}$, our fission-fusion hybrid will still breed considerable ${ }^{239} \mathrm{Pu}$ due to its large flux magnitude in the typical fast reactor range.

Figure 7.6 shows the monoenergetic conversion ratio in the fast energy range for three fissile isotopes: ${ }^{239} \mathrm{Pu},{ }^{237} \mathrm{~Np}$, and ${ }^{233} \mathrm{U} .{ }^{237} \mathrm{~Np}$ is bred primarily from the ${ }^{238} \mathrm{U}(\mathrm{n}, 2 \mathrm{n})$ reaction 
(see Figure 7.3), but it is also produced in much smaller amounts from successive (n, $\gamma$ ) on ${ }^{235} \mathrm{U}$ and the $\alpha$-decay of ${ }^{241} \mathrm{Am} .{ }^{233} \mathrm{U}$ is bred from the ${ }^{235} \mathrm{U}(\mathrm{n}, 3 \mathrm{n})$ reaction. Here the "enrichment" is $0.7 \%$, consistent with our natural uranium hybrid. As we saw in Figure 7.5, the ${ }^{239} \mathrm{Pu}$ conversion ratio is high below a few $\mathrm{MeV}$.

We are especially interested in ${ }^{237} \mathrm{~Np}$, as it is both fissile and a highly mobile waste product. In 1992, the U.S. government declassified the fact that ${ }^{237} \mathrm{~Np}$ can be used make nuclear weapons [34]. In 2002, researchers at Los Alamos National Laboratory performed a criticality experiment with a ${ }^{237} \mathrm{~Np}$ sphere to show that its critical mass is approximately $60 \mathrm{~kg}$, less than $20 \%$ larger than that of ${ }^{235} \mathrm{U}$ [35]. The $k_{\infty}$ value of pure ${ }^{237} \mathrm{~Np}$ metal is approximately 1.7 compared to 2.28 for ${ }^{235} \mathrm{U}[36]$. That ${ }^{237} \mathrm{~Np}$ is moderately fissile and a proliferation concern is somewhat surprising, as it is an exception to the general rule that fissile nuclides obey $2 Z-N=43 \pm 2$. Proliferation concerns aside, ${ }^{237} \mathrm{~Np}$ is also known as the most mobile of the actinide waste products, and so it is highly undesirable [37].

While pure fission reactors (thermal or fast) breed relatively little ${ }^{237} \mathrm{~Np}$ (because the $(\mathrm{n}, 2 \mathrm{n})$ threshold is nearly $7 \mathrm{MeV})$, the fusion-born neutrons in our hybrid will breed ${ }^{237} \mathrm{~Np}$ at an expeditious rate. As shown in Figure 7.6 , the monoenergetic ${ }^{237} \mathrm{~Np}$ conversion ratio at $14 \mathrm{MeV}$ exceeds 100. The fusion-born neutrons will also breed a non-negligible amount of ${ }^{233} \mathrm{U}$, something that usually only shows up in the thorium cycle. Although the ${ }^{233} \mathrm{U}$ conversion ratio never exceeds 0.2 , it is roughly equal to the ${ }^{239} \mathrm{Pu}$ conversion ratio at 14 $\mathrm{MeV}$.

Now that we have spent some time with monoenergetic conversion ratios to gain insight, let us turn to spectrum-averaged conversion ratios. We calculate these with a system of differential equations with one-group cross-sections as shown in Equations 7.8 and 7.9. For simplicity, we will assume a constant flux shape throughout the fuel, and we will use the hybrid spectrum from Figure 3.16. For perspective, we will also calculate conversion ratios for typical thermal and fast pure fission spectra. We will borrow a thermal spectrum from the MIT Reactor, and we will obtain an approximate fast spectrum from a simple MCNP input file with $\mathrm{UO}_{2}$ fuel rods surrounded by sodium coolant (see Appendix I.4). We plot these three spectra in Figure 7.7, where each has been normalized to the same total magnitude of 1.0. The flux magnitude is not especially important, as the equilibrium conversion ratio (refer back to Figure 7.4) depends only on the flux shape. The flux magnitude will determine how quickly the conversion ratio approaches its equilibrium value, but it will not affect that equilibrium value.

Table 7.1 shows conversion ratios for ${ }^{239} \mathrm{Pu},{ }^{237} \mathrm{~Np}$, and ${ }^{233} \mathrm{U}$ in the thermal, fast, and hybrid flux spectra. As expected, the fast spectrum achieves a ${ }^{239} \mathrm{Pu}$ conversion ratio of greater than 1.0 while the thermal spectrum does not. The hybrid spectrum achieves a prodigious ${ }^{239} \mathrm{Pu}$ conversion ratio of 22 , because it is not critical and contains very little ${ }^{235} \mathrm{U}$. As for ${ }^{237} \mathrm{~Np}$, the thermal and fast spectra produce very little. However, the hybrid 


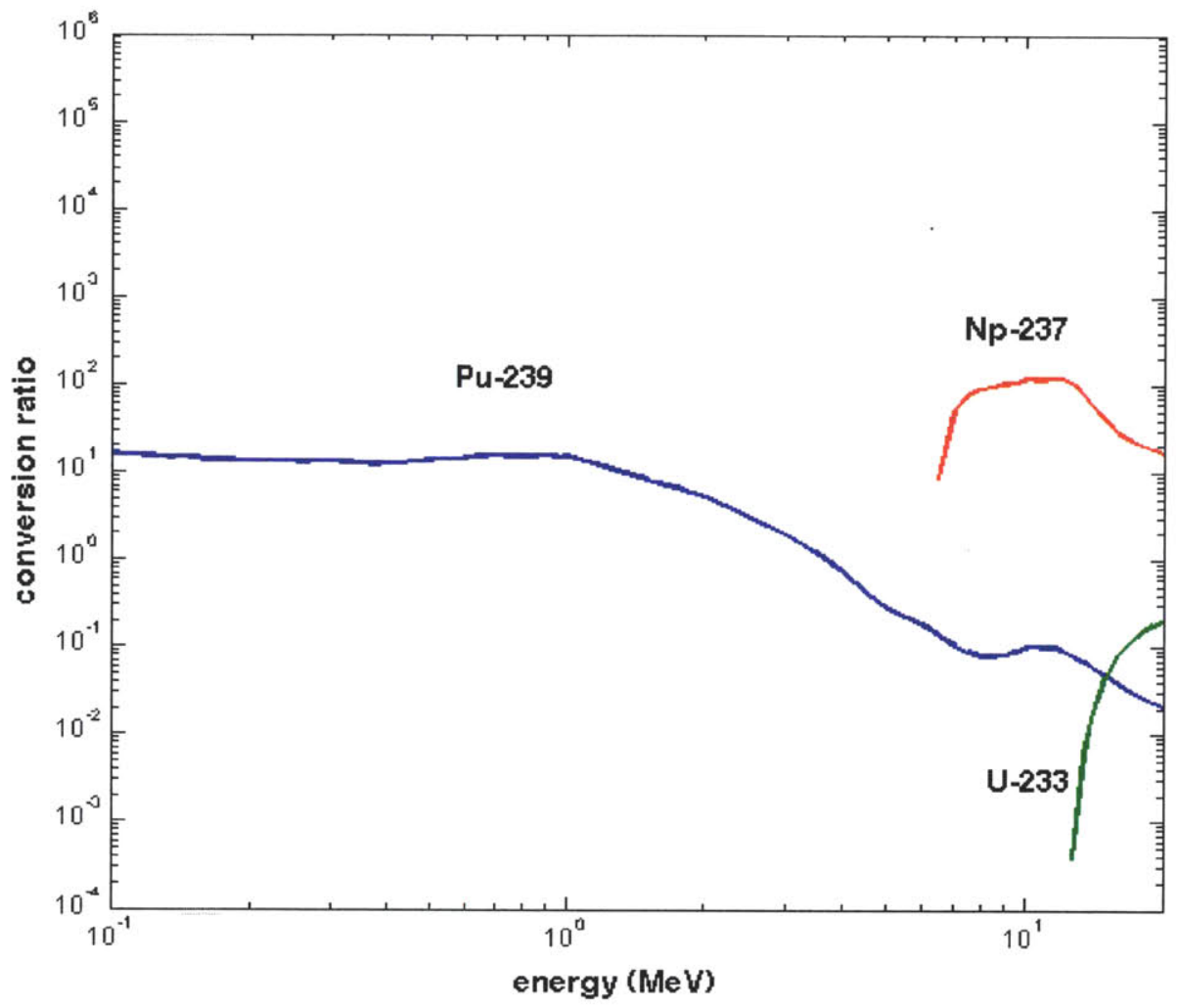

Figure 7.6: Monoenergetic conversion ratios for ${ }^{239} \mathrm{Pu},{ }^{237} \mathrm{~Np}$, and ${ }^{233} \mathrm{U}$. This is for natural uranium fuel with $0.7 \%{ }^{235} \mathrm{U}$. At $14 \mathrm{MeV}$, just as much ${ }^{233} \mathrm{U}$ as ${ }^{239} \mathrm{Pu}$ is produced per ${ }^{235} \mathrm{U}$ fissioned.

spectrum actually achieves a ${ }^{237} \mathrm{~Np}$ conversion ratio of greater than 1.0 ! Our fission-fusion hybrid "converts" ${ }^{235} \mathrm{U}$ into ${ }^{237} \mathrm{~Np}$ just as well as a typical fast reactor converts ${ }^{235} \mathrm{U}$ into ${ }^{239} \mathrm{Pu}$. None of the spectra produce a substantial amount of ${ }^{233} \mathrm{U}$, although the hybrid does produce about 1000 times as much as a fast reactor.

The consequence of this is that our fission-fusion hybrid is a prolific breeder. Depending on the cycle length, this hybrid could breed enormous quantities of ${ }^{239} \mathrm{Pu}$ for use in pure fission reactors. The ${ }^{237} \mathrm{~Np}$ it produces could also be used as fuel in theory, although it has not yet been proven to be a viable fuel. Fast reactors have been touted as sufficient to breed fissile fuel for future generations of nuclear power, but our hybrid reactor is clearly superior in this respect.

We should pause here to elucidate exactly how we perform these breeding calculations in MATLAB. We construct a unionized set of all cross-sections on a 248-group energy grid of constant lethargy width corresponding to our Monte Carlo flux, which is tallied per unit lethargy. Although this unionized set does not fully delineate the resonance region and 


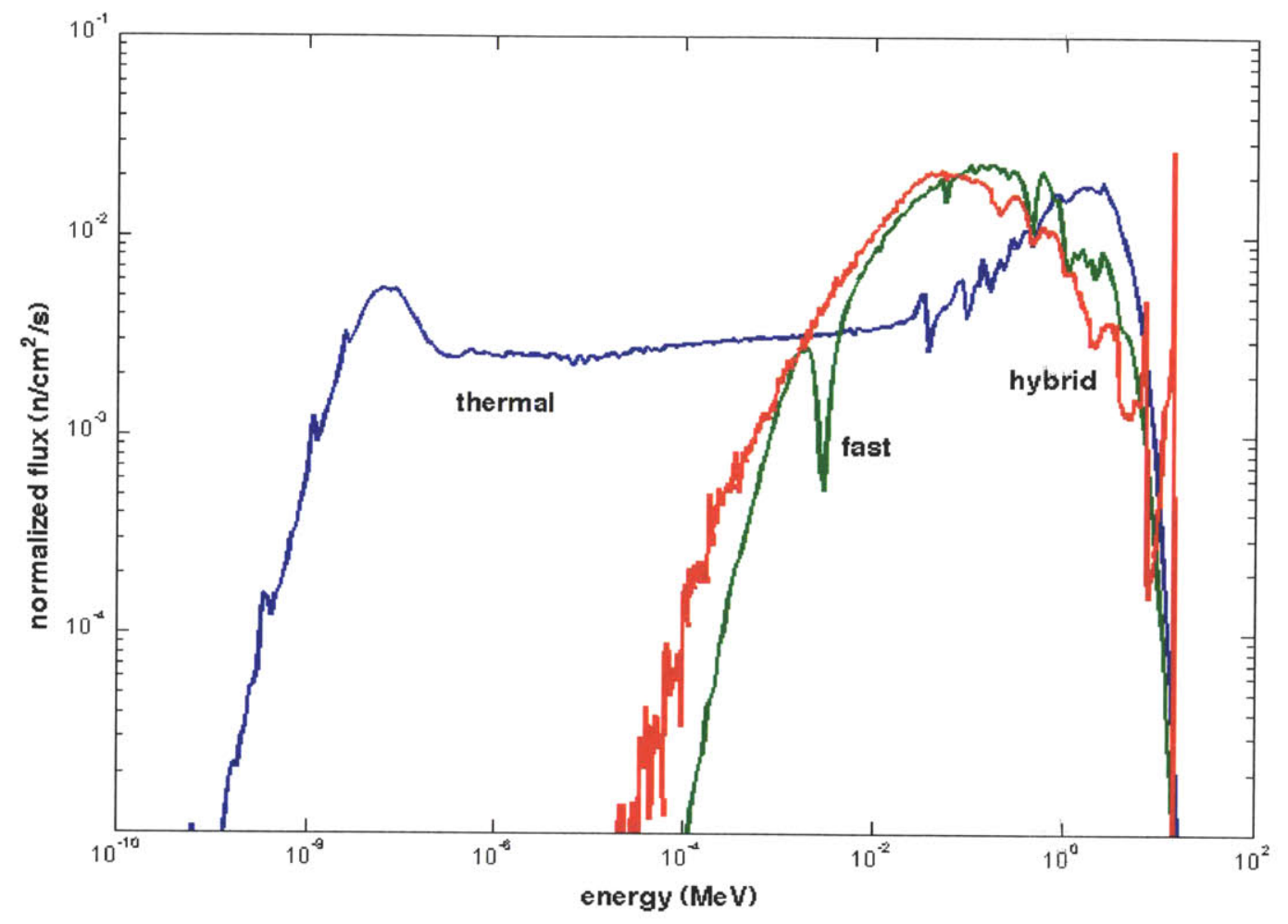

Figure 7.7: Our fission-fusion hybrid spectrum overlaid on typical spectra for fast and thermal reactors. All three spectra are normalized so that they have the same magnitude.

would not suffice for a full-scale design, it is adequate for this rudimentary calculation. The conversion ratios we cite for thermal and fast spectra in Table 7.1 are consistent with widely-known values. Once we have constructed this unionized set, we can simply sum the element-wise multiplication of the flux and each cross-section to obtain a value proportional to each reaction rate. For example, we can approximate the equilibrium conversion ratios for the three isotopes in Table 7.1 as

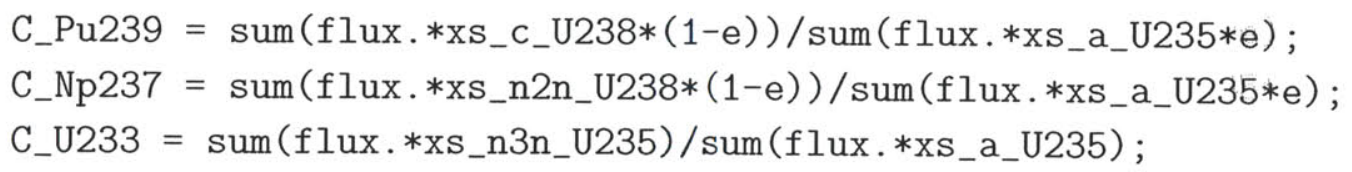

Here e is the enrichment by atomic fraction. This is a very convenient and efficient method for computing conversion ratios from unionized cross-section data when the flux is computed per unit lethargy with constant lethargy group width. The monoenergetic conversion ratios are similar except that we multiply scalars instead of summing element-wise array products. 
Table 7.1: Fissile Nuclide Conversion Ratios

$\begin{array}{lccc} & \text { thermal } & \text { fast } & \text { hybrid } \\ { }^{239} \mathrm{Pu} & 0.67 & 1.3 & 22 \\ { }^{237} \mathrm{~Np} & 0.0028 & 0.011 & 1.3 \\ { }^{233} \mathrm{U} & 5.9 \times 10^{-8} & 7.7 \times 10^{-7} & 7.6 \times 10^{-4}\end{array}$

For the numerical integration necessary to obtain the time-dependent conversion ratios in Figure 7.4, see Appendix H.

We should qualify this analysis further by stating that conversion ratio might not be the most suitable metric for evaluating the breeding capability of subcritical systems. Just as neglecting criticality obviates a host of fuel cycle constraints, it might also render certain fuel cycle metrics obsolete. Although conversion ratio still tracks the total fissile inventory, perhaps a different quantity, such as fissile nuclei bred per fusion event, might be more useful for comparing various subcritical systems. For example, using depleted uranium instead of natural uranium in our subcritical system would increase the conversion ratio by a factor of 2 to 4 , even though there would be little difference in reactor performance or the amount of plutonium produced. However, the change in plutonium nuclei bred per fusion event would be commensurately small. Future work on subcritical breeding should develop new metrics for (1) comparison between critical and subcritical systems and (2) comparison between different subcritical systems. 


\subsection{Viability of a Thorium Fuel Cycle}

Natural thorium is $100 \%{ }^{232} \mathrm{Th}$, which is fissionable but not fissile. However, the ${ }^{232} \mathrm{Th}(\mathrm{n}, \gamma)$ reaction breeds ${ }^{233} \mathrm{U}$, which is highly fissile. A thorium-fueled reactor would require an added initial concentration of fissile material such as ${ }^{239} \mathrm{Pu}$ to jump-start the fission chain reaction and achieve criticality long enough for ${ }^{233} \mathrm{U}$ to pick up the torch. Proponents of the thorium fuel cycle tout its dearth of actinides, lack of enrichment requirements, and greater natural abundance.

Thorium fuel in a fission-fusion hybrid is an intriguing idea. The fusion neutron source would obviate the need for initial fissile material - the fuel could be nothing but natural thorium. Hans Bethe mentioned this idea in 1979 [39], and it has been revisited in recent years at the MIT Plasma Science and Fusion Center [38] as well as at the University of Illinois [40].

Since fresh thorium fuel for our hybrid would contain no fissile material, we must employ the "breeding ratio" rather than the conversion ratio to evaluate its effectiveness. The breeding ratio is defined as the ratio of the production rate of fissile nuclides to the total rate of fission. For the thorium cycle, it is approximately

$$
B^{\mathrm{Th}} \approx \frac{\sigma_{c}^{\mathrm{Th} 232}}{\sigma_{f}^{\mathrm{Th} 232}}
$$

For the uranium cycle with atomic fraction enrichment $\epsilon$, it is

$$
B^{\mathrm{U}} \approx \frac{(1-\epsilon) \sigma_{c}^{\mathrm{U} 238}}{\epsilon \sigma_{f}^{\mathrm{U} 235}+(1-\epsilon) \sigma_{f}^{\mathrm{U} 238}}
$$

We can compare breeding ratios for each cycle using the same three spectra (thermal, fast, and hybrid) as in our uranium cycle conversion ratio analysis (see Figure 7.7). Table 7.2 shows breeding ratios for ${ }^{239} \mathrm{Pu}$ and ${ }^{237} \mathrm{~Np}$ in the uranium cycle and for ${ }^{233} \mathrm{U}$ and ${ }^{231} \mathrm{~Pa}$ in the thorium cycle. The breeding ratios for ${ }^{237} \mathrm{~Np}$ and ${ }^{231} \mathrm{~Pa}$ are defined in the same way as in Equations 7.12 and 7.13 except with $(n, 2 n)$ replacing $(n, \gamma) .{ }^{231} \mathrm{~Pa}$ is a peculiar isotope. Just barely non-fissile with $k_{\infty}=0.95$, it can still achieve criticality with a reflector or with an added moderator [36]. We have computed its breeding ratio here only because it is the thorium-cycle analog of uranium-cycle ${ }^{237} \mathrm{~Np}$.

Table 7.2 shows that in the uranium cycle, the hybrid spectrum breeds more of each fissile material than the fast or thermal spectra. However, in the thorium cycle, the hybrid spectrum breeds more ${ }^{231} \mathrm{~Pa}$ but significantly less ${ }^{233} \mathrm{U}$, which is the whole point of the thorium cycle.

Figure 7.8 shows the ${ }^{232} \mathrm{Th}(\mathrm{n}, \gamma)$ and ${ }^{238} \mathrm{U}(\mathrm{n}, \gamma)$ cross-sections as functions of energy. These are roughly equivalent to effective ${ }^{239} \mathrm{Pu}$ and ${ }^{233} \mathrm{U}$ "breeding cross-sections" in the uranium 
and thorium cycles. The thorium cycle breeds substantially more ${ }^{233} \mathrm{U}$ at thermal energies than at fast energies.

The main conclusion here is that our fast-spectrum fission-fusion hybrid is not optimally suited for the thorium cycle. The fixed neutron source is definitely highly favorable to the thorium cycle, but the hybrid spectrum should be thermalized in order to maximize ${ }^{233} \mathrm{U}$ production. Unlike our design, a fission-fusion hybrid thorium burner should have a moderator. We will now close this thorium discussion and press onward.

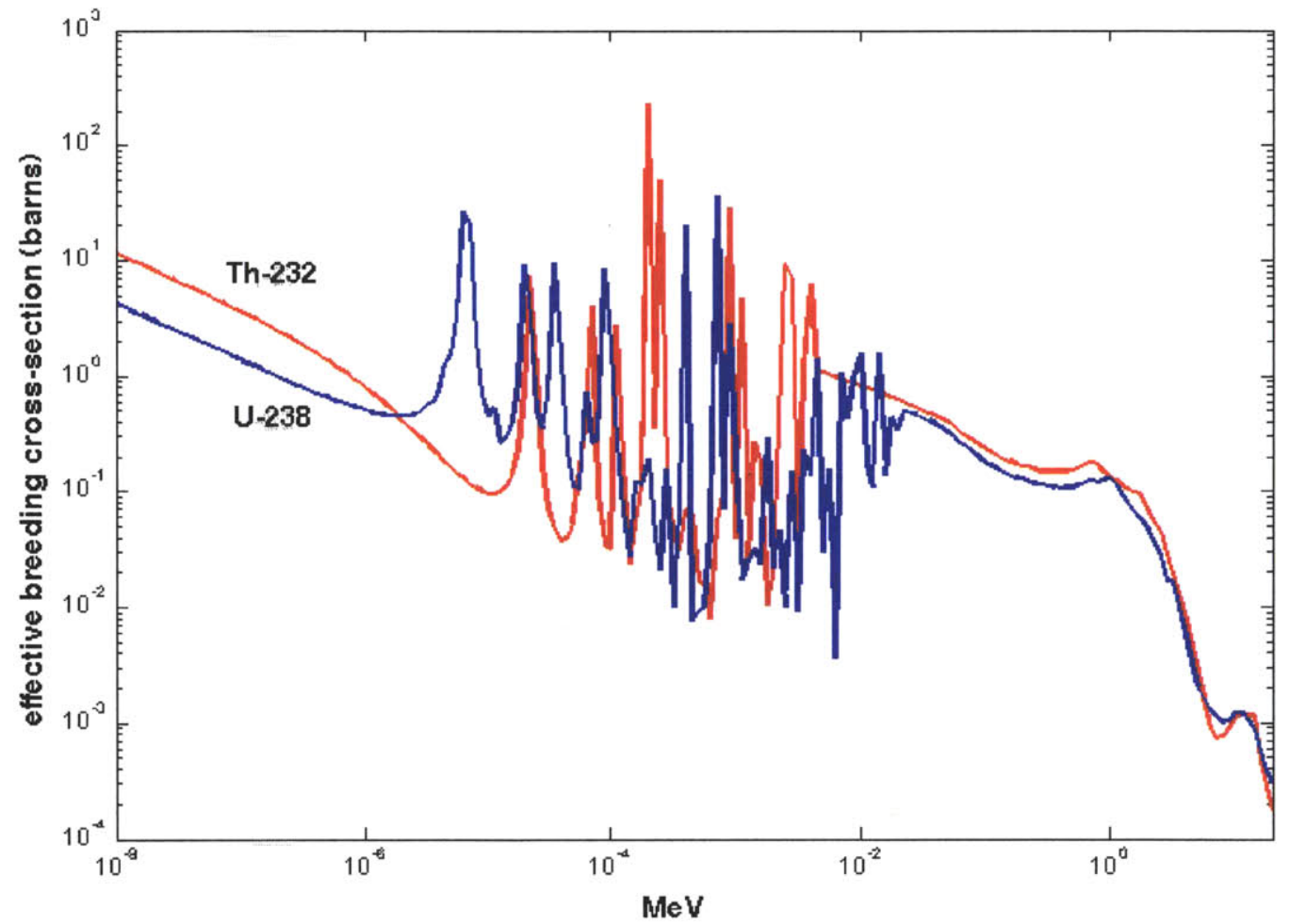

Figure 7.8: Effective ${ }^{239} \mathrm{Pu}$ and ${ }^{233} \mathrm{U}$ breeding cross-sections for uranium and thorium fuel, respectively. These are roughly equal to the ${ }^{238} \mathrm{U}(\mathrm{n}, \gamma)$ and ${ }^{232} \mathrm{Th}(\mathrm{n}, \gamma)$ cross-sections. 
Table 7.2: Comparison of Thorium and Uranium Breeding Ratios

$\begin{array}{cccc} & \text { thermal } & \text { fast } & \text { hybrid } \\ \text { thorium }\left({ }^{233} \mathrm{U}\right) & 36 & 32 & 19 \\ \text { thorium }\left({ }^{231} \mathrm{~Pa}\right) & 0.26 & 0.23 & 1.4 \\ \text { uranium }\left({ }^{239} \mathrm{Pu}\right) & 0.67 & 1.2 & 3.5 \\ \text { uranium }\left({ }^{237} \mathrm{~Np}\right) & 0.0028 & 0.0099 & 0.21\end{array}$




\subsection{Non-Proliferation}

Non-proliferation is an intricate subject, bringing together aspects of fuel cycle analysis, physical security of facilities, and political science, and even game theory. For the purposes of this work, we will only discuss some rudimentary fuel cycle aspects of the subject.

As we have already discussed at length, our fission-fusion hybrid is subcritical and thus requires no enrichment. Although pure fission reactors require levels of enrichment much lower than those required to construct weapons, it is the same enrichment process. The existence of this process (and people who have knowledge of it) is itself a proliferation risk, one that we willingly take on because it is the only way to achieve criticality in pure fission reactors (with the notable exception of the CANada Deuterium Uranium (CANDU) reactor, which requires deuterium enrichment).

One could imagine a world in which a few large fission-fusion hybrids use natural uranium to breed plutonium, which in turn fuels a fleet of fast and thermal fission reactors. This hypothetical world would require no enrichment facilities whatsoever, although it would introduce a different proliferation risk in terms of producing large quantities of plutonium and neptunium.

In thermal reactors, ${ }^{239} \mathrm{Pu}$ is the main proliferation risk due to its relatively high concentration and status as a highly fissile nuclide. However, plutonium in spent fuel always contains certain fractions of ${ }^{238} \mathrm{Pu}$ and ${ }^{240} \mathrm{Pu} .{ }^{238} \mathrm{Pu}$ undergoes $\alpha$-decay, which generates hazardous heat within the material. Much worse, ${ }^{240} \mathrm{Pu}$ undergoes spontaneous fission, which could pre-detonate or fizzle a weapon. Thus, weapons-grade plutonium must have sufficiently low concentrations of ${ }^{238} \mathrm{Pu}$ and ${ }^{240} \mathrm{Pu}$ [41].

As a crude measure of relative plutonium isotopic ratios, Table 7.3 shows one-group ${ }^{239} \mathrm{Pu}(\mathrm{n}, \gamma){ }^{240} \mathrm{Pu}$ and ${ }^{239} \mathrm{Pu}(\mathrm{n}, 2 \mathrm{n}){ }^{238} \mathrm{Pu}$ cross-sections for the same thermal, fast, and hybrid spectra utilized in the preceding sections. Clearly, thermal reactors produce far more ${ }^{240} \mathrm{Pu}$ per ${ }^{239} \mathrm{Pu}$ atom than fast reactors or fission-fusion hybrids, although they produce relatively little ${ }^{238} \mathrm{Pu}$. However, comparing fast reactors to hybrids shows that hybrids produce marginally more ${ }^{240} \mathrm{Pu}$ and substantially more ${ }^{238} \mathrm{Pu}$. Even though hybrids seem to best fast reactors, the very large ${ }^{239} \mathrm{Pu}(\mathrm{n}, \gamma)$ cross-section of thermal reactors seems to outweigh the advantage hybrids have with respect to ${ }^{239} \mathrm{Pu}(\mathrm{n}, 2 \mathrm{n})$. Of course, thermal reactors have ${ }^{239} \mathrm{Pu}$ breeding and conversion ratios of less than 1.0, and so they are not even breeders in the first place.

However, there is one additional source of ${ }^{238} \mathrm{Pu}$ we have neglected until now: ${ }^{237} \mathrm{~Np}(\mathrm{n}, \gamma)$. When ${ }^{237} \mathrm{~Np}$ captures a neutron, ${ }^{238} \mathrm{~Np}$ decays into ${ }^{238} \mathrm{Pu}$. As hybrids contain large portions of ${ }^{237} \mathrm{~Np}$, this will substantially add to the content of ${ }^{238} \mathrm{Pu}$ in plutonium. We would need to perform a detailed burnup analysis to determine the evolution of ${ }^{238} \mathrm{Pu}$ content.

${ }^{237} \mathrm{~Np}$ itself is also concerning. Although it can be chemically separated as a nearly pure nuclide (with only trace amounts of other neptunium isotopes), it does not match the 
proliferation potential of ${ }^{239} \mathrm{Pu}$, which is much more fissile (higher $k_{\infty}$ and lower critical mass). A 2005 Institute for Science and International Security (ISIS) report stated that "the proliferation risk currently posed by neptunium and americium remains relatively small" $[42]$.

The most salient point in this non-proliferation analysis should be that these fission-fusion hybrids will breed plutonium that is plausibly more proliferation-resistant than that bred by fast reactors. This fact coupled with a lack of enrichment requirements actually makes hybrids seem fairly attractive in the context of non-proliferation. However, more rigorous burnup and transmutation analysis would be necessary to confirm this.

Table 7.3: ${ }^{239} \mathrm{Pu}$ One-Group Cross-Sections (barns)

$\begin{array}{cccc} & \text { thermal } & \text { fast } & \text { hybrid } \\ \left.{ }^{239} \mathrm{Pu}(\mathrm{n}, \gamma)\right)^{240} \mathrm{Pu} & 56 & 0.44 & 0.49 \\ { }^{239} \mathrm{Pu}(\mathrm{n}, 2 \mathrm{n})^{238} \mathrm{Pu} & 0.0014 & 5.1 \times 10^{-4} & 0.0049\end{array}$




\subsection{Waste}

So far, we have discussed breeding and proliferation with respect to the actinides. Although not a huge proliferation risk, ${ }^{237} \mathrm{~Np}$ is perhaps the most problematic actinide present in waste. Although our fission-fusion hybrid produces far more ${ }^{237} \mathrm{~Np}$ than any pure fission reactor, it will also fission ${ }^{237} \mathrm{~Np}$ far more effectively than any pure fission reactor. Although moderately fissile, ${ }^{237} \mathrm{~Np}$ has a larger fission cross-section at $14 \mathrm{MeV}$ than it does at thermal energies. The hybrid will also fission (non-fissile) fissionable nuclides before they can produce fissile nuclides through transmutation. Fissioning unwanted actinides is usually preferable to transmuting them into other (potentially also unwanted) actinides. Various studies have attempted to quantify this, and proponents of inertial confinement hybrids with natural or depleted uranium claim that it is possible to burn $99 \%$ of all actinides [30]. This claim rests on the viability of a decades-long cycle time, which we will discuss in the following section. For the purposes of fair comparison between neutron spectra, we are currently discussing burnup and transmutation on time scales concordant with current light water reactor cycle times.

Beyond the actinides, our fission-fusion hybrid will also transmute problematic fission product waste. In order to quantify this, we will invent a new dimensionless parameter called the "fission product factor". We can evaluate this parameter for each individual fission product, and it is the ratio of production through fission to removal through neutron absorption. Here we define "neuron absorption" as $(\mathrm{n}, \gamma),(\mathrm{n}, 2 \mathrm{n}),(\mathrm{n}, \alpha)$, or any other neutron reaction that transmutes the nuclide. Of course, sometimes one fission product will transmute into another, but we are usually only interested in the long-lived and medium-lived hazardous fission products, which are all separated by at least two units of mass number $A$. If we assume that the uranium concentration $N_{\mathrm{U}}$ is relatively constant and that the initial fission product concentration $N_{\mathrm{FP}}$ is zero, this "fission product factor" is actually equal to the short-term equilibrium ratio $N_{\mathrm{FP}} / N_{\mathrm{U}}$.

$$
\frac{N_{\mathrm{FP}}}{N_{\mathrm{U}}}=\frac{\text { FP production rate per } \mathrm{U} \text { atom }}{\text { FP removal rate per FP atom }}
$$

If we know the fission product yield $Y_{\mathrm{FP}}$, then we can express $N_{\mathrm{FP}} / N_{\mathrm{U}}$ in terms of crosssections, the flux, and the atomic fraction enrichment.

$$
\frac{N_{\mathrm{FP}}}{N_{\mathrm{U}}}=\frac{Y_{\mathrm{FP}} \int_{0}^{\infty}\left[\epsilon \sigma_{f}^{\mathrm{U} 235}(E)+(1-\epsilon) \sigma_{f}^{\mathrm{U} 238}(E)\right] \phi(E) d E}{\int_{0}^{\infty} \sigma_{a}^{\mathrm{FP}}(E) \phi(E) d E}
$$

This "fission product factor" is a simple, reasonable way to quantify how well a reactor transmutes hazardous fission products. Lower values of $N_{\mathrm{FP}} / N_{\mathrm{U}}$ mean that fission products will generally have lower concentrations. Table 7.4 compares $N_{\mathrm{FP}} / N_{\mathrm{U}}$ for nine hazardous long-lived or medium-lived fission products with our usual three spectra. With the exception 
of ${ }^{99} \mathrm{Tc}$, every isotope exhibits its lowest $N_{\mathrm{FP}} / N_{\mathrm{U}}$ value in the hybrid spectrum. In some cases, the difference exceeds two orders of magnitude. Thus, it is reasonable to conclude that our fission-fusion hybrid will be highly effective at transmuting the most harmful fission product waste. We should note that this advantage is due almost entirely to $(n, 2 n)$ reactions on the fission products. These results are consistent with other recent studies [45].

This is crucial, because less long-lived fission product waste requires less storage space in geologic waste repositories. Yucca Mountain is a perpetual political issue, and it is one of the main reasons why scientists are taking a second look at hybrids. If Yucca Mountain and other geologic repositories are forever stymied, reducing the amount of waste will become exponentially more important.

Table 7.4: Fission Product Equilibrium Concentrations

$\begin{array}{lcccc}Y(\%) & & \text { thermal } N_{\mathrm{FP}} / N_{\mathrm{U}} & \text { fast } N_{\mathrm{FP}} / N_{\mathrm{U}} & \text { hybrid } N_{\mathrm{FP}} / N_{\mathrm{U}} \\ 6.911 & { }^{135} \mathrm{Cs} & 0.13 & 0.089 & 0.027 \\ 6.337 & { }^{137} \mathrm{Cs} & 4.1 & 0.88 & 0.11 \\ 6.139 & { }^{99} \mathrm{Tc} & 0.0078 & 0.026 & 0.0093 \\ 5.458 & { }^{93} \mathrm{Zr} & 0.17 & 0.095 & 0.028 \\ 4.505 & { }^{90} \mathrm{Sr} & 14 & 0.93 & 0.11 \\ 1.250 & { }^{107} \mathrm{Pd} & 0.0068 & 0.0032 & 0.0011 \\ 0.841 & { }^{129} \mathrm{I} & 0.0057 & 0.0055 & 0.0019 \\ 0.108 & { }^{126} \mathrm{Sn} & 0.16 & 0.027 & 0.0024 \\ 0.045 & { }^{79} \mathrm{Se} & 1.7 \times 10^{-4} & 3.2 \times 10^{-4} & 1.0 \times 10^{-4}\end{array}$




\subsection{Cycle Time}

The cycle time of pure fission reactors is limited by two factors: criticality and materials. After a certain portion of the heavy metal has fissioned, the reactor can no longer remain critical. After a certain amount of radiation damage, certain structural materials may fail. In contrast, since fission-fusion hybrids need not achieve criticality, only materials limit their cycle time.

We originally intended this hybrid to be a power-producing reactor, and it certainly is that. However, we have also shown that it is a prolific plutonium breeder. If we use it to breed fissile fuel for pure fission reactors in order to obviate enrichment as we described in Section 7.5, then the cycle time will be on the order of a typical fast reactor cycle time.

However, if we decide that breeding fissile fuel is not a main goal of this hybrid, if we merely desire to extract as much power from the fuel as possible, then the cycle time is limitless. In theory, the fusion source could continue to bombard the blanket with $14 \mathrm{MeV}$ neutrons until every single actinide has fissioned and then pointlessly continue to bombard the chaotic mass of fission products for all of eternity. Of course, there would come a point in time when the fission blanket power gain would become low enough such that further operation would not be worthwhile. This time scale would be decades. Many proponents of fission-fusion hybrids have touted this ultra-long cycle time. In the context of inertial confinement hybrids, $99 \%$ of all actinides would be fissioned in a cycle time of 50 years [30]. Tang's 2002 master's thesis proposes a 30-year cycle time [22]. Implementing such cycles would largely negate concerns over proliferation and actinide waste, as most actinides would be fissioned. Furthermore, as we have seen in Section 7.6, most harmful fission product waste would be transmuted. We could extract a huge portion of the total potential nuclear energy from the fuel. It would wring all the juice from the orange. Of course, as the density of actinides would substantially decrease after decades of operation, $k_{\text {eff }}$ would also decrease along with the fission blanket power multiplication. We could counter this by implementing the "variable fixed source" method we proposed in Section 7.2. The only obstacle to this wonderful ultra-long cycle time is material irradiation, which we do not analyze in this thesis. 


\subsection{Two Options}

In this burnup discussion, we have identified two main fuel cycle options:

- Burner: This would burn natural or depleted uranium. The cycle time would be decades. It would fission a large portion of all actinides and transmute a large portion of hazardous fission products. This would be a once-through burn-and-bury fuel cycle, achieving extremely high burnup and eventually fissioning a large portion of all the actinides. This is the primary mission we set forth in our introduction.

- Breeder: This would use natural or depleted uranium as fuel. It would breed ample fissile material during a relatively short cycle time. This fissile material would then be used as fuel for pure fission reactors. This would obviate the need for enrichment, and it would extract energy from the world's supply of depleted uranium. Though not our primary objective, this could become an attractive option if uranium reserves begin to run low in 50-100 years. As we have shown in Section 7.3, the breeding performance would be vastly superior to that of a fast reactor.

Essentially, this fission-fusion hybrid can burn or transmute anything by brute force - without the need for criticality, all it takes is a high-energy neutron source and plenty of time. It can burn and extract energy from anything that is fissionable. Currently, the nuclear industry goes to great lengths to obtain enriched uranium, put it in light water reactors that fission less than $10 \%$ of fissionable nuclides, and then fret over how to handle the remaining $90 \%$. We are inured to the status quo, but when we take a step back and think about the whole

picture, it seems pretty asinine. With fission-fusion hybrids, we could simply burn natural uranium until nearly all actinides have fissioned. It would be a like a car - you put the gas in, and you burn all of it. What a novel concept! 


\section{Pebble Structure, Thermal Hydraulics, and Safety}

Thus far, we have assumed that the spherical fuel pebbles are composed of nothing more than pure $\mathrm{UO}_{2}$. This fission model is appropriate - its simplicity is commensurate with the simplicity of our fusion model. It generates approximate results to shape our intuition. However, intuition for an unworkable system is pointless. For this work to be valuable, we must demonstrate that this system would be physically realizable given an appropriate design effort. The intention of this thesis is not any sort of intricate build-ready design, but we must perform just enough thermal hydraulic analysis to show that cooling would be practical and that the important materials would remain in their intended phases.

\subsection{Pebble Structure}

As far as the fuel pebbles are concerned, the main engineering issue is brittleness. Pure $\mathrm{UO}_{2}$ pebbles would crack under significant mechanical and thermal stresses. Existing light water reactors encase fuel elements within metal cladding to maintain structural integrity and to capture fission product gases (primarily Xenon and Krypton) in the fuel-cladding gap. However, a fuel-cladding gap would be severely problematic in spherical pebble geometry, because the fuel would always contact the cladding at only one point. In order to capture and store gaseous fission products within a cladded pebble, the pebble would need to contain a large central void.

Ryu and Sekimoto have studied uranium mononitride (UN) pebbles with a central void and stainless steel cladding [16]. The void comprises $1 / 4$ the volume of the pebble (excluding cladding), which reduces the homogenized uranium atom density by $25 \%$. However, UN is also more dense than $\mathrm{UO}_{2}\left(12.88\right.$ vs. $\left.10.97 \mathrm{~g} / \mathrm{cm}^{3}\right)$ and contains more uranium atoms per unit mass (one per $252 \mathrm{u}$ vs. one per $270 \mathrm{u}$ ). Neglecting porosity differences, the cumulative effect of these two facts is that UN has a uranium atom density $25 \%$ higher than that of $\mathrm{UO}_{2}$. So even though the central void reduces the fuel volume by $25 \%$, UN pebbles with central voids have a homogenized uranium atom density that is only about $6 \%$ lower than that of solid $\mathrm{UO}_{2}$ pebbles. The homogenized uranium atom density is tremendously important to us, as the total fission power gain is directly proportional to $k_{0}$, the neutron multiplication induced by the first generation of fusion-born neutrons (see Equations 7.1 and 7.2). This is a remarkable solution to our dilemma - ensure structural integrity of the fuel and confine gaseous fission products while sacrificing only $6 \%$ uranium density. UN also has the added advantage of less moderation than $\mathrm{UO}_{2}$, given that $\mathrm{N}$ has a smaller scattering cross-section than $\mathrm{O}$ and would have a smaller atom density in $\mathrm{UN}$ than $\mathrm{O}$ in $\mathrm{UO}_{2}$.

The other concerning issue is whether the cladding material can withstand helium temperatures approaching $1000^{\circ} \mathrm{C}$, which we will understand the need for in Section 7.2. Most stainless steels retain their stainless (oxidation resistant) quality up to only several hundred 
${ }^{\circ} \mathrm{C}$ under fast neutron irradiation. There have been many studies devoted to investigating various structural materials for high temperatures and/or fast neutron spectrums [26]. Potential cladding materials include ferritic or martensitic steels, austenitic stainless steels, nickel superalloys, or a multitude of ceramics. Of course, we should never rule out silicon carbide, which has nearly achieved panacea status these days. Unfortunately, $\mathrm{SiC}$ would be onerous to manufacture as a spherical shell. Tristructural-isotropic (TRISO) fuel particles in conventional pebble bed reactors do contain a spherical $\mathrm{SiC}$ coating, but coating a particle that is a couple of millimeters in diameter is orders of magnitude simpler than actually manufacturing a spherical shell that is a couple of centimeters in diameter. However, since this is not a materials thesis, we will not perform any detailed materials analysis. We will assume that if the much-lauded Very High Temperature Gas Reactor (VHTGR) can find suitable materials at near $1000^{\circ} \mathrm{C}$, so can we.

If we truly abhor a central void but accept that cladding is absolutely necessary for structural reasons, we must consider perforated cladding as the only potentially viable option to deal with the gaseous fission products. Quirkily enough, wiffle balls are the inspiration for this. See Figure 8.1 for an illustration. This general concept has actually been around for quite a while and is known as "vented fuel", although it has not been studied in depth for pebble fuel. In the context of sodium-cooled reactors, vented fuel is vaunted fuel, because the most problematic gaseous fission products react with sodium. Iodine, the most lethal gaseous fission product, is rendered innocuous as NaI. In the context of reactors cooled by inert gas, however, vented fuel would be significantly more challenging. There would need to be some sort of mechanism to remove the insidious fission products from the coolant. Even if such a mechanism could work, there would still be significant safety issues concerning lossof-coolant accident (LOCA) scenarios. However, this idea has been studied quantitatively and shown to be robust, most recently in Stephanie Kempf's 2008 MIT master's thesis [28]. Despite these challenges, perforated pebble cladding is probably the only way to achieve high fission power gain with $\mathrm{UO}_{2}$ fuel.

Since we have discussed vented fuel, we should at least quickly address the manufacturing of perforated cladding. First, the ratio of perforation diameter to pebble diameter should be small, much smaller than in the case of wiffle balls. This would prevent shards of fuel from passing through the perforations. Second, an important question is whether to manufacture the cladding with its perforations simultaneously or to drill the perforations at a later time, possibly even after the fuel has been secured within the cladding. We have mentioned the technical difficulty of manufacturing $\mathrm{SiC}$ as a stand-alone spherical shell. However, if we can coat TRISO particles with $\mathrm{SiC}$, it should not be completely unreasonable to coat our much larger fuel pebbles with $\mathrm{SiC}$. Once the $\mathrm{SiC}$ coating has cooled, it might be possible to drill many very small perforations into it so that its surface resembles the finest of cheese graters. Thus, $\mathrm{UO}_{2}$ pebble fuel encased in perforated $\mathrm{SiC}$ cladding without a central void could be 
viable, albeit cumbersome to manufacture.

In this section, we have discussed the pros and cons of various potential pebble structures. Beyond ensuring a feasible conceptual design, our parmount goal is to maximize the homogenized uranium atom density, which ultimately determines the hybrid power gain. Although we have performed our neutronics analysis assuming solid $\mathrm{UO}_{2}$ pebbles, which would require perforated cladding, many other possibilities warrant future work. The UN fuel with a central void proposed by Ryu and Sekimoto is an especially intriguing option [16]. While alternative fuel composition and cladding options will certainly alter our quantitative results, they would not significantly change the fundamental advantages of an L-mode tokamak fission-fusion hybrid with natural uranium. Also, the fast neutron spectrum tends to mitigate structural variation. For example, adding a fairly thick ( $10 \%$ of pebble radius) $\mathrm{SiC}$ cladding to our $\mathrm{UO}_{2}$ pebbles decreases $k_{0}$ (the fusion-born neutron multiplication) by about $10 \%$. That will in turn reduce the fission power gain by about $10 \%$. It makes a difference, but it does not fundamentally alter the concept. The purpose of this study is exploration of an idea, not an elaborate design. At this point in the hybrid debate, viable conceptual designs are persuasive while intricate build-ready designs are superfluous.

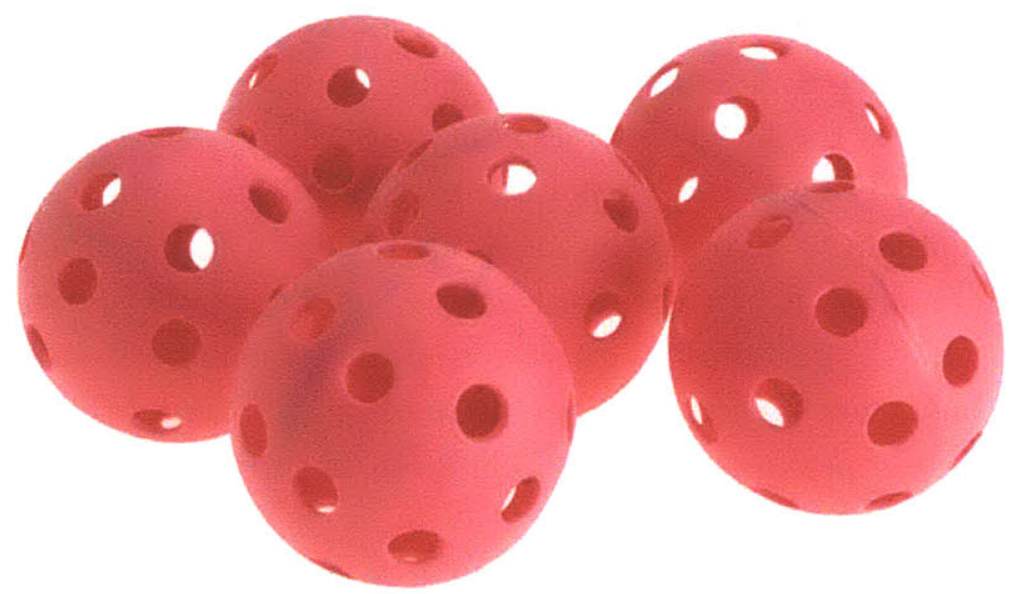

Figure 8.1: Pink wiffle balls. These will suffice as an illustration of perforated pebble cladding. The spherical cladding encases the fuel to ensure structural integrity but is perforated in order to vent the fuel of gaseous fission products. In practice, the perforations would be much smaller relative to the pebble diameter than the case of wiffle balls. 


\subsection{Thermal Hydraulic Analysis}

As should be evident, this thesis is primarily about fusion and reactor physics. However, we must perform enough basic thermal hydraulic analysis to ensure that it would be possible. We have developed a model based on pebble bed correlations to enstire reasonable coolant velocity, coolant pressure drop, and temperature extrema.

Figure 8.2 shows a conceptual hybrid schematic with the helium inboard and outboard flow paths. This is a poloidal cross-section of the tokamak. The entire device can be obtained by rotating this cross-section $360^{\circ}$ about a vertical axis to the right or left of the figure. Note that the dimensions (especially the blanket thickness) are not to scale. In gas reactors, the coolant usually flows downward to prevent positive acceleration due to heating. 


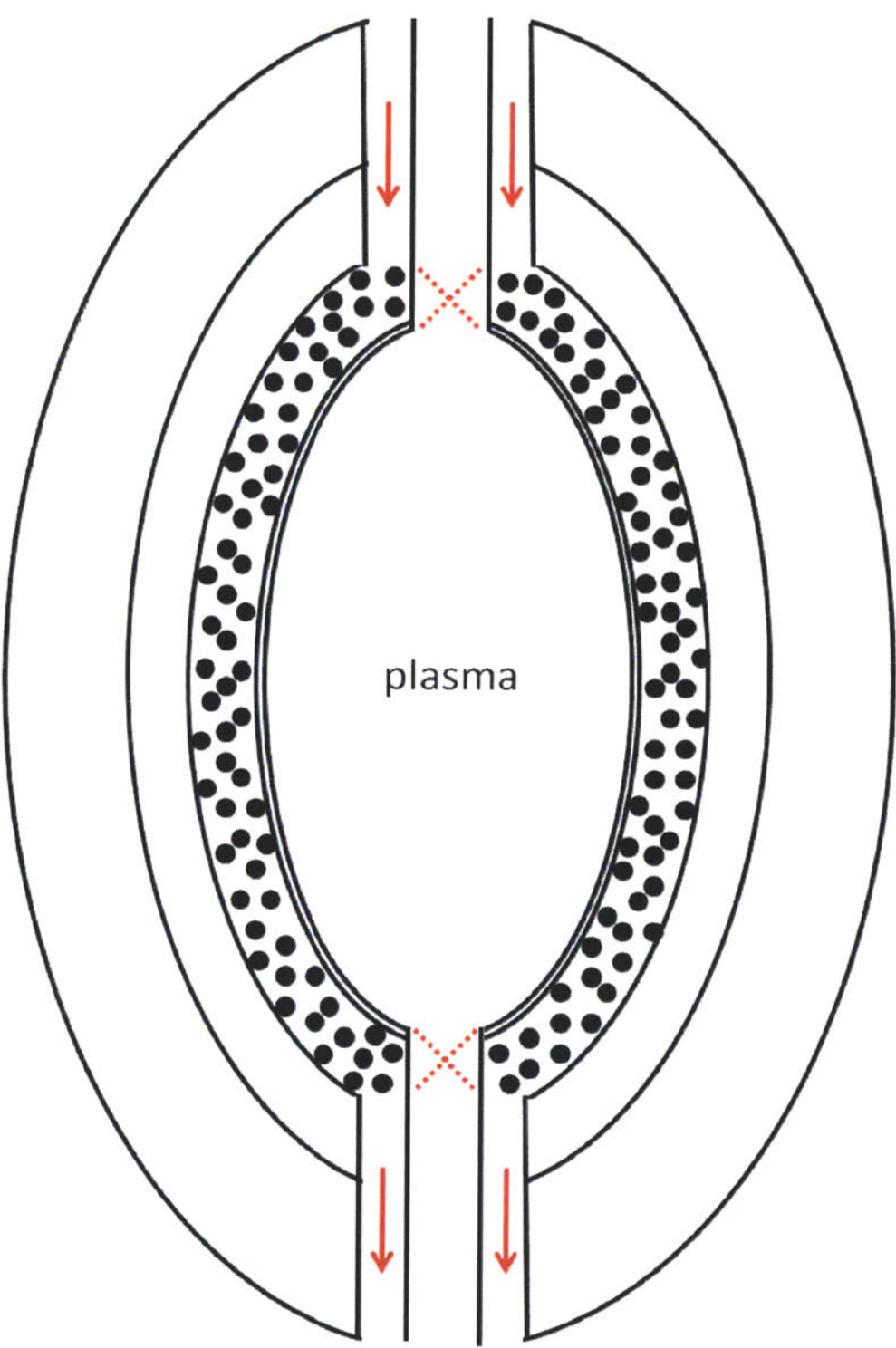

Figure 8.2: A basic schematic of the hybrid concept showing helium coolant flow paths. The helium, denoted with arrows, flows downward through the pebbles to reduce acceleration. The dotted X's mark the X-points in the magnetic topology where we must allow ample space for diverters. Exterior to the fuel pebble layer are the Li-Pb breeding and shielding layers, respectively. Although the cross-section here is elliptic, we can approximate it as circular for the purposes of our thermal analysis. 


\subsubsection{Coolant Flow Rate and Pressure Drop}

Let us begin the analysis. We can approximate the total power deposited in each half of the fuel region as $P_{\text {fuel }}$, which is the fusion power $P_{\text {fus }}$ multiplied by half the fission gain $Q_{\text {fis }}$. Of course, some of the power is deposited in the first wall, Li-Pb layer, and shielding layer, but we accept this overestimate as added assurance that the flow rate will not be overly optimistic. Also, as we have seen with toroidal neutron flux distributions, $P_{\text {fuel }}$ is not evenly divided between the inboard and outboard fuel regions - the outboard fuel generates more power.

$$
P_{\text {fuel }}=\frac{4}{5} P_{\text {fus }} Q_{\text {fis }} / 2
$$

Now, if we know the desired temperature drop, we can perform a simple energy balance to obtain the mass flow rate $\dot{m}$.

$$
P_{\text {fuel }}=\dot{m} c_{p}\left(T_{\text {out }}-T_{\text {in }}\right)
$$

The helium velocity is a bit more subtle. We can easily compute $v_{\text {empty }}$, the velocity the helium would have (given the same $\dot{m}$ ) without the pebbles obstructing its path. We can also define $\epsilon$, the pebble bed "void fraction", the fraction of the total fuel region volume that is not filled with fuel pebbles. Mathematical studies show that when spheres are stacked randomly in a volume, they will occupy about $64 \%$ of that volume [23]. Thus, $\epsilon$ is about 0.36 . Studies of various packed beds show that we can express the true fluid velocity as $v_{\text {empty }} / \epsilon[17]$.

$$
v=\frac{v_{\text {empty }}}{\epsilon}=\frac{\dot{m} / \rho A_{\text {flow }}}{\epsilon}
$$

However, we must first define the flow area $A_{\text {flow }}$. Referring back to Figure 8.2, we can approximate the elliptic annular regions as circular annular regions. There are four regions surrounding the plasma: the first-wall, the pebble fuel and helium coolant, the Li-Pb breeder, and the shield. Let the radii defining these poloidal annuli be $a_{1}, a_{2}, a_{3}, a_{4}$, and $a_{5}$. Clearly, the pebbles and coolant comprise the annulus between $a_{2}$ and $a_{3}$. Now the flow area is the product of the poloidal annular thickness and the toroidal circumference. Of course, the toroidal circumference varies as the coolant flows poloidally through the annulus, but we can approximate its average value as $2 \pi\left(R+a_{1}\right)$, where $R$ is the tokamak major radius.

$$
A_{\text {flow }}=2 \pi\left(R+a_{1}\right)\left(a_{3}-a_{2}\right)
$$

The hydraulic diameter for a packed bed is directly related to the pebble diameter [17].

$$
D_{h}=\frac{\epsilon}{1-\epsilon} D_{\text {pebble }}
$$


Now the Reynolds number is simple to compute.

$$
\operatorname{Re}=\frac{v \rho D_{h}}{\mu}
$$

Before proceeding further, we should note how we compute properties of helium. We determine helium density $\rho$ with the ideal gas law using average system temperature and pressure. It is true that $\rho$ will vary quite a bit if the helium temperature gain is large, but averaged parameters are necessary for the forthcoming pressure drop calculation. We determine the helium thermal conductivity as a function of temperature and pressure using a well-established correlation [25]. Here the pressure $p_{0}$ is in $\mathrm{Pa}$, temperature is in $\mathrm{K}$, and $k_{\mathrm{He}}$ is in $\mathrm{W} / \mathrm{m} / \mathrm{K}$.

$$
k_{\mathrm{He}}=\left(2.682 \times 10^{-3}\right)\left[1+\left(1.123 \times 10^{-3}\right)\left(\frac{p_{0}}{100000}\right)\right] T^{0.71\left[1-\left(2 \times 10^{-4}\right)\left(\frac{p_{0}}{100000}\right)\right]} ;
$$

The viscosity is simpler, as it depends mainly on temperature [25]. Here $\mathrm{T}$ is in $\mathrm{K}$, and $\mu_{\mathrm{He}}$ is in $\mathrm{Pa}^{*} \mathrm{~s}$.

$$
\mu_{\mathrm{He}}=\left(3.674 \times 10^{-7}\right) T^{0.7}
$$

Now we draw our pressure drop correlation from a computational fluid dynamics (CFD) pebble bed study [19]. Here $\Delta P$ is the total pressure drop accumulating over a flow length $\Delta H$. No integration of helium properties over temperature (which varies linearly with flow length, assuming uniform power density) is required.

$$
\frac{\Delta P}{\Delta x}=\Psi\left(\frac{1-\epsilon}{\epsilon^{3}}\right)\left(\frac{1}{2 \rho D_{\text {pebble }}}\right)\left(\frac{\dot{m}}{A_{\text {flow }}}\right)^{2}
$$

The pressure drop coefficient $\Psi$ is a sum of turbulent and laminar components.

$$
\Psi=\frac{505}{\operatorname{Re} /(1-\epsilon)}+\frac{0.1}{(\operatorname{Re} /(1-\epsilon))^{0.1}}
$$

With this relatively simple model, we can determine the useful properties $\Delta P, v$, and Re as functions of average pressure, temperature, and pebble size. We will not display all of these permutations, but just the few that are most instructive. Figure 8.3 shows the ratio of the helium pressure drop $\Delta P$ to the ambient pressure $P_{0}$ as a function of $P_{0}$. All other parameters, including the temperature increase, are fixed. We desire this ratio to be low, probably not more than a few percent, in order to achieve a high Brayton cycle efficiency. Clearly, the ambient helium pressure would need to exceed $5 \mathrm{MPa}$ for a ratio below $1 \%$.

Figure 8.4 shows this same pressure ratio as a function of fuel pebble diameter. As the fuel pebbles become larger, the flow area remains constant, but the hydraulic diameter 


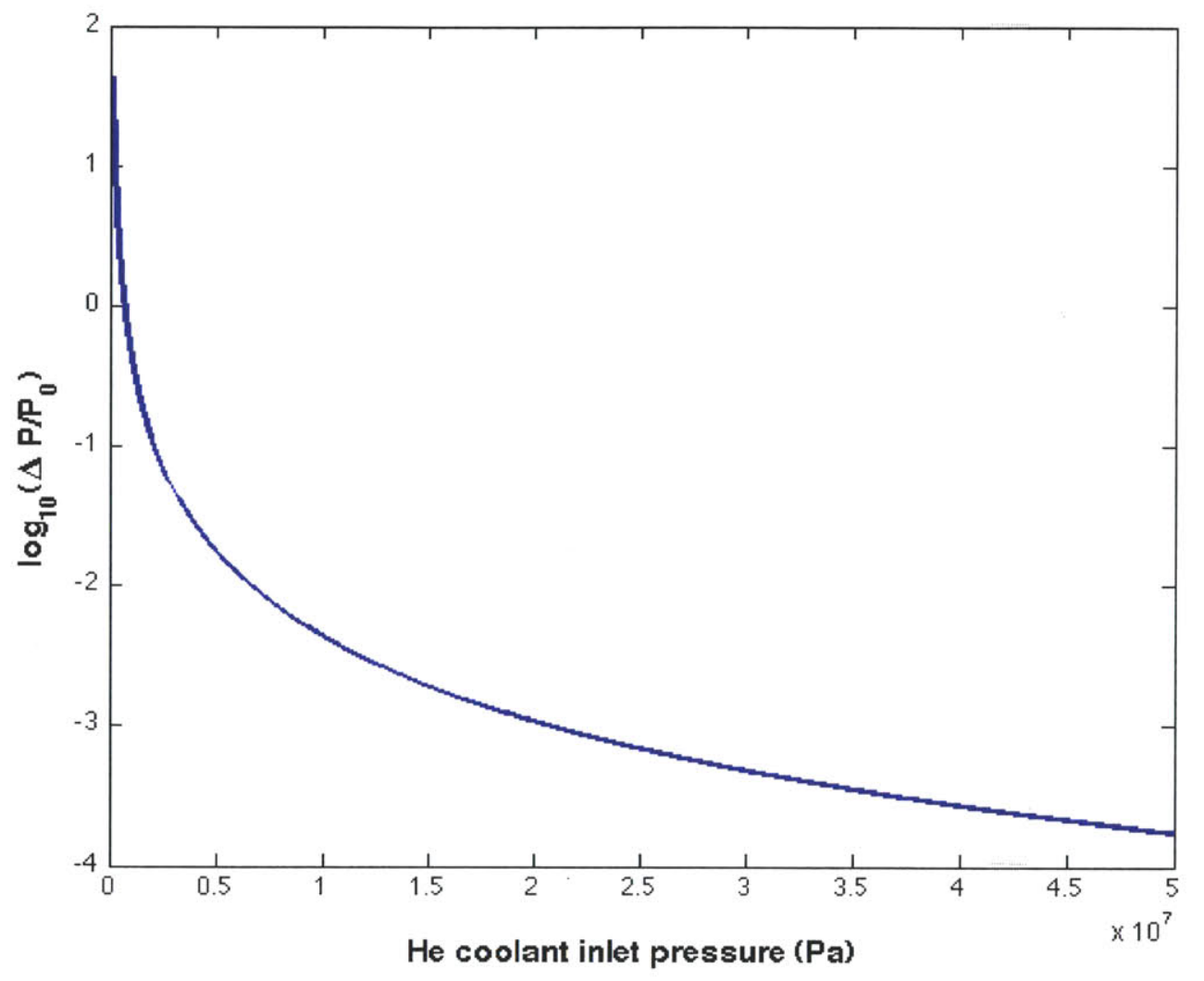

Figure 8.3: The ratio of the helium pressure drop $\Delta P$ to the ambient pressure $P_{0}$ as a function of $P_{0}$. Here $\Delta T$ is fixed at $500^{\circ} \mathrm{C}$, and the pebble diameter is $3 \mathrm{~cm}$. Note that at very low $P_{0}$, the ratio exceeds 1 , because the pressure drop correlation is not valid at such low pressures.

increases. It is "easier" for gas to flow between larger pebbles than between smaller pebbles. The gas does not need to be forced through tinier gaps between the pebbles, and so less pressure is lost.

Figure 8.5 shows the Reynolds number Re as a function of fuel pebble diameter. The relationship is linear, which results from the simple definition of Re. The important thing to note here is that at the necessary ambient pressure and temperature drop, the flow is always turbulent. Laminar flow is not a realistic possibility.

Figure 8.6 shows the helium coolant velocity as a function of its total temperature increase. For larger temperature increases, smaller mass flow rates are necessary. At low temperature increases of less than $200^{\circ} \mathrm{C}$, the required helium velocity is impossibly large. Thus, a large temperature increase is not only favorable for the Brayton cycle efficiency, but it is absolutely necessary to ensure a low coolant flow rate.

Figure 8.7 shows the helium velocity as a function of ambient pressure. Higher pressure 


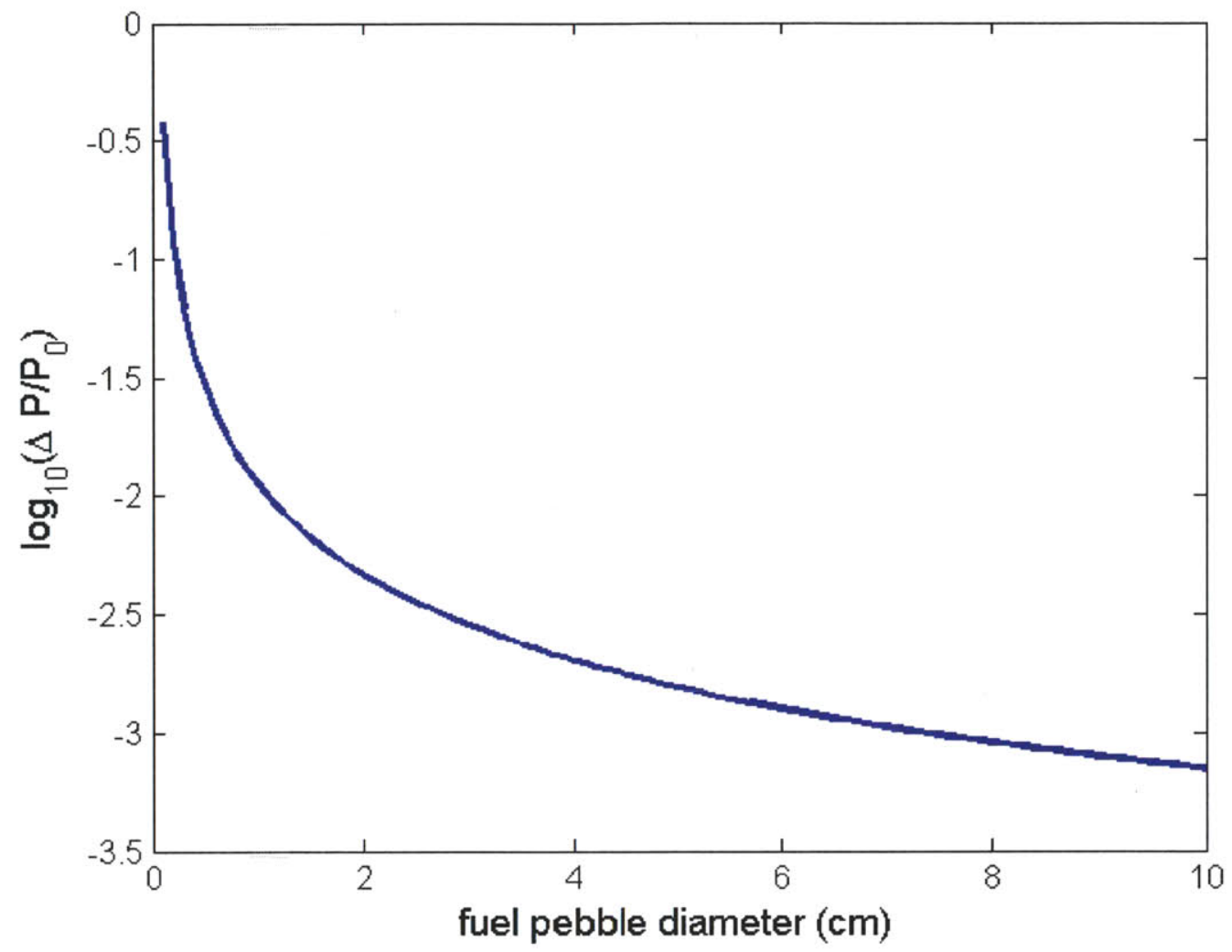

Figure 8.4: The ratio of the helium pressure drop $\Delta P$ to the ambient pressure $P_{0}$ as a function of fuel pebble diameter. Here $\Delta T$ is fixed at $500^{\circ} \mathrm{C}$, and the ambient pressure is $15 \mathrm{MPa}$.

condenses the helium, which allows it to absorb more heat at a lower mass flow rate. In order to keep the velocity below $10 \mathrm{~m} / \mathrm{s}$, the core must be pressurized to at least $25 \mathrm{MPa}$. Note that the flow velocity is independent of pebble size, because it is proportional to $v_{\text {empty }}$. Obviously, $\epsilon$ is independent of pebble size. 


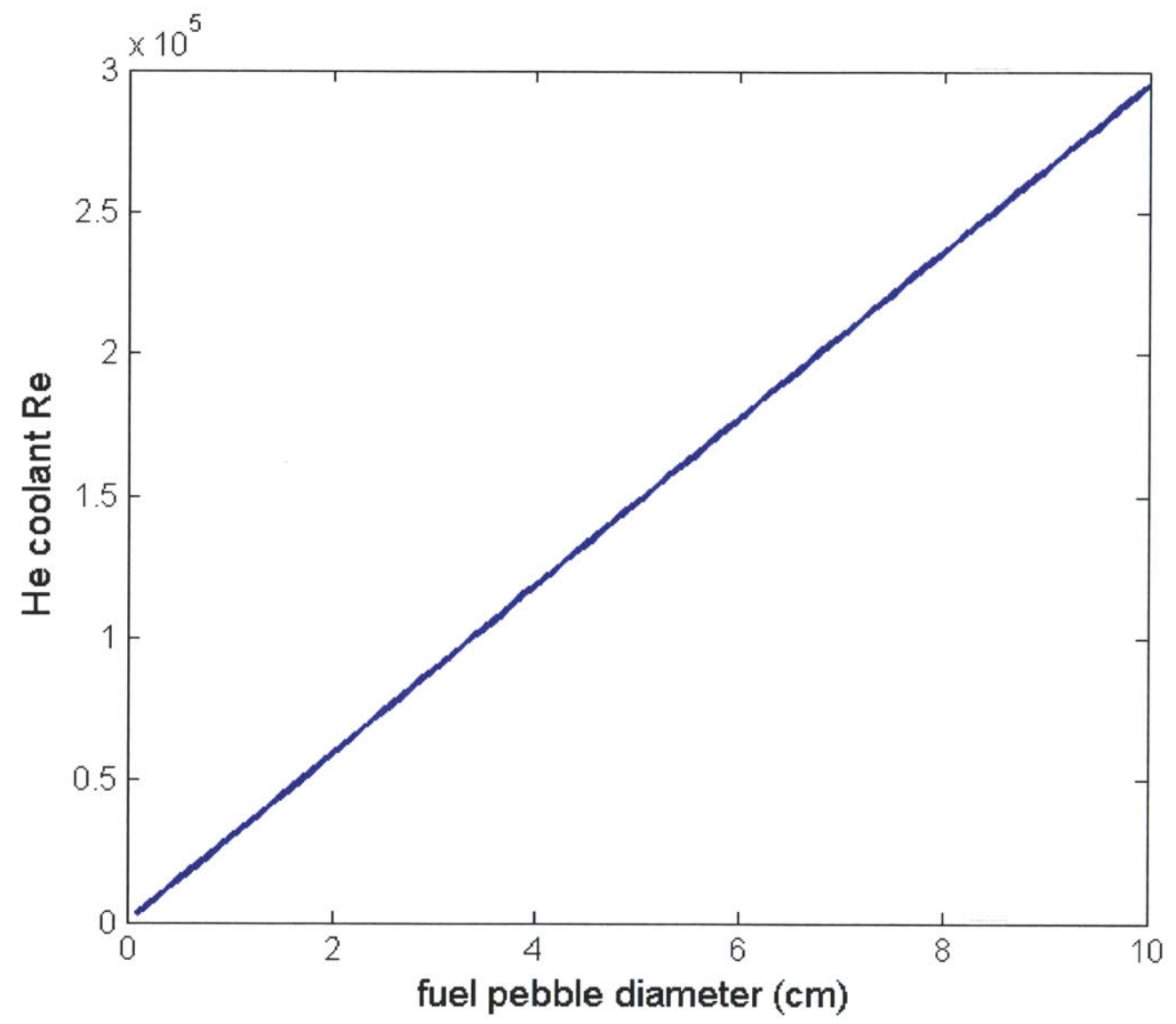

Figure 8.5: The Reynolds number as a function of pebble size. Here $\Delta T$ is fixed at $500^{\circ} \mathrm{C}$, and the ambient pressure is $15 \mathrm{MPa}$. The flow is clearly turbulent for all reasonable pebble sizes. 


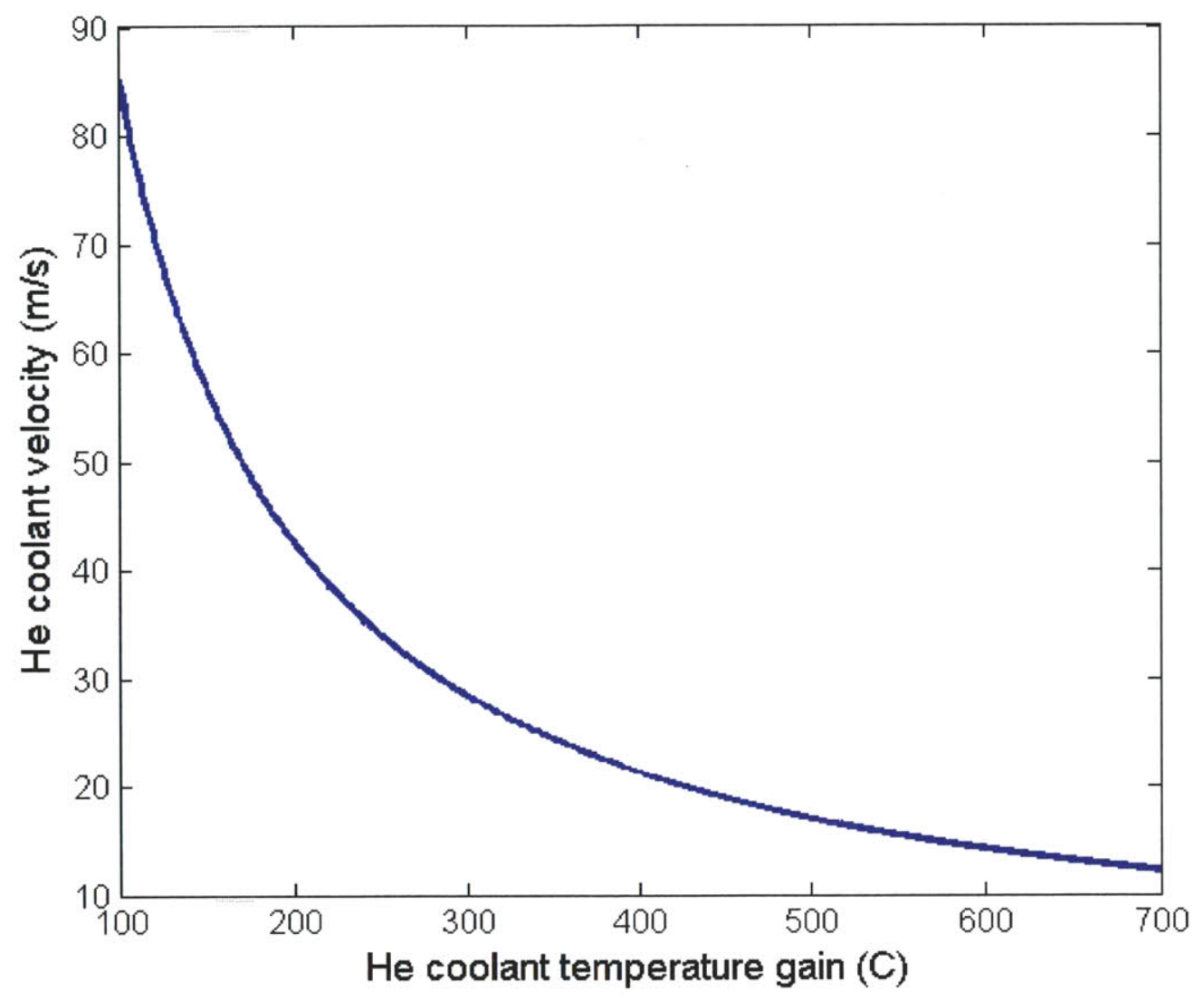

Figure 8.6: The helium coolant velocity as a function of its temperature increase through the core. Here $P_{0}$ is fixed at $15 \mathrm{MPa}$, and the pebble size is $3 \mathrm{~cm}$. 


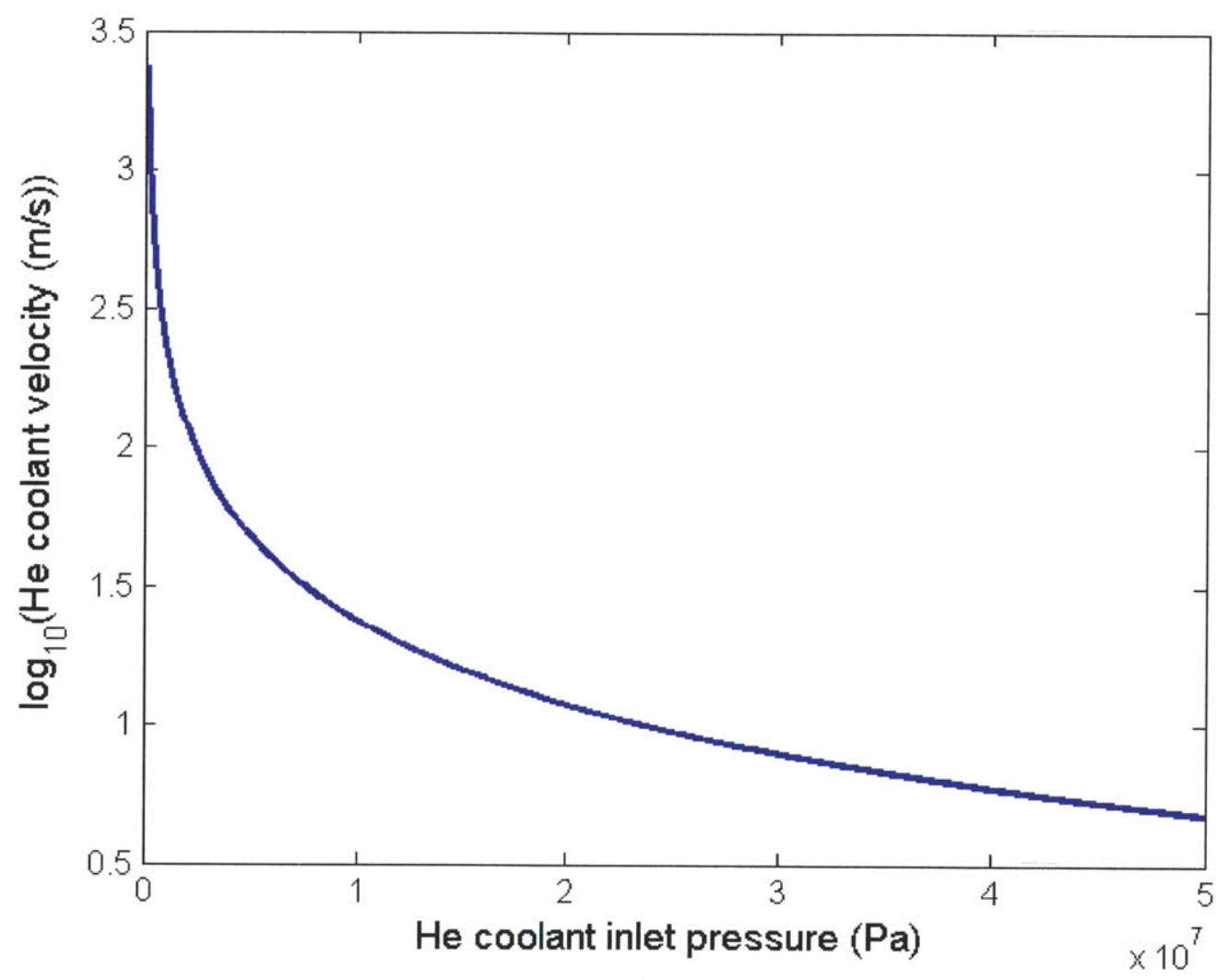

Figure 8.7: The helium coolant velocity as a function of ambient pressure. Here $\Delta T$ is fixed at $500^{\circ} \mathrm{C}$, and the pebble diameter is $3 \mathrm{~cm}$. 


\subsubsection{Heat Transfer and Temperature}

Now that we have examined flow rates and pressure drops, we must estimate the temperature extrema in the $\mathrm{UO}_{2}$ fuel and liquid $\mathrm{Li}-\mathrm{Pb}$ breeder. $\mathrm{UO}_{2}$ must not exceed a certain margin below its $2865^{\circ} \mathrm{C}$ melting point, and Li-Pb must remain between its melting and boiling points. Figure 8.8 shows a phase diagram for $\mathrm{Pb}$ content in $\mathrm{Li}$. The eutectic point is at $\mathrm{Pb}_{84} \mathrm{Li}_{16}$. However, we have shown with neutronics that such a eutectic contains far too much lead to achieve breeding ratio of 1.0 while still allowing for substantial fission power gain. In a pure fusion reactor, there is no problem with $\mathrm{Pb}_{84} \mathrm{Li}_{16}$, but the fission component usurps enough neutrons to ruin its efficacy. We could use pure lithium, which exhibits a lower melting point than the eutectic, but that would introduce an additional chemical hazard. Pure lithium is exorbitantly corrosive to the point that it is harmful to skin contact. In our neutronics analysis, we concluded that the lead content must be quite low at 10-15\% to breed sufficient tritium with a reasonable volume of $\mathrm{Li}-\mathrm{Pb}$. The natural question to ask now is, "How much lead content is necessary to negate the chemical hazard?" We have no answer, but we will assume that $10-15 \% \mathrm{~Pb}$ is sufficient. The rationale for $\mathrm{Pb}_{84} \mathrm{Li}_{16}$ was a low melting point, not that $84 \% \mathrm{~Pb}$ is necessary to fix the chemical problem. The melting point of $\mathrm{Pb}_{10} \mathrm{Li}_{90}$ is about $500^{\circ} \mathrm{C}$. We do not know its boiling point, but for this analysis we will assume it is equal to that of pure lithium $\left(1342^{\circ} \mathrm{C}\right)$. The true boiling point is probably substantially higher, so designing to $1342^{\circ} \mathrm{C}$ will ensure a large error margin. So, in summary, we wish to keep $\mathrm{UO}_{2}$ below $2865^{\circ} \mathrm{C}$ and $\mathrm{Li}-\mathrm{Pb}$ between $500^{\circ} \mathrm{C}$ and $1342^{\circ} \mathrm{C}$

We begin the heat transfer analysis by obtaining an expression for the Nusselt number $\mathrm{Nu}$ between the pebbles and helium coolant. In a pebble bed, the angle between the bulk coolant velocity and the pebble surface varies quite a bit. Thus, if we apply equations representing flow over flat planes, there are small "pockets" of space in which the helium is actually laminar. We can express the laminar and turbulent contributions to $\mathrm{Nu}$ as $\mathrm{Nu}_{l}$ and $\mathrm{Nu}_{t}[17]$.

$$
\begin{aligned}
\mathrm{Nu}_{l} & =0.664(\mathrm{Re} / \epsilon)^{1 / 2} \operatorname{Pr}^{1 / 3} \\
\mathrm{Nu}_{t} & =\frac{0.037(\mathrm{Re} / \epsilon)^{0.8} \operatorname{Pr}}{1+2.443(\mathrm{Re} / \epsilon)^{-0.1}\left(\operatorname{Pr}^{2 / 3}-1\right)}
\end{aligned}
$$

Then the total "spherical" $\mathrm{Nu}$ is $\mathrm{Nu}_{\mathrm{sp}}$. This makes intuitive sense if one thinks of $\mathrm{Nu}_{l}$ and $\mathrm{Nu}_{t}$ as $\sin \theta$ and $\cos \theta$. The constant value of 2 is the solution for quiescent fluid (zero flow rate).

$$
\mathrm{Nu}_{\mathrm{sp}}=2+\left(\mathrm{Nu}_{l}^{2}+\mathrm{Nu}_{t}^{2}\right)^{1 / 2}
$$

Now we can apply a correlation for the effective pebble bed $\mathrm{Nu}$ [17]. 


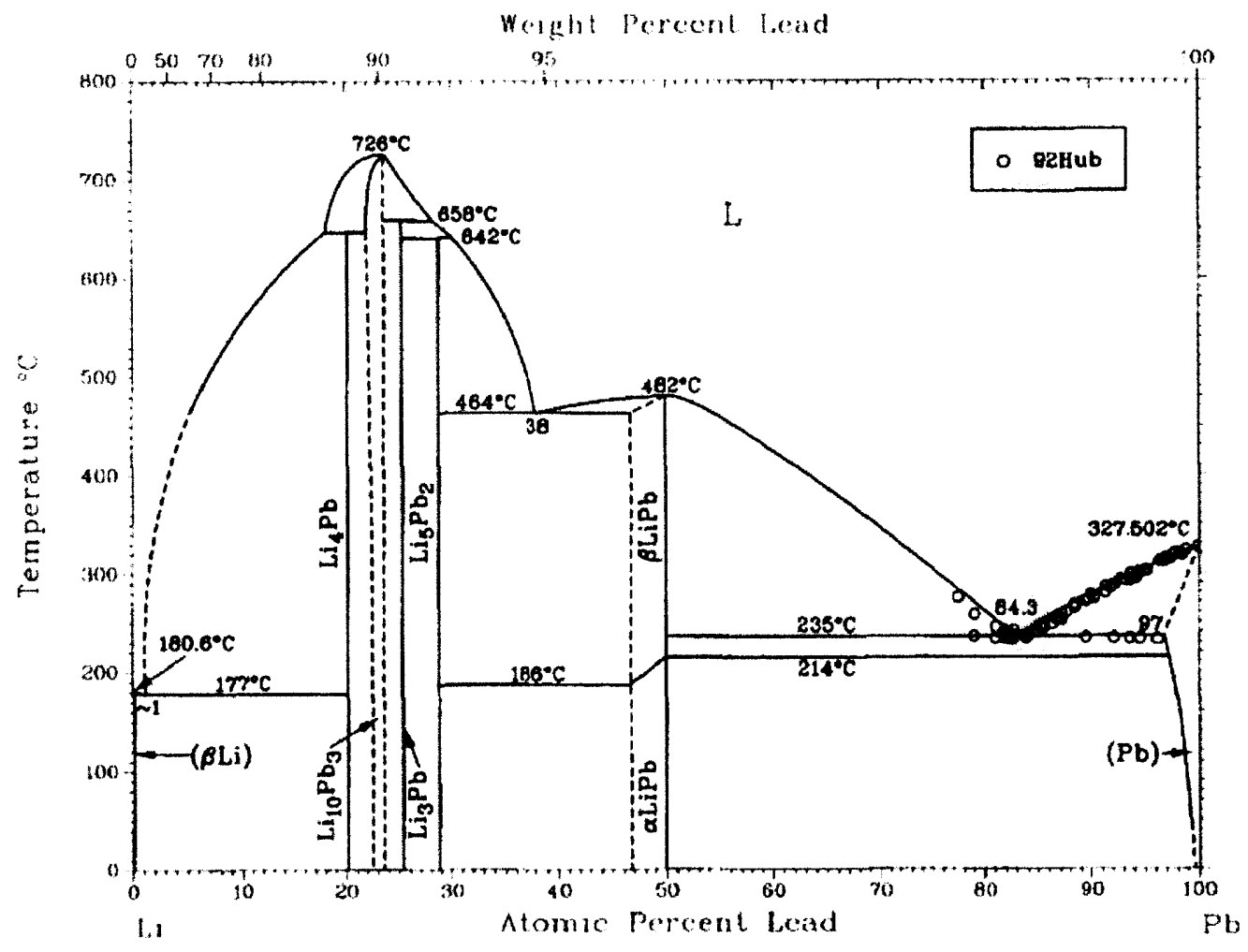

Figure 8.8: A phase diagram for $\mathrm{Pb}$ content in $\mathrm{Li}$. The eutectic point is at $\mathrm{Pb}_{84} \mathrm{Li}_{16}[20]$.

$$
\mathrm{Nu}=(1+1.5(1-\epsilon)) \mathrm{Nu}_{\mathrm{sp}}
$$

Of course, we define the dimensionless constants $\mathrm{Nu}$ and $\mathrm{Pr}$ as usual. Nu defines the heat transfer coefficient $h$ between the pebbles and coolant.

$$
\begin{aligned}
& \mathrm{Nu}=\frac{h D_{h}}{k} \\
& \mathrm{Pr}=\frac{c_{p} \mu}{k}
\end{aligned}
$$

Now let us analyze the fuel pebble temperature. We can approximate the volumetric heat generation simply by dividing the total power by the cumulative volume of all the $\mathrm{UO}_{2}$ pebbles.

$$
q_{\text {fuel }}^{\prime \prime \prime}=P_{\text {fuel }} / V_{\text {fuel }}
$$


That cumulative volume is the volume of the entire toroidal shell fuel region reduced by the factor $1-\epsilon$.

$$
V_{\text {fuel }}=\frac{\pi\left(a_{3}^{2}-a_{2}^{2}\right)(2 \pi R)}{1-\epsilon}
$$

Now we can solve the heat conduction equation in spherical geometry.

$$
k \nabla^{2} T=q^{\prime \prime \prime}
$$

The result, in terms of the maximum $\mathrm{UO}_{2}$ temperature $T_{\max }$, is

$$
T_{\text {fuel }}(r)=T_{\max }-\frac{q_{\text {fuel }}^{\prime \prime \prime} r^{2}}{6 k_{\text {fuel }}}
$$

Defining the heat flux $q^{\prime \prime}$ at the pebble surface, we can relate $T_{\text {fuel }}\left(r_{0}\right)$ to the heat transfer coefficient $h$. Here $r_{0}$ is the pebble radius, and we have introduced a temperature jump $\Delta T_{\text {clad }}$ for whatever sort of cladding is used. When we must compute temperature values, we will assume the cladding is $\mathrm{SiC}$ with a thickness $5 \%$ of the pebble radius.

$$
q_{\text {fuel }}^{\prime \prime}=\frac{q_{\text {fuel }}^{\prime \prime \prime} r_{0}}{3}=h\left[T_{\text {fuel }}\left(r_{0}\right)-\Delta T_{\text {clad }}-T_{\text {coolant }}\right]
$$

Now let us turn to the $\mathrm{Li}-\mathrm{Pb}$ heat transfer, which is significantly more complex and arbitrary. We will model the liquid Li-Pb as sitting in a cylindrical annulus between radii $a_{3}$ and $a_{4}$. In our neutronics analysis, we computed the total power deposited direction in the $\mathrm{Li}-\mathrm{Pb}$, which is about $40 \%$ of the fusion neutron power.

$$
\begin{aligned}
& P_{\mathrm{Li}}=\frac{4}{5} P_{\text {fus }}(0.4) \\
& q_{\mathrm{Li}}^{\prime \prime \prime}=P_{\mathrm{Li}} / V_{\mathrm{Li}}
\end{aligned}
$$

We solve the heat conduction equation again, this time in cylindrical coordinates.

$$
T_{\mathrm{Li}}(r)=-\frac{q_{\mathrm{Li}}^{\prime \prime \prime} r^{2}}{4 k_{\mathrm{Li}}}+C_{1} \ln (r)+C_{2}
$$

Now things become arbitrary. The helium flowing through the pebbles adjacent to this region cools the Li- $\mathrm{Pb}$. However, much heat is also conducted outward into the shield, which is often cooled with water. Many tokamak designs even cool the lithium directly with a separate coolant cycle. As an initial test, let us assume that the helium cools all the Li-Pb heat generation. This is equivalent to putting a perfect insulator between the $\mathrm{Li}-\mathrm{Pb}$ and the shield at $r=a_{4}$. Setting $d T_{\mathrm{Li}} / d r=0$ at that points yields 


$$
T_{\mathrm{Li}}(r)=\frac{q_{\mathrm{Li}}^{\prime \prime \prime}}{2 k_{\mathrm{Li}}}\left[\frac{a_{3}^{2}-r^{2}}{2}+a_{4}^{2} \ln \left(r / a_{3}\right)\right]+\Delta T_{\text {wall }}+T_{\text {coolant }}
$$

We again define an arbitrary temperature drop across the wall between the $\mathrm{Li}-\mathrm{Pb}$ and fuel pebbles. When we must compute temperature values, we will assume this wall is $\mathrm{SiC}$ (like the plasma first-wall). Naturally, this temperature distribution will yield the largest physically possible Li- $\mathrm{Pb}$ temperature at $r=a_{4}$. This is a high ceiling estimate of the maximum Li-Pb temperature.

Now let us consider a second scenario in which the heat flux at $r=a_{3}$ is equal to the heat flux at $r=a_{4}$. This means that just as much heat is conducted into the shield as into the helium coolant. It is a rather arbitrary choice, but it is a feasible scenario that could be engineered. We will not perform any analysis for the shield cooling, which is well-established in existing tokamak technology.

$$
-k_{\mathrm{Li}}\left[\frac{d T}{d r}\right]_{a_{3}}=k_{\mathrm{Li}}\left[\frac{d T}{d r}\right]_{a_{4}}
$$

These boundary conditions yield

$$
T_{\mathrm{Li}}(r)=\frac{q_{\mathrm{Li}}^{\prime \prime \prime}}{2 k_{\mathrm{Li}}}\left[\frac{a_{3}^{2}-r^{2}}{2}+a_{3} a_{4} \ln \left(r / a_{3}\right)\right]+\Delta T_{\text {wall }}+T_{\text {coolant }}
$$

The location of maximum temperature is

$$
r_{\max }=\sqrt{a_{3} a_{4}}
$$

This is a common elegant result in cylindrical heat conduction. The value of the maximum temperature at $r_{\max }$, however, is not so elegant.

$$
T_{\mathrm{Li}}\left(r_{\max }\right)=\frac{q_{\mathrm{Li}}^{\prime \prime \prime} a_{3}}{2 k_{\mathrm{Li}}}\left[\frac{a_{3}-a_{4}}{2}+a_{4} \ln \left(\sqrt{a_{4} / a_{3}}\right)\right]+\Delta T_{\text {wall }}+T_{\text {coolant }}
$$

Figure 8.9 shows $T_{\mathrm{Li}}(r)$ for both scenarios examined here near the helium coolant outlet, where the helium temperature is $900^{\circ} \mathrm{C}$. Clearly, the $\mathrm{Li}-\mathrm{Pb}$ exceeds the boiling point of pure Li by several hundred ${ }^{\circ} \mathrm{C}$ when no heat is conducted into the shield. Even when the shield cools the Li- $\mathrm{Pb}$ just as much as the helium, the $\mathrm{Li}-\mathrm{Pb}$ remains only $100^{\circ} \mathrm{C}$ below the boiling point. Of course, this is near the helium outlet. Near the helium inlet, $T_{\mathrm{Li}}(r)$ will be significantly lower. Our model does not consider transverse (parallel to helium flow) heat conduction, which would reduce the maximum $\mathrm{Pb}-\mathrm{Li}$ temperature. Also, $q_{\mathrm{Li}}^{\prime \prime \prime}$ is not evenly distributed throughout the Li-Pb volume in the same way $q_{\text {fuel }}^{\prime \prime \prime}$ is distributed throughout the pebble bed volume. In reality, $q_{\mathrm{Li}}^{\prime \prime \prime}$ will be higher near the fuel pebbles, where the neutron flux is higher. Indeed, the helium coolant could in fact cool most of the Li-Pb heat generation. 
Regardless, we will take our "half-and-half" heat transfer model as an overestimate of the maximum $\mathrm{Li}-\mathrm{Pb}$ temperature.

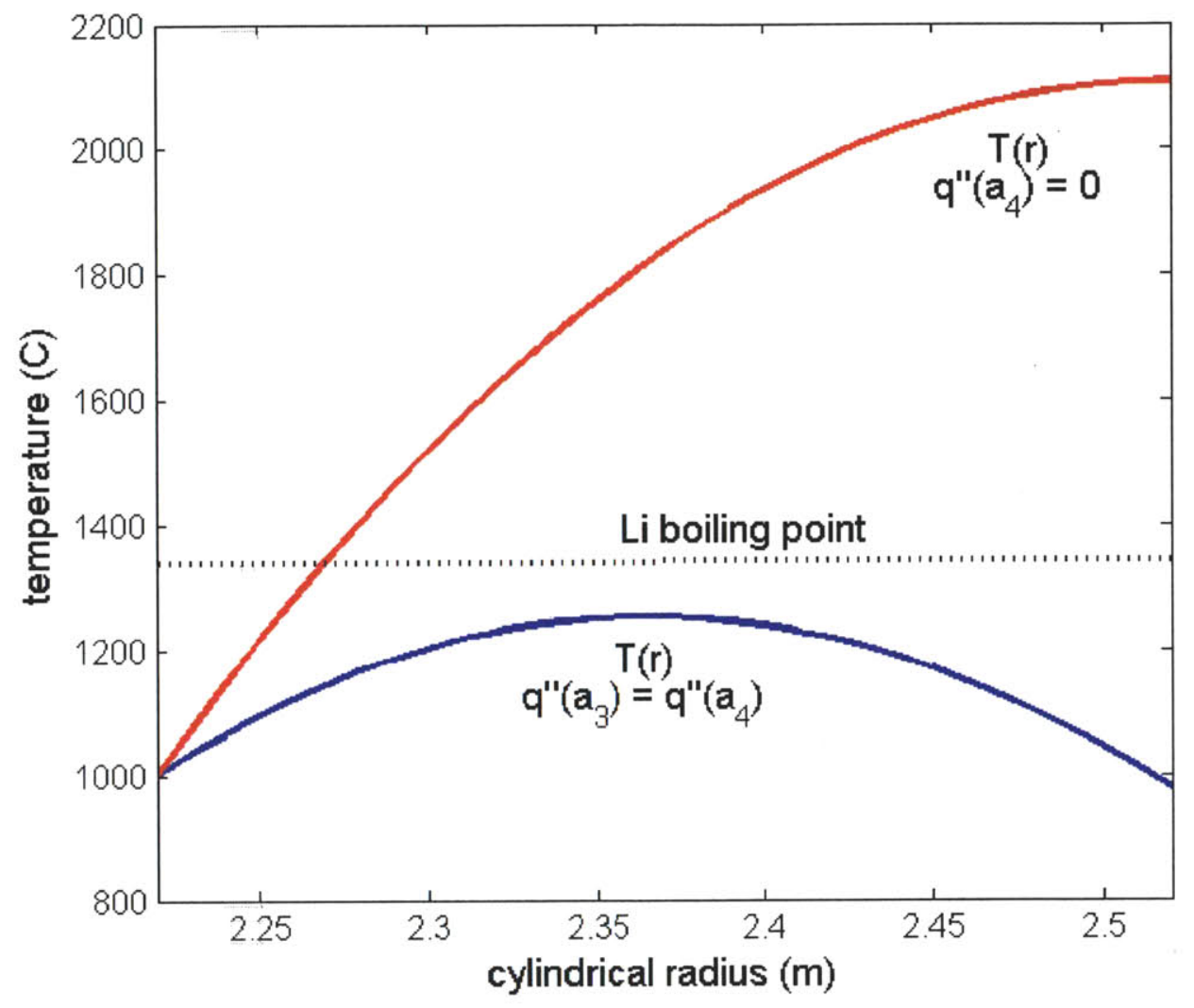

Figure 8.9: $T_{\mathrm{Li}}(r)$ near the coolant outlet for zero heat conduction at $r=a_{4}$ and for equal heat conduction at $r=a_{3}$ and $a_{4}$. The boiling point of pure $\mathrm{Li}$ is shown. Here the helium outlet temperature is $900^{\circ} \mathrm{C}$.

With our model settled, we can display the pertinent temperature extrema as functions of temperature drop and pebble size. To ensure that the lithium remains above its melting point, we set the inlet helium temperature equal to the $\mathrm{Pb}_{10} \mathrm{Li}_{90}$ melting temperature of $500^{\circ} \mathrm{C}$. Figure 8.10 shows the three temperature extrema of interest as a function of fuel pebble diameter. Pebble size does not affect $\mathrm{Li}-\mathrm{Pb}$ temperature, but the $\mathrm{UO}_{2}$ melting points clearly limits the pebble diameter to approximately $8 \mathrm{~cm}$.

Figure 8.11 shows the same temperature extrema as functions of helium temperature gain. If the helium begins at $500^{\circ} \mathrm{C}$, it cannot exceed $1000^{\circ} \mathrm{C}$ due to the lithium boiling point. 


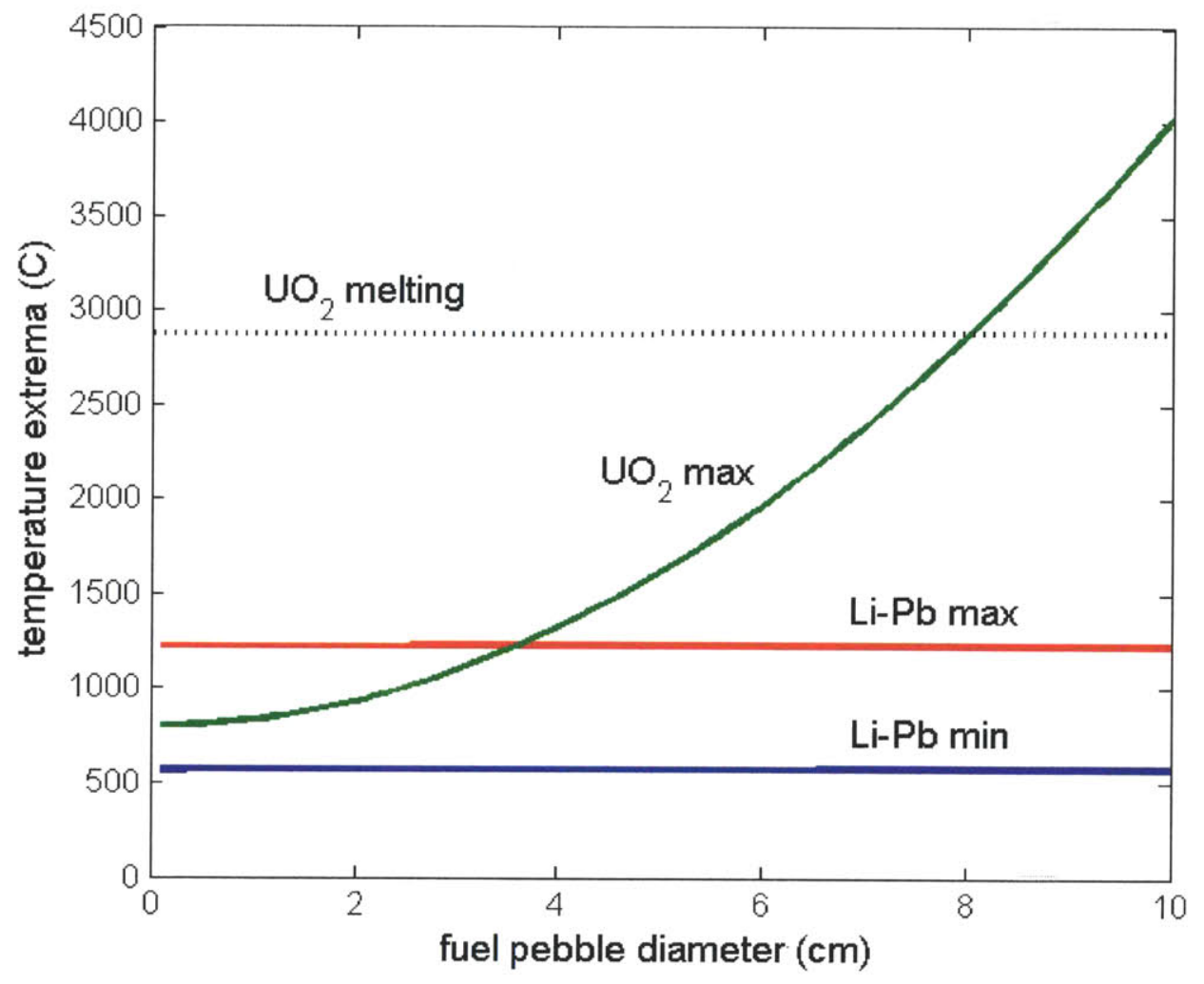

Figure 8.10: Temperature extrema as functions of fuel pebble diameter. Here the ambient pressure is $15 \mathrm{MPa}$, and the helium temperature gain is $500^{\circ} \mathrm{C}$. In this model, pebble size has no effect on the $\mathrm{Li}-\mathrm{Pb}$ temperatures. 


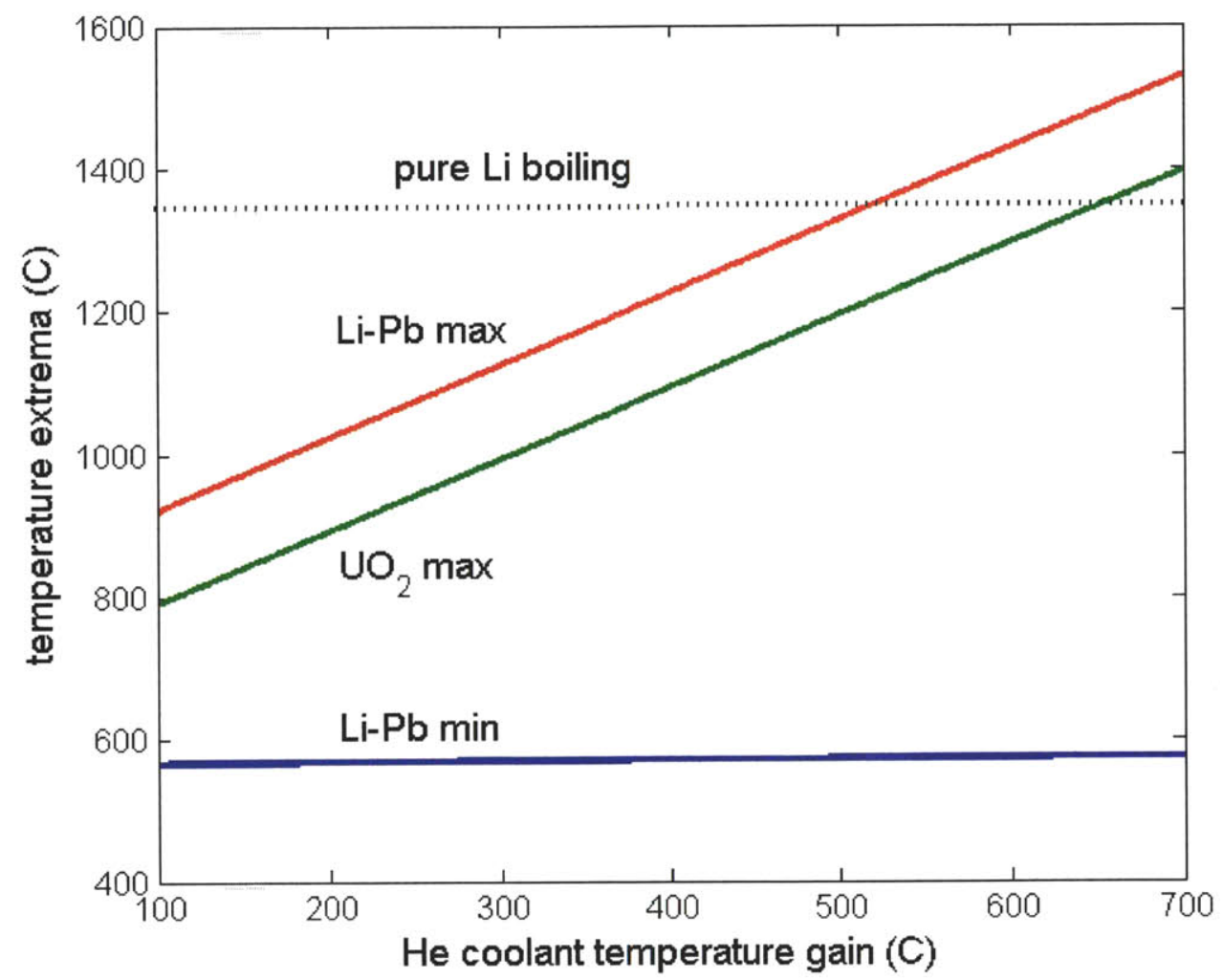

Figure 8.11: Temperature extrema as functions of helium temperature gain. Here the ambient pressure is $15 \mathrm{MPa}$, and the fuel pebble diameter is $3 \mathrm{~cm}$. 


\subsubsection{Summary}

The coolant is hot. This is a direct result of the high $\mathrm{Li}-\mathrm{Pb}$ melting temperature. Pure lithium would solve the problem, but that is a safety question that we cannot address in the scope of this thesis. Ideally, the lithium would be in some solid ceramic with a very high melting temperature. However, that would pose neutronics problems, as the lithium atom density must remain quite high in order to breed

Despite these difficulties, we have succeeded in demonstrating that the thermal hydraulics for this device are indeed feasible. The ambient pressure would need to be quite high $(\approx$ $20 \mathrm{MPa}$ ), and the helium temperature range would need to be $500-950^{\circ} \mathrm{C}$. Under these conditions, the helium velocity could be as low as $10 \mathrm{~m} / \mathrm{s}$. A pebble diameter in vicinity of 2 or $3 \mathrm{~cm}$ would be suitable. Larger is preferable, but it is prudent to keep the pebble size small relative to the entire pebble bed thickness $(\approx 20 \mathrm{~cm})$.

Table 8.1: Pebble Bed Blanket Thermal Hydraulic Parameters

$$
\begin{array}{cc}
P_{\text {tot }}=1.7 \mathrm{GW} & q_{\text {fuel }}^{\prime \prime \prime}=25.6 \mathrm{MW} / \mathrm{m}^{3} \\
T_{\text {in }}=375^{\circ} \mathrm{C} & T_{\text {out }}=825^{\circ} \mathrm{C} \\
p_{0}=25 \mathrm{MPa} & \Delta p / p_{0}=9 \times 10^{-4} \\
\dot{m}=730 \mathrm{~kg} / \mathrm{s} & \dot{V}=36 \mathrm{~m}^{3} / \mathrm{s} \\
\mathrm{Re}=66600 & v=9.7 \mathrm{~m} / \mathrm{s} \\
D_{\text {pebble }}=2.5 \mathrm{~cm}(1 \mathrm{inch}) & T_{\max , \mathrm{UO}_{2}}=1040^{\circ} \mathrm{C} \\
T_{\min , \mathrm{Li}-\mathrm{Pb}}=550^{\circ} \mathrm{C} & T_{\max , \mathrm{Li}-\mathrm{Pb}}=1250^{\circ} \mathrm{C}
\end{array}
$$




\subsection{Brayton Power Cycle and Electric Power}

Now that we have expounded on the helium coolant flow through the fission core, it would be prudent to perform some rudimentary analysis on the power conversion cycle. Since the coolant is a gas, so a Brayton cycle is the natural choice. Figure 8.12 shows a schematic and T-S diagram for a typical Brayton cycle with real components and duct pressure losses. We will assume that the reader is familiar with this and not bother to review basic thermodynamic cycles.

For the sake of simplicity commensurate with the rest of our thermal hydraulic analysis, we will assume an ideal Brayton cycle. This differs from Figure 8.12 in that (1) the transitions $1 \rightarrow 2$ and $3 \rightarrow 4$ are isentropic and (2) the transitions $2 \rightarrow 3$ and $4 \rightarrow 1$ are isobaric. In practice, the cycle might also include complications such as regeneration, reheating, or intercooling, but we will keep things simple. With only two pressures $p_{1}$ and $p_{2}$, the compression ratio $r_{p}$ is $p_{2} / p_{1}$. A standard textbook result is that the efficiency $\eta$ of an ideal Brayon cycle is

$$
\eta=1-r_{p}^{\frac{1-\gamma}{\gamma}}
$$

Here $\gamma=c_{p} / c_{v}=1.658$ for helium. Another standard result is the optimal compression ratio, the value of $r_{p}$ that yields the maximum $\eta$ for a fixed temperature difference.

$$
\left(r_{p}\right)_{\text {optimal }}=\left(\frac{T_{3}}{T_{1}}\right)^{\frac{\gamma}{2(\gamma-1)}}=\left(\frac{T_{3}}{T_{2}}\right)^{\frac{\gamma}{\gamma-1}}
$$

In this case, $T_{1}=375^{\circ} \mathrm{C}$ and $T_{2}=815^{\circ} \mathrm{C}$ so that $\left(r_{p}\right)_{\text {optimal }}=3.8$. Since our reactor core operates at $p_{2}=25 \mathrm{MPa}$, the heat exchanger pressure $p_{1}$ must be $6.6 \mathrm{MPa}$. This yields an efficiency of $41 \%$. Consequently, our minimum-scale steady-state L-mode hybrid will have an electrical power output of about $5 \mathrm{GW}$. This is too large for the current U.S. electrical grid, but, as we discussed in Section 6.5, this unfortunate conclusion is emblematic the conundrum of stable tokamak power production. 
Table 8.2: Ideal Brayton Cycle Parameters

$$
\begin{array}{cc}
T_{2}=375^{\circ} \mathrm{C} & T_{3}=825^{\circ} \mathrm{C} \\
T_{1}=110^{\circ} \mathrm{C} & T_{4}=375^{\circ} \mathrm{C} \\
p_{1}=6.6 \mathrm{MPa} & p_{2}=25 \mathrm{MPa} \\
r_{p}=3.8 & \gamma=1.658 \\
\dot{Q}_{R}=1.7 \mathrm{GW} & \dot{Q}_{H X}=1.0 \mathrm{GW} \\
\dot{W}_{T}=1.7 \mathrm{GW} & \dot{W}_{C P}=1.0 \mathrm{GW} \\
\eta=0.41 &
\end{array}
$$



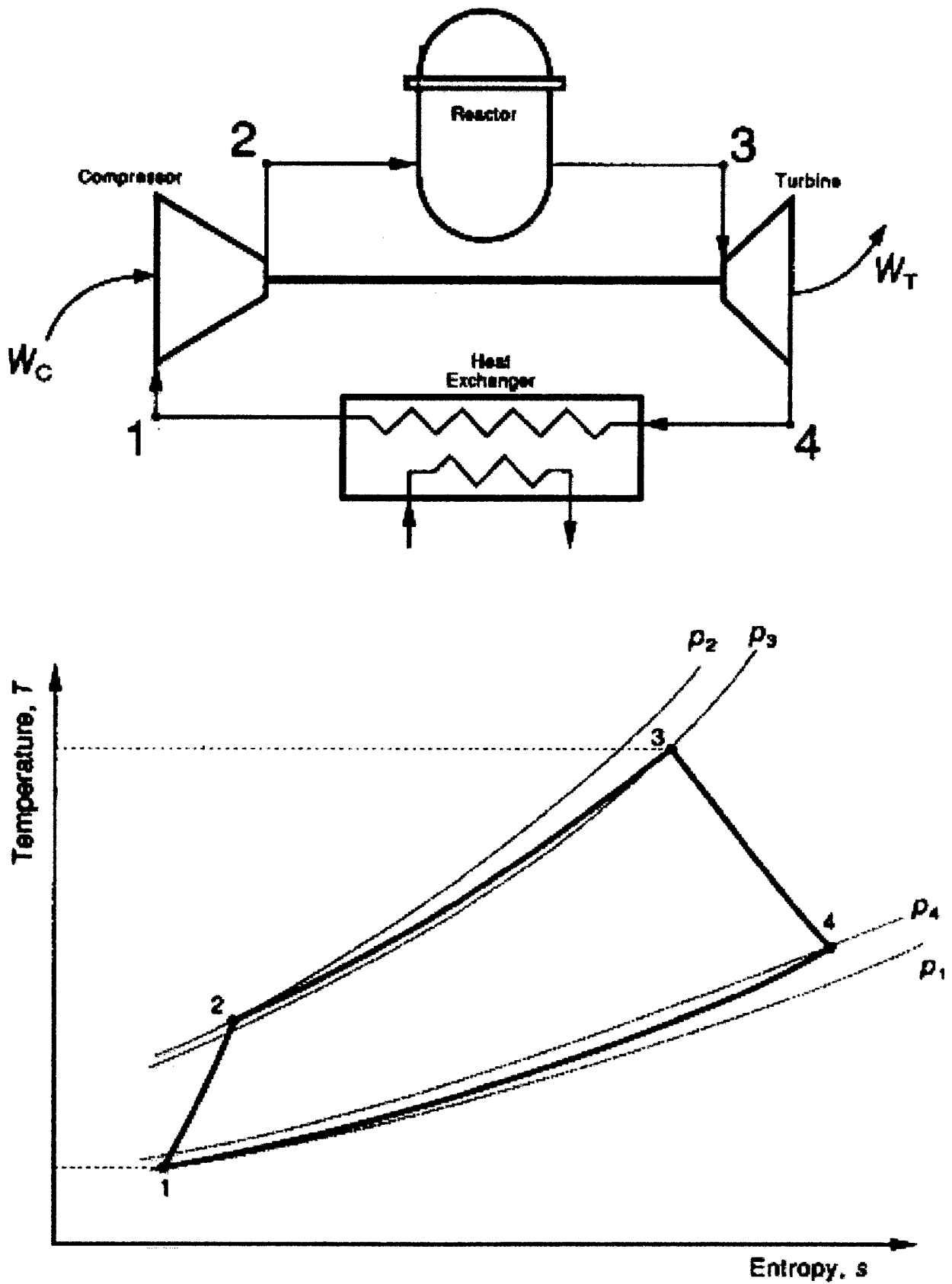

Figure 8.12: A generic Brayton power cycle with real components and duct pressure losses[29]. 


\subsection{Safety}

Here we will briefly discuss safety and address generic accident scenarios.

This fission-fusion hybrid is invulnerable to criticality accidents, as $k_{\text {eff }}$ is initially around 0.3 and never nears 1.0 despite prodigious ${ }^{239} \mathrm{Pu}$ breeding. No reactivity excursion could likely cause the fission blanket to go critical. There are no control rods, because the fusion reaction directly and entirely controls the fission reaction. This is the main safety advantage of subcritical hybrids over any critical fission reactor. In the event of a loss of coolant accident (LOCA) or loss of flow accident (LOFA), we should immediately quench the fusion reaction. This will completely shut down the fission chain reaction with virtually no chance of any lingering reactivity.

Nevertheless, the fuel will still generate decay heat following an emergency shutdown. In conventional pebble beds, the graphite matrix is virtually impervious to melting (carbon has the highest melting point of any pure element), and LOCA temperature transients have been analyzed in detail [44]. However, our pebbles are spheres of $\mathrm{UO}_{2}$. These spheres will cool more rapidly than traditional cylindrical $\mathrm{UO}_{2}$ fuel elements by virtue of geometry, but it is plausible that they could still melt if the helium coolant were to stagnate or depressurize. As we explained in Section 8.2 and will elaborate on in Section 9.1.1, $\mathrm{UO}_{2}$ spheres are strongly preferable over graphite matrix spheres due to their higher homogenized uranium atom density, to which the fission power multiplication is directly proportional. Thus, we are presented with a trade off. With a graphite matrix, we achieve passive safety but must settle for relatively low power gain. With $\mathrm{UO}_{2}$, we achieve superior power gain but must implement active safety systems. In this thesis, we choose the $\mathrm{UO}_{2}$ option, because we are primarily concerned with maximizing power gain. 


\section{Ramifications}

Our analysis is complete, and we have thoroughly discussed the advantages and disadvantages of various aspects of our conceptual design. Now we will compare it to other hybrid designs and put it into the context of the current hybrid debate.

\subsection{Comparison to Other Studies}

As we discussed in our introduction, there has been a renewed interest in fission-fusion hybrids within the past decade and more intensely within the past few years. Here we will discuss a few other of these design proposals in more detail and compare them to ours.

\subsubsection{Tokamak Pebble Bed Hybrids (ITER-PBR)}

We will begin with hybrid configurations most similar to ours - tokamaks with pebble bed blankets. Vincent Tang wrote a master's thesis in 2002 at MIT entitled "Preliminary Design of a Fusion-Fission Tokamak Pebble Bed Reactor" [22]. His proposed design, though also a tokamak with a pebble bed blanket, differs from ours in many ways. First, he uses lithium titanate $\left(\mathrm{Li}_{2} \mathrm{TiO}_{3}\right)$ pebbles for tritium breeding such that the device has two pebble bed zones. Second, he places these breeder pebbles between the plasma and the fissionable fuel pebbles. We have shown that this is not optimal (see Section 3.1.4), but he makes it work by adding beryllium to the breeder pebbles as a neutron multiplier. Third, he stipulates that the fissionable pebbles be comprised of spent fuel from the Pebble Bed Modular Reactor (PMBR). This spent fuel contains $96.3 \%{ }^{238} \mathrm{U}$ and $1.3 \%{ }^{235} \mathrm{U}$ by mass, so it is not too much unlike our choice of natural uranium. The remainder is $1.1 \%{ }^{236} \mathrm{U}$ and traces of a few plutonium isotopes. Tang's goal is for the hybrid to perform a deep burn of this spent fuel to extract more energy from it and dramatically reduce waste storage area. Fourth, he has his fuel in tristructural-isotropic (TRISO) particles embedded in a larger graphite matrix pebble, just as in the PBMR. Fifth, he does not perform any fusion scaling analysis - he assumes all the properties of ITER.

Tang achieves a fission blanket multiplication of no greater than 3.9, which differs significantly from our value of 7.7 (or 6.1, including the fusion $\alpha$-particle power). We can determine the reason for this disparity by comparing the fusion neutron multiplication $k_{0}$ and the fission neutron multiplication $k$. Tang's thesis has a $k$ value of 0.26 , virtually the same as our value of 0.27 . Thus, there is no substantial difference in fission neutron multiplication. However, Tang's $k_{0}$ value is a mere 0.63 compared to our 1.19. Now let us look at $Q_{\text {fis }}$ as a function of $k$ and $k_{0}$ in Equation 9.1. This is similar to Equation 7.2 and arises from the discussion in Section 7.2. Essentially, this describes Figure 3.13. 


$$
Q_{\text {fis }}=\left[\frac{193.9}{14.1}\right] k_{0}\left[\frac{1}{\bar{\nu}_{0}}+\frac{1}{\bar{\nu}}\left(\frac{1}{1-k}-1\right)\right]
$$

Here $\bar{\nu}_{0}$ and $\bar{\nu}$ are the average $\nu$ values for the fusion and fission neutrons, respectively. The main thing to take away from this is that the fission multiplication (and the total hybrid power) is proportional to $k_{0}$. We can easily show this, because the ratio of Tang's $k_{0}$ to our $k_{0}$ is nearly equal to the ratio of our $Q_{\text {fis }}$ to Tang's $Q_{\text {fis }}(0.63 / 1.19 \approx 3.9 / 7.7)$. The reason for Tang's much lower $k_{0}$ is that he uses graphite matrix pebbles, while we use $\mathrm{UO}_{2}$ pebbles. Since the fusion neutrons mostly bombard the uranium layer from one side, $k_{0}$ is highly dependent on the macroscopic fission cross-section at high energies. We have a much higher uranium atom density than Tang, and so our $k_{0}$ is much higher. This is the reason why we devoted so much discussion to the pebble structure - a high fissionable atom density is crucial for hybrids to achieve high power gain. TRISO particles embedded in graphite pebbles serve the purposes of critical reactors, but this is not a critical reactor - it is a bombardment.

This serves to show that subcritical hybrid reactors will usually require different fuel than critical fission reactors. In hybrid reactors, all neutron generations are not created equal as they are in fission reactors. The fusion-born neutrons are all-important to achieving a high power gain, and the fissionable atom density must be high along their path.

Table 9.1 summarizes the main advantages and disadvantages of graphite matrix and $\mathrm{UO}_{2}$ pebbles. Favorable properties are blue, while unfavorable properties are red. Beyond power gain, a number of other factors come into play. Even with helium coolant, the graphite matrix pebbles will moderate the neutrons to some extent. Tang classifies his spectrum as epithermal, while our spectrum is unequivocally fast. Graphite pebbles are passively safe, while $\mathrm{UO}_{2}$ pebbles might require active safety systems (such as emergency core cooling). TRISO particles have SiC coating, which contains fission products. As we discussed in Section 8.1, $\mathrm{UO}_{2}$ pebbles might require fission product release into the coolant, which would require removal systems. The other option is UN pebbles, which would have the same homogenized uranium atom density as $\mathrm{UO}_{2}$ pebbles but could contain fission products within a hollow center. In terms of manufacturing, both choices come with disadvantages. TRISO particles and graphite matrix pebbles require a complex manufacturing process, while perforated $\mathrm{SiC}$ cladding (or any other kind of pebble cladding) would have its own technical challenges. 
Table 9.1: Comparison of Graphite Matrix Pebbles to $\mathrm{UO}_{2}$ Pebbles

\begin{tabular}{|c|c|}
\hline graphite matrix & $\mathrm{UO}_{2}$ \\
\hline low power gain & high power gain \\
\hline soft spectrum & hard spectrum \\
\hline passive safety & active safety \\
\hline $\begin{array}{l}\text { fission products } \\
\text { contained within fuel }\end{array}$ & $\begin{array}{l}\text { fission product } \\
\text { removal necessary }\end{array}$ \\
\hline $\begin{array}{c}\text { complex fuel } \\
\text { manufacturing process }\end{array}$ & $\begin{array}{c}\text { complex cladding } \\
\text { manufacturing process }\end{array}$ \\
\hline
\end{tabular}




\subsubsection{Other Tokamak Hybrids (SABR)}

At the Georgia Institute of Technology, Professor Weston Stacey has led a number of interesting tokamak hybrid projects over the past decade. This most recent design is the Subcritical Advanced Burner Reactor (SABR), which differs substantially from the pebble blanket concept [32]. The SABR is essentially a slightly subcritical $(k=0.9$ to 0.95$)$ fast reactor arranged within an annulus around the outside of a tokamak core. The fuel is in standard rod configuration with sodium coolant so that the fission technology is not substantially different than what has already been studied in depth. Figure 9.1 shows a simple schematic of this.

The fission gain of SABR is given as a range anywhere from 6 to 30 . They did not analyze their neutronics in terms of $k_{0}$ and $k$, but we can do it for them. Supposing that $k$ is set at an optimistic 0.95 , the gain from all fission-born neutrons is $1 /(1-k)-1=19$. Then, given the total gain range of 6 to 30 , the corresponding $k_{0}$ range is approximately 0.07 to 0.35 . The relatively low $k_{0}$ is reasonable, given that the geometry of SABR is less favorable (in terms of capturing the fusion-born neutrons) than the pebble blanket designs. They state that only $39 \%$ of the fusion-born neutrons even reach the fission core, and so the fraction that actually spur fission must be substantially lower than that. Of the fusion-born neutrons that reach the uranium layer in our design, less than half spur fission.

The main drawback of SABR relative to our design is that the fission component is very similar to a pure fission reactor. In order to achieve $k=0.95$, substantial uranium enrichment is required. Even if SABR is intended primarily for waste transmutation, then it is part of a fuel cycle that requires enrichment. However, as we discussed in Section 7.8, our design could actually comprise an entire fuel cycle without enrichment. It begins with natural or depleted uranium as fresh fuel, produces a huge amount of commercial power, transmutes most of the hazardous waste as it is created, and could even breed fissile fuel for other purposes. Though it is difficult to compare two devices that are intended for different purposes, we feel that $k=0.95$ does not take full advantage of the flexibility that subcritical operation allows for. 


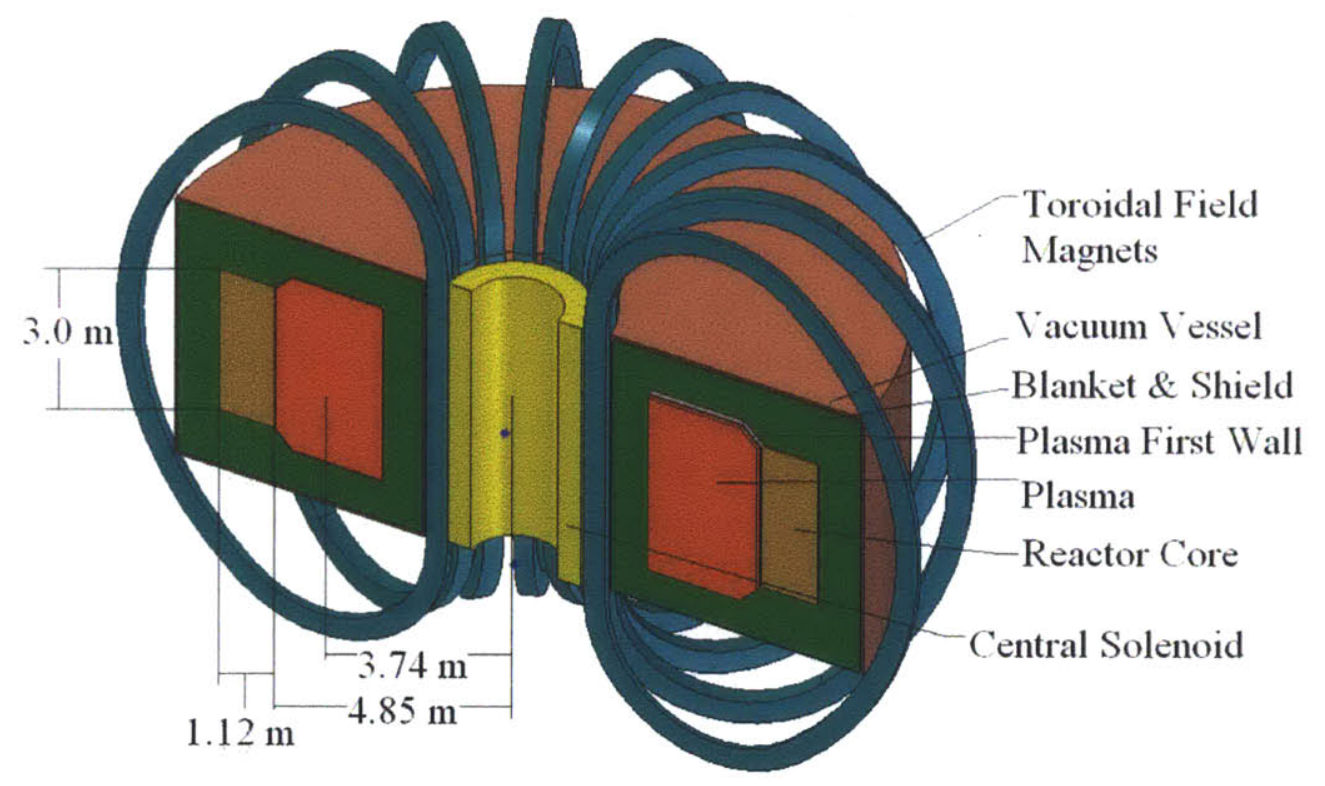

Figure 9.1: A schematic of the Subcritical Advanced Burner Reactor (SABR) conceived at the Georgia Institute of Technology [31]. 


\subsubsection{Inertial Confinement Hybrids (LIFE)}

The Lawrence Livermore National Laboratory (LLNL) in association with the University of California at Berkeley has conceived and designed the Laser Inertial Confinement FusionFission Energy (LIFE) Reactor [31]. See Figure 9.2, which happens to be glorious. Lasers induce fusion through inertial confinement in the hollow center, while the spherical shell contains natural uranium. The primary coolant is $2 \mathrm{LiF}^{-B e F_{2}}$ (FLiBe) molten salt, which doubles as a tritium breeder (and as a neutron poison in the case of ${ }^{6} \mathrm{Li}$ ). They also employ $\mathrm{Li}_{17} \mathrm{~Pb}_{83}$ as a first-wall coolant and additional tritium breeder.

As inertial confinement is fundamentally different than magnetic confinement, we will not compare the fusion component of this design to ours.

LIFE touts a $500 \mathrm{MW}$ fusion source with a depleted uranium blanket power multiplication of 4 to 8 . It can operate at a total power of $2 \mathrm{GW}$ for 50 years and burn $99 \%$ of all actinides. They bolster these lofty claims with some impressive neutronics burnup analysis. Initially, the fission blanket contains almost entirely depleted uranium. The spectrum is thermal. As burnup proceeds, the blanket breeds large quantities of ${ }^{239} \mathrm{Pu}$, and the power increases until it reaches a peak at around 10 years. Subsequently, the power slowly declines for the rest of the lifetime. In order to maintain constant power, they propose varying the concentration of ${ }^{6} \mathrm{Li}$, which is a neutron poison at thermal energies.

The interesting point here is that LIFE has a maximum depleted uranium power gain of 8 , which is consistent with our natural uranium power gain of 7.7. Of course, the geometry is spherical as opposed to toroidal, and there are different material selections. However, the same basic concept of a primarily ${ }^{238} \mathrm{U}$ blanket nearly completely enclosing a $14 \mathrm{MeV}$ neutron source is the same. Thus, we cite this as a corroborating data point. 


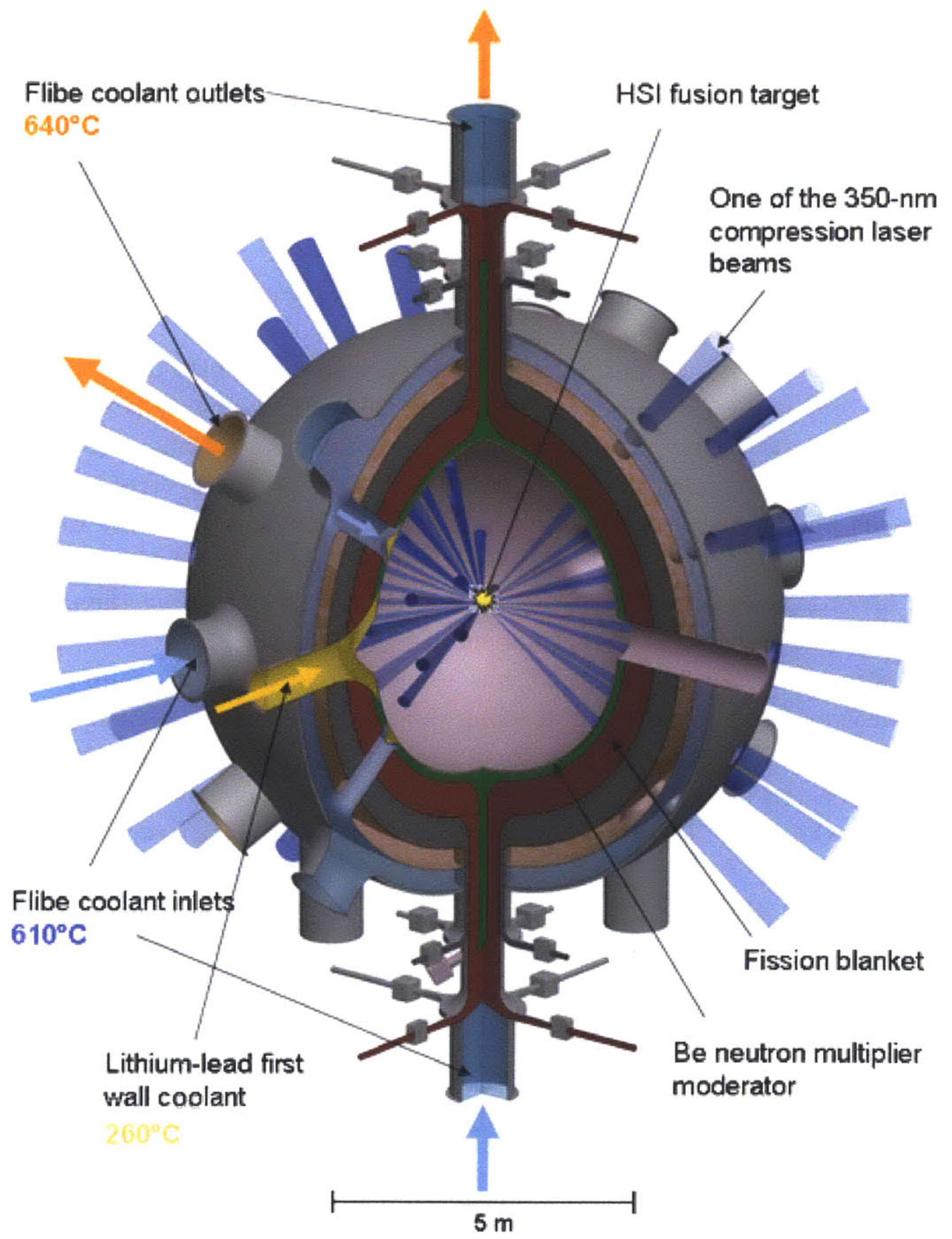

Figure 9.2: A schematic of the Laser Inertial Confinement Fusion-Fission Energy (LIFE) Reactor conceived at Lawrence Livermore National Laboratory (LLNL). 


\subsection{The Hybrid Debate}

In the fall of 2009, the U.S. Department of Energy's Office of Fusion Energy Sciences sponsored the "Research Needs for Fusion-Fission Hybrids" conference in Gaithersburg, Maryland [21]. This generated a report that was a part of the much larger Report of the Research Needs Workshop (ReNeW). This conference assessed the current state of hybrid research, the pros and cons of hybrids, and future research needs.

This conference included a panel of skeptics who highlighted the major challenges and drawbacks of fission-fusion hybrids. One of their main conclusions was that hybrids would complicate many current challenges of both fission and fusion. We have already discussed this issue at some length in Section 1, and the primary motivation for this entire thesis was to show that fission and fusion can be mutually beneficial in a hybrid relationship.

They also suggest that fast reactors can accomplish everything that hybrids can in terms of fissile fuel breeding and waste transmutation. This is true, but fast reactors accomplish these two missions far less effectively than hybrids. For example, we determined (in Section 7.3) that a typical fast reactor can breed ${ }^{239} \mathrm{Pu}$ with a conversion ratio of 1.2 . In sharp contrast, our hybrid can do so with a conversion ratio of 22! We also determined (in Section 7.6) that our hybrid can also transmute long-lived fission product waste more effectively than a typical fast reactor.

The third and final point we wish to contest is that of non-proliferation. The hybrid skeptics express concern that hybrids would pose a significant proliferation risk beyond that of pure fission reactors. While it is certainly true that proliferation would be more of a concern for hybrids than for light water reactors, we have shown (in Section 7.5) that hybrids would breed plutonium that contains larger portions of ${ }^{238} \mathrm{Pu}$ and ${ }^{240} \mathrm{Pu}$ than that bred by a typical fast reactor. Also, the absence of enrichment is favorable for non-proliferation. Although we would need to perform much more detailed fuel cycle analysis to state with confidence that hybrids are more favorable than fast reactors in this respect, it is premature to assume that hybrids will pose an elevated proliferation risk.

The ReNew hybrid report closes with a set of high-level research needs, which includes a fuel cycle comparison of hybrid systems to pure fission systems. We took the first step in this direction by comparing hybrids to thermal and fast reactors in the areas of fissile fuel breeding, waste transmutation, and non-proliferation. Although our analysis was quite basic, our findings suggest that hybrids could facilitate a more favorable fuel cycle than fast reactors. This should be the primary focus of future hybrid research.

\subsection{Overarching Conclusions}

Fission-fusion hybrids have the potential to ease the challenges of both fission and fusion by actualizing steady-state L-mode operation on the fusion side and subcritical natural 
or depleted uranium burning on the fission side. We have identified a maximum natural uranium tokamak blanket multiplication of 7.7, and we have also identified a corresponding steady-state L-mode tokamak minimum scale of 5.2 meters (5/6 the size of ITER). We are not so presumptuous as to declare this the long-hoped-for panacea of nuclear energy, but we do contend that it could be superior to pure fission systems while mitigating some of the most challenging aspects of fusion.

Furthermore, we have demonstrated that pressurized helium could cool the fission blanket with a flow rate of less than 10 meters per second. We have also performed basic fuel cycle analysis to show that this hybrid could be superior to pure fission reactors for the alternative missions of fissile fuel breeding and waste transmutation. Surprisingly, there could even be some non-proliferation advantages. Any future work we conduct on this subject will undoubtedly focus on its fuel cycle implications.

We dub this device the Steady-State L-Mode Non-Enriched Uranium Tokamak Hybrid (SLEUTH). Although no subterfuge was involved in the production of this work, we hope this serves as both a sobriquet and a mnemonic. We hope this work spurs interest in and further research on fission-fusion hybrids. Perhaps, incidentally, it will even spur a profusion of pro-fusion sentiment! 


\section{References}

[1] Bromberg, L., et. al. "Options for the use of high temperature superconductor in tokamak fusion reactor designs". Fusion Engineering and Design 54: 167-180. (2000).

[2] Campbell, M. and K. Schultz. "Fusion as a Source for Hydrogen Production". General Atomics. Fusion Power Associates Meeting. (2004).

[3] Chen. F. Plasma Physics and Controlled Fusion, Vol. 1, 2nd Ed. Springer (2006).

[4] Chow, J., et. al. "Large Scale Economic Production of Hydrogen Fuel from a SteadyState Tokamak Fusion Reactor: HYPERION". The MIT Nuclear Science and Engineering Department. Nuclear Systems Design Project. (2008).

[5] Dahlgren, F., et al. "ARIES-AT Magnet Systems". Fusion Engineering and Design 80: 139-160. (2006).

[6] Erckmann, V. and U. Gasparino. "Electron-cyclotron resonance heating and current drive in toroidal fusion plasmas". Plasma Physics and Controlled Fusion 36: 1869-962. (1994).

[7] Freidberg, J. Plasma Physics and Fusion Energy. Cambridge University Press (2007).

[8] Greenwald, M., et. al. Nuclear Fusion 37. (1997).

[9] Harvey, R. W., et. al. "Eletron cyclotron heating and current drive in ITER". Nuclear Fusion 37: 69-103. (1997).

[10] Huba, J.D. 2007 NRL Plasma Formulary. U.S. Naval Research Laboratory (2007).

[11] ITER choice of parameters. The ITER Project. (2009).

[12] Jardin, S. C., et.al. "Physics basis for a tokamak fuision power plant". Fusion Engineering and Design 48: 281-298. (2000).

[13] Raffray, A. R., et. al. "High performance blanket for ARIES-AT power plant". Fusion Engineering and Design 58-59: 549-553. (2001).

[14] Sheffield, J., et. al. "A study of options for the deployment of large fusion power plants". Fusion Science and Technology 40: 1-36. (2001).

[15] Wesson, J. and D. J. Campbell. Tokamaks. Oxford University Press. (2004).

[16] Ryu K. and H. Sekimoto. "A possibility of highly efficient uranium utilization with a pebble bed fast reactor". Annals of Nuclear Energy 27: 1139-45 (2000). 
[17] Achenbach, E. "Heat and Flow Characteristics of Packed Beds". Experimental Thermal and Fluid Science 10: 17-27 (1995).

[18] David, S., et. al. "Fast subcritical hybrid reactors for energy production: evolution of physical parameters and induced radiotoxicities". Nuclear Instruments and Methods in Physics Research A 443: 510-30 (2000).

[19] Brudieu, M. and A. Kadak. "Blind Benchmark Predictions of Nacok Air Ingress Tests Using Computational Fluid Dynamics". Proceedings HTR2006: $3^{\text {rd }}$ International Topical Meeting on High Temperature Reactor Technology, Johannesburg, South Africa (2006).

[20] Okamoto, H. "Li-Pb (Lithium-Lead)". Journal of Phase Equilibria Vol. 14 No. 6 (1993).

[21] Freidberg, J., et. al. "Research Needs for Fusion-Fission Hybrid Systems". Report of the Research Needs Workshop (ReNeW), Gaithersburg, Maryland (2009).

[22] Tang, V. and R. Parker. "Preliminary Design of a Fusion-Fission Tokamak Pebble Bed Reactor". Master of Science Thesis, Department of Nuclear Science and Engineering, Massachusetts Institute of Technology (2002).

[23] Song, C., P. Wang, and H. Makse. "A phase diagram for jammed matter". Nature 453: 629-32 (2008).

[24] Jaunch, U. and B. Schulz. "Thermophysical Properties in the Li-Pb System: Part III: Estimation of the thermophysical properties in the system Li-Pb". Kernforschungszentrum Karlsruhe 4144 (1986).

[25] Petersen, H. "The Properties of Helium: Density, Specific Heats, Viscosity, and Thermal Conductivity at Pressures from 1 to 100 bar and from Room Temperature to about 1800 K". Danish Atomic Energy Commission (1970).

[26] Murty, K. L. and I. Charit. "Structural materials for Gen-IV nuclear reactors: Challenges and opportunities". Journal of Nuclear Materials 383: 189-95 (2008).

[27] Salvat, F., et. al. "PENELOPE-2006: A Code System for Monte Carlo Simulation of Electron and Photon Transport". Organization for Economic Cooperation and Development (OECD): Nuclear Energy Agency (NEA) No. 6222 (2006).

[28] Reed, M. and D. Whyte. "A Steady-State L-Mode Tokamak Fusion Reactor: Large Scale and Minimum Scale". Bachelor of Science Thesis, Department of Physics, Massachusetts Institute of Technology (2009). 
[29]. McKee, S. A. and M. J. Driscoll. "Implementation of Vented Fuel Assemblies in the Supercritical $\mathrm{CO}_{2}$-Cooled Fast Reactor". Master of Science Thesis, Department of Nuclear Science and Engineering, Massachusetts Institute of Technology (2008).

[30] Todreas, N. E. and M. S. Kazimi. Nuclear Systems I: Thermal Hydraulic Fundamentals. Taylor \& Francis Group (1990).

[31] Kramer, K. J. "Neutron Transport and Nuclear Burnup Analysis for the Laser Inertial Confinement Fusion-Fission Energy (LIFE) Engine". Fusion Science and Technology 56: 625-31. (2009).

[32] Stacey, W. M., et. al. "A TRU-Zr Metal-Fuel Sodium-Cooled Fast Subcritical Advanced Burner Reactor". Nuclear Technology 162: 53-79. (2008).

[33] Hebert, A. Applied Reactor Physics. Presses Internationales Polytechnique (2009).

[34] Lidsky, L. M. "Fission-Fusion Systems: Hybrid, Symbiotic, and Augean". Nuclear Fusion 15, 151173 (1975).

[35] "Restricted Data Declassification Decisions: 1946 to the Present" (RDD-7). Office of Declassification, U.S. Department of Energy. (2001).

[36] Sanchez, R.G. et. al. "Criticality of a ${ }^{237}$ Np Sphere". Nuclear Science and Engineering 158: 1-14. (2008).

[37] Lavarenne, C., et. al. "Evaluation of nuclear criticality safety data and limits for actinides in transport". Departement de Prevention et D'Etude des Accidents, Institut de Radioprotection et de Surete Nucleaire. (2001).

[38] Friese, J. I., et. al. "Existing Evidence for the Fate of Neptunium in the Yucca Mountain Repository". Pacific Northwest National Laboratory (PNNL-14307). (2003).

[39] Tang, V. and R. Parker. "The Fusion-Fission Hybrid Revisited: Preliminary Analysis of a Fusion Driven Thorium Burner". MIT Plasma Science and Fusion Center. (2001).

[40] Bethe, H. A. "The Fusion Hybrid". Physics Today. (1979).

[41] Ragheb, M. and A. Nour Eldin. "The Fusion-Fission Thorium Hybrid". 1st Thorium Energy Alliance Conference, Washington, D.C. (2009).

[42] "Nonproliferation and Arms Control Assessment of Weapons-Usable Fissile Material Storage and Excess Plutonium Disposition Alternatives". U.S. Department of Energy. (1997). 
[43] Albright, D. and K. Kramer. "Neptunium 237 and Americium: World Inventories and Proliferation Concerns". Institute for Science and International Security (ISIS). (2005).

[44] Tang, V. and R. Parker. "Temperature transients of a fusion-fission ITER pebble bed reactor in loss of coolant accident". Fusion Engineering and Design 65: 11-26. (2003).

[45] Takibayev, A., et al. "Fusion-Driven Transmutation of Selected Long-Lived Fission Products". Progress in Nuclear Energy 47: 354-60. (2005).

[46] Freidberg, J. and A. Kadak. "Fission-Fusion Hybrids Revisited". Nature Physics 5: 3702. (2009).

[47] Fridman, E, E. Shwageraus, and A. Galperin, "Implementation of Multi-Group CrossSection Methodology in BGCore Monte Carlo-Depletion Code". Proceeding of PHYSOR 2008, Interlaken, Switzerland, September 14-19 (2008). 


\section{A Fusion Model}

\section{A.1 0-D Core Model}

This is a 0-D tokamak fusion core model, which relates various parameters such as $R, a$, $P_{F} / A_{S}, Q$, and $B$. We use this to generate our "plasma phase diagrams" in Section 6 .

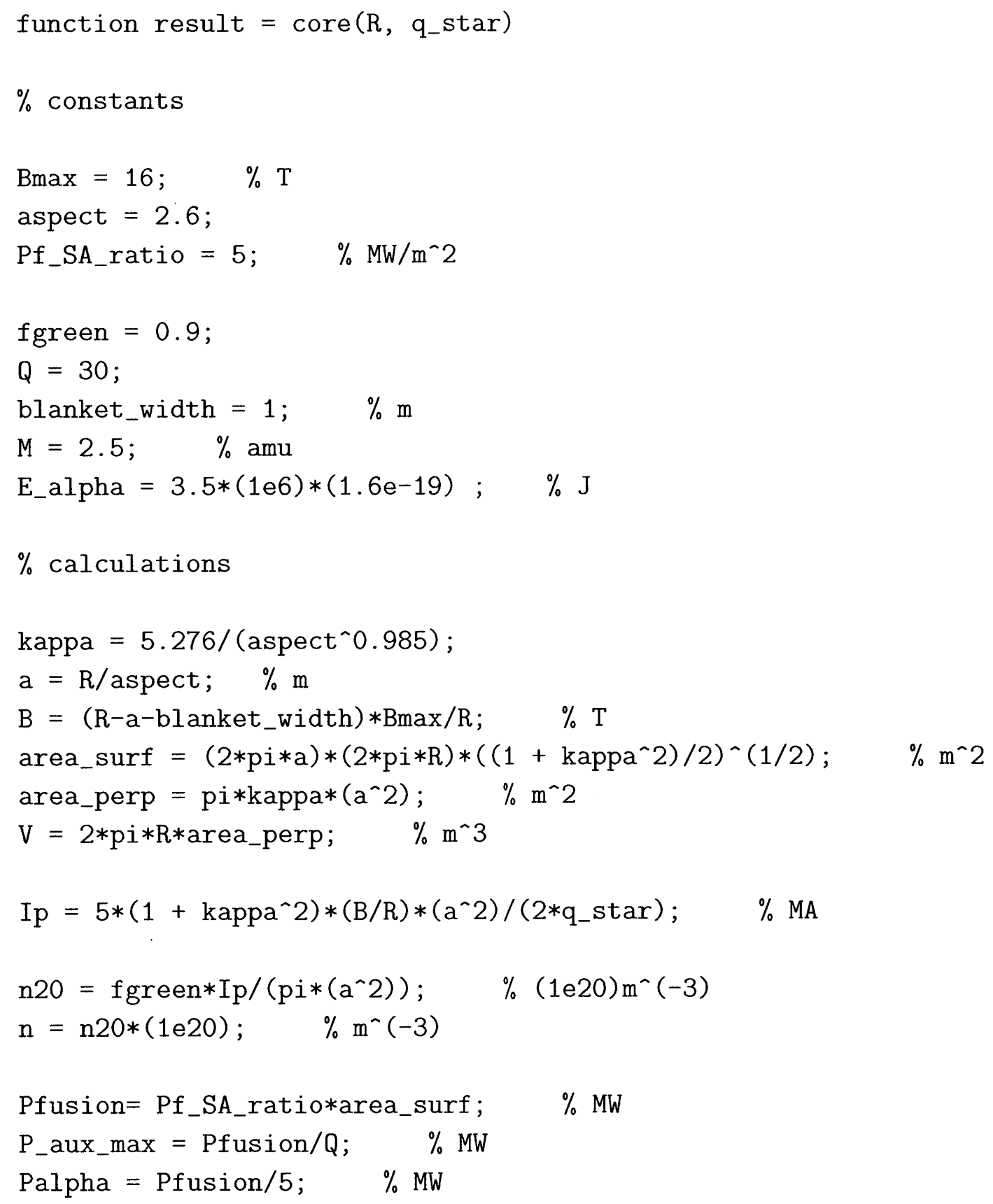




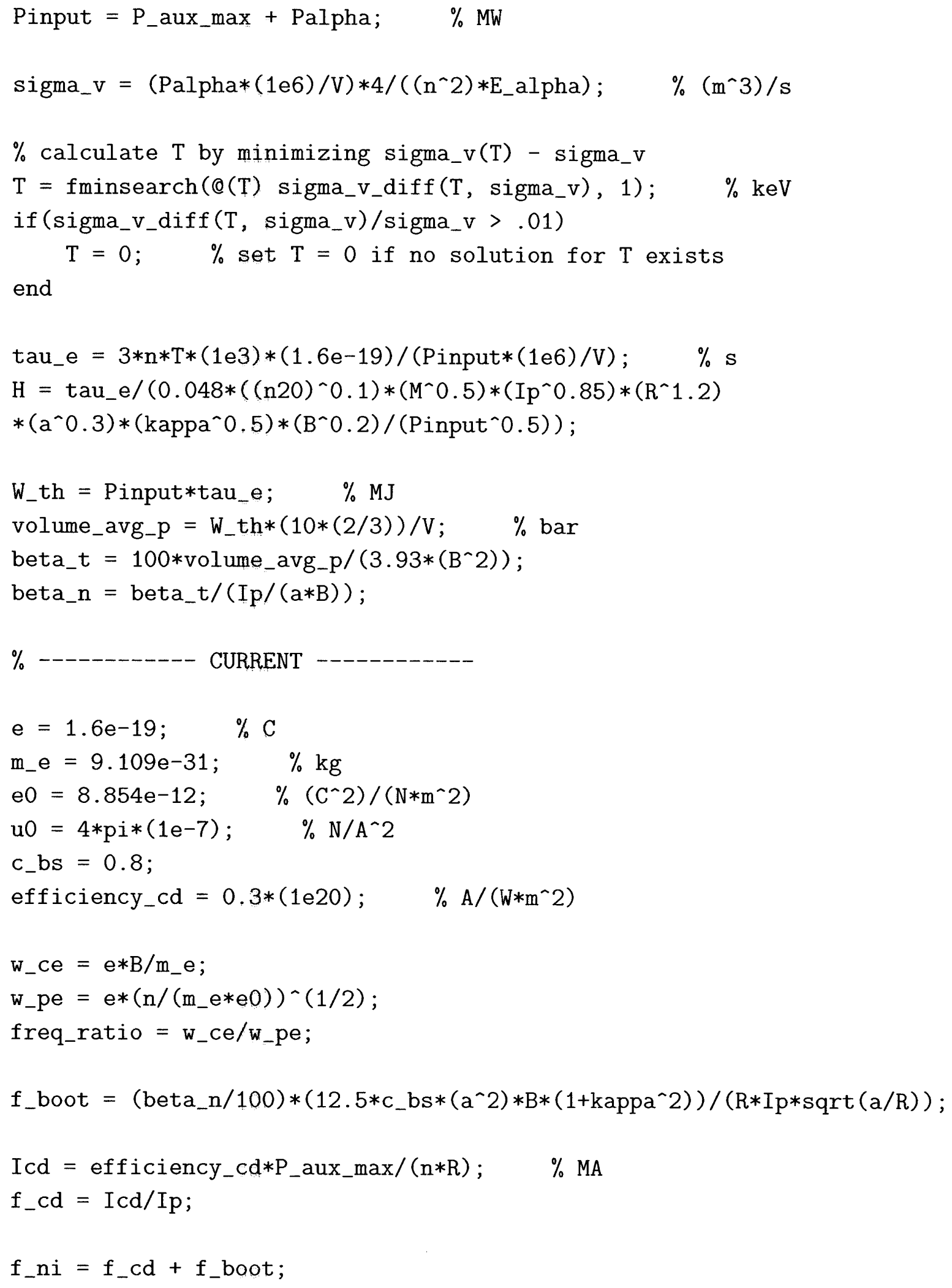




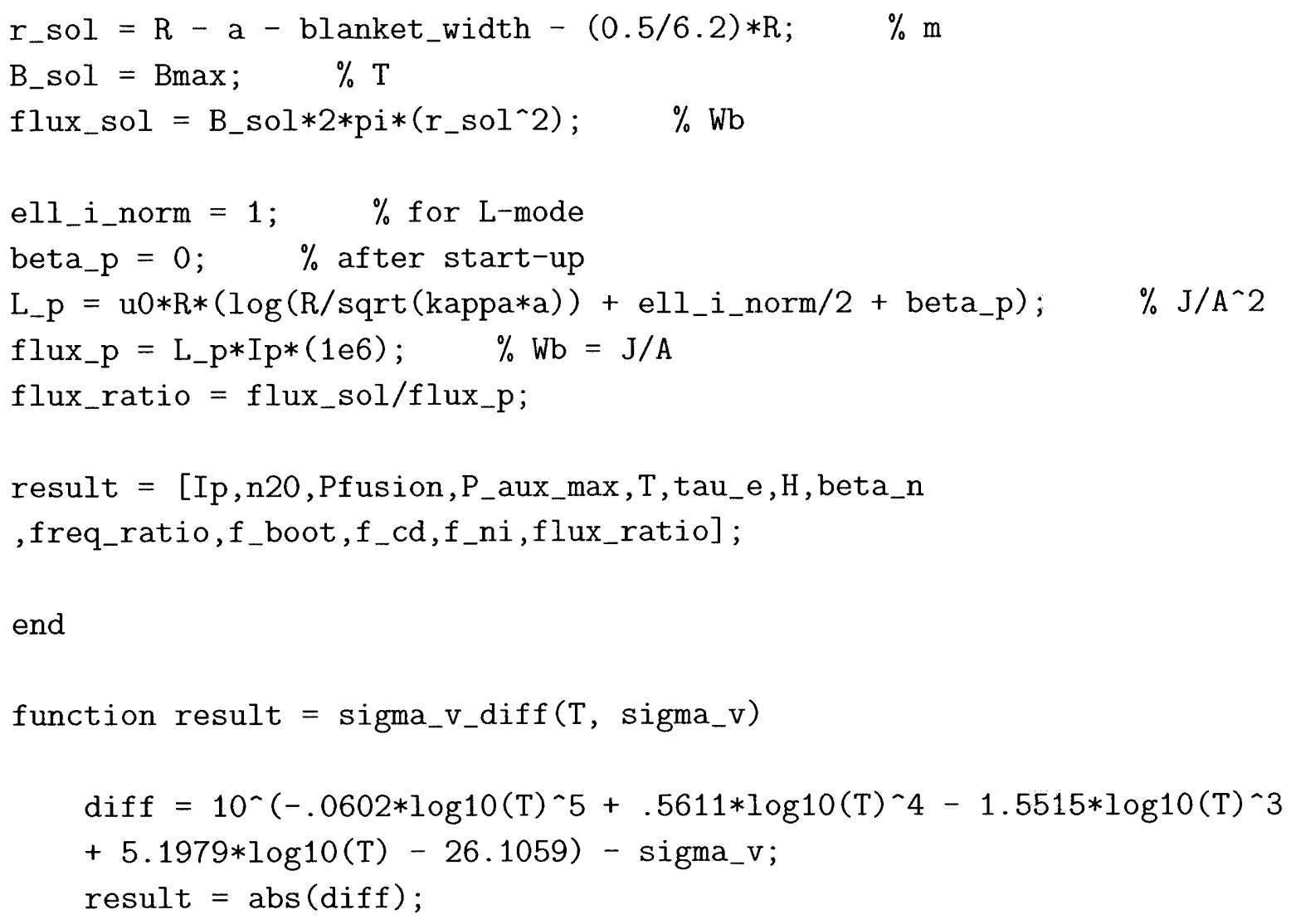

end 


\section{A.2 1-D Density, Temperature, and Power Profiles}

This is a method for computing the density, temperature, and power profiles in a 1-D spherical tokamak core model. This assumes a fixed set of 0-D parameters.

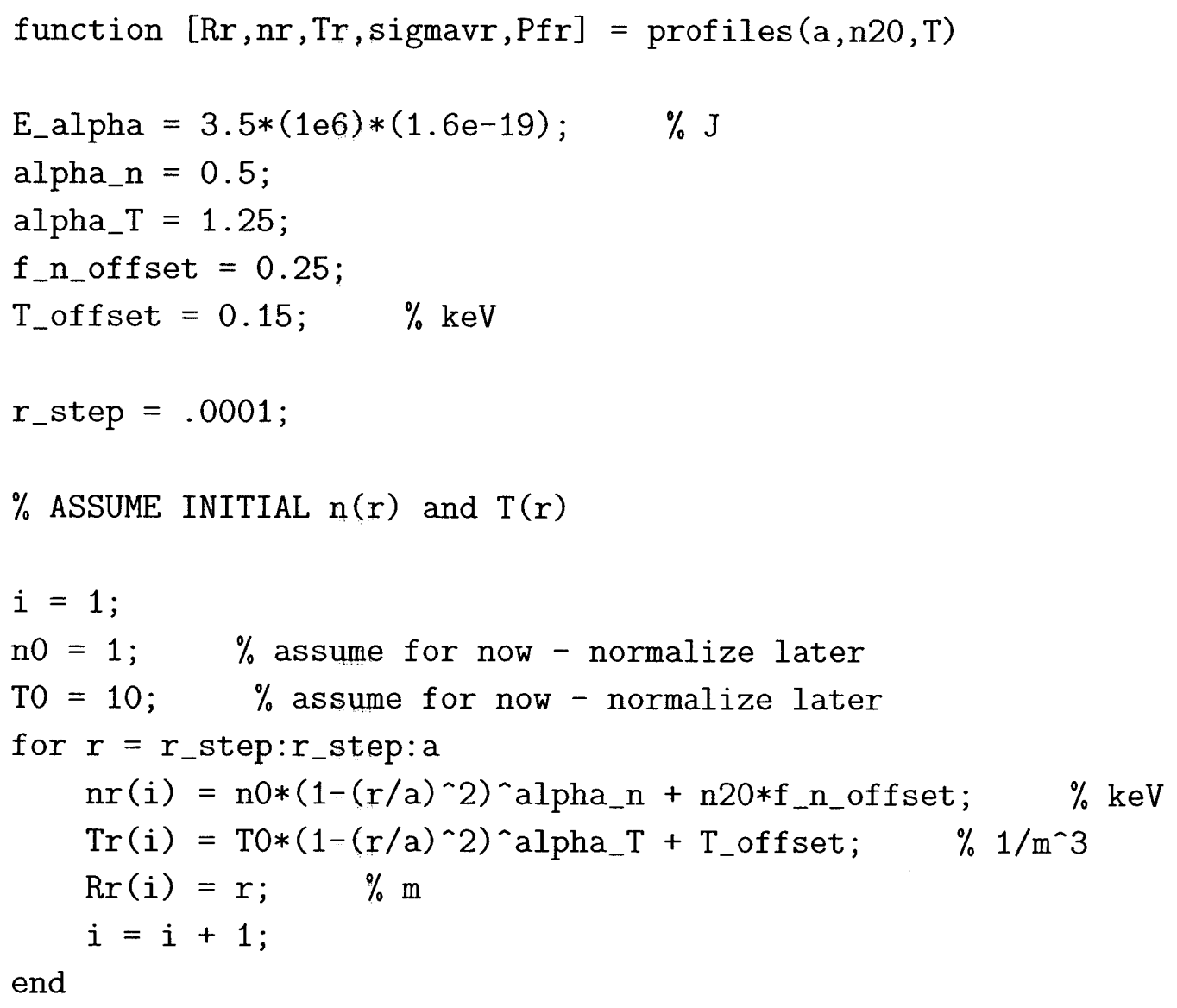

end

$\%$ NORMALIZE $\mathrm{n}(\mathrm{r})$ and $\mathrm{T}(\mathrm{r})$

n_tot $=0$;

T_tot $=0$;

for $i=1:$ length $(\mathrm{R} r)$

part_n $=\operatorname{nr}(i) * 2 * p i * \operatorname{Rr}(i) * r_{-}$step;

part_T $=\operatorname{Tr}(i) * 2 * p i * \operatorname{Rr}(i) * r_{-}$step;

n_tot $=\mathrm{n}_{-}$tot + part_n;

$\mathrm{T}_{\text {_tot }}=\mathrm{T}_{-}$tot + part_T ;

end

$\mathrm{n}_{-} \mathrm{avg}=\mathrm{n}_{-} \mathrm{tot} /\left(\mathrm{pi} *\left(\mathrm{a}^{\wedge} 2\right)\right)-\mathrm{n} 20 * \mathrm{f} \_\mathrm{n}_{-}$offset; 
$T_{-}$avg $=T_{-}$tot $/\left(\mathrm{pi} *\left(\mathrm{a}^{\wedge} 2\right)\right)-\mathrm{T}_{\text {_offset }}$;

norm_const_n0 $=\left(\mathrm{n} 20-\mathrm{n} 20 * \mathrm{f} \_n \_\right.$offset $) / \mathrm{n}$-avg;

norm_const_T0 $=\left(T-T_{-}\right.$offset $) / T \_$avg;

$\mathrm{nr}=\left(\mathrm{nr}-\mathrm{n} 20 * \mathrm{f} \_\mathrm{n} \_\right.$offset $) *$ norm_const_no $+\mathrm{n} 20 * \mathrm{f} \_\mathrm{n}$ off set;

$\operatorname{Tr}=\left(\operatorname{Tr}-T_{-}\right.$offset $) *$ norm_const_TO + T_offset;

$\%$ CALCULATE OTHER DISTRIBUTIONS

for $i=1: \operatorname{length}(\mathrm{Rr})$ $\operatorname{sigmavr}(i)=\operatorname{sigma}_{-} v(\operatorname{Tr}(i)) ; \quad \%\left(\mathrm{~m}^{\wedge} 3\right) / \mathrm{s}$

end

for $i=1: \operatorname{length}(\mathrm{Rr})$ $\operatorname{Pfr}(i)=0.25 *\left((\operatorname{nr}(i) *(1 \mathrm{e} 20))^{\wedge} 2\right) * \operatorname{sigmavr}(i) * E_{-}$alpha*5/(1e6); \% MW/m^3 end

end

function result $=$ sigma_v $(\mathrm{T})$

result $=10^{\wedge}\left(-.0602 * \log 10(\mathrm{~T})^{\wedge} 5+.5611 * \log 10(\mathrm{~T})^{\wedge} 4-1.5515 * \log 10(\mathrm{~T}) \wedge 3\right.$

$+5.1979 * \log 10(\mathrm{~T})-26.1059)$;

end 


\section{A.3 1-D Current Profiles}

This is a method for computing the various current profiles in a 1-D spherical tokamak core model. This assumes a fixed set of 0-D parameters.

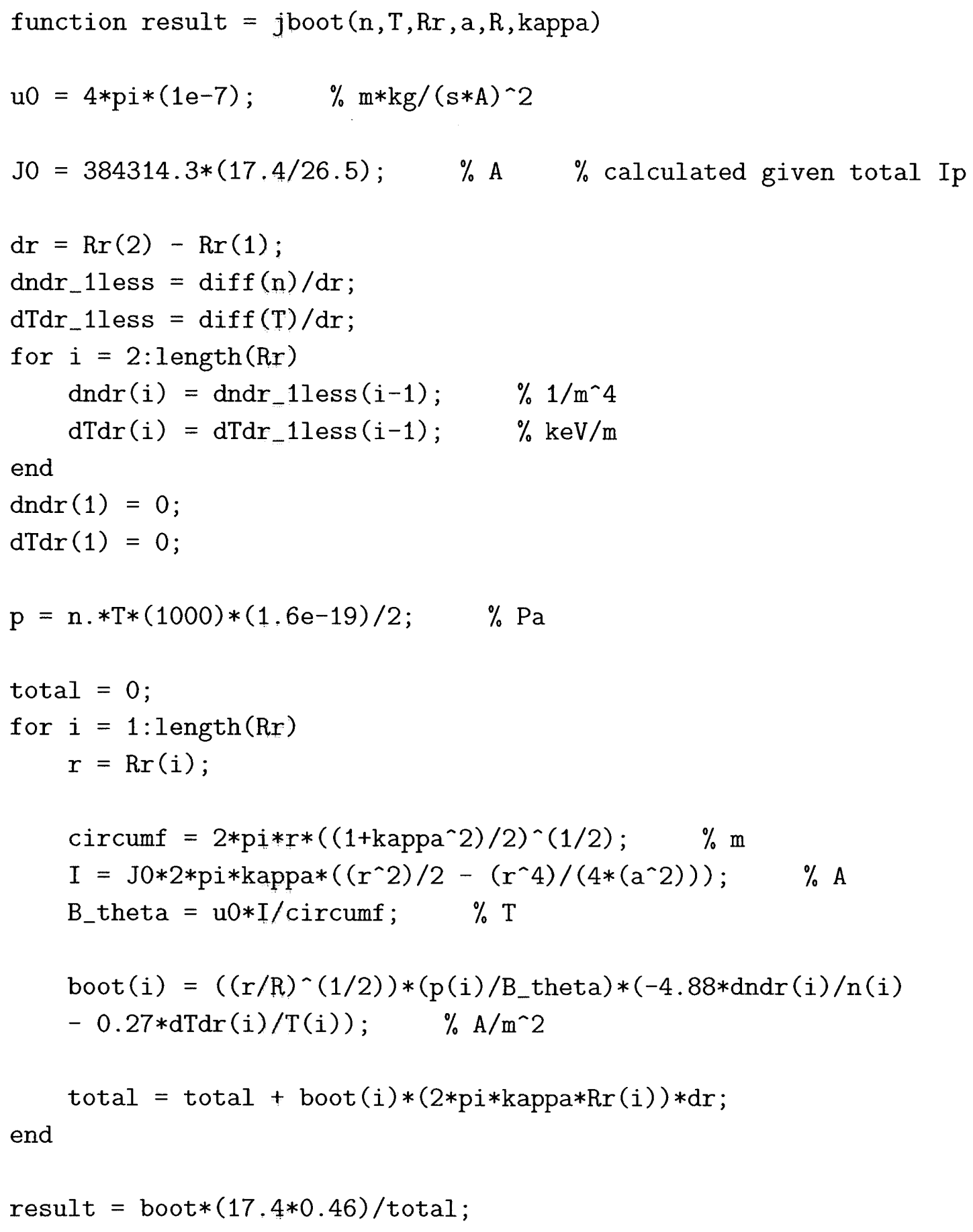


end 


\section{A.4 1-D q Profile}

This is a method for computing the safety factor $q(r)$ profile in a 1-D spherical tokamak core model. This assumes a fixed set of 0-D parameters.

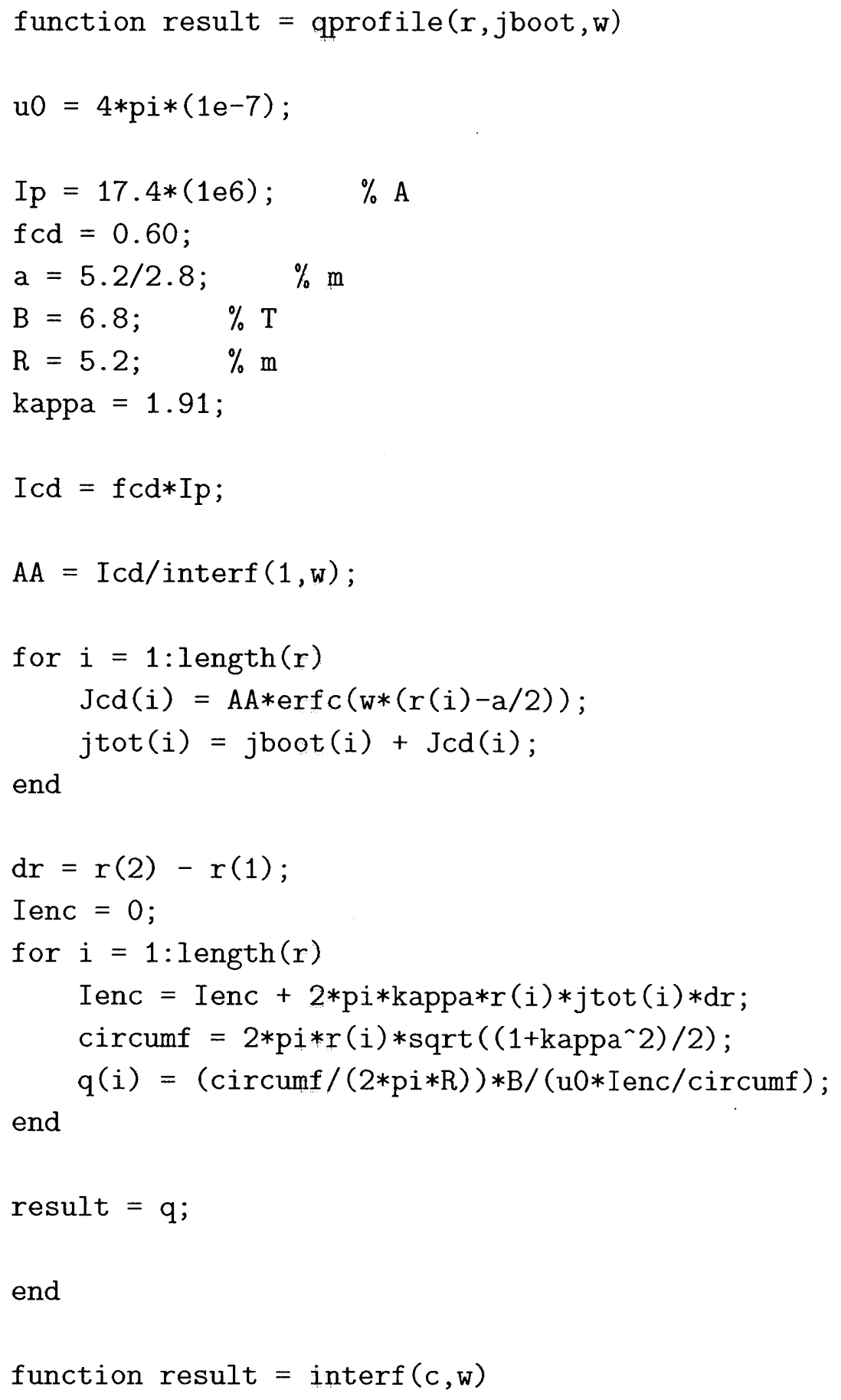




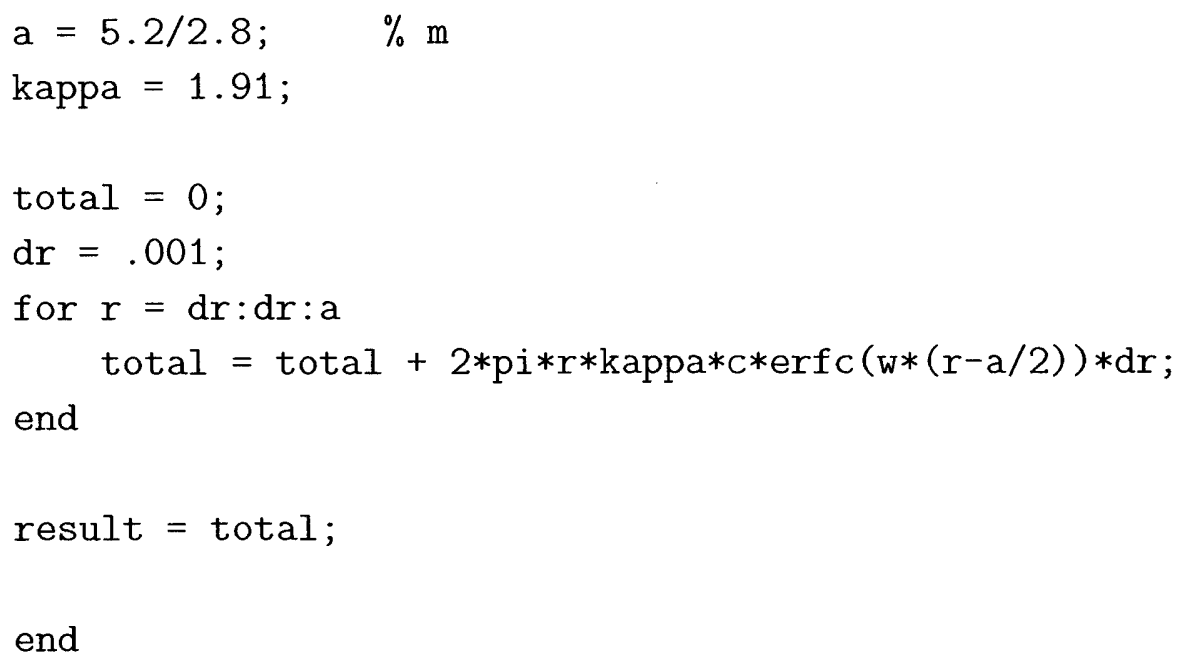




\section{A.5 Auxiliary Power for Operating Point Access}

This is a method for computing the 0-D auxiliary power as a function of density and temperature, which maps startup and shutdown processes.

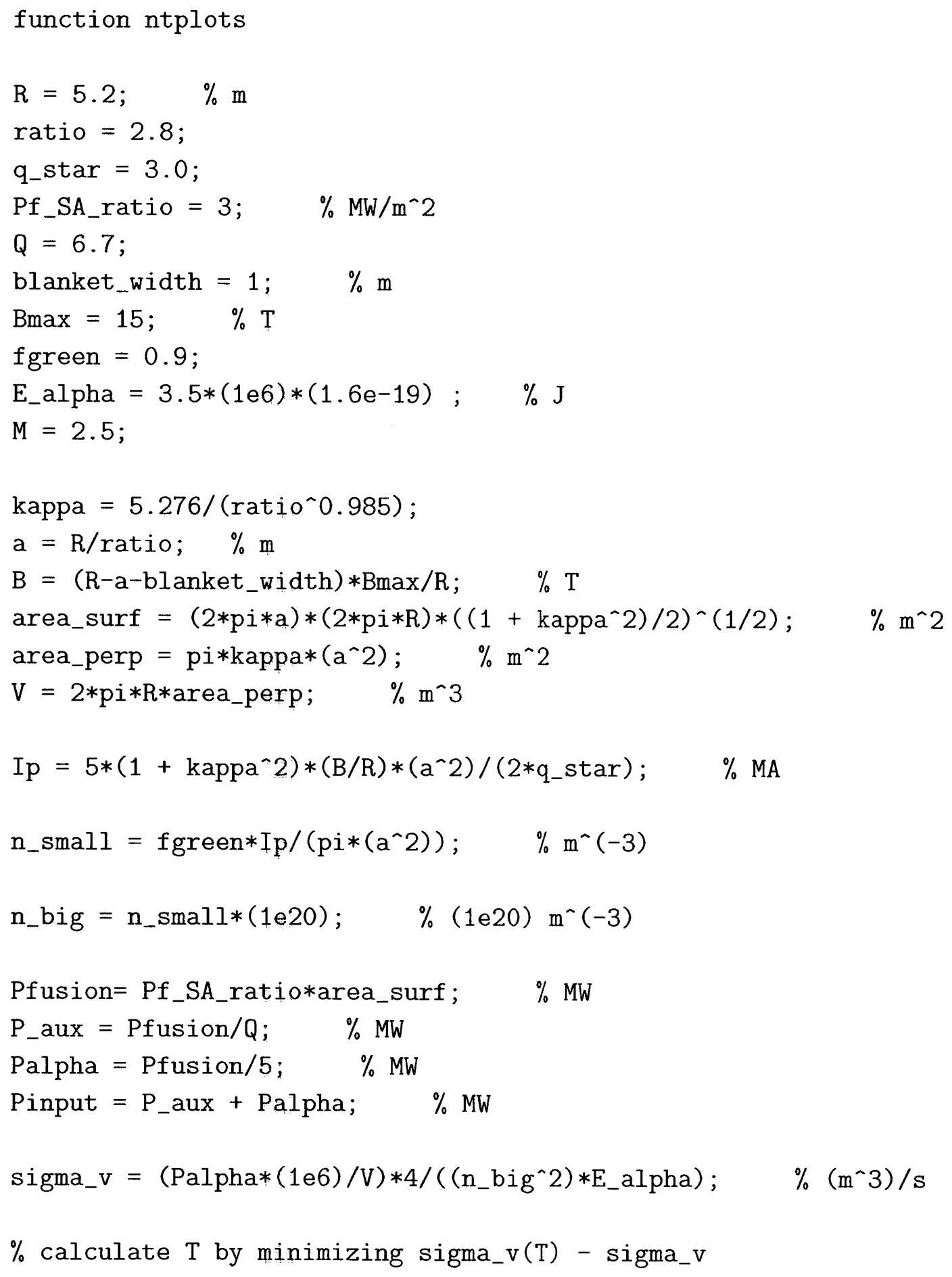




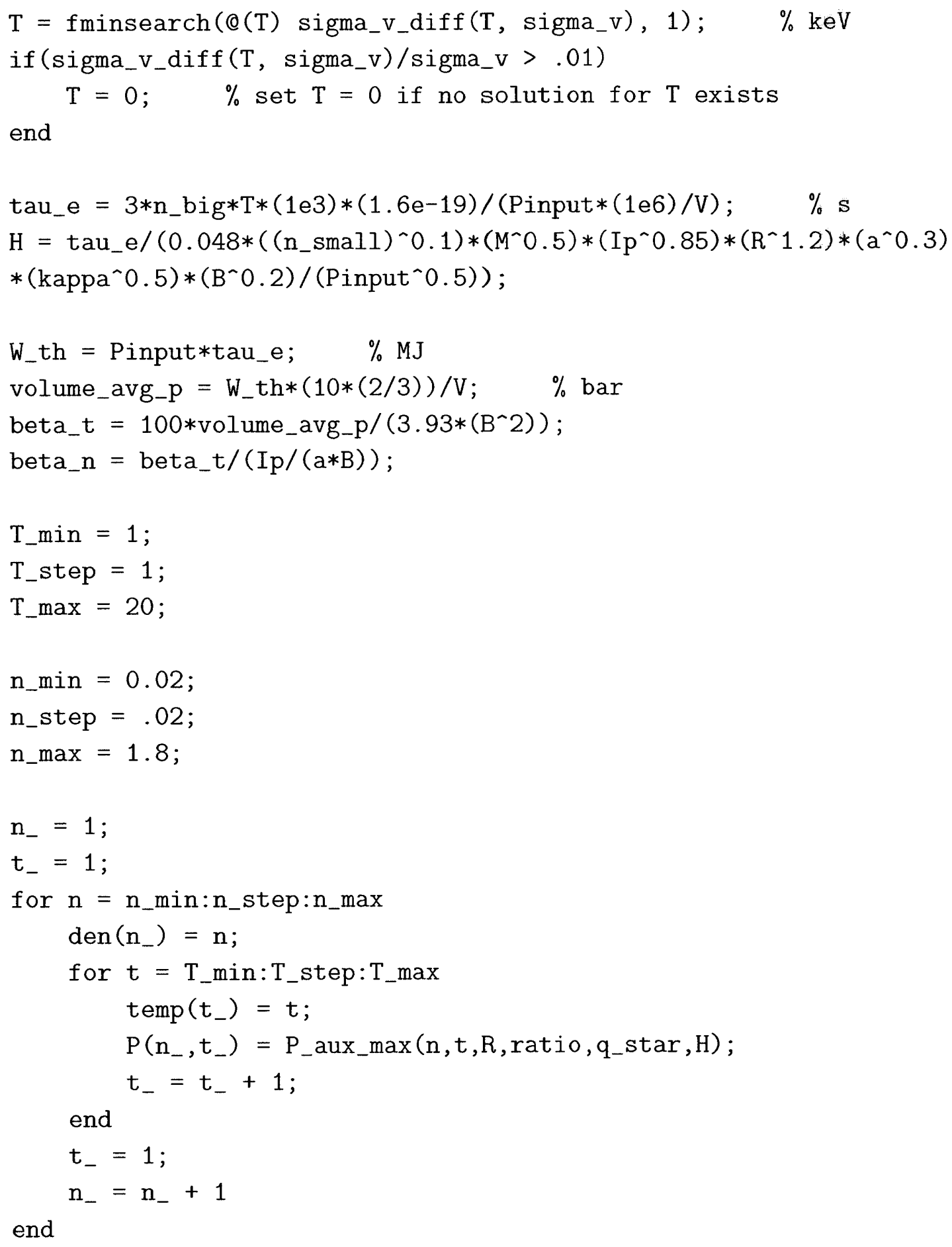


$\%$ plotting

contourlevels $=[0: .01: .9]$;

contourf (temp, den, P, contourlevels);

colorbar;

hold on;

contour (temp, den , P, [0,P_aux*.97/1000] , 'r' , 'LineWidth' ,4);

scatter ([T+.6], [n_small], 200, 'r' , 'LineWidth' ,4);

ylabel ('n (10^\{20\}/m^3)', 'FontSize', 12);

xlabel ('T (keV)', 'FontSize', 12);

title ('P_\{\mathrm\{\mathrm\{aux\}\}\} (n, T) (GW) ', 'FontSize', 12);

end

function result $=P_{-}$aux_max $\left(n_{-}\right.$small, $T, R$, ratio, q_star, $\left.H\right)$

$\%$ constants

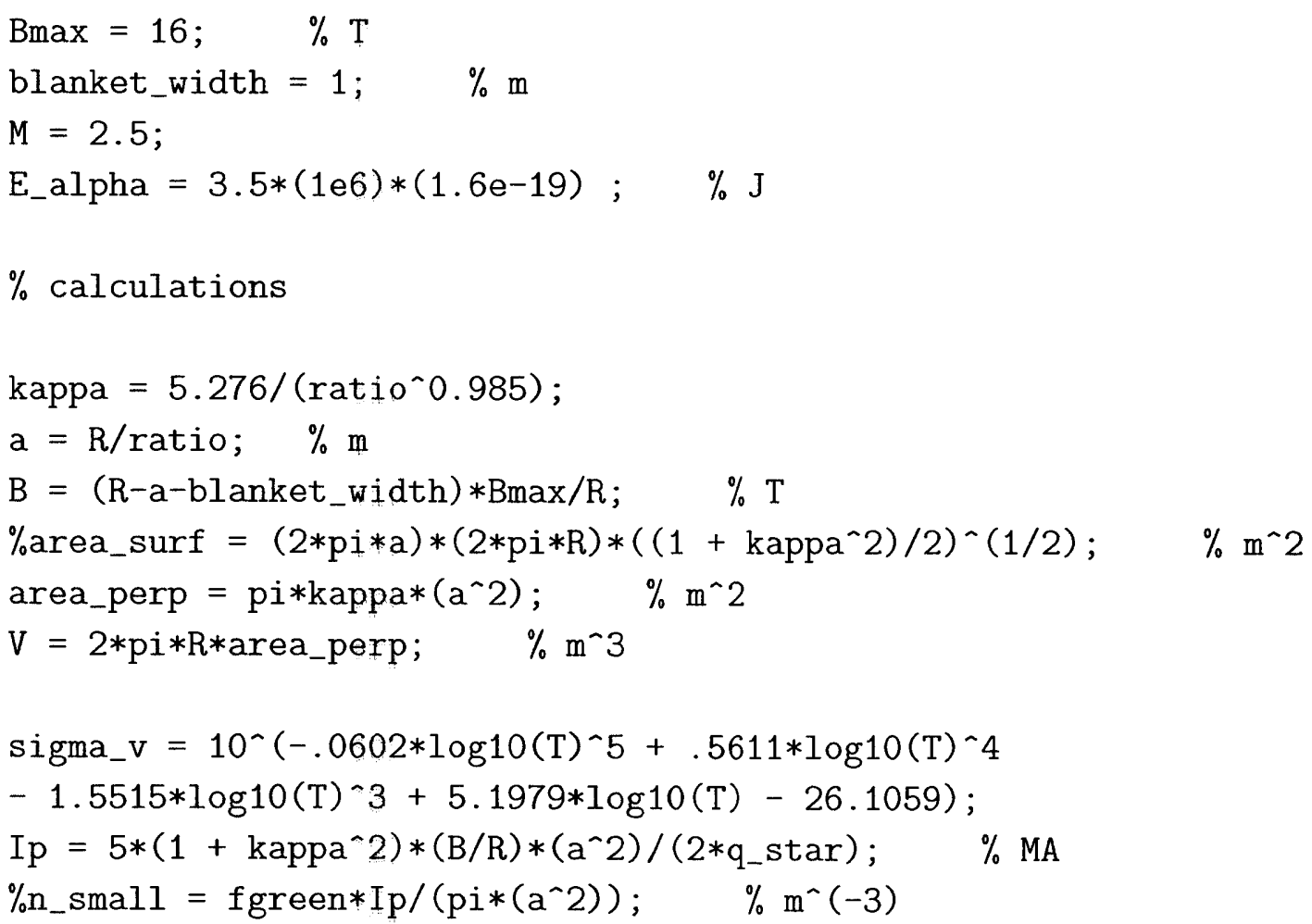




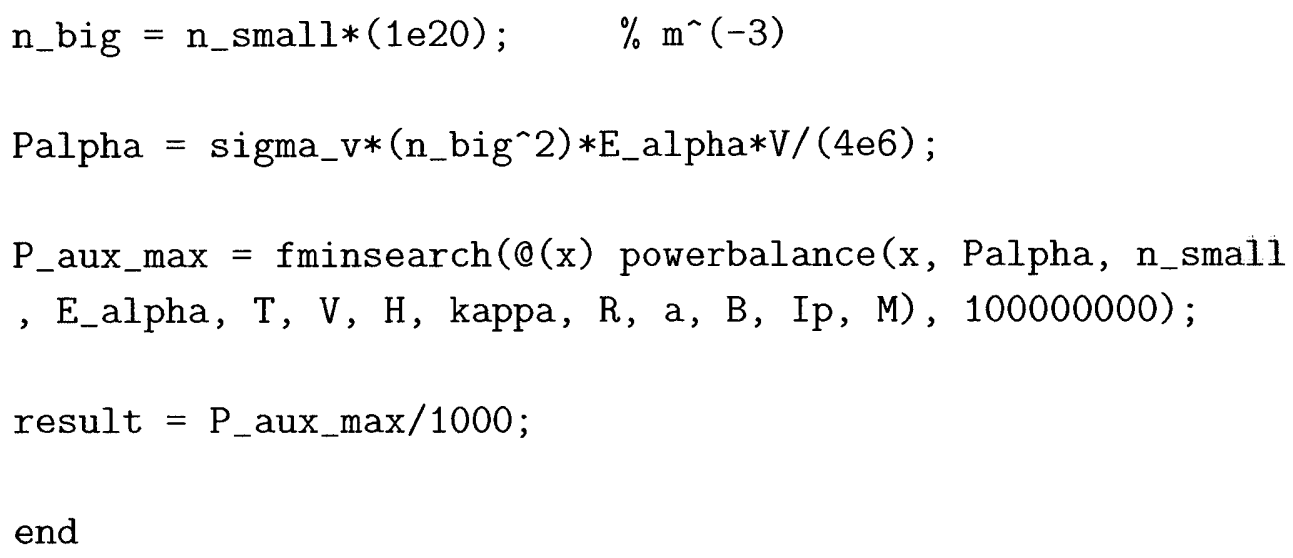

end

function result $=$ sigma_v_diff $(T$, sigma_v $)$

$\operatorname{diff}=10^{\wedge}\left(-.0602 * \log 10(\mathrm{~T})^{\wedge} 5+.5611 * \log 10(\mathrm{~T})^{\wedge} 4-1.5515 * \log 10(\mathrm{~T})^{\wedge} 3\right.$

$+5.1979 * \log 10(\mathrm{~T})-26.1059)$ - sigma_v;

result $=\operatorname{abs}(\operatorname{diff})$;

end 


\section{B Toroidal Monte Carlo Code}

This is the complete fission-fusion hybrid Monte Carlo code in toroidal geometry, which we developed from scratch. It samples a fixed neutron source within the toroidal plasma and tallies subcritical fission and tritium breeding within the blanket. We utilize ENDF cross-sections and analog Monte Carlo (except in particle splitting during fission).

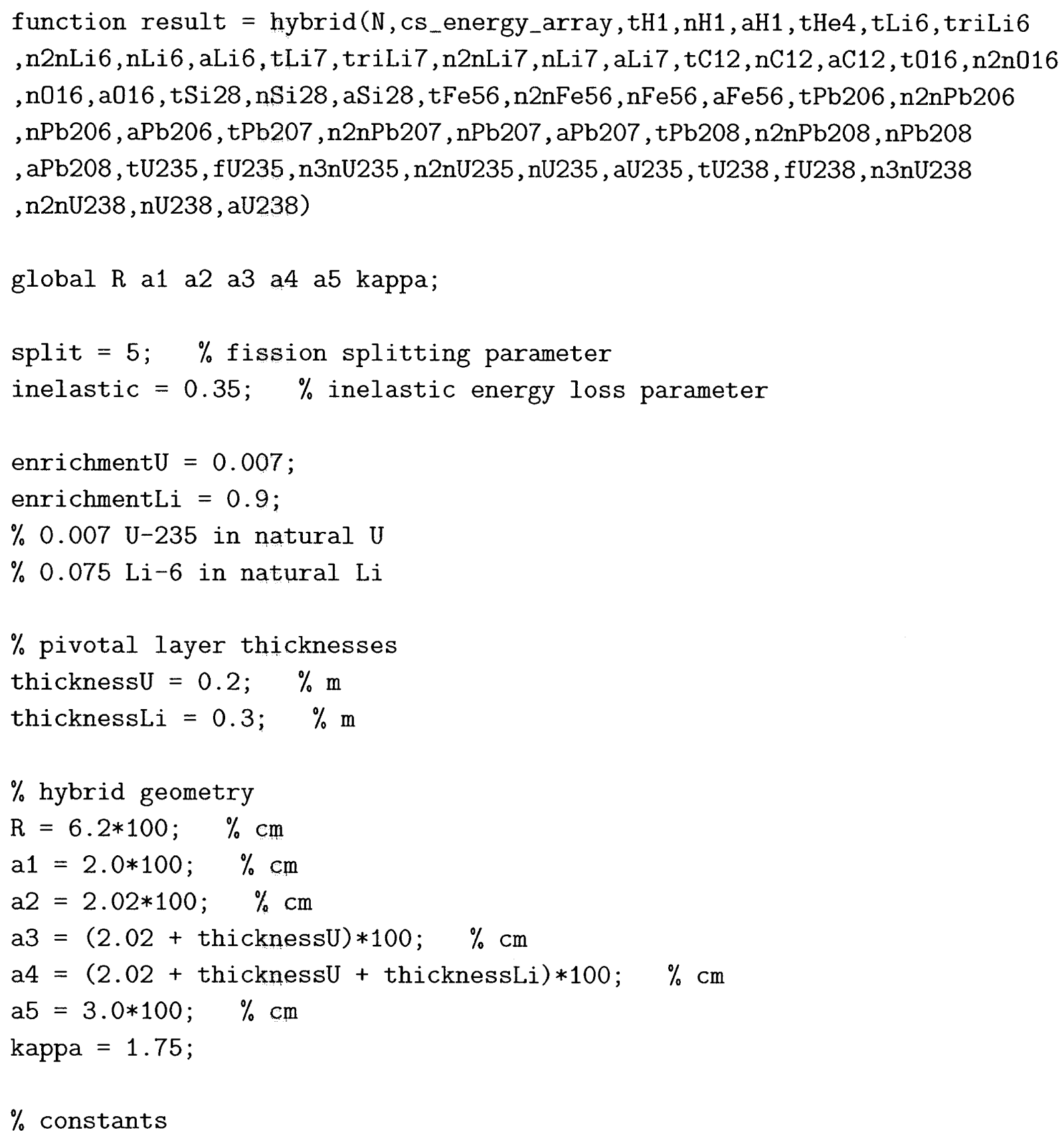




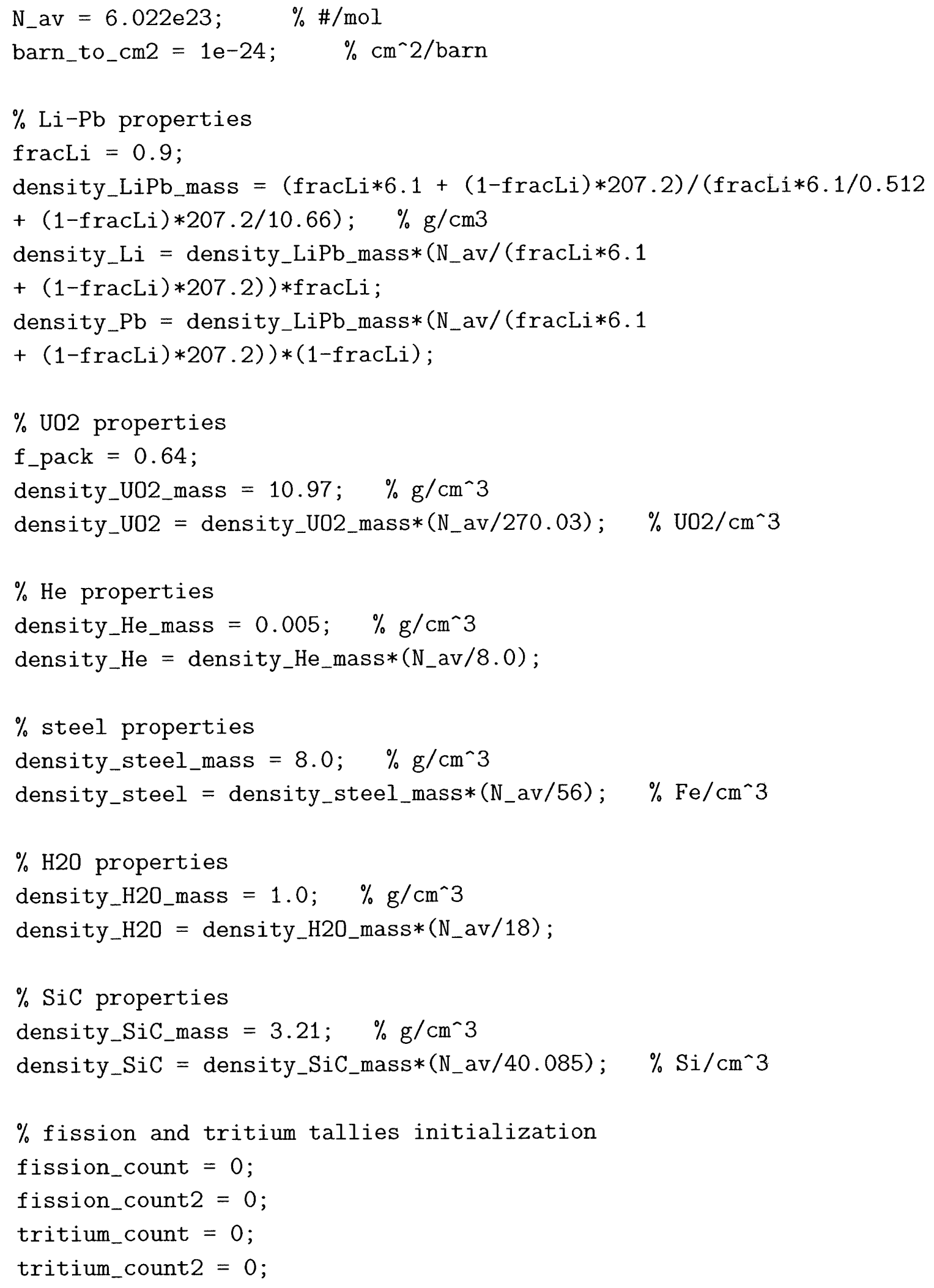


$\%$ count particle kills from rounding error round0ffErrorCount $=0$;

$\%$ neutron generation initialization

generations $=\operatorname{zeros}(1, N * 2)$;

generations $(1)=\mathrm{N}$;

generationsNum = generations;

countGen $=1$;

energies $=\operatorname{zeros}(1, N * 2)+14.1$;

weights $=\operatorname{zeros}(1, \mathrm{~N} * 2)+1.0$;

position $=\operatorname{zeros}(3, N * 2)$;

$\%$ sample fusion reaction sites

for $i=1: \mathrm{N}$

$\mathrm{x}=\mathrm{R} * 10$;

$\mathrm{y}=0$;

$z=a 1 * k a p p a * 10$

$\%$ toroidal rejection sampling loop

while (withintoroid $(\mathrm{x}, \mathrm{y}, \mathrm{z}, \mathrm{a1})==0$ )

$\mathrm{x}=(\mathrm{rand} * 2-1) * \mathrm{a} 1+\mathrm{R}$;

$z=(\operatorname{rand} * 2-1) *$ kappa $* a 1$;

end

$\operatorname{position}(1, i)=x$;

position $(2, i)=y$;

position $(3, i)=z$;

end

energiesNext = energies;

weightsNext = weights;

positionNext = position;

$\%$ neutron generation loop

while (generationsNum (countGen) >0)

countGen $=$ countGen +1 ;

$\%$ transfer stored energies, weights, and positions 


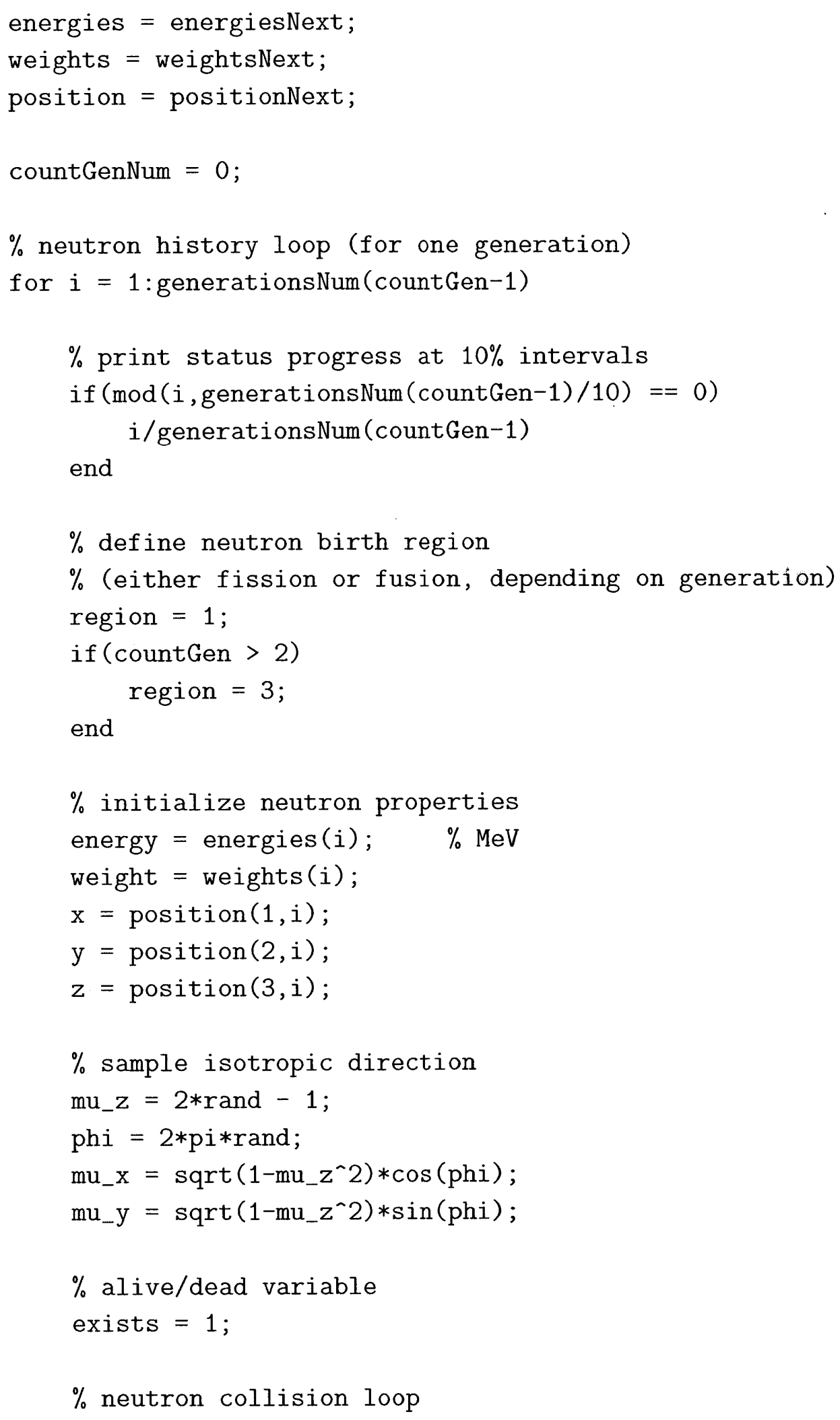




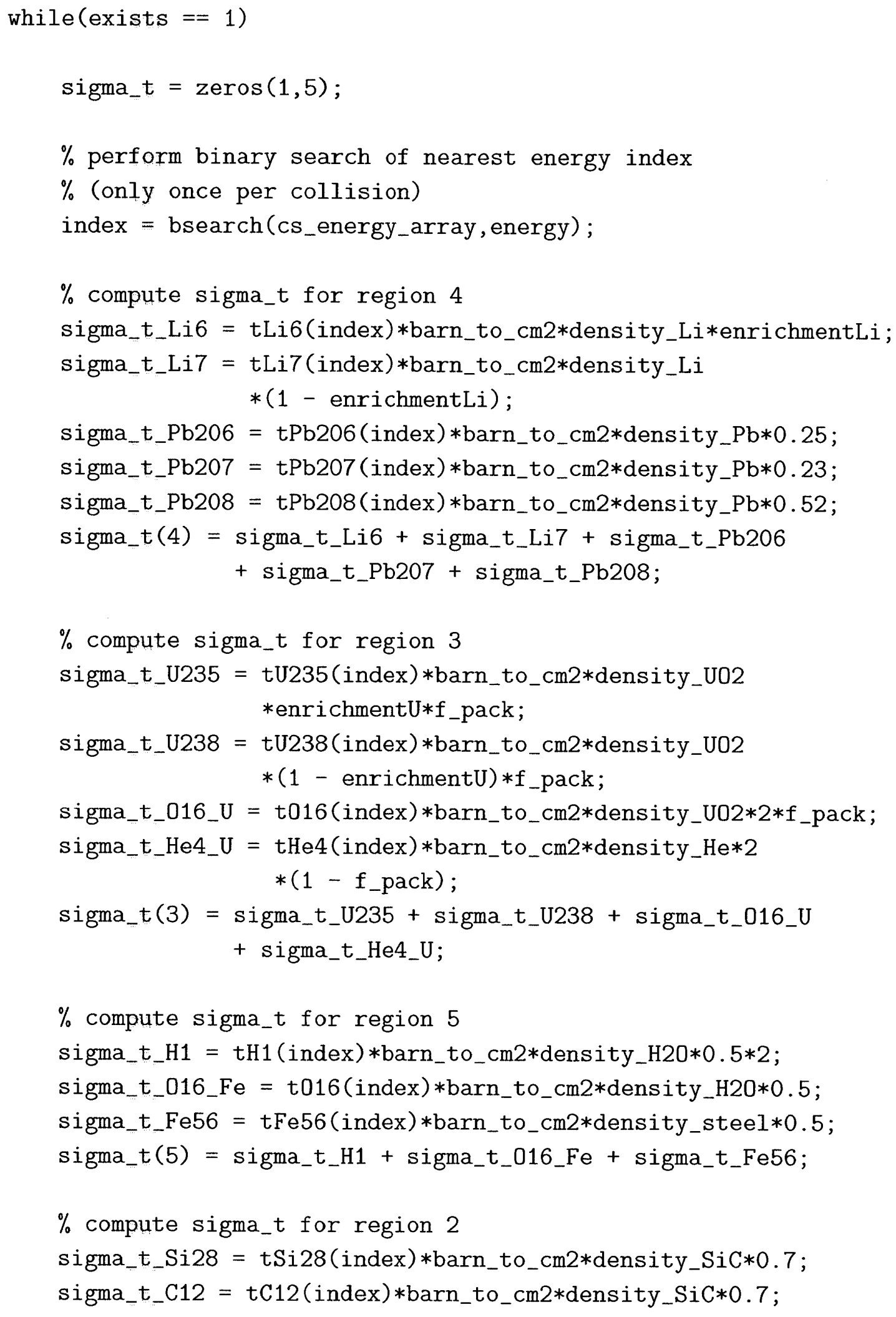




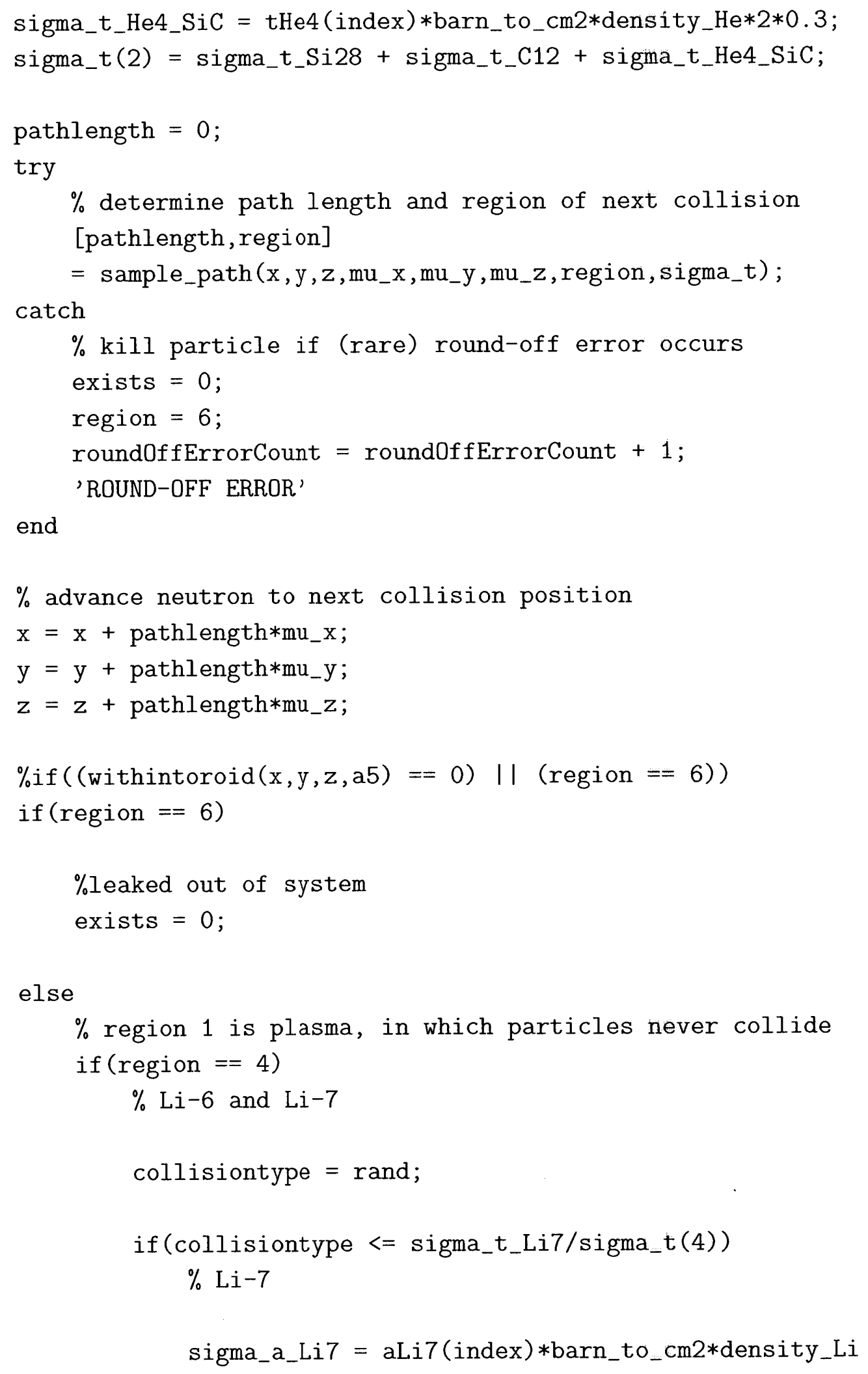




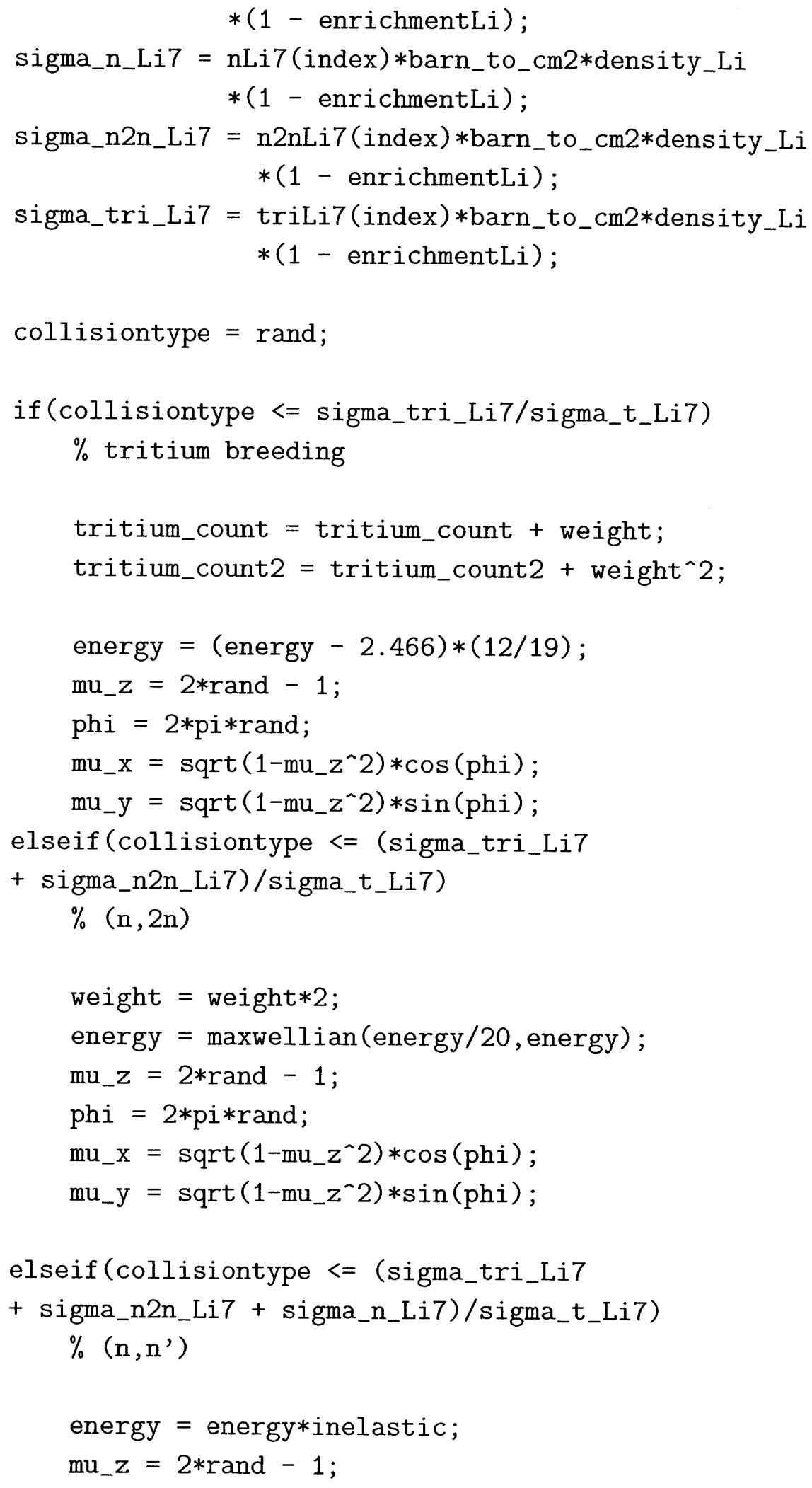




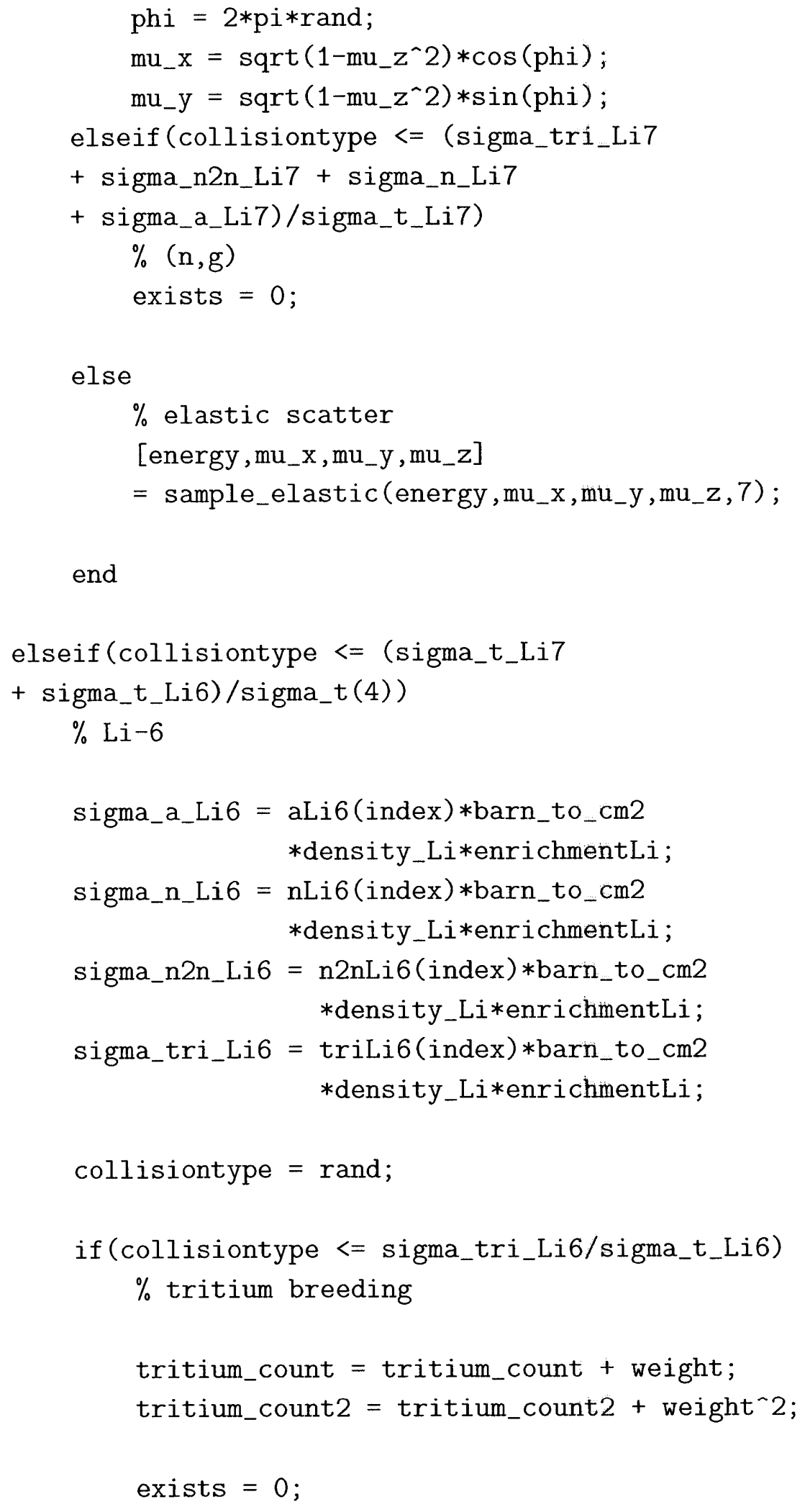

end 


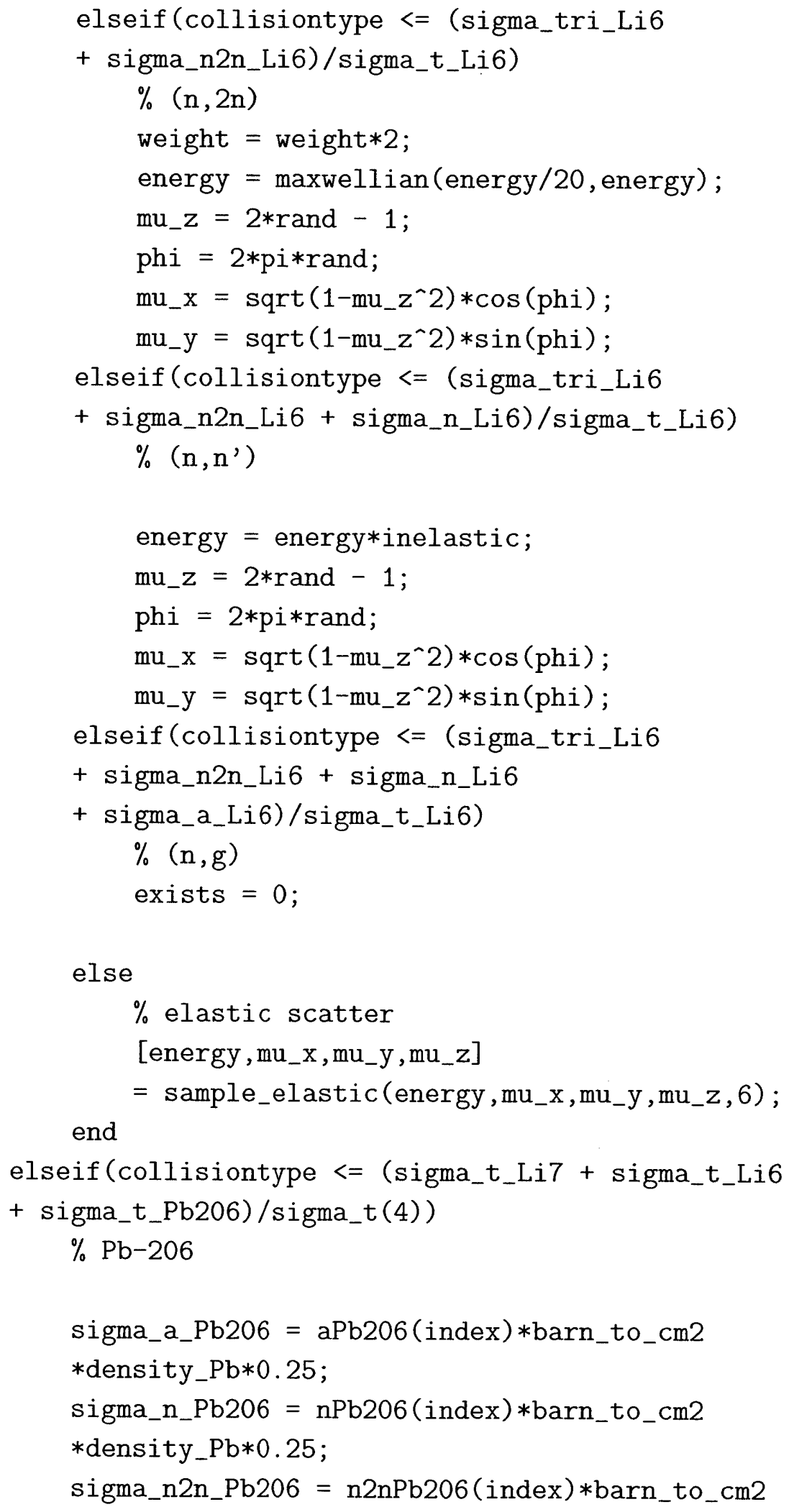




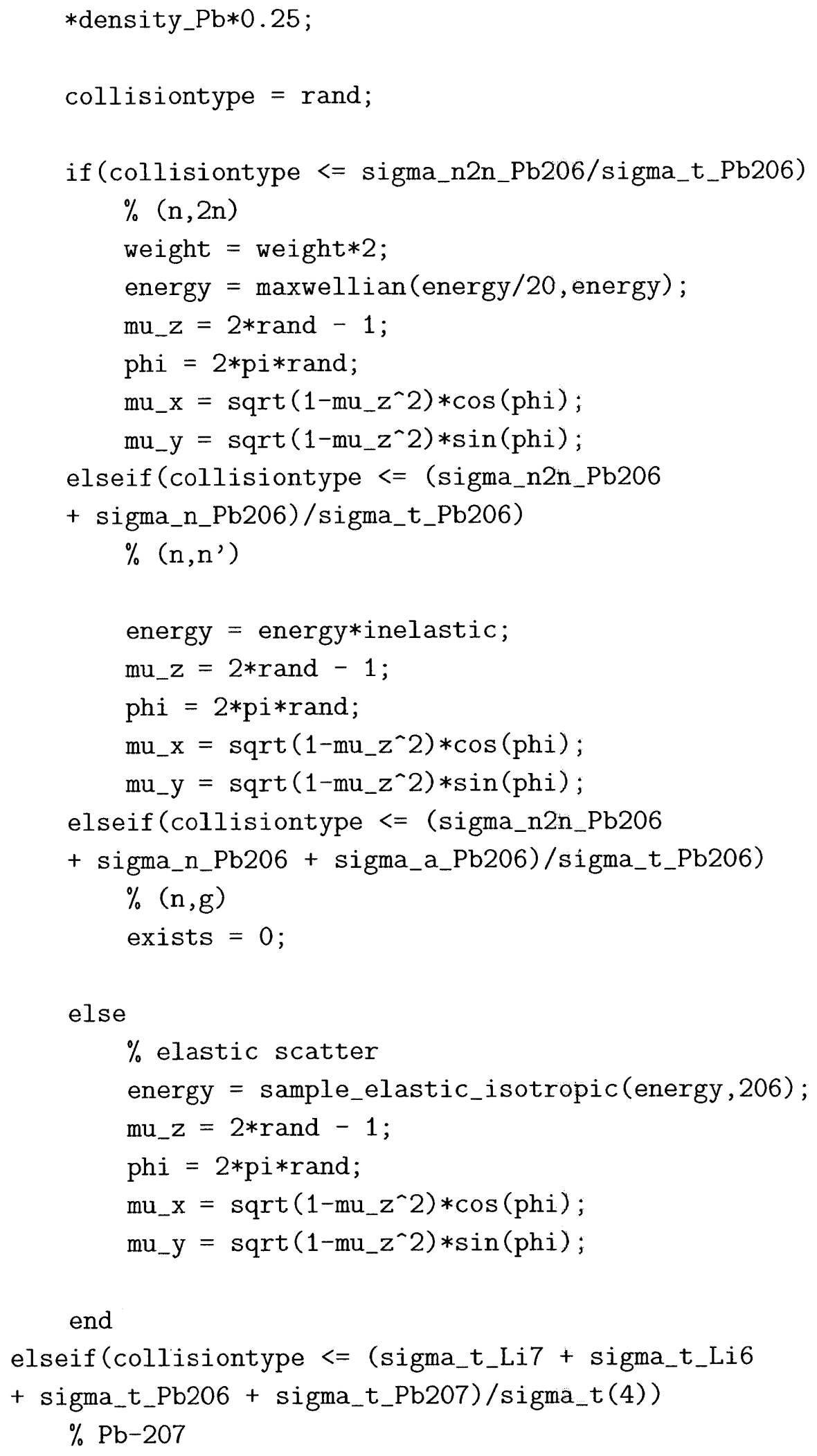




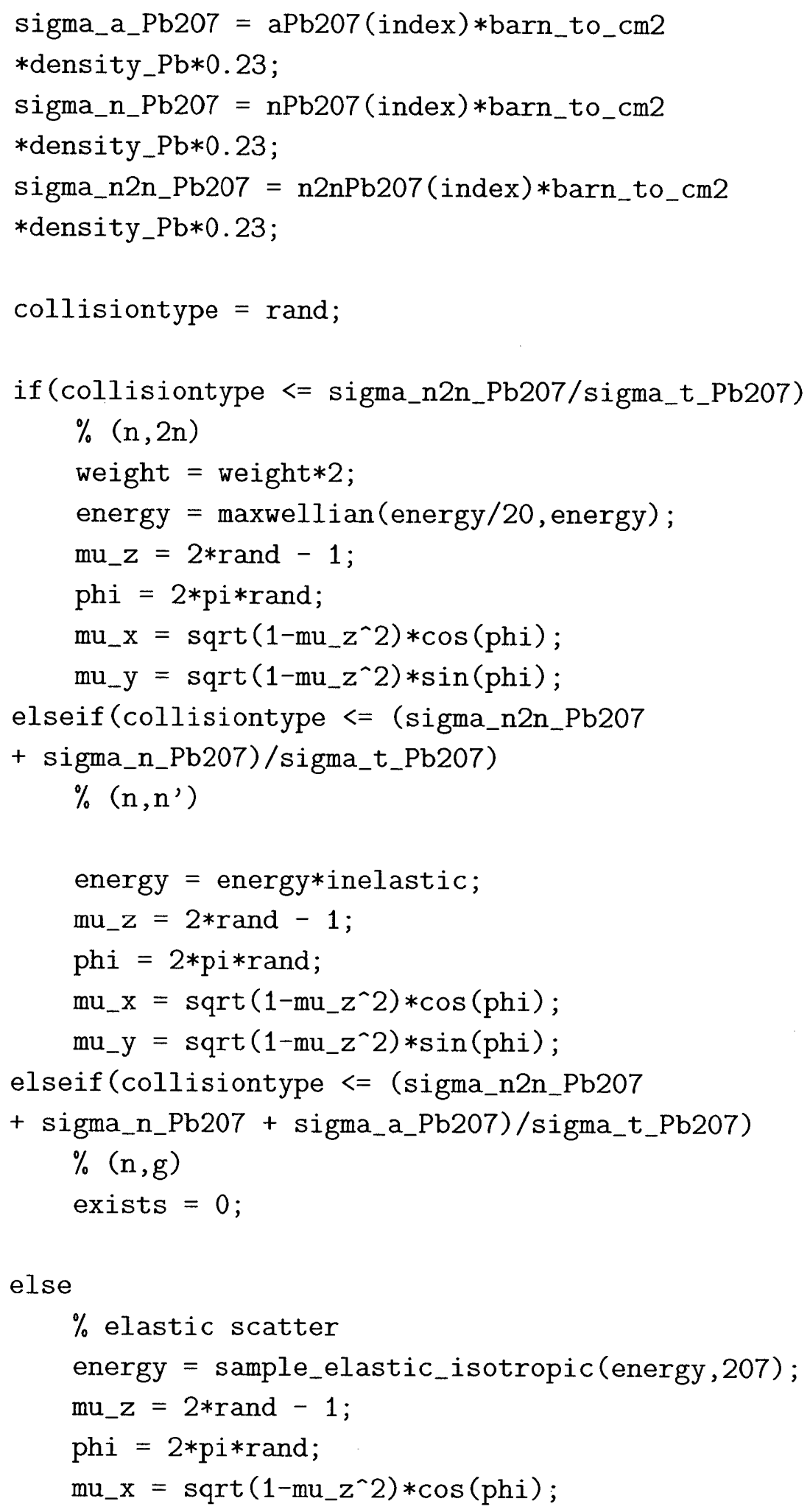




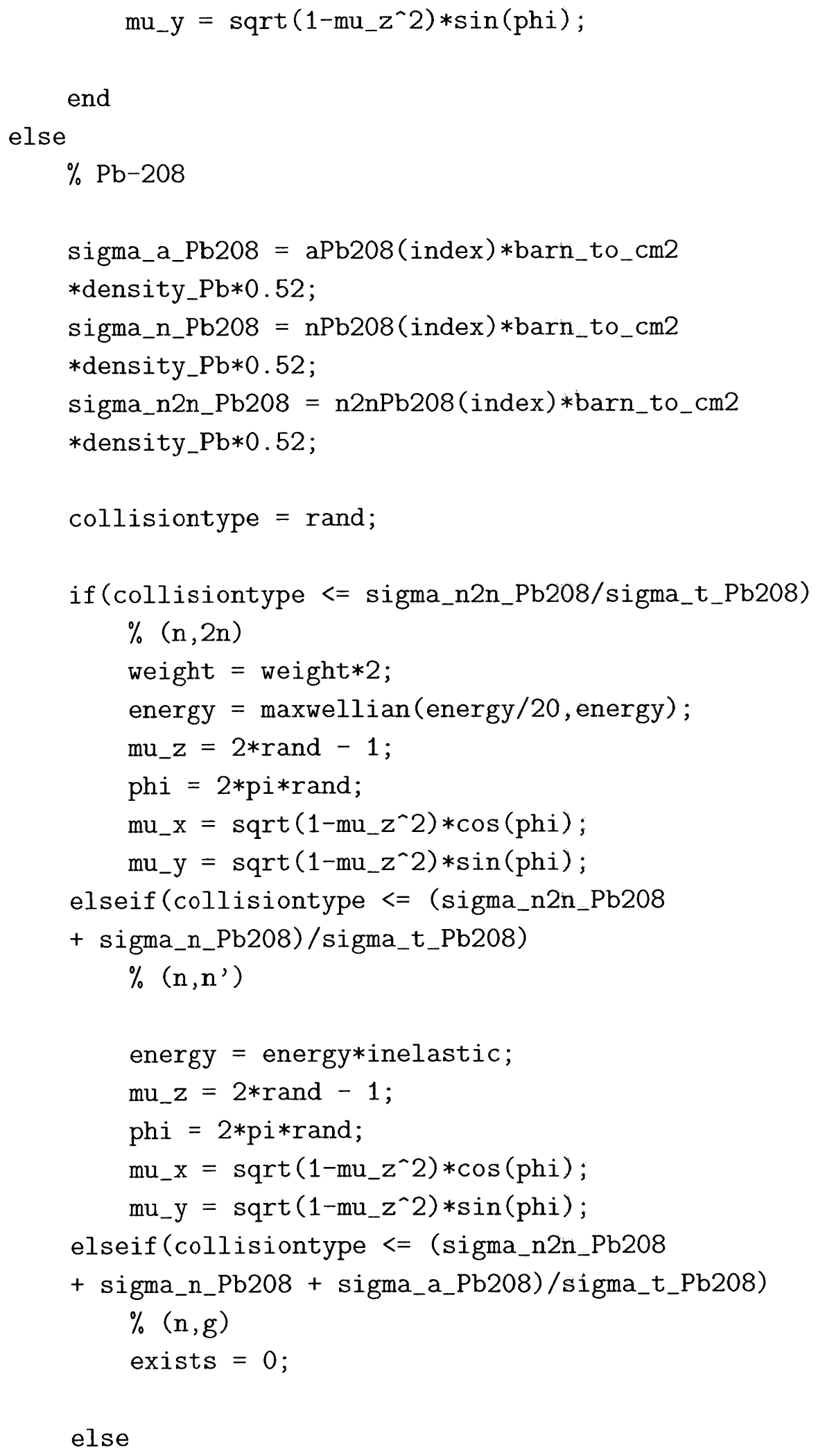




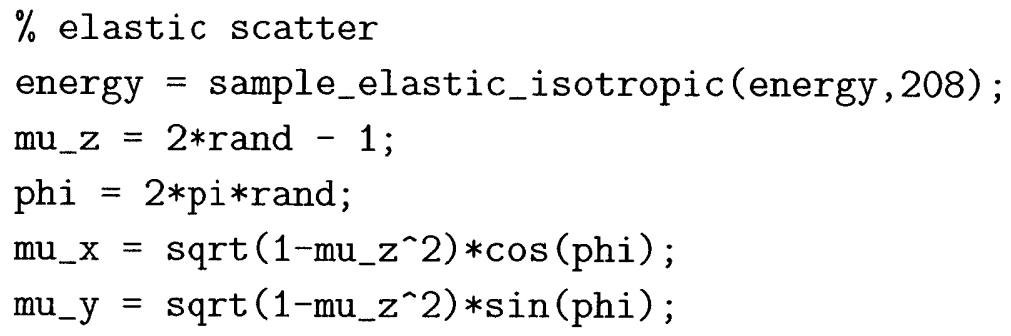

end

end

\section{elseif (region $==3$ )}

$\% \mathrm{U}-235, \mathrm{U}-238$, and $0-16$

$\% \mathrm{He}-4$ is neutron-transparent

collisiontype = rand;

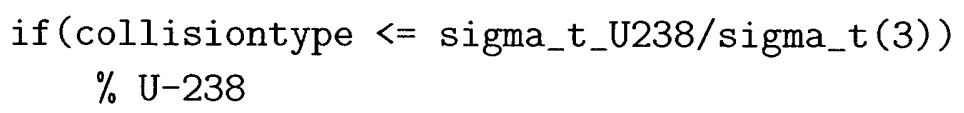




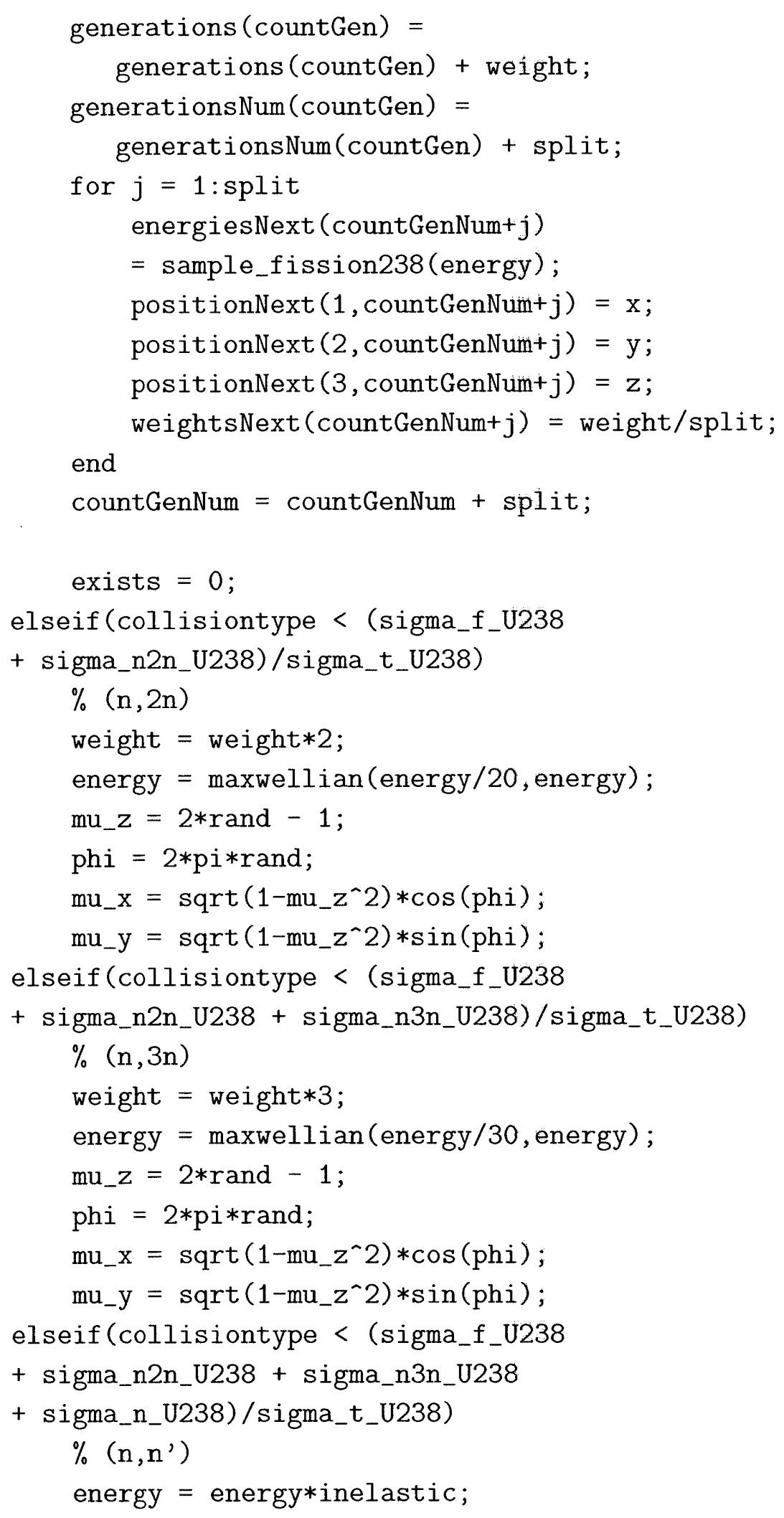




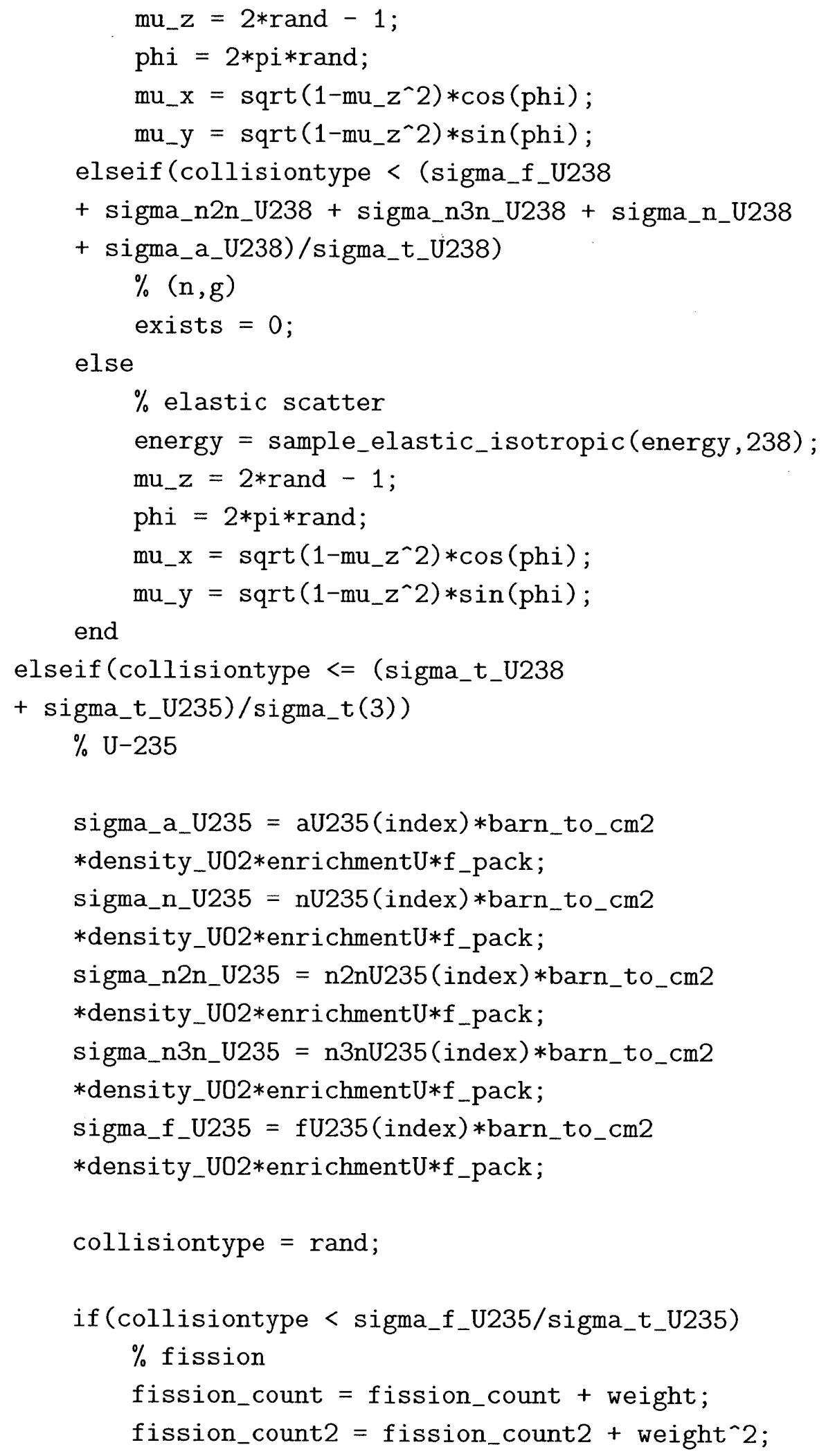




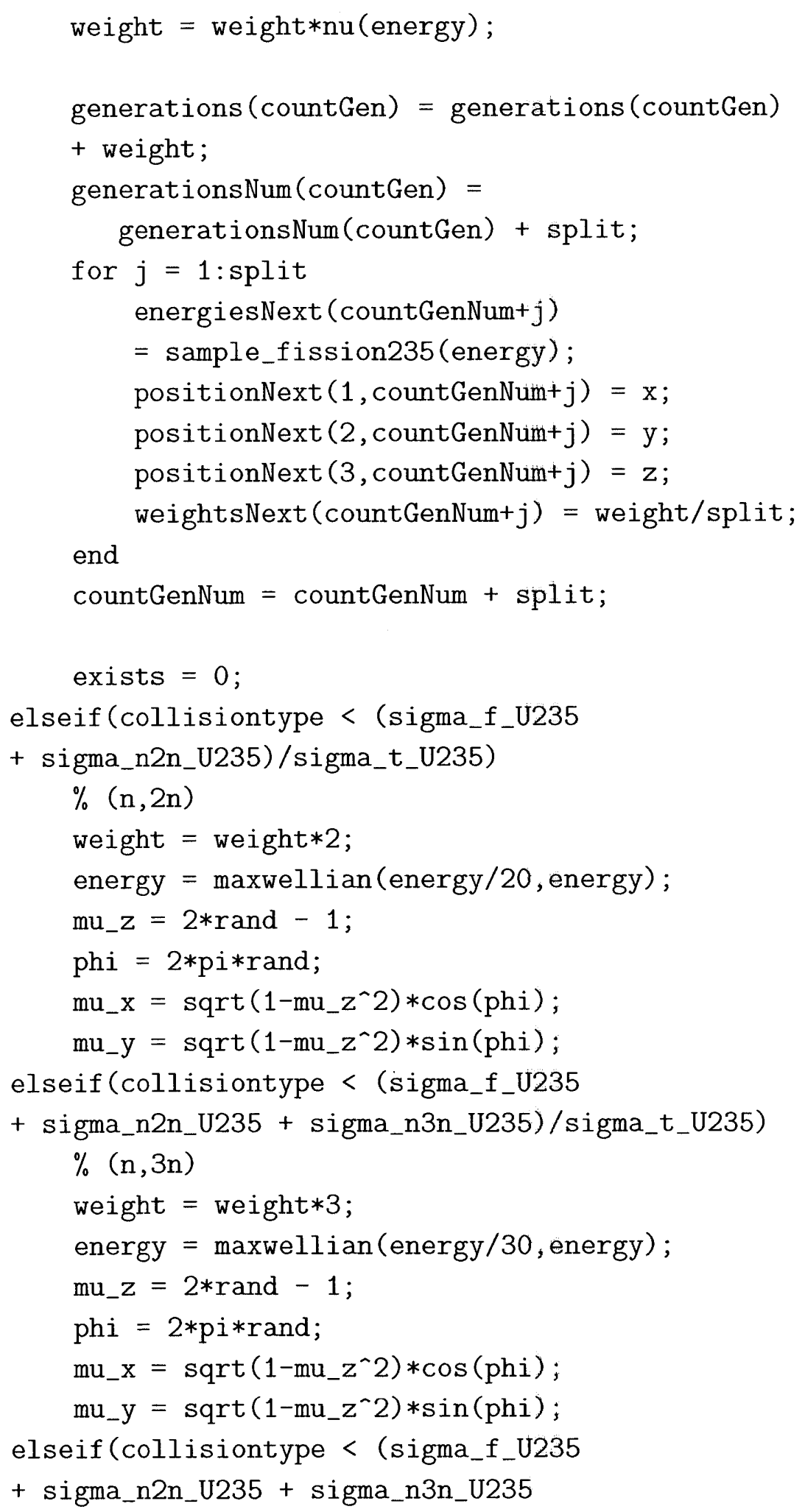




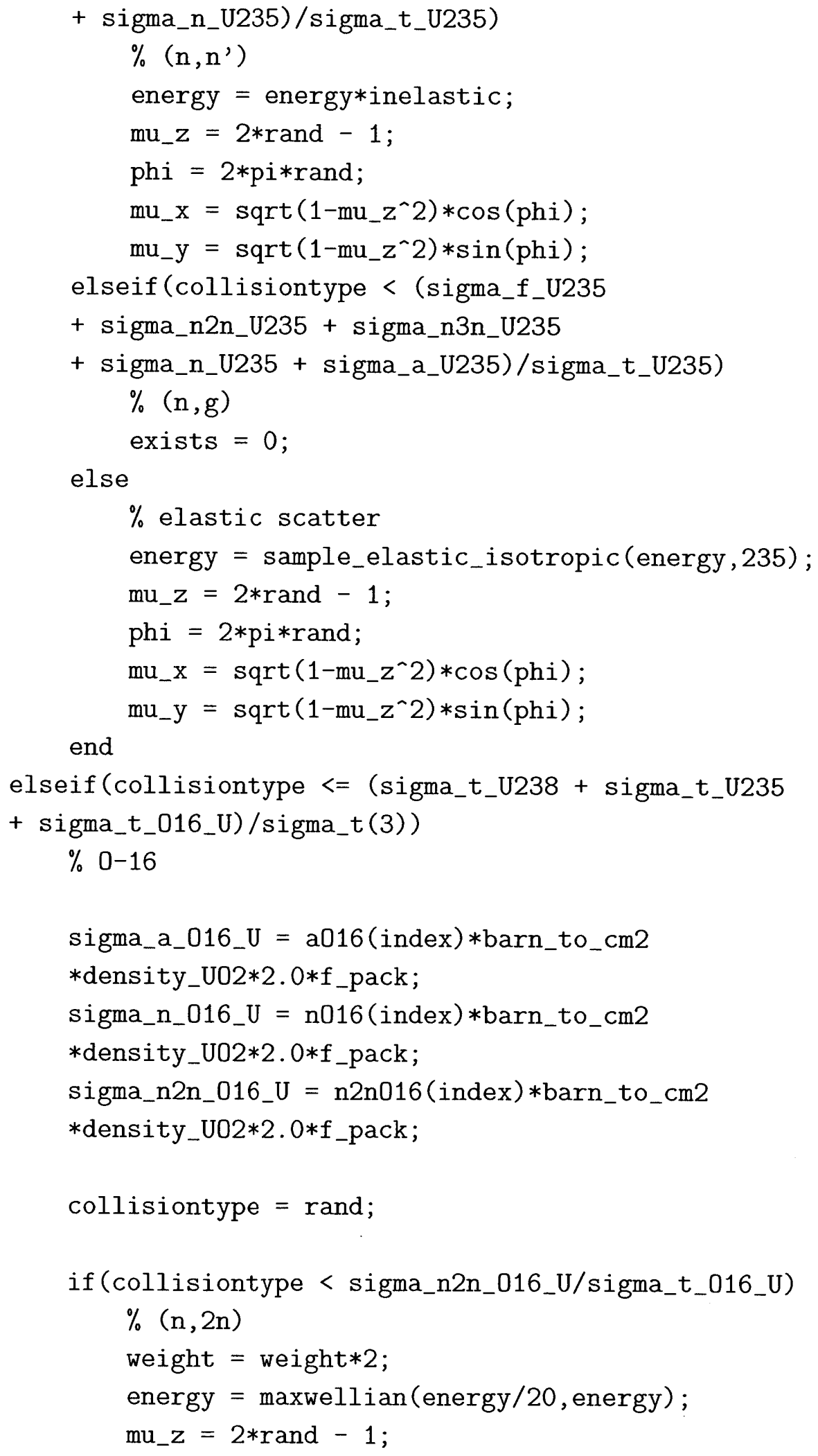




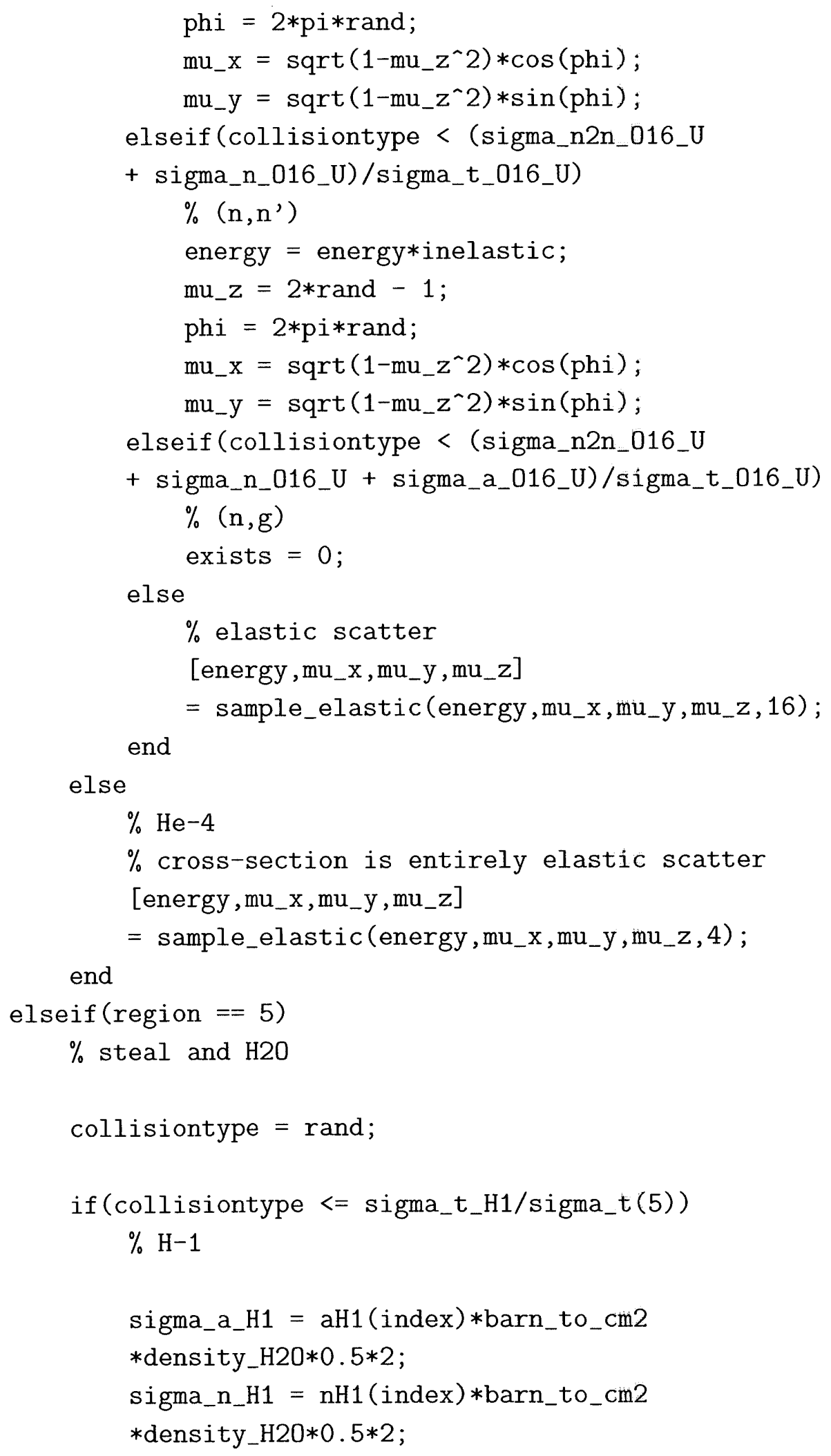




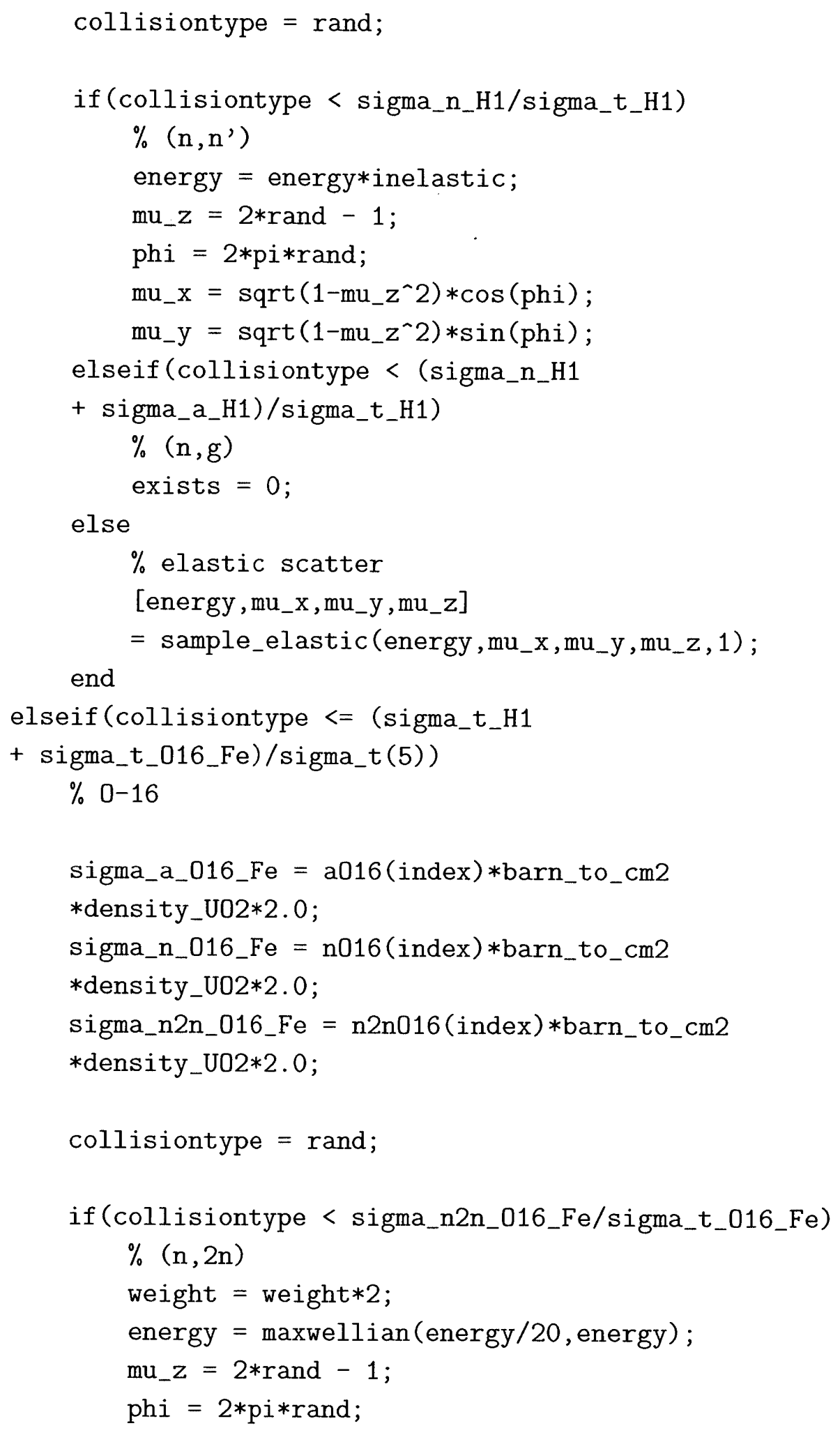




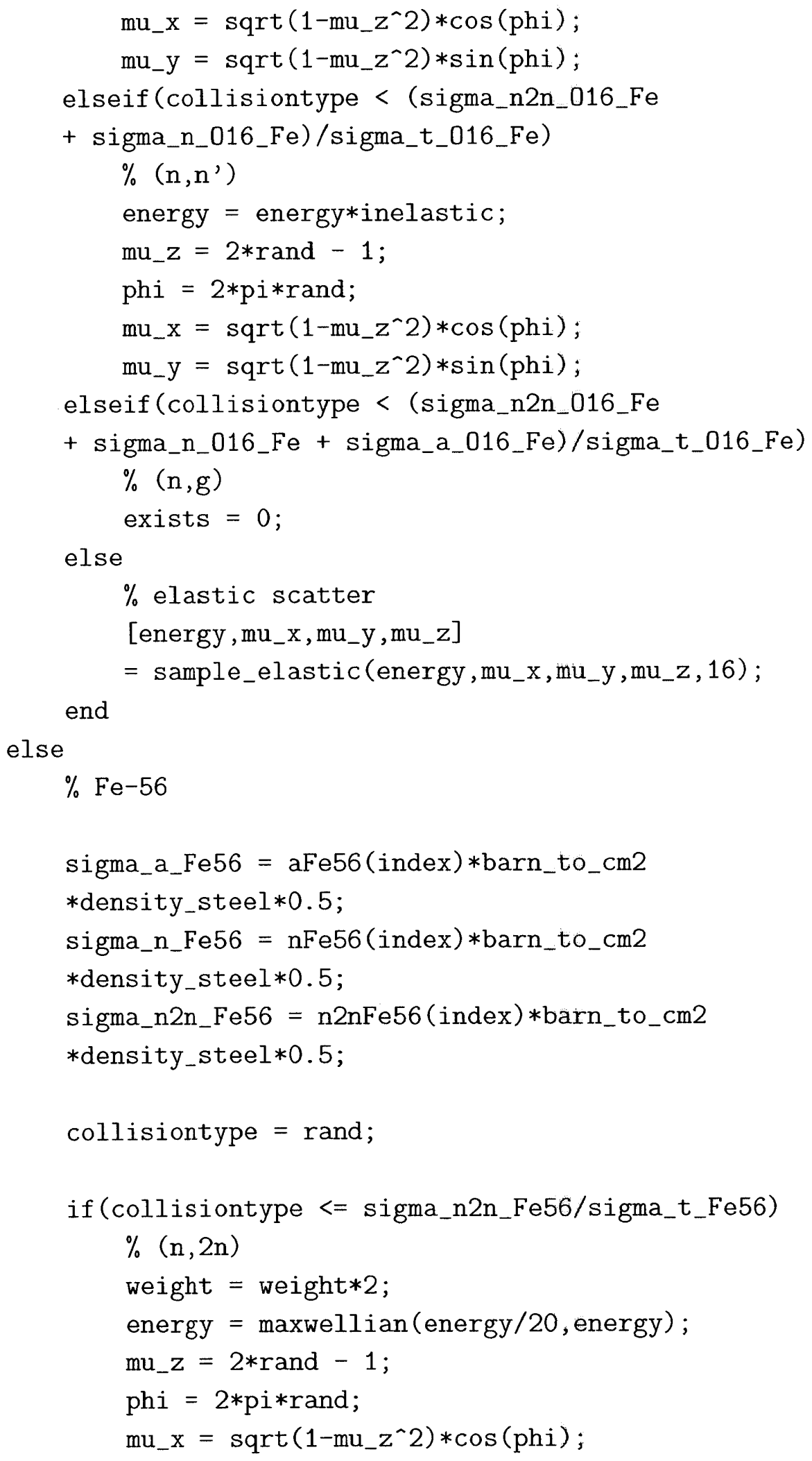




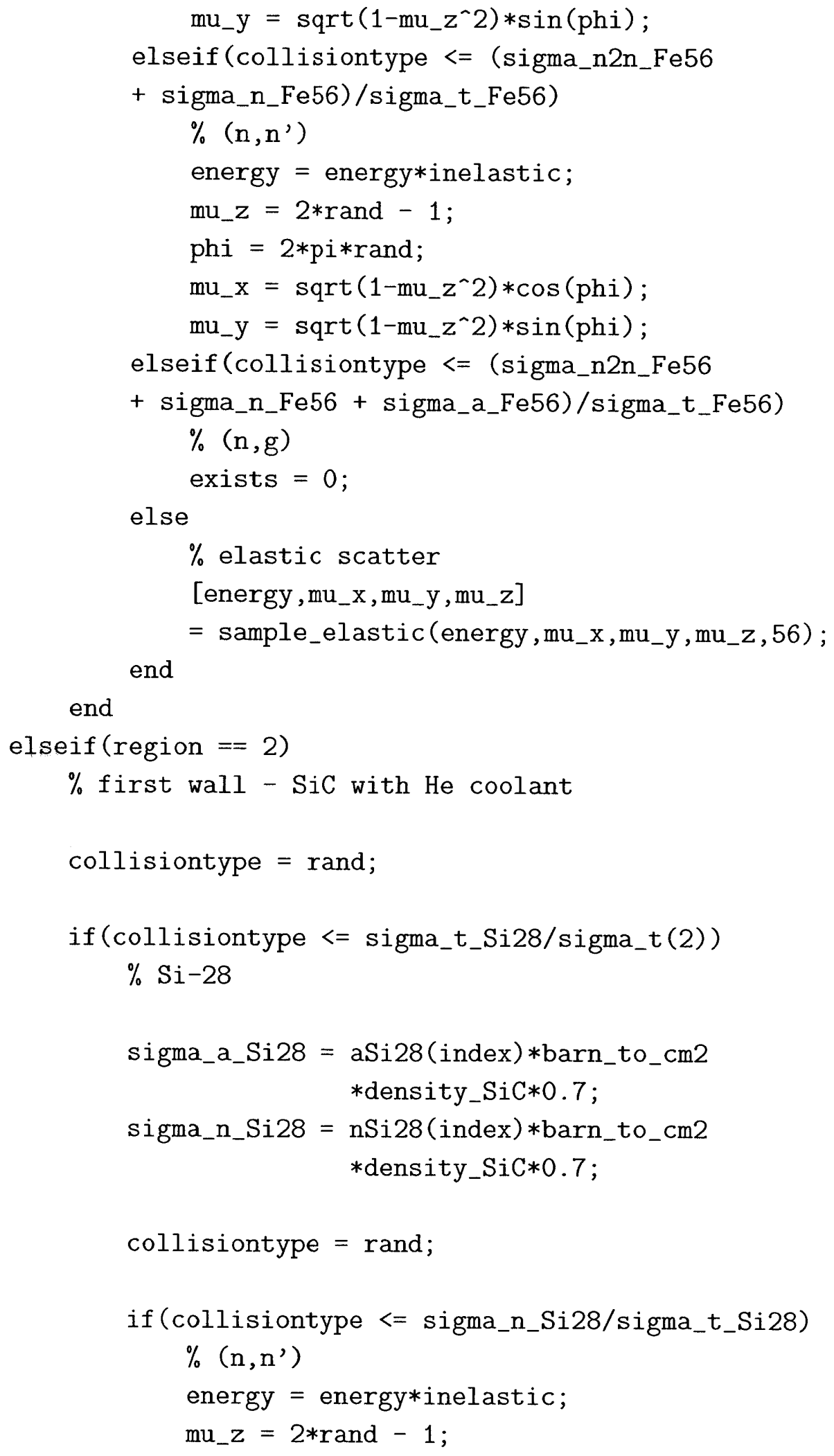




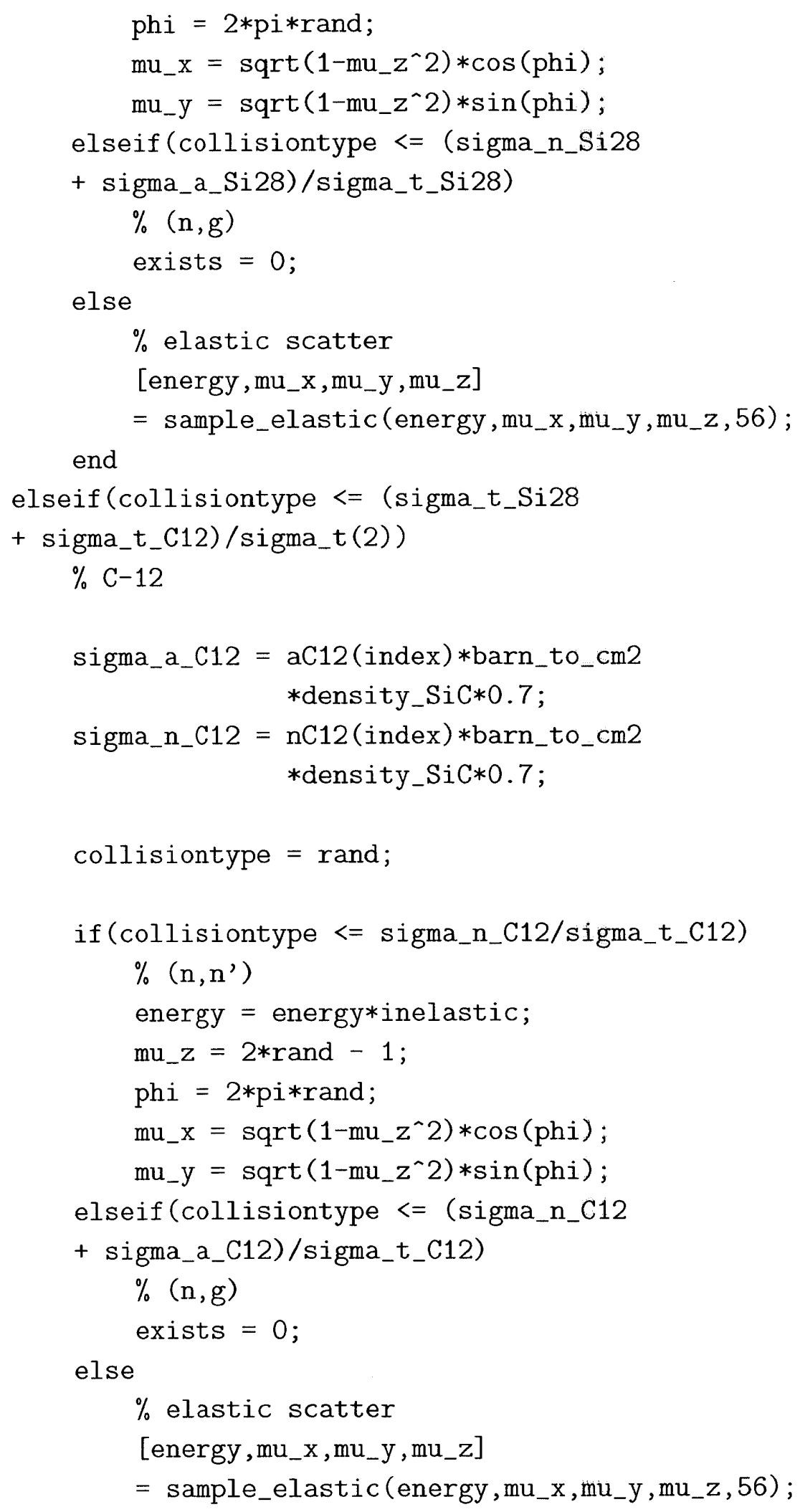




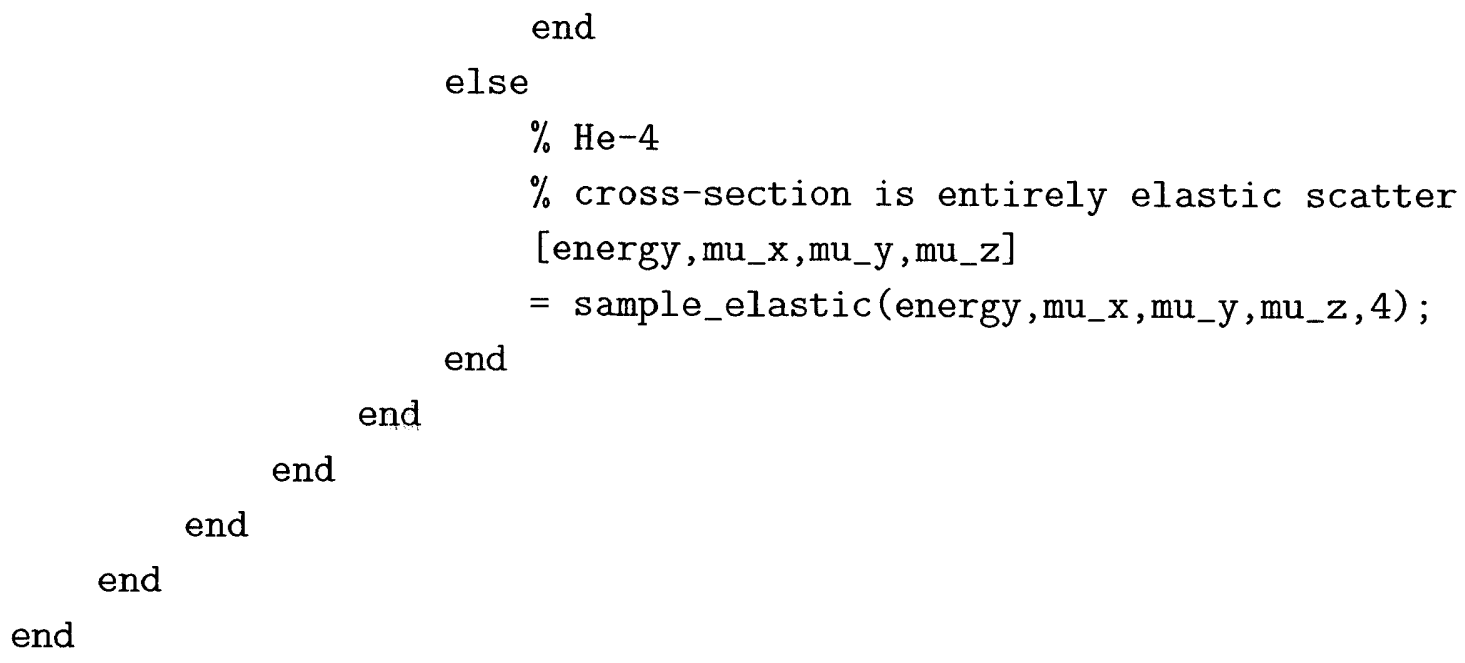


$\%$ output generation and $\mathrm{k}$ data

result $=[\mathrm{k}$; generations 2 ; generationsNum2];

end

$\%$ sample anisotropic elastic scattering

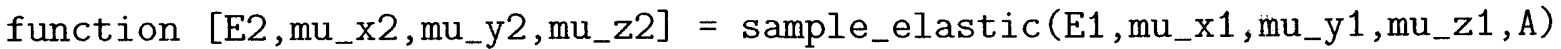

$\%$ sample angular shifts

alpha $=((A-1) /(A+1))^{\wedge} 2$;

$\mathrm{E} 2=(1-\mathrm{alpha}) * \mathrm{E} 1 * \mathrm{rand}+\mathrm{alpha} * \mathrm{E} 1$

dmu_cm $=(2 *(E 2 / E 1)-(1+$ alpha $)) /(1-$ alpha $)$;

$\mathrm{dmu}=\cos \left(\operatorname{atan} 2\left(\operatorname{sqrt}\left(1-\mathrm{dmu}_{-} \mathrm{cm}^{-2} 2\right), 1 / \mathrm{A}+\mathrm{dmu} \mathrm{cm}_{-}\right)\right) ;$

dphi $=2 *$ pi $*$ rand;

$\%$ compute new unit vectors

$m u_{-} \mathrm{x} 2=m u_{-} \mathrm{x} 1 * \mathrm{dmu}+\left(\operatorname{sqrt}\left(1-\mathrm{dmu} u^{\wedge} 2\right) / \operatorname{sqrt}\left(1-\mathrm{mu} \mathrm{z} 1^{\wedge} 2\right)\right)$

$*\left(m u \_x 1 * m u \_z 1 * \cos (d p h i)-m u_{-} y 1 * \sin (d p h i)\right)$;

$\mathrm{mu} \mathrm{y} 2=\mathrm{mu} \mathrm{y} \mathrm{y} 1 * \mathrm{dmu}+\left(\operatorname{sqrt}\left(1-\mathrm{dmu} \mathrm{a}^{\wedge}\right) / \operatorname{sqrt}\left(1-\mathrm{mu} z 1^{\wedge} 2\right)\right)$

$*\left(\mathrm{mu} \_\mathrm{y} 1 * \mathrm{mu} \_z 1 * \cos (\mathrm{dph} i)+\mathrm{mu} \_\mathrm{x} 1 * \sin (\mathrm{dph} i)\right)$;

$\mathrm{mu}_{z} z 2=\mathrm{mu} \_z 1 * \mathrm{dmu}-\operatorname{sqrt}\left(1-\mathrm{dmu}{ }^{\wedge} 2\right) * \operatorname{sqrt}\left(1-\mathrm{mu} \_z 1^{\wedge} 2\right) * \cos (\mathrm{dph} i) ;$

end

$\%$ sample isotropic elastic scattering

function $\mathrm{E} 2$ = sample_elastic_isotropic $(\mathrm{E} 1, \mathrm{~A})$

alpha $=((A-1) /(A+1))^{\wedge} 2$;

$\mathrm{E} 2=(1-\mathrm{al}$ pha $) * \mathrm{E} 1 * \mathrm{rand}+\mathrm{alpha} * \mathrm{E} 1 ;$

end

$\%$ sample path length in toroidal geometry

function [pathlength_tot,region]

$=\operatorname{sample} \_p a t h\left(x, y, z, m u_{-} x, m u_{-} y, m u_{-} z, r e g i o n\right.$, sigma_t $)$

global a1 a2 a3 a4 a5;

$\%$ obtain positive real quartic solutions for each torus points1 = solvequartic $\left(\mathrm{a} 1, \mathrm{x}, \mathrm{y}, \mathrm{z}, \mathrm{mu} \_\mathrm{x}, \mathrm{mu}\right.$ _y, $\left.\mathrm{mu} \_\mathrm{z}\right)$; 


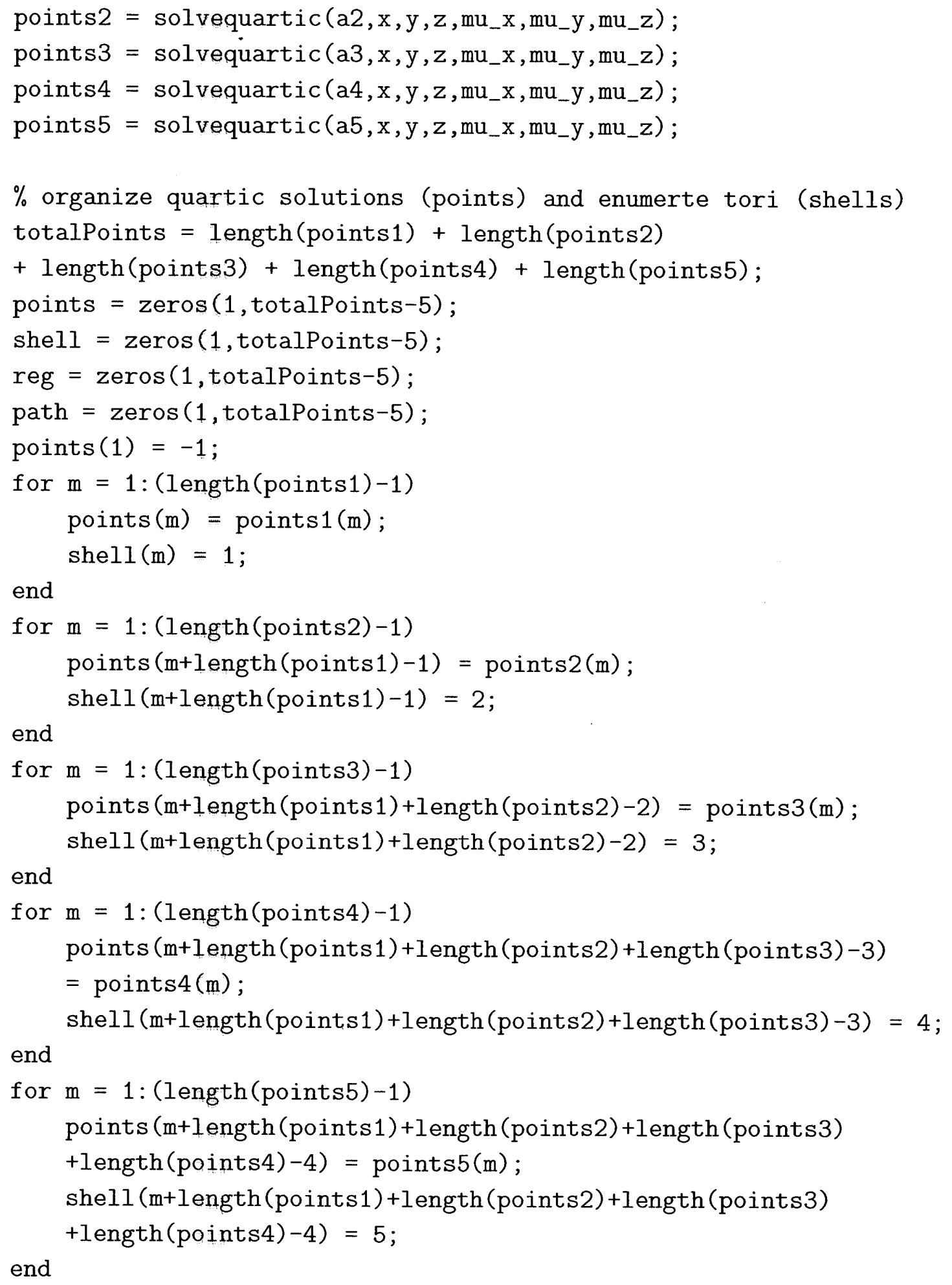




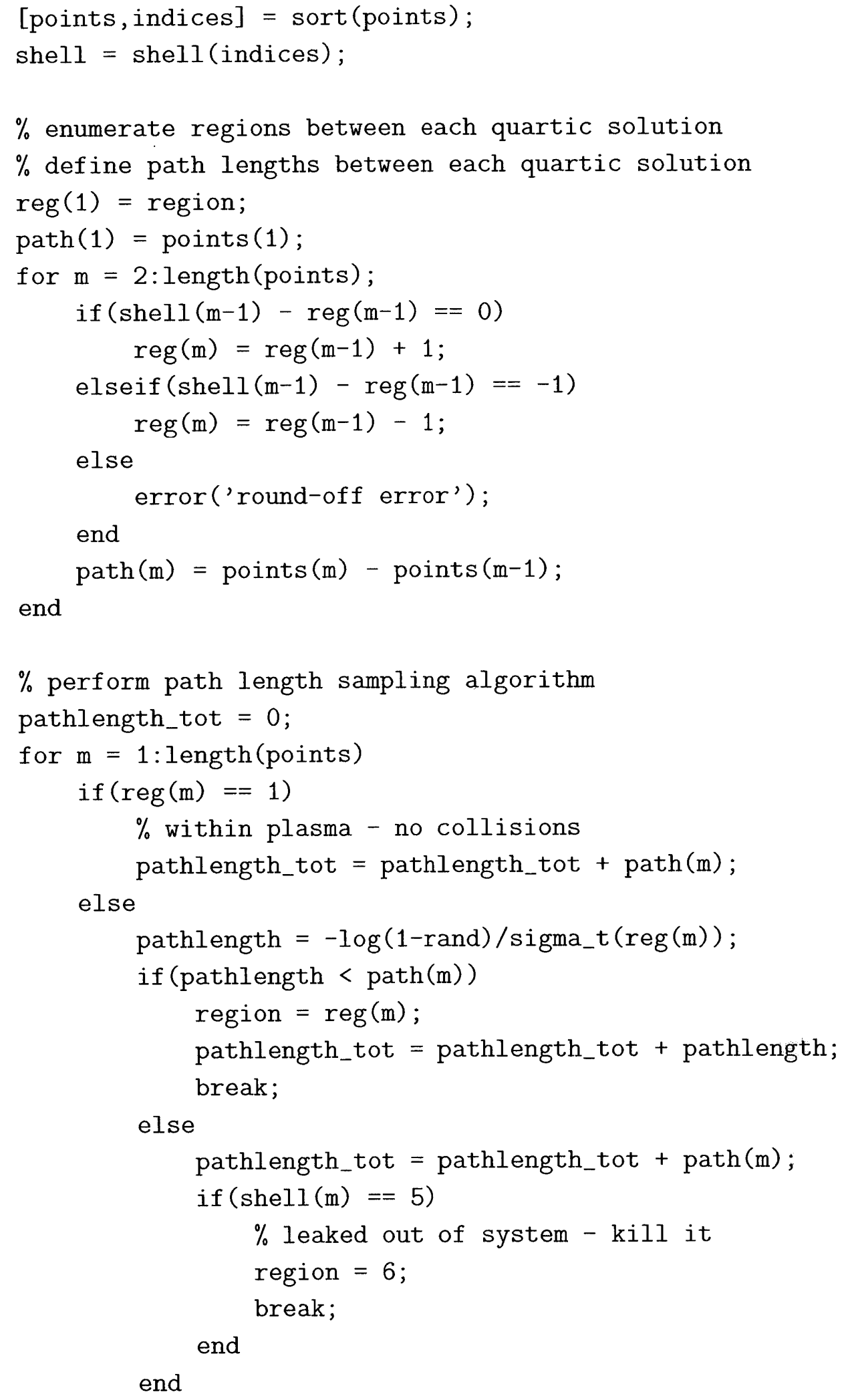


end

end

end

$\%$ determine positive real quartic solutions for one torus function radiiuseful2 $=\operatorname{solvequartic}(\mathrm{ra}, \mathrm{x} 0, \mathrm{y} 0, \mathrm{z} 0, \mathrm{x} 1, \mathrm{y} 1, \mathrm{z} 1)$

global R kappa;

$\mathrm{L}=\mathrm{R}^{\wedge} 2-\mathrm{ra}^{\wedge} 2+\mathrm{x} 0^{\wedge} 2+\mathrm{y}^{\wedge} 2+(\mathrm{z} 0 / \mathrm{kappa})^{\wedge} 2 ; \quad \% \mathrm{~cm}^{\wedge} 2$

$M=2 * x 0 * x 1+2 * y 0 * y 1+2 * z 0 * z 1 /\left(k^{2}{ }^{2}{ }^{\wedge} 2\right) ; \quad \% c m$

$\mathrm{N}=\mathrm{x}^{\wedge}{ }^{\wedge} 2+\mathrm{y} 1^{\wedge} 2+(\mathrm{z} 1 / \mathrm{kappa})^{\wedge} 2 ; \quad \%$ unitless

$\%$ define standard quartic coefficients

$\mathrm{A}=\mathrm{N}^{\wedge} 2 ; \quad \%$ unitless

$\mathrm{B}=2 * \mathrm{~N} * \mathrm{M} ; \quad \% 1 / \mathrm{cm}$

$\mathrm{C}=2 * \mathrm{~N} * \mathrm{~L}+\mathrm{M}^{\wedge} 2-4 *\left(\mathrm{R}^{\wedge} 2\right) *\left(\mathrm{x} 1^{\wedge} 2+\mathrm{y} 1^{\wedge} 2\right) ; \quad \% 1 / \mathrm{cm}^{\wedge} 2$

$\mathrm{D}=2 * \mathrm{M} * \mathrm{~L}-8 *\left(\mathrm{R}^{\wedge} 2\right) *(\mathrm{x} 0 * \mathrm{x} 1+\mathrm{y} 0 * \mathrm{y} 1) ; \% 1 / \mathrm{cm}^{\wedge} 3$

$\mathrm{E}=\mathrm{L}^{\wedge} 2-4 *\left(\mathrm{R}^{\wedge} 2\right) *\left(\mathrm{x}^{\wedge} 2+\mathrm{y} 0^{\wedge} 2\right) ; \quad \% 1 / \mathrm{cm}^{\wedge} 4$

$\%$ call Ferrari's method for solutions

radii $=$ quarticzeros $(A, B, C, D, E) ; \% m$

$\% \operatorname{radi} i=\operatorname{roots}([A, B, C, D, E]) ; \quad \% c m$

$\%$ select only positive real zeros

radiiuseful $=\operatorname{zeros}(1,4)$;

$\mathrm{m}=1$;

for $j=1: 4$

$r=\operatorname{radii}(j)$;

if $(($ real $(r)>0) \& \&(\operatorname{abs}($ imag $(r) / r e a l(r))<1 e-3))$

radiiuseful $(m)=\operatorname{real}(r)$;

$\mathrm{m}=\mathrm{m}+1$

end

end

$\operatorname{radiiuseful}(\mathrm{m})=-1$;

$\%$ convert solution into convenient output format

radiiuseful2 $=\operatorname{zeros}(1, \mathrm{~m})$; 


$$
\begin{aligned}
& \text { for } j=1: m \\
& \text { radiiuseful } 2(j)=\operatorname{radiiuseful}(j) ; \\
& \text { end }
\end{aligned}
$$

end

$\%$ Ferrari's quartic solution

function result $=$ quarticzeros $(A, B, C, D, E)$

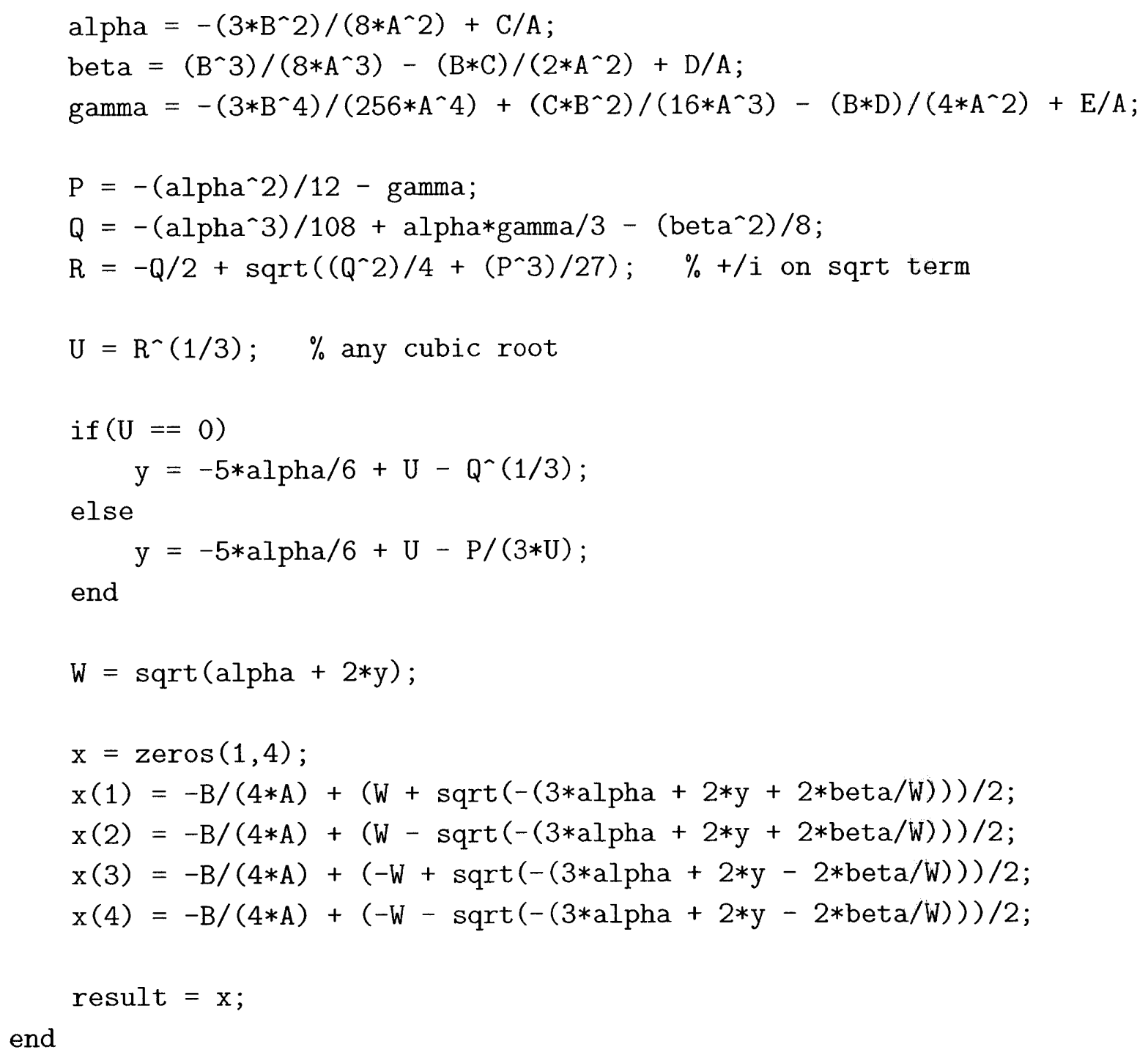


global R kappa;

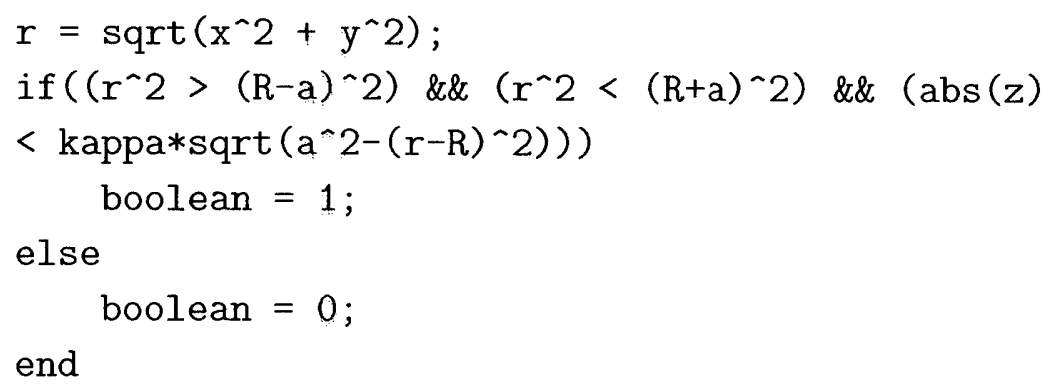

end

$\%$ evaluate fission nu

function value $=n u(E)$

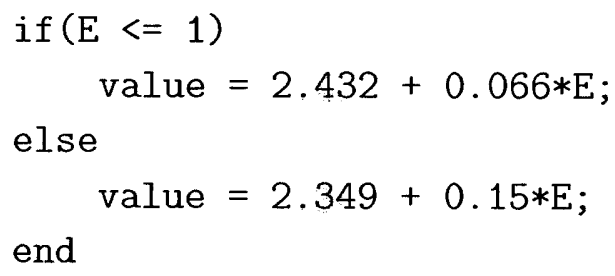

end

$\%$ sample truncated U-238 fission spectrum

$\%$ with rejection sampling

function $\mathrm{x}=$ sample_fission238(Ei)

$$
\begin{aligned}
& x=10 \\
& y=100 \\
& \text { while }(y>\operatorname{chi} 238(x, E i)) \\
& \qquad \begin{aligned}
x=\operatorname{rand} * 14 \\
\text { y }=\text { rand }
\end{aligned} \\
& \text { end }
\end{aligned}
$$

end

$\%$ sample truncated U-235 fission spectrum

$\%$ with rejection sampling 
function $\mathrm{x}=$ sample_fission235(Ei)

$$
\begin{aligned}
& \begin{array}{l}
x=10 \\
y=100
\end{array} \\
& \begin{array}{l}
\text { while }(y>\operatorname{chi} 235(x, E i)) \\
\quad x=\operatorname{rand} * 14 ; \\
y=\operatorname{rand} * 0.8
\end{array} \\
& \text { end }
\end{aligned}
$$

end

$\%$ evaluate fission chi for U-238

function value $=\operatorname{chi} 238(\mathrm{E}, \mathrm{Ei})$

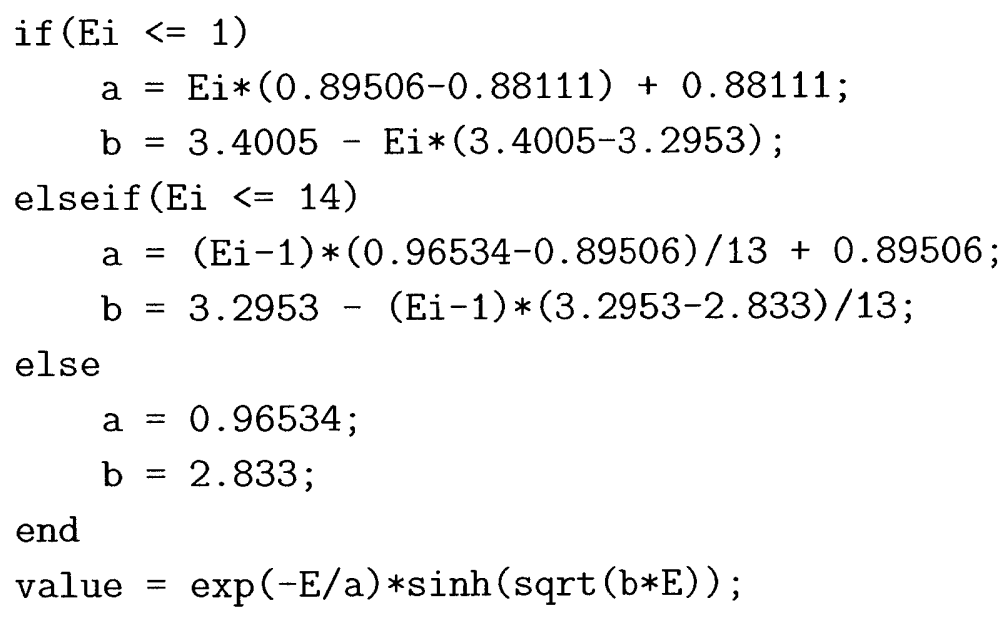

end

$\%$ evaluate fission chi for U-235

function value $=\operatorname{chi} 235(\mathrm{E}, \mathrm{Ei})$

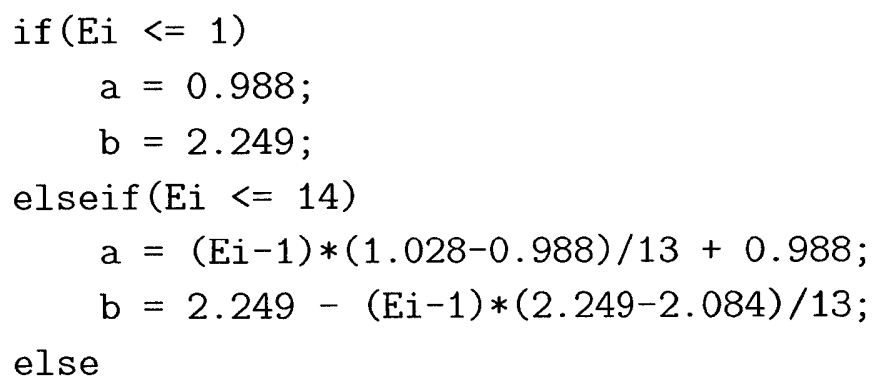




$$
\begin{aligned}
& \mathrm{a}=1.028 \text {; } \\
& \mathrm{b}=2.084 \text {; }
\end{aligned}
$$

end

$\%$ sample maxwellian distribution for $(n, x n)$

function $\mathrm{x}=\operatorname{maxwellian}(\mathrm{a}, \mathrm{EO})$

$$
\begin{aligned}
& \mathrm{x}=9999 \\
& \mathrm{y}=10 ; \\
& \operatorname{while}(\mathrm{y}>\mathrm{x} * \exp (-\mathrm{x} / \mathrm{a})) \\
& \qquad \begin{aligned}
& \mathrm{x}=\operatorname{rand} * \mathrm{E} 0 * 0.9 ; \\
& \mathrm{y}=\operatorname{rand} *(\mathrm{a} / \exp (1))
\end{aligned} \\
& \text { end }
\end{aligned}
$$

end

$\%$ open source binary search code (slightly modified)

$\% \operatorname{bsearch}(\mathrm{x}, \mathrm{var})$

$\%$ Written by Aroh Barjatya

$\%$ Binary search for values specified in vector 'var' within data vector ' $\mathrm{x}$ '

$\%$ The data has to be pre-sorted in ascending or decending order

$\%$ There is no way to predict how the function will behave if there

$\%$ are multiple numbers with same value.

$\%$ returns the index values of the searched numbers

function index $=\operatorname{bsearch}(x, \operatorname{var})$

$x$ Len $=$ length $(\mathrm{x})$;

[xRow $\mathrm{xCol}]=\operatorname{size}(\mathrm{x})$;

if $x(1)>x(x$ Len) $\%$ means $x$ is in descending order

if $x$ Row $==1$

$$
\mathrm{x}=\mathrm{fliplr}(\mathrm{x}) ;
$$

else

$$
x=f \operatorname{lipud}(x) ;
$$




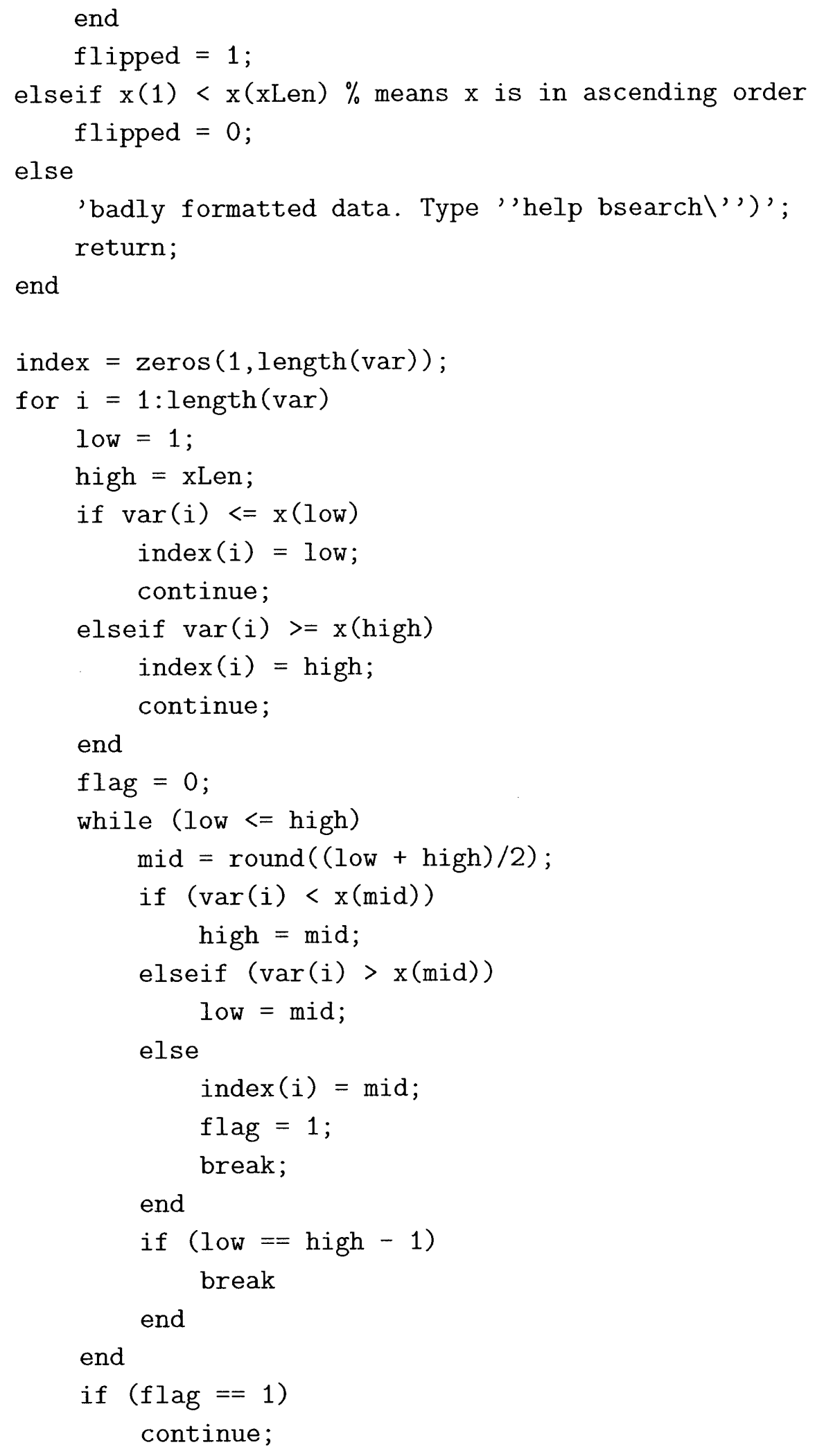




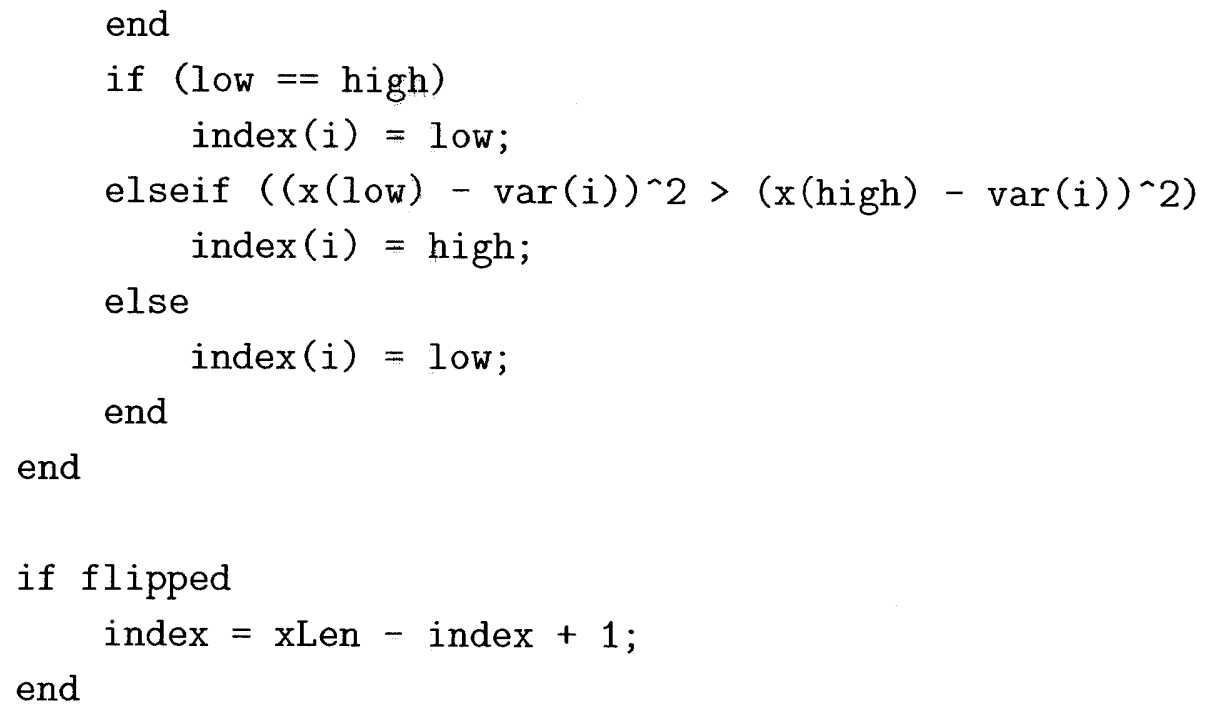




\section{Cylindrical Monte Carlo Code}

This is the path length sampling method for a fission-fusion hybrid Monte Carlo code in cylindrical geometry. This could replace the corresponding sample_path method in the toroidal code in Appendix B.

$\%$ sample path length in cylindrical geometry

function [pathlength_tot,region]

$=\operatorname{sample} e_{-}$ath $\left(\mathrm{x}, \mathrm{y}, \mathrm{z}, \mathrm{mu} \_\mathrm{x}, \mathrm{mu} \mathrm{y}_{-}, \mathrm{mu} \mathrm{z}_{-}\right.$, region, sigma_t $)$

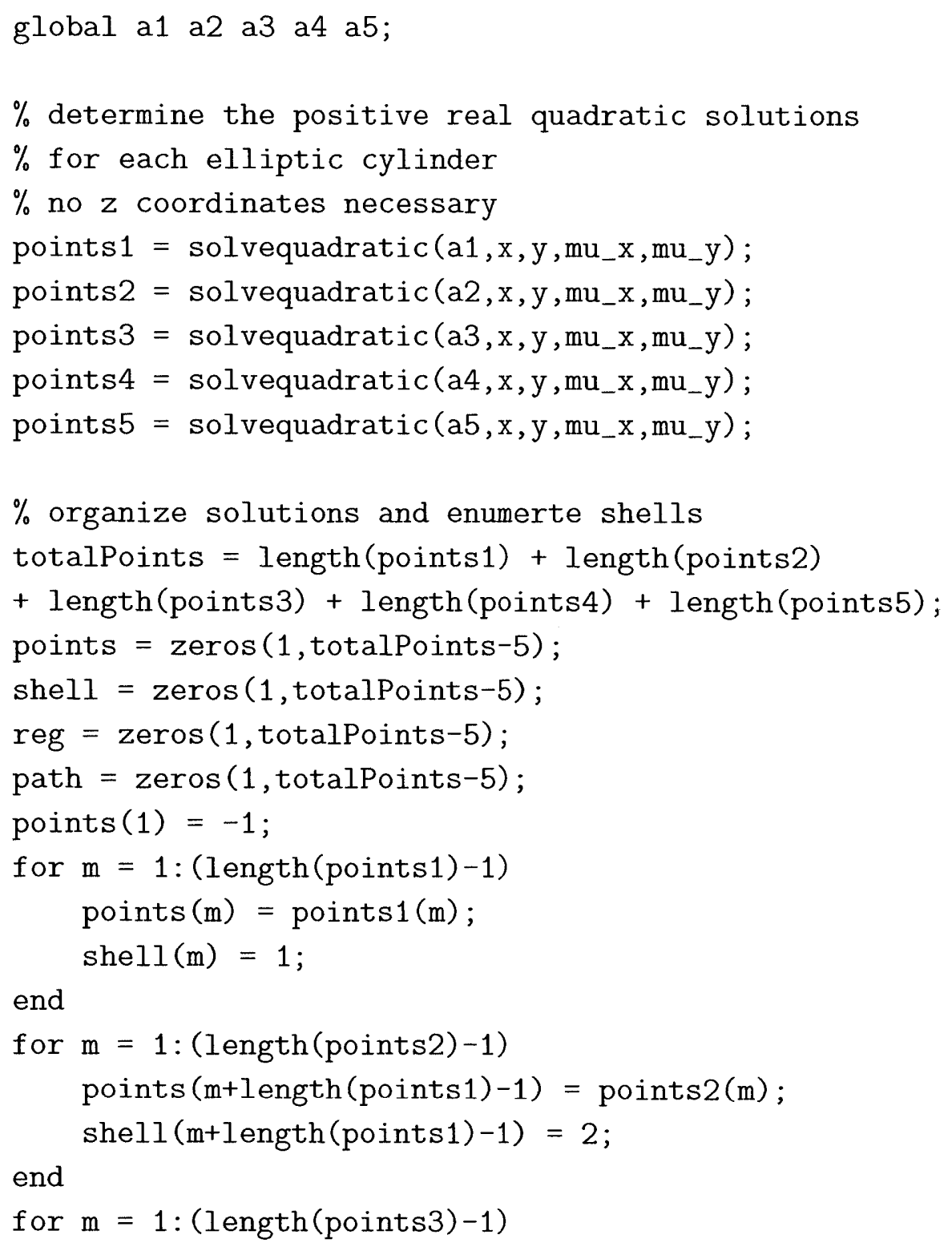

end 


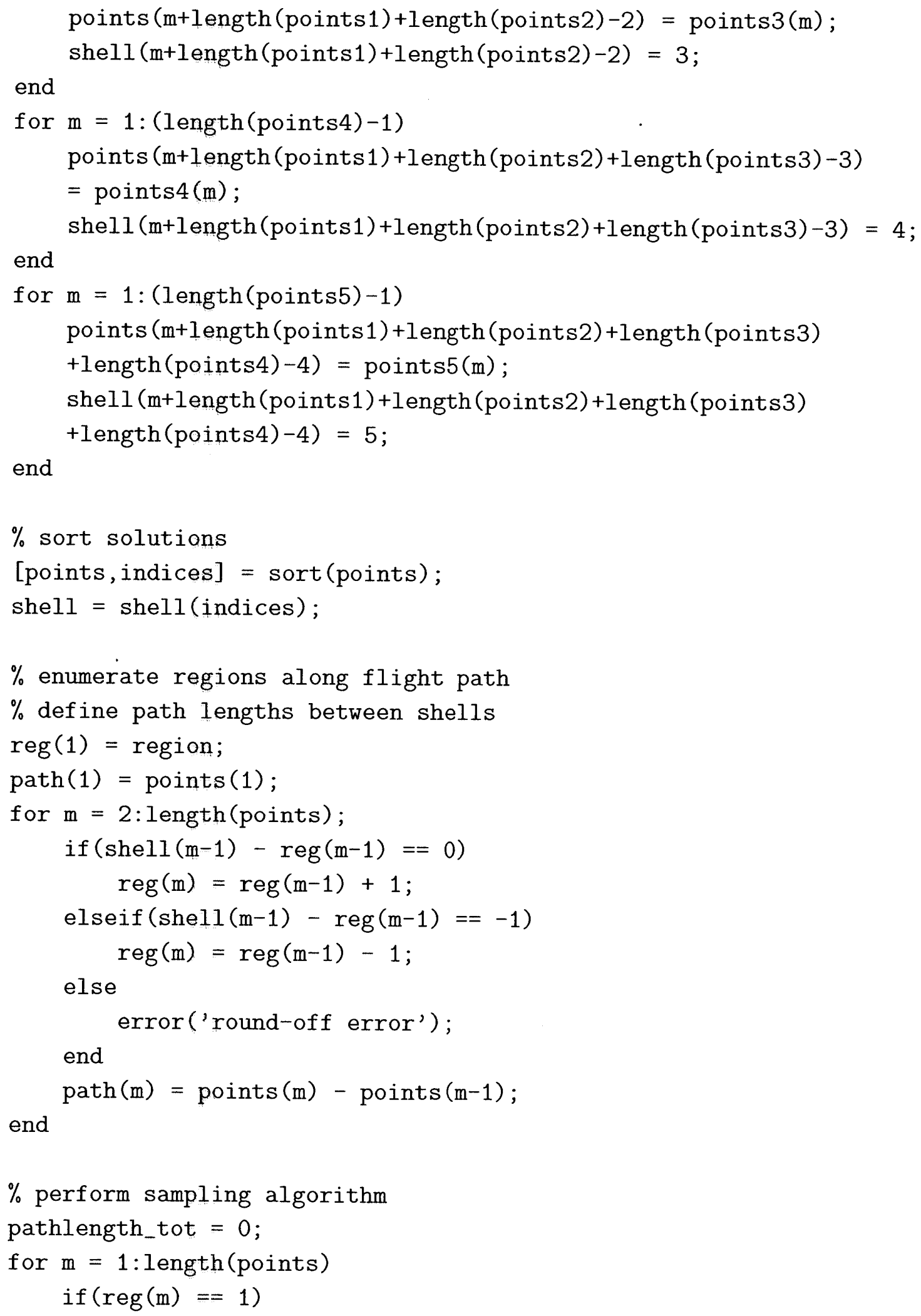




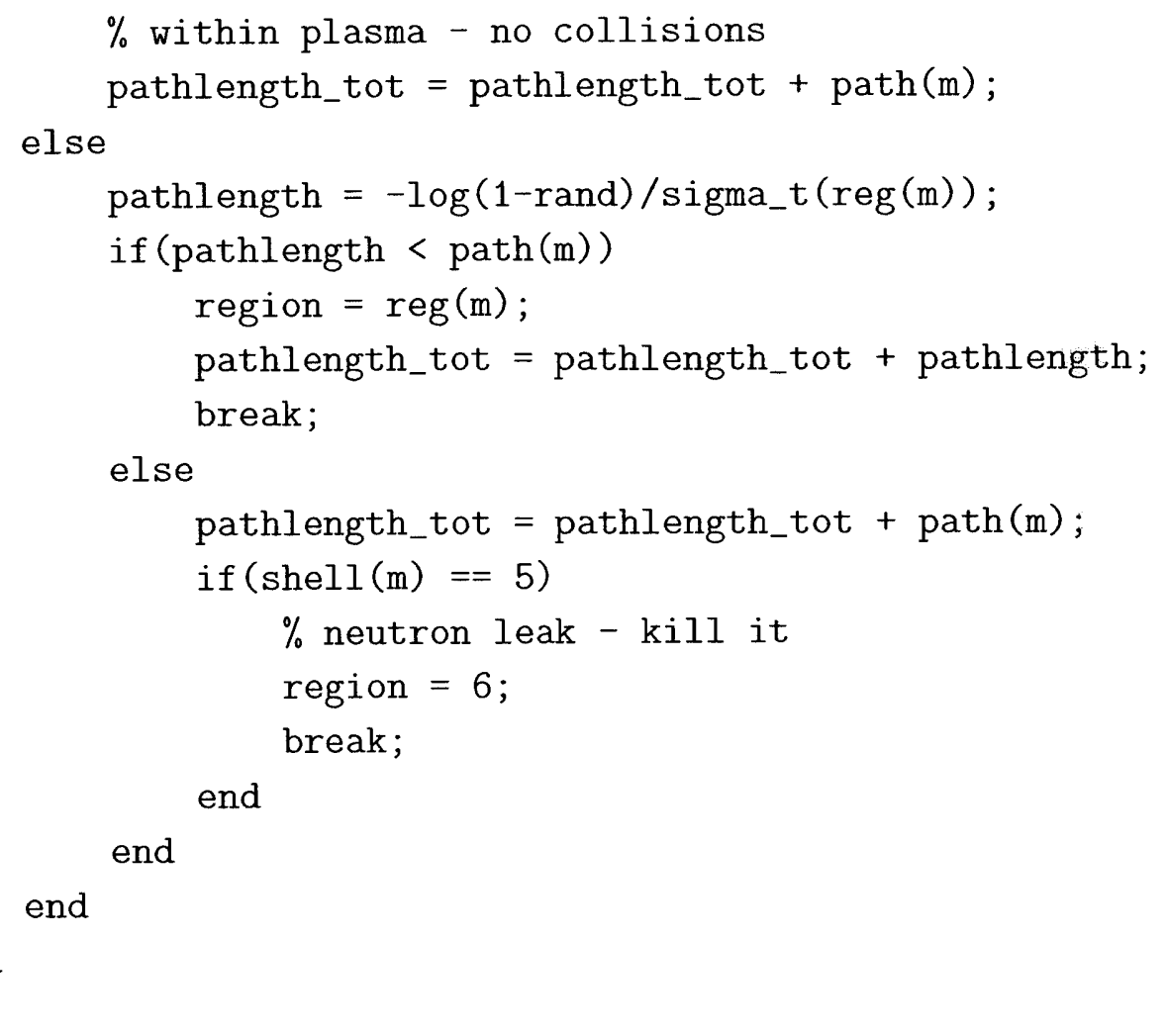

$\%$ solve quadratic equation for elliptic cylinder function radiiuseful2 $=\operatorname{solvequadratic}(\mathrm{ra}, \mathrm{x} 0, \mathrm{y} 0, \mathrm{x} 1, \mathrm{y} 1)$

global kappa;

$\%$ define standard quadradic coefficients

$A=x 1^{\wedge} 2+(y 1 / k a p p a)^{\wedge} 2$

$\mathrm{B}=2 *\left(\mathrm{x} 0 * \mathrm{x} 1+\mathrm{y} 0 * \mathrm{y} 1 / \mathrm{kappa}^{\wedge} 2\right)$;

$\mathrm{C}=\mathrm{xO}^{\wedge} 2+(\mathrm{y} 0 / \mathrm{kappa})^{\wedge} 2-\mathrm{ra}^{\wedge} 2$;

$\%$ the famed quadratic equation! $\operatorname{radii}(1)=\left(-B+\operatorname{sqrt}\left(B^{\wedge} 2-4 * A * C\right)\right) /(2 * A)$; $\operatorname{radii}(2)=\left(-B-\operatorname{sqrt}\left(B^{\wedge} 2-4 * A * C\right)\right) /(2 * A)$;

$\%$ determine the positive real solution radiiuseful $=\operatorname{zeros}(1,2)$;

$\mathrm{m}=1$;

for $j=1: 2$ 


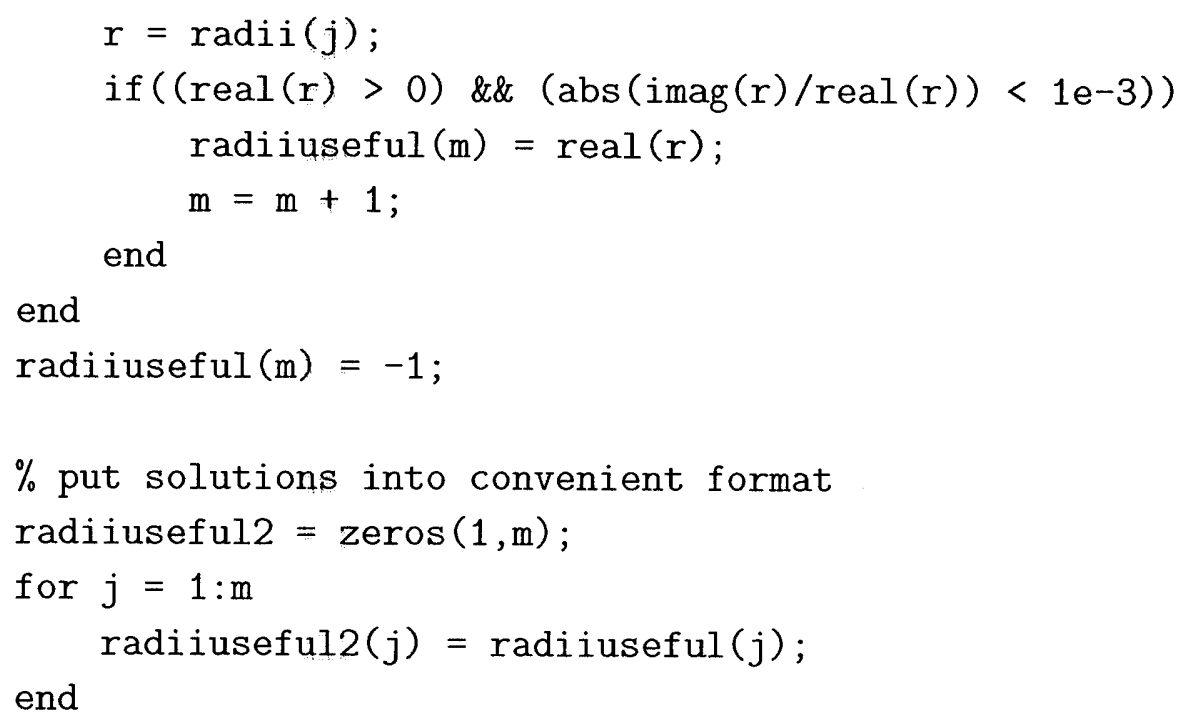




\section{Plasma Surface Neutron Flux Code}

\section{D.1 Toroidal Flux Monte Carlo}

A Monte Carlo code for toroidal fusion source sampling. Although only a part of the fullscale fission-fusion hybrid code, this stand-alone module can compute the scalar and angular neutron flux distributions at any point on the plasma surface.

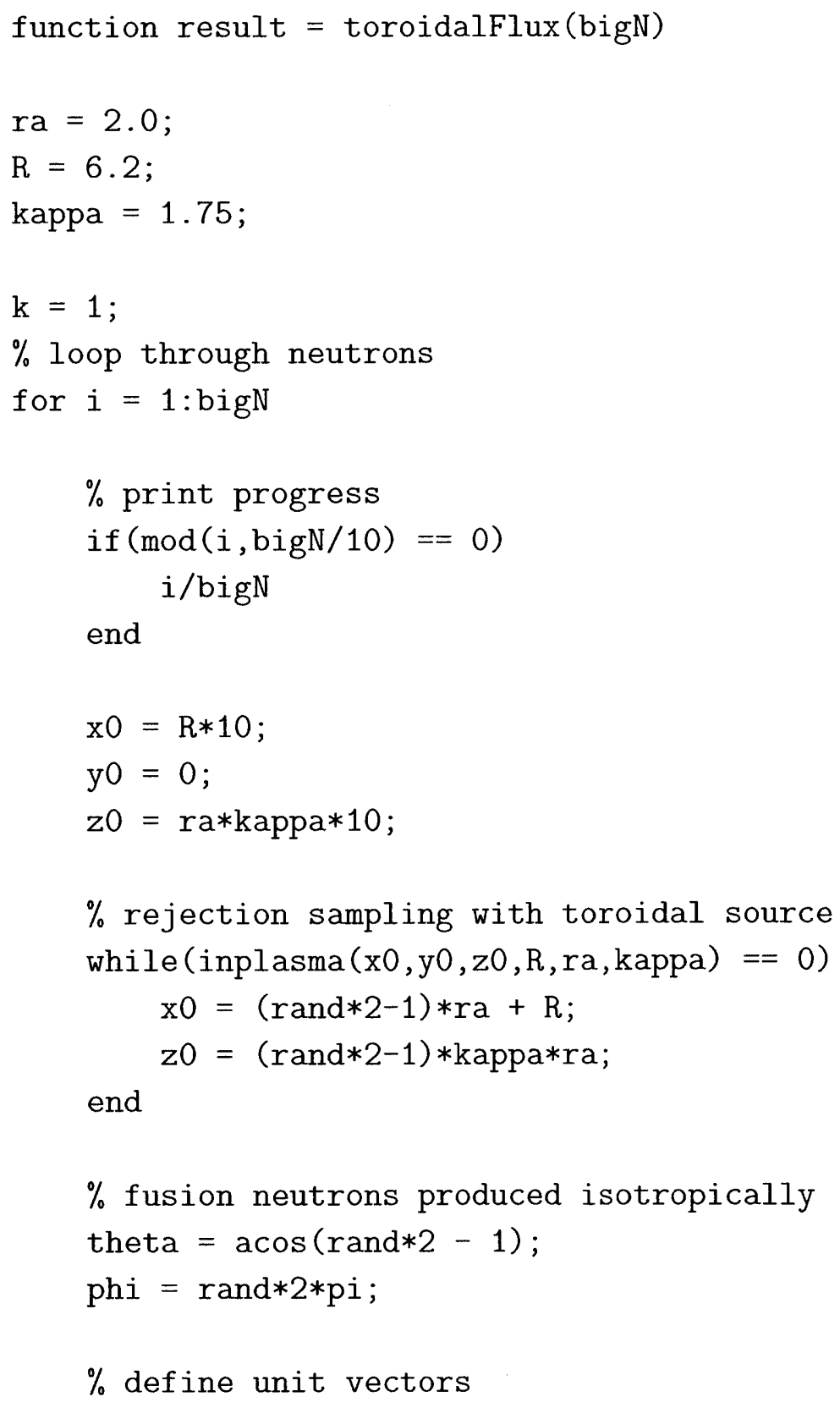




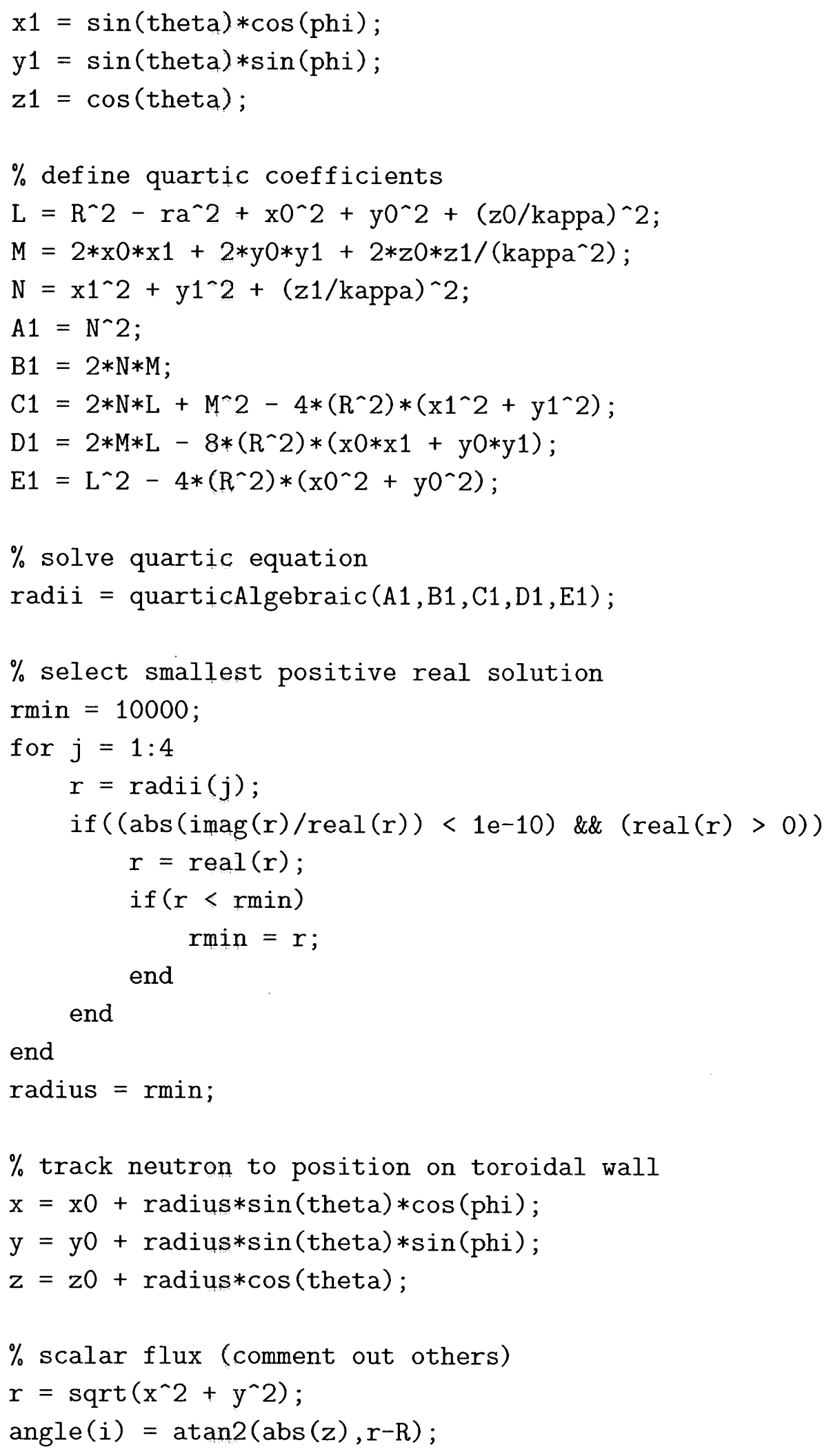




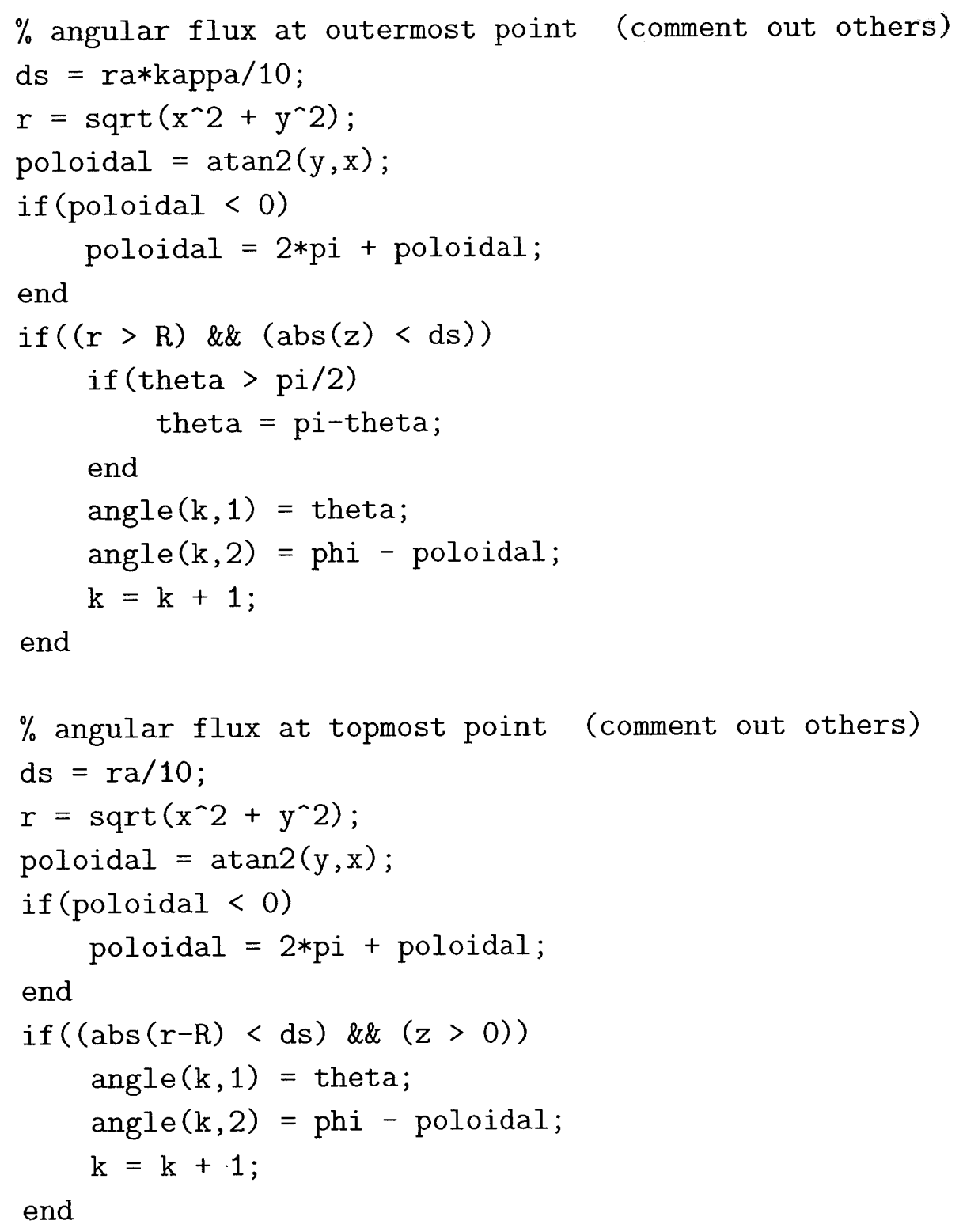

end

$\%$ plot histograms

hist (angle (:, 1) ,200);

[bar, $\mathrm{xbar}]=\operatorname{hist}($ angle, 100$)$;

$\%$ result $=[$ xbar $;$ bar $]$;

result = angle; 
end

$\%$ test whether a point is within a toroid

function result $=$ inplasma $(x, y, z, R, a, k a p p a)$

$x=\operatorname{sqrt}\left(x^{\wedge} 2+y^{\wedge} 2\right)$

if $\left(\left(r^{\wedge} 2>(R-a)^{\wedge} 2\right) \quad \& \&\left(r^{\wedge} 2<(R+a)^{\wedge} 2\right) \& \&\left(\operatorname{abs}(z)<\operatorname{kappa} * \operatorname{sqrt}\left(a^{\wedge} 2-(r-R)^{\wedge} 2\right)\right)\right)$ result $=1$;

else

$$
\text { result }=0 \text {; }
$$

end

end

$\%$ Ferrari's quartic equation solution

function result $=$ quarticAlgebraic $(A, B, C, D, E)$

alpha $=-\left(3 * B^{\wedge} 2\right) /\left(8 * A^{\wedge} 2\right)+C / A ;$

beta $=\left(\mathrm{B}^{\wedge} 3\right) /\left(8 * \mathrm{~A}^{\wedge} 3\right)-(\mathrm{B} * \mathrm{C}) /\left(2 * \mathrm{~A}^{\wedge} 2\right)+\mathrm{D} / \mathrm{A}$;

gamma $=-\left(3 * B^{\wedge} 4\right) /\left(256 * A^{\wedge} 4\right)+\left(C * B^{\wedge} 2\right) /\left(16 * A^{\wedge} 3\right)-(B * D) /\left(4 * A^{\wedge} 2\right)+E / A$;

$\mathrm{P}=-\left(\mathrm{alpha}{ }^{\wedge} 2\right) / 12-$ gamma;

$Q=-($ alpha^3)/108+alpha*gamma/3-(beta^2)/8;

$R=-Q / 2+\operatorname{sqrt}\left(\left(Q^{\wedge} 2\right) / 4+\left(P^{\wedge} 3\right) / 27\right) ;$

$U=R^{\wedge}(1 / 3)$

if $(U==0)$

$y=-5 * a l p h a / 6+U-Q^{\wedge}(1 / 3) ;$

else

$y=-5 * a l p h a / 6+U-P /(3 * U) ;$

end

$\mathrm{W}=\operatorname{sqrt}(\mathrm{alpha}+2 * \mathrm{y})$

$x(1)=-B /(4 * A)+(W+\operatorname{sqrt}(-(3 * a l p h a+2 * y+2 *$ beta $/ W))) / 2 ;$ 
$\mathrm{x}(2)=-\mathrm{B} /(4 * \mathrm{~A})+(\mathrm{W}-\operatorname{sqrt}(-(3 * \mathrm{alpha}+2 * \mathrm{y}+2 *$ beta $/ \mathrm{W}))) / 2 ;$

$\mathrm{x}(3)=-\mathrm{B} /(4 * \mathrm{~A})+(-\mathrm{W}+\operatorname{sqrt}(-(3 * a \mathrm{lpha}+2 * \mathrm{y}-2 *$ beta $/ \mathrm{W}))) / 2$;

$\mathrm{x}(4)=-\mathrm{B} /(4 * \mathrm{~A})+(-\mathrm{W}-\operatorname{sqrt}(-(3 * a l p h a+2 * \mathrm{y}-2 *$ beta $/ \mathrm{W}))) / 2$;

result $=\mathrm{x}$;

end 


\section{D.2 Toroidal Volume - Spiric Sections}

This function calculates the volume of toroid by integrating spiric sections from $x=R-a$ to $R+a$.

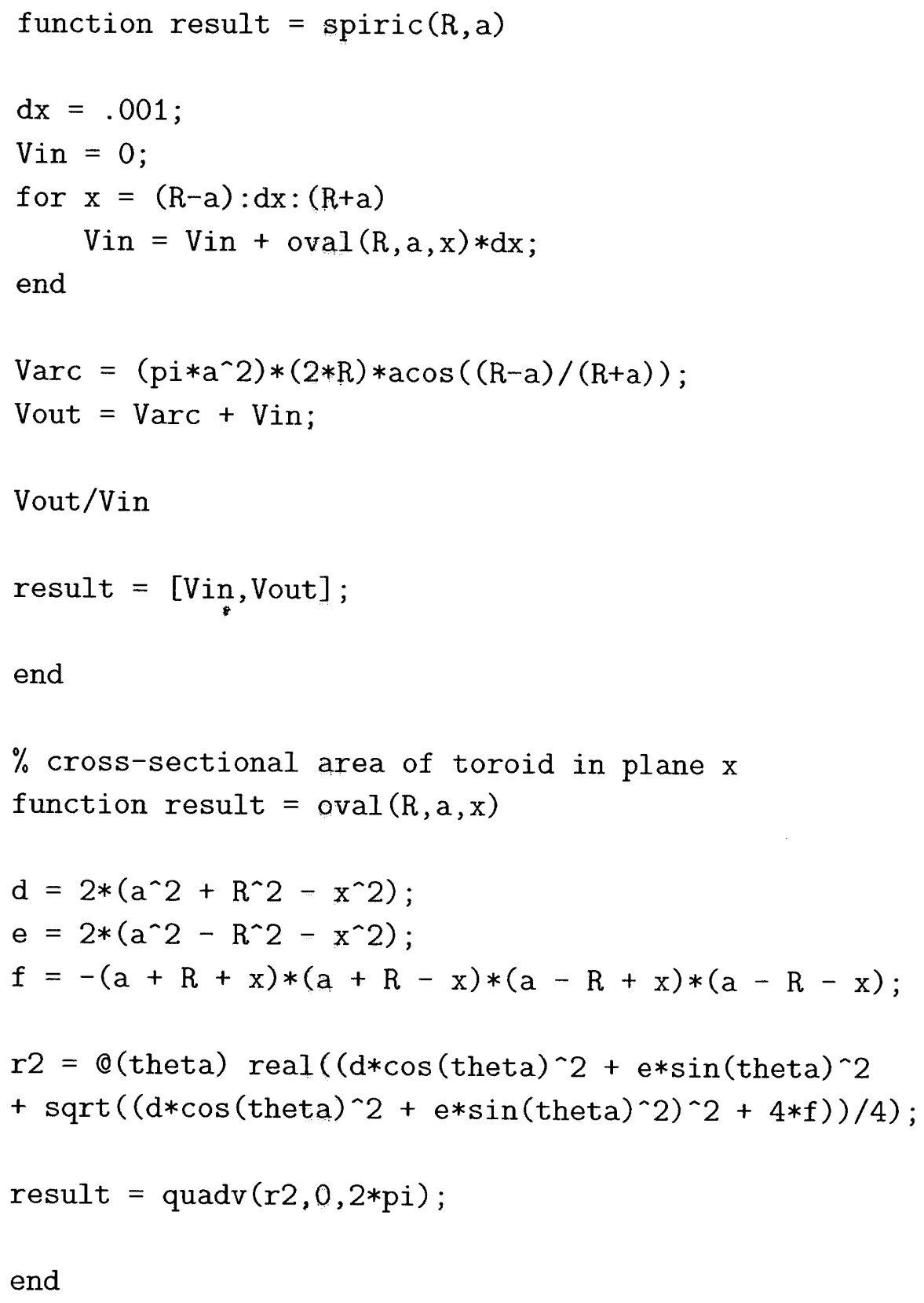




\section{E $\quad k_{\infty}$ Monte Carlo Code}

This is a geometry-independent Monte Carlo neutron transport code for calculating $k_{\infty}$. Here we apply it to $\mathrm{UO}_{2}$ for arbitrary enrichment.

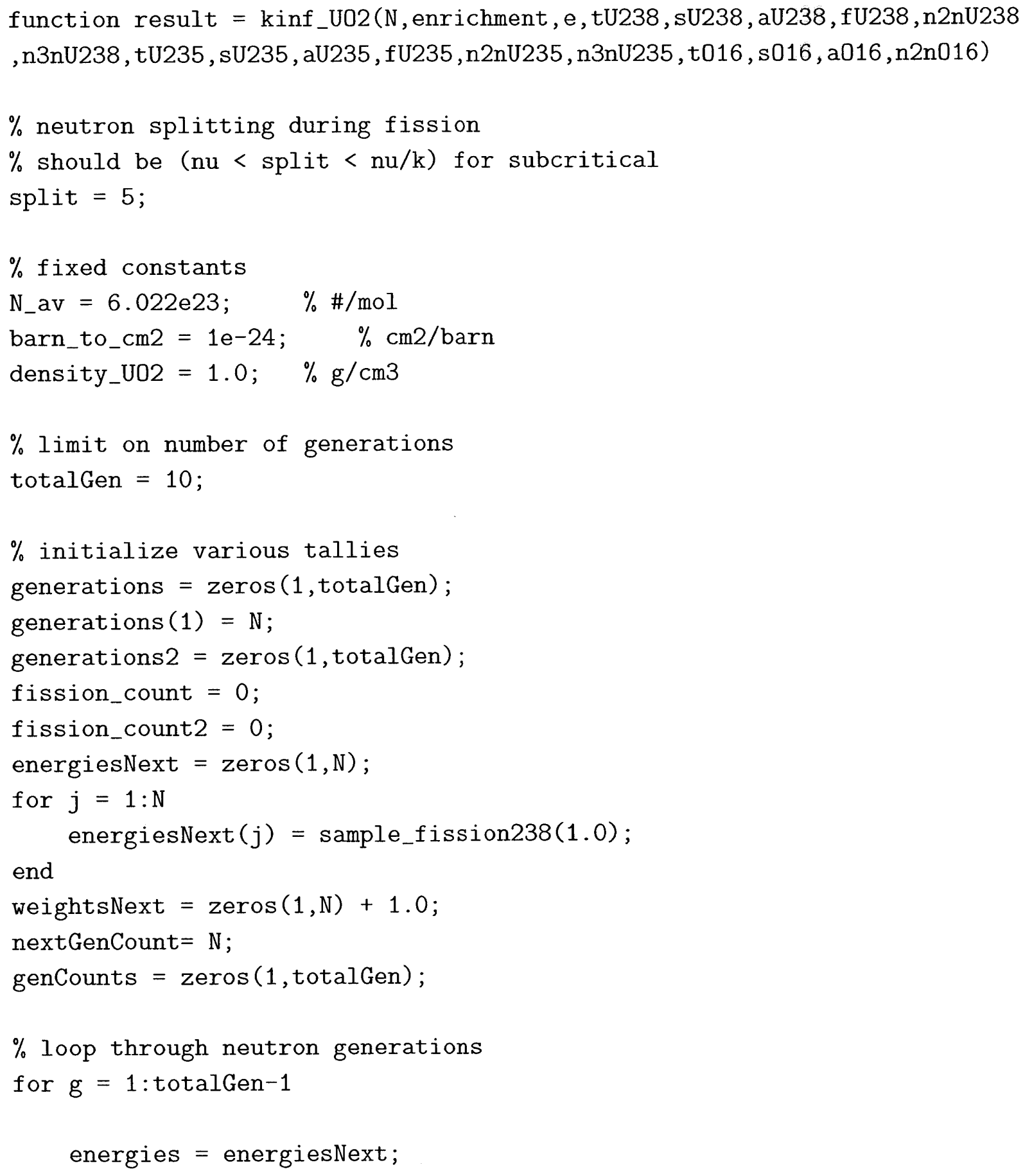




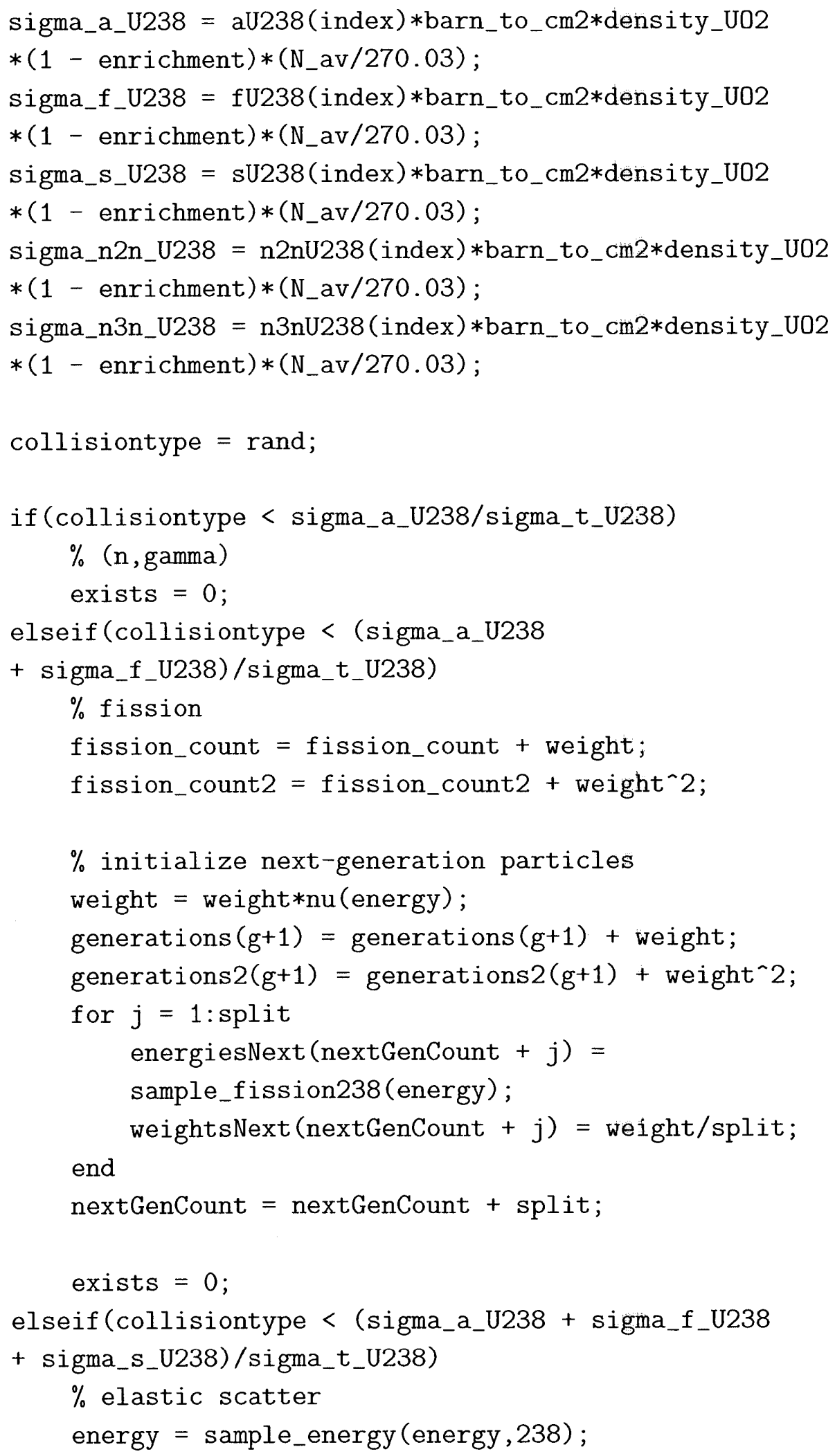




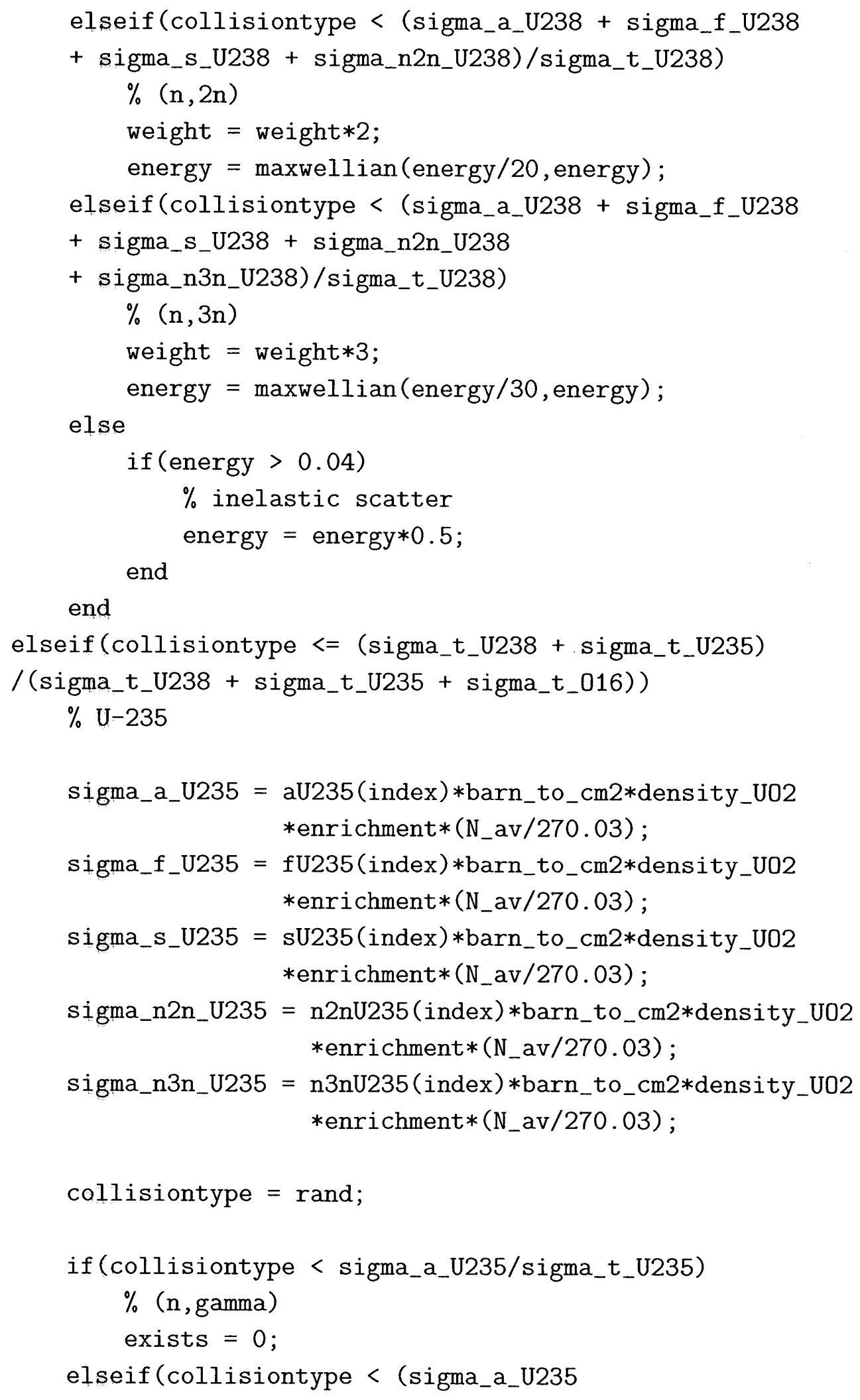




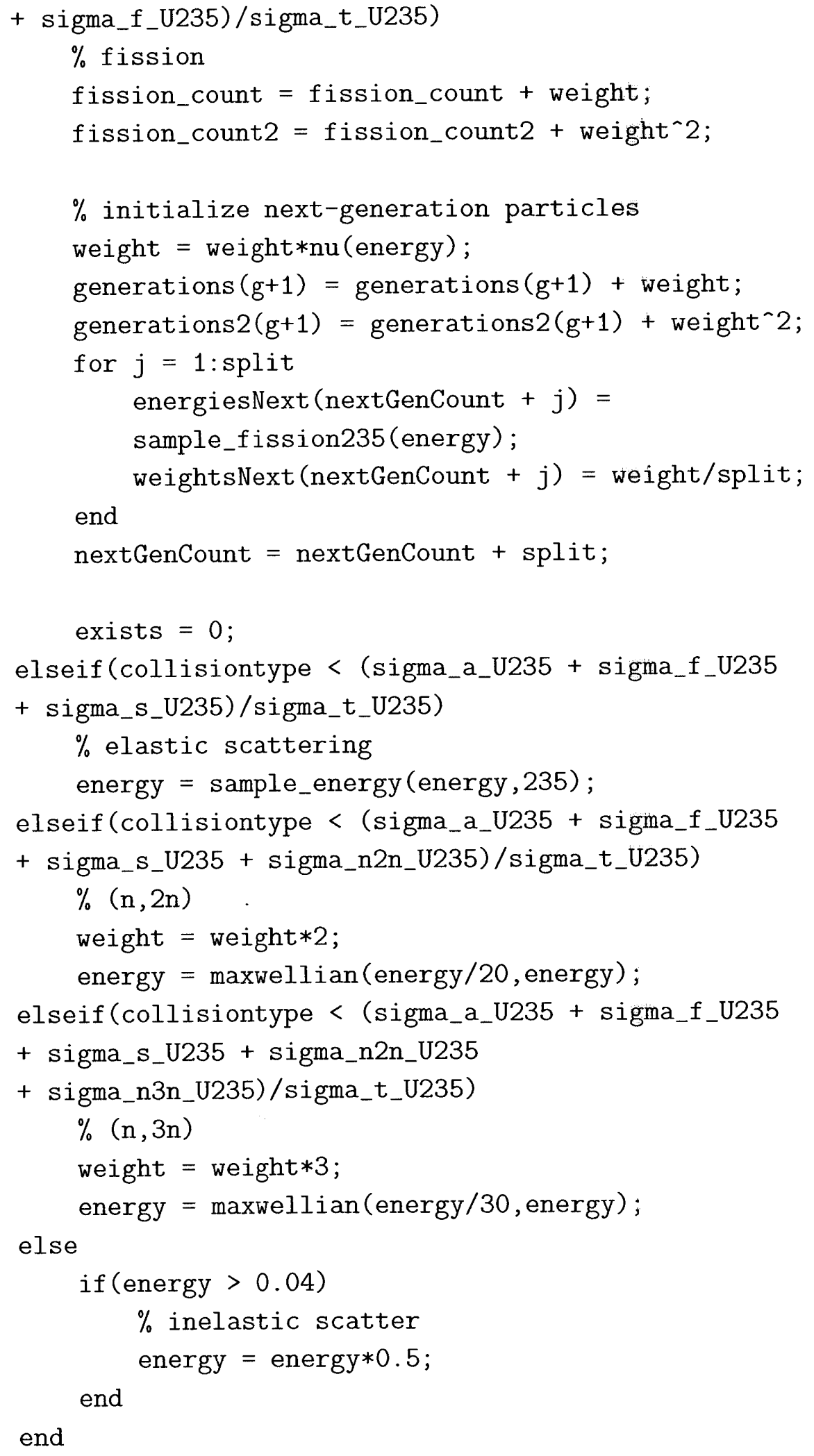




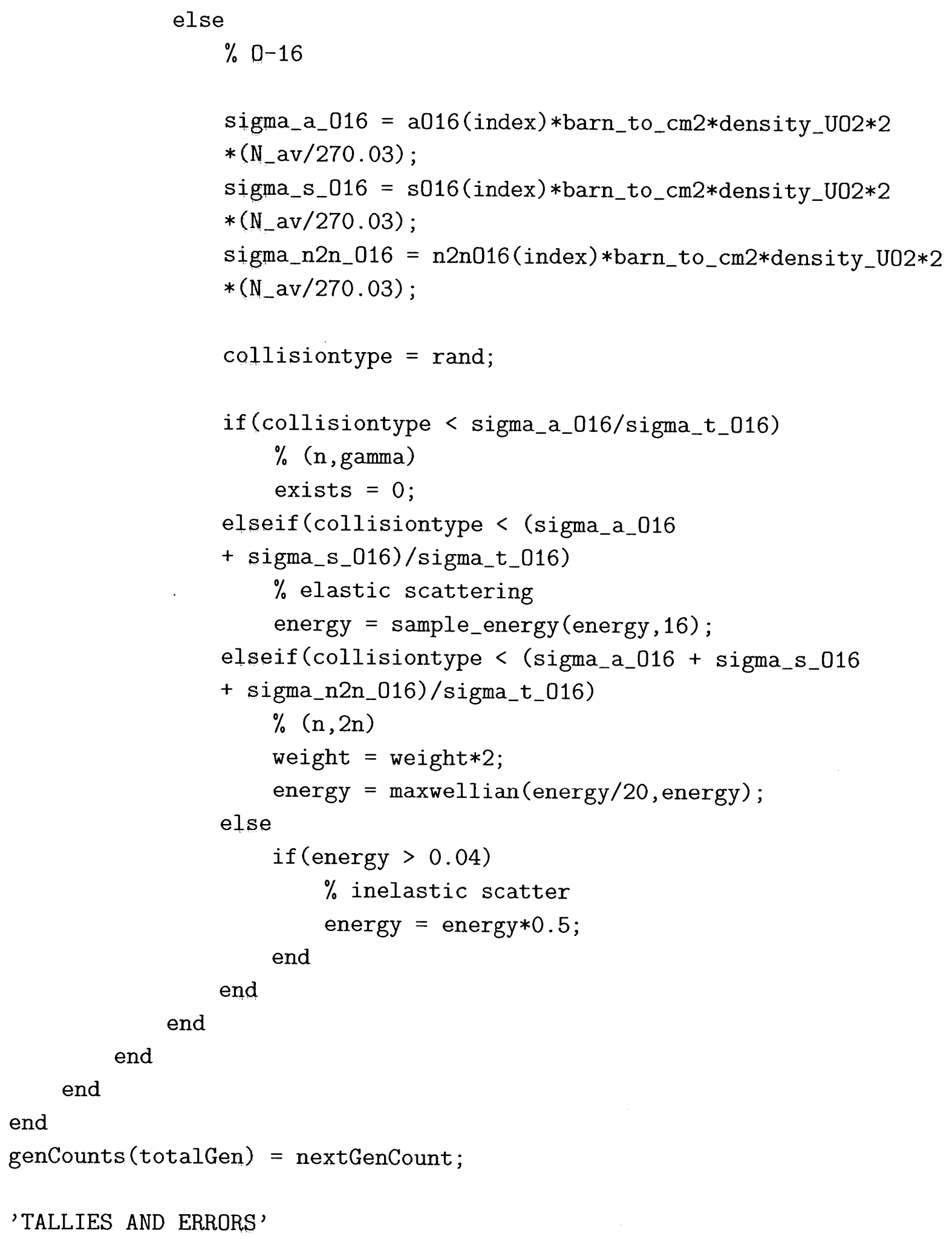




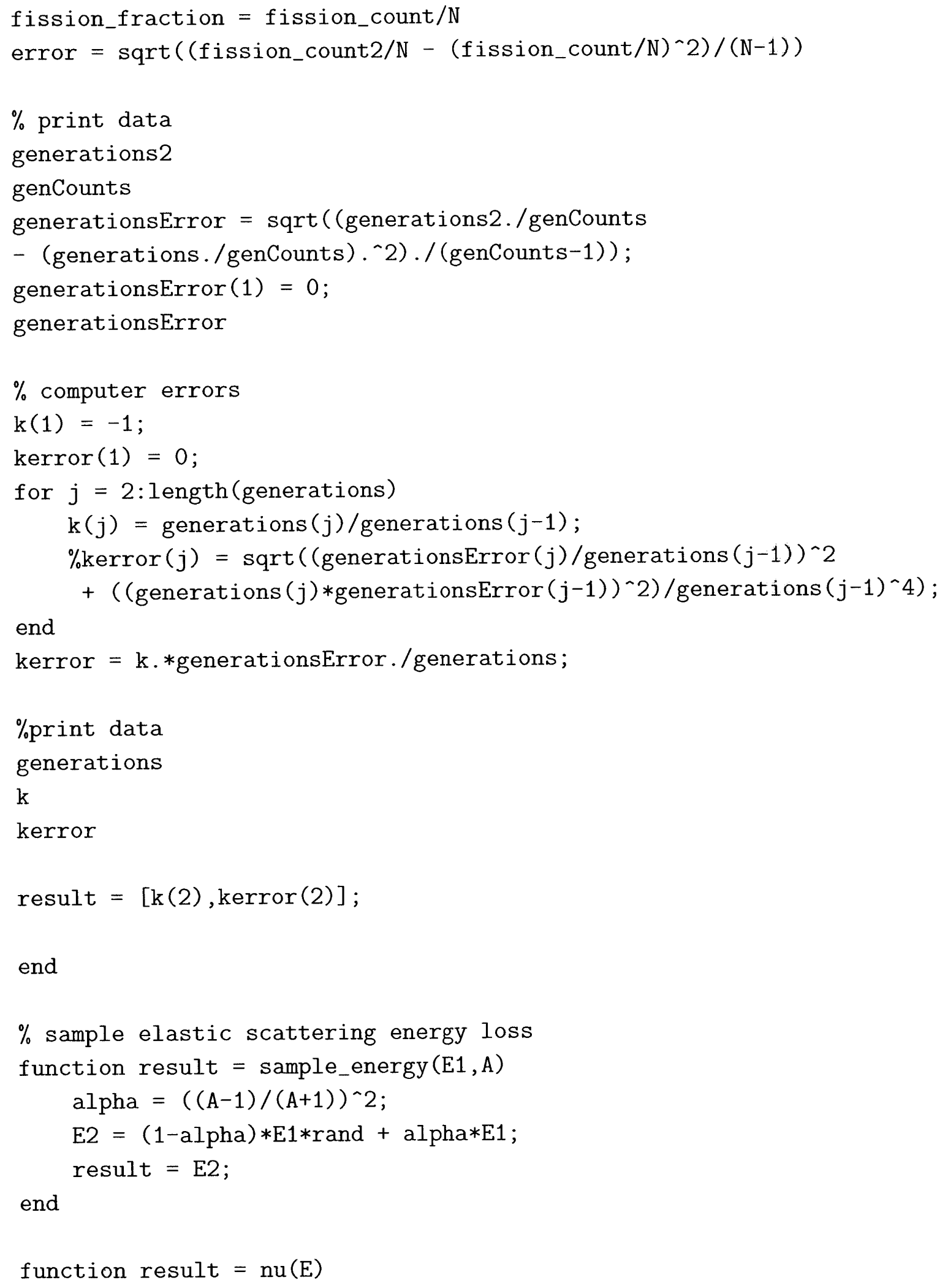




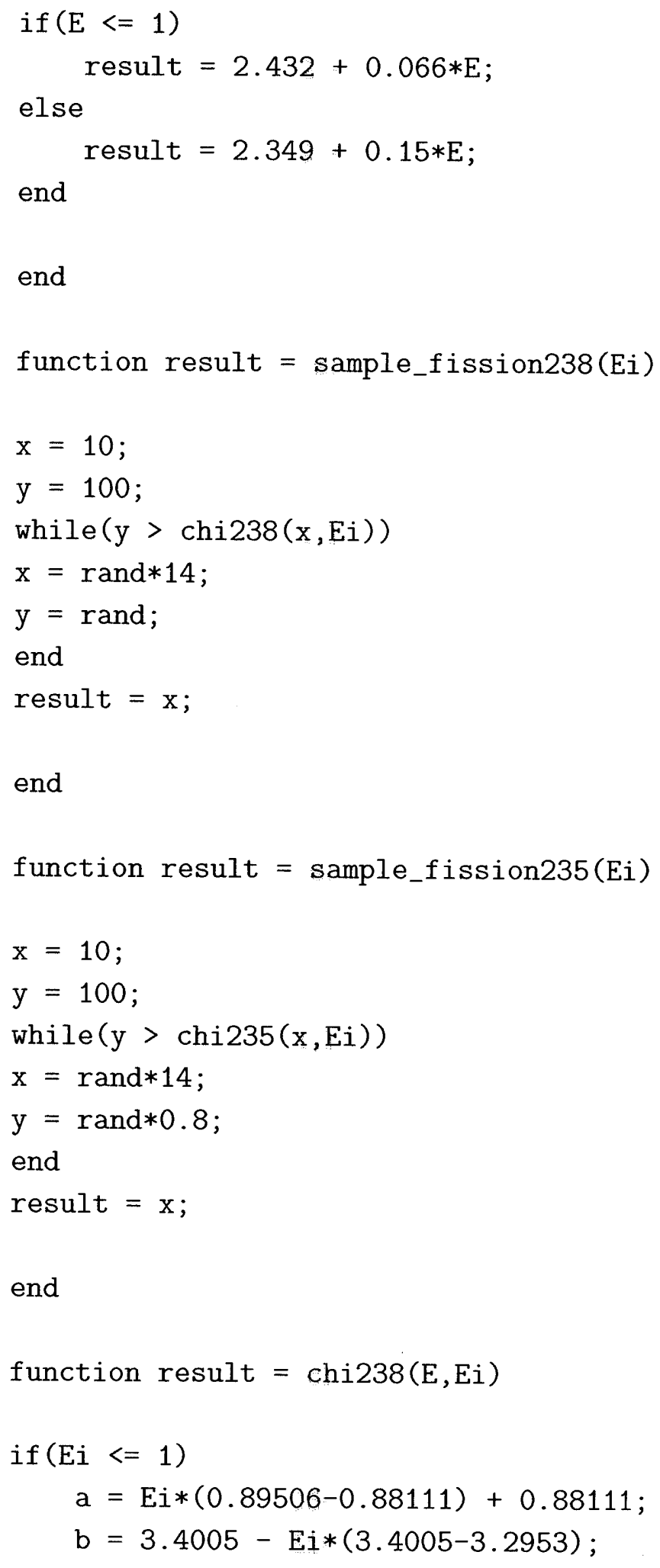




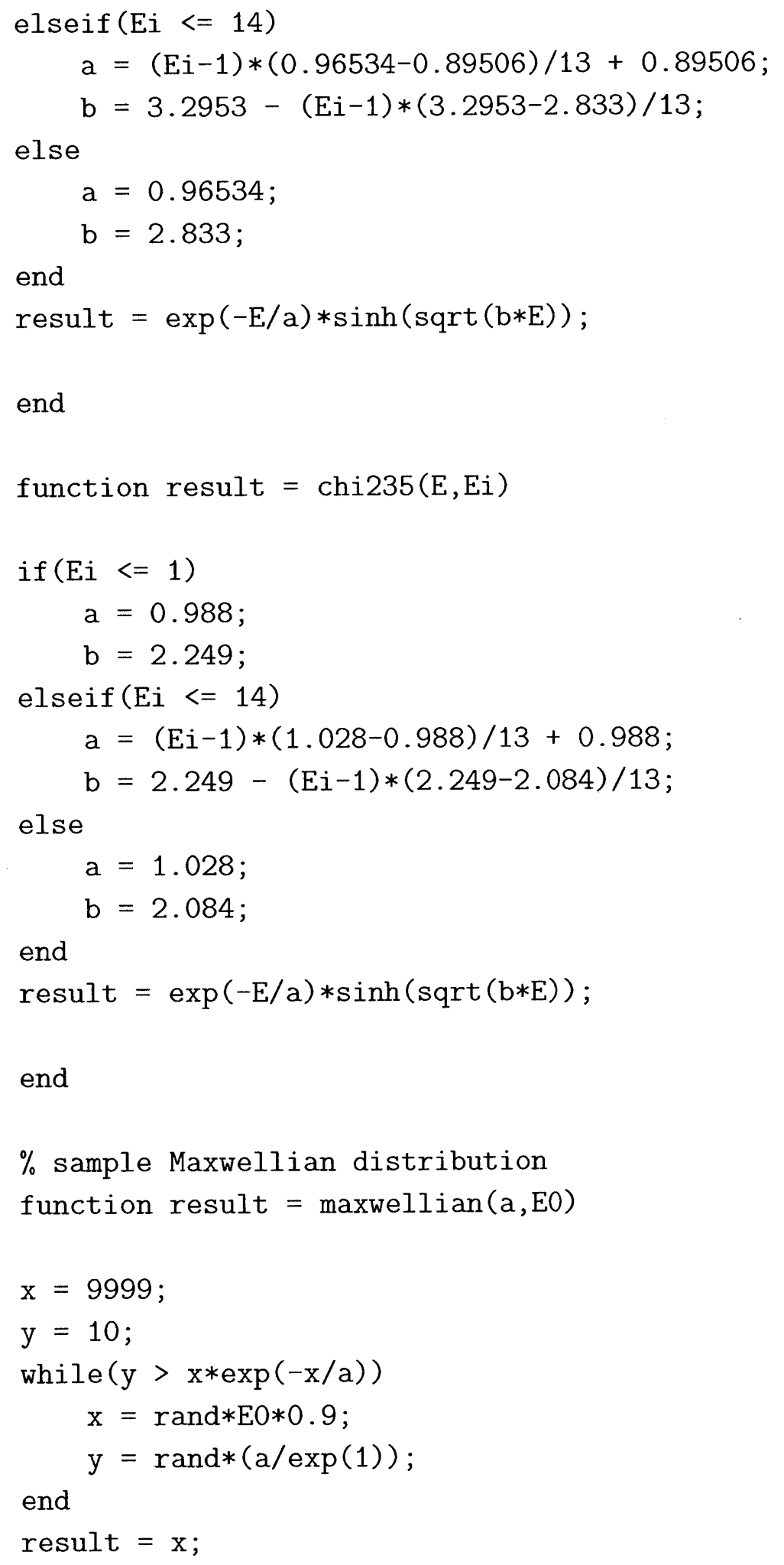


end 


\section{F Elastic Scattering}

Here is a simple Monte Carlo code for calculating elastic scattering angular probability distributions $P\left(\mu_{L}\right)$ for arbitrary nuclide mass $A$.

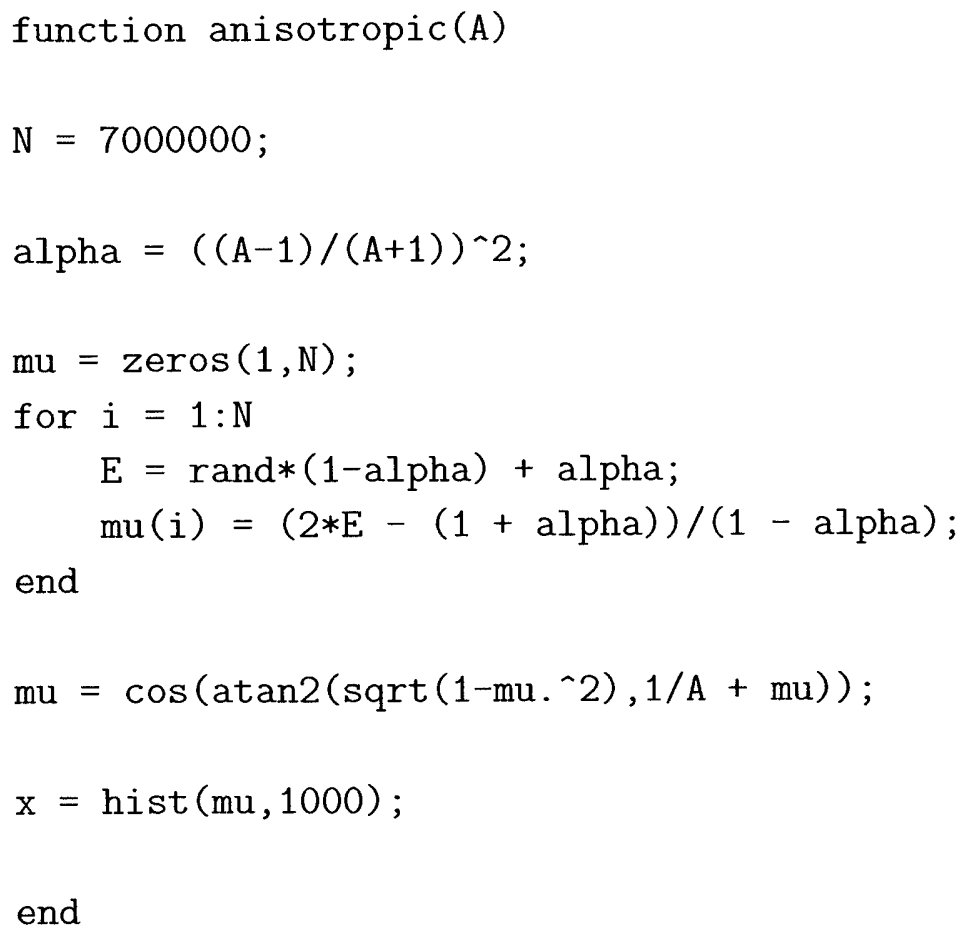




\section{G Thermal Hydraulics Model}

This is a gas-cooled pebble bed thermal hydraulics model in toroidal geometry.

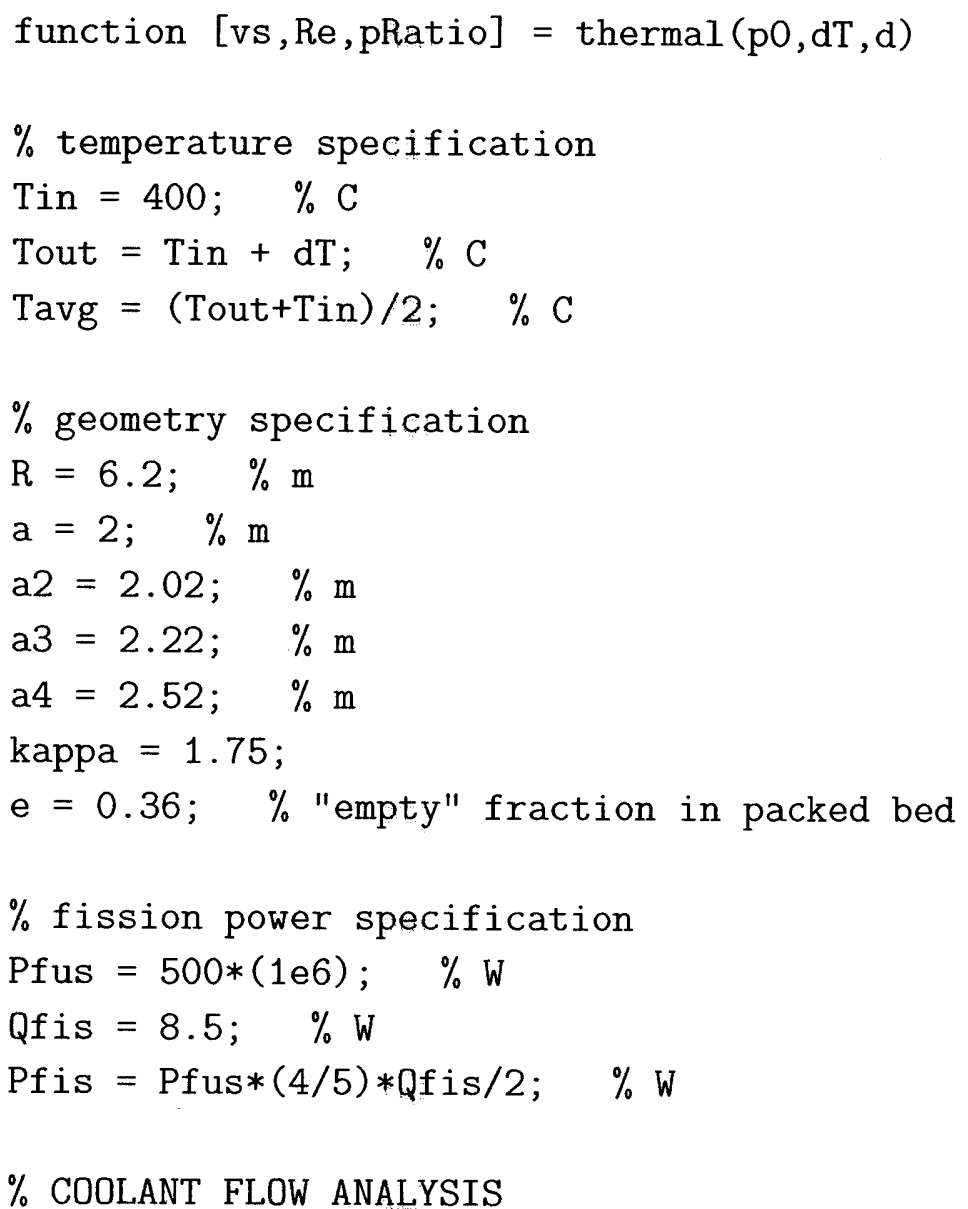

$\%$ mass/power balance

$\mathrm{cp}=5195 ; \quad \% \mathrm{~J} / \mathrm{kg} / \mathrm{K}$

$\mathrm{m}=\operatorname{Pfis} /(\mathrm{cp} * \mathrm{dT}) ; \quad \% \mathrm{~kg} / \mathrm{s}$

$\%$ He properties

rho $=0.1786 *(\mathrm{p} 0 / 101000) *(273 / \mathrm{Tavg}) ; \quad \% \mathrm{~kg} / \mathrm{m}^{\wedge} 3$

$\mathrm{mu}=(3.674 \mathrm{e}-7) *(\operatorname{Tavg}+273) \wedge 0.7 ; \quad \% \mathrm{~Pa} * \mathrm{~s}$

$\mathrm{k}_{-} \mathrm{He}=(2.682 \mathrm{e}-3) *(1+(1.123 \mathrm{e}-3) *(\mathrm{p} 0 / 100000))$

$*(\operatorname{Tavg}+273)^{\wedge}(0.71 *(1-(2 \mathrm{e}-4) *(\mathrm{p} 0 / 100000))) ; \quad \% \mathrm{~W} / \mathrm{m} / \mathrm{K}$

$\%$ flow rate 


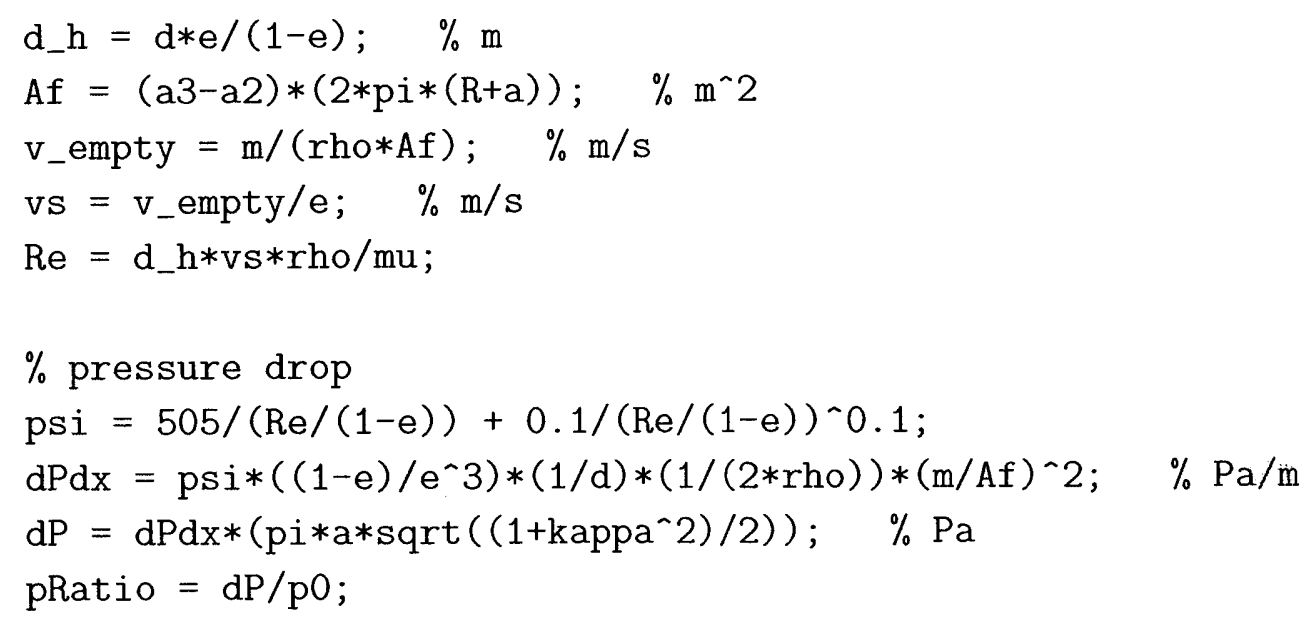

$\%$ FUEL PEBBLE ANALYSIS

$\%$ U02 properties

$\mathrm{k}_{-} \mathrm{U}=5.0 ; \quad \% \mathrm{~W} / \mathrm{m} / \mathrm{K}$

Tmelt_U $=2865 ; \quad \% \mathrm{C}$

$\%$ U02 power density

$\mathrm{V}_{-} \mathrm{U}=\mathrm{pi} *\left(\mathrm{a} 3^{\wedge} 2-\mathrm{a} 2 \_2\right) *(2 * \mathrm{pi} * \mathrm{R}) *(1-\mathrm{e}) ; \quad \% \mathrm{~m}^{\wedge} 3$

q3_U $=$ Pfis $/$ V_U; $\% \mathrm{~W} / \mathrm{m} \wedge 3$

$\%$ heat transfer coefficient

$\operatorname{Pr}=\mathrm{cp} * \mathrm{mu} / \mathrm{k}_{-} \mathrm{He}$;

$\mathrm{Nul}=0.664 *\left(\operatorname{Pr}^{\wedge}(1 / 3)\right) *\left((\operatorname{Re} / \mathrm{e})^{\wedge}(1 / 2)\right)$;

Nut $=\left(0.037 * \operatorname{Pr} *(\operatorname{Re} / e)^{\wedge} 0.8\right) /\left(1+2.443 *\left((\operatorname{Re} /)^{\wedge}(-0.1)\right) *\left(\operatorname{Pr}^{\wedge}(2 / 3)-1\right)\right)$;

Nusp $=2+\left(\mathrm{Nul}^{\wedge} 2+\mathrm{Nut}^{\wedge} 2\right)^{\wedge}(1 / 2)$

$\mathrm{Nu}=(1+1.5 *(1-\mathrm{e})) * \mathrm{Nusp}$

$\mathrm{h}=\mathrm{Nu} * \mathrm{k} \_\mathrm{He} / \mathrm{d} \_\mathrm{h} ; \quad \% \mathrm{~W} / \mathrm{m}^{\wedge} 2 / \mathrm{K}$

$\% \mathrm{SiC}$ cladding temperature drop

$\mathrm{k} \_\mathrm{SiC}=4.0 ; \quad \% \mathrm{~W} / \mathrm{m} / \mathrm{K}$

q2_U $=$ q3_U*d/6; $\% \mathrm{~W} / \mathrm{m}^{\wedge} 2$

$\mathrm{dTclad}=\mathrm{q} 2 \_\mathrm{U} *(\mathrm{~d} / 10) / \mathrm{k}_{-} \mathrm{SiC} ; \quad \% \mathrm{C}$

$\% \max$ U02 temperature

$\mathrm{T}_{-} \mathrm{Umax}=\mathrm{q} 3 \_\mathrm{U} *(\mathrm{~d} / 2) /(3 * \mathrm{~h})+\mathrm{Tout}+\mathrm{dTclad}+\mathrm{q} 3 \_\mathrm{U} *\left((\mathrm{~d} / 2)^{\wedge} 2\right) /\left(6 * \mathrm{k}_{-} \mathrm{U}\right) ; \quad \% \mathrm{C}$ Tmelt_U - T_Umax 


\section{$\%$ LITHIUM ANALYSIS}

$\% \mathrm{Li}-\mathrm{Pb}$ properties (approximate)

$\mathrm{k}_{\text {_Li }}=50.0 ; \quad \% \mathrm{~W} / \mathrm{m} / \mathrm{K}$

Tmelt_Li $=500 ; \% \mathrm{C}$

Tboil_Li $=1342 ; \quad \% \mathrm{C}$

$\% \mathrm{Li}-\mathrm{Pb}$ power density

V_Li $=p i *\left(a 4^{\wedge} 2-a 3^{\wedge} 2\right) *(2 * p i * R) ; \quad \% m^{\wedge} 3$

q3_Li $=$ Pfus $* 0.41 /$ V_Li $; \quad \% \mathrm{~W} / \mathrm{m}^{\wedge} 3$

$\%$ Zr separation wall temperature drop

$\mathrm{k} \_\mathrm{Zr}=23.0 ; \quad \% \mathrm{~W} / \mathrm{m} / \mathrm{K}$

q2_Li $=$ q3_Li*V_Li $/(2 * p i * a 3 * 2 * p i * R) / 2 ; \% \mathrm{~W} / \mathrm{m}^{\wedge} 2$

$\mathrm{dTwall}=\mathrm{q} 2 \_\mathrm{Li} * .02 / \mathrm{k} \_\mathrm{Zr}+\mathrm{q} 2 \_\mathrm{Li} / \mathrm{h} ; \% \mathrm{C}$

$\% \max \mathrm{Li}-\mathrm{Pb}$ temperature

$\mathrm{T}_{-} \mathrm{Limax}=\left(\left(\mathrm{q} 3 \_\mathrm{Li} * \mathrm{a} 3\right) /\left(2 * \mathrm{k}_{-} \mathrm{Li}\right)\right) *((\mathrm{a} 3-\mathrm{a} 4) / 2+\mathrm{a} 4 * \log (\operatorname{sqrt}(\mathrm{a} 4 / \mathrm{a} 3)))$

+ Tout + dTwall; $\% \mathrm{C}$

T_Limin = Tin + dTwall; $\% \mathrm{C}$

$\%$ print temperature results

Tboil_Li - T_Limax

T_Limin - Tmelt_Li

Tmelt_U - T_Umax

end 


\section{H Conversion Ratio Model}

This is a MATLAB code that numerically integrates the uranium transmutation-decay chain to compute conversion ratio as a function of time.

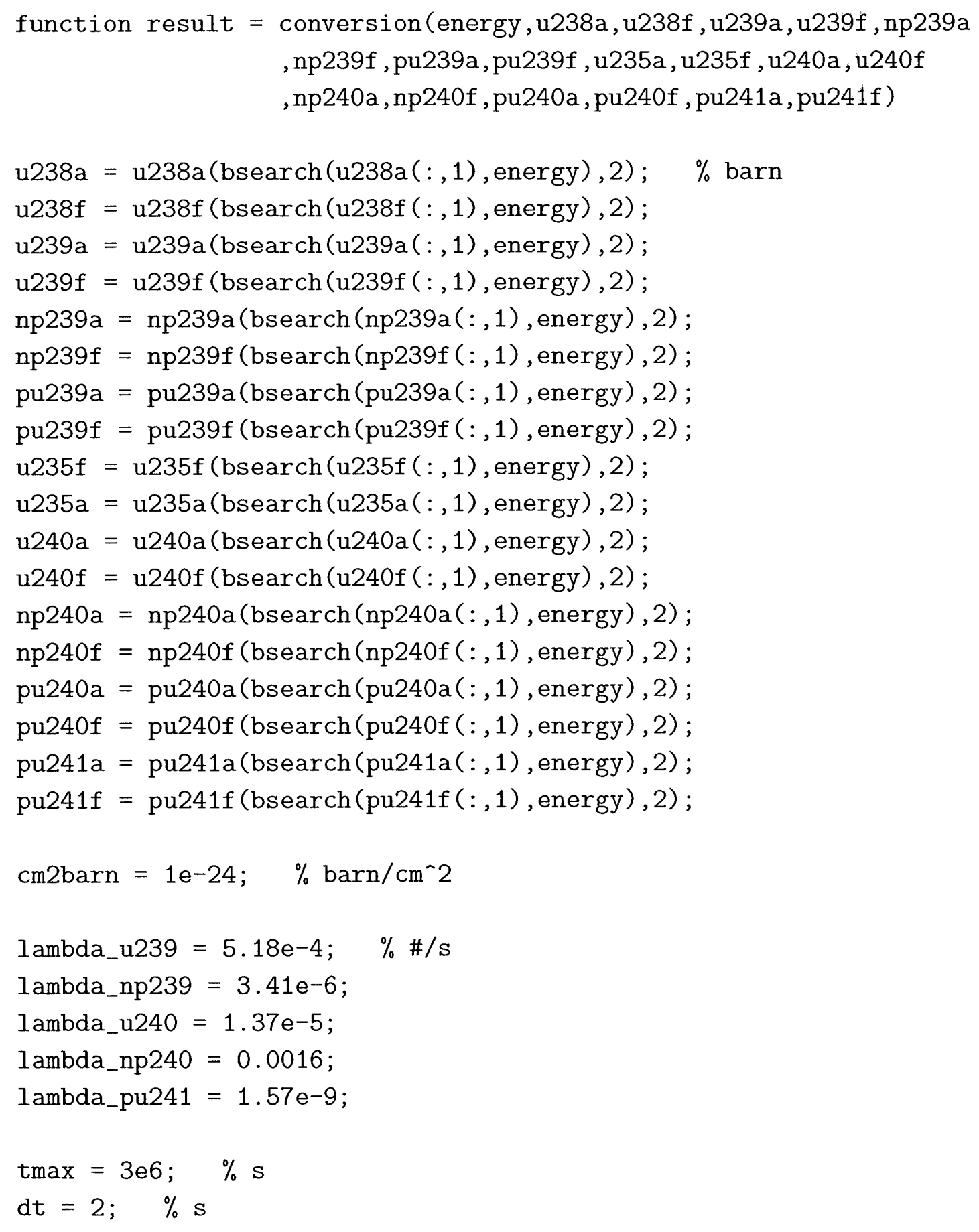


$\%$ number of time steps

num $=\mathrm{tmax} / \mathrm{dt}$;

$\%$ preallocate number densities

Nu238 $=\operatorname{zeros}(1$, num $)$;

Nu239 = $\operatorname{zeros}(1$, num $)$;

Nnp239 = zeros $(1$, num $)$;

Npu239 = zeros $(1$, num $)$;

Nu235 = $\operatorname{zeros}(1$, num $)$;

$\mathrm{Nu} 240=\operatorname{zeros}(1$, num $)$;

Nnp240 = zeros $(1$, num $)$;

$\operatorname{Npu240}=\operatorname{zeros}(1$, num $)$;

Npu241 = zeros $(1$, num);

$\%$ preallocate gains/losses

Nu235loss $=\operatorname{zeros}(1$, num $)$;

Npu239gain = zeros (1, num);

Npu241gain $=\operatorname{zeros}(1$, num $)$;

$\%$ chose arbitrary flux magnitude

flux $=1 \mathrm{e} 12 ; \quad \% \# / \mathrm{s} / \mathrm{cm}^{\wedge} 2$

$\%$ initialize number densities

$\mathrm{Nu} 238(1)=.95 ; \quad \% \# / \mathrm{cm}^{\wedge} 3$

$\mathrm{Nu} 239$ (1) = 0;

$\operatorname{Nnp} 239(1)=0$;

$\operatorname{Npu239(1)=0;~}$

$\operatorname{Nu} 235(1)=.05$;

$\mathrm{Nu} 240(1)=0$;

$\operatorname{Nnp} 240(1)=0$;

$\mathrm{Npu240}(1)=0$;

$\operatorname{Npu241(1)~=~} 0$;

$\operatorname{Nu} 235 \operatorname{loss}(1)=0$;

Npu239gain(1) $=0$;

$i=2$;

for $t=d t: d t: t \max$ 
$\%$ numerically integrate

$$
\begin{aligned}
& \operatorname{Nu} 238(i)=\operatorname{Nu} 238(i-1)-u 238 a * c m 2 \operatorname{barn} * N u 238(i-1) * f l u x * d t \\
& \text { - u238f*cm2barn } * N u 238(i-1) * f l u x * d t ; \\
& \mathrm{Nu} 239(\mathrm{i})=\mathrm{Nu} 239(\mathrm{i}-1)-\mathrm{u} 239 \mathrm{a} * \mathrm{~cm} 2 \operatorname{barn} * \mathrm{Nu} 239(\mathrm{i}-1) * \mathrm{flux} * \mathrm{dt} \\
& \text { - u239f*cm2barn*Nu239(i-1)*flux*dt } \\
& \text { - lambda_u239*Nu239(i-1)*dt } \\
& +\mathrm{u} 238 \mathrm{a} * \mathrm{~cm} 2 \mathrm{barn} * \mathrm{Nu} 238(\mathrm{i}-1) * \mathrm{flux} * \mathrm{dt} \text {; } \\
& \operatorname{Nnp} 239(i)=\operatorname{Nnp} 239(i-1)-\operatorname{np} 239 a * c m 2 b a r n * N n p 239(i-1) * f l u x * d t \\
& \text { - np239f*cm2barn*Nnp239 (i-1)*flux*dt } \\
& \text { - lambda_np239*Nnp239(i-1)*dt } \\
& + \text { lambda_u239*Nu239(i-1)*dt; } \\
& \operatorname{Npu239}(i)=\operatorname{Npu239}(i-1)-\operatorname{pu} 239 a * c m 2 b a r n * N p u 239(i-1) * f l u x * d t \\
& \text { - pu239f } * \text { cm2barn*Npu239 (i-1)*flux*dt } \\
& \text { + lambda_np239*Nnp239(i-1)*dt; } \\
& \operatorname{Nu} 240(i)=\operatorname{Nu} 240(i-1)+u 239 a * c m 2 b a r n * N u 239(i-1) * f l u x * d t \\
& \text { - u240f*cm2barn*Nu240(i-1)*flux*dt } \\
& \text { - lambda_u240*Nu240(i-1)*dt } \\
& \text { - u240a*cm2barn*Nu240(i-1)*flux*dt; } \\
& \operatorname{Nnp} 240(i)=\operatorname{Nnp} 240(i-1)+\text { np239a } * \text { cm2barn } * \operatorname{Nnp} 239(i-1) * f 1 u x * d t \\
& \text { - np240f*cm2barn*Nnp240 (i-1)*flux*dt } \\
& \text { - lambda_np240*Nnp240(i-1)*dt } \\
& \text { - np240a*cm2barn*Nnp240 (i-1)*flux*dt } \\
& \text { + lambda_u240*Nu240(i-1)*dt; } \\
& \operatorname{Npu} 240(i)=\operatorname{Npu} 240(i-1)+\operatorname{pu} 239 a * c m 2 b a r n * N p u 239(i-1) * f 1 u x * d t \\
& \text { - pu240f*cm2barn*Npu240 (i-1)*flux*dt } \\
& \text { - pu240a*cm2barn*Npu240(i-1)*flux*dt } \\
& \text { + lambda_np240*Nnp240(i-1)*dt; } \\
& \operatorname{Npu241}(i)=\operatorname{Npu241}(i-1)+\operatorname{pu} 240 a * c m 2 b a r n * N p u 240(i-1) * f 1 u x * d t \\
& \text { - pu241f*cm2barn*Npu241 (i-1)*flux*dt } \\
& \text { - pu241a*cm2barn*Npu241(i-1)*flux*dt } \\
& \text { - lambda_pu241*Npu241(i-1)*dt; } \\
& \text { Npu239gain }(i)=- \text { pu239a } * \operatorname{cm} 2 b a r n * N p u 239(i-1) * f l u x \\
& \text { - pu239f*cm2barn*Npu239(i-1)*flux } \\
& \text { + lambda_np239*Nnp239(i-1); } \\
& \text { Npu241gain }(i)=\operatorname{pu240a} * \text { cm2barn } * \text { Npu240 }(i-1) * f 1 u x
\end{aligned}
$$


- pu241f*cm2barn*Npu241(i-1)*flux

- pu241a*cm2barn*Npu241 (i-1)*flux

- lambda_pu241*Npu241(i-1);

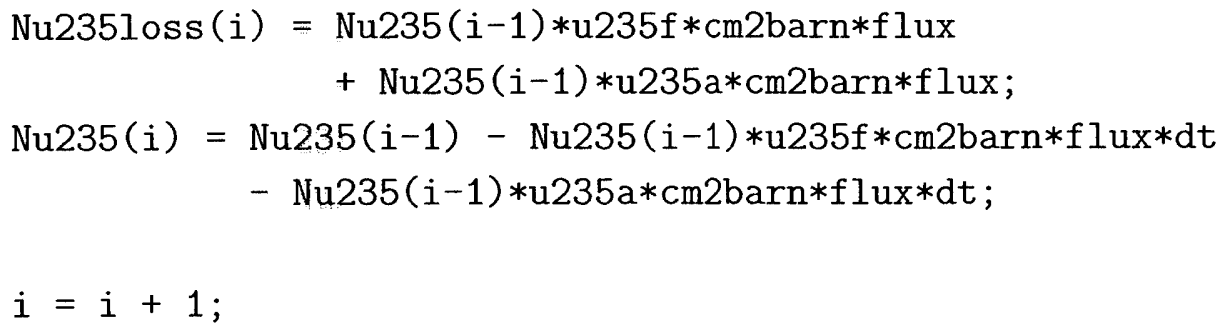

end 


\section{MCNP Input Files}

\section{I.1 Full Toroidal Hybrid Blanket}

This is an MCNP input file for a fission-fusion hybrid in toroidal geometry. This produces results consistent with the MATLAB Monte Carlo code in Appendix B.
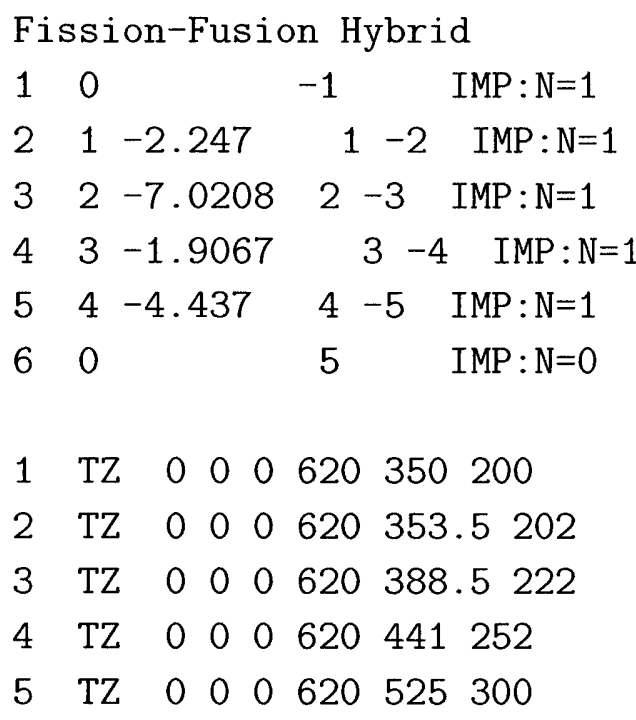

$\begin{array}{lllllll}M 1 & 6000.73 c & -0.29905 & 14028.73 c & -0.70028 & 2004.73 c & -0.00067\end{array}$

$\begin{array}{lllllll}\text { M2 } & 92235.73 c & -0.00619 & 92238.73 c & -0.87508 & 8016.73 c & -0.11847\end{array}$ $2004.73 c-0.00026$

M3 $3006.73 c-0.18542 \quad 3007.73 c-0.02404 \quad 82000 \quad-0.79054$

M4 $26056.73 c-0.8873 \quad 1001.73 c-0.0125 \quad 8016.73 c-0.1002$

SDEF $\mathrm{X}=\mathrm{d} 1 \quad \mathrm{Y}=\mathrm{d} 2 \quad \mathrm{Z}=\mathrm{d} 3 \quad \mathrm{ERG}=14.1 \quad \mathrm{PAR}=1 \quad \mathrm{CEL}=1$

SI1 -820. 820

SP1 $0 \quad 1$

SI2 -820. 820

SP2 $0 \quad 1$

SI3 -350. 350

SP3 $0 \quad 1$

MODE N

NPS 100000

$\mathrm{F} 1: \mathrm{N} 15$

F2:N 15

F6:N 3 
F7:N 3

$\mathrm{F} 4: \mathrm{N} 3$

KCODE 200001.0010 $\begin{array}{llll}\text { prdmp } & 0 & -0 & 1\end{array}$ 


\section{I.2 $\mathbf{k}_{\infty}$ for $\mathrm{U}$ and $\mathrm{UO}_{2}$}

This is an MCNP input file for computing $k_{\infty}$ for $\mathrm{UO}_{2}$. This produces results consistent with the MATLAB Monte Carlo code in Appendix E.

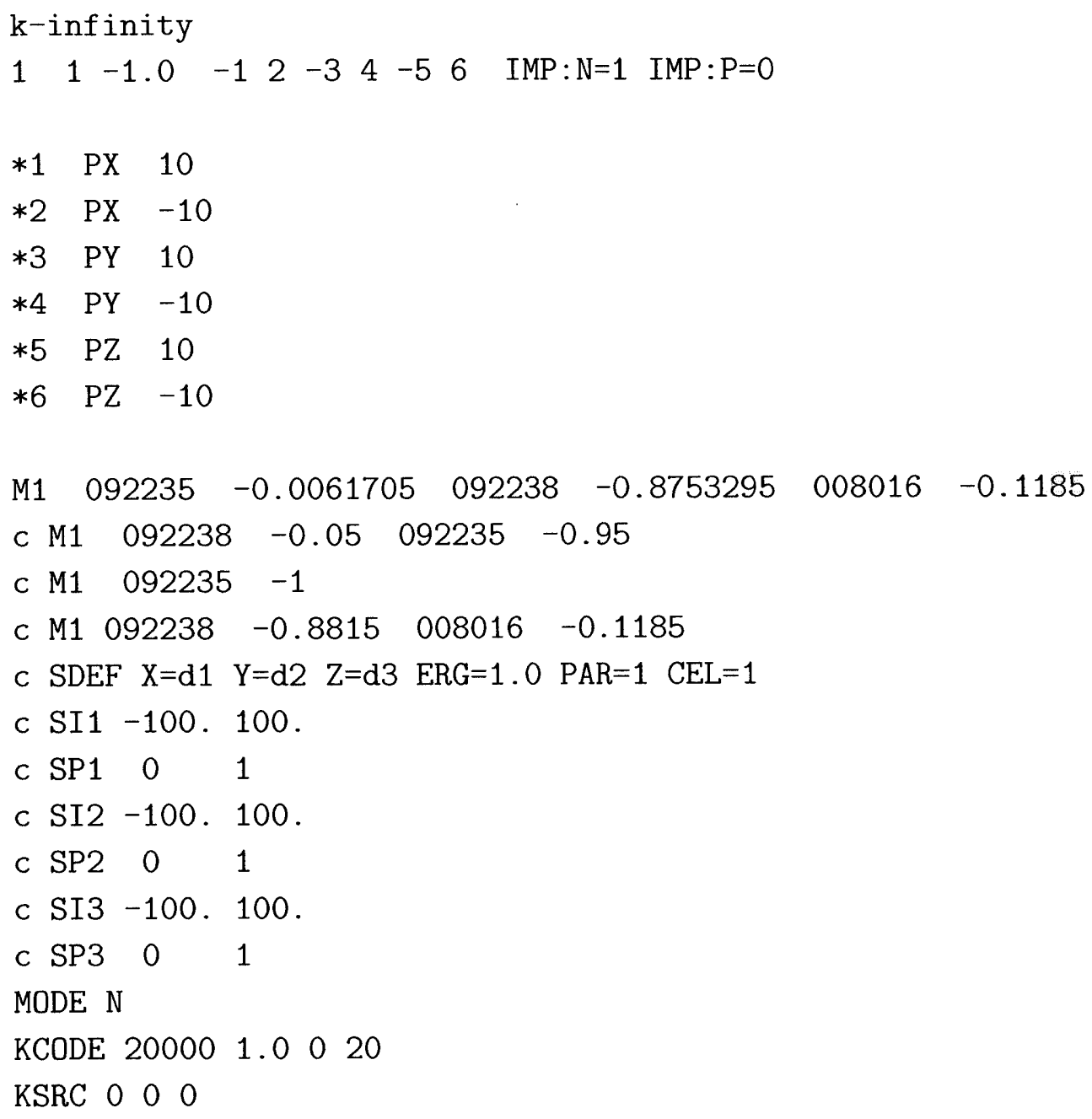




\section{I.3 Infinite Array of $\mathrm{UO}_{2}$ Pebbles in $\mathrm{H}_{2} \mathrm{O}$ Pool (Simple Cubic)}

This is a MCNP input file for computing $k_{\infty}$ for an infinite array of $\mathrm{UO}_{2}$ pebbles in a pool of $\mathrm{H}_{2} \mathrm{O}$. We can vary the pebble radius to determine $k_{\infty}$ as a function of it.

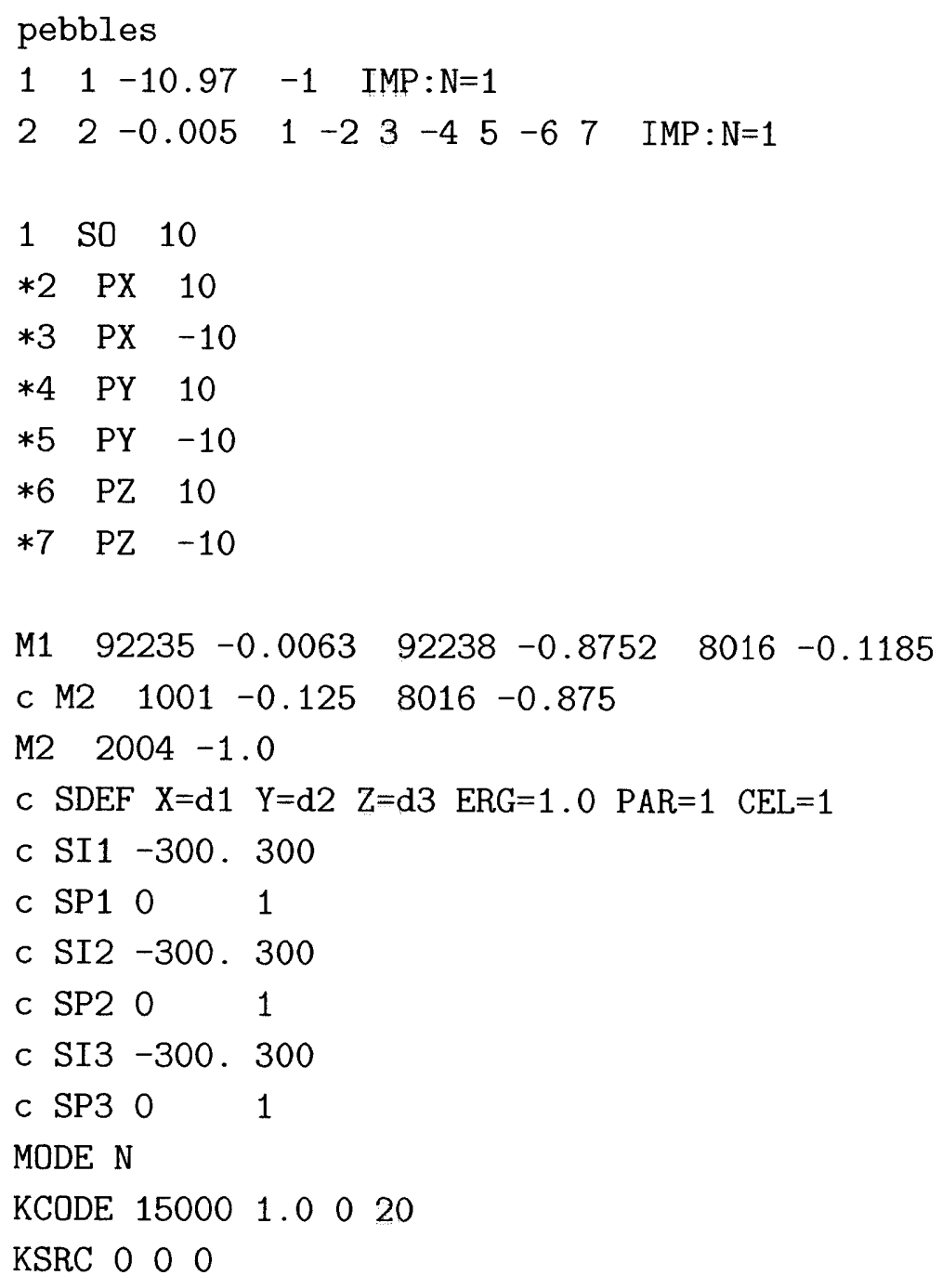




\section{I.4 Typical Fast Reactor Spectrum}

This is a MCNP input file for obtaining an approximate 248-group flux shape for a typical fast reactor. We construct a $3 \times 3$ array of $\mathrm{UO}_{2}$ pin cells immersed in $\mathrm{Na}$ coolant and adjust the physical dimensions such that $k=1.0$. We borrow 248 energy bins of equal lethargy width from the MIT Reactor research group.

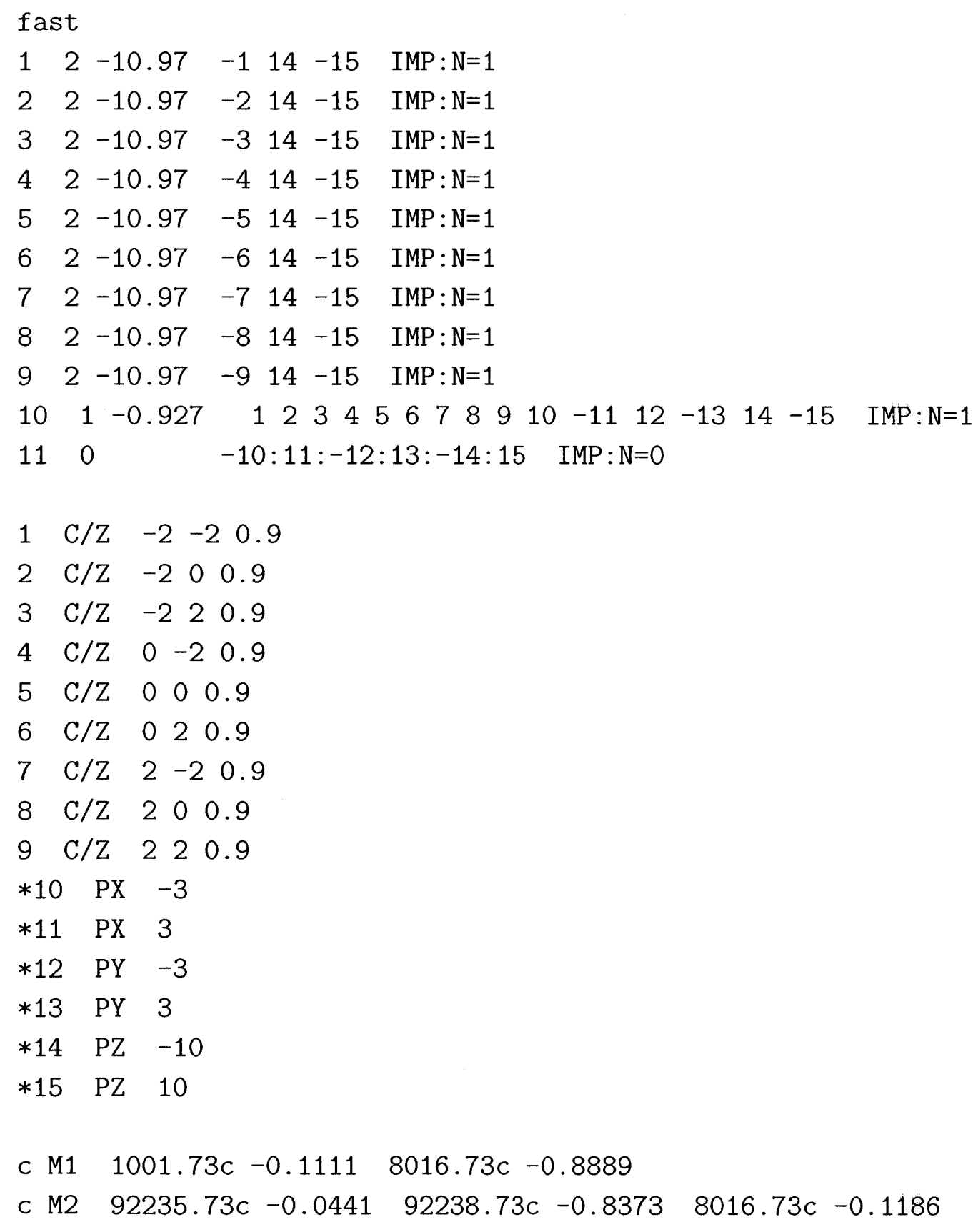




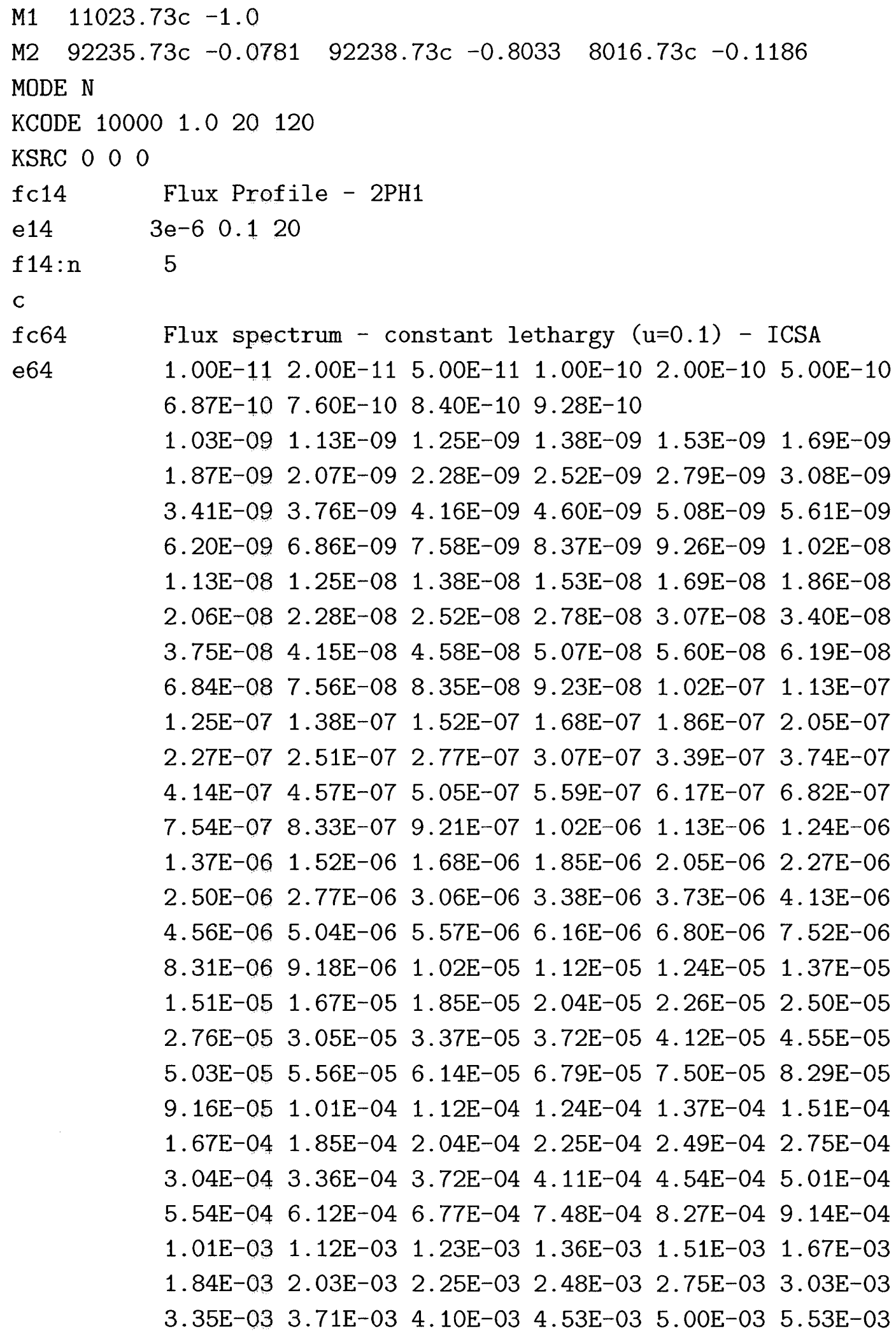


6.11E-03 6.75E-03 $7.46 \mathrm{E}-03 \quad 8.25 \mathrm{E}-03 \quad 9.11 \mathrm{E}-03 \quad 1.01 \mathrm{E}-02$

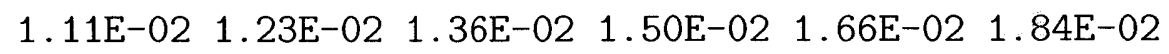

$\begin{array}{llllll}2.03 \mathrm{E}-02 & 2.24 \mathrm{E}-02 & 2.48 \mathrm{E}-02 & 2.74 \mathrm{E}-02 & 3.03 \mathrm{E}-02 & 3.34 \mathrm{E}-02\end{array}$

3.70E-02 4.08E-02 4.51E-02 4.99E-02 5.51E-02 6.09E-02

6.73E-02 7.44E-02 8.22E-02 9.09E-02 1.01E-01 $1.11 \mathrm{E}-01$

$\begin{array}{llllll}1.23 \mathrm{E}-01 & 1.36 \mathrm{E}-01 & 1.50 \mathrm{E}-01 & 1.66 \mathrm{E}-01 & 1.83 \mathrm{E}-01 & 2.02 \mathrm{E}-01\end{array}$

$2.24 \mathrm{E}-01 \quad 2.47 \mathrm{E}-01 \quad 2.73 \mathrm{E}-01 \quad 3.02 \mathrm{E}-01 \quad 3.34 \mathrm{E}-01 \quad 3.69 \mathrm{E}-01$

$\begin{array}{llllll}4.07 \mathrm{E}-01 & 4.50 \mathrm{E}-01 & 4.98 \mathrm{E}-01 & 5.50 \mathrm{E}-01 & 6.08 \mathrm{E}-01 & 6.72 \mathrm{E}-01\end{array}$

$\begin{array}{llllll}7.42 \mathrm{E}-01 & 8.20 \mathrm{E}-01 & 9.07 \mathrm{E}-01 & 1.00 \mathrm{E}+00 & 1.11 \mathrm{E}+00 & 1.22 \mathrm{E}+00\end{array}$

$\begin{array}{lllllll}1.35 \mathrm{E}+00 & 1.50 \mathrm{E}+00 & 1.65 \mathrm{E}+00 & 1.83 \mathrm{E}+00 & 2.02 \mathrm{E}+00 & 2.23 \mathrm{E}+00\end{array}$

$\begin{array}{lllllll}2.46 \mathrm{E}+00 & 2.72 \mathrm{E}+00 & 3.01 \mathrm{E}+00 & 3.33 \mathrm{E}+00 & 3.68 \mathrm{E}+00 & 4.06 \mathrm{E}+00\end{array}$

$\begin{array}{llllll}4.49 \mathrm{E}+00 & 4.96 \mathrm{E}+00 & 5.48 \mathrm{E}+00 & 6.06 \mathrm{E}+00 & 6.70 \mathrm{E}+00 & 7.40 \mathrm{E}+00\end{array}$

$\begin{array}{llllll}8.18 \mathrm{E}+00 & 9.04 \mathrm{E}+00 & 1.00 \mathrm{E}+01 & 1.11 \mathrm{E}+01 & 1.22 \mathrm{E}+01 & 1.35 \mathrm{E}+01\end{array}$

$\begin{array}{lll}1.49 \mathrm{E}+01 & 1.65 \mathrm{E}+01 \quad 1.82 \mathrm{E}+012.00 \mathrm{E}+01 \mathrm{~T}\end{array}$

$\mathrm{f} 64: \mathrm{n} \quad 5$

c THE END 